\title{
ANÁLISIS DE PROBLEMAS ELECTROMAGNÉTICOS MEDIANTE MÉTODOS MODALES Y MATRICES GENERALIZADAS, Y APLICACIONES
}

\author{
Vicente Enrique Boria Esbert \\ Director: Dr. Mariano Baquero Escudero
}

Tesis Doctoral presentada en la Universidad Politécnica de Valencia para la obtención del título de Doctor Ingeniero de Telecomunicación Valencia, Mayo 1997 

A mis padres, en agradecimiento a sus constantes sacrificios. 



\section{AGRADECIMIENTOS}

Recordar en unas pocas líneas a todas aquellas personas, o instituciones, que merecen mi agradecimiento resulta una tarea compleja; por lo que, caso de olvidar involuntariamente a alguna de ellas, pido disculpas de antemano.

En primer lugar, quiero agradecer a mis padres todo su apoyo y comprensión; especialmente durante la redacción de la tesis, pues sus palabras de ánimo me han ayudado a superar los momentos más difíciles. A ellos deseo agradecer además, muy especialmente, su gran preocupación por proporcionarme una buena educación (personal y académica); gran responsable, sin duda, de que por ejemplo esta tesis haya sido posible. Quisiera recordar, asimismo, la inestimable ayuda de mi hermana a lo largo de todos estos años; a quien siempre he tenido cerca de mí, y cuya determinación constituye constantemente un verdadero ejemplo a seguir. Dentro del ámbito personal, deseo agradecer sinceramente a una gran persona, Ana Vidal, su inestimable apoyo y aprecio personal; así como su continua disposición, siempre jovial y desinteresada, a ayudarme en todo aquello que de ella he necesitado para escribir esta tesis.

En el mundo universitario, es justo agradecer en primer lugar toda la ayuda y el apoyo (personal y profesional) que siempre he recibido de Miguel Ferrando; principal artífice, con sus ideas y su gran confianza en mí, del trabajo aquí presentado. Debo reconocer y agradecer también la ayuda prestada por Mariano Baquero; quien, con sus acertados comentarios, me ayudó a encauzar el trabajo sobre problemas cerrados realizado en Holanda, y cuya tesis constituye por otra parte el punto de partida de la actividad que he desarrollado en Valencia relacionada con problemas abiertos. Agradecer además muy especialmente, tanto a Miguel como a Mariano, su constante preocupación e interés, durante la realización de la tesis, no sólo por el trabajo desarrollado sino también por mi persona. Asimismo, debo recordar al resto de personas con las que he estado trabajando durante estos años en temas relacionados con la tesis, especialmente en la parte de problemas abiertos. Por otra parte, quisiera agradecer a Elías de los Reyes que haya pensado en mí para trabajar en el Grupo de Calentamiento por Microondas que dirige personalmente, y al que espero le sea de especial utilidad parte del trabajo contenido en esta tesis.

Finalmente, mostrar mi más sincero agradecimiento a Marco Guglielmi, responsable del Grupo de Componentes Pasivos de ESTEC (ESA), con quien he estado trabajando en problemas cerrados durante año y medio; cuyas constantes ideas nutren una buena parte de esta tesis, y cuyo afán investigador es digno de elogio. A su vez, quiero agradecer al Ministerio de Educación y Ciencia la concesión de una Beca de Formación de Personal Universitario e Investigador, dentro del Subprograma de Especialización en la Agencia Espacial Europea, que sufragó mi estancia en ESTEC (Holanda); fruto de la cual surgió parte de la presente tesis. Dar las gracias también al Departamento de Comunicaciones, al cual pertenezco, por haberme permitido simultanear la citada estancia investigadora en el extranjero con la actividad docente. 



\section{Índice General}

1 Introducción 1

2 Análisis Eficiente de Estructuras Pasivas de Microondas mediante la Matriz de Admitancias Generalizada

2.1 La Matriz de Admitancias Generalizada de Uniones Planares entre Guías Arbitrarias ................ 10

2.2 Acelaración del Cálculo de la Matriz de Admitancias Generalizada de Uniones Planares entre Guías Arbitrarias . . . . . . . . . . 16

2.2.1 Series Estática y Dinámica . . . . . . . . . . . 17

2.2.2 Tratamiento Refinado de la Serie Dinámica . . . . . . . . . 22

2.2.3 Eficiencia Computacional de la Técnica de Aceleración . . 28

2.2.4 Análisis de Septums Cortocircuitados y Cavidades de Reacción mediante la Técnica de Aceleración . . . . . . . . 31

2.3 Técnica Recursiva para la Resolución de Sistemas de Ecuaciones Lineales en Banda. . . . . . . . . . . . . . . 48

2.3.1 Formulación Básica del Problema . . . . . . . . . . . 49

2.3.2 Reducción a Una Matriz $Y$ Equivalente . . . . . . . . . 54

2.3.3 Descripción de la Técnica Recursiva . . . . . . . . . . 59

2.3.4 Eficiencia Computacional de la Técnica Recursiva . . . . . 64

3 Ejemplos de Análisis y Diseño Eficiente de Dispositivos Pasivos de Microondas Complejos

3.1 Análisis y Diseño de Filtros Paso Banda en Guía Rectangular con Ventanas de Acoplo Inductivas y Tornillos de Sintonía . . . . . . . 72

3.1.1 Representación de un Tornillo de Sintonía mediante un Tramo Uniforme de Guía Reentrante . . . . . . . . . . 75 Evaluación de las Integrales de Acoplamiento . . . . . . 77 Equivalencia entre un Tornillo de Sintonía y un Tramo Uniforme de Guía Reentrante . . . . . . . . 87

3.1.2 Investigación de Zonas de Sintonía . . . . . . . . . . 91 
3.1.3 Procedimiento de Diseño de Filtros Paso Banda en Guía Rectangular con Ventanas de Acoplo Inductivas y Tornillos de Sintonía . . . . . . . . . . . . . . 94

3.2 Análisis y Diseño de Filtros Paso Banda de Modo Dual en Guía Circular ..................... 112

3.2.1 Descripción de Soluciones para Implementar Filtros Paso Banda de Modo Dual . . . . . . . . . . . . . . . 114

3.2.2 Filtros Paso Banda de Modo dual en Guía Circular con Iris Elípticos . . . . . . . . . . . 120

3.2.3 Filtros Paso Banda de Modo dual en Guía Circular con Iris Elípticos y Guías Circulares Reentrantes . . . . . . . 129

4 Caracterización de Objetos Dispersores en Espacio Libre mediante Técnicas Espectrales

4.1 Matriz de Caracterización . . . . . . . . . . . . . 146

4.2 Matriz de Corrientes . . . . . . . . . . . . . . 154

4.2 .1 Óptica Física . . . . . . . . . . . . . . . . . . . . 159

Polarización $\mathrm{TM}^{\mathrm{z}} \ldots \ldots \ldots \ldots$. . . . . . . . 161

Polarización $\mathrm{TE}^{\mathrm{z}} \ldots \ldots \ldots \ldots \ldots . \ldots \ldots$

4.2 .2 Ecuación Integral . . . . . . . . . . . . . . . 164

Polarización TM $\mathrm{TM}^{\mathrm{z}} \ldots \ldots \ldots \ldots \ldots 6$

Polarización TE $\mathrm{TE}^{\mathrm{z}} \ldots \ldots \ldots \ldots$. . . . . . . 169

4.2.3 Estudio Comparativo entre Óptica Física y Método de los

Momentos . . . . . . . . . . . . 173

4.3 Matriz de Espectro . . . . . . . . . . . . . 176

4.3.1 Técnica basada en la Transformada Discreta de Fourier . . 177

Polarización $\mathrm{TM}^{\mathrm{z}} \ldots \ldots \ldots \ldots \ldots \ldots$

Polarización TEz . . . . . . . . . . . . 180

4.3.2 Técnica basada en el Teorema de Adición para las Funciones de Hankel . . . . . . . . . . . . . . . 180

Polarización $\mathrm{TM}^{\mathrm{z}} \ldots \ldots \ldots \ldots \ldots 18 \ldots \ldots$

Polarización TEz . . . . . . . . . . . . . 182

4.3.3 Estudio Comparativo entre Transformada Discreta de Fourier y Teorema de Adición para las Funciones de Hankel . 182

5 Caracterización de Objetos Dispersores Canónicos en Espacio Libre

5.1 Cilindro Metálico Infinito ante Incidencia TM². Solución Analítica 186

5.1.1 Cálculo Analítico de la Matriz de Caracterización . . . . . 186

5.1 .2 Resultados. . . . . . . . . . . . . . . 188

5.2 Cilindro Metálico Infinito ante Incidencia $\mathrm{TM}^{\mathrm{z}}$. Solución No Ana-

lítica . . . . . . . . . . . . . . . 190 
5.2.1 Cálculo de la Matriz de Corrientes mediante Óptica Física

5.2.2 Cálculo de la Matriz de Corrientes mediante el Método de los Momentos . . . . . . . . . . . . . 194

5.2 .3 Resultados . . . . . . . . . . . . . 196

5.3 Tira Metálica Infinita ante Incidencia $\mathrm{TM}^{\mathrm{z}} \ldots \ldots$. . . . . . . . 201

5.3.1 Cálculo de la Matriz de Corrientes mediante el Método de los Momentos ................ . 201

5.3 .2 Resultados....................... 204

5.4 Tira Metálica Infinita ante Incidencia TE $\ldots . . . . . . .207$

5.4.1 Cálculo de la Matriz de Corrientes mediante el Método de los Momentos . . . . . . . . . . . . 208

5.4 .2 Resultados . . . . . . . . . . . . 210

6 Caracterización Conjunta de Múltiples Objetos Dispersores en Espacio Libre mediante un Algoritmo Recursivo

6.1 Descripción de un Algoritmo Recursivo para la Caracterización Conjunta de Múltiples Objetos Dispersores . . . . . . . . . . . 214

6.1.1 Matrices de Caracterización Conjunta de Dos Objetos Dispersores .................. 216

6.1.2 Introducción de un Tercer Objeto Dispersor . . . . . . . 229

6.1.3 Generalización del Algoritmo a $N$ Objetos Dispersores . . 235

6.2 Refinamiento del Algoritmo Recursivo para la Caracterización Conjunta de Grupos de Objetos Dispersores . . . . . . . . 238

6.3 Limitaciones en la Caracterización Conjunta de Múltiples Objetos Dispersores y Soluciones . . . . . . . . . . . . . . . 243

6.3.1 Agrupación de Objetos Dispersores . . . . . . . . . . 244

6.3.2 Segmentación del Contorno de los Objetos Dispersores . . 249

6.3.3 Reconstrucción del Campo Incidente sobre Grupos de Objetos Dispersores . . . . . . . . . . . 253

6.3.4 Conclusiones. Automatización del Algoritmo Recursivo . . 254

6.4 Iteración Especial para Fuente Interna al Problema de Múltiples Objetos Dispersores . . . . . . . . . . . . . . . 258

6.5 Reconstrucción del Campo Dispersado por Múltiples Objetos Dis-

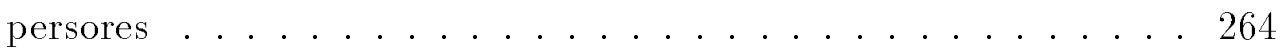

7 Ejemplos de la Dispersión Electromagnética producida por Múltiples Objetos Dispersores en Espacio Libre

7.1 Eficiencia Computacional de la Técnica de Análisis basada en la Segmentación del Contorno de un Objeto Dispersor . . . . . . . . 272

7.2 Múltiples Tiras Metálicas Infinitas ante Incidencia TM ${ }^{\mathrm{z}}$ y TE ${ }^{\mathrm{z}}$. 276 7.2.1 Una Tira Metálica Infinita Grande en Términos Eléctricos

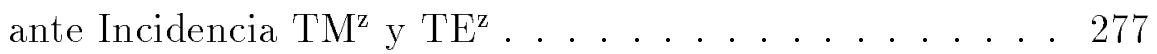


7.2.2 Dos Tiras Metálicas Infinitas Alejadas ante Incidencia TM 279

7.3 Reflectores ante Incidencia $\mathrm{TM}^{\mathrm{z}} \mathrm{y} \mathrm{TE}^{\mathrm{z}}$. . . . . . . . . . . . . . 283

7.3.1 Reflector Hiperbólico ante Incidencia TM ${ }^{\mathrm{z}}$. . . . . . . . . 284

7.3.2 Reflector Parabólico ante Incidencia $\mathrm{TM}^{\mathrm{z}}$. . . . . . . . . . 286

7.3.3 Reflector Parabólico ante Incidencia TE $\ldots$. . . . . . . . 299

7.4 Antenas Cassegrain ante Incidencia $\mathrm{TM}^{\mathrm{z}}$ y $\mathrm{TE}^{\mathrm{z}}$. . . . . . . . . . 301

7.4.1 Antena Cassegrain Simétrica ante Incidencia TM ${ }^{\mathrm{z}}$. . . . . 302

7.4.2 Antena Cassegrain Offset ante Incidencia TE $\mathrm{TE}^{\mathrm{z}}$. . . . . . . 307

7.5 Antena Bocina ante Incidencia $\mathrm{TM}^{\mathrm{z}} \ldots \ldots$. . . . . . . . . 310

8 Conclusiones

A Desarrollos en Serie de Taylor de la función $f_{r}(k) \quad 321$

A.1 Modos TE . . . . . . . . . . . . . . . . . 321

A.2 Modos TM. . . . . . . . . . . . . . . . 323

B DUMAS 3.0: Un Programa para el Análisis y el Diseño Eficiente de Estructuras Pasivas de Microondas $\quad 327$

B.1 Modos Accesibles y Localizados . . . . . . . . . . . . . . . . . . 328

B.2 Redes Ideales para el Diseño de Filtros . . . . . . . . . . . . . . 332

B.3 Análisis de Estructuras Pasivas de Microondas . . . . . . . . . . . 341

C Espectros Escalares de Ondas Planas y Cilíndricas. Transformaciones entre Espectros Plano y Cilíndrico $\quad 349$

C.1 Espectros Escalares. Concepto . . . . . . . . . . . . . 350

C.1.1 Espectro Escalar de Ondas Planas . . . . . . . . . 351

C.1.2 Espectro Escalar de Ondas Cilíndricas ......... . . 354

C.2 Transformaciones entre Espectros Plano y Cilíndrico . . . . . . 357

C.2.1 Espectro Cilíndrico de una Onda Plana Incidente en Problemas Bidimensionales. . . . . . . . . . . 358

C.2.2 Matriz de Transformación de Espectro Cilíndrico a Espectro Plano en Problemas Bidimensionales . . . . . . . 359

D Análisis del Giro de un Objeto Dispersor 363

D.1 Matrices de Giro. Concepto . . . . . . . . . . . . . . 364

D.2 Ejemplo de Aplicación de las Matrices de Giro . . . . . . . . . . 369

E Traslación de Espectros Cilíndricos $\quad 371$

E.1 Teorema de Adición para las Funciones de Bessel . . . . . . . . . 372

E.1.1 Matriz de Traslación de Espectro Incidente a Incidente . . 377

E.2 Teorema de Adición para las Funciones de Hankel . . . . . . . . 386

E.2.1 Matriz de Traslación de Espectro Emergente a Incidente . 393

E.2.2 Matriz de Traslación de Espectro Emergente a Emergente 406 
F Publicaciones en Revistas Internacionales 413

Bibliografía 


\section{Índice de Tablas}

2.1 Tiempo total de cálculo que requiere el análisis de la estructura mostrada en la figura 2.10 dividido por el número (100) de frecuencias consideradas. . . . . . . . . . . . . . .

2.2 Factor de mejora entre los diferentes métodos; definido como el cociente de los tiempos de cálculo requeridos por los diversos métodos propuestos en el análisis de la estructura mostrada en la figura 2.10 . . . . . .

2.3 Número de operaciones matriciales (multiplicaciones e inversiones de matrices con elementos complejos) requeridas para resolver el sistema lineal en banda que aparece al analizar un dispositivo de microondas constituido por $N$ guías arbitrarias. . . . . . . . . .

2.4 Dimensiones de las guías reentrantes centradas que se escogen como elementos de sintonía situados en las ventanas de acoplo del filtro de 4

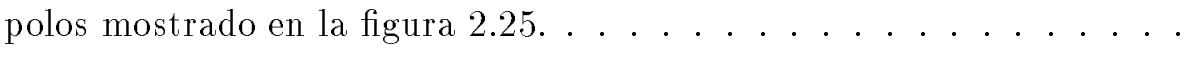

2.5 Dimensiones de las guías reentrantes centradas que se escogen como elementos de sintonía situados en las cavidades resonantes del filtro de 4 polos mostrado en la figura $2.25 \ldots$. . . . . . . . . .

2.6 Tiempo de cálculo requerido para resolver en cada frecuencia el sistema lineal en banda que aparece al analizar el filtro de 4 polos mostrado en la figura $2.25 \ldots \ldots \ldots \ldots \ldots \ldots$

3.1 Efecto de la penetración del tornillo de sintonía sobre la frecuencia central de la banda de paso del filtro mostrado en la figura 3.4 (apartado

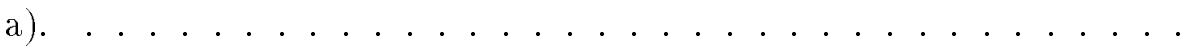

3.2 Tamaño del elemento de sintonía equivalente, con sección transversal cuadrada, en función de la penetración del tornillo de sintonía $(\phi=4 \mathrm{~mm})$ situado dentro de la cavidad de la estructura mostrada en la figura 3.4 (apartado a). . . . . . . . . . . .

3.3 Dimensiones optimizadas de las ventanas de acoplo inductivas y de las cavidades resonantes que integran los dos filtros paso banda en guía rectangular, sin elementos de sintonía, centrados respectivamente a $11 \mathrm{GHz}$ y $13 \mathrm{GHz}$ con un ancho de banda de $300 \mathrm{MHz}$ y respuesta tipo Chebyshev. . . . . . . . . . . . . . 
3.4 Penetraciones de los elementos de sintonía situados en las ventanas de acoplo de la estrucutra básica para conseguir los niveles de acoplamiento requeridos por los diseños a $11 \mathrm{GHz}$ y $13 \mathrm{GHz}$. . . . . . . .

3.5 Penetraciones de los elementos de sintonía situados en las cavidades de la estructura básica para centrar su respuesta a $11 \mathrm{GHz}$ y $13 \mathrm{GHz}$ respectivamente. . . . . . . . . . . . . . 101

3.6 Valores optimizados de las penetraciones de los elementos de sintonía situados en las ventanas de acoplo y en las cavidades de la estructura básica para centrar su respuesta a $11 \mathrm{GHz}$ y $13 \mathrm{GHz}$ respectivamente.

3.7 Valores optimizados de las dimensiones de los iris elípticos del filtro de modo dual mostrado en la figura 3.24 para centrar su respuesta con

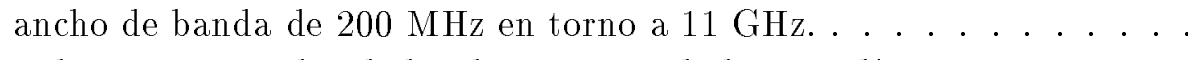

3.8 Valores optimizados de las dimensiones de los iris elípticos del filtro de modo dual mostrado en la figura 3.30 para centrar su respuesta con ancho de banda de aproximadamente $200 \mathrm{MHz}$ a $11 \mathrm{GHz}$. . . . . . . 134

3.9 Valores optimizados de las coordenadas de los puntos que definen tres inserciones metálicas, presentes en cada tramo de guía circular reentrante insertado dentro de cada cavidad resonante del filtro de modo dual mostrado en la figura 3.30, con el objeto de centrar la respuesta de dicha estructura con ancho de banda de aproximadamente $200 \mathrm{MHz}$ a $11 \mathrm{GHz} . \ldots \ldots \ldots \ldots \ldots \ldots$

E.1 Evolución de la amplitud asociada al elemento más grande de la matriz de traslación de espectro emergente a incidente en función del número de modos cilíndricos incidentes considerados. . . . . . . . . . . . 404 


\section{Índice de Figuras}

2.1 Unión planar entre dos guías arbitrarias en a); y representación de dicha unión mediante una red equivalente multimodal basada en la matriz de admitancias generalizada en b). . . . . . . . . . . 11

2.2 Estructura utilizada para evaluar los elementos $Y_{m, n}^{(1,1)}$ e $Y_{m, n}^{(2,1)}$; que se obtiene situando un cortocircuito en el puerto 2. . . . . . . . . 13

2.3 Estructura utilizada para evaluar los elementos $Y_{m, n}^{(2,2)}$; que se obtiene situando un cortocircuito en el puerto 1. . . . . . . . . . 15

2.4 Geometría de una unión planar offset entre guías rectangulares. . . . . 19

2.5 Convergencia de los elementos $Y_{m, m}^{(2,2)}(m=1,15,25,32)$ de la MAG de una unión planar entre una guía rectangular grande $(a=19.050$ $\mathrm{mm}, b=9.525 \mathrm{~mm})$ y una guía rectangular pequeña $(a=5.100 \mathrm{~mm}$, $b=7.000 \mathrm{~mm})$ con un offset $(c=4.000 \mathrm{~mm}, d=0.750 \mathrm{~mm}) . \ldots$.

2.6 Convergencia de las series estáticas $\hat{Y}_{m, m}^{(2,2)}(m=1,15,25,32)$ de la MAG de una unión planar entre una guía rectangular grande $(a=19.050$ $\mathrm{mm}, b=9.525 \mathrm{~mm})$ y una guía rectangular pequeña $(a=5.100 \mathrm{~mm}$, $b=7.000 \mathrm{~mm})$ con un offset $(c=4.000 \mathrm{~mm}, d=0.750 \mathrm{~mm}) . .$.

2.7 Convergencia de las series dinámicas $\tilde{Y}_{m, m}^{(2,2)}(m=1,15,25,32)$ de la MAG de una unión planar entre una guía rectangular grande $(a=19.050$ $\mathrm{mm}, b=9.525 \mathrm{~mm})$ y una guía rectangular pequeña $(a=5.100 \mathrm{~mm}$, $b=7.000 \mathrm{~mm})$ con un offset $(c=4.000 \mathrm{~mm}, d=0.750 \mathrm{~mm})$. En a) $m=1$, y en b) $m=15,25,32 \ldots \ldots \ldots \ldots$

2.8 Evolución de la función $f_{r}(k)$ frente a la variable normalizada $k / k_{t, r}^{(1)}$ para modos TE en a); y para modos TM en b). . . . . . . . . .

2.9 Convergencia de las partes estáticas $(p=1)$ de $\hat{\tilde{Y}}_{m, m}^{(2,2)}(m=1,15,25,32)$ de la MAG de una unión planar entre una guía rectangular grande $(a=19.050 \mathrm{~mm}, b=9.525 \mathrm{~mm})$ y una guía rectangular pequeña $(a=5.100 \mathrm{~mm}, b=7.000 \mathrm{~mm})$ con un offset $(c=4.000 \mathrm{~mm}, d=0.750$ mm). En a) $m=1$, y en b) $m=15,25,32 \ldots \ldots$. . . . . .

2.10 Estructura guiada constituida por uniones planares centradas entre guías rectangulares de diferentes dimensiones, y por transiciones entre guías rectangulares y guías reentrantes. . . . . . . . . . . . 
2.11 Septum cortocircuitado en plano E. Vista en perspectiva en a), y sección longitudinal en $b) . \ldots \ldots \ldots \ldots \ldots$

2.12 Conexión de las redes equivalentes multimodales, definidas mediante las correspondientes matrices de admitancias generalizadas, que corresponden a los elementos integrantes de un septum cortocircuitado en plano E como el mostrado en la figura 2.11. . . . . . . . . . .

2.13 Módulo del parámetro $S_{11}$ para diferentes valores (en $\mathrm{mm}$ ) de la dimensión $B_{2}$ de un septum cortocircuitado en plano $\mathrm{E}\left(A_{1}=22.86 \mathrm{~mm}\right.$, $\left.B_{1}=10.16 \mathrm{~mm}, T_{1}=T_{2}=0.035 \mathrm{~mm}, L=9.00 \mathrm{~mm}\right) \ldots \ldots \ldots$

2.14 Cavidad de reacción en plano E situada dentro de la guía rectangular en a), y cavidad de reacción en plano E situada fuera de la guía rectangular

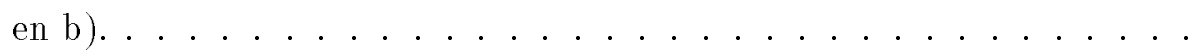

2.15 Módulo del parámetro $S_{11}$ para diferentes valores de las dimensiones $L_{1}$ y $L_{2}$ de una cavidad de reacción en plano $E$ situada dentro de la guía rectangular $\left(A_{1}=22.86 \mathrm{~mm}, B_{1}=10.16 \mathrm{~mm}, B_{2}=2.06 \mathrm{~mm}\right.$, $\left.W=0.10 \mathrm{~mm}, T_{1}=T_{2}=0.10 \mathrm{~mm}, L_{1}+L_{2}+W=18.10 \mathrm{~mm}\right) .$.

2.16 Sección longitudinal de un septum cortorcircuitado en plano E que constituye el elemento básico de las cavidades de reacción situadas fuera de la guía. . . . . . . . . . . . . . .

2.17 Conexión de las diferentes redes equivalentes multimodales, definidas mediante las correspondientes matrices de admitancias generalizadas, que integran un septum cortocircuitado en plano E como el mostrado en la figura $2.16 . \ldots \ldots \ldots \ldots . \ldots . \ldots$

2.18 Módulo del parámetro $S_{11}$ para diferentes valores de las dimensiones $L_{1}$ y $L_{2}$ de una cavidad de reacción en plano $E$ situada fuera de la guía rectangular $\left(A_{1}=22.86 \mathrm{~mm}, B_{1}=10.16 \mathrm{~mm}, B_{2}=2.00 \mathrm{~mm}\right.$, $\left.W=0.10 \mathrm{~mm}, T_{1}=0.10 \mathrm{~mm}, L_{1}+L_{2}+W=18.10 \mathrm{~mm}\right) . \ldots$.

2.19 Guía uniforme arbitraria en a); y representación de dicha guía mediante una red equivalente multimodal basada en la matriz de admitancias generalizada en b). . . . . . . . . . . . .

2.20 Unión planar entre dos guías arbitrarias en a); y representación de dicha unión mediante una red equivalente multimodal basada en la matriz de admitancias generalizada en $b) . \ldots \ldots \ldots$

2.21 Conexión de las redes equivalentes multimodales, definidas mediante las correspondientes matrices de admitancias generalizadas, que corresponden a los elementos integrantes de un dispositivo de microondas constituido por $N$ guías arbitrarias. . . . . . . . . .

2.22 Red equivalente multimodal, definida mediante la matriz de admitancias generalizada $Y_{\mathrm{ab}}$, que corresponde a la conexión en cascada de dos redes equivalentes multimodales, expresadas también en función de sus respectivas matrices de admitancias generalizadas $Y_{\mathrm{a}}$ e $Y_{\mathrm{b}} \ldots \ldots$. . . 
2.23 Red equivalente multimodal, definida mediante una matriz de admitancias generalizada, que corresponde al mismo dispositivo de microondas constituido por $N$ guías arbitrarias descrito electromagnéticamente en la figura $2.21 \ldots \ldots \ldots \ldots \ldots \ldots \ldots$

2.24 Red equivalente multimodal, asociada al dispositivo de microondas constituido por $N$ guías arbitrarias, cargada con las admitancias características de los modos correspondientes para obtener los parámetros de dispersión de dicho dispositivo. . . . . . . . . . . .

2.25 Filtro paso banda de 4 polos con ventanas de acoplo inductivas y elementos de sintonía. . . . . . . . . . . . . .

3.1 Filtro paso banda de 4 polos en guía rectangular con ventanas de acoplo

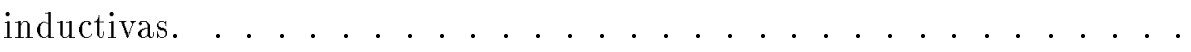

3.2 Representación de un tornillo de sintonía mediante un tramo uniforme de guía reentrante. . . . . . . . . . . . . .

3.3 Filtro paso banda de 4 polos en guía rectangular con ventanas de acoplo inductivas y elementos de sintonía. . . . . . . . . . . .

3.4 Estructura construida para medir el comportamiento electromagnético de un tornillo de sintonía situado en la cavidad resonante de un filtro paso banda en a), y estructura equivalente empleando un elemento de sintonía con sección transversal cuadrada en b). . . . . . . . .

3.5 Módulo de los parámetros $S_{21}$ y $S_{11}$ que presenta una estructura como la mostrada en la figura 3.4 (apartado b); cuyo elemento de sintonía tiene sección transversal cuadrada (lado igual a $2.820 \mathrm{~mm}$ ) y penetra $1.680 \mathrm{~mm}$ dentro de la cavidad resonante de la estructura. . . . . . . .

3.6 Estructura considerada para estudiar la evolución de la frecuencia central de la banda de paso de un filtro paso banda de un solo polo, con un elemento de sintonía en su cavidad resonante, en función de la penetración de dicho elemento. . . . . . . . . . . . .

3.7 Evolución de la frecuencia central de la banda de paso de la estructura mostrada en la figura 3.6 frente a la penetración del elemento de sintonía situado en la cavidad resonante de dicha estructura. . . . . . . . . .

3.8 Estructura escogida para analizar la evolución del ancho frecuencial de la banda de paso de un filtro paso banda de un solo polo, con elementos de sintonía en las ventanas de acoplo de entrada y salida del filtro, en función de la penetración de dichos elementos. . . . . . . . .

3.9 Evolución del ancho de banda relativo normalizado, que presenta la respuesta paso banda de la estructura mostrada en la figura 3.8, frente a la penetración del elemento de sintonía situado en las ventanas de acoplo de dicha estructura. . . . . . . . . . . . . . 
3.10 Respuesta electromagnética (módulo de los parámetros $S_{21}$ y $S_{11}$ ) que presenta un filtro paso banda simétrico de 4 polos, implementado en guía rectangular con ventanas de acoplo inductivas y respuesta tipo Chebyshev, centrado a $11 \mathrm{GHz}$ y con un ancho de banda de $300 \mathrm{MHz}$ en a); y mismo tipo de filtro paso banda centrado a $13 \mathrm{GHz}$ en b). . .

3.11 Estructura escogida para determinar el nivel de acoplamiento requerido por cada ventana de acoplo inductiva de los filtros sin elementos de sintonía en a), y estructura utilizada para obtener ese mismo nivel de acoplamiento empleando elementos de sintonía en la ventana correspondiente de la estructura básica en b). . . . . . . . . . .

3.12 Comparación entre las respuestas electromagnéticas (parámetros $S_{21}$ ) de las estructuras mostradas en la figura 3.11 (apartados a y b) para el caso menos crítico (primera ventana de acoplo de anchura $8.850 \mathrm{~mm}$ del filtro a $13 \mathrm{GHz}$ ) en a), e idéntica comparación para el caso más crítico (segunda ventana de acoplo de anchura $7.098 \mathrm{~mm}$ del filtro a $11 \mathrm{GHz}$ )

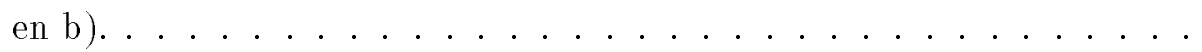

3.13 Estructura escogida para determinar las penetraciones del elemento de sintonía, a situar en la primera cavidad de la estructura básica, con el objeto de centrar la respuesta de esta primera cavidad a $11 \mathrm{GHz}$ y $13 \mathrm{GHz}$ respectivamente. . . . . . . . . . . . . .

3.14 Estructura escogida para determinar las penetraciones del elemento de sintonía, a situar en la segunda cavidad de la estructura básica, con el objeto de centrar la respuesta conjunta de las dos primeras cavidades de la estructura básica a $11 \mathrm{GHz}$ y $13 \mathrm{GHz}$ respectivamente. . . . . .

3.15 Comparación entre las respuestas eléctromagnéticas (parámetros $S_{21}$ ) de la estructura real y de la red ideal tras finalizar el diseño de las dos primeras cavidades de la estructura básica, con elementos de sintonía, para centrar su respuesta paso banda a $11 \mathrm{GHz}$ en a); y misma comparación una vez culminado el proceso de diseño de la primera cavidad de la estructura básica, incluyendo también elementos de sintonía, para centrar su respuesta a $13 \mathrm{GHz}$ en b). . . . . . . . . . . .

3.16 Simulación de la respuesta electromagnética (módulo de los parámetros $S_{21}$ y $\left.S_{11}\right)$ que presenta la estructura básica diseñada empleando los elementos de sintonía apropiados para centrar la respuesta de la estructura a $11 \mathrm{GHz}$ en a), simulación de dicha respuesta cuando se utilizan los elementos de sintonía para centrar la respuesta de la estructura a $13 \mathrm{GHz}$ en b), y medidas de las respuestas que ofrece la implementación real de la estructura básica centrada a ambas frecuencias en $\mathrm{c}$ ). . . . 106 
3.17 Simulación de la respuesta electromagnética en banda y fuera de banda (módulo de los parámetros $S_{21}$ y $S_{11}$ ) que presenta la estructura básica diseñada empleando los elementos de sintonía apropiados para centrar la respuesta paso banda de la estructura a $11 \mathrm{GHz}$ en a), y misma simulación cuando los elementos de sintonía centran la respuesta paso banda de la estructura a $13 \mathrm{GHz}$ en b). . . . . . . . . . 107

3.18 Vista frontal de la estructura básica de filtro paso banda con ventanas inductivas en a), y vista de la parte superior de dicha estructura en b).

3.19 Pieza con elementos de sintonía de sección transversal cuadrada (de lado igual a $2.000 \mathrm{~mm}$ o $4.000 \mathrm{~mm}$ ) cuyas penetraciones permiten centrar la respuesta paso banda de la estructura básica a $11 \mathrm{GHz}$ en a), y ensamblaje de esta pieza con la estructura básica para implementar el filtro con respuesta centrada a $11 \mathrm{GHz}$ en b). . . . . . . . .

3.20 Pieza con elementos de sintonía de sección transversal cuadrada (de lado igual a $2.000 \mathrm{~mm}$ o $4.000 \mathrm{~mm}$ ) cuyas penetraciones permiten centrar la respuesta paso banda de la estructura básica a $13 \mathrm{GHz}$ en a), y pieza con tornillos de sintonía de sección transversal circular (con diámetro $\phi=2$ $\mathrm{mm}$ o $\phi=5 \mathrm{~mm}$ ) cuyas penetraciones pueden ajustarse manualmente para centrar la respuesta paso banda de la estructura básica a cualquier frecuencia comprendida entre $11 \mathrm{GHz}$ y $13 \mathrm{GHz}$ en b). . . . . . .

3.21 Ensamblaje de la pieza que incluye tornillos de sintonía con la estructura básica construida para implementar un filtro paso banda cuya respuesta puede centrarse a cualquier frecuencia comprendida entre $11 \mathrm{GHz}$ y $13 \mathrm{GHz}$ en a), y vista conjunta de todas las piezas construidas en b). .

3.22 Medidas de la respuesta electromagnética (módulo de los parámetros $S_{21}$ y $\left.S_{11}\right)$ que presenta la estructura básica construida empleando tornillos de sintonía para centrar la respuesta a $11 \mathrm{GHz}$ en a), a $12 \mathrm{GHz}$ en b), y a $13 \mathrm{GHz}$ en c). . . . . . . . . . . .

3.23 Configuración de iris con sección transversal elíptica para implementar el ajuste de la frecuencia de resonacia asociada a cada modo en a), para obtener el nivel de acoplo entre modos duales dentro de cada cavidad en b), y para conseguir el acoplo entre cavidades adyacentes en c). . .

3.24 Configuración de un filtro de modo dual en guía circular de 4 polos, simétrico y con respuesta paso banda elíptica (2 ceros de transmisión), que emplea iris elípticos para conseguir los diferentes tipos de acoplo

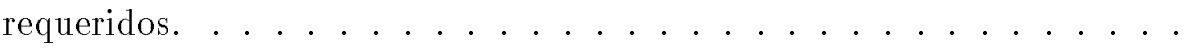

3.25 Simulación de la respuesta electromagnética (módulo de los parámetros $S_{21}$ y $S_{11}$ ) que presenta el filtro de modo dual propuesto en la figura 3.24 , diseñado para que su respuesta esté centrada en torno a $11 \mathrm{GHz}$ con un ancho de banda de $200 \mathrm{MHz}$, en a); y medidas de la respuesta que ofrece la implementación de dicho filtro en b). . . . . . . . . 
3.26 Iris elíptico utilizado para conseguir implementar los acoplos de entrada y salida en a), e iris elíptico escogido para obtener el acoplo entre modos duales situados dentro de cada cavidad en b). . . . . . . . 126

3.27 Iris elíptico que permite realizar en cada cavidad el ajuste frecuencial independiente de cada modo dual en a), e iris elíptico elegido para conseguir el acoplo requerido entre cavidades adyacentes en $b)$. . . .

3.28 Vista conjunta de todas las piezas construidas para implementar el filtro de modo dual diseñado en a), y aspecto final de dicho filtro una vez se ensamblan todas las piezas que lo integran en b). . . . . . . . .

3.29 Sección transversal de una guía circular reentrante, con tres inserciones metálicas en forma de cono, que pretende simular el efecto de los tornillos de sintonía tradicionalmente empleados en los filtros de modo dual en guía circular en a); y aspecto de la sección transversal de otra guía circular reentrante, obtenida a partir de la sección transversal de la guía original (apartado a) aplicando simetría respecto del eje $\hat{y}$, que se emplea junto a la guía original para implementar respuestas elípticas

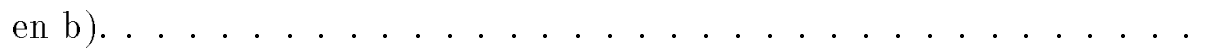

3.30 Configuración de un filtro de modo dual en guía circular de 4 polos, simétrico y con respuesta paso banda sin ceros de transmisión, que consta de iris elípticos y guías circulares reentrantes para implementar los diferentes requerimientos del filtro. . . . . . . . . . .

3.31 Simulación de la respuesta electromagnética (módulo de los parámetros $S_{21}$ y $\left.S_{11}\right)$ que presenta el filtro de modo dual propuesto en la figura 3.30 diseñado para que su respuesta paso banda, sin ceros de transmisión, esté centrada a $11 \mathrm{GHz}$ con un ancho de banda aproximado de $200 \mathrm{MHz}$ en a), simulación de la respuesta electromagnética (módulo de los parámetros $S_{21}$ y $S_{11}$ ) de la misma estructura mostrada en la figura 3.30 pero modificada para conseguir que su respuesta presente 2 ceros de transmisión en b), y medidas de la respuesta que ofrece la implementación real de la estructura recogida en la figura 3.30 en c). .

3.32 Iris elíptico utilizado para conseguir implementar los acoplos de entrada y salida en a), e iris elíptico escogido para obtener el acoplo entre parejas de modos duales situados en cavidades adyacentes en b). . . . . . .

3.33 Guía circular reentrante, con tres inserciones metálicas, que permite conseguir el acoplo requerido entre modos duales dentro de cada cavidad, así como ajustar independientemente la frecuencia de resonancia de cada uno de estos modos, junto con un tramo de guía circular equivalente en el que se insertan tres tornillos de sintonía en a); y vista conjunta de todas las piezas integrantes del filtro de modo dual diseñado, que emplea iris elípticos y guías circulares reentrantes, en b). . . . . 
3.34 Guía circular con tres tornillos de sintonía (diámetro $\phi=2 \mathrm{~mm}$ ), cuyas penetraciones pueden ajustarse manualmente para controlar de manera independiente la frecuencia de resonancia de cada modo y conseguir el acoplo entre modos requerido, en a), vista conjunta de todas las piezas construidas para implementar el filtro de modo dual diseñado, en el que se sustituyen las inserciones metálicas cónicas por tornillos de sintonía, en b), y aspecto final del filtro con tornillos de sintonía una vez ensambladas todas sus piezas en $\mathbf{c}$ ). . . . . . . . . .

3.35 Medidas de la respuesta electromagnética (módulo de los parámetros $S_{21}$ y $S_{11}$ ) del filtro de modo dual construido, con respuesta sin ceros de transmisión, empleando tornillos de sintonía en lugar de guías reentrantes en a); y medidas de la misma estructura, modificada para incorporar 2 ceros de transmisión en su respuesta, empleando nuevamente tornillos de sintonía en b). . . . . . . . . . . . .

4.1 Función de transferencia en sistemas electrónicos y en problemas de dispersión electromagnética. . . . . . . . . . . . . 147

4.2 Sistema de coordenadas utilizado en la caracterización de dispersores bidimensionales. . . . . . . . . . . . . . . . . . 148

4.3 Regiones para calcular el campo dispersado por un conductor perfecto. 154

4.4 Sección transversal de un dispersor metálico perfectamente conductor. 155

4.5 Equivalente físico de un dispersor metálico perfectamente conductor. . 156

4.6 Discretización de un objeto dispersor para calcular la distribución de corrientes ante incidencia $\mathrm{TM}^{\mathrm{z}}$. Vista longitudinal en a) y transversal

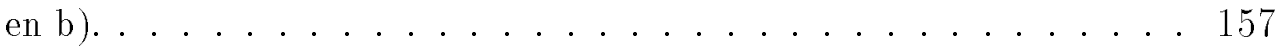

4.7 Equivalente físico de un conductor perfecto, plano e infinito. . . . . 159

4.8 Incidencia de una onda plana sobre un cilindro infinito. . . . . . . . 162

4.9 Sección de un dispersor infinito según $\hat{z}$. Coordenadas de la fuente y del punto de cálculo del campo dispersado. . . . . . . . . . 167

4.10 Sección de un dispersor infinito según $\hat{z}$. Densidad de corriente inducida por un campo incidente con polarización $\mathrm{TE}^{\mathrm{Z}} \ldots \ldots \ldots \ldots$. . . 170

4.11 Fuente puntual de corriente y coordenadas locales. . . . . . . . . . 172

4.12 Evolución del error cuadrático medio de la densidad superficial de corriente, calculada mediante Óptica Física y Método de los Momentos, con el radio del cilindro infinito. . . . . . . . . . . . . . . 174

4.13 Evolución del error cuadrático medio del campo dispersado en la zona de campo lejano, calculado mediante Óptica Física y Método de los Momentos, con el radio del cilindro infinito. . . . . . . . . . 175

4.14 Circunferencia de radio $a \lambda$ y disposición de los puntos donde se calcula el campo dispersado; para obtener su espectro mediante la Transformada Discreta de Fourier. . . . . . . . . . . . . 178 
5.1 Amplitud y fase de una onda plana incidente con dirección de avance $\beta=0 ;$ que se ha reconstruido a partir de su espectro cilíndrico. . . . . 188

5.2 Amplitud y fase del campo eléctrico dispersado por un cilindro metálico infinito, de radio $0.5 \lambda$, ante la incidencia de una onda plana $(\beta=0)$.

5.3 Amplitud y fase del campo eléctrico total; obtenido como la suma de la onda plana incidente $(\beta=0)$ y del campo eléctrico dispersado por un cilindro metálico infinito, de radio $0.5 \lambda$, ante dicha incidencia. . . . . .

5.4 Amplitud y fase del campo eléctrico total; obtenido como la suma de la onda plana incidente $(\beta=\pi / 4)$ y del campo eléctrico dispersado por un cilindro metálico infinito, de radio $0.5 \lambda$, ante dicha incidencia. . . . 190

5.5 Posición de los hilos de corriente sobre la superficie del cilindro. . . . . 193

5.6 Amplitud del campo eléctrico dispersado por un cilindro de radio $0.5 \lambda$ ante la incidencia de una onda plana $(\beta=0)$; calculado de forma analítica en a), mediante la aproximación de Óptica Física en b) y mediante el Método de los Momentos en c). . . . . . . . . . .

5.7 Fase del campo eléctrico dispersado por un cilindro de radio $0.5 \lambda$ ante la incidencia de una onda plana $(\beta=0)$; calculado de forma analítica en a), mediante la aproximación de Óptica Física en b) y mediante el Método de los Momentos en c). . . . . . . . . . . . .

5.8 Amplitud del campo eléctrico dispersado por un cilindro de radio $6 \lambda$ ante la incidencia de una onda plana $(\beta=0)$; calculado de forma analítica en a), mediante la aproximación de Óptica Física en b) y mediante el Método de los Momentos en c). . . . . . . . . . . . .

5.9 Fase del campo eléctrico dispersado por un cilindro de radio $6 \lambda$ ante la incidencia de una onda plana $(\beta=0)$; calculado de forma analítica en a), mediante la aproximación de Óptica Física en b) y mediante el Método de los Momentos en c). . . . . . . . . . . .

5.10 Amplitud y fase del campo eléctrico dispersado por una tira metálica infinita, de longitud $1 \lambda$ y orientada según el eje $\hat{y}$, anta la incidencia de una onda plana $(\beta=0) . \ldots \ldots \ldots \ldots$

5.11 Amplitud y fase del campo eléctrico total; obtenido como la suma de la onda plana incidente $(\beta=0)$ y del campo eléctrico dispersado ante dicha incidencia por una tira metálica infinita, de longitud $1 \lambda$ y orientada según el eje $\hat{y} . \ldots \ldots \ldots \ldots \ldots$

5.12 Amplitud y fase del campo eléctrico dispersado por una tira metálica infinita, de longitud $1 \lambda$ y orientada según el eje $\hat{y}$, anta la incidencia de una onda plana $(\beta=\pi / 6) \ldots \ldots \ldots \ldots$

5.13 Amplitud y fase del campo eléctrico total; obtenido como la suma de la onda plana incidente $(\beta=\pi / 6)$ y del campo eléctrico dispersado ante dicha incidencia por una tira metálica infinita, de longitud $1 \lambda \mathrm{y}$ orientada según el eje $\hat{y}$. . . . . . . . . . . . . 206 
5.14 Segmentación del contorno de un objeto dispersor con grosor infinitesimal para incidencia con polarización $\mathrm{TE}^{\mathrm{z}}$. . . . . . . . 207

5.15 Diagrama de radiación (onda plana incidente con dirección de avance $\beta=-\pi / 3$ y polarizada según $\mathrm{TE}^{\mathrm{z}}$ ) de una tira metálica infinita, de longitud $2 \lambda$ y orientada según el eje $\hat{x} \ldots \ldots \ldots 211$

6.1 Esquema general de un problema formado por dos dispersores $O_{1}$ y $O_{2} .217$

6.2 Campo dispersado por $O_{1}$ ante el campo incidente original. . . . . . 220

6.3 Campo dispersado por $O_{2}$ ante un campo incidente que procede de $O_{1}$. 222

6.4 Diagrama completo de la realimentación entre $O_{1}$ y $O_{2}$ cuando el campo incidente original, de espectro $E I$, afecta tan sólo a $O_{1}$. . . . . . 223

6.5 Diagrama completo de la realimentación entre $O_{1}$ y $O_{2}$ cuando el campo incidente original, de espectro $E I$, afecta tan sólo a $O_{2}$. . . . . . 225

6.6 Esquema general de un problema de realimentación múltiple entre tres dispersores $O_{1}, O_{2}$ y $O_{3} \ldots \ldots \ldots \ldots$. . . . . . . 230

6.7 Diagrama completo de la realimentación entre $O_{1}, O_{2}$ y $O_{3}$ cuando el campo incidente original, de espectro $I$, afecta tan sólo a $O_{1}$ y $O_{2}$. . .

6.8 Diagrama completo de la realimentación entre $O_{1}, O_{2}$ y $O_{3}$ cuando el campo incidente original, de espectro $I$, afecta tan sólo a $O_{3}$. . . . 235

6.9 Esquema general de un problema de acoplo electromagnético entre dos grupos de dispersores. . . . . . . . . . . . . . . . 241

6.10 Diagrama explicativo de la primera situación problemática que provoca errores en la aplicación del algoritmo recursivo. . . . . . . . .

6.11 Ejemplo práctico de la primera limitación del algoritmo recursivo que incorpora un solo objeto en cada iteración. Presentación del problema en a), y posible solución al mismo en b). . . . . . . . . .

6.12 Ejemplo práctico de la primera limitación del algoritmo recursivo que permite analizar agrupaciones de objetos dispersores. Presentación del problema en a), y posible solución al mismo en b). . . . . . . . . 248

6.13 Diagrama explicativo de la segunda situación problemática que provoca errores en la aplicación del algoritmo recursivo. . . . . . . . 250

6.14 Ejemplo práctico de la segunda limitación del algoritmo recursivo. Presentación del problema en a), y posible solución al mismo en b). . . . 251

6.15 Diagrama explicativo de la tercera situación problemática que provoca errores en la aplicación del algoritmo recursivo. . . . . . . . 253

6.16 Esquema general de un problema de acoplo electromagnético entre dos grupos de dispersores con fuente interna situada en $C_{f}$. . . . . . 259

6.17 División del espacio libre en tres regiones para la reconstrucción del campo dispersado por múltiples objetos. . . . . . . . . . 265 
7.1 Tiempos de cálculo en la caracterización de una tira metálica infinita, de longitud $4 \lambda$, ante incidencia polarizada según $\mathrm{TM}^{\mathrm{z}}$ en a); y ante incidencia polarizada según $\mathrm{TE}^{\mathrm{z}}$ en $\left.\mathrm{b}\right) \ldots \ldots \ldots \ldots \ldots$

7.2 Amplitud del campo eléctrico dispersado en zona de campo lejano por dos tiras metálicas infinitas, de grosor despreciable y longitudes $2 \lambda \mathrm{y}$ $10 \lambda$ respectivamente, ante la incidencia de una onda plana $(\beta=-\pi / 3)$ polarizada según $\mathrm{TM}^{\mathrm{z}}$ en a); y polarizada según $\mathrm{TE}^{\mathrm{z}}$ en b). . . . . . 278

7.3 Estrategia de análisis de la dispersión producida por dos tiras metálicas, alejadas entre sí, mediante su previa segmentación en dos grupos de objetos dispersores en a); y sin segmentación en b). . . . . . . . .

7.4 Amplitud y fase del campo eléctrico dispersado por dos tiras metálicas, obtenido mediante segmentación de cada tira en tres subtiras, ante la incidencia de una onda plana $(\beta=0)$ en a) y en $b)$; y amplitud y fase del campo eléctrico total, definido como la suma de los anteriores campos eléctricos incidente y dispersado, en c) y en $\mathrm{d}$ ). . . . . . . 281

7.5 Amplitud del campo eléctrico dispersado por dos tiras metálicas, obtenido sin segmentación, ante la incidencia de una onda plana $(\beta=0)$ en a); y amplitud del campo eléctrico total, definido como la suma de los anteriores campos eléctricos incidente y dispersado, en b). . . . . . .

7.6 Estrategia de análisis de la dispersión producida por un reflector hiperbólico $(c=\lambda, e=2, D=4 \lambda)$, aproximando su contorno mediante 11 tiras de igual longitud. $\ldots \ldots \ldots \ldots \ldots \ldots$

7.7 Amplitud y fase del campo eléctrico dispersado por un reflector hiperbólico $(c=\lambda, e=2, D=4 \lambda)$, ante la incidencia de una onda plana $(\beta=0)$, en a) y en $b)$; y amplitud del campo eléctrico total, definido como la suma de los anteriores campos eléctricos incidente y dispersado, en $c) \ldots \ldots \ldots \ldots \ldots \ldots \ldots \ldots \ldots \ldots \ldots$

7.8 Estrategia de análisis de la dispersión producida por un reflector parabólico $(D=10 \lambda, f=8 \lambda)$, aproximando su contorno mediante 20 tiras de igual longitud. . . . . . . . . . . . . .

Amplitud y fase del campo eléctrico dispersado por un reflector parabólico $(D=10 \lambda, f=8 \lambda)$, ante la incidencia de una onda plana $(\beta=0)$, en a) y en $\mathrm{b})$; y amplitud y fase del campo eléctrico total, definido como la suma de los anteriores campos eléctricos incidente y dispersado, en c) y en $\mathrm{d}) . \ldots \ldots \ldots \ldots$

7.10 Estrategia de análisis de la dispersión producida por un reflector parabólico ( $D=10 \lambda, f=8 \lambda$ ), aproximando su contorno mediante 20 tiras de igual longitud, cuando la fuente que genera el campo incidente está situada en el foco (punto $C_{f}$ ) del reflector parabólico. . . . . . . .

7.11 Amplitud y fase del campo eléctrico incidente sobre un reflector parabólico ( $D=10 \lambda, f=8 \lambda$ ), generado por una fuente situada en el foco $C_{f}$ de coordenadas cartesianas normalizadas $(-6,0) . \ldots . . .291$ 
7.12 Amplitud y fase del campo eléctrico dispersado por un reflector parabólico $(D=10 \lambda, f=8 \lambda)$, ante la incidencia de un campo generado por una fuente situada en el foco del reflector, en a) y en b); y amplitud y fase del campo eléctrico total, definido como la suma de los anteriores campos eléctricos incidente y dispersado, en c) y en $\mathrm{d}$ ). . . . . . . .

7.13 Estrategia de análisis de la dispersión producida por un reflector parabólico $(D=10 \lambda, f=8 \lambda)$, aproximando su contorno mediante 20 tiras de igual longitud, cuando la fuente que genera el campo incidente está situada $2 \lambda$ por encima del foco del reflector parabólico. . . . . . . .

7.14 Amplitud y fase del campo eléctrico incidente sobre un reflector parabólico $(D=10 \lambda, f=8 \lambda)$, generado por una fuente situada $2 \lambda$ por encima del foco en un punto $C_{f}$ de coordenadas cartesianas normalizadas $(-6,2) \ldots \ldots \ldots \ldots \ldots$

7.15 Amplitud y fase del campo eléctrico dispersado por un reflector parabólico $(D=10 \lambda, f=8 \lambda)$, ante un campo incidente generado por una fuente situada $2 \lambda$ por encima del foco del reflector, en a) y en $b$ ); $y$ amplitud y fase del campo eléctrico total, definido como la suma de los anteriores campos eléctricos incidente y dispersado, en c) y en d). . .

7.16 Amplitud del campo eléctrico incidente, dispersado y total en zona de campo lejano correspondiente al análisis de un reflector parabólico $(D=10 \lambda, f=8 \lambda)$, con fuente situada $2 \lambda$ por encima del foco. $\quad .$.

7.17 Amplitud del campo eléctrico dispersado en zona de campo lejano por un reflector parabólico $(D=10 \lambda, f=8 \lambda)$, calculada empleando el algoritmo recursivo y mediante el Método de la Distribución en la Apertura, ante un campo incidente $\left(\mathrm{TM}^{\mathrm{z}}\right)$ generado por una fuente omnidireccional situada en el foco del reflector. . . . . . . . . . . .

7.18 Amplitud del campo magnético dispersado en zona de campo lejano por un reflector parabólico $(D=15 \lambda, f=20 \lambda)$, calculada empleando el algoritmo recursivo y mediante el Método de la Distribución en la Apertura, ante un campo incidente $\left(\mathrm{TE}^{\mathrm{Z}}\right)$ generado por una fuente omnidireccional situada en el foco del reflector. . . . . . . . .

7.19 Estrategia de análisis de la dispersión electromagnética producida por una antena Cassegrain simétrica, cuando la fuente que genera el campo incidente está situada en el foco (punto $C_{f}$ ) del sistema multireflector, aproximando los contornos del reflector hiperbólico $(c=\lambda, e=2$, $D=4 \lambda)$ y del reflector parabólico $(D=14 \lambda, f=8 \lambda)$ que integran dicha antena mediante 7 y 11 tiras respectivamente. . . . . . . .

7.20 Amplitud y fase del campo eléctrico incidente sobre una antena Cassegrain simétrica, constituida por un reflector hiperbólico $(c=\lambda, e=2$, $D=4 \lambda)$ y un reflector parabólico $(D=14 \lambda, f=8 \lambda)$, que está generado por una fuente situada en el foco del sistema multireflector. . . . . . . 
7.21 Amplitud y fase del campo eléctrico dispersado por una antena Cassegrain simétrica, constituida por un reflector hiperbólico $(c=\lambda, e=2$, $D=4 \lambda)$ y un reflector parabólico $(D=14 \lambda, f=8 \lambda)$, ante una incidencia generada por una fuente situada en el foco en a) y en b); y amplitud y fase del campo eléctrico total, definido como la suma de los anteriores campos eléctricos incidente y dispersado, en $\mathrm{c}$ ) y en $\mathrm{d}$ ). . . . . . . .

7.22 Amplitud del campo eléctrico incidente, dispersado y total en zona de campo lejano correspondiente al análisis de una antena Cassegrain simétrica, constituida por un reflector hiperbólico $(c=\lambda, e=2, D=4 \lambda)$ y un reflector parabólico $(D=14 \lambda, f=8 \lambda)$, con fuente situada en el foco del sistema multireflector. . . . . . . . . . . . 306

7.23 Amplitud del campo magnético total en zona de campo lejano correspondiente al análisis de una antena Cassegrain offset, constituida por un reflector hiperbólico $(c=10 \lambda, e=1.66, D=3 \lambda)$ y un reflector parabólico $(D=15 \lambda, f=20 \lambda)$, ante un campo incidente $\left(\mathrm{TE}^{\mathrm{z}}\right)$ generado por una fuente directiva situada entre los dos reflectores que integran la antena Cassegrain. . . . . . . . . . . . .

7.24 Fase del campo magnético total; obtenido como la suma del campo magnético incidente y del campo magnético dispersado ante dicha incidencia por una antena Cassegrain offset, que está constituida por un reflector hiperbólico $(c=10 \lambda, e=1.66, D=3 \lambda)$ y un reflector parabólico $(D=15 \lambda, f=20 \lambda) \ldots \ldots \ldots \ldots \ldots \ldots$

7.25 Estrategia de análisis del campo generado por una antena del tipo bocina $(D=4 \lambda)$, aproximando su contorno mediante 10 tiras metálicas, cuando la fuente que excita la bocina está situada en el punto $C_{f}$. . .

7.26 Amplitud y fase de la excitación $\left(\mathrm{TM}^{\mathrm{z}}\right)$ de la antena bocina $(D=4 \lambda)$, generada por una fuente omnidireccional situada en el punto $C_{f}$, en a) y en b); y amplitud y fase del campo eléctrico generado por la bocina, definido como la suma de la excitación y el campo eléctrico dispersado por las tiras que definen la bocina ante dicha excitación, en c) y en d).

7.27 Amplitud del campo eléctrico dispersado en zona de campo lejano por las tiras metálicas que definen una antena bocina $(D=4 \lambda)$, ante una excitación $\left(\mathrm{TM}^{\mathrm{z}}\right)$ generada por una fuente situada en el punto $C_{f}$ de la

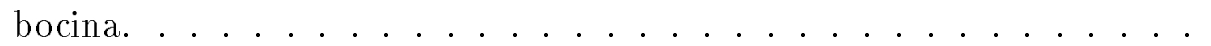

B.1 Representación de una unión planar entre dos guías arbitrarias mediante una red equivalente multimodal, basada en la matriz de admitancias generalizada original cuyos modos localizados están cargados con sus respectivas admitancias características, en a); y representación de la misma unión planar mediante otra red equivalente multimodal, basada en una matriz de admitancias equivalente a la original que sólo relaciona modos accesibles, en b). . . . . . . . . . . . . 
B.2 Imagen del programa DUMAS 3.0 durante la etapa de diseño de un filtro paso banda empleando el primer tipo de red ideal; concretamente cuando se está analizando la respuesta de la estructura real para diseñar su segunda cavidad junto con sus respectivas ventanas de acoplo. . . . 335

B.3 Imagen del programa DUMAS 3.0 durante la etapa de diseño de un filtro paso banda empleando el primer tipo de red ideal; concretamente tras analizar la respuesta de la estructura real una vez finalizado el diseño de su segunda cavidad junto con sus respectivas ventanas de acoplo. . . . . . . . . . . . . . .

B.4 Imagen del programa DUMAS 3.0 durante el análisis de una red ideal del tercer tipo; concretamente tras analizar la respuesta de dicha red ideal truncada de manera que contenga tres inductancias serie. . . . .

B.5 Diferentes tipos de uniones planares que pueden caracterizarse con el programa DUMAS $3.0 \ldots \ldots \ldots \ldots \ldots$

B.6 Imagen del programa DUMAS 3.0 durante el análisis de un filtro de modo dual en guía circular con iris elípticos y guías circulares reentrantes, e imagen del editor gráfico del programa ANAPLAN-W con el contorno de las guías circulares reentrantes empleadas en el mencionado filtro. .

B.7 Imagen del programa DUMAS 3.0 tras el análisis de un filtro de modo dual en guía circular con iris elípticos y guías circulares reentrantes; concretamente mostrando en una gráfica los resultados obtenidos con dicho análisis. . . . . . . . . . . . . . 346

C.1 Dirección de avance de una onda plana. . . . . . . . . . . . . 351

D.1 Posición original de un objeto dispersor en a), y posición de dicho objeto dispersor girado un ángulo $\phi_{g}$ respecto de la situación anterior en b). .

D.2 Amplitud del campo eléctrico total en un problema de dispersión electromagnética producida, ante la incidencia de una onda plana $(\beta=0)$, por una tira metálica infinita, de longitud $1 \lambda$; que se encuentra orientada según el eje $\hat{y}(\phi=\pi / 2)$ en a ), y orientada según la dirección angular $\phi=\pi / 4$ en b). . . . . . . . . . . . . . .

E.1 Significado gráfico de las variables $u, v, w, \alpha$ y $\chi$; que aparecen en el Teorema de Adición de Graf. . . . . . . . . . . . . . . . 373

E.2 Geometría de un sistema constituido por dos objetos dispersores $O_{1}$ y $\mathrm{O}_{2}$; que permite determinar los valores concretos de las variables $u, v, w, \alpha$ y $\chi$ presentes en el Teorema de Adición de Graf. . . . . . . 375

E.3 Expresión de un campo incidente mediante dos espectros cilíndricos referidos a distintos puntos; que se definen a través de sus coordenadas cartesianas globales. . . . . . . . . . . . . . 378 
E.4 Región de validez para la reconstrucción de un cierto campo incidente a partir de una versión trasladada del espectro asociado originalmente a dicho campo. . . . . . . . . . . . . . . . 380

E.5 Amplitud y fase de una onda plana incidente $(\beta=0)$. Reconstrucción a partir de su espectro cilíndrico centrado en $(0,0)$ con $N_{i_{1}}=k 5 \lambda$. . 382

E.6 Amplitud y fase de una onda plana incidente $(\beta=0)$. Reconstrucción a partir de su espectro cilíndrico centrado en $(2,0)$ con $N_{i_{2}}=k 2 \lambda$; obtenido mediante una traslación del espectro cilíndrico original centrado en $(0,0)$ con $N_{i_{1}}=k 5 \lambda \ldots \ldots \ldots \ldots \ldots$

E.7 Amplitud y fase de una onda plana incidente, con dirección de avance $\beta=0$, a lo largo del eje $\hat{x}(y=0)$. Reconstrucción completa a partir del espectro cilíndrico original asociado a dicha incidencia, y reconstrucción parcial a partir de la versión de dicho espectro trasladada al punto $(2,0)$ con $N_{i_{2}}=k 2 \lambda \ldots \ldots \ldots \ldots \ldots \ldots \ldots \ldots$

E.8 Amplitud y fase de una onda plana incidente $(\beta=0)$. Reconstrucción a partir de su espectro cilíndrico centrado en $(4.5,0)$ con $N_{i_{2}}=k 2 \lambda$; obtenido mediante una traslación del espectro cilíndrico original centrado en $(0,0)$ con $N_{i_{1}}=k 5 \lambda \ldots \ldots \ldots \ldots \ldots$

E.9 Amplitud y fase de una onda plana incidente, con dirección de avance $\beta=0$, a lo largo del eje $\hat{x}(y=0)$. Reconstrucción completa a partir del espectro cilíndrico original asociado a dicha incidencia, y reconstrucción parcial a partir de la versión de dicho espectro trasladada al punto $(4.5,0)$ con $N_{i_{2}}=k 2 \lambda \ldots \ldots \ldots \ldots \ldots \ldots$

E.10 Expresión del campo dispersado por $O_{1}$, definido por su espectro cilíndrico emergente, como incidencia sobre $\mathrm{O}_{2}$ utilizando el correspondiente espectro cilíndrico incidente. . . . . . . . . . . . . .

E.11 Región de validez para la reconstrucción de un campo dispersado por $O_{1}$, definido inicialmente en términos de su correspondiente espectro cilíndrico emergente, a partir de un espectro cilíndrico incidente centrado en el objeto $O_{2} \ldots \ldots \ldots$. . . . . . . . . . . . . 398

E.12 Amplitud y fase del campo eléctrico dispersado por un cilindro metálico infinito, de radio $2 \lambda$ y centrado en $(-4,0)$, ante la incidencia de una onda plana $(\beta=0)$. Reconstrucción a partir de su espectro cilíndrico emergente centrado en $(-4,0) \ldots \ldots \ldots \ldots$

E.13 Amplitud y fase del campo eléctrico dispersado por un cilindro metálico infinito, de radio $2 \lambda$ y centrado en $(-4,0)$, ante la incidencia de una onda plana $(\beta=0)$. Reconstrucción a partir del espectro cilíndrico incidente centrado en $(3,0)$, y constituido por $2 N_{i_{2}}+1$ componentes espectrales con $N_{i_{2}}=k 4 \lambda \ldots \ldots \ldots \ldots \ldots \ldots \ldots$. . . . . . 400 
E.14 Amplitud y fase del campo eléctrico dispersado por el cilindro, ante una onda plana $(\beta=0)$, a lo largo del eje $\hat{x}(y=0)$. Reconstrucción completa a partir del espectro emergente asociado originalmente a dicho campo, y reconstrucción parcial a partir del espectro incidente centrado en el punto $(3,0)$ con $N_{i_{2}}=k 4 \lambda \ldots \ldots$. . . . . . . . . 401

E.15 Amplitud y fase del campo eléctrico dispersado por un cilindro metálico infinito, de radio $2 \lambda$ y centrado en $(-4,0)$, ante la incidencia de una onda plana $(\beta=0)$. Reconstrucción a partir del espectro cilíndrico incidente centrado en $(3,0)$, y constituido por $2 N_{i_{2}}+1$ componentes espectrales

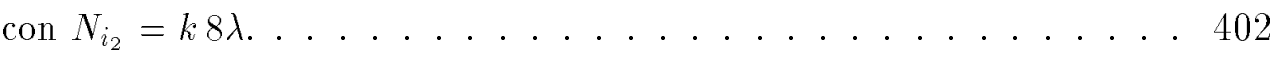

E.16 Amplitud y fase del campo eléctrico dispersado por el cilindro, ante una onda plana $(\beta=0)$, a lo largo del eje $\hat{x}(y=0)$. Reconstrucción completa a partir del espectro emergente asociado originalmente a dicho campo, y reconstrucción parcial a partir del espectro incidente centrado en el punto $(3,0)$ con $N_{i_{2}}=k 8 \lambda \ldots \ldots \ldots$. . . . . . 403

E.17 Expresión de un campo generado por una o varias fuentes mediante dos espectros cilíndricos emergentes referidos a distintos puntos; que se definen a través de sus coordenadas cartesianas globales. . . . . . . 407

E.18 Región de validez para la reconstrucción de un campo generado por una o varias fuentes de corriente, definido inicialmente en términos de su espectro cilíndrico emergente centrado en el punto $\left(x_{c_{1}}, y_{c_{1}}\right)$, a partir de una versión de dicho espectro trasladada al punto $\left(x_{c_{2}}, y_{c_{2}}\right)$. . . 410 


\section{Capítulo 1}

\section{Introducción}

El análisis de la dispersión electromagnética, tanto en sistemas guiados (problemas cerrados) como en espacio libre (problemas abiertos), constituye un tema que ha despertado tradicionalmente un enorme interés en la literatura; y que incluso hoy en día, debido a sus diversas y múltiples aplicaciones, continúa siendo objeto de nuevas investigaciones por parte de la comunidad científica internacional. Así por ejemplo, numerosas estructuras pasivas de microondas implementadas en guía, tales como filtros paso banda para comunicaciones, filtros de banda eliminada para aplicaciones de calentamiento por microondas, transductores ortomodales, o incluso giradores de polarización, basan su funcionamiento en fenómenos de dispersión generados en el interior de dichos dispositivos; mientras el comportamiento de un gran número de elementos radiantes en espacio libre, entre los que se encuentran las antenas reflectoras o los sistemas multireflectores, se debe a la dispersión electromagnética que producen en estos casos dichas estructuras. A su vez, la determinación de la sección recta radar (RCS) de blancos, el análisis de estructuras semicerradas como las antenas bocina, e incluso el estudio de modelos de propagación en entornos urbanos para su aplicación en comunicaciones móviles, constituyen problemas abiertos que pueden solucionarse completamente analizándolos como problemas de dispersión. Todas estas aplicaciones requieren pues de una caracterización precisa, al tiempo que eficiente, de los diferentes problemas de dispersión electromagnética que surgen en cada caso; debiendo emplear para ello el método que resulte más adecuado.

De entre las diferentes y numerosas técnicas que permiten resolver problemas de dispersión, tanto en sistemas guiados como en espacio libre, conviene considerar aquéllas basadas en soluciones modales; cuyos principios generales ya se encuentran recogidos con detalle en [1]. Los métodos modales resultan especialmente interesantes al analizar la dispersión producida por geometrías sencillas; pues permiten obtener de manera relativamente simple soluciones analíticas exactas a dichos problemas. Ahora bien, cuando la geometría del objeto dispersor es arbitraria, no es posible obtener soluciones analíticas exactas para la dispersión 
producida por la estructura bajo análisis; resultando necesario en estos casos combinar las mencionadas técnicas de análisis modal con métodos numéricos, tales como por ejemplo el Método de los Momentos o el Método de los Elementos Finitos, que se ocupan de resolver las ecuaciones integrodiferenciales derivadas de las correspondientes condiciones de contorno.

Los métodos modales recién mencionados vienen utilizándose tradicionalmente en la resolución de problemas de dispersión electromagnética en sistemas guiados; pues ya a finales de la década de los 60 y comienzos de los 70 , tal y como se recoge en [2-5], se plantean dichos métodos (conocidos en inglés como modematching) para caracterizar uniones planares entre guías con secciones transversales diferentes. Estos métodos modales permiten caracterizar la dispersión producida por las citadas discontinuidades, con bastante precisión por cierto, mediante matrices de dispersión (en inglés scattering) generalizadas; cuya combinación adecuada, empleando la teoría de circuitos desarrollada para tratar redes de microondas, permite analizar numerosos dispositivos de microondas integrados por la conexión en cascada de diferentes guías. Ahora bien, esta técnica clásica de análisis presenta varios inconvenientes; destacando entre ellos la baja eficiencia computacional asociada a su implementación, debido a la complejidad inherente al método en cuestión, así como el problema de la convergencia relativa expuesto en $[6,7]$. Con el objeto de evitar estos inconvenientes, en [8] se propone recientemente un nuevo método de análisis modal, basado en matrices de inmitancias (admitancias o impedancias según el caso) generalizadas, para caracterizar discontinuidades en sistemas guiados; método que constituye una adaptación de una técnica clásica propuesta a finales de los años 60 en [9], empleada en su momento para caracterizar uniones entre guías rectangulares en forma de T. Este nuevo método permite caracterizar con una gran precisión, al tiempo que eficientemente, las diversas discontinuidades que integran normalmente los dispositivos pasivos de microondas; sin embargo, su eficiencia comienza a disminuir a medida que se incrementa la complejidad del dispositivo bajo análisis, por lo que parece interesante esforzarse en intentar mejorar la citada eficiencia del método.

Así pues, como primer objetivo general de la tesis, se plantea el desarrollo de técnicas que permitan mejorar la eficiencia de los métodos de análisis modal, en concreto de aquellas versiones basadas en matrices de inmitancias generalizadas; que, tal y como se termina de mencionar, permiten caracterizar la dispersión causada por las discontinuidades presentes entre las guías integrantes de los dispositivos de microondas. Una vez alcanzado este primer objetivo, haciendo uso de la nueva técnica optimizada, se plantea su aplicación al análisis y al diseño de estructuras pasivas de microondas complejas, empleadas de forma tradicional en diversos campos, que hasta el momento no habían podido caracterizarse completamente debido a su complejidad, o requerían en su caracterización de importantes esfuerzos computacionales. En concreto, entre otras muchas posibles aplicaciones, se propone emplear la nueva técnica para analizar filtros de banda 
eliminada implementados con septums y cavidades de reacción, de amplia utilidad en aplicaciones de calentamiento por microondas, para caracterizar el efecto de los tornillos de sintonía empleados tradicionalmente en el ajuste fino de la respuesta de los filtros paso banda, o para estudiar el comportamiento de nuevas estructuras paso banda de modo dual en guía circular, de amplia utilización en la carga útil de los satélites de comunicaciones. En estas dos últimas aplicaciones, al tiempo que se pretende realizar su análisis electromagnético completo, constituye también objetivo de esta tesis el desarrollo de estrategias que permitan el diseño óptimo de los mencionados dispositivos.

En cuanto a los problemas de dispersión electromagnética en espacio libre, su resolución mediante técnicas de análisis modal no se encuentra tan sistematizada como en el caso de los problemas cerrados; no encontrando en la literatura clásica referencias a matrices generalizadas como las utilizadas al resolver problemas de dispersión en sistemas guiados. En concreto, el análisis tradicional de los mencionados problemas abiertos se ha realizado aplicando a cada problema particular, y ante una determinada incidencia, la técnica que resulta más apropiada; entre las que se encuentran por ejemplo las conocidas técnicas de alta frecuencia, como Óptica Física, Óptica Geométrica, Teoría Física de la Difracción, Teoría Geométrica de la Difracción, o los métodos catalogados clásicamente como numéricos, tales como el Método del Unimomento, el Método de los Elementos de Contorno, o el Método del Gradiente Conjugado con Transformada Rápida de Fourier. La utilización de matrices generalizadas para resolver problemas de dispersión en espacio abierto constituye un tema relativamente reciente; encontrando una de las primeras referencias al respecto en [10], así como posteriores trabajos sobre el tema en $[11,12]$. En esta línea de investigación, conviene destacar los avances recogidos en [13,14]; donde se propone caracterizar el comportamiento dispersor de objetos en espacio libre mediante matrices de caracterización individual, cuyo concepto es similar al de las matrices de dispersión (en inglés scattering) generalizadas que se utilizan en sistemas guiados. Estas nuevas matrices de caracterización, definidas en el dominio espectral, relacionan de hecho espectros de campo dispersado por los objetos con espectros de campo incidente; constituyendo dichas matrices verdaderas funciones de transferencia asociadas respectivamente a cada objeto dispersor. La principal ventaja de este método consiste en tener caracterizado, mediante una simple matriz, el comportamiento dispersor de un objeto en espacio libre ante cualquier incidencia; lo que permite sistematizar la resolución de todos aquéllos problemas donde intervengan uno o incluso varios de estos objetos dispersores.

Consecuentemente, un objetivo esencial de la presente tesis consiste en desarrollar de forma adecuada el concepto de la citada matriz de caracterización; así como plantear una técnica general que permita el cálculo de esta matriz para cualquier objeto dispersor. Esta nueva técnica, con la intención de poder caracterizar cualquier objeto dispersor mediante su correspondiente matriz, debe 
complementarse con los métodos clásicos de alta frecuencia y con los métodos numéricos tradicionales mencionados en el párrafo anterior; debiendo seleccionar en cada problema concreto el procedimiento más adecuado para obtener la citada matriz de caracterización. Por tanto, resulta necesario comprobar cómo se comportan todos estos métodos en combinación con la novedosa técnica de análisis modal propuesta; habiendo escogido para ello en este trabajo, a modo de ejemplo, la aproximación de Óptica Física y el Método de los Momentos. Aunque esta comprobación se ha realizado para problemas bidimensionales, por razones de simplicidad, el concepto de la matriz de caracterización introducido resulta igualmente válido para problemas tridimensionales; cuyo análisis mediante la nueva técnica tan sólo requerirá de un mayor esfuerzo computacional. Una vez caracterizado el comportamiento dispersor de un objeto en espacio libre, mediante su correspondiente matriz de caracterización individual, es posible calcular la dispersión producida por dicho objeto ante cualquier posible incidencia; lo que permite pensar en la posible utilización de dichas matrices para resolver problemas de acoplo electromagnético entre múltiples objetos dispersores.

El análisis de problemas de dispersión electromagnética producida por múltiples objetos, debido al gran número de posibles aplicaciones donde es necesario resolver este tipo de problemas, es un tema recogido ampliamente en la literatura; donde se proponen diversos algoritmos para la completa resolución de las múltiples interacciones que tienen lugar entre los objetos dispersores. Entre dichos algoritmos, se encuentran aquéllos no muy eficientes en términos computacionales que se aproximan de forma iterativa a la solución final buscada, o aquéllos otros que emplean técnicas recursivas de gran complejidad, e incluso los que utilizan teoría de grafos recomendados únicamente cuando el número de objetos dispersores es bajo. Como otro gran objetivo del trabajo a desarrollar, se plantea la deducción de un nuevo algoritmo para resolver problemas de acoplo entre múltiples objetos; algoritmo que combina, gracias al empleo del concepto de la matriz de caracterización individual introducido previamente, una estrategia recursiva con teoría de grafos para sistemas realimentados. Este nuevo algoritmo, eficiente y de gran sencillez conceptual, caracteriza finalmente el comportamiento dispersor de cada objeto, teniendo en cuenta las interacciones múltiples que se producen entre todos los objetos del problema, mediante una nueva matriz de caracterización conjunta; cuyo concepto es similar al de la matriz que caracterizaba anteriormente el comportamiento dispersor aislado de ese mismo objeto.

A continuación, una vez deducido el nuevo algoritmo de análisis de problemas de dispersión producida por múltiples objetos, se particulariza dicho algoritmo al análisis de diversas situaciones prácticas bidimensionales. Una primera situación donde resulta interesante aplicar este nuevo algoritmo está asociada al análisis de objetos dispersores grandes en términos eléctricos; que tradicionalmente, por razones de eficiencia computacional, se han caracterizado empleando técnicas clásicas de alta frecuencia, a pesar de que el análisis preciso de estas 
estructuras debiera realizarse empleando algún método numérico. En estos problemas puede seguirse una estrategia de segmentación del contorno del objeto dispersor en elementos de menor tamaño, cuya caracterización individual pueda implementarse con precisión utilizando alguno de los mencionados métodos numéricos, para posteriormente analizar el comportamiento de la estructura global mediante el nuevo algoritmo recursivo propuesto. El análisis de sistemas radiantes multireflectores, integrados normalmente por dos elementos reflectores grandes en términos eléctricos, constituye sin duda otra posible aplicación directa del nuevo algoritmo recursivo deducido en este trabajo; cuya implementación suele ir precedida también, tal y como se termina de mencionar para dispersores individuales, de una adecuada segmentación del contorno de los elementos que integran la estructura multireflectora considerada. Finalmente, de entre el gran número de posibles aplicaciones que pueden resolverse con el nuevo algoritmo recursivo, éste se aplica con éxito en la presente tesis al análisis de una antena bocina, que constituye una estructura radiante semicerrada, incluyendo en dicho análisis la presencia de la guía alimentadora de la antena.

Con el objeto de presentar las soluciones propuestas para cumplir con los diversos objetivos planteados, se ha dividido la tesis ofrecida a continuación en una serie de capítulos y apéndices; cuyo contenido se describe, con la mayor brevedad posible, en los siguientes párrafos.

Comenzando con la aplicación de los métodos modales para resolver problemas de dispersión en sistemas guiados, en el capítulo 2 se describe una técnica que permite acelerar enormemente el cálculo de las matrices de inmitancias (admitancias o impedancias según el caso), empleadas recientemente para caracterizar de manera precisa la dispersión que generan las discontinuidades presentes en las estructuras pasivas de microondas. Dicha técnica, expuesta con detalle en el mencionado capítulo 2, se basa simplemente en la extracción de la dependencia frecuencial asociada a cietas series infinitas; extracción que requiere desarrollar una determinada función en serie de Taylor, cuyos coeficientes se ofrecen con detalle en el apéndice A. Con el objeto de corroborar el buen funcionamiento de esta nueva técnica, se ofrecen resultados correspondientes al análisis de septums cortocircuitados en plano E, y de cavidades de reacción integradas por dichos septums; concluyendo de estos resultados la posible aplicación de los septums y de las cavidades de reacción como redes adaptadoras y como filtros de banda eliminada, de gran interés en aplicaciones de calentamiento por microondas. Una vez acelerada la computación de las matrices de inmitancias, se descubre que el principal esfuerzo computacional asociado al análisis de dispositivos complejos, integrados por la conexión en cascada de diferentes tramos uniformes de guía, se centra en la resolución del correspondiente sistema de ecuaciones lineales; cuya matriz de coeficientes, al haber empleado matrices de inmitancias en la caracterización individual de las discontinuidades, presenta estructura en banda. En el intento de reducir el tiempo que supone resolver este sistema de ecuaciones, se propone una 
nueva técnica recursiva muy eficiente que permite resolver el mencionado sistema; explotando para ello su naturaleza en banda.

En el capítulo 3, se ofrecen ejemplos de análisis y diseño eficiente de dos tipos de estructuras pasivas de microondas complejas; para lo cual se hace uso de una herramienta de programación especialmente optimizada, denominada comercialmente DUMAS 3.0 y descrita brevemente en el apéndice B. Con esta herramienta, que incorpora las dos nuevas técnicas propuestas en el capítulo 2 para analizar eficientemente problemas de dispersión en sistemas guiados, se ha diseñado en concreto una estructura básica de filtro paso banda en guía rectangular, cuya respuesta puede sintonizarse empleando los tradicionales tornillos de sintonía; así como dos filtros paso banda de modo dual en guía circular, uno de ellos implementado utilizando sólo iris elípticos, y el otro empleando iris elípticos y nuevamente tornillos de sintonía. Junto a la descripción de las estructuras diseñadas, en el mencionado capítulo 3 se detallan también las estrategias seguidas en el diseño de los mencionados dispositivos.

Por lo que respecta al análisis de problemas de dispersión en espacio libre, el capítulo 4 describe con profundidad el concepto del nuevo tipo de matriz generalizada, denominada matriz de caracterización individual, con la que se pretende caracterizar los citados problemas abiertos. Esta matriz, que se define en el dominio espectral, debe relacionar pues los espectros asociados a los campos incidentes y dispersados del problema bajo análisis; detallando el concepto de espectro asociado a una solución electromagnética en el apéndice C. A su vez, en el capítulo 4 se propone una estrategia genérica para obtener la matriz de caracterización individual de un objeto dispersor; que consiste en el cálculo de dos nuevas matrices, denominadas respectivamente matriz de corrientes y matriz de espectro, cuyo producto determina la matriz de caracterización buscada. Los conceptos asociados a cada una de estas dos nuevas matrices se recogen también en el citado capítulo 4; donde además se proponen diferentes técnicas para el cálculo de dichas matrices, particularizadas por motivos de sencillez al análisis de objetos dispersores bidimensionales.

Una vez expuesto el concepto de la matriz de caracterización individual de un objeto dispersor en espacio libre, así como las posibles técnicas para implementar el cálculo de dicha matriz, en el capítulo 5 se caracteriza la dispersión electromagnética producida por dos objetos canónicos, como son el cilindro y la tira, ambos metálicos e invariantes en una dimensión; con el objeto de corroborar pues el buen comportamiento de esta nueva técnica propuesta, y de comprobar asimismo algunas de las principales ventajas que presenta la nueva matriz definida. En la caracterización de alguno de estos objetos dispersores, girado respecto de una posición original en la que previamente ya se había caracterizado su comportamiento dispersor, se obtiene la nueva matriz asociada al objeto girado a partir de aquélla deducida en la situación original; empleando para ello las correspondientes matrices de giro, cuyo concepto se detalla en el apéndice D, que evitan 
repetir para el mismo objeto girado todo el proceso de cálculo de su matriz de caracterización individual descrito en el capítulo 4.

Haciendo uso del concepto de la matriz de caracterización individual asociada a cada objeto dispersor, así como de unas nuevas matrices que permiten trasladar espectros cilíndricos, expuestas con todo detalle en el apéndice E, se plantea en el capítulo 6 un algoritmo recursivo basado en teoría de grafos para analizar la dispersión producida por múltiple objetos; cuya implementación genera una matriz de caracterización conjunta asociada a cada objeto del problema bajo análisis, que determina su comportamiento dispersor teniendo en cuenta las múltiples realimentaciones entre todos los objetos. En el citado capítulo 6 , se exponen diferentes versiones del mismo algoritmo recursivo; cada una de las cuales permite resolver diferentes problemas de acoplo electromagnético, como por ejemplo cuando la fuente generadora del campo incidente a los objetos es externa a todos ellos, o cuando dicha fuente se encuentra situada entre dichos objetos. A su vez, se plantean situaciones problemáticas que pueden causar errores en la aplicación directa del correspondiente algoritmo recursivo; y se proponen ciertas acciones preventivas cuya observación previa al empleo del algoritmo elimina cualquier tipo de problema. Finalmente, una vez resuelto el problema de dispersión múltiple, es necesario determinar el campo dispersado por todos los objetos en cualquier región del espacio; para lo cual, en este capítulo 6 se divide el espacio en tres regiones, y se determinan las expresiones a utilizar para evaluar el campo dispersado total en cada una de estas tres regiones.

Tras deducir en el capítulo 6 un algoritmo recursivo, basado en teoría de grafos, que permite analizar la dispersión electromagnética generada por múltiples objetos dispersores; dicho algoritmo, concretamente su versión más adecuada para cada caso, se particulariza en el capítulo 7 a situaciones problemáticas bidimensionales cuyo análisis requiere considerar interacciones entre diversos objetos dispersores. En primer lugar, se ofrece un estudio de la eficiencia computacional asociada a la técnica de análisis de objetos dispersores grandes en términos eléctricos basada en segmentación; revelando dicho estudio el importante ahorro que introduce esta técnica, descrita anteriormente, frente a los métodos clásicos de análisis de la dispersión que produce el objeto original (sin segmentación de su contorno). Seguidamente, aplicando segmentación, se analizan diversos problemas constituidos por varias tiras metálicas grandes eléctricamente cuyas soluciones se conocen mediante otros métodos tradicionales; con la intención de comparar estos resultados conocidos con los proporcionados por la técnica basada en segmentación, y confirmar pues de esta manera la validez de dicha técnica. A continuación, tras comprobar que la segmentación del contorno de un objeto grande en términos eléctricos permite caracterizar su comportamiento dispersor con gran precisión y de manera eficiente, se muestran resultados obtenidos con la citada técnica correspondientes a la dispersión electromagnética producida por reflectores hiperbólicos, por reflectores parabólicos, y por antenas Cassegrain 
constituidas por ambos tipos de reflectores individuales; resultados que permiten corroborar un gran número de propiedades muy conocidas de los mencionados elementos radiantes. Para finalizar, con el objeto de manifestar la potencia de análisis del método propuesto, que emplea la citada técnica de segmentación junto con la versión adecuada del algoritmo recursivo descrito en el capítulo 6 , se aplica con éxito dicho método a la caracterización del comportamiento de una antena tipo bocina.

Por último, en el capítulo 8 se ofrecen las conclusiones generales de la tesis, así como las futuras líneas de investigación que sugiere el presente trabajo; mientras que en el apéndice $\mathrm{F}$ se recogen algunas de las publicaciones más representativas fruto del trabajo desarrollado en esta tesis, concretamente aquéllas que en el momento actual ya han sido publicadas (o aceptadas para su próxima publicación) en revistas internacionales. 


\section{Capítulo 2}

\section{Análisis Eficiente de Estructuras Pasivas de Microondas mediante la Matriz de Admitancias Generalizada}

La Matriz de Admitancias Generalizada (MAG), calculada empleando teoría de líneas de transmisión, se ha utilizado tradicionalmente en la caracterización electromagnética de uniones planares entre guías arbitrarias; ya que empleando dicha matriz se obtienen resultados muy precisos con una gran eficiencia computacional. A pesar de los esfuerzos realizados para desarrollar procedimientos que permitan calcular, de manera sencilla y eficiente, los elementos de la MAG; todavía resulta interesante esforzarse en reducir el tiempo de computación requerido en el cálculo de dichas matrices, especialmente cuando se analizan dispositivos de microondas complejos. Asimismo, utilizando la MAG para describir el comportamiento de las uniones entre guías, se observa que el análisis de dispositivos constituidos por diferentes guías uniformes, conectadas en cascada, requiere de la resolución de sistemas de ecuaciones lineales en banda. Así pues, tras haber optimizado el cálculo de la MAG, será necesario encontrar también un método eficiente y preciso que resuelva los mencionados sistemas de ecuaciones; para de esta forma analizar eficientemente estructuras pasivas complejas.

En este capítulo, se introduce en primer lugar el procedimiento clásico de cálculo de los elementos de la MAG; para descubrir que el método requiere de un mayor esfuerzo computacional en la implementación de unas sumas infinitas asociadas a ciertos elementos de esta matriz. A continuación, se describe un nuevo procedimiento, simple y rápido, para calcular estos elementos de la MAG; que se basa en extraer de las citadas sumas infinitas su dependencia frecuencial, y por tanto implementar dichas sumas con muy pocos términos en cada frecuencia de análisis. Una vez expuesta esta nueva técnica que acelera la computación de las 
mencionadas series infinitas, y tras comprobar la mejora que supone en la eficiencia computacional del cálculo de la MAG; se ha utilizado dicha técnica en el análisis electromagnético de septums cortocircuitados en plano E, y de cavidades de reacción en plano $\mathrm{E}$ implementadas con septums. Tras acelerar la computación de los elementos de la MAG para uniones planares entre guías arbitrarias, se descubre que el principal esfuerzo computacional requerido en el análisis de dispositivos de microondas complejos, constituidos por diferentes guías uniformes conectadas en cascada, está ligado a la resolución de los sistemas de ecuaciones lineales en banda que aparecen al forzar las correspondientes condiciones de contorno; sistemas cuyo tamaño es mucho mayor, y por tanto su solución más costosa, a medida que crece la complejidad del dispositivo. Por tanto, en el presente capítulo se ofrece también un procedimiento muy eficiente para resolver este tipo de sistemas; empleando para ello una técnica recursiva que explota la estructura en banda de la matriz de coeficientes asociada al sistema de ecuaciones a resolver. Finalmente, se presenta un estudio comparativo, en términos de eficiencia computacional, entre la técnica recursiva propuesta y otros métodos clásicos de análisis de dispositivos integrados por guías conectadas en cascada; revelando dicho análisis que el método recursivo propuesto en el capítulo requiere un menor esfuerzo computacional que las otras dos técnicas clásicas.

\subsection{La Matriz de Admitancias Generalizada de Uniones Planares entre Guías Arbitrarias}

El análisis del comportamiento electromagnético de uniones planares entre guías arbitrarias, como la mostrada en la figura 2.1 (apartado a), constituye un tema que ha suscitado un enorme interés en el pasado. Así por ejemplo, en [15] se representa la citada unión mediante una red equivalente; aunque dicha red sólo tiene en cuenta las interacciones asociadas al modo fundamental, y por tanto no resulta apropiada su utilización en el diseño de las actuales componentes de microondas. Debido a la complejidad de estos dispositivos, el análisis de las uniones planares entre guías requiere de algoritmos de simulación que permitan considerar las interacciones entre modos de orden superior, y que además puedan implementarse eficientemente desde el punto de vista computacional. Tradicionalmente, estas interacciones que se producen entre los modos de orden superior se caracterizan mediante procedimientos basados en adaptación modal [2-5], denominados de manera genérica en la literatura como mode-matching; los cuales proporcionan resultados bastante precisos, pero con un elevado coste computacional.

Recientemente, en [16] se plantea una red equivalente multimodal, basada en la formulación de una ecuación integral, para representar las uniones planares entre guías arbitrarias. Los elementos integrantes de la matriz de acoplamiento multimodal, que constituye la red equivalente, se calculan tras resolver la 


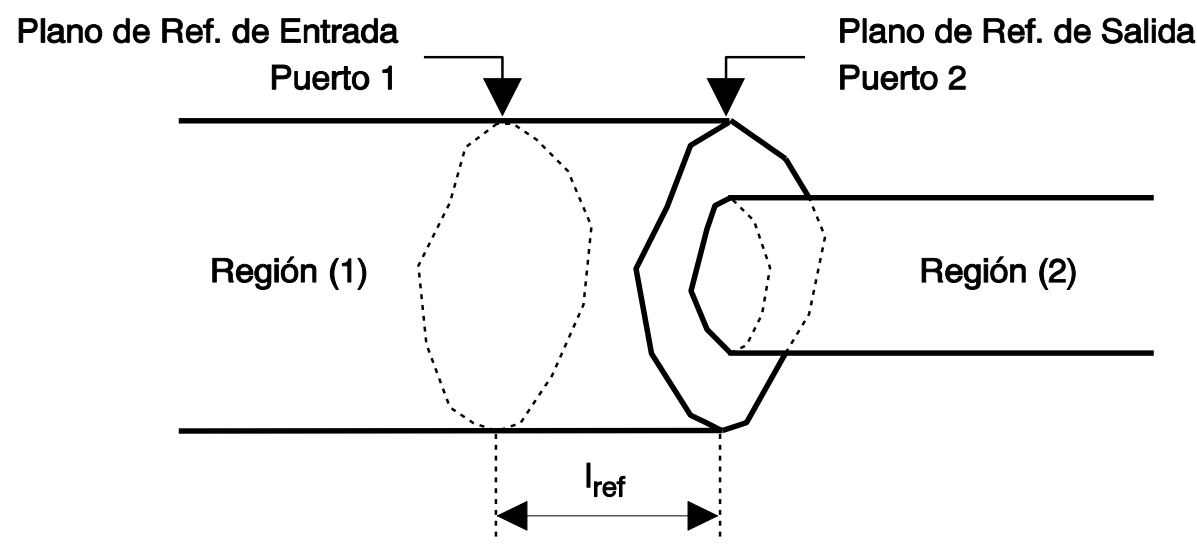

a)

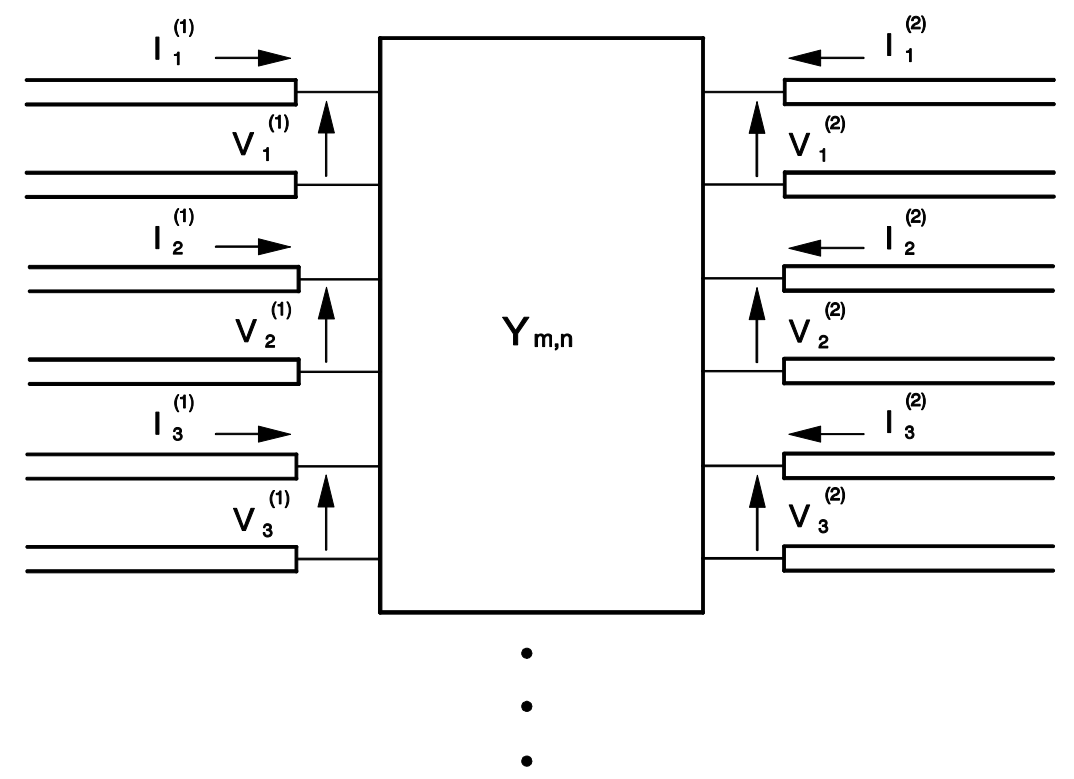

b)

Figura 2.1: Unión planar entre dos guías arbitrarias en a); y representación de dicha unión mediante una red equivalente multimodal basada en la matriz de admitancias generalizada en b).

correspondiente ecuación integral obtenida al imponer las condiciones de contorno. Aunque este método puede implementarse computacionalmente de manera eficiente, requiere de un trabajo analítico previo bastante arduo; por lo que se impone buscar una técnica más sencilla para calcular los elementos de la citada matriz de acoplamiento.

En esta sección, se describe un método simple para obtener la red equivalente multimodal, en términos de la matriz de admitancias generalizada (MAG), de 
uniones planares entre guías arbitrarias. Este método, planteado originalmente en [9] para caracterizar uniones entre guías rectangulares en forma de $\mathrm{T}$, parte de los resultados finales a obtener, es decir de la red equivalente multimodal que representa la unión planar, para evaluar los distintos elementos de la MAG asociada a dicha unión. Este nuevo procedimiento, descrito de forma detallada en [8], se basa en la teoría general de redes, y equivale a medir directamente el valor de los elementos de la MAG; lo que conduce, como se recoge a continuación, a expresiones analíticas elegantes y simples que son válidas para cualquier unión planar entre guías arbitrarias.

La estructura a caracterizar consta de una unión planar entre dos guías arbitrarias, tal y como se muestra en la figura 2.1 (apartado a). El primer paso del método propuesto consiste en definir unos planos de referencia de entrada y salida (ver apartado a en fig. 2.1), separados una distancia $l_{\text {ref, }}$ y escribir formalmelnte el resultado final buscado de la forma

$$
I_{m}^{(\delta)}=\sum_{n=1}^{\infty} Y_{m, n}^{(\delta, 1)} \cdot V_{n}^{(1)}+\sum_{n=1}^{\infty} Y_{m, n}^{(\delta, 2)} \cdot V_{n}^{(2)}
$$

donde $I_{m}^{(\delta)}$ y $V_{n}^{(\delta)}$ representan respectivamente corrientes y voltajes modales, y $\delta$ puede tomar un valor 1 para la región (1), o 2 para la región (2); tal y como se muestra en la figura 2.1 (apartado a). Las corrientes y los voltajes modales $I_{m}^{(\delta)}$ y $V_{n}^{(\delta)}$ constituyen respectivamente las amplitudes de las funciones vectoriales modales normalizadas $\vec{h}_{m}^{(\delta)}$ y $\vec{e}_{n}^{(\delta)}$; funciones vectoriales asociadas a la sección transversal de la guía $(\delta)$, y definidas por ejemplo en [15]. El sistema de ecuaciones lineales planteado en (2.1) se puede representar gráficamente mediante una red equivalente multimodal, recogida en la figura 2.1 (apartado b), que puede utilizarse para analizar estructuras pasivas de microondas constituidas por uniones planares entre guías. Concretamente, en el análisis de dispositivos complejos de microondas, integrados por un cierto número de uniones planares conectadas por tramos de guías uniformes, tan sólo se requiere definir un sistema de ecuaciones lineales más grande; cuya matriz de coeficientes, integrada por diversos bloques, presenta una estructura en banda (ver detalles en sección 2.3). En el citado sistema de ecuaciones en banda, cada uno de los bloques que integran la matriz de coeficientes representa bien la MAG asociada a una unión planar entre guías, o bien la MAG de un tramo de guía que conecta uniones; bloques que se distribuyen en la citada matriz de coeficientes de forma adecuada, como se indica en la sección 2.3, tras imponer las correspondientes condiciones de contorno.

El aspecto clave del método descrito en esta sección consiste en observar que es posible deducir, a partir del sistema de ecuaciones lineales recogido en (2.1), la siguiente expresión formal para los elementos de la MAG

$$
Y_{m, n}^{(\delta, \gamma)}=\frac{I_{m}^{(\delta)}}{V_{n}^{(\gamma)}} ; \quad \forall V_{i}^{(\xi)}=0, \text { con } i \neq n, \xi \neq \gamma
$$




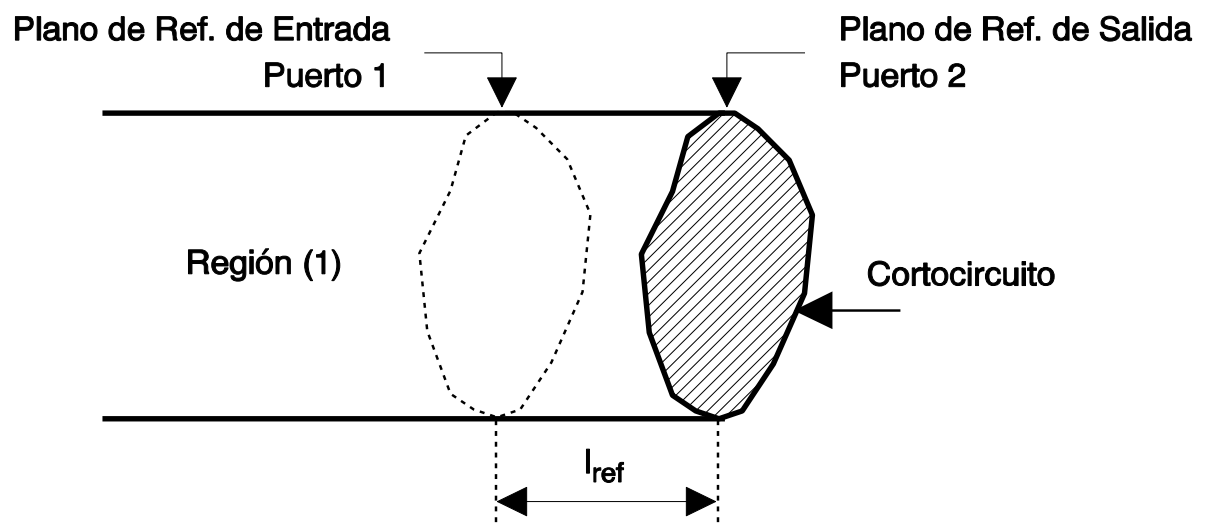

Figura 2.2: Estructura utilizada para evaluar los elementos $Y_{m, n}^{(1,1)}$ e $Y_{m, n}^{(2,1)}$; que se obtiene situando un cortocircuito en el puerto 2.

En (2.2), $\gamma$ y $\xi$ pueden tomar de nuevo un valor 1 para la región (1), o un valor 2 para la región (2), respectivamente. Asimismo, esta expresión recién deducida en (2.2) constituye la definición, en términos de teoría general de redes, de los elementos $Y_{m, n}^{(\delta, \gamma)}$ de la MAG; y puede utilizarse también para obtener de manera directa, como se indica a continuación, el valor de los citados elementos $Y_{m, n}^{(\delta, \gamma)}$

$$
Y_{m, n}^{(\delta, \gamma)}=\frac{\int_{s t(\delta)} \vec{H}^{(\delta)}\left[V_{n}^{(\gamma)}\right] \cdot \vec{h}_{m}^{(\delta)} \mathrm{d} s^{\prime}}{V_{n}^{(\gamma)}}
$$

donde $\vec{H}^{(\delta)}\left[V_{n}^{(\gamma)}\right]$ representa el campo magnético transversal generado en el puerto $\delta$ por el $n$-ésimo modo incidente, de amplitud $V_{n}^{(\gamma)}$, escogido como excitación en el puerto $\gamma$.

Para explicar con detalle como utilizar las ecuaciones (2.2) y (2.3) en la deducción de los elementos de la MAG, seguidamente se expone como obtener los elementos $Y_{m, n}^{(1,1)}$ de dicha matriz. La obtención de estos elementos requiere, según la definición de los mismos recogida en (2.2), una excitación en el puerto 1 asociada al $n$-ésimo modo incidente (que corresponde a la condición $V_{n}^{(1)} \neq 0$ y resto de voltajes en el puerto 1 iguales a cero), y un cortocircuito físico situado en el puerto 2 (correspondiente a la condición de que todos los voltajes en el puerto 2 deben ser cero). Como consecuencia de imponer estas condiciones, la estructura resultante que se obtiene para evaluar los elementos $Y_{m, n}^{(1,1)}$ se muestra en la figura 2.2, donde se observa que no existe discontinuidad alguna; permitiendo pues deducir directamente, a partir de (2.3) y empleando teoría de líneas de transmisión [15], la siguiente expresión para los mencionados elementos $Y_{m, n}^{(1,1)}$

$$
Y_{m, n}^{(1,1)}=(-j) \cdot Y_{0 n}^{(1)} \cdot \cot \left(\beta_{n}^{(1)} \cdot l_{\mathrm{ref}}\right) \cdot \delta_{m, n}
$$

donde $\delta_{m, n}$ representa la delta de Kronecker; cuyo valor, sobradamente conocido, 
se determina del siguiente modo

$$
\delta_{m, n}= \begin{cases}1 & \text { para } m=n \\ 0 & \text { para } m \neq n\end{cases}
$$

Procediendo de igual manera con los elementos $Y_{m, n}^{(2,1)}$ de la MAG, según la definición de dichos elementos recogida en (2.2), se requiere un único modo incidente (el $n$-ésimo) desde el puerto 1 y un cortocircuito físico en el puerto 2 ; lo que supone considerar de nuevo la misma estructura recogida en la figura 2.2, empleada anteriormente al deducir los elementos $Y_{m, n}^{(1,1)}$. En esta ocasión, sin embargo, para obtener los elementos $Y_{m, n}^{(2,1)}$ se necesita determinar la corriente inducida en el puerto 2 por el modo incidente desde el puerto 1; para lo cual, se hará uso de la propiedad de ortogonalidad que presentan las funciones vectoriales modales y de la teoría de líneas de transmisión [15]. Una vez obtenida la citada corriente en el puerto 2 (cortocircuito físico), y tras sustituir su valor en (2.3); es posible implementar el cálculo de los citados elementos $Y_{m, n}^{(2,1)}$ de la siguiente manera

$$
Y_{m, n}^{(2,1)}=j \cdot Y_{0 n}^{(1)} \cdot \csc \left(\beta_{n}^{(1)} \cdot l_{\mathrm{ref}}\right) \cdot<\vec{h}_{n}^{(1)} \vec{h}_{m}^{(2)}>
$$

expresión que también puede utilizarse para calcular los elementos $Y_{n, m}^{(1,2)}$; pues las uniones planares consideradas son recíprocas.

Finalmente, empleando de nuevo la definición de los elementos de la MAG ofrecida en (2.2), se deduce que para obtener los elementos $Y_{m, n}^{(2,2)}$ de la citada matriz se necesita un solo modo incidente (el n-ésimo) desde el puerto 2, y un cortocircuito físico situado en esta ocasión en el puerto 1; condiciones que definen una estructura como la mostrada en la figura 2.3. De acuerdo con la expresión recogida en (2.3), para evaluar los elementos $Y_{m, n}^{(2,2)}$ se requiere determinar la corriente inducida en el puerto 2 por el modo incidente generado en dicho puerto. El valor de esta corriente inducida se obtiene pues empleando teoría de líneas de transmisión [15], y continuidad del campo magnético transversal en el puerto 2; deducidendo de esta manera, tras sustituir la mencionada corriente inducida en (2.3), la siguiente expresión para los elementos $Y_{m, n}^{(2,2)}$ de la MAG

$$
Y_{m, n}^{(2,2)}=(-j) \cdot \sum_{r=1}^{\infty} Y_{0 r}^{(1)} \cdot \cot \left(\beta_{r}^{(1)} \cdot l_{\mathrm{ref}}\right) \cdot<\vec{e}_{r}^{(1)} \vec{e}_{n}^{(2)}>\cdot<\vec{h}_{r}^{(1)} \vec{h}_{m}^{(2)}>
$$

En las expresiones que permiten obtener los elementos de la MAG, deducidas anteriormente en $(2.4),(2.6)$ y $(2.7), Y_{0 n}^{(1)}$ e $Y_{0 r}^{(1)}$ representan respectivamente la admitancia característica del $n$-ésimo y del $r$-ésimo modo en la región (1); mientras $\beta_{n}^{(1)}$ y $\beta_{r}^{(1)}$ son respectivamente la constante de propagación asociada al $n$-ésimo y al $r$-ésimo modo de la región (1). Por lo que respecta al parámetro $l_{\text {ref, }}$, presente también en las mencionadas expresiones, éste representa la separación 


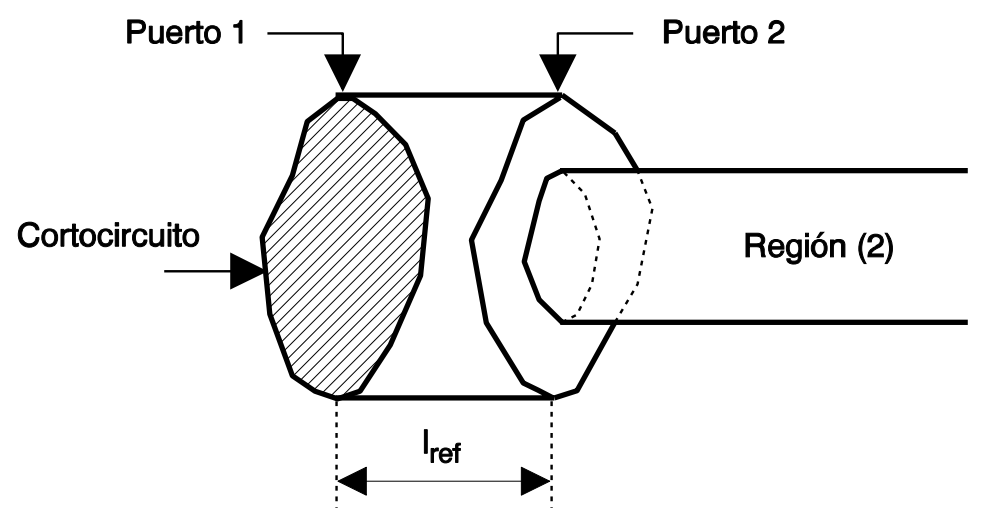

Figura 2.3: Estructura utilizada para evaluar los elementos $Y_{m, n}^{(2,2)}$; que se obtiene situando un cortocircuito en el puerto 1.

entre los planos de referencia de entrada y salida; tal y como se recoge de manera gráfica en la figura 2.1 (apartado a). Por último, el símbolo $<>$ que aparece en (2.6) y (2.7) representa un producto interior; el cual se define normalmente como la integral de acoplamiento, debido a condiciones de ortogonalidad, del producto escalar de las funciones vectoriales modales normalizadas. Las expresiones analíticas concretas de los citados productos interiores suelen ser bastante simples; aunque dependen, lógicamente, de las guías específicas que definen la unión planar bajo análisis.

Una vez deducidas las expresiones formales que permiten calcular los distintos elementos de la MAG, resulta conveniente observar la estructura global de dicha matriz; que puede subdividirse en cuatro bloques bien diferenciados. Uno de estos bloques, concretamente el asociado a los elementos $Y_{m, n}^{(1,1)}$, consta tan sólo de elementos en la diagonal principal; que se calculan de manera rápida implementando las operaciones descritas en (2.4). Existen también otros dos bloques, aquéllos asociados respectivamente a los elementos $Y_{m, n}^{(2,1)}$ e $Y_{m, n}^{(1,2)}$, cuyos elementos se deducen rápidamente a partir de la expresión recogida en (2.6); aunque en este caso debe calcularse el valor de todos los elementos de dichos bloques (no diagonales). Sin embargo, el cálculo de los elementos $Y_{m, n}^{(2,2)}$ de la MAG, que constituyen el cuarto bloque de la citada matriz de admitancias, requiere determinar según (2.7) el valor de unos sumatorios; cuyos índices, que afectan tan sólo a los modos de la región (1), determinan la necesidad de sumar en prinicpio infinitos términos. Lógicamente, la implementación computacional de estos sumatorios requiere truncar el límite superior de los mismos en un valor finito; para lo cual, al caracterizar cada unión planar entre guías mediante su correspondiente matriz de admitancias, debe realizarse un estudio de la convergencia de los elementos $Y_{m, n}^{(2,2)}$ que integran dicha matriz. Es interesante reseñar que la convergencia de estos elementos puede verse afectada por el valor escogido para el parámetro $l_{\text {ref; }}$; aunque empíricamente se ha comprobado que un valor en torno a los $5 \mathrm{~mm}$ re- 
sulta apropiado para la mayoría de las aplicaciones. Como resulta evidente de los comentarios anteriores, el principal esfuerzo computacional requerido en la obtención de la MAG de una unión planar entre guías procede del cálculo de los elementos $Y_{m, n}^{(2,2)}$; ya que implica sumatorios que deben realizarse para cada frecuencia a la que se pretende caracterizar la unión planar bajo estudio. Debido a esta razón, en la próxima sección se describe una técnica eficiente que permite acelerar enormemente el cálculo de los citados elementos $Y_{m, n}^{(2,2)}$.

Para concluir, simplemente comentar que el método expuesto en la presente sección permite obtener, de manera simple y elegante, la red equivalente multimodal de una unión planar entre guías arbitrarias en términos de una matriz de admitancias generalizada. Las expresiones derivadas para los elementos de la MAG pueden emplearse para cualquier tipo de guías que integran la unión planar bajo estudio; aunque resultan especialmente simples, y rápidas de implementar, cuando los modos de la región (1) se conocen de forma analítica. En el supuesto caso de que dichos modos se definieran sólo numéricamente, y que los modos de la región (2) presentaran expresiones analíticas conocidas; resultaría más eficiente, desde el punto de vista computacional, caracterizar la unión planar mediante una red equivalente multimodal expresada en términos de la matriz de impedancias generalizada, tal y como se recoge con detalle en [8].

\subsection{Acelaración del Cálculo de la Matriz de Ad- mitancias Generalizada de Uniones Plana- res entre Guías Arbitrarias}

La caracterización electromagnética de uniones planares entre guías arbitrarias mediante una red equivalente multimodal, basada en la matriz de admitancias generalizada (MAG), constituye un tema de enorme interés que ha sido objeto de diversas publicaciones $[8,9,17]$. Como se puede comprobar en las expresiones deducidas en la sección anterior, el principal esfuerzo computacional requerido en el cálculo de la MAG de una unión planar entre guías, siguiendo la formulación expuesta en [8], se concentra en la evaluación de unas series infinitas asociadas a los elementos $Y_{m, n}^{(2,2)}$ de la citada matriz de admitancias; proceso que debe repetirse para cada frecuencia a la que se pretende caracterizar la unión planar bajo estudio. Si fuera posible extraer la dependencia frecuencial de las mencionadas series, y se pudieran evaluar por tanto fuera del bucle en frecuencia; se conseguiría reducir sustancialmente el tiempo empleado en la determinación de los elementos de la MAG de una unión planar.

En la presente sección, se describe un método muy eficiente que permite acelerar el cálculo de los elementos $Y_{m, n}^{(2,2)}$ de la MAG de uniones planares entre guías arbitrarias; extrayendo para ello la dependencia frecuencial de las series infinitas 
que deben evaluarse al calcular dichos elementos. Este nuevo procedimiento, recogido de manera detallada en [18], conduce a unas expresiones finales para los elementos $Y_{m, n}^{(2,2)}$ que son muy generales; y en las que tan sólo se requiere sumar muy pocos términos por cada frecuencia de análisis. Para determinar la mejora que supone, en términos de eficiencia computacional, el empleo de la técnica de aceleración en el cálculo de los elementos $Y_{m, n}^{(2,2)}$ de la MAG, se ofrece un estudio comparativo entre esta nueva técnica y otros métodos clásicos que también pueden emplearse en la obtención de los citados elementos; revelando dicho estudio que la técnica propuesta en esta sección resulta de hecho la más eficiente. Finalmente, con el objeto de validar el nuevo procedimiento expuesto para calcular la MAG de uniones planares, éste se ha empleado en el análisis electromagnético de septums cortocircuitados en plano $\mathrm{E}$, y de cavidades de reacción en plano $\mathrm{E}$ integradas por septums; que pueden utilizarse como elementos básicos para el diseño de redes adaptadoras y filtros de banda eliminada. Los resultados obtenidos al simular el comportamiento electromagnético de estos elementos básicos, empleando en las simulaciones la nueva técnica de aceleración propuesta, se comparan con otros resultados de los mismos dispositivos, recogidos en la literatura y deducidos empleando adaptación modal (mode-matching); concluyendo de esta comparación que el nuevo procedimiento de cálculo de los elementos $Y_{m, n}^{(2,2)}$ de la citada MAG genera también resultados muy precisos.

\subsubsection{Series Estática y Dinámica}

Con la intención de presentar la nueva técnica que permite acelerar el cálculo de los elementos $Y_{m, n}^{(2,2)}$ de la MAG de uniones planares, se considerará la misma unión planar entre guías arbitrarias (ver apartado a en fig. 2.1) utilizada en la sección anterior para exponer el concepto de la matriz de admitancias generalizada. El comportamiento electromagnético de dicha unión planar puede definirse, tal y como se ha explicado en la sección 2.1, en términos de una matriz de admitancias generalizada; que determina una red equivalente multimodal (ver apartado b en fig. 2.1). Los elementos de la MAG de una unión planar entre guías arbitrarias se calculan, siguiendo el procedimiento descrito en la sección anterior, mediante las expresiones $(2.4),(2.6)$ y (2.7); que presentan el siguiente aspecto

$$
\begin{gathered}
Y_{m, n}^{(1,1)}=(-j) \cdot Y_{0 n}^{(1)} \cdot \cot \left(\beta_{n}^{(1)} \cdot l_{\mathrm{ref}}\right) \cdot \delta_{m, n} \\
Y_{m, n}^{(2,1)}=Y_{n, m}^{(1,2)}=j \cdot Y_{0 n}^{(1)} \cdot \csc \left(\beta_{n}^{(1)} \cdot l_{\mathrm{ref}}\right) \cdot<\vec{h}_{n}^{(1)} \vec{h}_{m}^{(2)}> \\
Y_{m, n}^{(2,2)}=(-j) \cdot \sum_{r=1}^{\infty} Y_{0 r}^{(1)} \cdot \cot \left(\beta_{r}^{(1)} \cdot l_{\mathrm{ref}}\right) \cdot<\vec{e}_{r}^{(1)} \vec{e}_{n}^{(2)}>\cdot<\vec{h}_{r}^{(1)} \vec{h}_{m}^{(2)}>
\end{gathered}
$$

donde todos los parámetros que intervienen se encuentran perfectamente descritos en la mencionada sección 2.1. Además, en (2.8), (2.9) y (2.10) los subíndices $m$ 
y $n$ designan respectivamente modos TE y TM en los puertos 1 y 2 ; mientras el índice $r$ representa modos TE y TM en la región (1). Asimismo, conviene reseñar que los modos TE y TM, tanto los que se definen en los puertos de la red equivalente multimodal como los que intervienen en el sumatorio presente en (2.10), se encuentran ordenados según el sentido creciente del valor de sus respectivos números de onda de corte.

Como resulta evidente de las expresiones (2.8), (2.9) y (2.10), el principal esfuerzo computacional requerido en la obtención de los elementos de la MAG procede del cálculo de los elementos $Y_{m, n}^{(2,2)}$; que requiere la evaluación de las correspondientes series en cada frecuencia de interés. Con el objetivo de reducir el coste computacional que supone evaluar las citadas series, puede llevarse a cabo una primera acción consistente en extraer de cada una de dichas series una parte independiente de la frecuencia $[19,20]$. Esta acción se implementa sumando y restando en (2.10) una serie estática; que se define del siguiente modo

$$
\hat{Y}_{m, n}^{(2,2)}=(-j) \cdot \sum_{r=1}^{\infty} \hat{Y}_{0 r}^{(1)} \cdot \cot \left(\hat{\beta}_{r}^{(1)} \cdot l_{\mathrm{ref}}\right) \cdot\left\langle\vec{e}_{r}^{(1)} \vec{e}_{n}^{(2)}>\cdot<\vec{h}_{r}^{(1)} \vec{h}_{m}^{(2)}>\right.
$$

obteniendo pues para el elemento $Y_{m, n}^{(2,2)}$ de la MAG la siguiente expresión

$$
Y_{m, n}^{(2,2)}=\hat{Y}_{m, n}^{(2,2)}-\tilde{Y}_{m, n}^{(2,2)}
$$

donde el segundo término en la parte derecha de esta última igualdad constituye la denominada serie dinámica; cuyo aspecto se ofrece a continuación

$$
\begin{gathered}
\tilde{Y}_{m, n}^{(2,2)}=(-j) \cdot \sum_{r=1}^{\infty}\left[\hat{Y}_{0 r}^{(1)} \cdot \cot \left(\hat{\beta}_{r}^{(1)} \cdot l_{\mathrm{ref}}\right)-Y_{0 r}^{(1)} \cdot \cot \left(\beta_{r}^{(1)} \cdot l_{\mathrm{ref}}\right)\right] \\
\cdot<\vec{e}_{r}^{(1)} \vec{e}_{n}^{(2)}>\cdot<\vec{h}_{r}^{(1)} \vec{h}_{m}^{(2)}>
\end{gathered}
$$

En $(2.11)$ y $(2.13)$, los términos $\hat{Y}_{0 r}^{(1)}$ y $\hat{\beta}_{r}^{(1)}$ representan respectivamente el comportamiento asintótico, para grandes valores del número de onda de corte transversal $k_{t, r}^{(1)}$, de la admitancia característica $Y_{0 r}^{(1)}$ y de la constante de propagación $\beta_{r}^{(1)}$; presentando dichas expresiones asintóticas los siguientes valores

$$
\begin{aligned}
& \hat{Y}_{0 r}^{(1)}= \begin{cases}(-j) \cdot \frac{k_{t, r}^{(1)}}{\omega \cdot \mu} & \text { para modos TE } \\
j \cdot \frac{\omega \cdot \varepsilon}{k_{t, r}^{(1)}} & \text { para modos TM }\end{cases} \\
& \hat{\beta}_{r}^{(1)}=(-j) \cdot k_{t, r}^{(1)} \text { para modos TE y TM }
\end{aligned}
$$

Siguiendo esta nueva formulación recién descrita, para obtener los elementos $Y_{m, n}^{(2,2)}$ de la MAG según (2.12), es necesario calcular el valor de las respectivas 


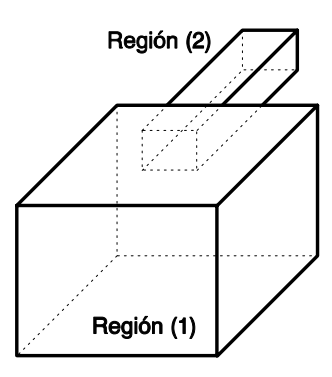

Offset en $\mathrm{x}=\mathrm{c}$

Offset en $y=d$

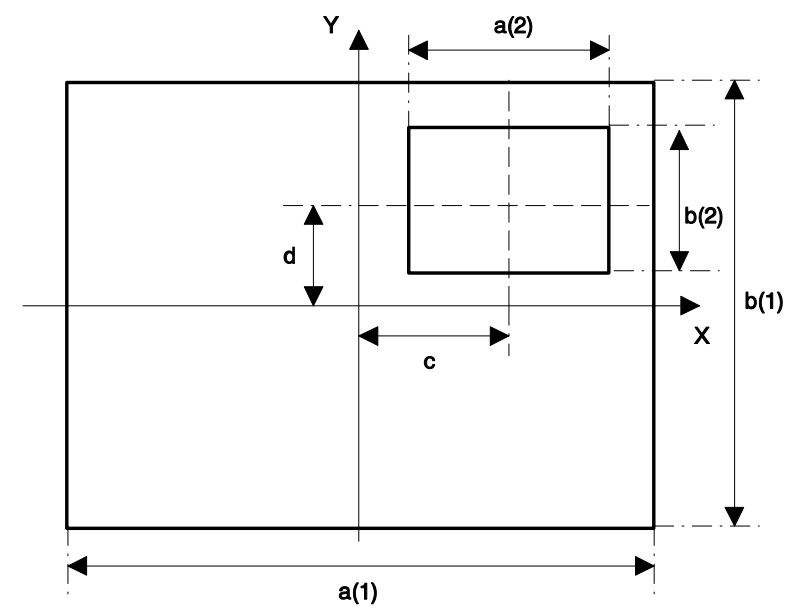

Figura 2.4: Geometría de una unión planar offset entre guías rectangulares.

contribuciones estáticas $\hat{Y}_{m, n}^{(2,2)}$ y dinámicas $\hat{Y}_{m, n}^{(2,2)}$. Por lo que respecta a las series estáticas, tal y como se deduce de (2.11), (2.14) y (2.15), es posible calcular sus valores una sola vez antes de comenzar el bucle en frecuencia; mientras que las correspondientes series dinámicas, cuyo cálculo se implementa según (2.13), deben computarse para cada valor de la frecuencia. Ahora bien, estas series dinámicas convergen mucho más rápidamente que las series originales asociadas a los elementos $Y_{m, n}^{(2,2)}$; debido a que el término entre corchetes en (2.13) tiende a cero de manera muy rápida cuando el índice $r$ del sumatorio crece, a diferencia del término sin corchetes en (2.10) que tiende a cero más lentamente.

Con el objeto de verificar la mejora que introduce la técnica presentada en este apartado, se ofrece seguidamente un estudio típico de la convergencia de algunos de los elementos $Y_{m, n}^{(2,2)}$, así como de las correspondientes series estáticas $\hat{Y}_{m, n}^{(2,2)} \mathrm{y}$ dinámicas $\tilde{Y}_{m, n}^{(2,2)}$, de la MAG de una unión planar offset entre una guía rectangular grande $(a=19.050 \mathrm{~mm}, b=9.525 \mathrm{~mm})$ y una guía rectangular pequeña $(a=5.100 \mathrm{~mm}, b=7.000 \mathrm{~mm})$ con un offset $(c=4.000 \mathrm{~mm}, d=0.750 \mathrm{~mm})$; tipo de unión cuyo aspecto genérico se recoge en la figura 2.4.

En primer lugar, se estudia la convergencia de algunos elementos $Y_{m, n}^{(2,2)}$ de la MAG de la mencionada unión; calculados utilizando la expresión recogida en (2.10). Los resultados correspondientes al estudio de la convergencia de los elementos $Y_{m, m}^{(2,2)}(m=1,15,25,32)$ se ofrecen en la figura 2.5 ; donde $m=1,15,25,32$ hacen referencia respectivamente a los modos $\mathrm{TE}_{01}, \mathrm{TM}_{22}, \mathrm{TM}_{32}$ y $\mathrm{TM}_{15}$ de la guía rectangular pequeña. Como puede observarse en la figura 2.5, para que los elementos $Y_{m, n}^{(2,2)}$ de la MAG presenten resultados numéricamente estables, en el caso de la unión planar offset considerada, es necesario sumar al menos 400 términos en (2.10).

A continuación, se realiza un estudio de la convergencia de las series estáticas $\hat{Y}_{m, m}^{(2,2)}(m=1,15,25,32)$ de la MAG de la misma unión planar definida anterior- 


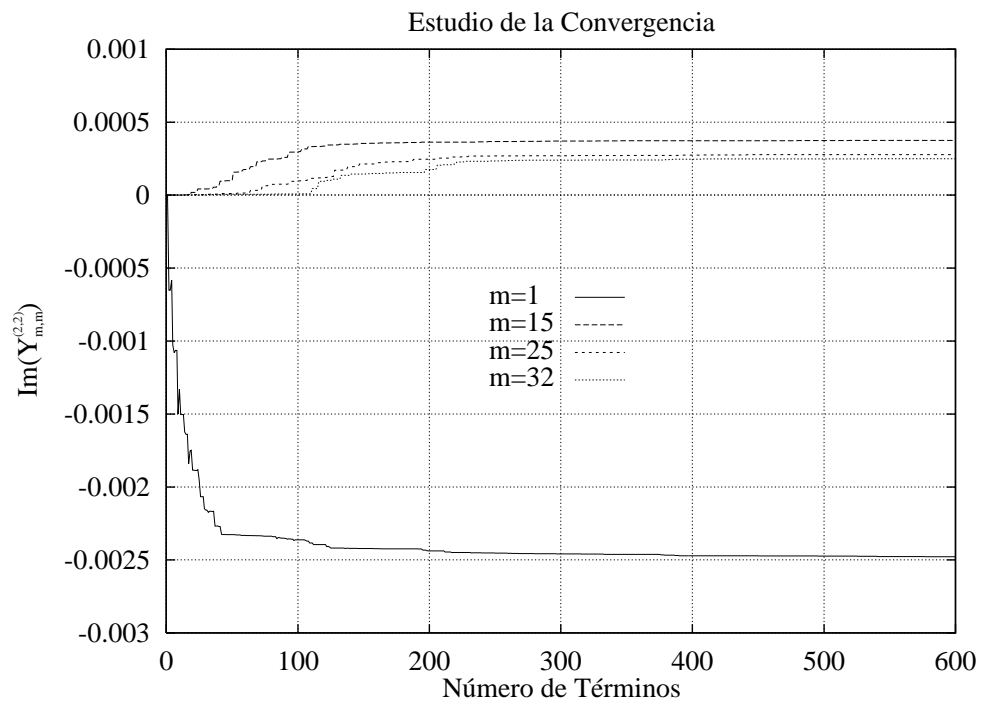

Figura 2.5: Convergencia de los elementos $Y_{m, m}^{(2,2)}(m=1,15,25,32)$ de la MAG de una unión planar entre una guía rectangular grande $(a=19.050 \mathrm{~mm}, b=9.525 \mathrm{~mm})$ y una guía rectangular pequeña $(a=5.100 \mathrm{~mm}, b=7.000 \mathrm{~mm})$ con un offset $(c=4.000 \mathrm{~mm}$, $d=0.750 \mathrm{~mm}$ ).

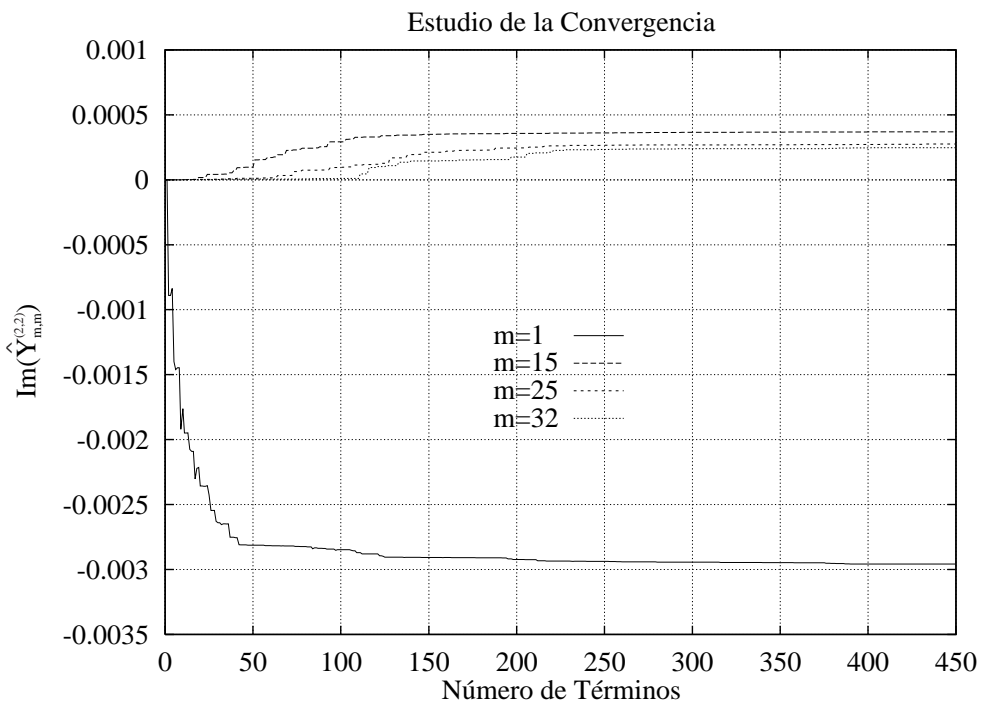

Figura 2.6: Convergencia de las series estáticas $\hat{Y}_{m, m}^{(2,2)}(m=1,15,25,32)$ de la MAG de una unión planar entre una guía rectangular grande $(a=19.050 \mathrm{~mm}$, $b=9.525 \mathrm{~mm})$ y una guía rectangular pequeña $(a=5.100 \mathrm{~mm}, b=7.000 \mathrm{~mm})$ con un offset $(c=4.000 \mathrm{~mm}, d=0.750 \mathrm{~mm})$.

mente; calculando dichas series según (2.11). La evolución del valor de las mencionadas series estáticas, en función del número de términos sumados en (2.11), se muestra en la figura 2.6; donde los significados del subíndice $m$ son los mismos 


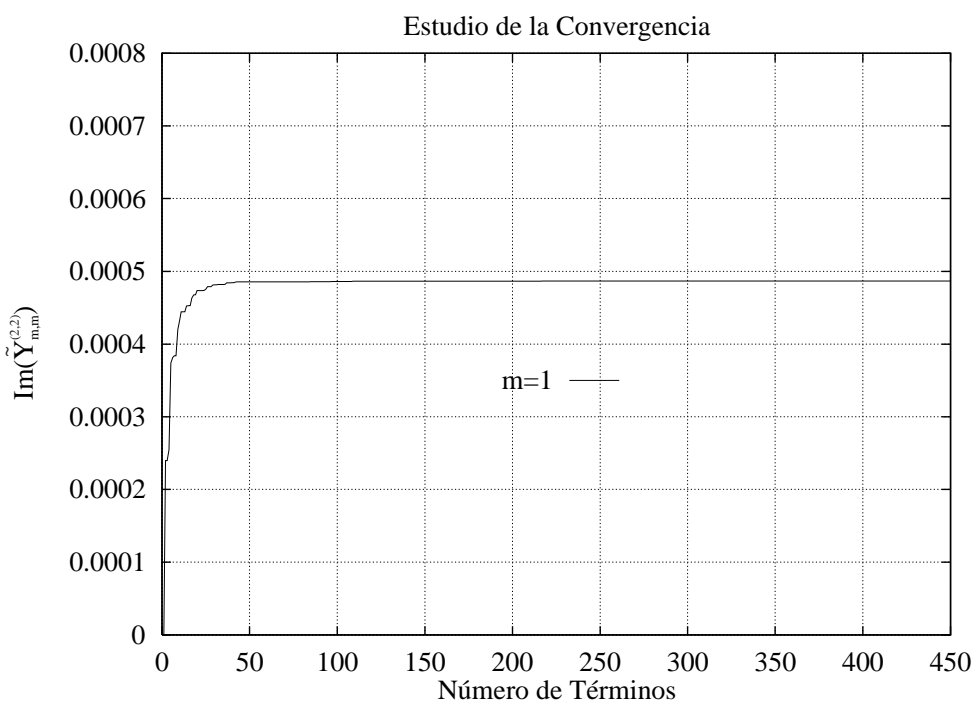

a)

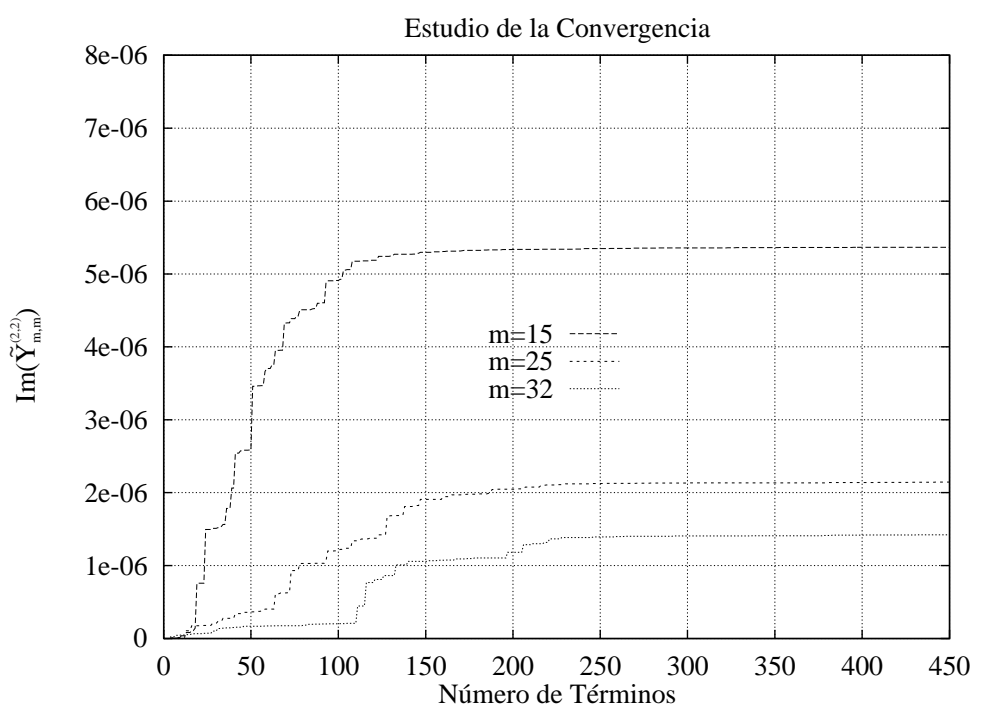

b)

Figura 2.7: Convergencia de las series dinámicas $\tilde{Y}_{m, m}^{(2,2)}(m=1,15,25,32)$ de la MAG de una unión planar entre una guía rectangular grande $(a=19.050 \mathrm{~mm}$, $b=9.525 \mathrm{~mm})$ y una guía rectangular pequeña $(a=5.100 \mathrm{~mm}, b=7.000 \mathrm{~mm})$ con un offset $(c=4.000 \mathrm{~mm}, d=0.750 \mathrm{~mm})$. En a) $m=1$, y en b) $m=15,25,32$.

que en el caso anterior. Observando la figura 2.6, se descubre que para obtener resultados convergentes al evaluar las series estáticas $\hat{Y}_{m, n}^{(2,2)}$ hacen falta sumar de nuevo 400 términos; tal y como era previsible.

Por último, se ofrece un análisis de la convergencia que presentan las series dinámicas $\tilde{Y}_{m, m}^{(2,2)}(m=1,15,25,32)$ de la MAG de la misma unión planar offset 
considerada previamente; obteniendo los valores de las citadas series dinámicas mediante la expresión deducida en (2.13). Los resultados obtenidos tras realizar este estudio de convergencia se resumen en la figura 2.7 ( $m=1$ en apartado a y $m=15,25,32$ en apartado b); donde los modos asociados a los distintos valores del subíndice $m$ son los mismos que en los dos casos anteriores. En la figura 2.7, se descubre que tan sólo se requiere sumar 250 términos en (2.13) para que las series dinámicas $\tilde{Y}_{m, n}^{(2,2)}$ alcancen valores estables.

Del estudio de convergencia recién presentado, se deduce que la técnica propuesta en este apartado para calcular los elementos $Y_{m, n}^{(2,2)}$ de la MAG de uniones planares, basada en el concepto de las series estática y dinámica, reduce de hecho el esfuerzo computacional que supone calcular los citados elementos $Y_{m, n}^{(2,2)}$; pues en dicha técnica sólo es necesario evaluar por cada punto en frecuencia las correspondientes series dinámicas, y éstas convergen más rápidamente que las expresiones originales recogidas en (2.10) para calcular los distintos elementos $Y_{m, n}^{(2,2)}$. A pesar de la reducción que introduce la técnica recién descrita en el coste computacional asociado al cálculo de los elementos $Y_{m, n}^{(2,2)}$, ésta no es su principal ventaja; pues la descomposición de dichos elementos en sus respectivas series estáticas y dinámicas permite, tal y como se demuestra en el próximo apartado mediante una adecuada manipulación de las series dinámicas, continuar reduciendo el tiempo requerido para calcular los elementos $Y_{m, n}^{(2,2)}$ de la MAG de uniones planares entre guías arbitrarias.

\subsubsection{Tratamiento Refinado de la Serie Dinámica}

Aunque la técnica de extracción en frecuencia, recién descrita en el apartado 2.2.1, reduce el esfuerzo computacional asociado al cálculo de los elementos $Y_{m, n}^{(2,2)}$ de la MAG de uniones planares; todavía es posible, mediante un tratamiento adecuado de las series dinámicas de los elementos $Y_{m, n}^{(2,2)}$, continuar reduciendo el coste computacional que requiere la obtención de dichos elementos. Así pues, considerando el elemento $Y_{m, n}^{(2,2)}$, la primera acción a tomar sobre su correspondiente serie dinámica $\tilde{Y}_{m, n}^{(2,2)}$ consiste en dividirla en dos nuevos términos: uno de ellos, el primero, que contiene las contribuciones proporcionadas a dicha serie por todos los modos que se propagan en la región (1) a la frecuencia de interés, y también las contribuciones procedentes de los primeros modos que a la citada frecuencia se encuentran al corte en dicha región (1); mientras el segundo término en el que se divide la serie dinámica $\tilde{Y}_{m, n}^{(2,2)}$ contiene las contribuciones del resto de modos en la región (1) que, a la frecuencia de análisis, se encuentran en corte. De esta manera, la serie dinámica $\tilde{Y}_{m, n}^{(2,2)}$ se expresa del siguiente modo

$$
\tilde{Y}_{m, n}^{(2,2)}=\tilde{\tilde{Y}}_{m, n}^{(2,2)}+\hat{\tilde{Y}}_{m, n}^{(2,2)}
$$

En $(2.16), \tilde{\tilde{Y}}_{m, n}^{(2,2)}$ e $\hat{\tilde{Y}}_{m, n}^{(2,2)}$ representan los dos términos descritos en el párrafo 
anterior; que se definen respectivamente mediante las siguientes expresiones

$$
\begin{gathered}
\tilde{\tilde{Y}}_{m, n}^{(2,2)}=(-j) \cdot \sum_{r=1}^{R}\left[\hat{Y}_{0 r}^{(1)} \cdot \cot \left(\hat{\beta}_{r}^{(1)} \cdot l_{\mathrm{ref}}\right)-Y_{0 r}^{(1)} \cdot \cot \left(\beta_{r}^{(1)} \cdot l_{\mathrm{ref}}\right)\right] \\
\cdot<\vec{e}_{r}^{(1)} \vec{e}_{n}^{(2)}>\cdot<\vec{h}_{r}^{(1)} \vec{h}_{m}^{(2)}> \\
\hat{\hat{Y}}_{m, n}^{(2,2)}=(-j) \cdot \sum_{r=R+1}^{\infty} \hat{Y}_{0 r}^{(1)} \cdot \cot \left(\hat{\beta}_{r}^{(1)} \cdot l_{\mathrm{ref}}\right) \cdot\left[1-\frac{Y_{0 r}^{(1)} \cdot \cot \left(\beta_{r}^{(1)} \cdot l_{\mathrm{ref}}\right)}{\hat{Y}_{0 r}^{(1)} \cdot \cot \left(\hat{\beta}_{r}^{(1)} \cdot l_{\mathrm{ref}}\right)}\right] \\
\cdot<\vec{e}_{r}^{(1)} \vec{e}_{n}^{(2)}>\cdot<\vec{h}_{r}^{(1)} \vec{h}_{m}^{(2)}>
\end{gathered}
$$

donde el valor del índice de truncamiento $R$, que divide la serie dinámica original en los dos términos recién expuestos, se escoge según el criterio

$$
k_{t, R+1}^{(1)}>\left.2 \cdot k\right|_{\omega=\omega_{\max }}
$$

Observando (2.19), se descubre que al determinar el valor de $R$ el número de onda $k$ se evalúa a la frecuencia máxima dentro de la banda de interés; pues de esta forma se garantiza, a cualquier frecuencia en la que se pretende caracterizar la unión planar bajo análisis, que todos los términos de la serie presente en (2.18) corresponden a modos en corte en la región (1).

En el intento de reducir el coste computacional que supone evaluar la serie dinámica $\tilde{Y}_{m, n}^{(2,2)}$ mediante $(2.16),(2.17)$ y $(2.18)$, conviene centrarse en el término encerrado entre corchetes presente en la última de estas tres expresiones. Dicho término, designado como $f_{r}(k)$, presenta pues el siguiente aspecto

$$
f_{r}(k)=1-\frac{Y_{0 r}^{(1)} \cdot \cot \left(\beta_{r}^{(1)} \cdot l_{\mathrm{ref}}\right)}{\hat{Y}_{0 r}^{(1)} \cdot \cot \left(\hat{\beta}_{r}^{(1)} \cdot l_{\mathrm{ref}}\right)}
$$

donde $Y_{0 r}^{(1)}$ y $\beta_{r}^{(1)}$ representan respectivamente la admitancia característica y la constante de propagación del $r$-ésimo modo en la región (1); cuyas expresiones, recogidas por ejemplo en [15], se ofrecen a continuación

$$
\begin{array}{ll}
Y_{0 r}^{(1)}= \begin{cases}(-j) \cdot \frac{\sqrt{\left(k_{t, r}^{(1)}\right)^{2}-(k)^{2}}}{\omega \cdot \mu} & \text { para modos TE } \\
j \cdot \frac{\omega \cdot \varepsilon}{\sqrt{\left(k_{t, r}^{(1)}\right)^{2}-(k)^{2}}} & \text { para modos TM }\end{cases} \\
\beta_{r}^{(1)}=(-j) \cdot \sqrt{\left(k_{t, r}^{(1)}\right)^{2}-(k)^{2}} \text { para modos TE y TM }
\end{array}
$$

Sustituyendo pues en (2.20) $Y_{0 r}^{(1)}$ y $\beta_{r}^{(1)}$ por sus respectivos valores recién mostrados en (2.21) y (2.22), así como $\hat{Y}_{0 r}^{(1)}$ y $\hat{\beta}_{r}^{(1)}$ por las correspondientes expresiones 
asintóticas recogidas en $(2.14)$ y $(2.15)$, es posible escribir la función $f_{r}(k)$ de la siguiente manera

$$
f_{r}(k)= \begin{cases}1-\frac{\sqrt{1-\left(k / k_{t, r}^{(1)}\right)^{2}} \cdot \operatorname{coth}\left(c \cdot \sqrt{1-\left(k / k_{t, r}^{(1)}\right)^{2}}\right)}{\operatorname{coth}(c)} & \text { para modos TE } \\ 1-\frac{\operatorname{coth}\left(c \cdot \sqrt{1-\left(k / k_{t, r}^{(1)}\right)^{2}}\right)}{\sqrt{1-\left(k / k_{t, r}^{(1)}\right)^{2}} \cdot \operatorname{coth}(c)} & \text { para modos TM }\end{cases}
$$

donde la constante $c$ se define como $c=\left(k_{t, r}^{(1)} \cdot l_{\text {ref }}\right)$. Con el objeto de observar el comportamiento de la función $f_{r}(k)$, tanto para modos TE como para modos $\mathrm{TM}$, en función de la variable normalizada $k / k_{t, r}^{(1)}$; se han evaluado las expresiones recogidas en (2.23) para un valor típico de la constante $c$, como puede ser $c=10$, en unos valores de la variable normalizada $k / k_{t, r}^{(1)}$ comprendidos entre 0 y 1 . Los resultados obtenidos se encuentran representados en la figura 2.8 (caso TE en apartado a y caso TM en apartado b); donde se observa que para valores pequeños de la variable normalizada $k / k_{t, r}^{(1)}$ (cuando $r \rightarrow \infty \Rightarrow k / k_{t, r}^{(1)} \rightarrow 0$ ) estas dos curvas presentan una evolución muy suave. Debido a este comportamiento de la función $f_{r}(k)$ en la zona correspondiente a valores de la variable normalizada $k / k_{t, r}^{(1)}$ próximos a 0 , es posible aproximar en dicha zona la citada función $f_{r}(k)$ por un simple desarrollo en serie de Taylor centrado en $k / k_{t, r}^{(1)}=0$; que se expresa tal y como se indica a continuación

$$
f_{r}(k)=\sum_{p=0}^{\infty} b_{p, r} \cdot\left(\frac{k}{k_{t, r}^{(1)}}\right)^{p}
$$

En (2.24), debe tenerse en cuenta que el desarrollo en serie de Taylor consta de dos posibles conjuntos de coeficientes $\left(b_{p, r}\right)$; que corresponden respectivamente al caso en el que $r$ hace referencia a un modo TE, y al caso en el que $r$ se refiere a un modo TM. Estudiando asimismo la expresión original de la función $f_{r}(k)$, recogida en (2.23), se descubre que dicha función tiene un comportamiento par tanto para modos TE como para modos TM, y que además presenta un valor igual a cero cuando $k / k_{t, r}^{(1)}=0$. De estas propiedades que presenta la función $f_{r}(k)$, es posible deducir de antemano que los coeficientes $b_{p, r}$ en (2.24) deben ser iguales a cero para valores impares del índice $p$ y cuando $p=0$. Las expresiones explícitas de los coeficientes $b_{p, r}$ no nulos del desarrollo en serie de Taylor de la citada función $f_{r}(k)$ pueden encontrarse en el apéndice A.

Utilizando pues en (2.18) el desarrollo en serie de Taylor de la función $f_{r}(k)$ presentado en (2.24), y haciendo uso de las consideraciones recién expuestas sobre 


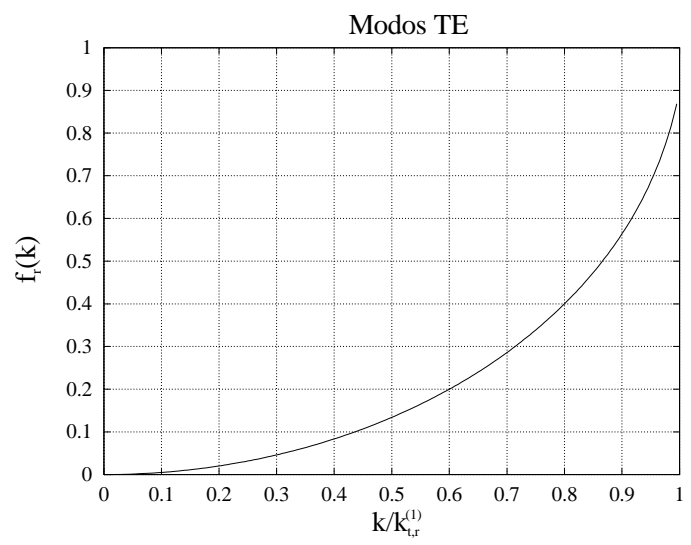

a)

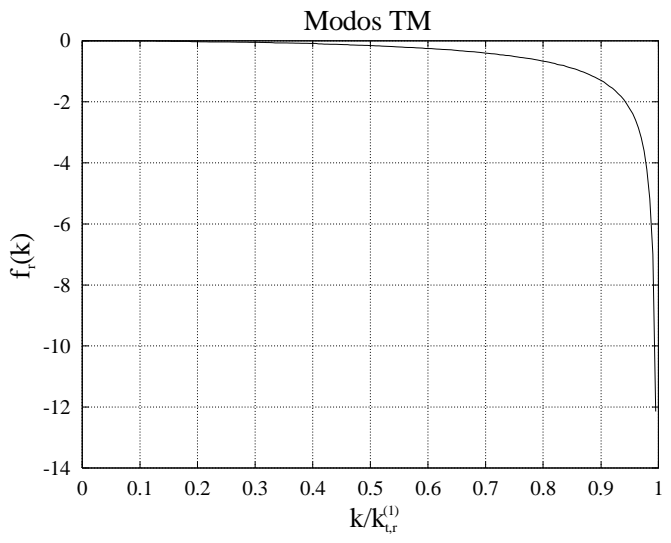

b)

Figura 2.8: Evolución de la función $f_{r}(k)$ frente a la variable normalizada $k / k_{t, r}^{(1)}$ para modos TE en a); y para modos TM en b).

los coeficientes de dicho desarrollo, el término $\hat{\tilde{Y}}_{m, n}^{(2,2)}$ se reescribe como

$$
\begin{aligned}
\hat{\tilde{Y}}_{m, n}^{(2,2)}=(-j) \cdot \sum_{p=1}^{\infty} k^{(2 p)} & \cdot \sum_{r=R+1}^{\infty} \frac{b_{2 p, r}}{\left(k_{t, r}^{(1)}\right)^{(2 p)}} \cdot \hat{Y}_{0 r}^{(1)} \cdot \cot \left(\hat{\beta}_{r}^{(1)} \cdot l_{\mathrm{ref}}\right) \\
\cdot & <\vec{e}_{r}^{(1)} \vec{e}_{n}^{(2)}>\cdot\left\langle\vec{h}_{r}^{(1)} \vec{h}_{m}^{(2)}>\right.
\end{aligned}
$$

Las series asociadas al índice $r$ en (2.25) ya no dependen de la frecuencia de análisis; y por tanto se pueden calcular, al igual que la serie estática (recordar apartado 2.2.1), una sola vez antes de iniciar el bucle en frecuencia. Por esta razón, a dichas series se las denominará partes estáticas de la serie dinámica. En cuanto a la primera serie que aparece en (2.25), con índice $p$, ésta debe calcularse sin embargo para cada posible valor de la frecuencia. No obstante, debido a la elección del valor del índice de truncamiento $R$ siguiendo el criterio definido en (2.19), dicha serie asociada en (2.25) al índice $p$ puede calcularse sumando tan sólo 4 términos; ya que este truncamiento del número de términos a sumar para calcular la citada serie, a causa de la aproximación de la función $f_{r}(k)$ por su correspondiente desarrollo en serie de Taylor mostrado en (2.24), supone cometer un error máximo al evaluar dicha función $f_{r}(k)$ de un orden de magnitud $O\left(0.5 \cdot 10^{-9}\right)$.

Así pues, el término $\hat{\tilde{Y}}_{m, n}^{(2,2)}$ se expresa finalmente, tras implement
operación de truncamiento recién descrita, del siguiente modo
\[ \hat{\tilde{Y}}_{m, n}^{(2,2)}=(-j) \cdot \sum_{p=1}^{4} k^{(2 p)} \cdot \sum_{r=R+1}^{\infty} \frac{b_{2 p, r}}{\left(k_{t, r}^{(1)}\right)^{(2 p)}} \cdot \hat{Y}_{0 r}^{(1)} \cdot \cot \left(\hat{\beta}_{r}^{(1)} \cdot l_{\text {ref }}\right) \]

$$
\cdot<\vec{e}_{r}^{(1)} \vec{e}_{n}^{(2)}>\cdot<\vec{h}_{r}^{(1)} \vec{h}_{m}^{(2)}>
$$


Para estudiar la convergencia de las partes estáticas de la serie dinámica que aparecen en (2.26), se considera el mismo tipo de unión planar offset empleada en los análisis de convergencia realizados en el apartado 2.2.1; es decir, una unión planar como la mostrada en la figura 2.4 entre una guía rectangular grande $(a=19.050 \mathrm{~mm}, b=9.525 \mathrm{~mm})$ y una guía rectangular pequeña $(a=5.100 \mathrm{~mm}$, $b=7.000 \mathrm{~mm})$ con un offset $(c=4.000 \mathrm{~mm}, d=0.750 \mathrm{~mm})$. Los resultados de este estudio de convergencia de las partes estáticas $(p=1)$ de algunos términos descritos según (2.26) se muestran en la figura 2.9; donde, nuevamente, los valores $m=1,15,25,32$ determinan respectivamente los modos $\mathrm{TE}_{01}, \mathrm{TM}_{22}, \mathrm{TM}_{32}$ y $\mathrm{TM}_{15}$ de la guía rectangular pequeña. De estos resultados recogidos en la figura 2.9, se concluye que para obtener valores numéricamente estables en las mencionadas partes estáticas $(p=1)$ de las series dinámicas, en el caso de la unión planar offset considerada, tan sólo es necesario sumar unos 250 términos en (2.26). Tal y como era de esperar, este número de términos es básicamente idéntico al que se dedujo en el apartado 2.2.1 para las series dinámicas originales; aunque, en el caso de las partes estáticas de dichas series dinámicas, el cálculo de sus respectivos valores puede implementarse una sola vez y fuera del bucle en frecuencia, a diferencia de lo que ocurre con las series dinámicas. Por lo que respecta a la convergencia del resto de partes estáticas $(p=2,3,4)$ de las series dinámicas, presentes también en (2.26), es más rápida que la asociada a la parte estática $(p=1)$; y por tanto, para no extender en demasía el contenido de este trabajo, no se ha considerado interesante incluir los correspondientes estudios de convergencia de las mencionadas partes estáticas $(p=2,3,4)$.

El estudio de convergencia recién expuesto revela la conveniencia de calcular las series dinámicas, asociadas a los elementos $Y_{m, n}^{(2,2)}$ de la MAG de uniones planares, mediante la técnica descrita en el presente apartado; puesto que la nueva técnica propuesta, complementada con el procedimiento descrito en el apartado 2.2.1 para calcular las series estáticas de los citados elementos $Y_{m, n}^{(2,2)}$, reduce considerablemente el esfuerzo computacional requerido en el cálculo de dichos elementos. Así pues, combinando las relaciones recogidas en (2.12) y (2.16), se obtiene la siguiente expresión final que permite calcular los elementos $Y_{m, n}^{(2,2)}$ de la MAG de una unión planar

$$
Y_{m, n}^{(2,2)}=\hat{Y}_{m, n}^{(2,2)}-\hat{\tilde{Y}}_{m, n}^{(2,2)}-\tilde{\tilde{Y}}_{m, n}^{(2,2)}
$$

donde las series $\hat{Y}_{m, n}^{(2,2)}$, definidas según (2.11), y las series con índice $r$, que están asociadas a los términos descritos mediante (2.26), representan contribuciones estáticas al valor de los elementos $Y_{m, n}^{(2,2)}$; ya que dichas series deben calcularse una sola vez antes de iniciar el bucle de frecuencias de análisis de la unión planar bajo estudio. El único esfuerzo computacional que debe realizarse en cada frecuencia de análisis está asociado a la evaluación de los sumatorios finitos presentes en los términos obtenidos según (2.26), y a la obtención de los términos de las series 


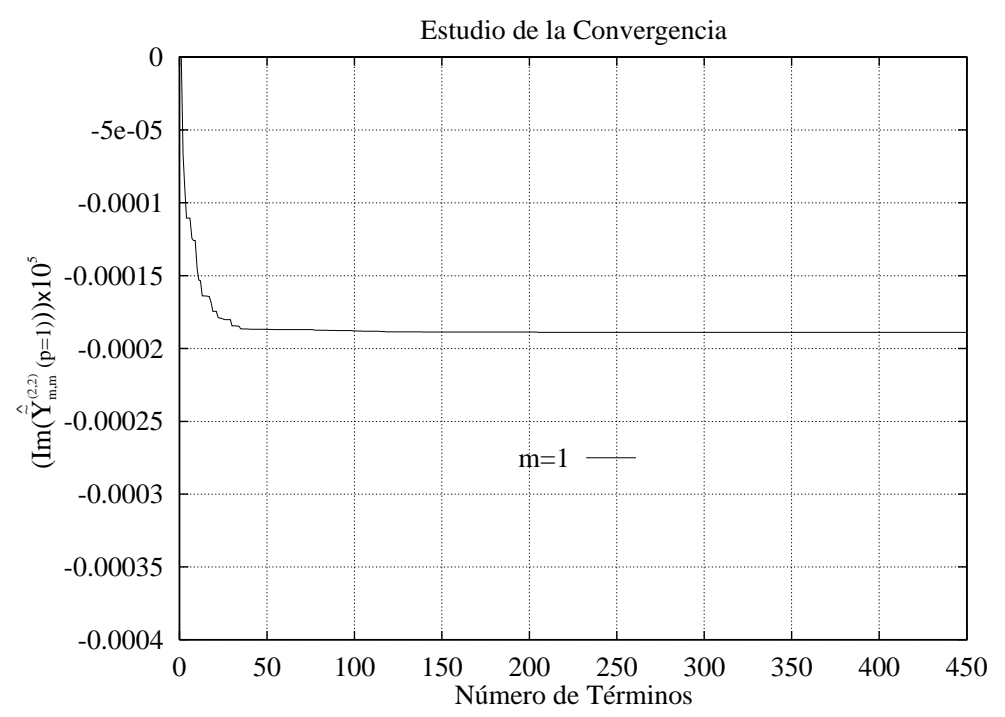

a)

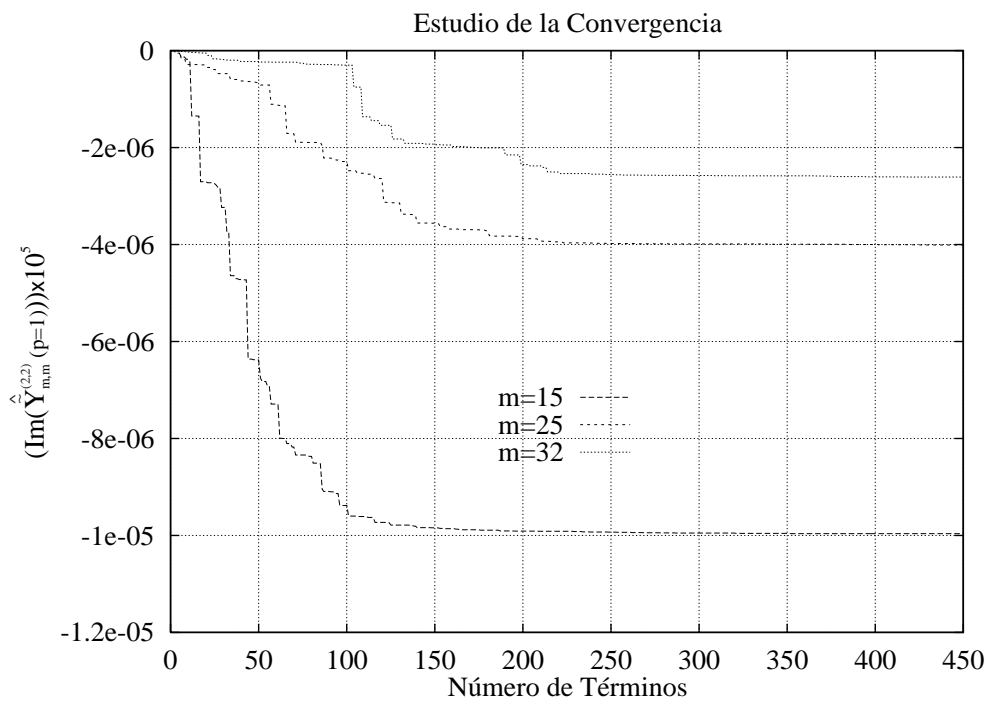

b)

Figura 2.9: Convergencia de las partes estáticas $(p=1)$ de $\hat{\tilde{Y}}_{m, m}^{(2,2)}(m=1,15,25,32)$ de la MAG de una unión planar entre una guía rectangular grande $(a=19.050 \mathrm{~mm}$, $b=9.525 \mathrm{~mm})$ y una guía rectangular pequeña $(a=5.100 \mathrm{~mm}, b=7.000 \mathrm{~mm})$ con un offset $(c=4.000 \mathrm{~mm}, d=0.750 \mathrm{~mm})$. En a) $m=1$, y en b) $m=15,25,32$.

dinámicas expresados mediante (2.17). No obstante, estos cálculos que deben implementarse en cada frecuencia de análisis requieren sumar, como máximo, 10 términos; lo que reduce enormemente el coste computacional requerido al caracterizar en frecuencia a la unión planar. 
Para concluir, conviene observar que esta técnica recién expuesta para caracterizar uniones planares mediante su correspondiente MAG es realmente independiente del tipo de guías que integran la unión bajo análisis; y que por tanto dicha técnica puede utilizarse en la caracterización electromagnética de uniones planares entre guías cuyos modos sólo pueden obtenerse numéricamente. Asimismo, comparando esta nueva técnica acelerada del cálculo de la MAG con el método basado en adaptación modal [2-5], se descubre que esta técnica clásica de análisis conocida también como mode-matching requiere realizar una inversión matricial en cada frecuencia; mientras la nueva técnica propuesta en este apartado evalúa las correspondientes series infinitas fuera del bucle en frecuencia, resultando pues mucho más eficiente desde el punto de vista computacional que el procedimiento de análisis basado en adaptación modal.

\subsubsection{Eficiencia Computacional de la Técnica de Acelera- ción}

Para demostrar la mejora que representa, en términos de eficiencia computacional, el empleo de la técnica propuesta en el apartado 2.2.2 para calcular los elementos $Y_{m, n}^{(2,2)}$ de la MAG de uniones planares, en este apartado se presenta un estudio comparativo del coste temporal que supone analizar una estructura típica de microondas; empleando en dicho análisis la nueva técnica descrita, así como los otros dos procedimientos de cálculo de los elementos $Y_{m, n}^{(2,2)}$ expuestos en la sección 2.1 y en el apartado 2.2.1. La estructura escogida para realizar el mencionado estudio comparativo se muestra en la figura 2.10; donde las guías de entrada y salida corresponden a guías normalizadas WR-75 $(a=19.050 \mathrm{~mm}$, $b=9.525 \mathrm{~mm}$ ), las ventanas de acoplo de entrada y salida presentan una sección transversal rectangular $(a=8.700 \mathrm{~mm}, b=9.525 \mathrm{~mm})$ con una profundidad de $2.000 \mathrm{~mm}$, los elementos de sintonía situados en ambas ventanas de acoplo tienen sección transversal cuadrada $(2.000 \mathrm{~mm} \times 2.000 \mathrm{~mm})$ y una penetración igual a $3.650 \mathrm{~mm}$, y finalmente la cavidad resonante corresponde de nuevo a una guía normalizada WR-75 de longitud $13.886 \mathrm{~mm}$. El análisis electromagnético de la estructura propuesta requiere pues, tras observar detalladamente la figura 2.10 , la caracterización previa de diversas uniones planares centradas entre guías rectangulares con diferentes dimensiones, así como la caracterización de varias transiciones entre guías rectangulares y guías reentrantes (en inglés ridge). En el estudio de estas últimas transiciones se precisa tener conocimiento del espectro modal de las guías reentrantes, así como de las integrales de acoplamiento entre los modos de dichas guías y los modos de las correspondientes guías rectangulares; modos e integrales que se han obtenido de forma numérica empleando el método propuesto en [21] y [22], resumido en el apartado 3.1.1 del capítulo 3.

Con el objeto de implementar el estudio comparativo recién descrito, se han calculado los elementos $Y_{m, n}^{(2,2)}$ de las MAGs de las distintas uniones planares que 


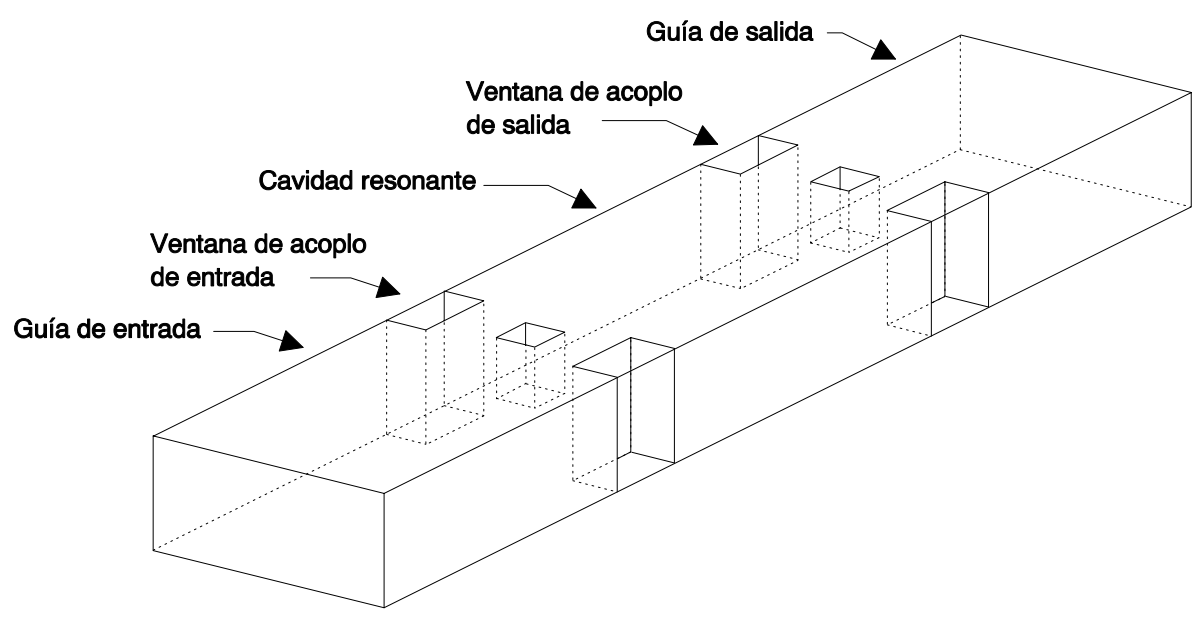

Figura 2.10: Estructura guiada constituida por uniones planares centradas entre guías rectangulares de diferentes dimensiones, y por transiciones entre guías rectangulares y guías reentrantes.

integran la estructura bajo estudio (ver figura 2.10) de tres maneras diferentes: la primera de ellas consiste en calcular los elementos $Y_{m, n}^{(2,2)}$ de cada unión empleando la definición original de dichos elementos recogida en (2.7), la segunda manera consiste en calcular los citados elementos $Y_{m, n}^{(2,2)}$ descomponiendo su cálculo en las series estáticas y dinámicas definidas según (2.11) y (2.13), y la tercera manera se basa en calcular los elementos $Y_{m, n}^{(2,2)}$ mediante la técnica propuesta en el apartado 2.2 .2 que utiliza las expresiones (2.11), (2.17) y (2.26). Los parámetros eléctricos calculados, siguiendo las tres estrategias expuestas, han sido los parámetros de dispersión (en inglés scattering) asociados al modo fundamental que presenta la estructura escogida (recordar fig. 2.10). Estos parámetros de dispersión se obtienen tras resolver un sistema lineal en banda; el cual surge al conectar en cascada, como se indica en la sección 2.3, las correspondientes MAGs individuales de las distintas uniones y tramos de guía que integran el dispositivo a caracterizar. En cuanto a la inversión del sistema en banda, se ha utilizado la técnica de eliminación Gaussiana con retrosustitución [23], y se ha tenido en cuenta además la naturaleza en banda del sistema a invertir; lo que reduce el coste computacional de la citada técnica de inversión de sistemas. Así pues, el análisis de la respuesta electromagnética de la estructura mostrada en la figura 2.10 se ha realizado para 100 valores de la frecuencia en la banda comprendida entre $10.80 \mathrm{GHz}$ y $11.20 \mathrm{GHz}$; habiendo realizado las diferentes simulaciones en una plataforma IBM Risc-6000. En la tabla 2.1, se ofrecen los diferentes tiempos totales (divididos por el número de frecuencias de análisis) empleados por cada método en el análisis de la estructura mostrada en la figura 2.10, incluyendo en dicho tiempo las diversas computaciones estáticas realizadas, cuando se consideran 20 y 32 modos de orden superior en todas las redes equivalentes multimodales 


\begin{tabular}{|l|c|c|}
\hline \hline \multirow{2}{*}{$\begin{array}{c}\text { Método implementado } \\
\text { para calcular } \mathbf{Y}_{\mathbf{m}, \mathbf{n}}^{(\mathbf{2})}\end{array}$} & \multicolumn{2}{|c|}{ Número de modos } \\
\cline { 2 - 3 } & $\mathbf{2 0}$ & $\mathbf{3 2}$ \\
\hline 1. Cálculo directo de las series & $5.713 \mathrm{seg}$ & $16.590 \mathrm{seg}$ \\
\hline 2. Series estáticas y dinámicas & $2.558 \mathrm{seg}$ & $6.824 \mathrm{seg}$ \\
\hline 3. Nueva técnica de aceleración & $0.536 \mathrm{seg}$ & $1.666 \mathrm{seg}$ \\
\hline \hline
\end{tabular}

Tabla 2.1: Tiempo total de cálculo que requiere el análisis de la estructura mostrada en la figura 2.10 dividido por el número (100) de frecuencias consideradas.

de la estrucutra analizada. En dicha tabla, los métodos designados como 1, 2 y 3 hacen referencia a los tres procedimientos seguidos en la computación de los elementos $Y_{m, n}^{(2,2)}$ de las distintas MAGs; procedimientos que se encuentran descritos respectivamente en la sección 2.1, en el apartado 2.2.1, y en el apartado 2.2.2. Observando pues los resultados mostrados en la tabla 2.1, se comprueba que la nueva técnica propuesta en el apartado 2.2.2 precisa de un menor esfuerzo computacional, tal y como era previsible, al analizar el comportamiento electromagnético del dispositivo seleccionado.

En el intento de cuantificar con mayor claridad los resultados recogidos en la tabla 2.1, se define un factor de mejora entre los diferentes métodos empleados al caracterizar la estructura mostrada en la figura 2.10; que se obtiene dividiendo entre sí los distintos tiempos empleados por dichos métodos en la caracterización electromagnética de la citada estructura. Los resultados correspondientes a este análisis comparativo de los distintos métodos propuestos, en función del recientemente definido factor de mejora, se muestran en la tabla 2.2; concluyendo, a partir de estos resultados, que la técnica propuesta en el apartado 2.2 .2 para calcular los elementos $Y_{m, n}^{(2,2)}$ de la MAG de uniones planares introduce, en el caso de la estructura considerada (recordar fig. 2.10), un factor de mejora de valor 10 con respecto a la técnica clásica descrita en la sección 2.1 para obtener los citados elementos $Y_{m, n}^{(2,2)}$. Conviene resaltar que este importante ahorro computacional, en un factor de valor 10, se ha obtenido en el análisis electromagnético de una estructura pasiva de microondas como la mostrada en la figura 2.10; que tan sólo consta de 8 uniones planares. Consecuentemente, si esta misma técnica de cálculo de los elementos $Y_{m, n}^{(2,2)}$ de uniones planares se aplica a estructuras más complejas, como por ejemplo al análisis de un filtro completo de microondas o a una red conformadora de haces, el citado factor de mejora aumentará en consonancia con la complejidad del dispositivo a caracterizar; lo que permite pensar pues, empleando la mencionada técnica, en el análisis e incluso en el diseño de dispositivos en la banda de microondas realmente complejos.

Así pues, tras el estudio de eficiencia computacional recién presentado, es posible concluir que el procedimiento descrito en el apartado 2.2.2 para acelerar el cálculo de los elementos $Y_{m, n}^{(2,2)}$ reduce, de manera sustancial, el tiempo empleado 


\begin{tabular}{|c|c|c|}
\hline \hline \multirow{2}{*}{ Factor de Mejora } & \multicolumn{2}{|c|}{ Número de modos } \\
\cline { 2 - 3 } & $\mathbf{2 0}$ & $\mathbf{3 2}$ \\
\hline$F_{2,1}$ & 2.233 & 2.431 \\
\hline$F_{3,2}$ & 4.772 & 4.096 \\
\hline$F_{3,1}$ & 10.659 & 9.958 \\
\hline \hline
\end{tabular}

Tabla 2.2: Factor de mejora entre los diferentes métodos; definido como el cociente de los tiempos de cálculo requeridos por los diversos métodos propuestos en el análisis de la estructura mostrada en la figura 2.10 .

por el método original (ver sección 2.1) en la obtención de dichos elementos de la MAG de uniones planares; resultando por tanto muy interesante la incorporación de este procedimiento de cálculo acelerado a herramientas de programación, basadas en el concepto de la matriz de admitancias generalizada, que permiten analizar y diseñar estructuras pasivas de microondas.

\subsubsection{Análisis de Septums Cortocircuitados y Cavidades de Reacción mediante la Técnica de Aceleración}

Tras haber comprobado la mejora que supone, en términos de eficiencia computacional, la aplicación de la técnica descrita en el apartado 2.2.2 para calcular los elementos $Y_{m, n}^{(2,2)}$ de la MAG de uniones planares, en el presente apartado se pretende validar dicha técnica mediante su aplicación al análisis electromagnético de septums cortocircuitados en plano $\mathrm{E}$, y de cavidades de reacción en plano $\mathrm{E}$ integradas por los mencionados septums; elementos que se emplean en el diseño de redes adaptadoras y filtros de banda eliminada. La respuesta electromagnética de estas dos estructuras ya se ha determinado en la literatura empleando otras técnicas clásicas de análisis, tales como por ejemplo adaptación modal (modematching) en [24]; permitiendo pues, mediante una simple comparación de los resultados ofrecidos en este apartado con los recogidos en [24], comprobar la precisión numérica que se obtiene al analizar estructuras pasivas de microondas utilizando la técnica de aceleración presentada anteriormente.

La primera estructura a analizar corresponde a un septum cortocircuitado en plano E; cuyo aspecto puede observarse en la figura 2.11 (vista en perspectiva en apartado a). Observando la sección longitudinal de dicho septum (ver apartado b en fig. 2.11), se descubre que consta de dos discontinuidades básicas: una de ellas consistente en una bifurcación en plano E de la guía de entrada (con dimensiones $A_{1}$ y $B_{1}$ ) en dos guías intermedias (de dimensiones $A_{1}$ y $B_{2}, A_{1}$ y $B_{3}$, respectivamente), y una segunda discontinuidad constituida por un simple cambio de altura entre una guía intermedia (aquélla con dimensiones $A_{1}$ y $B_{3}$ ) y la guía de salida del septum (nuevamente de dimensiones $A_{1}$ y $B_{1}$ ). Adicionalmente a estas 


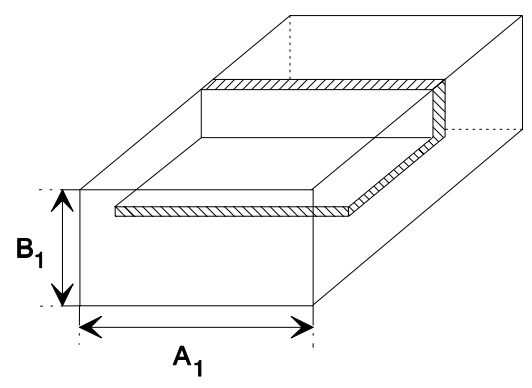

a)

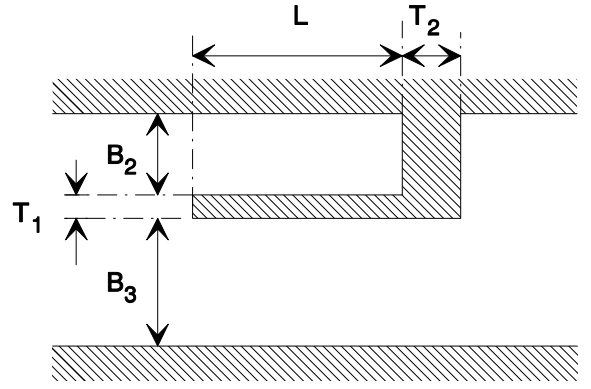

b)

Figura 2.11: Septum cortocircuitado en plano E. Vista en perspectiva en a), y sección longitudinal en $b$ ).

dos discontinuidades, para completar la estructura a caracterizar, es necesario considerar (ver de nuevo apartado b en fig. 2.11) dos tramos de guía uniforme: uno de ellos, de longitud $L$ y terminado en un cortocircuito físico, que está asociado a una de las guías intermedias de la estructura (en concreto a la de dimensiones $A_{1}$ y $\left.B_{2}\right)$; y un segundo tramo, en este caso de longitud $L+T_{2}$ y asociado a la segunda de las guías intermedias (aquélla con dimensiones $A_{1}$ y $B_{3}$ ), que conecta las dos discontinuidades de la estructura descritas previamente.

Para determinar la respuesta electromagnética del septum bajo análisis, se requiere en primer lugar construir las redes equivalentes multimodales, expresadas mediante las correspondientes matrices de admitancias generalizadas, que corresponden a los elementos (discontinuidades y tramos de guía) integrantes del septum. Comenzando pues por la primera de las discontinuidades (o transiciones) descritas en el párrafo anterior, concretamente con aquélla asociada a la bifurcación en plano E de la guía de entrada, se obtienen las siguientes expresiones (recordar sección 2.1) para los elementos $Y_{m, n t 1}$ de su MAG

$$
\begin{gathered}
Y_{m, n t 1}^{(1,1)}=(-j) \cdot Y_{0 n}^{(1)} \cdot \cot \left(\beta_{n}^{(1)} \cdot l_{\mathrm{ref}}\right) \cdot \delta_{m, n} \\
Y_{m, n t 1}^{(2,1)}=Y_{n, m t 1}^{(1,2)}=j \cdot Y_{0 n}^{(1)} \cdot \csc \left(\beta_{n}^{(1)} \cdot l_{\mathrm{ref}}\right) \cdot<\vec{h}_{n}^{(1)} \vec{h}_{m}^{(2)}> \\
Y_{m, n t 1}^{(3,1)}=Y_{n, m t 1}^{(1,3)}=j \cdot Y_{0 n}^{(1)} \cdot \csc \left(\beta_{n}^{(1)} \cdot l_{\mathrm{ref}}\right) \cdot<\vec{h}_{n}^{(1)} \vec{h}_{m}^{(3)}> \\
Y_{m, n t 1}^{(2,2)}=(-j) \cdot \sum_{r=1}^{\infty} Y_{0 r}^{(1)} \cdot \cot \left(\beta_{r}^{(1)} \cdot l_{\mathrm{ref}}\right) \cdot<\vec{e}_{r}^{(1)} \vec{e}_{n}^{(2)}>\cdot<\vec{h}_{r}^{(1)} \vec{h}_{m}^{(2)}> \\
Y_{m, n t 1}^{(3,2)}=Y_{n, m t 1}^{(2,3)}=(-j) \cdot \sum_{r=1}^{\infty} Y_{0 r}^{(1)} \cdot \cot \left(\beta_{r}^{(1)} \cdot l_{\mathrm{ref}}\right) \cdot<\vec{e}_{r}^{(1)} \vec{e}_{n}^{(2)}>\cdot<\vec{h}_{r}^{(1)} \vec{h}_{m}^{(3)}> \\
Y_{m, n t 1}^{(3,3)}=(-j) \cdot \sum_{r=1}^{\infty} Y_{0 r}^{(1)} \cdot \cot \left(\beta_{r}^{(1)} \cdot l_{\mathrm{ref}}\right) \cdot<\vec{e}_{r}^{(1)} \vec{e}_{n}^{(3)}>\cdot<\vec{h}_{r}^{(1)} \vec{h}_{m}^{(3)}>
\end{gathered}
$$


En (2.28)-(2.33), los términos $Y_{0 n}^{(1)}, Y_{0 r}^{(1)}, \beta_{n}^{(1)}$ y $\beta_{r}^{(1)}$ representan las admitancias características y las constantes de propagación asociadas respectivamente al $n$-ésimo y $r$-ésimo modo de la guía de entrada del septum (ver fig. 2.11); mientras la longitud $l_{\text {ref }}$ determina la separación entre dos planos de referencia, situado uno de ellos en la mencionada guía de entrada, y el otro en el plano donde se produce la bifurcación en plano E de dicha guía. En cuanto al término $\delta_{m, n}$, que tan sólo aparece en (2.28), representa la función delta de Kronecker; cuyo valor se encuentra recogido en (2.5). Por lo que respecta a las funciones vectoriales modales normalizadas $\vec{h}_{p}^{(\delta)}$ y $\vec{e}_{q}^{(\delta)}(p=m, n, r, q=n, r$ y $\delta=1,2,3)$ presentes en (2.28)-(2.33), definidas por ejemplo en [15], determinan respectivamente los campos magnético y eléctrico transversales asociados a los correspondientes modos ( $p$ o $q$ según el caso), normalizados en potencia, que pertenecen a la guía $\delta\left(\delta=1\right.$ para la guía de entrada con dimensiones $A_{1}$ y $B_{1}, \delta=2$ para la guía intermedia de dimensiones $A_{1}$ y $B_{2}$, y $\delta=3$ para la guía intermedia de dimensiones $A_{1}$ y $\left.B_{3}\right)$. Finalmente, los símbolos $\langle>$ en $(2.29)-(2.33)$ designan productos interiores entre las anteriores funciones vectoriales normalizadas; cuyas expresiones analíticas definitivas son bastante simples, las integrales de acoplamiento se reducen a integrar productos de funciones seno y funciones coseno, y por tanto no se incluyen en el presente trabajo para no extenderlo en demasía.

Esta primera discontinuidad del septum, a diferencia de las uniones planares consideradas en la sección 2.1, se caracteriza mediante una MAG constituida por nueve bloques; ya que dicha discontinuidad implica tres puertos o regiones. El cálculo de los elementos $Y_{m, n t 1}^{(1,1)}, Y_{m, n t 1}^{(2,1)}, Y_{m, n t 1}^{(1,2)}, Y_{m, n t 1}^{(3,1)}$ e $Y_{m, n t 1}^{(1,3)}$, al igual que ocurría con los elementos $Y_{m, n}^{(1,1)}, Y_{m, n}^{(2,1)}$ e $Y_{m, n}^{(1,2)}$ de la MAG de uniones planares entre dos guías arbitrarias, se realiza de manera muy rápida implementando en esta ocasión las operaciones descritas en (2.28)-(2.30). Sin embargo, para calcular los elementos $Y_{m, n t 1}^{(2,2)}, Y_{m, n t 1}^{(3,2)}, Y_{m, n t 1}^{(2,3)}$ e $Y_{m, n t 1}^{(3,3)}$, al igual que sucedía con los elementos $Y_{m, n}^{(2,2)}$ de la MAG de uniones planares entre dos guías, resulta necesario evaluar unos sumatorios que en este caso se definen mediante las expresiones recogidas en (2.31)-(2.33); operación que, tal y como se mencionó en la sección 2.1, supone un importante coste computacional en la determinación de los citados elementos de la MAG. Ahora bien, debido a la similitud de las expresiones (2.31)-(2.33) con aquélla mostrada en (2.7) para los elementos $Y_{m, n}^{(2,2)}$ de la MAG de uniones planares entre dos guías, es posible aplicar la técnica de aceleración descrita en el apartado 2.2 .2 al cálculo de los citados elementos $Y_{m, n t 1}^{(2,2)}, Y_{m, n t 1}^{(3,2)}, Y_{m, n t 1}^{(2,3)}$ e $Y_{m, n t 1}^{(3,3)}$; lo que reducirá enormemente el coste computacional asociado al análisis del septum, cuya parte más costosa resulta precisamente la caracterización de esta primera discontinuidad. De esta manera, aplicando la mencionada técnica de aceleración, se ha obtenido la MAG asociada a la primera de las dos transiciones que integran el septum a caracterizar.

Una vez obtenidos los elementos de la MAG asociada a la primera discontinuidad del septum bajo análisis, observando detalladamente la figura 2.11, se 
descubre que es posible obtener la MAG de la segunda discontinuidad (o transición) de dicho septum, correspondiente al cambio de altura entre una de las guías intermedias (aquélla con dimensiones $A_{1}$ y $B_{3}$ ) y la guía de salida del septum (de dimensiones $A_{1}$ y $B_{1}$ ), a partir de los elementos obtenidos previamente para la MAG de la primera transición. Así pues, considerando en esta segunda transición la guía intermedia como región (1) de esta unión planar y la guía de salida como región (2), se deducen las siguientes expresiones para los elementos de la MAG de esta segunda discontinuidad

$$
\begin{aligned}
Y_{m, n t 2}^{(1,1)} & =Y_{m, n t 1}^{(3,3)} \\
Y_{m, n t 2}^{(2,1)} & =Y_{n, m t 2}^{(1,2)}=Y_{m, n t 1}^{(1,3)} \\
Y_{m, n t 2}^{(2,2)} & =Y_{m, n t 1}^{(1,1)}
\end{aligned}
$$

Por lo que respecta al primero de los dos tramos de guía uniforme que integran el septum en plano E bajo estudio, concretamente aquél de longitud $L$ constituido por una guía intermedia (de dimensiones $A_{1}$ y $B_{2}$ ) terminada en cortocircuito, los elementos de la MAG que definen su correspondiente red equivalente multimodal se construyen [25] de la siguiente manera

$$
\begin{aligned}
& Y_{m, n g 1}^{(1,1)}=Y_{m, n g 1}^{(2,2)}=(-j) \cdot Y_{0 n}^{(1)} \cdot \cot \left(\beta_{n}^{(1)} \cdot l_{g 1}\right) \cdot \delta_{m, n} \\
& Y_{m, n g 1}^{(2,1)}=Y_{m, n g 1}^{(1,2)}=j \cdot Y_{0 n}^{(1)} \cdot \csc \left(\beta_{n}^{(1)} \cdot l_{g 1}\right) \cdot \delta_{m, n}
\end{aligned}
$$

donde $Y_{0 n}^{(1)}$ y $\beta_{n}^{(1)}$ hacen referencia a la admitancia característica y a la constante de propagación del $n$-ésimo modo en el tramo de guía considerado (aquél con dimensiones $A_{1}$ y $B_{2}$ ), el término $\delta_{m, n}$ designa nuevamente una función delta de Kronecker definida según (2.5), y el parámetro $l_{g 1}$ corresponde a la longitud $(L)$ de dicho tramo de guía uniforme del septum.

Para concluir con las redes equivalentes multimodales de los diferentes elementos (transiciones y tramos de guía) que integran el septum a analizar, se requiere determinar los elementos de la MAG del segundo tramo de guía de dicho septum, de longitud $L+T_{2}$ y asociado a la segunda de las guías intermedias (aquélla con dimensiones $A_{1}$ y $\left.B_{3}\right)$; cuyas expresiones, muy similares a las recién mostradas en $(2.37)$ y (2.38), presentan el siguiente aspecto

$$
\begin{aligned}
& Y_{m, n g 2}^{(1,1)}=Y_{m, n g 2}^{(2,2)}=(-j) \cdot Y_{0 n}^{(1)} \cdot \cot \left(\beta_{n}^{(1)} \cdot l_{g 2}\right) \cdot \delta_{m, n} \\
& Y_{m, n g 2}^{(2,1)}=Y_{m, n g 2}^{(1,2)}=j \cdot Y_{0 n}^{(1)} \cdot \csc \left(\beta_{n}^{(1)} \cdot l_{g 2}\right) \cdot \delta_{m, n}
\end{aligned}
$$

donde el significado de $Y_{0 n}^{(1)}$ y $\beta_{n}^{(1)}$ es idéntico al atribuido a estos mismos parámetros en (2.37) y (2.38), aunque en este caso se refieren a los modos de la segunda guía intermedia del septum (de dimensiones $A_{1}$ y $B_{3}$ ), y de nuevo los posibles 
valores de la función $\delta_{m, n}$ (delta de Kronecker) se resumen en (2.5). En cuanto al parámetro $l_{g 2}$, en este segundo tramo de guía uniforme representa precisamente la longitud $\left(L+T_{2}\right)$ de dicho tramo.

Tras caracterizar el comportamiento de los distintos elementos (transiciones y tramos de guía) del septum bajo análisis, mediante sus correspondientes matrices de admitancias generalizadas, para determinar la respuesta electromagnética del septum es necesario conectar estas redes equivalentes multimodales tal y como se indica en la figura 2.12; conexiones que surgen al imponer las correspondientes condiciones de continuidad, resumidas a continuación en términos de los voltajes y corrientes modales, de los campos eléctricos y magnéticos transversales definidos en los puertos de las diferentes redes equivalentes multimodales

$$
\begin{aligned}
& V_{t 1}^{(2)}=V_{g 1}^{(1)} \\
& I_{t 1}^{(2)}=-I_{g 1}^{(1)} \\
& V_{t 1}^{(3)}=V_{g 2}^{(1)} \\
& I_{t 1}^{(3)}=-I_{g 2}^{(1)} \\
& V_{g 1}^{(2)}=O \\
& V_{g 2}^{(2)}=V_{t 2}^{(1)} \\
& I_{g 2}^{(2)}=-I_{t 2}^{(1)}
\end{aligned}
$$

donde los diferentes términos $V$ e $I$ que aparecen en las condiciones de contorno recién expuestas representan vectores columna, constituidos respectivamente por voltajes o corrientes modales, cuyas dimensiones dependen del número de modos escogidos para representar los campos eléctricos y magnéticos en las diferentes guías del septum; mientras $O$ designa un vector columna nulo (todos los elementos iguales a 0), de dimensión igual al número de modos considerados para describir los campos eléctrico y magnético existentes en la primera de las guías intermedias (con dimensiones $A_{1}$ y $B_{2}$ ).

Con el objeto de plantear un conjunto de ecuaciones cuya resolución permita determinar el comportamiento electromagnético del septum, es necesario seleccionar inicialmente una serie de incógnitas; que en este caso se corresponden con los voltajes modales (vectores columna) $V_{t 1}^{(1)}, V_{t 1}^{(2)}, V_{t 1}^{(3)}, V_{g 2}^{(2)}$ y $V_{t 2}^{(2)}$ asociados a distintos puertos de las redes equivalentes multimodales (ver fig. 2.12). Al pretender resolver 5 incógnitas, será necesario plantear el mismo número de ecuaciones; debiendo utilizar para ello las condiciones de continuidad planteadas en (2.42), (2.44) y (2.47) en función de las respectivas corrientes modales (vectores columna). Ahora bien, dichas condiciones permiten obtener tan sólo 3 de las 5 ecuaciones matriciales requeridas; deduciendo las 2 restantes de las relaciones 


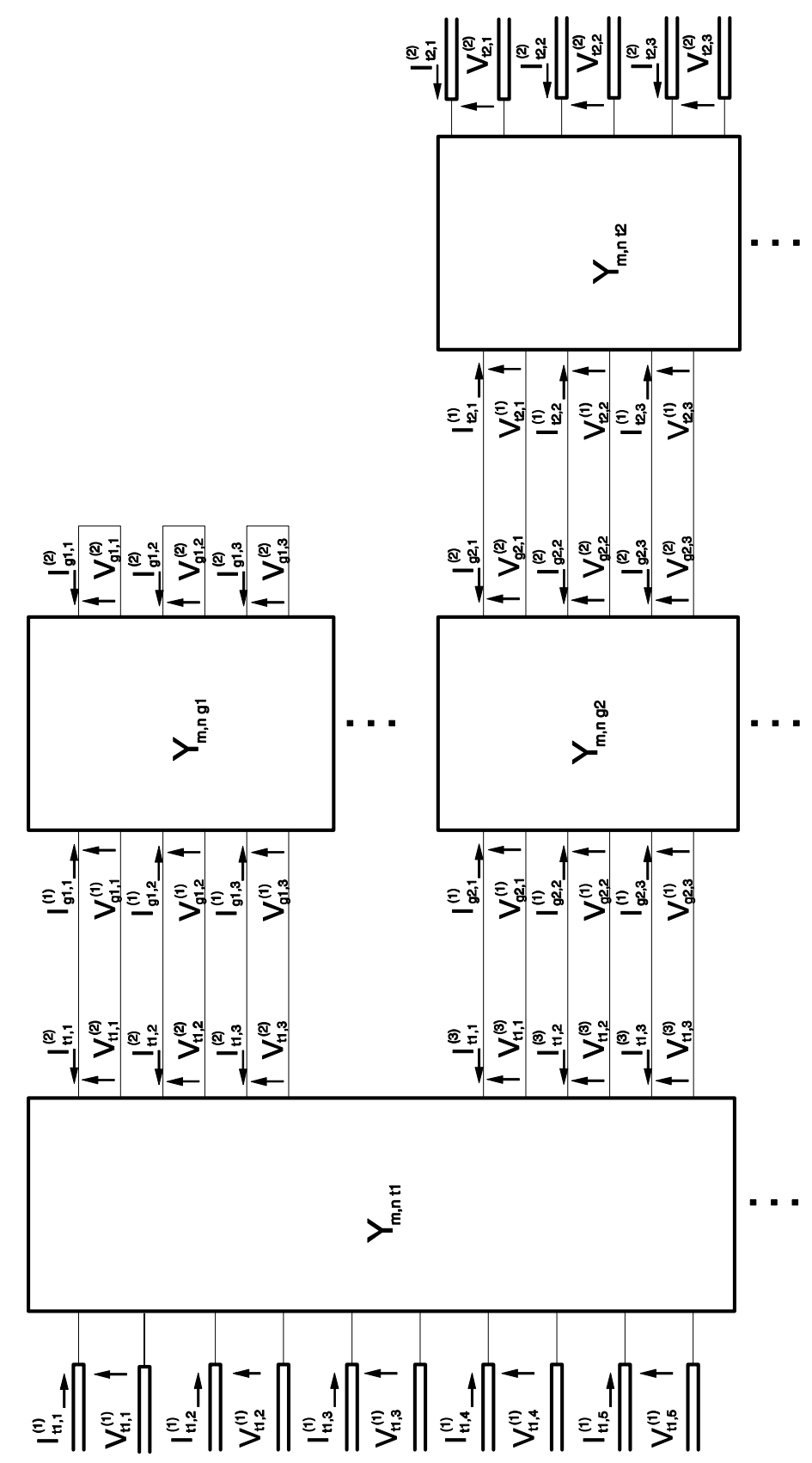

Figura 2.12: Conexión de las redes equivalentes multimodales, definidas mediante las correspondientes matrices de admitancias generalizadas, que corresponden a los elementos integrantes de un septum cortocircuitado en plano E como el mostrado en la figura 2.11 .

entre voltajes y corrientes modales (ambos vectores columna) que determinan las matrices $Y_{m, n t 1}$ e $Y_{m, n t 2}$. Utilizando pues las mencionadas relaciones matriciales, así como las condiciones de continuidad expuestas mediante las expresiones (2.41), 
(2.43), (2.45) y (2.46) en términos de los voltajes modales (vectores columna), es posible deducir el siguiente conjunto de ecuaciones que definen el comportamiento del septum a analizar

$$
\begin{aligned}
I_{t 1}^{(1)}= & Y_{t 1}^{(1,1)} \cdot V_{t 1}^{(1)}+Y_{t 1}^{(1,2)} \cdot V_{t 1}^{(2)}+Y_{t 1}^{(1,3)} \cdot V_{t 1}^{(3)} \\
O= & Y_{t 1}^{(2,1)} \cdot V_{t 1}^{(1)}+\left(Y_{t 1}^{(2,2)}+Y_{g 1}^{(1,1)}\right) \cdot V_{t 1}^{(2)}+Y_{t 1}^{(2,3)} \cdot V_{t 1}^{(3)} \\
O= & Y_{t 1}^{(3,1)} \cdot V_{t 1}^{(1)}+Y_{t 1}^{(3,2)} \cdot V_{t 1}^{(2)}+\left(Y_{t 1}^{(3,3)}+Y_{g 2}^{(1,1)}\right) \cdot V_{t 1}^{(3)} \\
& +Y_{g 2}^{(1,2)} \cdot V_{g 2}^{(2)} \\
O= & Y_{g 2}^{(2,1)} \cdot V_{t 1}^{(3)}+\left(Y_{g 2}^{(2,2)}+Y_{t 2}^{(1,1)}\right) \cdot V_{g 2}^{(2)}+Y_{t 2}^{(1,2)} \cdot V_{t 2}^{(2)} \\
I_{t 2}^{(2)}= & Y_{t 2}^{(2,1)} \cdot V_{g 2}^{(2)}+Y_{t 2}^{(2,2)} \cdot V_{t 2}^{(2)}
\end{aligned}
$$

Para determinar la respuesta electromagnética del septum considerado, se ha calculado el parámetro de dispersión $S_{11}$ asociado al modo fundamental que presenta dicho septum. El cálculo de este parámetro de dispersión requiere cargar en la figura 2.12, con la admitancia característica del modo apropiado en cada caso, todos los accesos libres de las redes equivalentes multimodales asociadas a las guías de entrada y salida del septum; a excepción claro está del acceso correspondiente en la red multimodal de la guía de entrada al modo fundamental, que representa la excitación del dispositivo. Al imponer esta condición de carga en el conjunto de ecuaciones recogidas en (2.48)-(2.52), de manera similar a como se describe con detalle en la sección 2.3 (apartados 2.3.2 y 2.3.3), se obtiene un nuevo sistema de 5 ecuaciones con 5 incógnitas; donde las 5 incógnitas representan ahora vectores columna integrados por unos elementos denominados autoimpedancia y transimpedancias, que se definen (ver concepto en apartado 2.3.2) como cocientes entre voltajes modales y la corriente asociada al modo fundamental. En cuanto a la naturaleza de este sistema de ecuaciones lineales a resolver para analizar el septum, debido al tipo de elementos (discontinuidades y tramos de guía) que integran dicho dispositivo y a su disposición, el mencionado sistema de ecuaciones presenta una estructura en banda; empleando por tanto en su resolución una técnica basada en eliminación Gausssiana con retrosustitución [23] que tiene en cuenta la citada estructura en banda del sistema.

Los resultados correspondientes al módulo del parámetro de dispersión $S_{11}$, respecto del modo fundamental $\left(\mathrm{TE}_{10}\right)$ en la guía de entrada del septum, se recogen en la figura 2.13 para diferentes valores en la dimensión $B_{2}\left(B_{2}=0.5 \mathrm{~mm}\right.$, $0.8 \mathrm{~mm}, 1.2 \mathrm{~mm}, 1.6 \mathrm{~mm}, 2.4 \mathrm{~mm}$ ) de un septum cortocircuitado en plano $\mathrm{E}$ $\left(A_{1}=22.86 \mathrm{~mm}, B_{1}=10.16 \mathrm{~mm}, T_{1}=T_{2}=0.035 \mathrm{~mm}, L=9.00 \mathrm{~mm}\right)$. En dicha figura, se observa que para una longitud fija $(L)$ del septum cortocircuitado, a medida que la altura $\left(B_{2}\right)$ del septum crece, la frecuencia de resonancia en los resultados (aquélla a la que $\left|S_{11}\right|=0 \mathrm{~dB}$ ) disminuye. De estos resultados (ver fig. 2.13), puede concluirse que el septum cortocircuitado en plano E se comporta 


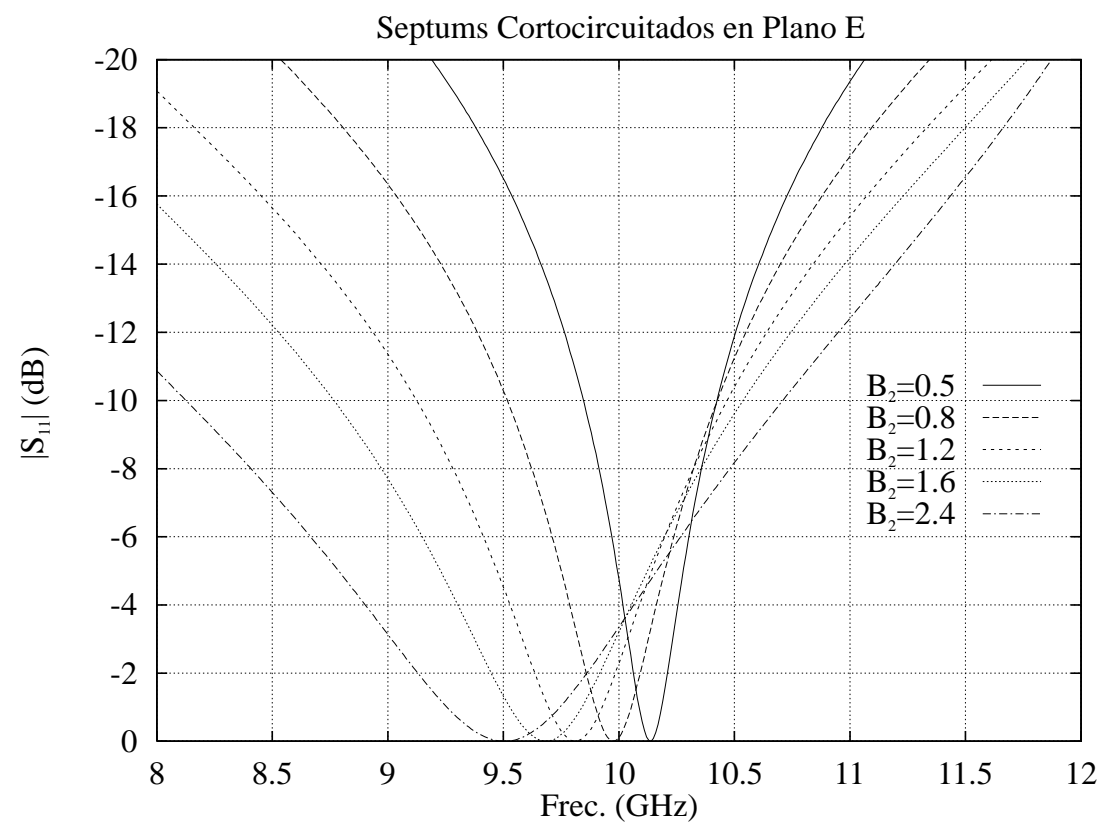

Figura 2.13: Módulo del parámetro $S_{11}$ para diferentes valores (en $\mathrm{mm}$ ) de la dimensión $B_{2}$ de un septum cortocircuitado en plano E $\left(A_{1}=22.86 \mathrm{~mm}, B_{1}=10.16 \mathrm{~mm}\right.$, $T_{1}=T_{2}=0.035 \mathrm{~mm}, L=9.00 \mathrm{~mm}$ ).

como un tramo de línea (en inglés stub) terminado en cortocircuito; el cual se encuentra situado en serie con la línea de transmisión equivalente [15] asociada al modo fundamental de la guía de entrada del septum analizado. Lógicamente, toda la señal que incide sobre el septum se refleja $\left(\left|S_{11}\right|=0 \mathrm{~dB}\right)$ a un valor de la frecuencia tal que la longitud $(L)$ del septum es aproximadamente igual a $\lambda_{g 1} / 4$; donde $\lambda_{g 1}$ representa la longitud de onda asociada al modo fundamental en el tramo de guía cortocircuitado (con dimensiones $A_{1}$ y $B_{2}$ ). Debido a este tipo de comportamiento electromagnético del septum en plano $\mathrm{E}$ cortorcircuitado, esta estructura puede utilizarse como elemento integrante de redes adaptadoras en circuitos pasivos o incluso activos de microondas.

Con el objeto de comprobar la precisión conseguida en los resultados de la figura 2.13, calculados empleando matrices de admitancias generalizadas en algunas de las cuales ciertos elementos se han obtenido siguiendo el procedimiento expuesto en el apartado 2.2.2, dichos resultados se comparan con aquéllos presentados en [24, pág. 3]; que corresponden a los mismos septums analizados anteriormente, pero deducidos empleando matrices de dispersión generalizadas obtenidas mediante adaptación modal. De esta comparación, se observa que ambos resultados son exáctamente idénticos; lo que permite concluir que la técnica propuesta en el apartado 2.2.2 para calcular de manera acelerada algunos de los elementos de la MAG de uniones planares genera resultados, no sólo con un menor coste computacional que la técnica clásica descrita en la sección 2.1 para obtener 
dichos elementos, sino con la misma precisión que otros métodos tradicionales de análisis de discontinuidades en guías (como por ejemplo el método basado en matrices de dispersión genealizadas deducidas empleando adaptación modal). En cuanto al coste computacional asociado a la generación de los resultados de la figura 2.13, habiendo realizado las simulaciones en una plataforma HP-9000/735, éste ha sido de aproximadamente unos 5 seg para cada frecuencia de análisis. Este tiempo de cálculo, siendo aún relativamente bajo en comparación con el coste computacional que hubiera supuesto emplear la técnica original descrita en la sección 2.1, presenta este valor al haber empleado en las simulaciones 40 modos en todos los puertos de las diferentes redes equivalente multimodales que integran la estructura analizada (recordar fig. 2.12); número de modos relativamente alto, pero requerido para obtener resultados convergentes. Una posible explicación a este elevado número de modos requeridos para obtener resultados convergentes procede del tipo de modos escogidos, $\mathrm{TE}^{\mathrm{z}}$ y $\mathrm{TM}^{\mathrm{z}}$, para representar los campos eléctricos y magnéticos en el septum; que se han elegido para poder incluir dicha estructura en dispositivos pasivos de microondas que excitan todos los modos, tanto los $\mathrm{TE}^{\mathrm{z}}$ como los $\mathrm{TM}^{\mathrm{z}}$. Realmente, si se pretendiera analizar el comportamiento de septums aislados o incluidos en estructuras que tan sólo presentan discontinuidades en plano E, tal y como se recoge en [24], deberían considerarse únicamente aquellos modos $\left(\mathrm{TE}_{\mathrm{mn}}^{\mathrm{x}}\right)$ que se excitan ante la incidencia del modo fundamental $\left(\mathrm{TE}_{10}^{\mathrm{z}}\right)$; lo que reduciría, al haber escogido el juego de modos óptimos que se excitan en la estructura, el número de dichos modos necesarios para obtener resultados convergentes. Aunque [24] no presenta tiempos de computación con los que poder comparar los obtenidos empleando el método descrito en el apartado 2.2.2, debido a las diversas inversiones que debe realizar el método expuesto en [24] para cada frecuencia de análisis, parece lógico pensar que el coste computacional de esta técnica será superior al asociado a la técnica de aceleración empleada al generar los resultados mostrados en la figura 2.13. Adicionalmente, otro inconveniente asociado al análisis de discontinuidades empleando la matriz de dispersión generalizada calculada mediante adaptación modal es el conocido fenómeno de la convergencia relativa [6,7]; que requiere, para obtener resultados convergentes en el análisis de las discontinuidades, unas combinaciones de modos en las distintas redes equivalentes multimodales con las mismas relaciones de proporcionalidad que presentan las dimensiones de las guías implicadas en dichas discontinuidades. La técnica de análisis propuesta en este capítulo, basada en la caracterización de las discontinuidades mediante matrices de admitancias generalizadas, no presenta el problema de la convergencia relativa; es decir, alcanzado un número de modos suficiente en cada uno de los puertos de las redes equivalentes multimodales, cualquier posible combinación de números de modos en dichos puertos genera resultados igualmente correctos.

A continuación, tras haber analizado la respuesta de un septum cortocircuitado en plano E (recordar fig. 2.11), se pretende caracterizar el comportamiento 


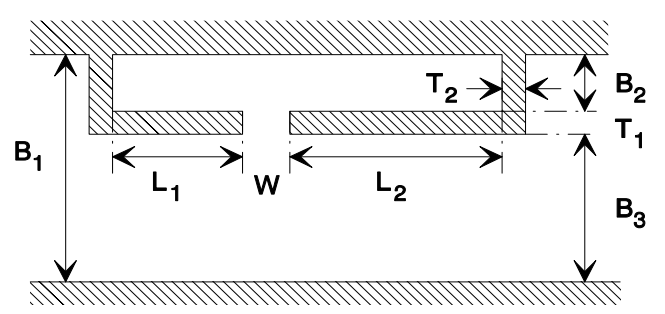

a)

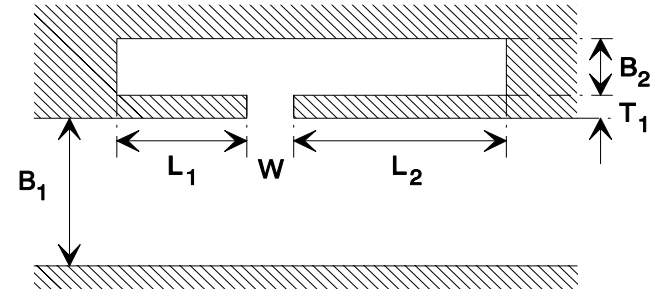

b)

Figura 2.14: Cavidad de reacción en plano E situada dentro de la guía rectangular en a), y cavidad de reacción en plano E situada fuera de la guía rectangular en b).

electromagnético de dos tipos de cavidades de reacción mostradas en la figura 2.14; una de ellas situada dentro de la guía rectangular (ver apartado a en fig. 2.14), y la otra situada fuera de la guía rectangular (ver apartado b en fig. 2.14). Considerando inicialmente la primera de las dos cavidades de reacción, se descrubre que dicha estructura consta de dos septums cortocircuitados en plano E como los analizados anteriormente, situados en direcciones opuestas, que se conectan entre sí mediante una guía intermedia (con dimensiones $A_{1}$ y $B_{1}$ ) de longitud $W$.

Así pues, para analizar la cavidad de reacción considerada inicialmente (apartado a en fig. 2.14), es necesario conectar las redes equivalentes multimodales de los elementos (discontinuidades y tramos de guía) que integran los dos septums de la cavidad de reacción. En cuanto a las redes multimodales asociadas a los elementos de cada septum, éstas se conectan de igual manera que en la figura 2.12; mientras estos dos conjuntos de redes, que se corresponden respectivamente con cada uno de los dos septums, deben conectarse entre sí en cascada haciendo uso de la red equivalente multimodal del tramo de guía situado entre dichos septums. Los elementos de la matriz de admitancias, asociada a esta última red equivalente multimodal del tramo de guía que conecta los dos septums, se calculan utilizando por ejemplo las mismas expresiones recogidas en (2.37) y (2.38) para el primer tramo de guía de los septums; teniendo en cuenta que en este caso las admitancias características y las constantes de propagación hacen referencia a los modos del tramo de guía intermedio de la cavidad (aquél con dimensiones $A_{1}$ y $B_{1}$ ), y que la longitud a utilizar para este tramo de guía se obtiene sustrayendo a la longitud física $W$ del tramo de guía original las longitudes $l_{\text {ref }}$, escogidas para separar los planos de referencia utilizados en el análisis de la primera discontinuidad de los dos septums que integran la cavidad (recordar fig. 2.1).

La respuesta electromagnética de esta primera cavidad de reacción se obtiene calculando el parámetro de dispersión $S_{11}$, asociado al modo fundamental ( $\mathrm{TE}_{10}^{\mathrm{z}}$ ), que presenta dicha cavidad. La obtención de este parámetro requiere, de igual manera que en el caso del septum analizado previamente, cargar todos los accesos de las redes multimodales asociadas a las guías de entrada y salida de la cavidad con 


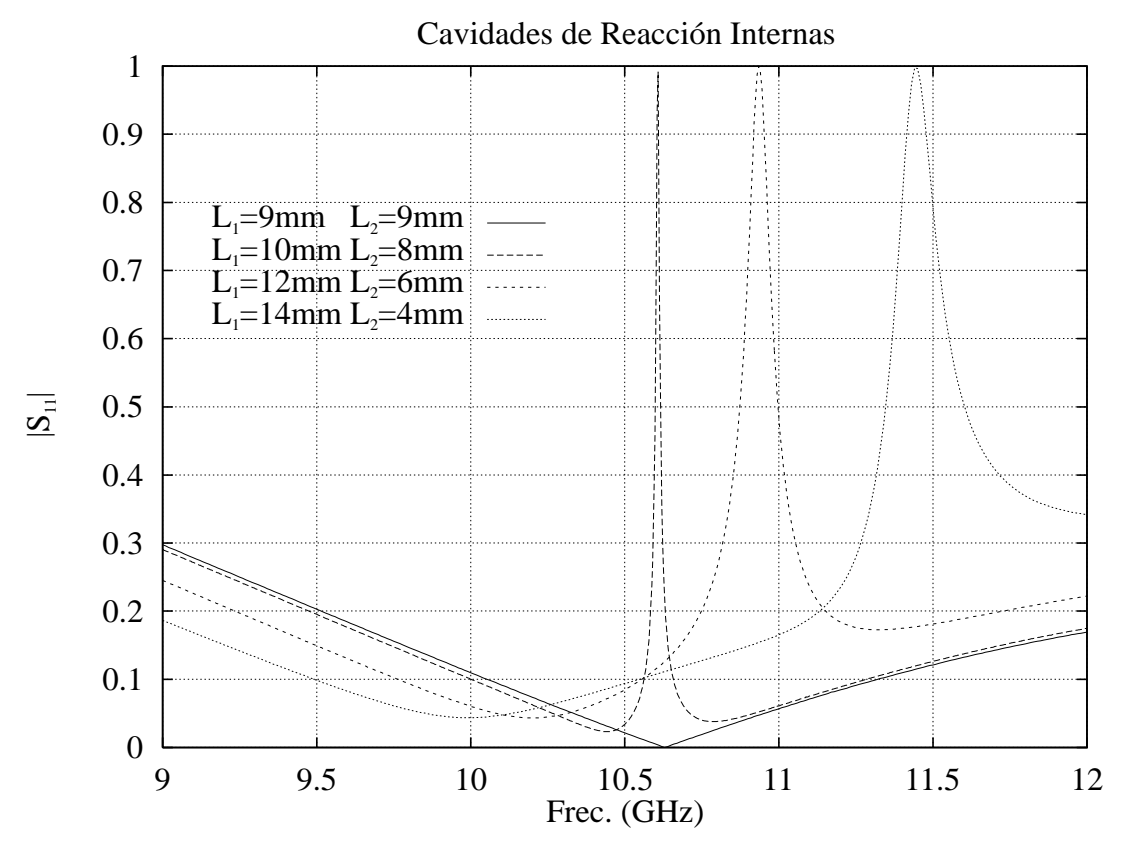

Figura 2.15: Módulo del parámetro $S_{11}$ para diferentes valores de las dimensiones $L_{1}$ y $L_{2}$ de una cavidad de reacción en plano E situada dentro de la guía rectangular $\left(A_{1}=22.86 \mathrm{~mm}, B_{1}=10.16 \mathrm{~mm}, B_{2}=2.06 \mathrm{~mm}, W=0.10 \mathrm{~mm}, T_{1}=T_{2}=0.10 \mathrm{~mm}\right.$, $\left.L_{1}+L_{2}+W=18.10 \mathrm{~mm}\right)$.

las admitancias características de los respectivos modos; a excepción del acceso asociado al modo fundamental en la guía de entrada, que constituye la excitación del dispositivo a caracterizar. De esta manera, se plantea un sistema de ecuaciones matriciales, con un mayor número de ecuaciones e incógnitas que el planteado anteriormente para el caso del septum, cuya resolución permite encontrar el parámetro de dispersión buscado. La resolución de este sistema de ecuaciones, al presentar de nuevo una estructura en banda, se implementa utilizando un método basado en eliminación Gaussiana con retrosustitución [23] que tiene en cuenta la naturaleza en banda del sistema. En la figura 2.15, se representa el módulo del parámetro $S_{11}$, calculado respecto del modo fundamental $\left(\mathrm{TE}_{10}^{\mathrm{z}}\right)$ de la guía de entrada de la cavidad, que presenta una cavidad de reacción en plano E situada dentro de la guía $\left(A_{1}=22.86 \mathrm{~mm}, B_{1}=10.16 \mathrm{~mm}, B_{2}=2.06 \mathrm{~mm}, W=0.10 \mathrm{~mm}\right.$, $T_{1}=T_{2}=0.10 \mathrm{~mm}$ ) para diferentes combinaciones en los valores de las longitudes $L_{1}$ y $L_{2}\left(L_{1}=L_{2}=9 \mathrm{~mm}, L_{1}=10 \mathrm{~mm}\right.$ y $L_{2}=8 \mathrm{~mm}, L_{1}=12 \mathrm{~mm}$ y $L_{2}=6 \mathrm{~mm}$, $L_{1}=14 \mathrm{~mm}$ y $L_{2}=4 \mathrm{~mm}$ ); observando en dicha figura que para la misma longitud total de la cavidad $\left(L_{1}+L_{2}+W=18.10 \mathrm{~mm}\right)$, la frecuencia de resonancia depende fuertemente de la posición en la cavidad del tramo de guía intermedio que conecta los dos septums de la cavidad. Es interesante observar además como, para el caso en el que este tramo de guía intermedio está situado en el centro de la cavidad de reacción $\left(L_{1}=L_{2}=9 \mathrm{~mm}\right.$ ), la respuesta de la cavidad no presenta 
ningún tipo de selectividad con la frecuencia; debido a que en dicha situación no se produce acoplo entre las guías de entrada y salida y la propia cavidad. Para el resto de casos considerados en la figura 2.15, la respuesta en frecuencia permite concluir que esta primera cavidad de reacción puede emplearse en el diseño de filtros de banda eliminada; los cuales, debido a la ubicación de la cavidad dentro de la guía, presentarán un tamaño pequeño, un peso bajo, y consecuentemente un coste reducido.

Los resultados mostrados en la figura 2.15 se han obtenido, tal y como se ha descrito con anterioridad, utilizando redes equivalentes multimodales de los elementos integrantes de la cavidad basadas en las correspondientes matrices de admitancias generalizadas; en algunas de las cuales ciertos elementos se han obtenido empleando el procedimiento descrito en el apartado 2.2.2. Para validar nuevamente este método, es posible comparar los resultados ofrecidos en la figura 2.15 con aquéllos recogidos en [24, pág. 4] para los mismos casos considerados en dicha figura; aunque el procedimiento seguido en [24] para obtener los resultados emplea matrices de dispersión generalizadas, obtenidas mediante adaptación modal. Comparando pues ambos resultados, se observa que son idénticos; lo que refuerza la conclusión de que el método de cálculo acelerado de algunos elementos de la MAG, no sólo ahorra tiempo de cálculo, sino que también genera resultados tan precisos como los obtenidos siguiendo otros métodos clásicos (por ejemplo el método basado en adaptación modal empleado en [24]). En el análisis de esta primera cavidad de reacción, para definir los campos eléctricos y magnéticos en los diferentes elementos que integran dicha cavidad, se han vuelto a emplear modos $\mathrm{TE}^{\mathrm{z}}$ y modos $\mathrm{TM}^{\mathrm{z}}$; pensando principalmente en la posible utilización de la cavidad de reacción en circuitos con discontinuidades que puedan excitar dichos modos. Concretamente, en la simulación de los resultados recogidos en la figura 2.15, se han empleado 55 modos en las guías de entrada, salida, y en la guía intermedia de la cavidad que conecta los dos septums; mientras en los tramos de guía cortocircuitados (con dimensiones $A_{1}$ y $B_{2}$ ), así como en los otros tramos de guía (con dimensiones $A_{1}$ y $B_{3}$ ), se han utilizado tan sólo 35 modos. Estos números de modos se escogen, lógicamente, tras realizar los correspondientes estudios de convergencia absoluta de los resultados a obtener; habiendo supuesto un coste computacional de las diferentes simulaciones, realizadas en una plataforma HP-9000/735, de aproximadamente $6.7 \mathrm{seg}$ por cada frecuencia de análisis.

Una vez analizada la primera de las dos posibles cavidades de reacción consideradas (recordar fig. 2.14), conviene caracterizar la segunda configuración mostrada en la figura 2.14 (apartado b); que corresponde al caso en el que la cavidad de reacción está situada fuera de la guía rectangular. Observando esta nueva estructura, se descubre que consta nuevamente de dos septums cortocircuitados en plano E situados en direcciones opuestas; los cuales están conectados por una guía intermedia (con dimensiones $A_{1}$ y $B_{1}+T_{1}+B_{2}$ ) de longitud $W$. Ahora bien, los septums que integran esta segunda cavidad de reacción presentan un 


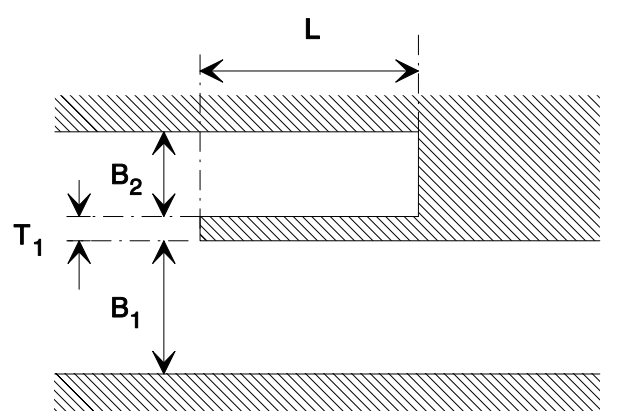

Figura 2.16: Sección longitudinal de un septum cortorcircuitado en plano E que constituye el elemento básico de las cavidades de reacción situadas fuera de la guía.

aspecto (ver sección longitudinal en figura 2.16) ligeramente diferente al que tienen los septums de la primera cavidad de reacción (recordar fig. 2.11). Siguiendo pues la misma estrategia de análisis empleada con la primera cavidad de reacción, para analizar esta segunda cavidad se requiere caracterizar en principio el comportamiento de los septums que integran dicha cavidad.

Así pues, el septum a caracterizar (ver fig. 2.16) está constituido por una sola discontinuidad; que consiste en una bifurcación en plano E de la guía de entrada (de dimensiones $A_{1}$ y $B_{1}+T_{1}+B_{2}$ ) en dos guías intermedias (cuyas dimensiones son $A_{1}$ y $B_{2}, A_{1}$ y $B_{1}$, respectivamente). De manera adicional, la estructura de este tipo de septum se compone (ver de nuevo fig. 2.16) de dos tramos de guía uniforme: el primero, de longitud $L$ y terminado en un cortocircuito físico, que se encuentra asociado a una de las guías intermedias del septum (concretamente a la de dimensiones $A_{1}$ y $B_{2}$ ); y el segundo, de longitud igual a la del anterior tramo y asociado a la segunda guía intermedia del septum (aquélla con dimensiones $A_{1}$ y $B_{1}$ ), que se introduce para trasladar el plano de referencia de salida del septum al mismo plano físico donde está situado el cortocircuito físico.

La caracterización del septum bajo análisis requiere construir en primer lugar las redes equivalentes multimodales, basadas en las correspondientes matrices de admitancias generalizadas, que definen el comportamiento electromagnético de los diferentes elementos (bifurcación en plano E y tramos de guía) integrantes del septum. Por tanto, empezando por la única discontinuidad o transición (bifurcación en plano E) de la que consta el septum considerado, se descubre que es idéntica a la primera de las discontinuidades del septum analizado previamente; razón por la cual pueden emplearse las expresiones recogidas en (2.28)-(2.33) para obtener los elementos de la MAG de la bifurcación en plano E. Todos los parámetros presentes en dichas expresiones tienen los mismos significados recogidos anteriormente para la primera bifurcación en plano $\mathrm{E}$ analizada; aunque debe tenerse en cuenta que en esta ocasión el puerto 1 de la nueva red equivalente multimodal, asociada a la nueva bifurcación en plano E, corresponde a la guía de entrada del septum (con dimensiones $A_{1}$ y $B_{1}+T_{1}+B_{2}$ ), el puerto 2 de 
la citada red se corresponde con la primera guía intermedia del septum (aquélla de dimensiones $A_{1}$ y $B_{2}$ ), y finalmente el tercer puerto de dicha red se refiere a la segunda guía intermedia del septum (de dimensiones $A_{1}$ y $B_{1}$ ). Por lo que respecta a los productos interiores que aparecen también en (2.28)-(2.33), al igual que en el caso de la anterior bifurcación en plano E, presentan de nuevo expresiones bastante simples; que corresponden a las mismas integrales de productos de funciones seno y coseno resueltas en el caso anterior, no incluyéndose por tanto en el presente trabajo. En cuanto al cálculo de los elementos de la MAG asociada a la discontinuidad considerada (matriz que denominaremos $Y_{m, n t 1}$ ), al presentar dicha matriz el mismo aspecto que aquélla deducida para la misma discontinuidad en el primer septum analizado, la estrategia seguida en el cálculo de los citados elementos es exactamente idéntica a la descrita con anterioridad; haciendo uso por tanto del método propuesto en el apartado 2.2.2 para calcular los elementos $Y_{m, n t 1}^{(2,2)}, Y_{m, n t 1}^{(3,2)}, Y_{m, n t 1}^{(2,3)}$ e $Y_{m, n t 1}^{(3,3)}$ de la mencionada MAG.

En lo referente a los dos tramos de guía uniforme que forman parte del septum a caracterizar, ambos de igual longitud $L$, para obtener los elementos de sus correspondientes matrices de admitancias generalizadas (designadas respectivamente como $Y_{m, n g 1}$ e $\left.Y_{m, n g 2}\right)$, que definen las respectivas redes equivalentes multimodales, pueden utilizarse las mismas expresiones recogidas en (2.37) y (2.38); las cuales se dedujeron para los elementos de la MAG del primer tramo de guía del septum caracterizado anteriormente, aunque pueden emplearse para cualquier tramo de guía uniforme. Lógicamente, en dichas expresiones (2.37) y (2.38), las admitancias características que aparecen, así como las constantes de propagación, deben hacer referencia a los modos del correspondiente tramo de guía; y el parámetro $l_{g 1}$ debe sustituirse por la longitud física de cada tramo de guía uniforme, en este caso de igual valor $(L)$ en los dos tramos a caracterizar.

Después de caracterizar el comportamiento electromagnético de los diferentes elementos (bifurcación en plano E y tramos de guía uniforme) que constituyen el septum bajo estudio, para determinar su respuesta es necesario conectar de forma adecuada, tal y como se recoge en la figura 2.17, las redes equivalentes multimodales asociadas a cada uno de los mencionados elementos del septum; conexiones que surgen de forzar las correspondientes condiciones de continuidad de los campos eléctricos y magnéticos transversales en distintos puertos de las citadas redes equivalentes. Estas condiciones de continuidad, de igual manera que aquéllas recogidas en (2.41)-(2.47), pueden expresarse en función de voltajes y corrientes modales (ambos vectores columna); permitiendo obtener, tras realizar las sustituciones correspondientes en dichas condiciones de continuidad, un conjunto de ecuaciones matriciales cuya resolución determina el comportamiento del septum que se pretende analizar. Escogiendo pues como incógnitas del sistema a plantear los voltajes modales (vectores columna) $V_{t 1}^{(1)}, V_{t 1}^{(2)}, V_{t 1}^{(3)}$ y $V_{g 2}^{(2)}$ asociados a diferentes puertos de las redes equivalentes multimodales mostradas en la figura 2.17, y haciendo uso asimismo de las mencionadas condiciones de 


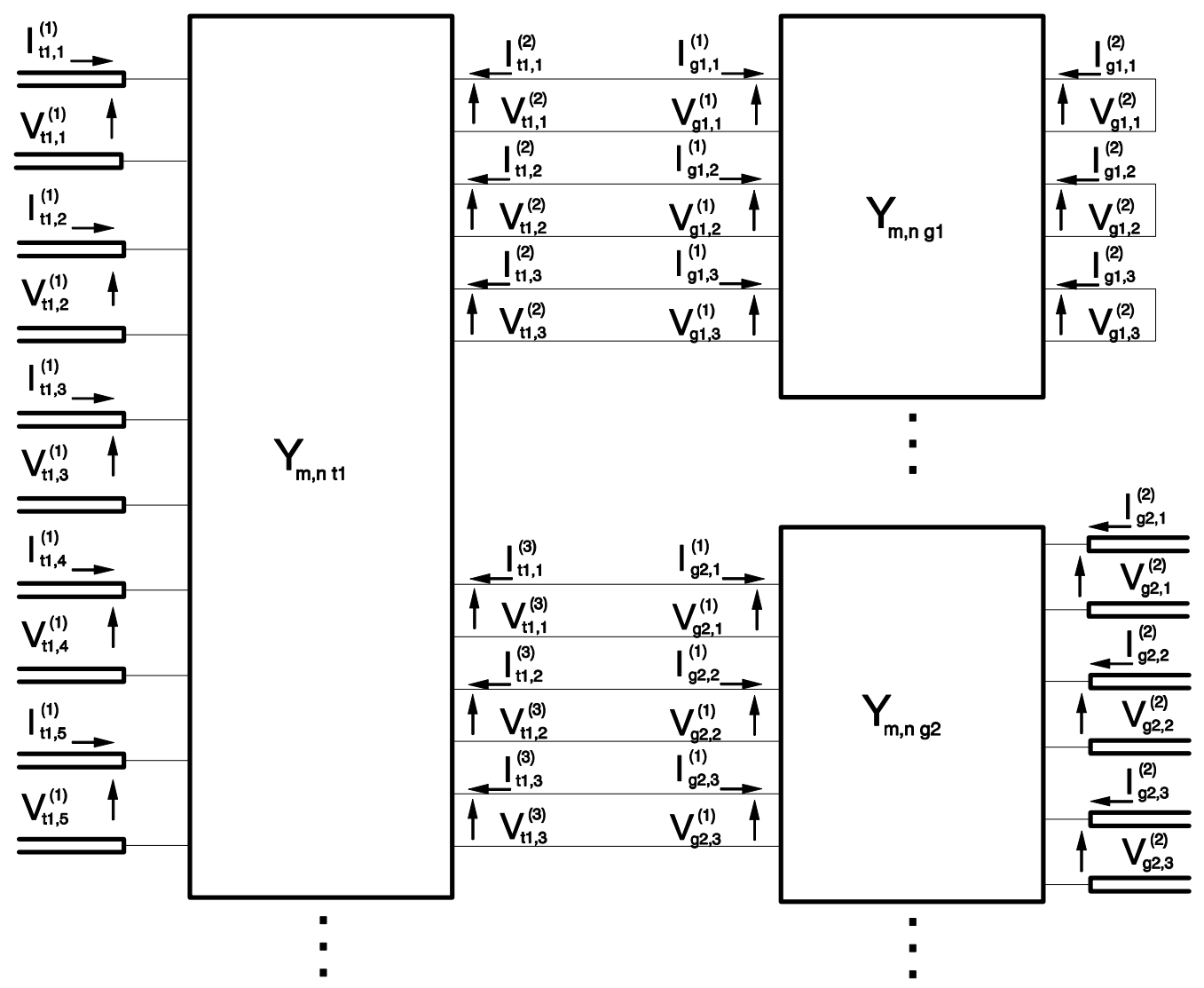

Figura 2.17: Conexión de las diferentes redes equivalentes multimodales, definidas mediante las correspondientes matrices de admitancias generalizadas, que integran un septum cortocircuitado en plano E como el mostrado en la figura 2.16.

continuidad y de algunas relaciones entre voltajes y corrientes modales (ambos vectores columna) que definen las matrices $Y_{m, n t 1}$ e $Y_{m, n g 2}$, se deduce el siguiente conjunto de ecuaciones matriciales a resolver para caracterizar el comportamiento del septum considerado

$$
\begin{aligned}
I_{t 1}^{(1)}= & Y_{t 1}^{(1,1)} \cdot V_{t 1}^{(1)}+Y_{t 1}^{(1,2)} \cdot V_{t 1}^{(2)}+Y_{t 1}^{(1,3)} \cdot V_{t 1}^{(3)} \\
O= & Y_{t 1}^{(2,1)} \cdot V_{t 1}^{(1)}+\left(Y_{t 1}^{(2,2)}+Y_{g 1}^{(1,1)}\right) \cdot V_{t 1}^{(2)}+Y_{t 1}^{(2,3)} \cdot V_{t 1}^{(3)} \\
O= & Y_{t 1}^{(3,1)} \cdot V_{t 1}^{(1)}+Y_{t 1}^{(3,2)} \cdot V_{t 1}^{(2)}+\left(Y_{t 1}^{(3,3)}+Y_{g 2}^{(1,1)}\right) \cdot V_{t 1}^{(3)} \\
& \quad+Y_{g 2}^{(1,2)} \cdot V_{g 2}^{(2)} \\
I_{g 2}^{(2)}= & Y_{g 2}^{(2,1)} \cdot V_{t 1}^{(3)}+Y_{g 2}^{(2,2)} \cdot V_{g 2}^{(2)}
\end{aligned}
$$

La respuesta electromagnética del septum que integra la segunda cavidad de reacción a estudiar, expresada en términos de los correspondientes parámetros de dispersión, se obtendría tras cargar adecuadamente todos los accesos de las 
guías de entrada y salida del septum, excepto el acceso donde se define la excitación del dispositivo, con las respectivas admitancias características; y resolver posteriormente el nuevo sistema de ecuaciones matriciales que surgiría al imponer dichas condiciones de carga en (2.53)-(2.56). Ahora bien, retornando al análisis del segundo tipo de cavidad de reacción (recordar apartado b en fig. 2.14), su caracterización requiere conectar previamente entre sí los dos conjuntos de redes equivalentes multimodales correspondientes a cada uno de los dos septums de esta segunda cavidad; cuyas redes equivalentes, asociadas a los elementos integrantes de dichos septums, se conectan como se indica en la figura 2.17. La conexión en cascada de estos dos conjuntos de redes se realiza mediante la red equivalente multimodal asociada al tramo de guía uniforme que conecta los mencionados septums de la cavidad; cuya matriz de admitancias generalizada se deduce utilizando por ejemplo las mismas expresiones recogidas en (2.37) y (2.38). En dichas expresiones, lógicamente, las admitancias características y las constantes de progación que aparecen están asociadas en este caso al tramo de guía intermedio de esta segunda cavidad (aquél de dimensiones $A_{1}$ y $B_{1}+T_{1}+B_{2}$ ); mientras la longitud a emplear para este tramo de guía se obtiene sustrayendo a la longitud física $W$ del tramo de guía original las longitudes $l_{\text {ref }}$, que se escogen para separar los planos de referencia utilizados en el análisis de la única discontinuidad de los dos septums que integran esta segunda cavidad (recordar fig. 2.1).

El comportamiento de la segunda cavidad de reacción se determina nuevamente calculando el parámetro de dispersión $S_{11}$, asociado al modo fundamental $\left(\mathrm{TE}_{10}^{\mathrm{z}}\right)$ de la guía de entrada, que presenta dicha cavidad. El cálculo de este parámetro, al igual que en el análisis del septum integrante de esta segunda cavidad, se implementa cargando en primer lugar los accesos de las redes equivalentes multimodales de las guías de entrada y salida de la cavidad, exceptuando el primer acceso de la guía de entrada que constituye la excitación de la estructura; y resolviendo a continuación el sistema de ecuaciones que resulta de imponer las mencionadas condiciones de carga. Este sistema de ecuaciones asociado a la cavidad bajo análisis, que consta de un mayor número de ecuaciones e incógnitas que los sistemas asociados a los septums de dicha cavidad, presenta también una estructura en banda; aplicando por tanto en su resolución una técnica basada en eliminación Gaussiana con retrosustitución [23] que tiene en cuenta la estructura en banda del sistema. En la figura 2.18, se muestran los resultados correspondientes al módulo del parámetro de dispersión $S_{11}$, calculado respecto del modo fundamental ( $\left.\mathrm{TE}_{10}^{\mathrm{z}}\right)$ de la guía de entrada de la cavidad, que presenta una cavidad de reacción en plano E situada fuera de la guía $\left(A_{1}=22.86 \mathrm{~mm}, B_{1}=10.16 \mathrm{~mm}, B_{2}=2.00 \mathrm{~mm}\right.$, $\left.W=0.10 \mathrm{~mm}, T_{1}=0.10 \mathrm{~mm}\right)$ para diferentes combinaciones de los valores de las longitudes $L_{1}$ y $L_{2}\left(L_{1}=L_{2}=9 \mathrm{~mm}, L_{1}=10 \mathrm{~mm}\right.$ y $L_{2}=8 \mathrm{~mm}, L_{1}=12 \mathrm{~mm}$ y $L_{2}=6 \mathrm{~mm}, L_{1}=14 \mathrm{~mm}$ y $L_{2}=4 \mathrm{~mm}$ ); observando en dicha figura que para una misma longitud total de la cavidad $\left(L_{1}+L_{2}+W=18.10 \mathrm{~mm}\right)$, la frecuencia de resonancia depende totalmente de la posición en la cavidad del tramo de guía 


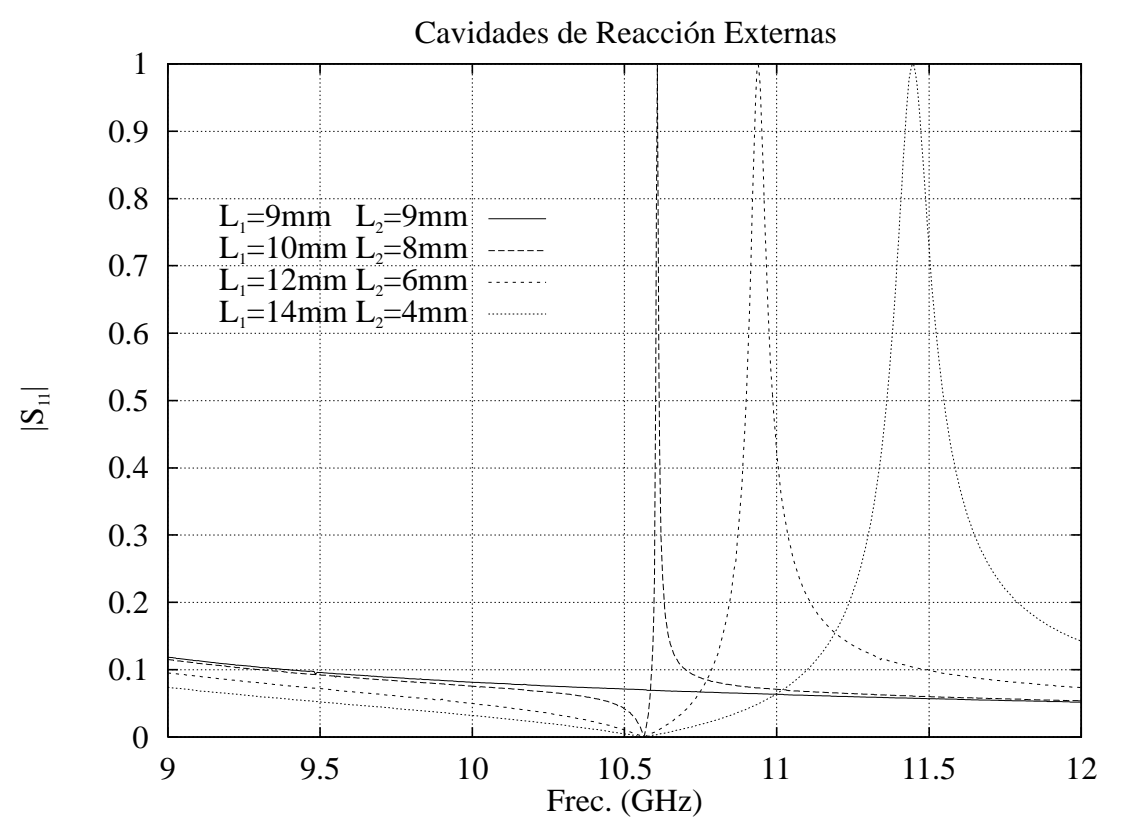

Figura 2.18: Módulo del parámetro $S_{11}$ para diferentes valores de las dimensiones $L_{1}$ y $L_{2}$ de una cavidad de reacción en plano E situada fuera de la guía rectangular $\left(A_{1}=22.86 \mathrm{~mm}, B_{1}=10.16 \mathrm{~mm}, B_{2}=2.00 \mathrm{~mm}, W=0.10 \mathrm{~mm}, T_{1}=0.10 \mathrm{~mm}\right.$, $\left.L_{1}+L_{2}+W=18.10 \mathrm{~mm}\right)$.

intermedio que conecta los dos septums que integran dicha cavidad, al igual que ocurría anteriormente cuando la cavidad de reacción estaba situada fuera de la guía (recordar fig. 2.15). Asimismo, para el caso en el que el tramo de guía intermedio se sitúa en el centro de la cavidad $\left(L_{1}=L_{2}=9 \mathrm{~mm}\right)$, tal y como sucedía con la primera cavidad de reacción (recordar de nuevo fig. 2.15), la respuesta en frecuencia de la segunda cavidad no presenta en absoluto un comportamiento selectivo con la frecuencia; debido a que, como ya se comentó con la primera cavidad, no se produce interacción alguna entre las guías de entrada y salida y la propia cavidad de reacción. Para el resto de combinaciones de las longitudes $L_{1}$ y $L_{2}$ consideradas en la figura 2.18, las correspondientes respuestas en frecuencia revelan que este segundo tipo de cavidad de reacción también puede emplearse como elemento integrante de filtros de banda eliminada; observando en este segundo tipo de cavidad que el módulo del parámetro $S_{11}$ presenta, a frecuencias diferentes de aquélla a la que se produce la reflexión total $\left(\left|S_{11}\right|=0 \mathrm{~dB}\right)$, valores más bajos que los obtenidos a dichas frecuencias en el caso de la cavidad de reacción situada dentro de la guía (comparar figuras 2.15 y 2.18).

Los resultados mostrados en la figura 2.18 se han obtenido, tal y como se ha descrito anteriormente, empleando las redes equivalentes multimodales de los elementos integrantes de la cavidad basadas en las respectivas matrices de admitancias generalizadas; habiendo utilizado en la obtención de ciertos elementos de 
algunas de estas matrices el método propuesto en el apartado 2.2.2. Con el objeto de validar pues dichos resultados, éstos se pueden comparar con los ofrecidos en [24, pág. 5] para los mismos casos considerados en la figura 2.18; aunque para obtener los resultados recogidos en [24] se han usado matrices de dispersión generalizadas, deducidas mediante la técnica conocida como adaptación modal. De este comparación, se comprueba la precisión conseguida en los resultados de la figura 2.18, que son prácticamente idénticos a los recogidos en [24, pág. 5]; lo que permite corroborar una vez más que la técnica descrita en el apartado 2.2.2 para calcular de forma acelerada algunos elementos de la MAG de uniones planares, empleada en el análisis de esta segunda cavidad de reacción, genera resultados correctos al igual que otros métodos tradicionalmente empleados en la caracterización de dichas discontinuidades (por ejemplo el método de adaptación modal utilizado en [24]). Nuevamente, al analizar esta segunda cavidad de reacción, se han empleado modos $\mathrm{TE}^{\mathrm{z}}$ y modos $\mathrm{TM}^{\mathrm{z}}$ para describir los campos eléctricos y magnéticos en las distintas secciones de guía que integran la citada cavidad; debido básicamente a la posible utilización de dicha cavidad de reacción en otros dispositivos que exciten los mencionados modos. En particular, para generar los resultados de la figura 2.18 , se han utilizado 50 modos en todos los tramos correspondientes a la guía (con dimensiones $A_{1}$ y $B_{1}$ ) sobre la que se sitúa la cavidad de reacción, 70 modos en la guía intermedia (con dimensiones $A_{1}$ y $B_{1}+T_{1}+B_{2}$ ) que conecta los dos septums de la cavidad, y 30 modos en los tramos de guía cortocircuitados (con dimensiones $A_{1}$ y $B_{2}$ ) de longitudes $L_{1}$ y $L_{2}$; números de modos escogidos tras realizar los correspondientes estudios de convergencia absoluta en los resultados a deducir. Estas simulaciones, empleando los modos recién mencionados y realizadas en una plataforma HP-9000/735, han requerido un coste computacional de aproximadamente $10 \mathrm{seg}$ por cada frecuencia de análisis; lo que representa un coste bastante aceptable teniendo en cuenta la complejidad de la estructura analizada.

\subsection{Técnica Recursiva para la Resolución de Sis- temas de Ecuaciones Lineales en Banda}

Tras acelerar el cálculo de los elementos de la MAG de uniones planares arbitrarias, empleando para ello el método recién descrito en la sección 2.2, se descubre que el principal esfuerzo computacional requerido en el análisis de dispositivos de microondas complejos procede de la resolución de un sistema de ecuaciones lineales; cuyo tamaño, y consecuentemente el coste asociado a su resolución, crecen muy rápidamente a medida que la complejidad del dispositivo a caracterizar aumenta. Recientemente, con el objeto de reducir este importante esfuerzo computacional, en [26] y [27] se plantean diversas estrategias a seguir para acelerar la resolución de los mencionados sistemas de ecuaciones lineales; estrategias que se 
basan esencialmente en una explotación adecuada de las características de la matriz de coeficientes del sistema a resolver. En este sentido, el empleo del método basado en matrices de admitancias generalizadas para caracterizar dispositivos de microondas integrados por guías uniformes conectadas en cascada, descrito anteriormente en la sección 2.2 o recogido por ejemplo en [8] y [18], resulta especialmente ventajoso; pues dicho método conduce a un sistema de ecuaciones lineales a resolver cuya matriz de coeficientes presenta una estructura en banda, característica que puede explotarse adecuadamente para incrementar la eficiencia computacional asociada a la resolución del citado sistema.

Así pues, en esta sección se describe una técnica muy eficiente, basada en un algoritmo recursivo, para resolver los sistemas de ecuaciones lineales en banda que surgen al analizar dispositivos de microondas, constituidos por diferentes guías uniformes conectadas en cascada, empleando matrices de admitancias generalizadas para caracterizar las uniones planares y los tramos de guía que integran dicho dispositivo. Este nuevo procedimiento recursivo de resolución, expuesto con detalle en [28], hace uso de la naturaleza en banda que presenta el sistema de ecuaciones lineales a resolver; generando unas expresiones analíticas simples de implementar que permiten obtener la solución final del sistema. Asimismo, con el objeto de comprobar el ahorro en número de operaciones (multiplicaciones e inversiones de matrices con elementos complejos) que supone el empleo de esta nueva técnica de resolución, se describe también un método para resolver los mencionados sistemas tradicionalmente utilizado cuando las uniones se caracterizan mediante matrices de dispersión generalizadas [5]. Por último, para poner de manifiesto la mejora en términos de eficiencia computacional que supone el empleo de la citada técnica recursiva, se presenta un estudio comparativo del coste computacional que supone resolver el sistema de ecuaciones asociado al análisis de una estructura típica de microondas; empleando para resolver dicho sistema un método de eliminación Gaussiana con retrosustitución [23] que tiene en cuenta la estructura en banda del sistema a resolver, una traslación del método propuesto en [5] cuando se utilizan matrices de dispersión generalizadas al caso que nos ocupa, y finalmente la técnica recursiva expuesta en la presente sección.

\subsubsection{Formulación Básica del Problema}

El comportamiento de un dispositivo de microondas, constituido por la conexión en cascada de diferentes secciones de guía uniforme, queda completamente descrito si todas las uniones planares entre dichas guías están perfectamente caracterizadas. Conectando adecuadamente las redes equivalentes multimodales de estas uniones planares y de los tramos de guía uniforme, definidas en función de las correspondientes matrices de admitancias generalizadas, se obtiene un sistema lineal de ecuaciones que permite deducir el comportamiento electromagnético del dispositivo completo; y cuya matriz de coeficientes presenta una estructura en 


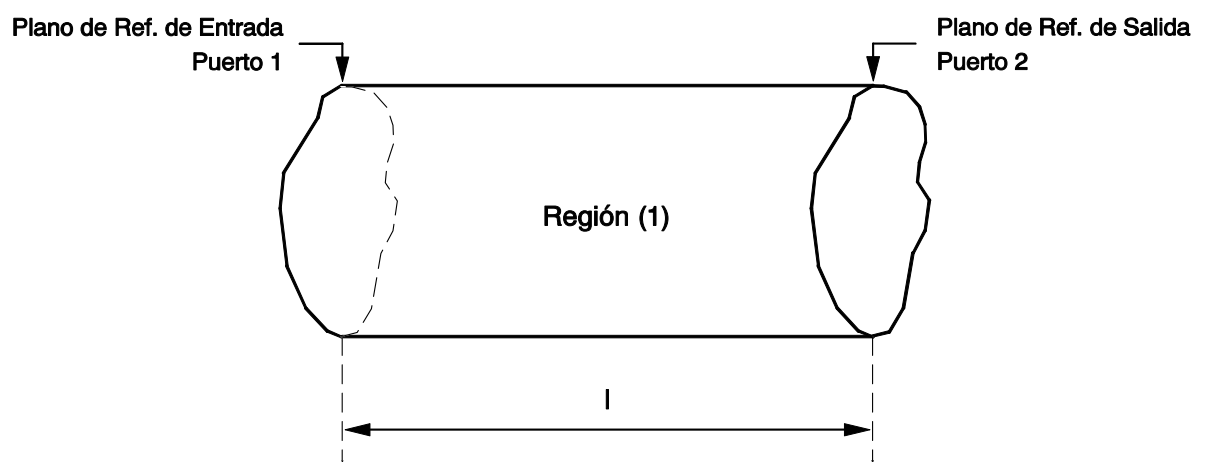

a)

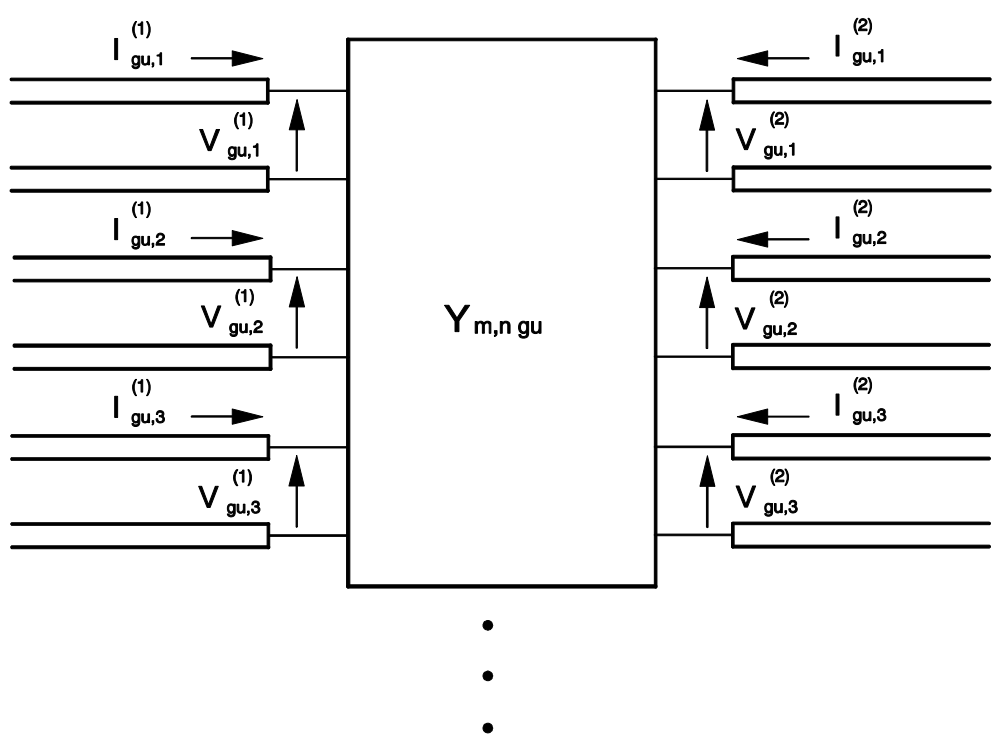

b)

Figura 2.19: Guía uniforme arbitraria en a); y representación de dicha guía mediante una red equivalente multimodal basada en la matriz de admitancias generalizada en b).

banda [8]. Por su parte, esta matriz de coeficientes está constituida por diferentes bloques; cada uno de los cuales se encuentra asociado bien a un tramo de guía uniforme o bien a una unión planar entre guías.

El comportamiento de un tramo de guía uniforme de longitud $l$ (ver apartado a en figura 2.19) puede describirse fácilmente mediante una red equivalente multimodal (ver apartado b en fig. 2.19); basada en una matriz de admitancias generalizada cuyos elementos se obtienen [25] del siguiente modo

$$
\begin{aligned}
& Y_{m, n \text { gu }}^{(1,1)}=Y_{m, n \text { gu }}^{(2,2)}=(-j) \cdot Y_{0 n}^{(1)} \cdot \cot \left(\beta_{n}^{(1)} \cdot l\right) \cdot \delta_{m, n} \\
& Y_{m, n \text { gu }}^{(2,1)}=Y_{m, n \text { gu }}^{(1,2)}=j \cdot Y_{0 n}^{(1)} \cdot \csc \left(\beta_{n}^{(1)} \cdot l\right) \cdot \delta_{m, n}
\end{aligned}
$$




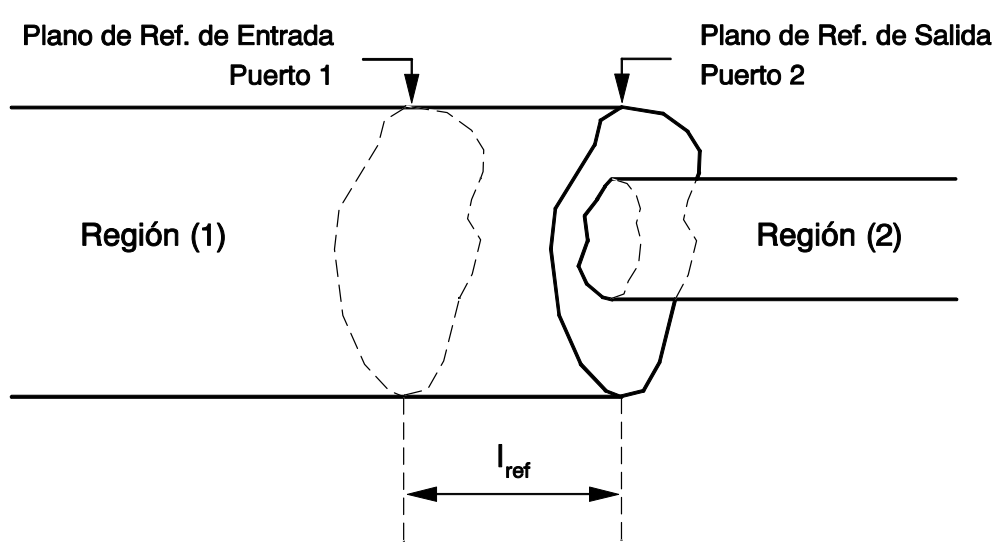

a)

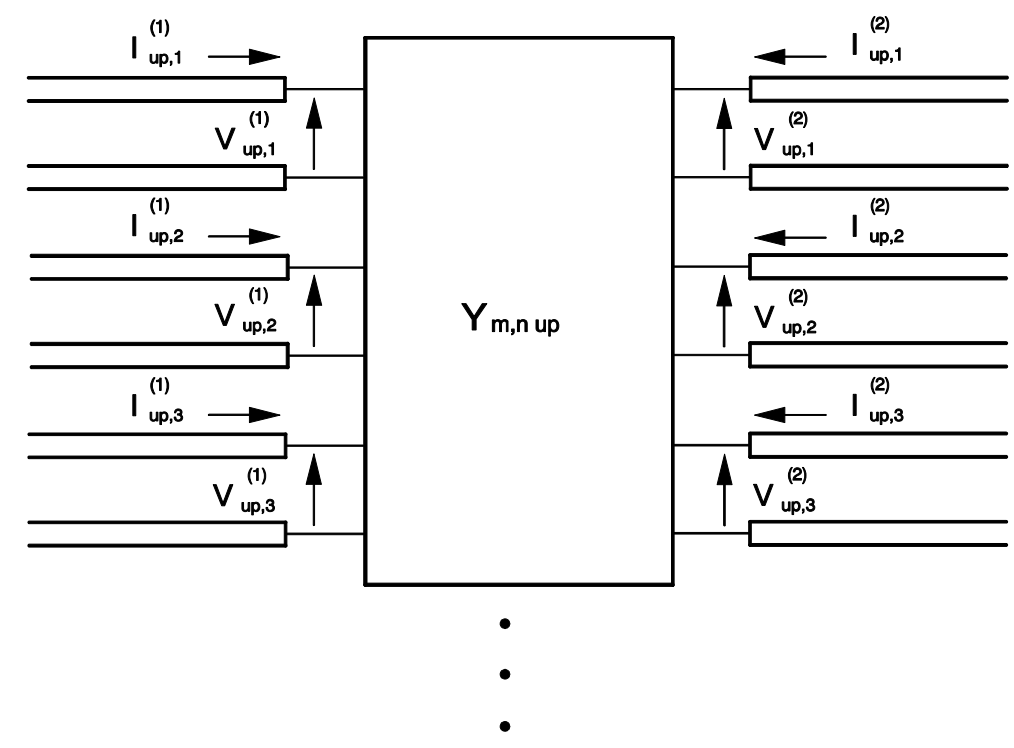

b)

Figura 2.20: Unión planar entre dos guías arbitrarias en a); y representación de dicha unión mediante una red equivalente multimodal basada en la matriz de admitancias generalizada en $b$ ).

En (2.57) y (2.58), $Y_{0 n}^{(1)}$ y $\beta_{n}^{(1)}$ hacen referencia respectivamente a la admitancia característica y a la constante de propagación del $n$-ésimo modo en el tramo de guía caracterizado; parámetros cuyas expresiones analíticas pueden encontrarse por ejemplo en [15]. En cuanto al término $\delta_{m, n}$, presente también en (2.57) y (2.58), éste representa la función delta de Kronecker expuesta en (2.5).

Una unión planar entre dos guías arbitrarias, como la que se muestra en la figura 2.20 (apartado a), puede caracterizarse perfectamente mediante su correspondiente red equivalente multimodal (ver apartado b en fig. 2.20), definida 
nuevamente en términos de una matriz de admitancias generalizada. Los elementos de esta matriz, tal y como se ha descrito en la sección 2.1, se obtienen implementando las siguientes expresiones

$$
\begin{gathered}
Y_{m, n \text { up }}^{(1,1)}=(-j) \cdot Y_{0 n}^{(1)} \cdot \cot \left(\beta_{n}^{(1)} \cdot l_{\text {ref }}\right) \cdot \delta_{m, n} \\
Y_{m, n \text { up }}^{(2,1)}=Y_{n, m \text { up }}^{(1,2)}=j \cdot Y_{0 n}^{(1)} \cdot \csc \left(\beta_{n}^{(1)} \cdot l_{\text {ref }}\right) \cdot<\vec{h}_{n}^{(1)} \vec{h}_{m}^{(2)}> \\
Y_{m, n \text { up }}^{(2,2)}=(-j) \cdot \sum_{r=1}^{\infty} Y_{0 r}^{(1)} \cdot \cot \left(\beta_{r}^{(1)} \cdot l_{\text {ref }}\right) \cdot\left\langle\vec{e}_{r}^{(1)} \vec{e}_{n}^{(2)}>\cdot<\vec{h}_{r}^{(1)} \vec{h}_{m}^{(2)}>\right.
\end{gathered}
$$

donde $\vec{e}^{(\gamma)}$ y $\vec{h}^{(\gamma)}$ representan las funciones vectoriales modales normalizadas que están asociadas a los campos eléctrico y magnético transversales de la región $(\gamma)$, donde $\gamma=1$ para la región (1) o $\gamma=2$ para la región (2). En (2.59)-(2.61), los parámetros $Y_{0 n}^{(1)}, Y_{0 r}^{(1)}, \beta_{n}^{(1)}$ y $\beta_{r}^{(1)}$ se refieren a las admitancias características y a las constantes de propagación correspondientes respectivamente al $n$-ésimo y al $r$-ésimo modo de la región (1), el término $\delta_{m, n}$ representa de nuevo la función delta de Kronecker, y el parámetro $l_{\text {ref }}$ determina la separación entre los planos de referencia de entrada y salida (ver apartado a en fig. 2.20).

Un dispositivo de microondas, constituido por $\mathrm{N}$ guías arbitrarias conectadas en cascada, puede describirse mediante la conexión de $\mathrm{N}-1$ uniones planares y N tramos de guía uniforme. Haciendo uso de las redes equivalentes multimodales que representan cada uno de los citados elementos, definidas en función de las correspondientes matrices de admitancias generalizadas, es posible obtener una representación equivalente multimodal global cuyo aspecto se ofrece en la figura 2.21; representación a partir de la cual puede deducirse el comportamiento electromagnético del dispositivo considerado. En términos matemáticos, esta representación global determina un sistema de ecuaciones lineales en banda [8]; que debe resolverse para obtener la respuesta del dispositivo a caracterizar. A medida que aumenta la complejidad de estos dispositivos de microondas, el tamaño de dichos sistemas crece considerablemente; resultando por tanto interesante esforzarse en reducir el coste computacional que supone resolver el mencionado sistema de ecuaciones lineales en banda.

Una posible técnica a utilizar en la resolución del citado sistema se basa en un procedimiento de eliminación Gaussiana con retrosustitución [23]; que para mejorar la eficiencia computacional asociada a la resolución del sistema debería tener en cuenta su naturaleza en banda. Un procedimiento alternativo, tradicionalmente empleado cuando las uniones planares y los tramos de guía se caracterizan mediante matrices de dispersión generalizadas [5], también puede emplearse en la resolución del mencionado sistema; procedimiento que se basa en reducir dos matrices de admitancias conectadas en cascada a una matriz equivalente, y en repetir dicha reducción de forma sucesiva hasta que se obtiene una sola matriz de admitancias que representa al dispositivo completo. La adaptación de este 


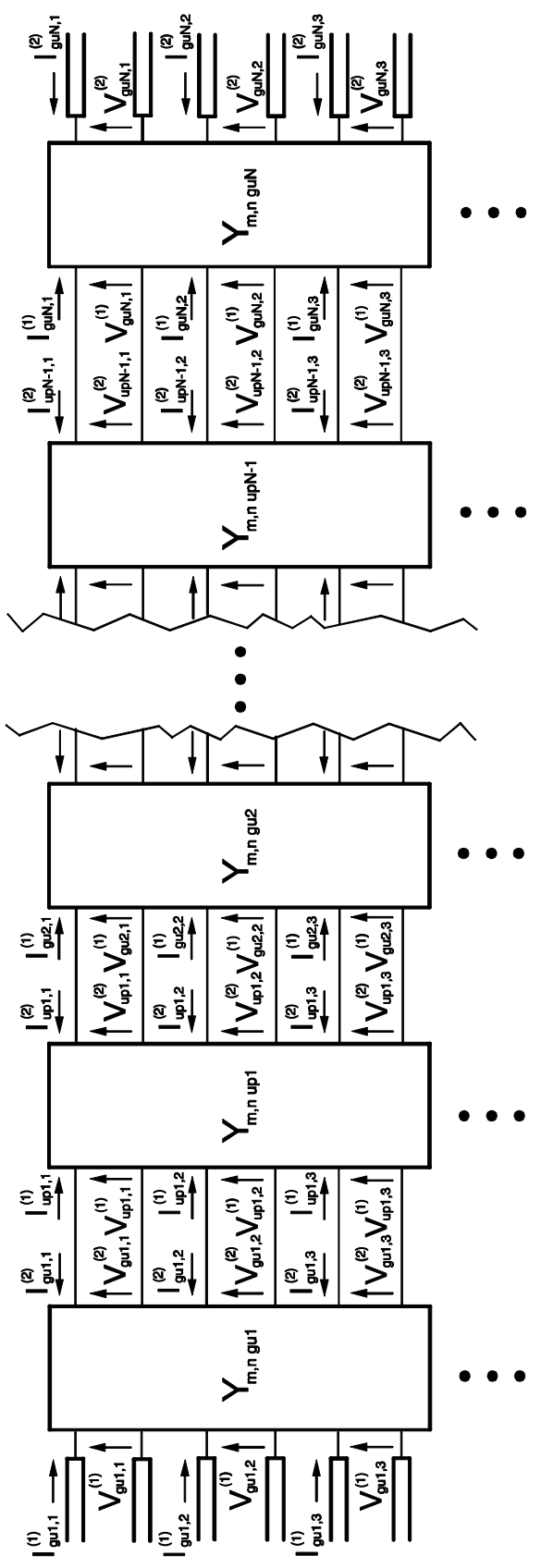

Figura 2.21: Conexión de las redes equivalentes multimodales, definidas mediante las correspondientes matrices de admitancias generalizadas, que corresponden a los elementos integrantes de un dispositivo de microondas constituido por $N$ guías arbitrarias.

procedimiento reductivo al caso que nos ocupa se expone en el siguiente apartado para poder comparar posteriormente el número de operaciones, multiplicaciones e inversiones de matrices con elementos complejos, que requiere este procedimiento 
y la nueva técnica recursiva descrita en el apartado 2.3.3. Asimismo, tal y como se comprueba en el apartado 2.3.4, los dos métodos propuestos inicialmente para resolver los sistemas de ecuaciones lineales en banda que surgen al caracterizar dispositivos de microondas resultan menos eficientes, desde el punto de vista computacional, que la nueva técnica recursiva propuesta en esta sección para resolver dichos sistemas de ecuaciones.

\subsubsection{Reducción a Una Matriz $Y$ Equivalente}

Para conseguir reducir a una sola matriz de admitancias equivalente la representación global de un dispositivo de microondas mostrada en la figura 2.21, es necesario adaptar al problema bajo análisis la técnica propuesta en [5] para el caso en el que se utilizan matrices de dispersión generalizadas. Esta técnica, trasladada al caso en el que se emplean matrices de admitancias generalizadas, consiste en reducir sucesivamente dos de estas matrices a una matriz equivalente del mismo tipo. Así por ejemplo, en la figura 2.22 se tienen dos redes equivalentes multimodales, definidas mediante sus correspondientes matrices de admitancias generalizadas $Y_{\mathrm{a}}$ e $Y_{\mathrm{b}}$, que están conectadas en cascada. Dichas matrices relacionan los voltajes y las corrientes modales (vectores columna) en los puertos de entrada y salida de las correspondientes redes multimodales (ver figura 2.22) mediante las siguientes expresiones

$$
\begin{aligned}
& {\left[\begin{array}{c}
I_{\mathrm{a}}^{(1)} \\
I_{\mathrm{a}}^{(2)}
\end{array}\right]=\left[\begin{array}{cc}
Y_{\mathrm{a}}^{(1,1)} & Y_{\mathrm{a}}^{(1,2)} \\
Y_{\mathrm{a}}^{(2,1)} & Y_{\mathrm{a}}^{(2,2)}
\end{array}\right] \cdot\left[\begin{array}{c}
V_{\mathrm{a}}^{(1)} \\
V_{\mathrm{a}}^{(2)}
\end{array}\right]} \\
& {\left[\begin{array}{c}
I_{\mathrm{b}}^{(1)} \\
I_{\mathrm{b}}^{(2)}
\end{array}\right]=\left[\begin{array}{cc}
Y_{\mathrm{b}}^{(1,1)} & Y_{\mathrm{b}}^{(1,2)} \\
Y_{\mathrm{b}}^{(2,1)} & Y_{\mathrm{b}}^{(2,2)}
\end{array}\right] \cdot\left[\begin{array}{c}
V_{\mathrm{b}}^{(1)} \\
V_{\mathrm{b}}^{(2)}
\end{array}\right]}
\end{aligned}
$$

La matriz de admitancias equivalente $Y_{\mathrm{ab}}$, asociada a la red equivalente multimodal que representa la conexión en cascada de las dos redes multimodales definidas mediante sus respectivas matrices $Y_{\mathrm{a}}$ e $Y_{\mathrm{b}}$ (ver figura 2.22), debe satisfacer las siguientes relaciones matriciales

$$
\left[\begin{array}{c}
I_{\mathrm{a}}^{(1)} \\
I_{\mathrm{b}}^{(2)}
\end{array}\right]=\left[\begin{array}{cc}
Y_{\mathrm{ab}}^{(1,1)} & Y_{\mathrm{ab}}^{(1,2)} \\
Y_{\mathrm{ab}}^{(2,1)} & Y_{\mathrm{ab}}^{(2,2)}
\end{array}\right] \cdot\left[\begin{array}{c}
V_{\mathrm{a}}^{(1)} \\
V_{\mathrm{b}}^{(2)}
\end{array}\right]
$$

Con el objeto de expresar los bloques de la matriz equivalente $Y_{\mathrm{ab}}$ en función de los bloques de las matrices $Y_{\mathrm{a}}$ e $Y_{\mathrm{b}}$, deben imponerse las condiciones de continuidad de los campos eléctrico y magnético transversales definidos en el puerto común que conecta las dos redes equivalentes multimodales (ver figura 2.22); condiciones de continuidad que pueden escribirse, tal y como se indica 


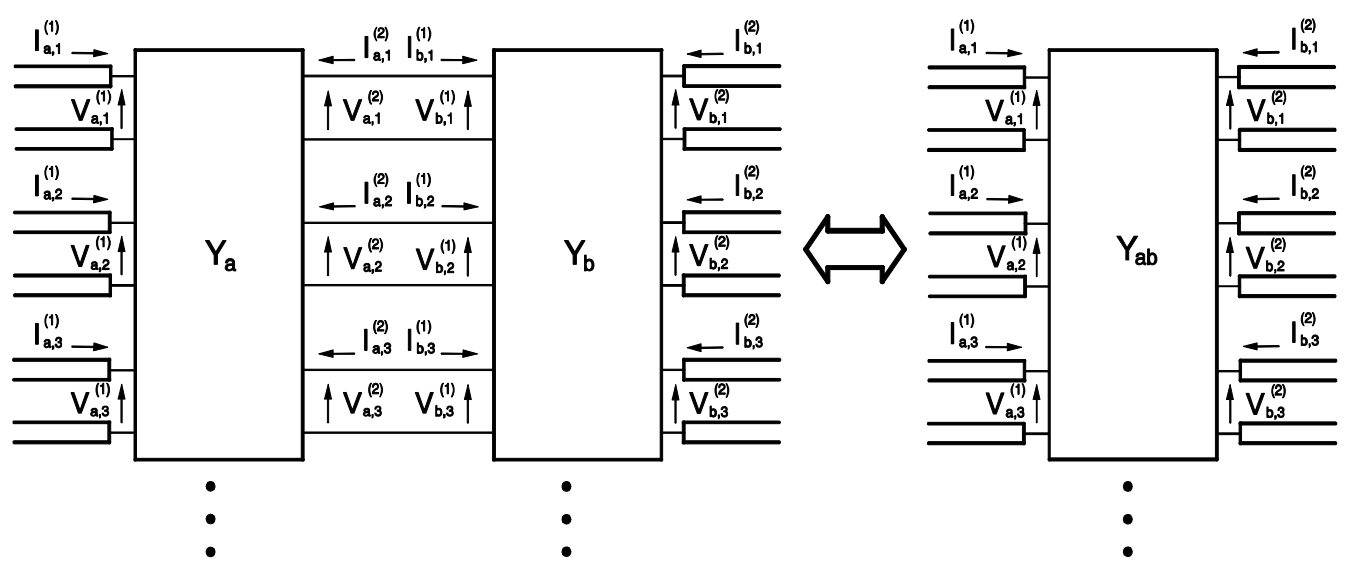

Figura 2.22: Red equivalente multimodal, definida mediante la matriz de admitancias generalizada $Y_{\mathrm{ab}}$, que corresponde a la conexión en cascada de dos redes equivalentes multimodales, expresadas también en función de sus respectivas matrices de admitancias generalizadas $Y_{\mathrm{a}}$ e $Y_{\mathrm{b}}$.

a continuación, en términos de los vectores columna cuyos elementos representan voltajes y corrientes modales

$$
\begin{aligned}
& V_{\mathrm{a}}^{(2)}=V_{\mathrm{b}}^{(1)} \\
& I_{\mathrm{a}}^{(2)}=-I_{\mathrm{b}}^{(1)}
\end{aligned}
$$

De estas condiciones de contorno, es posible deducir las siguientes expresiones; que permiten obtener los diferentes bloques de la matriz equivalente $Y_{\mathrm{ab}}$ a partir de los bloques de las matrices $Y_{\mathrm{a}}$ e $Y_{\mathrm{b}}$

$$
\begin{aligned}
& Y_{\mathrm{ab}}^{(1,1)}=Y_{\mathrm{a}}^{(1,1)}-Y_{\mathrm{a}}^{(1,2)} \cdot\left(Y_{\mathrm{a}}^{(2,2)}+Y_{\mathrm{b}}^{(1,1)}\right)^{-1} \cdot Y_{\mathrm{a}}^{(2,1)} \\
& Y_{\mathrm{ab}}^{(1,2)}=-Y_{\mathrm{a}}^{(1,2)} \cdot\left(Y_{\mathrm{a}}^{(2,2)}+Y_{\mathrm{b}}^{(1,1)}\right)^{-1} \cdot Y_{\mathrm{b}}^{(1,2)} \\
& Y_{\mathrm{ab}}^{(2,1)}=-Y_{\mathrm{b}}^{(2,1)} \cdot\left(Y_{\mathrm{a}}^{(2,2)}+Y_{\mathrm{b}}^{(1,1)}\right)^{-1} \cdot Y_{\mathrm{a}}^{(2,1)} \\
& Y_{\mathrm{ab}}^{(2,2)}=Y_{\mathrm{b}}^{(2,2)}-Y_{\mathrm{b}}^{(2,1)} \cdot\left(Y_{\mathrm{a}}^{(2,2)}+Y_{\mathrm{b}}^{(1,1)}\right)^{-1} \cdot Y_{\mathrm{b}}^{(1,2)}
\end{aligned}
$$

Para aplicar el procedimiento recién descrito al análisis del dispositivo constituido por $\mathrm{N}$ guías conectadas en cascada, se requiere obtener en primer lugar la matriz de admitancias equivalente de las dos últimas matrices $\left(Y_{m, n \text { upN-1 }}\right.$ e $\left.Y_{m, n g u N}\right)$ de la representación global mostrada en la figura 2.21 ; lo que origina una nueva red equivalente multimodal global asociada al dispositivo que está simplificada, cuyas dos últimas matrices han sido reemplazadas por la correspondiente matriz de admitancias equivalente. Procediendo de esta misma manera con la nueva red simplificada, y repitiendo dicho proceso hasta realizar $(2 \mathrm{~N}-2)$ iteraciones, se obtiene una sola matriz de admitancias equivalente $\left(Y_{\text {eq }}\right)$ asociada 


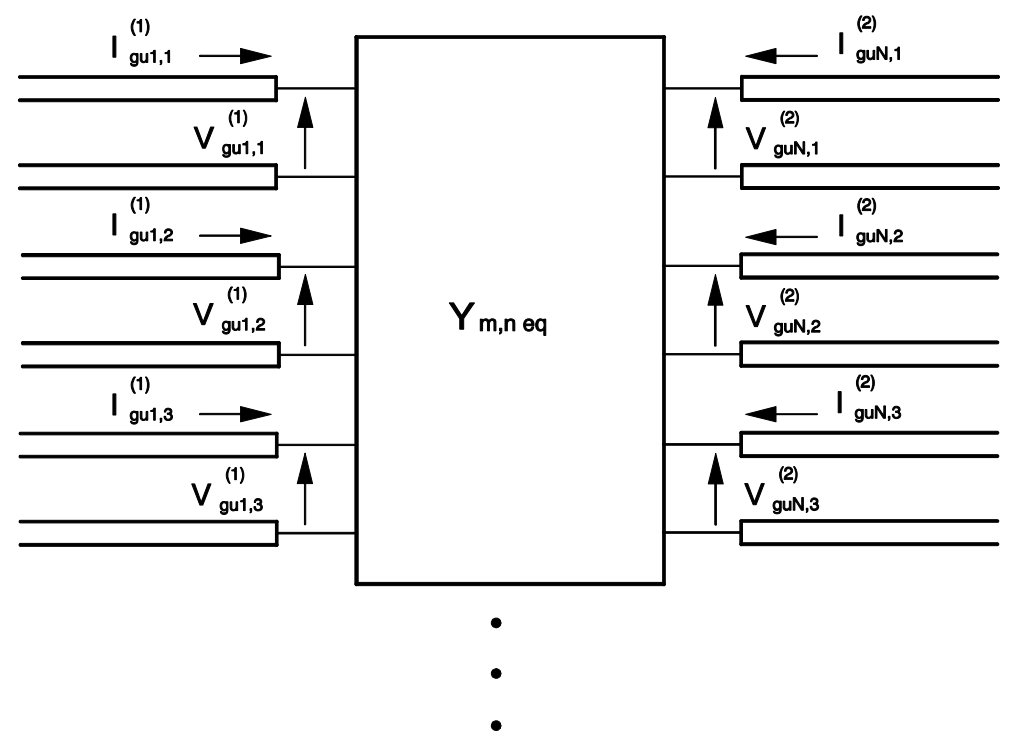

Figura 2.23: Red equivalente multimodal, definida mediante una matriz de admitancias generalizada, que corresponde al mismo dispositivo de microondas constituido por $N$ guías arbitrarias descrito electromagnéticamente en la figura 2.21 .

a la red equivalente multimodal (ver figura 2.23) que representa al dispositivo de microondas bajo análisis.

Una vez obtenida la red equivalente multimodal del dispositivo de microondas a caracterizar, cuyo comportamiento se define mediante la matriz de admitancias equivalente $\left(Y_{\mathrm{eq}}\right)$ deducida siguiendo el procedimiento descrito anteriormente, el cálculo de los parámetros de dispersión de dicho dispositivo, asociados por ejemplo al modo fundamental de las guías de entrada (guía 1) y salida (guía N), requiere cargar todos los accesos de los puertos de entrada y salida de la red equivalente multimodal con las admitancias características de los modos correspondientes; exceptuando claro está el primer acceso del puerto de entrada, que constituye la excitación del dispositivo. Cargando pues de esta manera, tal y como se indica gráficamente en la figura 2.24, la red equivalente multimodal asociada al dispositivo de microondas que se pretende analizar, es posible plantear un sistema de ecuaciones lineales expresado matricialmente del siguiente modo

$$
\left[\begin{array}{cc}
Y_{\mathrm{eq}}^{(1,1)}+Y_{\text {ogu1 }} & Y_{\mathrm{eq}}^{(1,2)} \\
Y_{\mathrm{eq}}^{(2,1)} & Y_{\mathrm{eq}}^{(2,2)}+Y_{\text {oguN }}
\end{array}\right] \cdot\left[\begin{array}{c}
Z_{\mathrm{gu} 1}^{(1)} \\
Z_{\mathrm{guN}}^{(2)}
\end{array}\right]=\left[\begin{array}{c}
I \\
O
\end{array}\right]
$$

donde la excitación del sistema de ecuaciones lineales viene definida mediante los vectores columna $I$ y $O$. Por lo que respecta al vector columna $I$, su dimensión depende del número de modos escogidos para representar los campos eléctrico y magnético en la guía de entrada del dispositivo, número representado por el parámetro NM(1); y sus elementos son todos iguales a 0 excepto el primer elemen- 


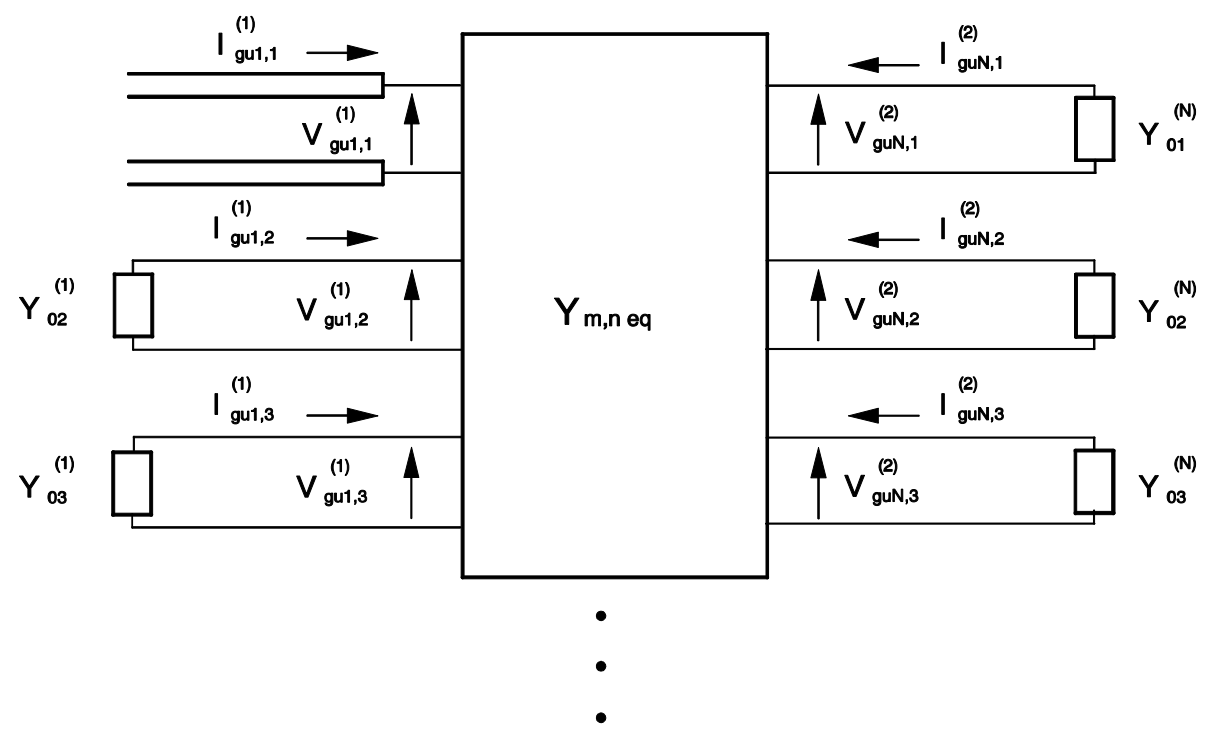

Figura 2.24: Red equivalente multimodal, asociada al dispositivo de microondas constituido por $N$ guías arbitrarias, cargada con las admitancias características de los modos correspondientes para obtener los parámetros de dispersión de dicho dispositivo.

to, de valor igual a 1, que está asociado a la excitación considerada para calcular los parámetros de dispersión del dispositivo. Por su parte, el vector columna $O$ presenta una dimensión asociada al número de modos elegidos para describir en esta ocasión los campos eléctrico y magnético en la guía de salida del dispositivo, número que designaremos mediante el parámetro $\mathrm{NM}(\mathrm{N})$; y todos los elementos de dicho vector $O$ presentan en este caso un valor nulo.

En cuanto a las incógnitas del sistema de ecuaciones planteado matricialmente en $(2.71), Z_{\mathrm{gu} 1}^{(1)}$ representa un vector columna, de dimensión igual a $\mathrm{NM}(1)$, cuyos elementos representan una autoimpedancia (asociada al primer modo) y transimpedancias (asociadas al resto de modos); que se definen como el cociente entre el voltaje asociado a cada modo en el puerto de entrada de la guía $1 \mathrm{y}$ la corriente asociada al modo fundamental de dicha guía en el mismo puerto de entrada, presentando pues el vector columna $Z_{\text {gu1 }}^{(1)}$ el siguiente aspecto

$$
Z_{\mathrm{gu} 1}^{(1)}=\left[\begin{array}{c}
\frac{V_{\mathrm{gu} 1,1}^{(1)}}{I_{\mathrm{gu} 1,1}^{(1)}} \\
\frac{V_{\mathrm{gu} 1,2}^{(1)}}{I_{\mathrm{gu} 1,1}^{(1)}} \\
\vdots \\
\frac{V_{\mathrm{gu} 1, \mathrm{NM}(1)}^{(1)}}{I_{\mathrm{gu} 1,1}^{(1)}}
\end{array}\right]
$$

Por lo que respecta al resto de incógnitas a resolver en el sistema de ecuaciones propuesto en términos matriciales en (2.71), éstas se encuentran asociadas al 
vector columna $Z_{\mathrm{guN}}^{(2)}$, de dimensión en este caso igual a $\mathrm{NM}(\mathrm{N})$, cuyos elementos representan todos ellos transimpedancias; que se definen como el cociente entre el voltaje asociado a cada modo en el puerto de salida de la guía $N$ y la corriente asociada al modo fundamental en el puerto de entrada de la guía 1, y se expresan matemáticamente de la siguiente manera

$$
Z_{\mathrm{guN}}^{(2)}=\left[\begin{array}{c}
\frac{V_{\mathrm{guN}, 1}^{(2)}}{I_{\mathrm{gu}(1,1}^{(1)}} \\
\frac{V_{\mathrm{guN}, 2}^{(2)}}{I_{\mathrm{gu} 1,1}^{(1)}} \\
\vdots \\
\frac{V_{\mathrm{guN}, \mathrm{NM}(\mathrm{N})}^{(2)}}{I_{\mathrm{gu} 1,1}^{(1)}}
\end{array}\right]
$$

Finalmente, las matrices $Y_{0 \mathrm{gu} 1}$ e $Y_{0 \mathrm{guN}}$, presentes en el sistema de ecuaciones propuesto en (2.71), aparecen como consecuencia de imponer las condiciones de carga en los puertos de entrada y salida de la red equivalente multimodal del dispositivo a caracterizar. Concretamente, la matriz $Y_{0 g u 1}$ es una matriz diagonal que contiene un 0 como primer elemento de su diagonal principal (el acceso asociado a la excitación del dispositivo no se carga), y las admitancias características de los respectivos modos considerados en la guía de entrada en el resto de elementos de dicha diagonal principal (condición de carga en el puerto de entrada de la guía 1); ofreciendo un aspecto que se muestra a continuación

$$
Y_{\text {0gu1 }}=\left[\begin{array}{cccc}
0 & 0 & \cdots & 0 \\
0 & Y_{02}^{(1)} & \cdots & 0 \\
0 & 0 & \ddots & 0 \\
0 & 0 & \cdots & Y_{0 \mathrm{NM}(1)}^{(1)}
\end{array}\right]
$$

Por otra parte, la matriz $Y_{0 \text { guN }}$ es nuevamente una matriz diagonal; que en este caso contiene en todos los elementos de su diagonal principal las correspondientes admitancias características de los modos escogidos en la guía de salida (condición de carga en el puerto de salida de la guía $N$ ). Así pues, dicha matriz $Y_{0 g u N}$ se construye como se indica seguidamente

$$
Y_{0 \mathrm{guN}}=\left[\begin{array}{cccc}
Y_{01}^{(\mathrm{N})} & 0 & \cdots & 0 \\
0 & Y_{02}^{(\mathrm{N})} & \cdots & 0 \\
0 & 0 & \ddots & 0 \\
0 & 0 & \cdots & Y_{0 \mathrm{NM}(\mathrm{N})}^{(\mathrm{N})}
\end{array}\right]
$$


Tras resolver pues el sistema de ecuaciones lineales planteado en (2.71), resulta posible obtener fácilmente los parámetros de dispersión $S_{11}$ y $S_{21}$ asociados al dispositivo de microondas que se pretende caracterizar; deduciendo dichos parámetros de dispersión a partir de las siguientes expresiones, que hacen uso de la autoimpedancia $Z_{\mathrm{gu} 1,1}^{(1)}$ y la transimpedancia $Z_{\mathrm{guN}, 1}^{(2)}$ obtenidas previamente

$$
\begin{aligned}
& S_{11}=\frac{Z_{\mathrm{gu} 1,1}^{(1)}-Z_{01}^{(1)}}{Z_{\mathrm{gu} 1,1}^{(1)}+Z_{01}^{(1)}} \\
& S_{21}=\frac{Z_{\mathrm{guN}, 1}^{(2)}}{\sqrt{Z_{01}^{(1)} \cdot Z_{01}^{(\mathrm{N})}}} \cdot\left(1-S_{11}\right)
\end{aligned}
$$

De esta forma, se ha caracterizado la respuesta electromagnética del dispositivo de microondas constituido por la conexión en cascada de $\mathrm{N}$ guías arbitrarias; habiendo empleado en la resolución del sistema de ecuaciones lineales asociado al dispositivo una técnica reductiva, basada en un procedimiento tradicionalmente utilizado cuando las uniones planares y los tramos de guía del dispositivo se caracterizan mediante matrices de dispersión generalizadas [5].

\subsubsection{Descripción de la Técnica Recursiva}

La técnica reductiva propuesta en el apartado anterior para caracterizar un dispositivo de microondas integrado por $\mathrm{N}$ guías en cascada, basada en una simple adaptación al caso que nos ocupa del método utilizado en [5] cuando se emplean matrices de dispersión generalizadas, no ha explotado en ningún momento la estructura en banda que presenta la matriz de coeficientes del sistema de ecuaciones lineales asociado al análisis de la estructura global ofrecida en la figura 2.21; razón por la cual parece lógico pensar que la técnica expuesta anteriormente para analizar los dipositivos de microondas considerados en esta sección no debe ser la más eficiente. En el presente apartado, haciendo uso de un algoritmo recursivo original, se expone una nueva técnica para resolver los sistemas de ecuaciones lineales en banda que surgen al analizar los citados dispositivos de microondas; con la esperanza de que al ajustarse dicho procedimiento a las características del sistema de ecuaciones lineales a resolver, se consiga reducir sustancialmente el esfuerzo computacional requerido en la resolución del mencionado sistema de ecuaciones lineales en banda.

Para exponer el nuevo método recursivo propuesto en este apartado, es necesario plantear inicialmente el sistema de ecuaciones lineales, con estructura en banda, que debe resolverse en el análisis de un dispositivo de microndas constituido por la conexión en cascada de $\mathrm{N}$ guías arbitrarias. Así pues, dicho sistema se obtiene imponiendo condiciones de continuidad de los campos eléctrico y magnético transversales en los puertos comunes de la estructura global mostrada 
en la figura 2.21; que pueden expresarse, tal y como se recoge a continuación, empleando unos vectores columna cuyos elementos constituyen los voltajes y las corrientes modales en los respectivos puertos comunes

$$
\begin{array}{cc}
V_{\mathrm{gu} 1}^{(2)}= & V_{\mathrm{up} 1}^{(1)} \\
I_{\mathrm{gu} 1}^{(2)} & =-I_{\mathrm{up} 1}^{(1)} \\
V_{\mathrm{up} 1}^{(2)} & =V_{\mathrm{gu} 2}^{(1)} \\
I_{\mathrm{up} 1}^{(2)} & =-I_{\mathrm{gu} 2}^{(1)} \\
\vdots & \vdots \\
V_{\mathrm{gu} i}^{(2)}= & V_{\mathrm{up} i}^{(1)} \\
I_{\mathrm{gu} i}^{(2)}= & -I_{\mathrm{up} i}^{(1)} \\
V_{\mathrm{up} i}^{(2)}= & V_{\mathrm{gu} i+1}^{(1)} \\
I_{\mathrm{up} i}^{(2)}= & -I_{\mathrm{gu} i+1}^{(1)} \\
\vdots & \vdots \\
V_{\mathrm{guN}-1}^{(2)}= & V_{\mathrm{upN}-1}^{(1)} \\
I_{\mathrm{guN}-1}^{(2)}= & -I_{\mathrm{upN}-1}^{(1)} \\
V_{\mathrm{upN}-1}^{(2)}= & V_{\mathrm{guN}}^{(1)} \\
I_{\mathrm{upN}-1}^{(2)}= & -I_{\mathrm{guN}}^{(1)}
\end{array}
$$

El planteamiento del sistema de ecuaciones lineales a resolver requiere seleccionar en principio un conjunto de incógnitas; escogiendo en este caso los voltajes modales (vectores columna) $V_{\mathrm{gu} 1}^{(1)}, V_{\mathrm{gu} 1}^{(2)}, V_{\mathrm{gu} 2}^{(1)}, \ldots, V_{\mathrm{guN}-1}^{(2)}, V_{\mathrm{guN}}^{(1)}, V_{\mathrm{guN}}^{(2)}$, que determinan los campos eléctricos transversales en los puertos de entrada y salida y en los puertos comunes de la estructura global del dipositivo bajo análisis (recordar fig. 2.21). Como se pretenden resolver $2 \mathrm{~N}$ incógnitas (vectores columna), conviene plantear el mismo número de ecuaciones matriciales; debiendo hacer uso para ello de las condiciones de continuidad de las corrientes modales (vectores columna) expuestas en (2.78)-(2.89). Ahora bien, dichas condiciones de continuidad permiten plantear $(2 \mathrm{~N}-2)$ ecuaciones de las $2 \mathrm{~N}$ ecuaciones necesarias para determinar el valor de todas las incógnitas propuestas; por lo que resulta necesario plantear dos ecuaciones adicionales, deducidas a partir de las relaciones matriciales entre voltajes modales (vectores columna) y corrientes modales (vectores columna) que definen las matrices $Y_{m, n \text { gu1 }}$ e $Y_{m, n \text { guN }}$. Una vez se plantean las ecuaciones de este 
sistema, haciendo uso de todas las mencionadas relaciones matriciales así como de las condiciones de continuidad propuestas en (2.78)-(2.89) para los voltajes modales (vectores columna), deben calcularse los parámetros de dispersión del dispositivo bajo análisis. Escogiendo los parámetros de dispersión asociados al modo fundamental de las guías de entrada (guía 1) y salida (guía $N$ ) del dispositivo a caracterizar, su cálculo requiere cargar todos los accesos libres de las redes equivalentes multimodales de las guías de entrada y salida del dispositivo (ver fig. 2.21) con las admitancias características de los modos correspondientes; exceptuando claro está el primer acceso de la red equivalente multimodal asociada a la guía de entrada, que corresponde al modo fundamental de dicha guía escogido como excitación del dispositivo. Tras imponer pues esta condición de carga al conjunto de ecuaciones planteadas anteriormente, se obtiene de forma definitiva el siguiente sistema constituido por $2 \mathrm{~N}$ ecuaciones

$$
\begin{aligned}
I_{\mathrm{gu} 1}= & \left(Y_{\mathrm{gu} 1}^{(1,1)}+Y_{0 \mathrm{gu} 1}\right) \cdot Z_{\mathrm{gu} 1}^{(1)}+Y_{\mathrm{gu} 1}^{(1,2)} \cdot Z_{\mathrm{gu} 1}^{(2)} \\
O_{\mathrm{gu} 1}= & Y_{\mathrm{gu} 1}^{(2,1)} \cdot Z_{\mathrm{gu} 1}^{(1)}+\left(Y_{\mathrm{gu} 1}^{(2,2)}+Y_{\mathrm{up} 1}^{(1,1)}\right) \cdot Z_{\mathrm{gu} 1}^{(2)}+Y_{\mathrm{up} 1}^{(1,2)} \cdot Z_{\mathrm{gu} 2}^{(1)} \\
O_{\mathrm{gu} 2}= & Y_{\mathrm{up} 1}^{(2,1)} \cdot Z_{\mathrm{gu} 1}^{(2)}+\left(Y_{\mathrm{up} 1}^{(2,2)}+Y_{\mathrm{gu} 2}^{(1,1)}\right) \cdot Z_{\mathrm{gu} 2}^{(1)}+Y_{\mathrm{gu} 2}^{(1,2)} \cdot Z_{\mathrm{gu} 2}^{(2)} \\
\vdots & \vdots \\
O_{\mathrm{gu} i}= & Y_{\mathrm{gu} i}^{(2,1)} \cdot Z_{\mathrm{gu} i}^{(1)}+\left(Y_{\mathrm{gu} i}^{(2,2)}+Y_{\mathrm{up} i}^{(1,1)}\right) \cdot Z_{\mathrm{gu} i}^{(2)}+Y_{\mathrm{up} i}^{(1,2)} \cdot Z_{\mathrm{gu} i+1}^{(1)} \\
O_{\mathrm{gu} i+1}= & Y_{\mathrm{up} i}^{(2,1)} \cdot Z_{\mathrm{gu} i}^{(2)}+\left(Y_{\mathrm{up} i}^{(2,2)}+Y_{\mathrm{gu} i+1}^{(1,1)}\right) \cdot Z_{\mathrm{gu} i+1}^{(1)}+Y_{\mathrm{gu} i+1}^{(1,2)} \cdot Z_{\mathrm{gu} i+1}^{(2)} \\
\vdots & \vdots \\
O_{\mathrm{guN}-1}= & Y_{\mathrm{guN}-1}^{(2,1)} \cdot Z_{\mathrm{guN}-1}^{(1)}+\left(Y_{\mathrm{guN}-1}^{(2,2)}+Y_{\mathrm{upN}-1}^{(1,1)}\right) \cdot Z_{\mathrm{guN}-1}^{(2)} \\
& +Y_{\mathrm{upN}-1}^{(1,2)} \cdot Z_{\mathrm{guN}}^{(1)} \\
O_{\mathrm{guN}}= & Y_{\mathrm{upN}-1}^{(2,1)} \cdot Z_{\mathrm{guN}-1}^{(2)}+\left(Y_{\mathrm{upN}-1}^{(2,2)}+Y_{\mathrm{guN}}^{(1,1)}\right) \cdot Z_{\mathrm{guN}}^{(1)} \\
& +Y_{\mathrm{guN}}^{(1,2)} \cdot Z_{\mathrm{guN}}^{(2)} \\
O_{\mathrm{guN}=} & Y_{\mathrm{guN}}^{(2,1)} \cdot Z_{\mathrm{guN}}^{(1)}+\left(Y_{\mathrm{guN}}^{(2,2)}+Y_{\mathrm{gguN}}\right) \cdot Z_{\mathrm{guN}}^{(2)}
\end{aligned}
$$

donde la excitación de este sistema de ecuaciones lineales se define mediante los vectores columna $I_{\text {gu1 } 1}$ y $O_{\text {gu } i}$, con $i \in[1, \ldots, N]$. El vector columna $I_{\text {gu1 }}$, cuya dimensión es igual al número de modos NM(1) elegidos en la guía de entrada del dispostivo, presenta un valor igual a 1 en su primer elemento, que está asociado a la excitación del dispositivo, y valores nulos en el resto de sus elementos; mientras $O_{\text {gu } i}$, con $i \in[1, \ldots, N]$, designa un conjunto de vectores nulos (todos los elementos de cada uno de estos vectores iguales a 0), cuyas dimensiones corresponden a los números de modos escogidos en las correspondientes guías del dispositivo 
bajo análisis. En cuanto a las matrices $Y_{0 \mathrm{gu} 1}$ e $Y_{0 \mathrm{guN}}$, presentes respectivamente en (2.90) y (2.97), constituyen unas matrices diagonales que surgen al imponer las condiciones de carga requeridas para calcular los parámetros de dispersión del dispositivo a caracterizar; cuyos elementos en la diagonal principal son las admitancias características de los modos de las guías de entrada (guía 1) y salida (guía N) del dispositivo considerado, presentando dichas matrices el mismo aspecto mostrado anteriormente en (2.74) y (2.75). Por último, en lo referente a las incógnitas a resolver en el sistema de ecuaciones definido según (2.90)-(2.97), éstas se encuentran recogidas en los vectores columna $Z_{\mathrm{gu} i}^{(\gamma)}$, con $i \in[1, \ldots, N]$ y $\gamma=1,2$; vectores constituidos por unos elementos que representan transimpedancias (a excepción del primer elemento del vector columna $Z_{\mathrm{gu} 1}^{(1)}$ que designa una autoimpedancia), y definidos del siguiente modo

$$
Z_{\mathrm{gu} i}^{(\gamma)}=\left[\begin{array}{c}
\frac{V_{\mathrm{gu} i, 1}^{(\gamma)}}{I_{\mathrm{gu} 1,1}^{(1)}} \\
\frac{V_{\mathrm{gu} i, 2}^{(\gamma)}}{I_{\mathrm{gu} 1,1}^{(1)}} \\
\vdots \\
\frac{V_{\mathrm{gu} i, \mathrm{NM}(i)}^{(\gamma)}}{I_{\mathrm{gu} 1,1}^{(1)}}
\end{array}\right]
$$

La resolución del sistema de ecuaciones lineales con estructura en banda, propuesto en (2.90)-(2.97), puede resolverse mediante la aplicación de un algoritmo recursivo; que comienza examinando la última de las ecuaciones de dicho sistema, aquélla definida según (2.97), de la que es posible deducir la siguiente relación entre los vectores columna $Z_{\mathrm{guN}}^{(2)}$ y $Z_{\mathrm{guN}}^{(1)}$

$$
Z_{\mathrm{guN}}^{(2)}=-Y_{2 \mathrm{~N}, 2 \mathrm{~N}-1}^{\prime} \cdot Z_{\mathrm{guN}}^{(1)}
$$

donde la matriz $Y_{2 \mathrm{~N}, 2 \mathrm{~N}-1}^{\prime}$, presente en la relación entre vectores columna recién expuesta, se define de la siguiente manera

$$
Y_{2 \mathrm{~N}, 2 \mathrm{~N}-1}^{\prime}=\left(Y_{\mathrm{guN}}^{(2,2)}+Y_{0 \mathrm{guN}}\right)^{-1} \cdot Y_{\mathrm{guN}}^{(2,1)}
$$

Utilizando a continuación la relación definida mediante (2.99) en la ecuación del sistema recogida en (2.96), se puede establecer una relación en este caso entre los vectores columna $Z_{\mathrm{guN}}^{(1)}$ y $Z_{\mathrm{guN}-1}^{(2)}$; cuyo aspecto se muestra seguidamente

$$
Z_{\mathrm{guN}}^{(1)}=-Y_{2 \mathrm{~N}-1,2 \mathrm{~N}-2}^{\prime} \cdot Z_{\mathrm{guN}-1}^{(2)}
$$

donde la matriz $Y_{2 \mathrm{~N}-1,2 \mathrm{~N}-2}^{\prime}$, que aparece en esta última relación definida entre los vectores columna $Z_{\mathrm{guN}}^{(1)}$ y $Z_{\mathrm{guN}-1}^{(2)}$, viene expresada como

$$
Y_{2 \mathrm{~N}-1,2 \mathrm{~N}-2}^{\prime}=\left(Y_{\mathrm{upN}-1}^{(2,2)}+Y_{\mathrm{guN}}^{(1,1)}-Y_{\mathrm{guN}}^{(1,2)} \cdot Y_{2 \mathrm{~N}, 2 \mathrm{~N}-1}^{\prime}\right)^{-1} \cdot Y_{\mathrm{upN}-1}^{(2,1)}
$$


Procediendo recursivamente de la manera indicada con anterioridad, se alcanza la $(2 i+1)$-ésima ecuación del sistema recogida en $(2.94)$; deduciendo de dicha ecuación del sistema una relación entre los vectores columna $Z_{\mathrm{gu} i+1}^{(1)}$ y $Z_{\mathrm{gu} i}^{(2)}$, que presenta el siguiente aspecto

$$
Z_{\mathrm{gu} i+1}^{(1)}=-Y_{2 i+1,2 i}^{\prime} \cdot Z_{\mathrm{gu} i}^{(2)}
$$

donde la matriz $Y_{2 i+1,2 i}^{\prime}$, que relaciona los vectores columna $Z_{\mathrm{gu} i+1}^{(1)} \mathrm{y} Z_{\mathrm{gu} i}^{(2)}$, viene definida según la siguiente expresión

$$
Y_{2 i+1,2 i}^{\prime}=\left(Y_{\mathrm{up} i}^{(2,2)}+Y_{\mathrm{gu} i+1}^{(1,1)}-Y_{\mathrm{gu} i+1}^{(1,2)} \cdot Y_{2 i+2,2 i+1}^{\prime}\right)^{-1} \cdot Y_{\mathrm{up} i}^{(2,1)}
$$

Del mismo modo, haciendo uso de la relación expuesta en (2.103) entre los vectores columna $Z_{\mathrm{gu} i+1}^{(1)}$ y $Z_{\mathrm{gu} i}^{(2)}$ en la (2i)-ésima ecuación del sistema descrita en (2.93), se obtiene una nueva relación en este caso entre los vectores columna $Z_{\mathrm{gu} i}^{(2)}$ y $Z_{\text {gu } i}^{(1)}$; que se expresa de la siguiente manera

$$
Z_{\mathrm{gu} i}^{(2)}=-Y_{2 i, 2 i-1}^{\prime} \cdot Z_{\mathrm{gu} i}^{(1)}
$$

donde la matriz $Y_{2 i, 2 i-1}^{\prime}$, que define esta nueva relación, se expresa a su vez como se indica a continuación

$$
Y_{2 i, 2 i-1}^{\prime}=\left(Y_{\mathrm{gu} i}^{(2,2)}+Y_{\mathrm{up} i}^{(1,1)}-Y_{\mathrm{up} i}^{(1,2)} \cdot Y_{2 i+1,2 i}^{\prime}\right)^{-1} \cdot Y_{\mathrm{gu} i}^{(2,1)}
$$

Repitiendo de igual manera el proceso anteriormente descrito hasta alcanzar la primera de las ecuaciones del sistema, definida según (2.90), se obtiene la siguiente expresión que permite determinar el valor del vector columna $Z_{\mathrm{gu} 1}^{(1)}$

$$
Z_{\mathrm{gu} 1}^{(1)}=Y_{1,1}^{\prime} \cdot I_{\mathrm{gu} 1}
$$

donde la matriz $Y_{1,1}^{\prime}$, requerida en la obtención del vector columna $Z_{\mathrm{gu1}}^{(1)}$, se deduce empleando la siguiente expresión analítica

$$
Y_{1,1}^{\prime}=\left(Y_{\mathrm{gu} 1}^{(1,1)}+Y_{0 \mathrm{gu} 1}-Y_{\mathrm{gu} 1}^{(1,2)} \cdot Y_{2,1}^{\prime}\right)^{-1}
$$

Una vez se conoce el valor del vector columna $Z_{\mathrm{gu} 1}^{(1)}$, empleando de forma adecuada las relaciones entre vectores columna incógnita recogidas en (2.103) y (2.105), es posible proceder de forma recursiva en sentido inverso al seguido en el procedimiento descrito hasta este momento; y deducir por tanto los valores del resto de vectores columna $Z_{\text {gui }}^{(\gamma)}$, con $i \in[1, \ldots, N]$ y $\gamma=1,2$ (excepto $Z_{\text {gu1 }}^{(1)}$ cuyo valor se ha obtenido anteriormente). Tras determinar el valor de todas las incógnitas (autoimpedancia y transimpedancias definidas en los respectivos accesos de los puertos de la estructura global mostrada en la figura 2.21) del sistema 
de ecuaciones a resolver, entre ellas $Z_{\mathrm{gu1}, 1}^{(1)}$ y $Z_{\mathrm{guN}, 1}^{(2)}$, los parámetros de dispersión del dispositivo bajo análisis se calculan empleando las expresiones deducidas previamente en (2.76) y (2.77).

Así pues, el método recién descrito constituye una técnica novedosa [28], basada en un algoritmo recursivo, que permite resolver el tipo de sistemas de ecuaciones lineales en banda asociados a la caracterización de dispositivos de microondas constituidos por N guías arbitrarias conectadas en cascada. Esta nueva técnica recursiva, tal y como se comprueba en el siguiente apartado, debe resultar más eficiente que la descrita en el apartado 2.3.2; pues el algoritmo recursivo propuesto explota la naturaleza en banda del sistema que se pretende resolver.

\subsubsection{Eficiencia Computacional de la Técnica Recursiva}

La caracterización de dispositivos de microondas constituidos por la conexión en cascada de $\mathrm{N}$ guías uniformes requiere resolver un sistema de ecuaciones lineales en banda; en cuya resolución pueden emplearse, entre otras posibles técnicas, aquélla descrita en el apartado 2.3 .2 basada en una reducción progresiva de la estructura global del dispositivo mostrada en la figura 2.21, y aquella otra técnica recursiva propuesta en el apartado 2.3.3 que hace uso de la estructura en banda del citado sistema. Con el objeto de comprobar el ahorro en número de operaciones matriciales que supone esta técnica recursiva, se ofrece en primer lugar un estudio comparativo del número de multiplicaciones e inversiones de matrices (con elementos complejos) requeridas por las citadas técnicas al resolver el mencionado sistema de ecuaciones lineales.

Considerando inicialmente la técnica propuesta en el apartado 2.3.2, consistente en reducir de manera progresiva dos matrices de admitancias a una matriz equivalente hasta representar la estructura global ofrecida en la figura 2.21 mediante una sola matriz $\left(Y_{\text {eq }}\right)$, se observa que para implementar las expresiones recogidas en (2.67)-(2.70) es necesario realizar 6 multiplicaciones y 1 inversión de matrices complejas en cada etapa del análisis reductivo; debiendo realizar, tal y como se indica en el apartado 2.3.2, $(2 \mathrm{~N}-2)$ de estas etapas reductivas para obtener la matriz de admitancias equivalente $\left(Y_{\mathrm{eq}}\right)$ que representa a la estructura global mostrada en la figura 2.21. Finalmente, para obtener los parámetros de dispersión que presenta el dispositivo bajo análisis, se requiere resolver el sistema de ecuaciones lineales planteado en términos matriciales en (2.71); para lo cual debe llevarse a cabo una última inversión de una matriz cuyas dimensiones son respectivamente el doble de las que presentan las matrices invertidas en cada una de las etapas reductivas. En la tabla 2.3, se recogen de forma resumida estos números de operaciones con matrices complejas (multiplicaciones e inversiones) que requiere la técnica de reducción progresiva descrita con detalle en el apartado 2.3.2; técnica que constituye una traslación al tipo de análisis propuesto en este capítulo, basado en matrices de admitancias generalizadas, del método 


\begin{tabular}{|l|c|c|}
\hline \hline \multirow{2}{*}{$\begin{array}{c}\text { Método implementado para } \\
\text { resolver el sistema lineal }\end{array}$} & \multicolumn{2}{|c|}{ Número de operaciones matriciales } \\
\cline { 2 - 3 } & Multiplicaciones & Inversiones \\
\hline 1. Técnica de reducción & $6 \cdot(2 N-2)$ & $2 N-1$ \\
\hline 2. Técnica recursiva & $3 \cdot(2 N-1)$ & $2 N$ \\
\hline \hline
\end{tabular}

Tabla 2.3: Número de operaciones matriciales (multiplicaciones e inversiones de matrices con elementos complejos) requeridas para resolver el sistema lineal en banda que aparece al analizar un dispositivo de microondas constituido por $N$ guías arbitrarias.

tradicionalmente utilizado en la caracterización de dispositivos de microondas empleando matrices de dispersión generalizadas [5].

Si se estudia a continuación la técnica recursiva expuesta en el apartado 2.3.3, que hace uso de la naturaleza en banda del sistema de ecuaciones lineales a resolver, observando (2.100), (2.104), (2.106) y (2.108), se deduce fácilmente que en cada iteración de dicho algoritmo recursivo se requiere una inversión de una matriz con elementos complejos; de manera que para resolver el sistema en banda será necesario realizar un número total de $2 \mathrm{~N}$ inversiones. En cuanto a estas inversiones, resulta interesante reseñar que todas las matrices a invertir presentan el mismo tamaño (dimensiones) que el de las matrices a invertir en cada etapa reductiva de la otra técnica; tal y como se puede comprobar recordando las ecuaciones que definen cada una de dichas etapas recogidas en (2.67)-(2.70). Por lo que respecta al número de multiplicaciones de matrices complejas a implementar, en (2.99) y (2.100) es necesario realizar 2 multiplicaciones de este tipo, las expresiones generales (2.103)-(2.106) determinan la necesidad de llevar a cabo 3 multiplicaciones en cada iteración del algoritmo recursivo, mientras la ecuación (2.108) requiere de una sola de estas multiplicaciones. Un resumen del número total de inversiones y multiplicaciones de matrices con elementos complejos que requiere esta técnica recursiva, descrita detalladamente en el apartado 2.3.3, se recoge de nuevo por razones comparativas en la tabla 2.3.

Del estudio comparativo ofrecido en la tabla 2.3, resulta fácil determinar la eficiencia computacional que presentan las dos técnicas descritas hasta el momento; concluyendo que la técnica basada en una reducción progresiva requiere casi el doble de multiplicaciones de matrices con elementos complejos que la técnica recursiva, mientras ambas técnicas precisan prácticamente del mismo número de inversiones complejas. En realidad, aunque la técnica de reducción progresiva realiza una inversión menos que la técnica recursiva, la última matriz a invertir por la técnica de reducción progresiva tiene el doble de tamaño (dimensiones) que el del resto de matrices previamente invertidas en dicho método, y que el de todas las matrices a invertir cuando se emplea la técnica recursiva. En definitiva, al analizar estructuras de microondas complejas constituidas por la conexión en cascada de $\mathrm{N}$ guías arbitrarias $(\mathrm{N} \gg 1)$, el coste computacional asociado a la 


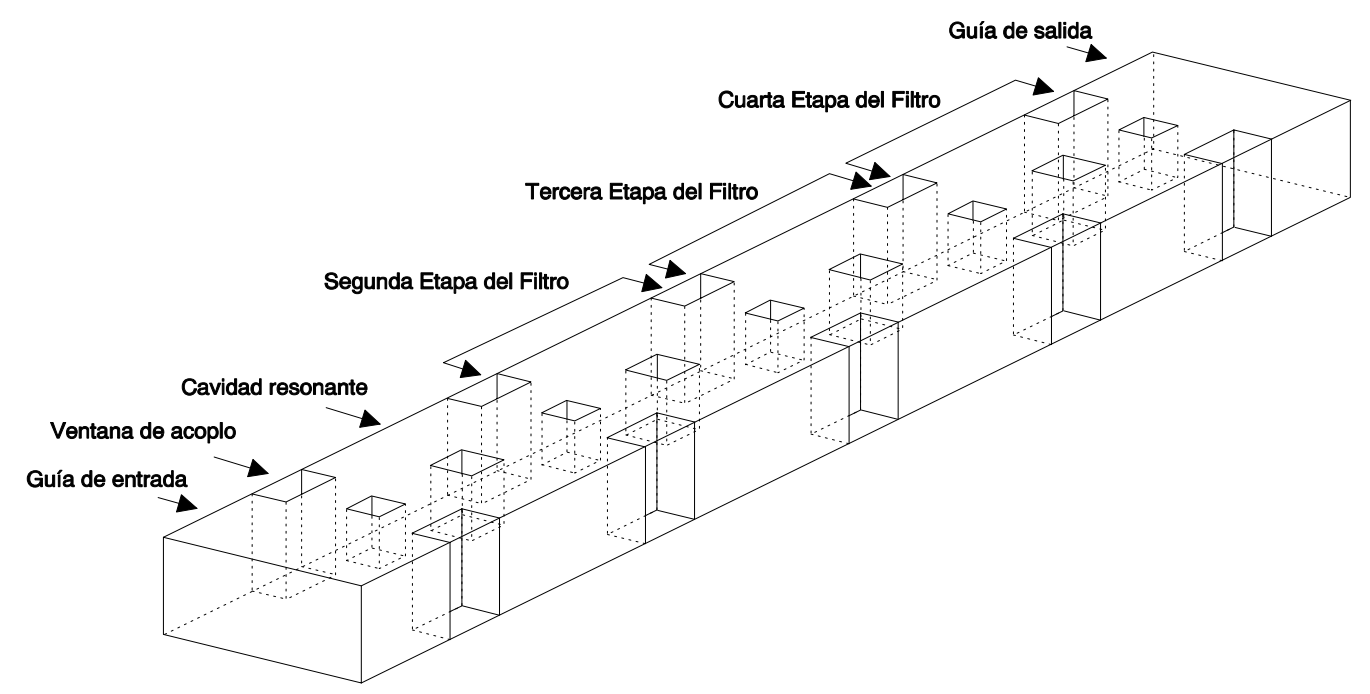

Figura 2.25: Filtro paso banda de 4 polos con ventanas de acoplo inductivas y elementos de sintonía.

resolución del mencionado sistema en banda mediante la técnica de reducción progresiva será al menos el doble que utilizando la técnica recursiva.

Con el objeto de determinar la eficiencia computacional de cada una de las técnicas propuestas para resolver el sistema en banda asociado al análisis de un dispositivo de microondas, se presenta a continuación un estudio comparativo del coste computacional requerido por dichas técnicas en la resolución del sistema asociado a un filtro paso banda de 4 polos, implementado con ventanas inductivas y elementos de sintonía tal y como se muestra en la figura 2.25; habiendo utilizado para resolver el citado sistema un procedimiento de eliminación Gaussiana con retrosustitución [23] que hace uso de la naturaleza en banda del sistema a resolver, la técnica de reducción progresiva expuesta en el apartado 2.3.2, y la nueva técnica recursiva propuesta en el apartado 2.3.3 que explota también la estructura en banda del sistema. La estructura escogida (ver figura 2.25) corresponde a un filtro paso banda simétrico de 4 polos, centrado en $11 \mathrm{GHz}$ y con un ancho de banda de $300 \mathrm{MHz}$, que está constituido lógicamente por 4 etapas (igual número de etapas que polos de la respuesta del filtro); donde la posición en frecuencia de la respuesta del filtro, así como el ancho de banda deseado, se consiguen situando elementos de sintonía (de sección transversal cuadrada) en las ventanas de acoplo y en las cavidades del filtro. Como guías de entrada y salida, se han escogido guías normalizadas WR-75 $(a=19.050 \mathrm{~mm}, b=9.525 \mathrm{~mm})$; mientras las ventanas de acoplo presentan unas secciones transversales cuyas dimensiones se recogen en la tabla 2.4, todas con una profundidad de $2.000 \mathrm{~mm}$. En cuanto a los elementos de sintonía presentes en las ventanas de acoplo, todos ellos tienen sección transversal cuadrada $(2.000 \mathrm{~mm} \times 2.000 \mathrm{~mm})$ y sus penetraciones, necesarias para conseguir el ancho de banda requerido en la respuesta del filtro, se resumen nuevamente 


\begin{tabular}{|c|c|c|c|c|}
\hline \multirow{2}{*}{$\begin{array}{c}\text { Elemento de sintonía } \\
\text { situado en }\end{array}$} & \multicolumn{4}{|c|}{ Guía reentrante } \\
\hline & $\mathrm{a}(\mathrm{mm})$ & $\mathrm{b}(\mathrm{mm})$ & $\mathrm{w}(\mathrm{mm})$ & $\mathrm{h}(\mathrm{mm})$ \\
\hline 1a ventana de acoplo & 8.707 & 9.525 & 2.000 & 3.366 \\
\hline $2^{\mathrm{a}}$ ventana de acoplo & 5.107 & 9.525 & 2.000 & 4.078 \\
\hline $3^{\underline{a}}$ ventana de acoplo & 5.109 & 9.525 & 2.000 & 3.570 \\
\hline 4a ventana de acoplo & 5.107 & 9.525 & 2.000 & 4.078 \\
\hline $5^{\text {a }}$ ventana de acoplo & 8.707 & 9.525 & 2.000 & 3.366 \\
\hline
\end{tabular}

a

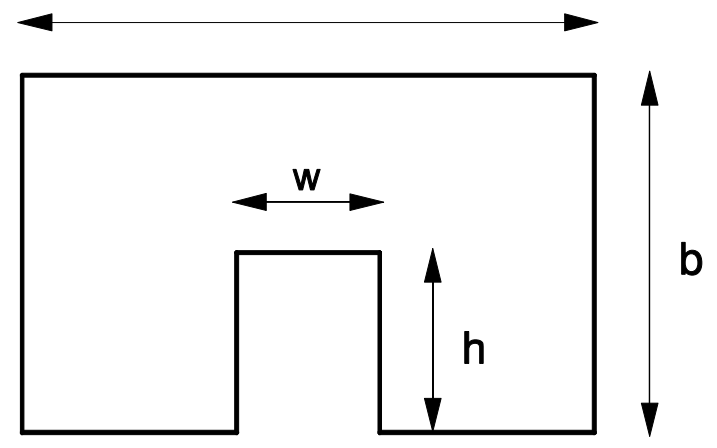

Tabla 2.4: Dimensiones de las guías reentrantes centradas que se escogen como elementos de sintonía situados en las ventanas de acoplo del filtro de 4 polos mostrado en la figura 2.25 .

en la tabla 2.4. Por lo que respecta a las cavidades resonantes, sus secciones transversales corresponden también a las de la guía normalizada WR-75 (ver tabla 2.5); y las longitudes de la primera y segunda cavidad son respectivamente $10.500 \mathrm{~mm}$ y $13.300 \mathrm{~mm}$, que determinan a su vez las longitudes de la tercera y cuarta cavidad del diseño debido a la simetría del filtro. Por último, los elementos de sintonía insertados en las cavidades tienen otra vez sección transversal cuadrada (en este caso de dimensiones $4.000 \mathrm{~mm} \times 4.000 \mathrm{~mm}$ ) con unas penetraciones, responsables ahora de la ubicación en frecuencia de la respuesta del filtro, resumidas en la tabla 2.5. La estructura propuesta (ver figura 2.25) está constituida por la conexión en cascada de 29 guías uniformes; que se dividen en guías rectangulares y guías reentrantes (en inglés ridge). Para analizar la citada estructura, resulta necesario caracterizar diversas uniones planares centradas entre guías rectangulares, y varias transiciones entre guías rectangulares y guías reentrantes; empleando para ello las correspondientes matrices de admitancias generalizadas, cuyos elementos $Y_{m, n}^{(2,2)}$ se calculan mediante el procedimiento acelerado propuesto en el apartado 2.2.2. En el análisis de las transiciones entre guías rectangulares y guías reentrantes se precisa conocer el espectro modal de las guías reentrantes, así como las integrales de acoplamiento entre dichos modos 


\begin{tabular}{|c|c|c|c|c|}
\hline \multirow{2}{*}{$\begin{array}{c}\text { Elemento de sintonía } \\
\text { situado en }\end{array}$} & \multicolumn{4}{|c|}{ Guía reentrante } \\
\hline & $\mathrm{a}(\mathrm{mm})$ & $\mathrm{b}(\mathrm{mm})$ & $\mathrm{w}(\mathrm{mm})$ & $\mathrm{h}(\mathrm{mm})$ \\
\hline 1므 cavidad resonante & 19.050 & 9.525 & 4.000 & 3.298 \\
\hline $2^{\text {a }}$ cavidad resonante & 19.050 & 9.525 & 4.000 & 2.972 \\
\hline $3^{\text {a }}$ cavidad resonante & 19.050 & 9.525 & 4.000 & 2.972 \\
\hline $4^{\mathrm{a}}$ cavidad resonante & 19.050 & 9.525 & 4.000 & 3.298 \\
\hline
\end{tabular}

a

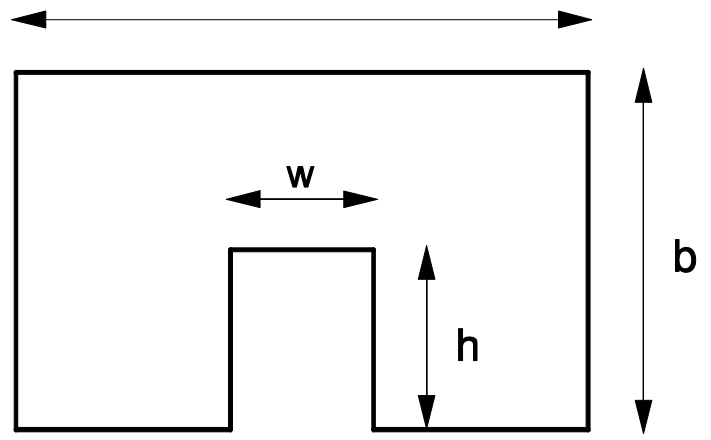

Tabla 2.5: Dimensiones de las guías reentrantes centradas que se escogen como elementos de sintonía situados en las cavidades resonantes del filtro de 4 polos mostrado en la figura 2.25 .

y los modos de las correspondientes guías rectangulares; en cuya obtención, tanto de los modos como de las citadas integrales, se ha seguido el método numérico propuesto en [21] y [22] que se resume en el apartado 3.1.1 del capítulo 3.

Para realizar el estudio comparativo recién descrito, se han determinado los parámetros de dispersión (en inglés scattering) asociados al modo fundamental que presenta el filtro paso banda de 4 polos escogido (recordar fig. 2.25); en cuyo cálculo se han seguido, por razones comparativas, las tres estrategias descritas en el párrafo anterior para resolver el sistema en banda que surge al analizar el mencionado filtro. La obtención del citado sistema en banda se debe, como se ha demostrado anteriormente en esta sección, a la conexión en cascada de las matrices de admitancias generalizadas que representan las distintas uniones planares y tramos de guía del dispositivo bajo análisis; resultando por tanto necesario el cálculo previo de dichas matrices, cuyos elementos $Y_{m, n}^{(2,2)}$ se han obtenido mediante la técnica acelerada (recordar apartado 2.2.2) para reducir el esfuerzo dedicado a su computación. En cuanto al número de modos requeridos para describir los campos eléctricos y magnéticos en las guías del filtro analizado, debido a la complejidad que presenta esta estructura con numerosas discontinuidades abruptas, ha sido necesario considerar 90 modos en cada una de dichas guías para obtener resultados convergentes. La respuesta electromagnética del filtro se ha obtenido 


\begin{tabular}{|l|c|}
\hline \hline $\begin{array}{c}\text { Método implementado para } \\
\text { resolver el sistema lineal }\end{array}$ & Coste computacional \\
\hline 1. Técnica de reducción & $43.50 \mathrm{seg}$ \\
\hline 2. Eliminación Gaussiana & $23.75 \mathrm{seg}$ \\
\hline 3. Técnica recursiva & $15.50 \mathrm{seg}$ \\
\hline \hline
\end{tabular}

Tabla 2.6: Tiempo de cálculo requerido para resolver en cada frecuencia el sistema lineal en banda que aparece al analizar el filtro de 4 polos mostrado en la figura 2.25 .

para 200 valores de la frecuencia en la banda comprendida entre $10.50 \mathrm{GHz}$ y $11.50 \mathrm{GHz}$; habiendo empleado en la realización de todas las simulaciones una plataforma IBM Risc-6000. Los resultados obtenidos, correspondientes al tiempo que supone resolver por cada valor de la frecuencia el sistema de ecuaciones lineales asociado al análisis del filtro considerado, se recogen en la tabla 2.6; donde el método designado como 1 hace referencia a la técnica de reducción progresiva expuesta con detalle en el apartado 2.3.2, el método etiquetado con el número 2 se refiere a un procedimiento típico de eliminación Gaussiana con retrosustitución [23] que hace uso de la naturaleza en banda del sistema, y finalmente el método asociado al número 3 corresponde a la nueva técnica recursiva descrita en el apartado 2.3.3 para resolver el mencionado sistema en banda. De los resultados ofrecidos en la tabla 2.6, se concluye que la técnica recursiva propuesta en la presente sección resulta la más eficiente (menos coste computacional) en comparación con las otras dos técnicas consideradas; resultando interesante destacar que dicha técnica recursiva, como era previsible del estudio comparativo realizado anteriormente en función del número de operaciones matriciales requeridas, reduce casi en un factor de valor 3 el tiempo empleado por la técnica reductiva en la resolución del sistema asociado al filtro analizado.

Como consecuencia del estudio de eficiencia computacional recién presentado, se extrae la conclusión de que los métodos de resolución de los mencionados sistemas en banda que tienen en cuenta la naturaleza de dichos sistemas, tales como los métodos 2 y 3 recogidos en la tabla 2.6, son más eficientes que aquellos otros (como el método 1 considerado también en la citada tabla 2.6) que no hacen uso de la característica en banda de los sistemas. Asimismo, como se desprende de los resultados recogidos en la tabla 2.6, la técnica de resolución del sistema en banda basada en un algoritmo recursivo resulta más eficiente que el procedimiento de eliminación Gaussiana con retrosustitución [23]; por lo que parece interesante, con el objeto de reducir todavía más el esfuerzo computacional dedicado al análisis y al diseño de dispositivos pasivos de microondas, incorporar la técnica recursiva propuesta (ver apartado 2.3.3) para resolver los mencionados sistemas en banda a las herramientas de programación, basadas en el cálculo acelerado de las matrices de admitancias generalizadas (recordar sección 2.2), que analizan y diseñan 
los citados dispositivos de microondas. Para concluir, el estudio comparativo de técnicas de resolución de sistemas ofrecido en el presente apartado confirma las ventajas del análisis de dispositivos de microondas empleando matrices de admitancias generalizadas; que generan sistemas de ecuaciones lineales a resolver con estructura en banda, y por consiguiente permiten utilizar métodos eficientes en la resolución de dichos sistemas que aprovechan su naturaleza en banda. Por contra, empleando otros métodos clásicos de análisis basados en matrices de dispersión generalizadas, conocidos como adaptación modal [2-5], se obtienen sistemas de ecuaciones lineales que no presentan una estructura en banda; en cuya resolución, la técnica empleada (muy similar a la técnica reductiva descrita en el apartado 2.3.2) supone un coste computacional (recordar tabla 2.6) casi 3 veces mayor que la técnica recursiva utilizada al emplear matrices de admitancias generalizadas. Podría pensarse no obstante en construir un sistema en banda cuando se emplean matrices de dispersión generalizadas; aunque esta acción supondría un coste adicional de una inversión, en cada valor de la frecuencia de análisis, de un bloque de las matrices de dispersión generalizadas por cada una de las ecuaciones matriciales a obtener, lo que implicaría sin duda un mayor esfuerzo computacional frente al ahorro conseguido posteriormente resolviendo el sistema en banda obtenido mediante la mencionada técnica recursiva. 


\section{Capítulo 3}

\section{Ejemplos de Análisis y Diseño Eficiente de Dispositivos Pasivos de Microondas Complejos}

El desarrollo de herramientas de programación que permiten analizar y diseñar filtros paso banda en guía rectangular, empleando en dichos filtros ventanas de acoplo inductivas entre las cavidades, ha alcanzado un punto en el que es posible diseñar este tipo de filtros en tiempo real con una gran precisión. Ahora bien, la correcta implementación de estos diseños, sin utilizar ningún elemento de sintonía, requiere de unas tolerancias en su fabricación ciertamente muy restrictivas; repercutiendo pues en un incremento sustancial del coste de estos filtros. Por otra parte, en determinadas aplicaciones resulta interesante poder desplazar la respuesta de uno de estos filtros dentro de una banda de frecuencias; lo que supone, al igual que para corregir las deficiencias en los diseños debidas a las recién mencionadas tolerancias, el empleo de tornillos de sintonía en los citados filtros diseñados con ventanas inductivas. El análisis electromagnético completo de estos filtros, que incorporan tornillos de sintonía, resulta especialmente costoso desde el punto de vista computacional; por lo que resulta interesante analizar y diseñar dichos filtros con alguna herramienta de programación muy eficiente. De igual manera, existen otros filtros paso banda utilizados especialmente en la carga útil de los satélites de comunicaciones, conocidos como filtros de modo dual (en inglés dual mode filters), cuya configuración resulta especialmente compleja; razón por la cual, en el análisis y diseño de estas estructuras se precisa nuevamente de una herramienta de programación especialmente optimizada.

En el presente capítulo, se analizan y diseñan filtros paso banda implementados en guía rectangular con ventanas inductivas y tornillos de sintonía, así como filtros paso banda de modo dual construidos en guía circular; empleando para ello una herramienta de programación optimizada, denominada comercialmente DUMAS 3.0 y documentada en el apéndice B, que utiliza la técnica descrita en el 
capítulo 2 (sección 2.2) para acelerar el cálculo de los elementos de las matrices de admitancias generalizadas de las uniones planares integrantes de los dispositivos, y que emplea también la técnica eficiente de resolución de sistemas de ecuaciones lineales en banda propuesta en el capítulo 2 (sección 2.3). Concretamente, haciendo uso del mencionado programa de análisis y diseño de dispositivos pasivos de microondas, se analiza en primer lugar el comportamiento electromagnético de los tornillos de sintonía situados en el interior de filtros paso banda en guía rectangular con ventanas inductivas; encontrando una equivalencia entre el comportamiento de estos tornillos y unos elementos de sección transversal cuadrada. Seguidamente, se investiga la zona de sintonía que es posible conseguir con dichos elementos cuando éstos se sitúan dentro de las ventanas inductivas y de las cavidades resonantes de los filtros; presentando a continuación una técnica novedosa para diseñar una estructura básica de filtro con ventanas inductivas que puede sintonizarse en una cierta banda de frecuencias. Tanto la técnica de diseño propuesta, como la equivalencia entre los tornillos de sección circular y los elementos de sección cuadrada, se corroboran con medidas de los parámetros de dispersión (en inglés scattering) correspondientes a una implementación práctica de una de estas estructuras. De igual manera, empleando de nuevo la mencionada herramienta de programación optimizada para el análisis y el diseño de estructuras pasivas de microondas, se presentan posteriormente dos nuevos diseños de filtros de modo dual en guía circular; diseños que se validan midiendo la respuesta electromagnética de las dos implementaciones de dichos dispositivos.

\subsection{Análisis y Diseño de Filtros Paso Banda en Guía Rectangular con Ventanas de Acoplo Inductivas y Tornillos de Sintonía}

El estado del arte de las herramientas avanzadas de programación que permiten analizar y diseñar filtros paso banda en guía rectangular con ventanas de acoplo inductivas, cuyo aspecto en el caso concreto de un filtro de 4 polos se ofrece en la figura 3.1, ha alcanzado un nivel en el que las implementaciones de dichos filtros sin elementos de sintonía están perfectamente garantizadas para cualquier frecuencia central de la banda de paso y para cualquier ancho de banda [29-31]. Estos filtros, al no incorporar ningún elemento de sintonía en su implementación, presentan la ventaja de que no es necesario realizar ningún tipo de ajuste manual posterior a su construcción. De manera adicional, la respuesta de estos filtros puede predecirse con gran precisión en una banda de frecuencias muy amplia; lo que resulta especialmente atractivo al permitir diseñar filtros que satisfagan una serie de especificaciones de rechazo fuera de la banda de trabajo del filtro, y que cumplan al mismo tiempo los requisitos impuestos dentro de la banda de paso 


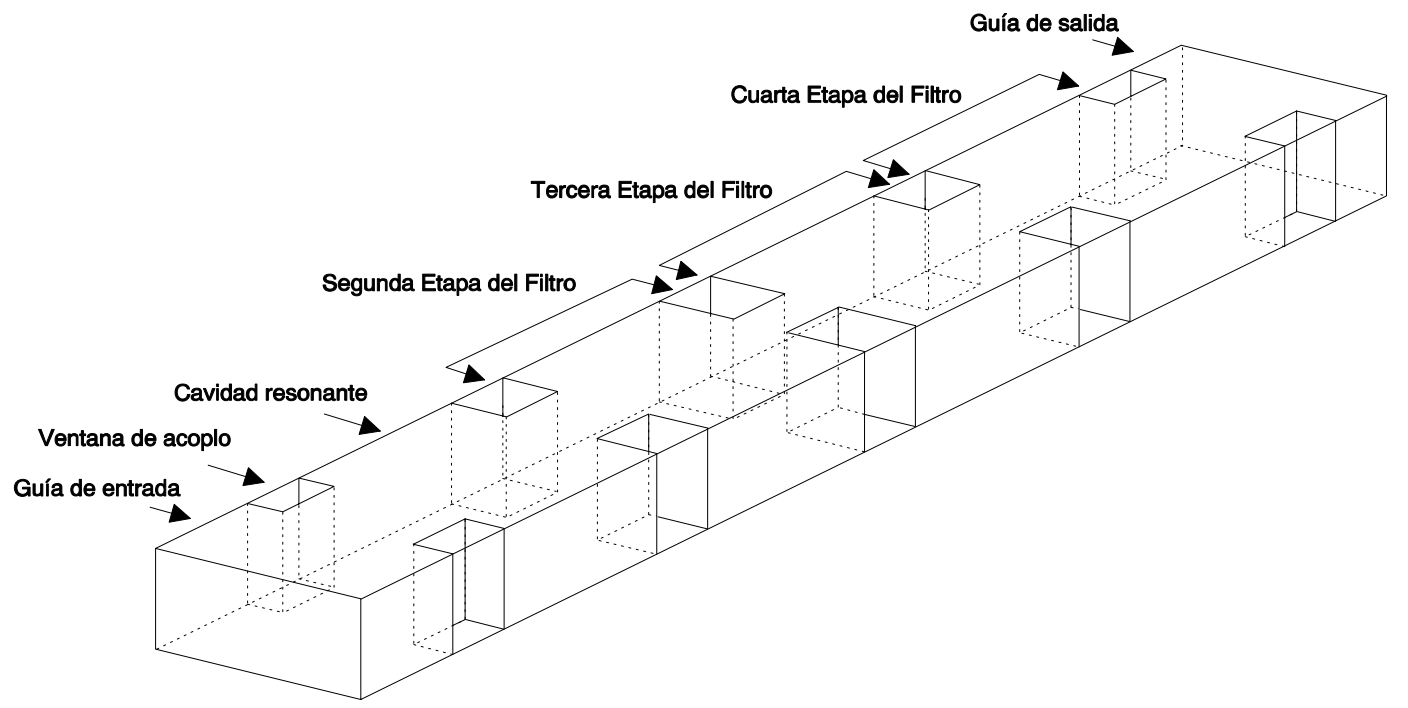

Figura 3.1: Filtro paso banda de 4 polos en guía rectangular con ventanas de acoplo inductivas.

del filtro, con implementaciones muy compactas que evitan el uso de filtros paso bajo adicionales.

Por contra, uno de los principales inconvenientes de las implementaciones sin elementos de sintonía está asociado al hecho de que, en determinadas situaciones, las tolerancias a observar durante la construcción del dispositivo son muy severas; provocando un incremento importante en el coste económico relativo a la construcción del filtro. Alternativamente, por diversas razones, puede resultar necesario centrar la respuesta de un filtro en diferentes frecuencias dentro de una banda; para lo cual, diseñando una estructura básica de filtro con ventanas inductivas, que pueda sintonizarse a cualquier frecuencia dentro de dicha banda mediante elementos de sintonía, se consigue reducir sustancialmente el coste que supondría implementar cada una de las respuestas con un filtro distinto.

Tradicionalmente, los dos problemas identificados en el párrafo anterior se evitan empleando tornillos de sintonía [32,33]. Sin embargo, aunque empleando dichos tornillos siempre es posible recuperar la respuesta del filtro en la banda de paso, no puede realizarse la misma consideración para la respuesta del filtro fuera de dicha banda. Por lo tanto, el desarrollo de una técnica que permita analizar completamente la respuesta de los filtros con elementos de sintonía, tanto dentro de la banda de paso como fuera de la misma, constituye un tema que hoy en día continúa suscitando un enorme interés.

Así pues, en esta sección se presenta inicialmente el análisis electromagnético de los tornillos de sintonía situados dentro de un filtro en guía rectangular con ventanas inductivas; empleando para ello el método de caracterización de uniones planares entre guías arbitrarias basado en matrices de admitancias generalizadas 

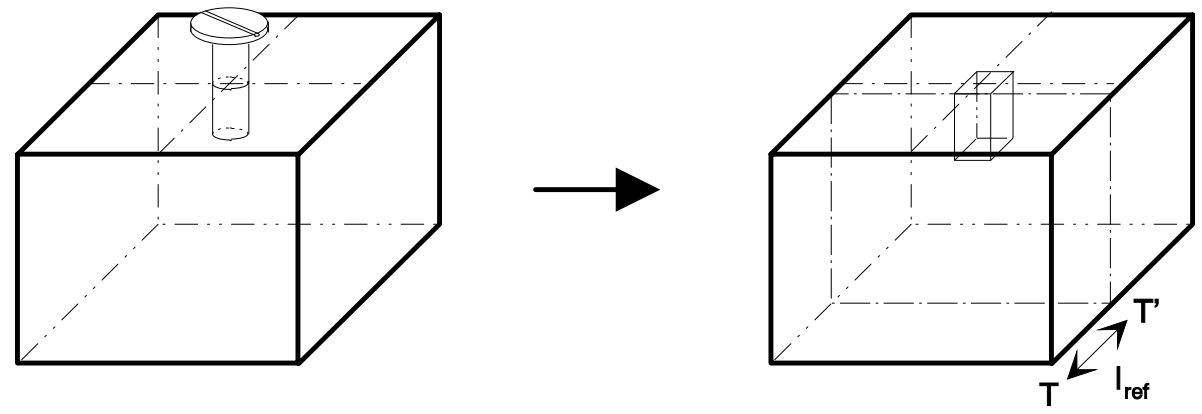

Figura 3.2: Representación de un tornillo de sintonía mediante un tramo uniforme de guía reentrante.

descrito en el capítulo 2 (sección 2.2), que calcula de forma acelerada los elementos $Y_{m, n}^{(2,2)}$ de las citadas matrices. En el análisis de los citados filtros con ventanas inductivas y tornillos de sintonía, estos últimos elementos se representan mediante tramos de guías reentrantes (en inglés ridge) uniformes; tal y como se recoge de manera gráfica en la figura 3.2. El análisis de los tornillos de sintonía requiere pues determinar en principio los modos de las diferentes guías reentrantes; y posteriormente obtener las integrales de acoplamiento entre dichos modos y los modos de las correspondientes guías rectangulares. El procedimiento seguido para obtener estos modos y estas integrales de acoplamiento, descrito con detalle en [21] y [22], se basa en un procedimiento numérico muy eficiente; que se ha considerado interesante exponer con brevedad en esta sección, debido básicamente a su generalidad y posible utilización en otras muchas aplicaciones. Este método numérico consiste básicamente en plantear una ecuación integral de manera que, tras aplicar el Método de los Momentos (procedimiento Galerkin), se obtiene un problema matricial lineal de autovalores; lo que conduce a códigos de programación rápidos y eficientes utilizando paquetes clásicos de tratamiento de matrices. Una vez caracterizado el comportamiento de los tornillos, se plantea un procedimiento empírico para determinar la equivalencia entre dichos tornillos, de sección transversal circular, y unos elementos de sintonía cuya sección transversal es cuadrada. A continuación, se ofrece un estudio de la zona de sintonía que es posible conseguir situando dichos elementos en las ventanas de acoplo inductivas y en las cavidades resonantes de los filtros paso banda; proporcionando de esta manera unas pautas para introducir los mencionados elementos de sintonía con el objeto de compensar imprecisones en la construcción de los filtros. Finalmente, se presenta una técnica novedosa y original que permite diseñar una estructura básica de filtro con ventanas inductivas; que pueda sintonizarse, empleando los correspondientes tornillos de sintonía en las ventanas de acoplo inductivas y en las cavidades resonantes de dicha estructura básica, dentro de una determinada banda de frecuencias. Con el objeto de validar esta técnica, así como el modelo seguido en la caracterización del comportamiento de los tornillos de sintonía, se 
ha construido una implementación práctica; confirmando, tras medir su respuesta electromagnética, los resultados de la etapa de diseño.

\subsubsection{Representación de un Tornillo de Sintonía mediante un Tramo Uniforme de Guía Reentrante}

Para diseñar pues filtros paso banda con tornillos de sintonía, resulta necesario representar inicialmente el comportamiento electromagnético de dichos tornillos. En principio, podría pensarse en caracterizar este comportamiento mediante el método de los elementos finitos; aunque en la práctica el tiempo de computación que requiere este método desaconseja su utilización en aplicaciones de diseño en tiempo real. Recientemente, en [34] se propone un método que permite modelar postes conductores en guías rectangulares empleando la conocida técnica de adaptación modal (en inglés mode-matching). Ahora bien, dicha técnica de adaptación modal presenta diversos inconvenientes; entre ellos aquél relacionado con el fenómeno de la convergencia relativa [6,7], y también el asociado al elevado coste computacional requerido en la solución rigurosa de la ecuación matricial que surge al aplicar el citado método de adaptación modal. En este apartado, con el objeto de simplificar la caracterización del tornillo de sintonía, se propone representar dicho tornillo situado en el interior de una guía rectangular mediante un elemento cilíndrico que presenta sección transversal cuadrada; tal y como se indica gráficamente en la figura 3.2. Observando esta misma figura, se descubre que la estructura a analizar puede descomponerse en dos uniones entre guía rectangular y guía reentrante conectadas en cascada. De esta forma, el análisis de un filtro paso banda en guía rectangular con ventanas de acoplo inductivas y tornillos de sintonía, como por ejemplo el filtro de 4 polos sintonizado que se muestra en la figura 3.3, se reduce a estudiar un número de uniones planares entre guías arbitrarias conectadas entre sí mediante tramos de guía uniforme.

La caracterización de las diferentes uniones planares, así como de los tramos de guía uniforme, que constituyen el filtro paso banda con tornillos de sintonía puede realizarse empleando matrices de admitancias generalizadas. Las expresiones analíticas de los elementos de las citadas matrices, correspondientes a todas las uniones planares que integran el dispositivo mostrado en la figura 3.3 , se expresan recordando (2.4), (2.6) y (2.7) de la siguiente manera

$$
\begin{gathered}
Y_{m, n \text { up }}^{(1,1)}=(-j) \cdot Y_{0 n}^{(1)} \cdot \cot \left(\beta_{n}^{(1)} \cdot l_{\text {ref }}\right) \cdot \delta_{m, n} \\
Y_{m, n \text { up }}^{(2,1)}=Y_{n, m \text { up }}^{(1,2)}=j \cdot Y_{0 n}^{(1)} \cdot \csc \left(\beta_{n}^{(1)} \cdot l_{\text {ref }}\right) \cdot<\vec{h}_{n}^{(1)} \vec{h}_{m}^{(2)}> \\
Y_{m, n \text { up }}^{(2,2)}=(-j) \cdot \sum_{r=1}^{\infty} Y_{0 r}^{(1)} \cdot \cot \left(\beta_{r}^{(1)} \cdot l_{\text {ref }}\right) \cdot<\vec{e}_{r}^{(1)} \vec{e}_{n}^{(2)}>\cdot<\vec{h}_{r}^{(1)} \vec{h}_{m}^{(2)}>
\end{gathered}
$$

donde, para el caso de una unión planar entre una guía rectangular y una guía reentrante, $\vec{e}^{(1)}$ y $\vec{h}^{(1)}$ representan respectivamente los campos eléctrico y magnético 


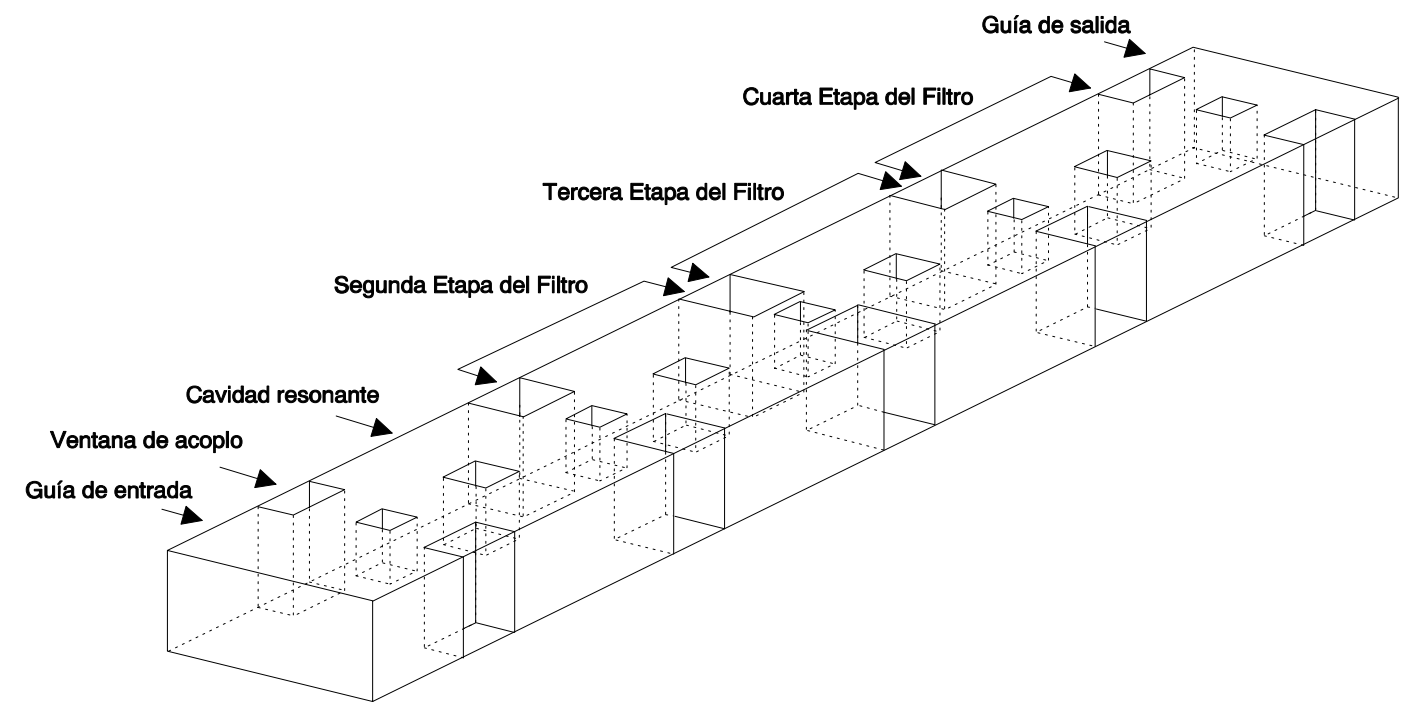

Figura 3.3: Filtro paso banda de 4 polos en guía rectangular con ventanas de acoplo inductivas y elementos de sintonía.

transversales en la guía rectangular, $\vec{e}^{(2)}$ y $\vec{h}^{(2)}$ constituyen respectivamente los campos eléctrico y magnético transversales en la guía reentrante; mientras $l_{\text {ref }}$ determina la longitud de separación entre los planos de referencia escogidos al caracterizar esta unión planar, denominados $\mathrm{T}$ y $\mathrm{T}^{\prime}$ en la figura 3.2. En lo referente al resto de parámetros presentes en (3.1), (3.2) y (3.3), sus respectivos significados se encuentran recogidos en el capítulo 2 (sección 2.1).

Una vez caracterizadas las uniones planares y los tramos de guía uniforme que integran el filtro bajo análisis (ver fig. 3.3), conectando en cascada las matrices de admitancias generalizadas que representan dichas uniones y tramos, se obtiene un sistema lineal de ecuaciones con estructura en banda; que surge tras imponer las correspondientes condiciones de continuidad de los campos eléctricos y magnéticos transversales. La resolución de dicho sistema en banda, tal y como se recoge en el capítulo 2 (sección 2.3), se realiza empleando una técnica recursiva muy eficiente que aprovecha la naturaleza en banda del sistema a resolver.

Como resulta evidente de las expresiones recogidas en (3.2) y (3.3), para caracterizar las uniones planares entre guía rectangular y guía reentrante es necesario obtener en primer lugar los modos de la guía reentrante; con el objeto de calcular posteriormente las integrales de acoplamiento entre los modos de la guía reentrante y los modos de la guía rectangular, integrales asociadas a los productos interiores definidos en dichas expresiones mediante los símbolos $\langle>$. Inicialmente, en este apartado se describe el método escogido para calcular las mencionadas integrales de acoplamiento; detallando asimismo la técnica utilizada en la obtención de las expresiones analíticas concretas de los modos de la guía reentrante. A continuación, se presenta un criterio de equivalencia empírico entre un tornillo 
de sintonía real, con sección transversal circular, y un elemento de sintonía que presenta una sección transversal cuadrada.

\section{Evaluación de las Integrales de Acoplamiento}

El cálculo de las integrales de acoplamiento entre los modos de una guía reentrante y una guía rectangular se ha realizado siguiendo el método propuesto en [22]; que constituye un método genérico para obtener un gran número de integrales de acoplamiento entre una guía rectangular (o circular) y una guía de tamaño más pequeño y contorno arbitrario. Este método requiere obtener inicialmente los modos de la guía pequeña; empleando para ello una técnica propuesta con anterioridad en [21], que puede utilizarse para encontrar los modos de estructuras obtenidas al perturbar una guía de sección transversal $S_{0}$ rectangular (o circular) con una (o varias) láminas conductoras cilíndricas axiales. Esta(s) lámina(s) intersecciona(n) con la sección transversal básica rectangular (o circular); produciendo una $(\mathrm{s})$ línea(s) conductora(s) que representaremos genéricamente mediante la variable $\sigma$, la cual se define a través de la siguiente ecuación paramétrica

$$
\vec{s}=\vec{s}(l)
$$

donde el extremo del vector $\vec{s}$ denota un punto sobre la(s) línea(s) $\sigma$; mientras $l$ representa una abscisa escogida de manera adecuada sobre dicha(s) línea(s).

El campo eléctrico en un punto de observación genérico $\vec{r}$, situado dentro de la guía rectangular (o circular), puede expresarse de la siguiente manera

$$
\vec{E}(\vec{r})=-j \eta k \int_{\sigma} \bar{G}_{e}\left(\vec{r}, \vec{s}^{\prime}, k\right) \cdot \vec{J}_{\sigma}\left(l^{\prime}\right) d l^{\prime}
$$

donde $\vec{s}^{\prime}=\vec{s}\left(l^{\prime}\right)$ determina la posición de una fuente puntual de corriente sobre la(s) línea(s) $\sigma, k=\omega \sqrt{\mu \epsilon}$ representa el número de onda, $\eta=\sqrt{\mu / \epsilon}$ define la impedancia característica, $\bar{G}_{e}$ constituye la función de Green diádica bidimensional del tipo eléctrico, y finalmente $\vec{J}_{\sigma}$ se refiere a la densidad de corriente inducida sobre la lámina(s) conductora(s). Conviene reseñar que en (3.5) debe tomarse el valor principal de la integral presente en dicha expresión; debido a la singularidad que presenta $\bar{G}_{e}$ cuando $\vec{r}$ determina un punto sobre la(s) línea(s) $\sigma$, tal y como se discute por ejemplo en [35]. Debido a la naturaleza de la función de Green usada en [21], el campo que proporciona la expresión recogida en (3.5) satisface la correspondiente condición de contorno sobre el contorno exterior rectangular (o circular) de la guía. De esta manera, es posible establecer una ecuación integral para la corriente incógnita $\vec{J}_{\sigma}$ imponiendo que la componente tangencial del campo eléctrico $\vec{E}$ se anule sobre $\sigma$. Esta ecuación presenta soluciones no triviales; suponiendo para ello que el número de onda $k$ en dicha ecuación constituye un número de onda resonante de la estructura perturbada, es decir un número de onda de corte asociado a un modo de la guía perturbada. 
La expresión general de la función de Green diádica $\bar{G}_{e}$ presente en (3.5), tanto para una guía con sección transversal rectangular como circular, se encuentra recogida en [36]; presentando dicha expresión el siguiente aspecto

$$
\bar{G}_{e}\left(\vec{r}, \vec{r}^{\prime}, k\right)=\bar{G}_{e t}\left(\vec{r}, \vec{r}^{\prime}, k\right)+\hat{z} \hat{z} G_{e z z}\left(\vec{r}, \vec{r}^{\prime}, k\right)
$$

donde $\hat{z}$ representa un vector unitario dirigido según el eje de la guía normal a su sección transversal; y $\bar{G}_{t t}$ constituye una función de Green diádica transversal al eje $\hat{z}$, definida como se indica a continuación

$$
\begin{aligned}
\bar{G}_{e t}\left(\vec{r}, \vec{r}^{\prime}, k\right)= & -\frac{1}{k^{2}} \nabla \nabla^{\prime} g\left(\vec{r}, \vec{r}^{\prime}\right)+\bar{G}_{s t}\left(\vec{r}, \vec{r}^{\prime}\right) \\
& +\sum_{m=1}^{\infty} \frac{k^{2}}{k_{m}^{2}\left(k_{m}^{2}-k^{2}\right)} \vec{e}_{m}(\vec{r}) \vec{e}_{m}\left(\vec{r}^{\prime}\right)
\end{aligned}
$$

Por su parte, el coeficiente $G_{e z z}$ asociado en (3.6) a la díada $\hat{z} \hat{z}$ se expresa del siguiente modo

$$
G_{e z z}\left(\vec{r}, \vec{r}^{\prime}, k\right)=g\left(\vec{r}, \vec{r}^{\prime}\right)+\sum_{m=1}^{\infty} \frac{k^{2}}{k_{m}^{\prime 2}\left(k_{m}^{\prime 2}-k^{2}\right)} \psi_{m}(\vec{r}) \psi_{m}\left(\vec{r}^{\prime}\right)
$$

En (3.7) y (3.8), los vectores $\vec{r}$ y $\vec{r}^{\prime}$ definen respectivamente el punto de observación y la posición de la fuente puntual de corriente dentro de la sección transversal de la guía rectangular (o circular), g constituye la función de Green escalar bidimensional que es solución de la ecuación de Poisson sujeta al cumplimiento de la condición $g=0$; mientras $k_{m}$ y $k_{m}^{\prime}$ se refieren respectivamente a los números de onda de corte del $m$-ésimo modo TE y del $m$-ésimo modo TM de la guía con sección transversal rectangular (o circular). Asimismo, el vector $\vec{e}_{m}$ representa en (3.7) el campo eléctrico transversal asociado al $m$-ésimo modo TE de la mencionada guía rectangular (o circular); y la función escalar $\psi_{m}$, presente en (3.8), determina la componente axial del campo eléctrico correspondiente al $m$-ésimo modo TM de la citada guía rectangular (o circular). Finalmente, el término $\bar{G}_{s t}$ empleado en (3.7) hace referencia a la función de Green diádica solenoidal, normal al contorno de la guía rectangular (o circular), que debe satisfacer la siguiente ecuación

$$
\nabla \times \nabla \times \bar{G}_{s t}\left(\vec{r}, \vec{r}^{\prime}\right)=\bar{I}_{t} \delta\left(\vec{r}-\vec{r}^{\prime}\right)+\nabla \nabla^{\prime} g\left(\vec{r}, \vec{r}^{\prime}\right)
$$

donde $\bar{I}_{t}$ designa una función diádica unitaria transversal, y $\delta\left(\vec{r}-\vec{r}^{\prime}\right)$ representa la función delta de Dirac bidimensional. En cuanto a las funciones $g$ y $\bar{G}_{s t}$, utilizadas en (3.7) y (3.8), presentan ambas un comportamiento singular logarítmico cuando los vectores $\vec{r}$ y $\vec{r}^{\prime}$ coinciden. Concretamente, en el caso de una guía con sección transversal circular, se dispone de expresiones analíticas conocidas en forma cerrada tanto para $g$ como para $\bar{G}_{s t}$; mientras en el caso de la guía con 
sección transversal rectangular, dichas funciones $g$ y $\bar{G}_{s t}$ se expresan mediante series convergentes de un único índice en las que la singularidad logarítmica está asociada al primer término de dichas series. Las expresiones concretas de estas funciones $g$ y $\bar{G}_{s t}$, tanto en el caso de la guía rectangular como de la guía circular, se encuentran recogidas en [21]. Por último, reseñar que las funciones modales $\vec{e}_{m}$ y $\psi_{m}$, presentes ambas también en (3.7) y (3.8), deben normalizarse como se indica seguidamente

$$
\begin{aligned}
\int_{S_{0}} \vec{e}_{m} \cdot \vec{e}_{m} d s & =1 \\
\int_{S_{0}} \psi_{m}^{2} d s & =1
\end{aligned}
$$

En lo referente a las expresiones explícitas de estas funciones modales normalizadas $\vec{e}_{m}$ y $\psi_{m}$, así como las de sus correspondientes números de onda de corte $k_{m}$ y $k_{m}^{\prime}$ a utilizar en (3.7) y (3.8), pueden encontrase por ejemplo en [15].

La densidad de corriente $\vec{J}_{\sigma}$ inducida sobre la lámina(s) conductora(s), que aperece en la definición del campo eléctrico $\vec{E}$ recogida en (3.5), puede descomponerse en una componente transversal y en una componente longitudinal; presentando pues el siguiente aspecto

$$
\vec{J}_{\sigma}\left(l^{\prime}\right)=J_{t}\left(l^{\prime}\right) \vec{t}\left(l^{\prime}\right)+J_{z}\left(l^{\prime}\right) \hat{z}
$$

donde el vector $\vec{t}$ representa un vector unitario tangente a la(s) línea(s) $\sigma$; mientras $J_{t}, J_{z}$ constituyen funciones incógnitas a determinar. Al sustituir pues en (3.5) $\bar{G}_{\epsilon}, \bar{G}_{t t}$ y $G_{e z z}$ por sus correspondientes expresiones mostradas en (3.6), (3.7) y (3.8), y empleando (3.12) para expresar el vector $\vec{J}_{\sigma}$, las componentes tangencial y longitudinal del campo eléctrico $\vec{E}$ se escriben de la siguiente manera

$$
\begin{aligned}
\vec{E}_{t}(\vec{r})= & j \frac{\eta}{k} \int_{\sigma} \nabla \frac{\partial g\left(\vec{r}, \vec{s}^{\prime}\right)}{\partial l^{\prime}} J_{t}\left(l^{\prime}\right) d l^{\prime} \\
& -j \eta k \int_{\sigma} \bar{G}_{s t}\left(\vec{r}, \vec{s}^{\prime}\right) \cdot \vec{t}\left(l^{\prime}\right) J_{t}\left(l^{\prime}\right) d l^{\prime} \\
& -j \eta k^{3} \sum_{m=1}^{\infty} \frac{\vec{e}_{m}(\vec{r})}{k_{m}^{2}\left(k_{m}^{2}-k^{2}\right)} \int_{\sigma} \vec{e}_{m}\left(\vec{s}^{\prime}\right) \cdot \vec{t}\left(l^{\prime}\right) J_{t}\left(l^{\prime}\right) d l^{\prime} \\
E_{z}(\vec{r})= & -j \eta k \int_{\sigma} g\left(\vec{r}, \vec{s}^{\prime}\right) J_{z}\left(l^{\prime}\right) d l^{\prime} \\
& -j \eta k^{3} \sum_{m=1}^{\infty} \frac{\psi_{m}(\vec{r})}{k_{m}^{\prime 2}\left(k_{m}^{\prime 2}-k^{2}\right)} \int_{\sigma} \psi_{m}\left(\vec{s}^{\prime}\right) J_{z}\left(l^{\prime}\right) d l^{\prime}
\end{aligned}
$$

De las dos ecuaciones recién deducidas en (3.13) y (3.14), la primera de ellas resulta apropiada para representar el campo eléctrico de los modos TE que se encuentran al corte; ya que en dicho caso la corriente inducida y el campo eléctrico 
son ambos transversales. Por otra parte, la segunda de las mencionadas ecuaciones resulta especialmente útil cuando se describen modos TM; pues para este tipo de modos el campo eléctrico y la corriente son longitudinales. Imponiendo en primer lugar la condición de contorno $\vec{E}_{t} \cdot \vec{t}=0$ sobre la(s) línea(s) $\sigma$, se obtiene la siguiente ecuación relativa a los modos TE

$$
\begin{gathered}
\frac{1}{k^{2}} \frac{\partial}{\partial l} \int_{\sigma} g\left(\vec{s}, \vec{s}^{\prime}\right) \frac{\partial J_{t}\left(l^{\prime}\right)}{\partial l^{\prime}} d l^{\prime}+\int_{\sigma} \vec{t}(l) \cdot \bar{G}_{s t}\left(\vec{s}, \vec{s}^{\prime}\right) \cdot \vec{t}\left(l^{\prime}\right) J_{t}\left(l^{\prime}\right) d l^{\prime} \\
+\sum_{m=1}^{\infty} \frac{\vec{t}(l) \cdot \vec{e}_{m}(\vec{s})}{k_{m}^{2}} a_{m}=0
\end{gathered}
$$

donde los coeficientes $a_{m}$, presentes en esta última ecuación recién deducida, se definen tal y como se indica seguidamente

$$
a_{m}=\frac{k^{2}}{k_{m}^{2}-k^{2}} \int_{\sigma} \vec{e}_{m}\left(\vec{s}^{\prime}\right) \cdot \vec{t}\left(l^{\prime}\right) J_{t}\left(l^{\prime}\right) d l^{\prime}
$$

Por contra, si la condición de contorno que se impone sobre la(s) línea(s) $\sigma$ es $E_{z}=0$, se obtiene la siguiente ecuación asociada a los modos TM

$$
\int_{\sigma} g\left(\vec{s}, \vec{s}^{\prime}\right) J_{z}\left(l^{\prime}\right) d l^{\prime}+\sum_{m=1}^{\infty} \frac{\psi_{m}(\vec{s})}{k_{m}^{\prime 2}} a_{m}^{\prime}=0
$$

donde los coeficientes $a_{m}^{\prime}$, que aparecen en la última ecuación propuesta, vienen definidos en esta ocasión del siguiente modo

$$
a_{m}^{\prime}=\frac{k^{2}}{k_{m}^{\prime 2}-k^{2}} \int_{\sigma} \psi_{m}\left(\vec{s}^{\prime}\right) J_{z}\left(l^{\prime}\right) d l^{\prime}
$$

Para determinar los modos TE de la guía perturbada, la componente tangencial $J_{t}$ de la densidad de corriente $\vec{J}_{\sigma}$ se representa como

$$
J_{t}\left(l^{\prime}\right)=\sum_{n=1}^{N} b_{n} w_{n}\left(l^{\prime}\right)
$$

donde $\mathbf{b}=\left(b_{1}, b_{2}, \ldots, b_{N}\right)_{t}$ constituye un vector columna formado por $N$ coeficientes numéricos; y $\{w\}$ designa un conjunto de $N$ funciones base definidas sobre la(s) linea $(\mathrm{s}) \sigma$.

En cuanto al sumatorio presente en la ecuación definida según (3.15), su cálculo requiere sumar en principio infinitos términos. No obstante, con el objeto de implementar dicho sumatorio computacionalmente, el número de términos a considerar en su cálculo puede truncarse en un valor igual a $M$; número de términos que permite obtener un valor bastante aproximado al real para el sumatorio en cuestión, suponiendo que el valor de $k$ es bastante más pequeño que 
el de $k_{M}$. Por razones también de implementación computacional, la(s) línea(s) $\sigma$ debe(n) dividirse en $N$ elementos; cada uno de los cuales está constituido por un cierto número de segmentos, cuyas longitudes pueden a su vez diferir unas de otras. En cuanto a las funciones $w_{n}$, tal y como se indica en [21], se recomienda el uso de funciones parabólicas a tramos; cuyas primeras derivadas son consecuentemente funciones lineales a tramos. La elección del tramo de función $w_{n}$, correspondiente a cada segmento del $n$-ésimo elemento de la(s) línea(s) $\sigma$, debe tener en cuenta las peculiaridades de la función incógnita $J_{t}$ en ciertos puntos de $\sigma$; tales como por ejemplo que la corriente puede tomar un valor no nulo en los extremos de $\sigma$ que además pertenecen al contorno de la sección transversal $S_{0}$ mientras la derivada de dicha corriente debe valer cero en dichos puntos, o que la corriente debe ser igual a cero en los bordes de $\sigma$ y su derivada no tiene por qué anularse en estos bordes (teóricamente o vale cero o diverge).

Sustituyendo pues (3.19) en (3.15) y (3.16), y aplicando en la ecuación definida según (3.15) el Método de los Momentos (procedimiento de Galerkin), se obtiene el siguiente problema algebraico de autovalores

$$
\left\{\left[\begin{array}{cc}
\mathbf{U} & \mathbf{O}_{t} \\
\mathbf{O} & \mathbf{C}
\end{array}\right]-k^{2}\left[\begin{array}{cc}
\mathbf{D} & \mathbf{R}_{t} \\
\mathbf{R} & \mathbf{L}
\end{array}\right]\right\} \cdot\left[\begin{array}{l}
\mathbf{a} \\
\mathbf{b}
\end{array}\right]=0
$$

En $(3.20), \mathbf{a}=\left(a_{1}, a_{2}, \ldots, a_{M}\right)_{t}$ representa un vector columna constituido por los coeficientes $a_{m}$, U designa una matriz unitaria de orden $M, \mathrm{O}$ constituye una matriz nula de dimensiones $N \times M$; y finalmente los elementos de las matrices D, C, L y $\mathbf{R}$ se obtienen mediante las siguientes expresiones

$$
\begin{aligned}
\mathbf{D} & =\operatorname{diag}\left(k_{1}^{-2}, k_{2}^{-2}, \ldots, k_{M}^{-2}\right) \\
\mathrm{C}_{i j} & =\int_{\sigma} \int_{\sigma} g\left(\vec{s}, \vec{s}^{\prime}\right) \frac{\partial w_{i}(l)}{\partial l} \frac{\partial w_{j}\left(l^{\prime}\right)}{\partial l^{\prime}} d l d l^{\prime} \\
\mathrm{L}_{i j} & =\int_{\sigma} \int_{\sigma} w_{i}(l) w_{j}\left(l^{\prime}\right) \vec{t}(l) \cdot \bar{G}_{s t}\left(\vec{s}, \vec{s}^{\prime}\right) \cdot \vec{t}\left(l^{\prime}\right) d l d l^{\prime} \\
\mathrm{R}_{i m} & =\frac{1}{k_{m}^{2}} \int_{\sigma} w_{i}(l) \vec{t}(l) \cdot \vec{e}_{m}(\vec{s}) d l \\
i, j & =1,2, \ldots, N \quad m=1,2, \ldots, M
\end{aligned}
$$

Una vez resuelto el problema de autovalores recogido en (3.20), los autovectores obtenidos deben normalizarse empleando la condición expuesta en [37]; que se resume a continuación

$$
\left[\begin{array}{l}
\mathbf{a} \\
\mathbf{b}
\end{array}\right]_{t} \cdot\left[\begin{array}{cc}
\mathbf{D} & \mathbf{R}_{t} \\
\mathbf{R} & \mathbf{L}
\end{array}\right] \cdot\left[\begin{array}{l}
\mathbf{a} \\
\mathbf{b}
\end{array}\right]=\mathbf{U}
$$

Utilizando pues los autovectores normalizados según el procedimiento que se termina de exponer, y tras imponer la condición de normalización del campo 
eléctrico transversal sobre la sección de la guía perturbada, se deduce la siguiente expresión final para el campo eléctrico transversal asociado al q-ésimo modo TE de la mencionada guía perturbada

$$
\begin{aligned}
\vec{E}_{q}(\vec{r})= & \frac{1}{\kappa_{q}} \sum_{n=1}^{N} b_{n q} \nabla_{t} \int_{\sigma} g\left(\vec{r}, \vec{s}^{\prime}\right) \frac{\partial w_{n}\left(l^{\prime}\right)}{\partial l^{\prime}} d l^{\prime} \\
& +\kappa_{q} \sum_{n=1}^{N} b_{n q} \int_{\sigma} \vec{G}_{s t}\left(\vec{r}, \vec{s}^{\prime}\right) \cdot \vec{t}\left(l^{\prime}\right) w_{n}\left(l^{\prime}\right) d l^{\prime} \\
& +\kappa_{q} \sum_{m=1}^{M} \frac{a_{m q}}{k_{m}^{2}} \vec{e}_{m}(\vec{r})
\end{aligned}
$$

donde $\kappa_{q}$ representa el número de onda de corte del $q$-ésimo modo TE, que corresponde al q-ésimo autovalor del problema planteado en (3.20); mientras los coeficientes $a_{m q}$ y $b_{n q}$ constituyen los elementos del $q$-ésimo autovector asociado al citado problema, autovector normalizado según la condición recogida anteriormente en (3.25).

A continuación, con el objeto de obtener los modos TM de la guía perturbada, la componente longitudinal $J_{z}$ de la densidad de corriente $\vec{J}_{\sigma}$ debe expandirse de la siguiente manera

$$
J_{z}\left(l^{\prime}\right)=\sum_{n=1}^{N^{\prime}} b_{n}^{\prime} u_{n}\left(l^{\prime}\right)
$$

donde $\mathbf{b}^{\prime}=\left(b_{1}^{\prime}, b_{2}^{\prime}, \ldots, b_{N^{\prime}}^{\prime}\right)_{t}$ representa un vector columna integrado por $N^{\prime}$ coeficientes; mientras $\{u\}$ determina un conjunto de $N^{\prime}$ funciones base que se definen sobre la(s) línea(s) $\sigma$.

En relación al sumatorio de infinitos términos que aparece en la ecuación definida mediante (3.17), nuevamente por razones de implementación computacional, dicho sumatorio debe truncarse en un número finito de términos a sumar; habiendo escogido en esta ocasión un número igual a $M^{\prime}$, que permite obtener un valor bastante aproximado para el citado sumatorio suponiendo que $k$ es mucho más pequeño que $k_{M^{\prime}}$. Por lo que respecta a la(s) línea(s) $\sigma$, ésta(s) se divide(n) en $N^{\prime}$ elementos, tantos como funciones base escogidas para expresar la corriente longitudinal $J_{z}$ mediante el desarrollo mostrado en (3.27); elementos que están constituidos respectivamente por segmentos cuyas longitudes no tienen por qué ser iguales. Para el caso que nos ocupa (modos TM), en [21] se recomienda el uso de funciones triangulares como funciones base $u_{n}$ en todos los elementos considerados; a excepción de aquéllos que incorporan algún borde de $\sigma$, en cuyo caso se propone la utilización de funciones "medio triángulo" (ver [21]) como funciones base para aproximar la posible singularidad de $J_{z}$ en dicho borde.

Empleando en (3.17) y (3.18) el desarrollo de la corriente longitudinal $J_{z}$ propuesto en (3.27), y tras aplicar en la ecuación integral descrita según (3.17) 
el Método de los Momentos (procedimiento de Galerkin), el problema asociado a la obtención de los modos TM de una guía perturbada se expresa del siguiente modo en forma matricial

$$
\left\{\left[\begin{array}{ll}
\mathbf{U} & \mathbf{O}_{t} \\
\mathbf{O} & \mathbf{O}^{\prime}
\end{array}\right]-k^{2}\left[\begin{array}{ll}
\mathbf{D}^{\prime} & \mathbf{R}_{t}^{\prime} \\
\mathbf{R}^{\prime} & \mathbf{L}^{\prime}
\end{array}\right]\right\} \cdot\left[\begin{array}{l}
\mathbf{a}^{\prime} \\
\mathbf{b}^{\prime}
\end{array}\right]=0
$$

En el problema de autovalores recién expuesto en $(3.28), \mathbf{a}^{\prime}=\left(a_{1}^{\prime}, a_{2}^{\prime}, \ldots, a_{M^{\prime}}^{\prime}\right)_{t}$ constituye un vector columna integrado por los coeficientes $a_{m}^{\prime}$, U define una matriz unitaria de orden $M^{\prime}$, $\mathrm{O}$ y $\mathbf{O}^{\prime}$ designan sendas matrices nulas de dimensiones $N^{\prime} \times M^{\prime}$ y $N^{\prime} \times N^{\prime}$ respectivamente; mientras los elementos de las matrices $\mathbf{D}^{\prime}$, $\mathbf{L}^{\prime}$ y $\mathbf{R}^{\prime}$ se deduce empleando las siguientes expresiones

$$
\begin{aligned}
\mathbf{D}^{\prime} & =\operatorname{diag}\left(k_{1}^{\prime-2}, k_{2}^{\prime-2}, \ldots, k_{M^{\prime}}^{\prime-2}\right) \\
\mathrm{L}_{i j}^{\prime} & =\int_{\sigma} \int_{\sigma} u_{i}(l) g\left(\vec{s}, \vec{s}^{\prime}\right) u_{j}\left(l^{\prime}\right) d l d l^{\prime} \\
\mathrm{R}_{i m}^{\prime} & =\frac{1}{k_{m}^{\prime 2}} \int_{\sigma} u_{i}(l) \psi_{m}(\vec{s}) d l \\
i, j & =1,2, \ldots, N^{\prime} \quad m=1,2, \ldots, M^{\prime}
\end{aligned}
$$

Observando el problema de autovalores mostrado en (3.28), se descubre que admite $N^{\prime}$ soluciones independientes con $k=0, a^{\prime}=0$ y $b^{\prime} \neq 0$ que no deben considerarse. De cualquier manera, el citado problema expuesto en (3.28) puede transformarse fácilmente en un problema típico de autovalores y autovectores; que se expresa tal y como se indica a continuación

$$
\left(\mathbf{D}^{\prime}-\mathbf{R}_{t}^{\prime} \cdot \mathbf{L}^{\prime-1} \cdot \mathbf{R}^{\prime}\right) \cdot \mathbf{a}^{\prime}=k^{-2} \mathbf{a}^{\prime}
$$

La transformación del problema original de autovalores, definido según (3.28), en el problema recién expuesto en (3.32) es posible gracias a que la matriz $\mathbf{L}^{\prime}$ presenta siempre un comportamiento no singular (ver [21]). Asimismo, conviene reseñar que este último problema de autovalores, propuesto en (3.32), admite sólo $M^{\prime}$ autovalores y autovectores; deduciendo el vector $\mathbf{b}^{\prime}$, una vez obtenido el vector $\mathbf{a}^{\prime}$, mediante la siguiente relación

$$
\mathbf{b}^{\prime}=-\mathbf{L}^{\prime-1} \cdot \mathbf{R}^{\prime} \cdot \mathbf{a}^{\prime}
$$

De nuevo, tras resolver el problema de autovalores y autovectores recogido en (3.32), deben normalizarse los autovectores obtenidos; empleando para ello la condición de normalización propuesta en [38], que se expresa como

$$
\sum_{m=1}^{M^{\prime}}\left|a_{m q}^{\prime}\right|^{2}=\kappa_{q}^{\prime 2}
$$


En $(3.34), \kappa_{q}^{\prime}$ representa el número de onda de corte del $q$-ésimo modo TM de la guía perturbada; que se corresponde directamente con el valor del q-ésimo autovalor del problema propuesto en (3.32). Una vez normalizados los autovectores obtenidos siguiendo el procedimiento recién expuesto, e imponiendo la condición de normalización del correspondiente potencial escalar sobre la superficie de la guía perturbada, se obtiene la siguiente expresión definitiva para el potencial escalar asociado al q-ésimo modo TM de la citada guía perturbada

$$
\phi_{q}(\vec{r})=\kappa_{q}^{\prime}\left[\sum_{n=1}^{N^{\prime}} b_{n q}^{\prime} \int_{\sigma} g\left(\vec{r}, \vec{s}^{\prime}\right) u_{n}\left(l^{\prime}\right) d l^{\prime}+\sum_{m=1}^{M^{\prime}} \frac{a_{m q}^{\prime}}{k_{m}^{\prime 2}} \psi_{m}(\vec{r})\right]
$$

donde los coeficientes $a_{m q}^{\prime}$ son los elementos del $q$-ésimo autovector del problema de autovalores definido según (3.32); autovector que se encuentra normalizado mediante la condición expresada en (3.34). Por otra parte, los coeficientes $b_{n q}^{\prime}$ constituyen los elementos del vector columna $\mathbf{b}_{q}^{\prime}$; que se encuentra relacionado con el vector columna $\mathbf{a}_{q}^{\prime}$ a través de la relación expuesta en (3.33).

Finalmente, la expresión analítica del campo eléctrico transversal asociado al $q$-ésimo modo TM de la guía perturbada se deduce, tal y como se indica seguidamente, a partir del potencial escalar correspondiente a dicho modo y definido con anterioridad en (3.35)

$$
\vec{E}_{q}^{\prime}(\vec{r})=-\frac{\nabla_{t} \phi_{q}(\vec{r})}{\kappa_{q}^{\prime}}
$$

Así pues, mediante el procedimiento descrito es posible obtener los modos de una guía cuyo contorno básico rectangular (o circular) se encuentra modificado. Una vez se conocen dichos modos, se procede a calcular las integrales de acoplamiento entre estos modos y los correspondientes modos de una guía rectangular (o circular), cuyo contorno básico no modificado coincide con el de la guía perturbada. Dichas integrales de acoplamiento se definen del siguiente modo

$$
\begin{aligned}
I_{p q}^{\mathrm{TE}, \mathrm{TE}} & =\int_{S} \vec{e}_{p}(\vec{r}) \cdot \vec{E}_{q}(\vec{r}) d s \\
I_{p q}^{\mathrm{TE}, \mathrm{TM}} & =\int_{S} \vec{e}_{p}(\vec{r}) \cdot \vec{E}_{q}^{\prime}(\vec{r}) d s \\
I_{p q}^{\mathrm{TM}, \mathrm{TE}} & =\int_{S} \vec{e}_{p}^{\prime}(\vec{r}) \cdot \vec{E}_{q}(\vec{r}) d s \\
I_{p q}^{\mathrm{TM}, \mathrm{TM}} & =\int_{S} \vec{e}_{p}^{\prime}(\vec{r}) \cdot \vec{E}_{q}^{\prime}(\vec{r}) d s
\end{aligned}
$$

donde $S$ designa la sección transversal de la guía perturbada, $\vec{e}_{p}\left(\vec{e}_{p}^{\prime}\right)$ representa el campo eléctrico transversal normalizado correspondiente al $p$-ésimo modo TE(TM) de la guía rectangular (o circular) cuyo contorno básico no se ha modificado, y finalmente $\vec{E}_{q}\left(\vec{E}_{q}^{\prime}\right)$ se refiere al campo eléctrico transversal normalizado asociado al q-ésimo modo TE(TM) de la guía perturbada. 
Con la intención pues de evaluar las integrales de acoplamiento definidas según (3.37)-(3.40), es necesario determinar previamente los campos eléctricos transverales $\vec{E}_{q}$ y $\vec{E}_{q}^{\prime}$ haciendo uso de las expresiones recogidas en $(3.26),(3.35)$ y (3.36); para lo cual las funciones de Green $g$ y $\bar{G}_{s t}$ se expresan, tal y como se indica en [39] y [21] respectivamente, mediante las siguientes expansiones de funciones modales normalizadas de la guía rectangular (o circular)

$$
\begin{aligned}
g\left(\vec{r}, \vec{s}^{\prime}\right) & =\sum_{i=1}^{\infty} \frac{\psi_{i}(\vec{r}) \psi_{i}\left(\vec{s}^{\prime}\right)}{k_{i}^{\prime 2}} \\
\bar{G}_{s t}\left(\vec{r}, \vec{s}^{\prime}\right) & =\sum_{i=1}^{\infty} \frac{\vec{e}_{i}(\vec{r}) \vec{e}_{i}\left(\vec{s}^{\prime}\right)}{k_{i}^{2}}
\end{aligned}
$$

Sustituyendo pues en (3.26) y (3.35) las expansiones de las funciones de Green $g$ y $\bar{G}_{s t}$ recién expuestas en $(3.41)$ y (3.42), y recordando la relación $\vec{e}_{i}^{\prime}=-\nabla_{t} \psi_{i} / k_{i}^{\prime}$, se obtienen las siguientes expresiones para los campos eléctricos transversales normalizados $\vec{E}_{q}\left(\vec{E}_{q}^{\prime}\right)$ correspondientes al $q$-ésimo modo TE(TM) de la guía perturbada

$$
\begin{aligned}
\vec{E}_{q}(\vec{r})= & -\frac{1}{\kappa_{q}} \sum_{n=1}^{N} b_{n q} \sum_{i=1}^{\infty} \frac{\vec{e}_{i}^{\prime}(\vec{r})}{k_{i}^{\prime}} \int_{\sigma} \psi_{i}\left(\vec{s}^{\prime}\right) \frac{\partial w_{n}\left(l^{\prime}\right)}{\partial l^{\prime}} d l^{\prime} \\
& +\kappa_{q} \sum_{n=1}^{N} b_{n q} \sum_{i=1}^{\infty} \frac{\vec{e}_{i}(\vec{r})}{k_{i}^{2}} \int_{\sigma} \vec{e}_{i}\left(\vec{s}^{\prime}\right) \cdot \vec{t}\left(l^{\prime}\right) w_{n}\left(l^{\prime}\right) d l^{\prime} \\
& +\kappa_{q} \sum_{m=1}^{M} \frac{a_{m q}}{k_{m}^{2}} \vec{e}_{m}(\vec{r}) \\
\vec{E}_{q}^{\prime}(\vec{r})= & \sum_{n=1}^{N^{\prime}} b_{n q}^{\prime} \sum_{i=1}^{\infty} \frac{\vec{e}_{i}^{\prime}(\vec{r})}{k_{i}^{\prime}} \int_{\sigma} \psi_{i}\left(\vec{s}^{\prime}\right) u_{n}\left(l^{\prime}\right) d l^{\prime} \\
& +\sum_{m=1}^{M^{\prime}} \frac{a_{m q}^{\prime}}{k_{m}^{\prime}} \vec{e}_{m}^{\prime}(\vec{r})
\end{aligned}
$$

Además, como se apunta en [21], las expresiones ofrecidas en (3.26), (3.35) y (3.36) para los campos eléctricos transversales normalizados $\vec{E}_{q}$ y $\vec{E}_{q}^{\prime}$, y en consecuencia estas últimas expresiones propuestas en (3.43) y (3.44) para dichos campos, proporcionan valores nulos de campo en aquellos puntos que están situados entre la sección transversal $\left(S_{0}\right)$ de la guía rectangular (o circular) no modificada y la sección transversal $(S)$ de la guía perturbada. Por tanto, las integrales de acoplamiento entre los modos de ambas guías, definidas según (3.37)-(3.40), pueden evaluarse integrando en el dominio definido por la sección transversal $\left(S_{0}\right)$ de la guía rectangular (o circular) cuyo contorno no está modificado; lo que resulta 
especialmente interesante al poder utilizar la propiedad de ortonormalidad de los vectores $\vec{e}$ y $\vec{e}^{\prime}$. De esta manera, se obtienen finalmente las siguientes expresiones para las mencionadas integrales de acoplamiento

$$
\begin{aligned}
I_{p q}^{\mathrm{TE}, \mathrm{TE}} & =\int_{S_{0}} \vec{e}_{p}(\vec{r}) \cdot \vec{E}_{q}(\vec{r}) d s=\kappa_{q}\left[\sum_{n=1}^{N} \mathrm{R}_{n p} b_{n q}+\frac{a_{p q}}{k_{p}^{2}}\right] \\
I_{p q}^{\mathrm{TE}, \mathrm{TM}} & =\int_{S_{0}} \vec{e}_{p}(\vec{r}) \cdot \vec{E}_{q}^{\prime}(\vec{r}) d s=0 \\
I_{p q}^{\mathrm{TM}, \mathrm{TE}} & =\int_{S_{0}} \vec{e}_{p}^{\prime}(\vec{r}) \cdot \vec{E}_{q}(\vec{r}) d s=-\frac{1}{\kappa_{q}} \sum_{n=1}^{N} \mathrm{R}_{n p}^{\prime \prime} b_{n q} \\
I_{p q}^{\mathrm{TM}, \mathrm{TM}} & =\int_{S_{0}} \vec{e}_{p}^{\prime}(\vec{r}) \cdot \vec{E}_{q}^{\prime}(\vec{r}) d s=k_{p}^{\prime} \sum_{n=1}^{N^{\prime}} \mathrm{R}_{n p}^{\prime} b_{n q}^{\prime}+\frac{a_{p q}^{\prime}}{k_{p}^{\prime}}
\end{aligned}
$$

donde los coeficientes $\mathrm{R}_{n p}, \mathrm{R}_{n p}^{\prime} \mathrm{y} \mathrm{R}_{n p}^{\prime \prime}$, presentes en las expresiones recién deducidas, se definen del siguiente modo

$$
\begin{aligned}
\mathrm{R}_{n p} & =\frac{1}{k_{p}^{2}} \int_{\sigma} \vec{e}_{p}\left(\vec{s}^{\prime}\right) \cdot \vec{t}\left(l^{\prime}\right) w_{n}\left(l^{\prime}\right) d l^{\prime} \\
\mathrm{R}_{n p}^{\prime} & =\frac{1}{k_{p}^{\prime 2}} \int_{\sigma} \psi_{p}\left(\vec{s}^{\prime}\right) u_{n}\left(l^{\prime}\right) d l^{\prime} \\
\mathrm{R}_{n p}^{\prime \prime} & =\frac{1}{k_{p}^{\prime}} \int_{\sigma} \psi_{p}\left(\vec{s}^{\prime}\right) \frac{\partial w_{n}\left(l^{\prime}\right)}{\partial l^{\prime}} d l^{\prime}
\end{aligned}
$$

Por supuesto, al evaluar todas las integrales de acoplamiento mediante las expresiones (3.45)-(3.48) se asume que $p \leq M$ cuando $p$ designa un modo TE, y que $p \leq M^{\prime}$ cuando $p$ se refiere a un modo TM. Asimismo, resulta interesante observar que los coeficientes $\mathrm{R}_{n p}$ y $\mathrm{R}_{n p}^{\prime}$, empleados respectivamente en (3.45) y (3.48), constituyen los elementos de las matrices $\mathbf{R}$ and $\mathbf{R}^{\prime}$ utilizadas en la obtención de los modos TE y TM de la guía perturbada; como se puede comprobar recordando las definiciones de los elementos de dichas matrices recogidas en (3.24) y (3.31). Por tanto, para determinar el valor de las citadas integrales de acoplamiento sólo es necesario calcular los elementos $\mathrm{R}_{n p}^{\prime \prime}$ presentes en (3.47), lo que implica resolver simples integrales de línea; pues el resto de parámetros necesarios para implementar (3.45)-(3.48) se conocen una vez obtenidos los modos de la guía perturbada.

El método recién descrito para obtener los modos de una guía cuyo contorno básico rectangular (o circular) está modificado, así como las integrales de acoplamiento entre dichos modos y los de la correspondiente guía rectangular (o circular) sin modificar, se encuentra implementado comercialmente en un programa denominado ANAPLAN-W, que se ejecuta en plataformas SUN, y desarrollado bajo 


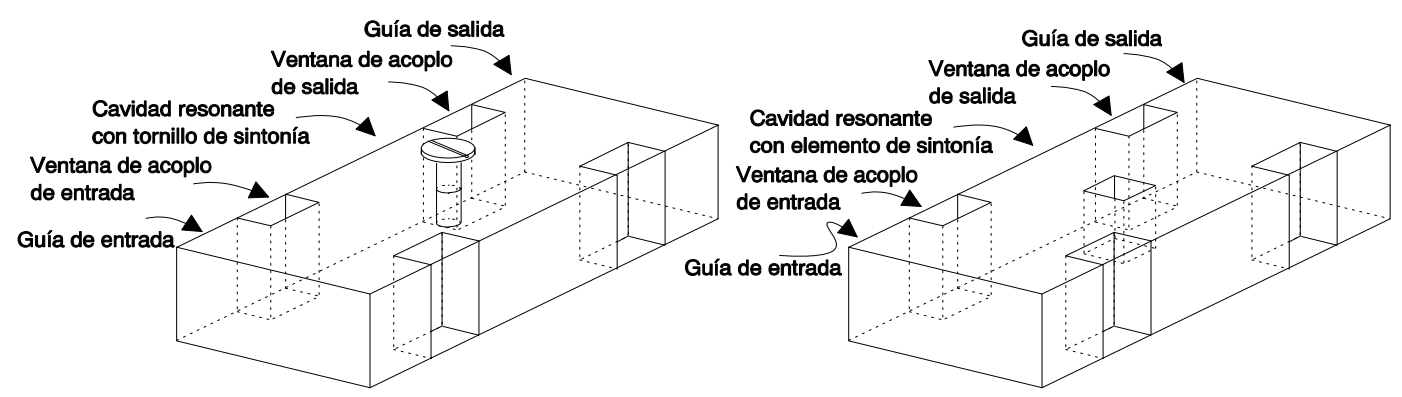

a)

b)

Figura 3.4: Estructura construida para medir el comportamiento electromagnético de un tornillo de sintonía situado en la cavidad resonante de un filtro paso banda en a), y estructura equivalente empleando un elemento de sintonía con sección transversal cuadrada en b).

el contrato 10966/94/NL/NB firmado entre la Universidad de Pavía y el Centro Europeo de Tecnología e Investigación Espacial (ESTEC) de la Agencia Espacial Europea (ESA). Todos los resultados ofrecidos en el presente capítulo, correspondientes claro está a dispositivos que utilizan elementos de sintonía, han sido generados empleando los valores que proporciona el citado programa ANAPLAN-W para las integrales de acoplamiento entre los modos de las correspondientes guías de contorno básico modificado y sin modificar.

\section{Equivalencia entre un Tornillo de Sintonía y un Tramo Uniforme de Guía Reentrante}

A continuación, se describe un procedimiento empírico para determinar la equivalencia entre el diámetro de un tornillo de sintonía, de sección transversal circular, y el lado de la sección transversal cuadrada de la inserción metálica de un tramo uniforme de guía reentrante. En dicho procedimiento, la altura del elemento de sintonía con sección transversal cuadrada, equivalente al tornillo de sintonía bajo análisis, se considera siempre igual a la altura del citado tornillo.

Así pues, con el objeto de tener conocimiento del comportamiento electromagnético de los tornillos de sintonía utilizados en los filtros paso banda, se ha considerado en primer lugar un tornillo de sección circular (diámetro $\phi=4 \mathrm{~mm}$ ) situado en una cavidad resonante acoplada con las guías de entrada y salida mediante sendas ventanas de acoplo inductivas; tal y como se muestra gráficamente en la figura 3.4 (apartado a). Las guías de entrada y salida escogidas en esta estructura son guías normalizadas WR-75 ( $a=19.050 \mathrm{~mm}, b=9.525 \mathrm{~mm})$, las ventanas de acoplo inductivas de entrada y salida presentan ambas sección transversal rectangular $(a=7.000 \mathrm{~mm}, b=9.525 \mathrm{~mm})$ con una profundidad de $1.000 \mathrm{~mm}, \mathrm{y}$ la cavidad resonante de la estructura está constituida de nuevo por 


\begin{tabular}{|c|c|}
\hline \hline $\begin{array}{c}\text { Penetración del Tornillo } \\
\text { de Sintonía ( } \mathbf{m m})\end{array}$ & $\begin{array}{c}\text { Frecuencia Central de la } \\
\text { Banda de Paso del Filtro (GHz) }\end{array}$ \\
\hline 0.000 & 14.530 \\
\hline 1.680 & 13.775 \\
\hline 2.520 & 13.322 \\
\hline 2.940 & 13.211 \\
\hline \hline
\end{tabular}

Tabla 3.1: Efecto de la penetración del tornillo de sintonía sobre la frecuencia central de la banda de paso del filtro mostrado en la figura 3.4 (apartado a).

una guía normalizada WR-75 $(a=19.050 \mathrm{~mm}, b=9.525 \mathrm{~mm})$ que presenta una longitud igual a $9.970 \mathrm{~mm}$.

Para determinar la respuesta electromagnética de la estructura mostrada en la figura 3.4 (apartado a), se han medido los parámetros de dispersión (en inglés scattering) que presenta dicho dispositivo para diferentes penetraciones del tornillo de sintonía situado en la cavidad resonante (ver apartado a en fig. 3.4); habiendo realizado las medidas con un analizador de redes $\mathrm{HP} 8510 \mathrm{C}$ en el Área de Microondas del Laboratorio de Sistemas de Radiofrecuencia que la ESA tiene en ESTEC. Como es bien sabido, a medida que aumenta la penetración del tornillo situado en la cavidad resonante, la frecuencia central de la banda de paso del filtro disminuye. En la tabla 3.1, se ofrecen los valores medidos de esta frecuencia central de la banda de paso del dispositivo construido (recordar apartado a en fig. 3.4) para diferentes penetraciones del tornillo de sintonía considerado.

Una vez medida la respuesta de la estructura mostrada en la figura 3.4 (apartado a) para diferentes penetraciones del tornillo de sintonía, haciendo uso de las herramientas de programación denominadas DUMAS 3.0 (ver apéndice B) y ANAPLAN-W, se simula el comportamiento electromagnético (parámetros de dispersión) de una estructura similar a aquélla cuya respuesta se ha medido previamente; estructura en la que en lugar de emplear el tornillo de sintonía considerado originalmente, éste se sustituye en cada uno de los casos presentados en la tabla 3.1 por un elemento de sección transversal cuadrada e igual altura que el tornillo (ver apartado b en fig. 3.4). Desde el punto de vista del análisis electromagnético de la nueva estructura recogida en la figura 3.4 (apartado b), el tramo de cavidad que contiene al elemento de sintonía con sección transversal cuadrada se considera como un tramo uniforme de guía reentrante; cuya inserción metálica (elemento de sintonía) presenta una sección transversal cuadrada (anchura de la inserción metálica igual a su profundidad). El tamaño de la inserción metálica cuadrada debe determinarse, para cada una de las distintas penetraciones recogidas en la tabla 3.1, con el objeto de centrar la respuesta paso banda de la estructura equivalente a la misma frecuencia obtenida empleando el tornillo de sintonía real. Así por ejemplo, para el caso en el que el tornillo penetra 


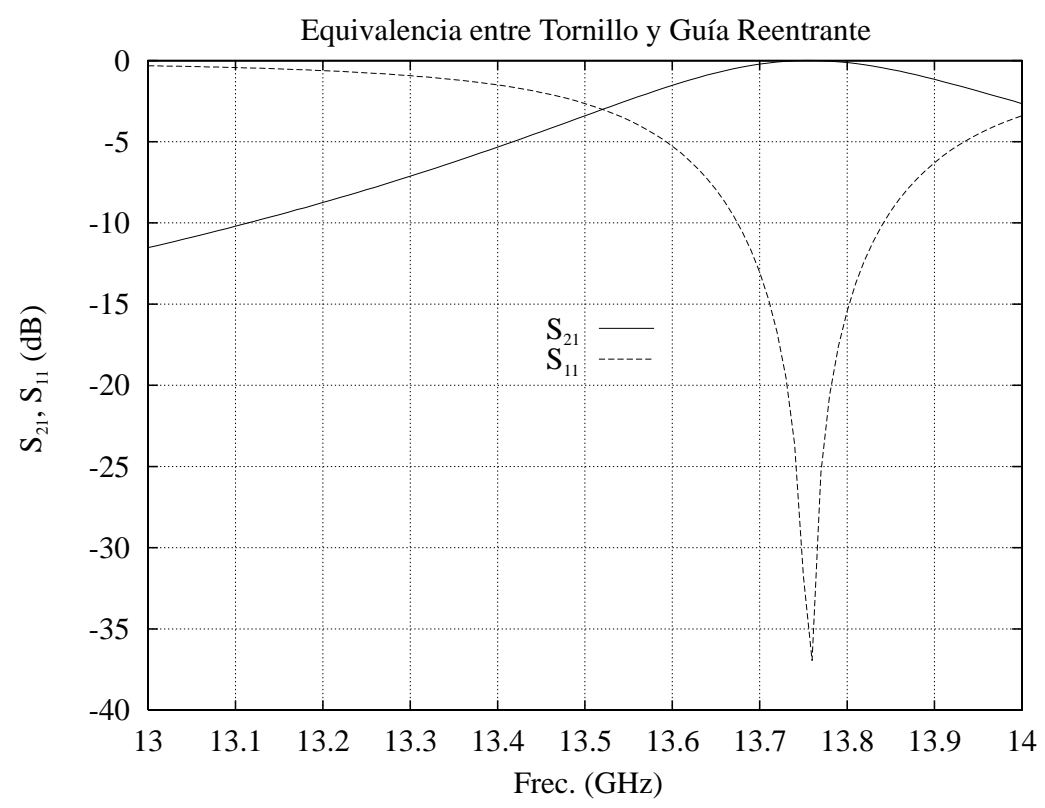

Figura 3.5: Módulo de los parámetros $S_{21}$ y $S_{11}$ que presenta una estructura como la mostrada en la figura 3.4 (apartado b); cuyo elemento de sintonía tiene sección transversal cuadrada (lado igual a $2.820 \mathrm{~mm}$ ) y penetra $1.680 \mathrm{~mm}$ dentro de la cavidad resonante de la estructura.

$1.680 \mathrm{~mm}$ dentro de la cavidad resonante, en la figura 3.5 se presenta la respuesta electromagnética (parámetros $S_{21}$ y $S_{11}$ ) de la estructura equivalente ofrecida en la figura 3.4 (apartado b); habiendo utilizado como elemento de sintonía un cilindro de sección cuadrada (lado igual a $2.820 \mathrm{~mm}$ ) y con la misma altura que el tornillo de sintonía real. En la figura 3.5, se observa que el elemento de sección cuadrada escogido centra efectivamente la respuesta paso banda de la estructura equivalente a la misma frecuencia central, concretamente a $13.775 \mathrm{GHz}$, a la que se encuentra centrada la respuesta paso banda de la estructura real original con el tornillo de sintonía (recordar tabla 3.1). En la tabla 3.2, se ofrece el tamaño que debe presentar cada elemento de sintonía equivalente, concretamente el valor del lado de su sección transversal cuadrada, para recuperar con la estructura mostrada en la figura 3.4 (apartado b) la misma respuesta que se ha obtenido previamente, empleando el tornillo de sintonía real, con unas penetraciones de dicho tornillo recogidas en la tabla 3.1 .

Observando los resultados ofrecidos en la tabla 3.2 , se descubre que a mayor penetración del tornillo de sintonía situado en la cavidad resonante de la estructura considerada (recordar apartado a en fig. 3.4), el tamaño del elemento de sintonía equivalente (el lado de su sección transversal cuadrada) requerido es cada vez menor; comportamiento que puede explicarse considerando el efecto perturbador introducido por los ángulos del mencionado elemento de sintonía 


\begin{tabular}{|c|c|}
\hline \hline $\begin{array}{c}\text { Penetración del Tornillo } \\
\text { de Sintonía }(\mathbf{m m})\end{array}$ & $\begin{array}{c}\text { Tamaño del Elemento de } \\
\text { Sintonía Equivalente }(\mathbf{m m})\end{array}$ \\
\hline 1.680 & 2.820 \\
\hline 2.520 & 1.700 \\
\hline 2.940 & 1.096 \\
\hline \hline
\end{tabular}

Tabla 3.2: Tamaño del elemento de sintonía equivalente, con sección transversal cuadrada, en función de la penetración del tornillo de sintonía $(\phi=4 \mathrm{~mm})$ situado dentro de la cavidad de la estructura mostrada en la figura 3.4 (apartado a).

equivalente. Así pues, al aumentar la penetración del tornillo de sintonía real, la perturbación electromagnética que producen los ángulos del elemento de sintonía equivalente introducido crece; por lo que el tamaño requerido en el elemento de sintonía equivalente es menor para conseguir el mismo efecto producido por el tornillo original. Empleando pues los valores mostrados en la tabla 3.2, y obteniendo además algunos otros valores adicionales para penetraciones mayores del tornillo de sintonía (hasta que el tornillo penetre aproximadamente la mitad de la altura de la cavidad resonante), es posible obtener una curva mediante mínimos cuadrados por ejemplo que se ajuste bien a todos los valores obtenidos. Una vez construida dicha curva, en ella se podrá leer el valor del tamaño del elemento de sintonía equivalente (lado de su sección transversal cuadrada) que corresponde a cada penetración del tornillo real considerado, aquél de diámetro $\phi=4 \mathrm{~mm}$, y situado dentro de la cavidad resonante del filtro paso banda de un solo polo implementado. Con el objeto de disponer de un juego apropiado de este tipo de gráficas, el proceso recién descrito debería repetirse para otros tornillos (diferentes penetraciones) situados en la misma estructura previamente considerada; y posteriormente situando todos los tornillos empleados en el proceso de medidas en estructuras similares (filtros paso banda de un solo polo), pero con dimensiones de sus elementos integrantes (ventanas de acoplo inductivas y cavidades resonantes) diferentes a las del ejemplo planteado anteriormente.

En un filtro paso banda implementado con ventanas de acoplo inductivas, tal y como ya se ha comentado con anterioridad, los tornillos de sintonía no sólo se sitúan en las cavidades resonantes para ajustar la frecuencia central de la banda de paso; sino que también se colocan en las ventanas de acoplo inductivas del filtro para conseguir el ancho frecuencial deseado en la citada banda de paso. Los efectos de los tornillos de sintonía en los mencionados filtros pueden simularse fácilmente con los programas DUMAS 3.0 y ANAPLAN-W; empleando para ello lógicamente elementos de sintonía con sección transversal cuadrada. Tras simular con dichos programas la respuesta deseada del filtro paso banda, es necesario determinar el valor de los diámetros de los tornillos de sintonía reales a emplear en el dispositivo a construir; para lo cual deben utilizarse gráficas de equivalencia 


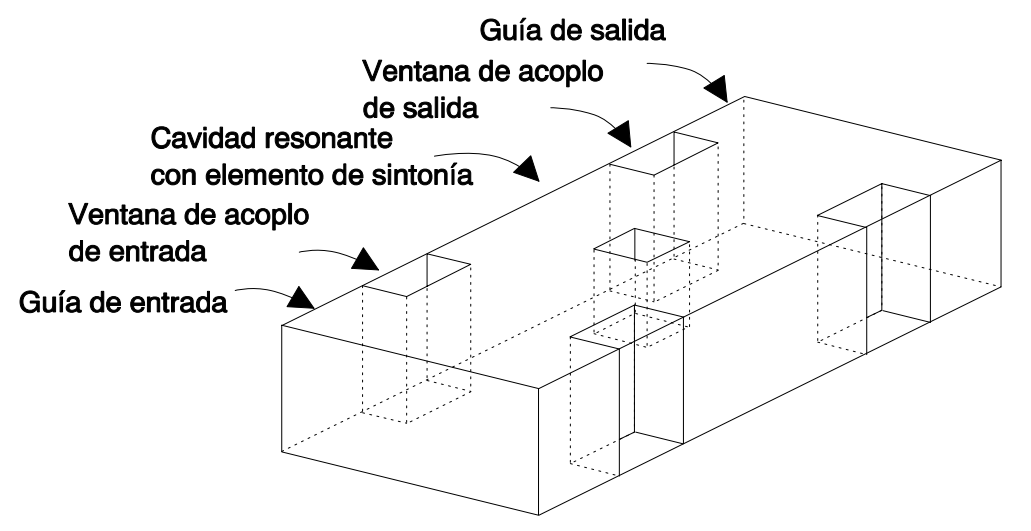

Figura 3.6: Estructura considerada para estudiar la evolución de la frecuencia central de la banda de paso de un filtro paso banda de un solo polo, con un elemento de sintonía en su cavidad resonante, en función de la penetración de dicho elemento.

similares a aquélla descrita en el párrafo anterior. En concreto, deben utilizarse dos de las mencionadas gráficas; una de ellas para obtener los diámetros de los tornillos a situar dentro de las cavidades resonantes del filtro, y la otra para deducir los diámetros de los tornillos a colocar en las ventanas de acoplo del citado diseño. De esta manera, utilizando los tornillos de sintonía cuyos diámetros se obtienen mediante dichas gráficas, el filtro paso banda implementado presentará una respuesta electromagnética muy similar a la obtenida en simulación con elementos de sintonía de sección transversal cuadrada.

\subsubsection{Investigación de Zonas de Sintonía}

Como primera aplicación del modelo desarrollado en el apartado 3.1.1 para representar el comportamiento electromagnético de los tornillos de sintonía, en este apartado se investiga en primer lugar el efecto que supone introducir un elemento de sintonía (con sección transversal cuadrada) en una cavidad resonante; que está acoplada con las guías de entrada y salida de la estructura considerada mediante sendas ventanas de acoplo inductivas (ver estructura en figura 3.6). Las dimensiones nominales de la sección transversal de las guías de entrada y salida escogidas $(a=47.550 \mathrm{~mm}, b=22.150 \mathrm{~mm})$ corresponden a las de la guía normalizada WR-187; mientras como ventanas de acoplo se eligen guías con sección transversal rectangular $(a=20.000 \mathrm{~mm}, b=22.150 \mathrm{~mm})$ de profundidad $4.000 \mathrm{~mm}$. La cavidad resonante del filtro viene representada de nuevo por una guía normalizada WR-187 de longitud $22.000 \mathrm{~mm}$; y el elemento de sintonía centrado en dicha cavidad presenta una sección transversal cuadrada de lado $10.000 \mathrm{~mm}$.

Una estructura como la considerada en el ejemplo bajo análisis, constituida por una cavidad resonante acoplada a las guías de entrada y salida mediante ventanas de acoplo inductivas (recordar fig. 3.6), constituye un filtro paso banda 


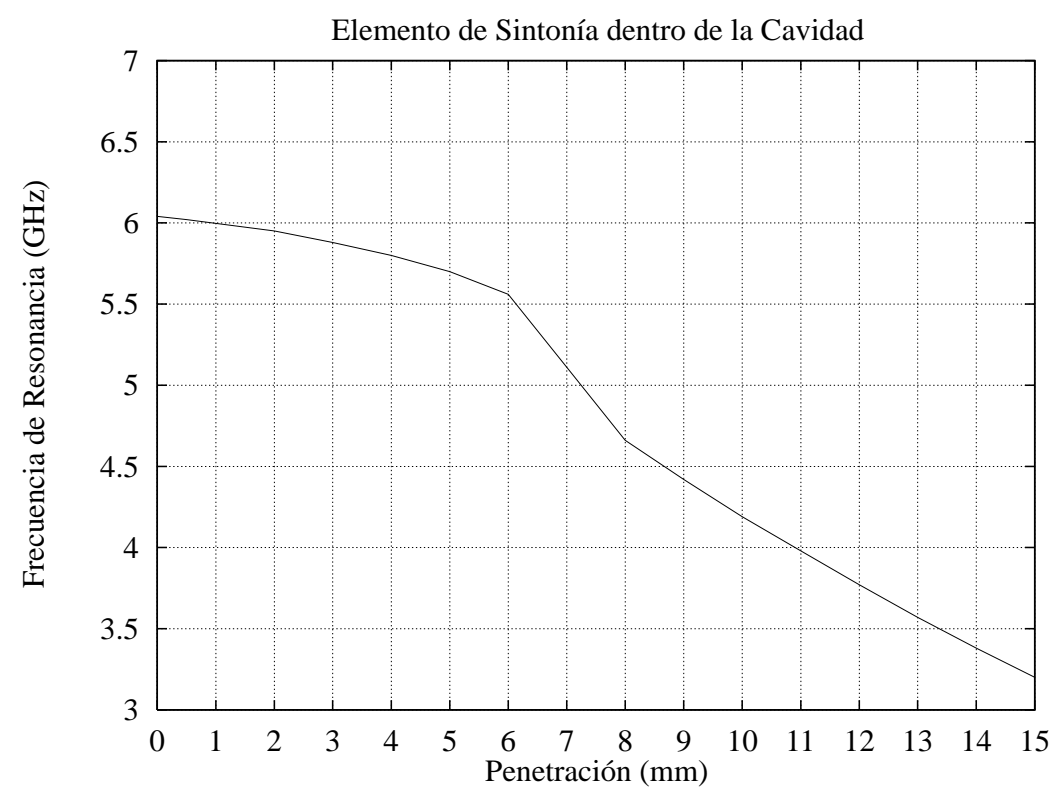

Figura 3.7: Evolución de la frecuencia central de la banda de paso de la estructura mostrada en la figura 3.6 frente a la penetración del elemento de sintonía situado en la cavidad resonante de dicha estructura.

en guía rectangular de un solo polo (una sola cavidad); cuya respuesta electromagnética (parámetro $S_{21}$ ) presenta una banda de paso centrada a una determinada frecuencia con un cierto ancho frecuencial. En la figura 3.7, se muestra la variación de la frecuencia central de la banda de paso del filtro considerado para diferentes penetraciones del elemento de sintonía situado en su cavidad resonante; observando, como era previsible, que dicha frecuencia central disminuye al aumentar la penetración del elemento de sintonía. De los resultados mostrados en la figura 3.7, para un elemento de sintonía que presenta sección transversal cuadrada (lado igual a $10.000 \mathrm{~mm}$ ) situado en la cavidad resonante de la estructura analizada, es posible determinar fácilmente la penetración requerida y la variación necesaria de dicha penetración para compensar imprecisiones en la fabricación de filtros paso banda, o incluso para sintonizar estos filtros a diferentes frecuencias pertenecientes a una determinada banda de interés.

De manera similar a como se ha estudiado previamente el efecto que supone situar un elemento de sintonía en la cavidad resonante de un filtro paso banda de un solo polo, seguidamente se procede a determinar como afecta colocar elementos de sintonía en las ventanas de acoplo del citado filtro paso banda; empleando para ello una estructura como la mostrada en la figura 3.8. En esta ocasión, la estructura considerada es idéntica (mismas dimensiones de los elementos que la integran) a la empleada en el caso anterior; exceptuando la profundidad de las ventanas de acoplo, cuyo valor se elige en este caso igual a $6.000 \mathrm{~mm}$. Como elemento de sintonía a situar en ambas ventanas (ver fig. 3.8), se ha escogido 


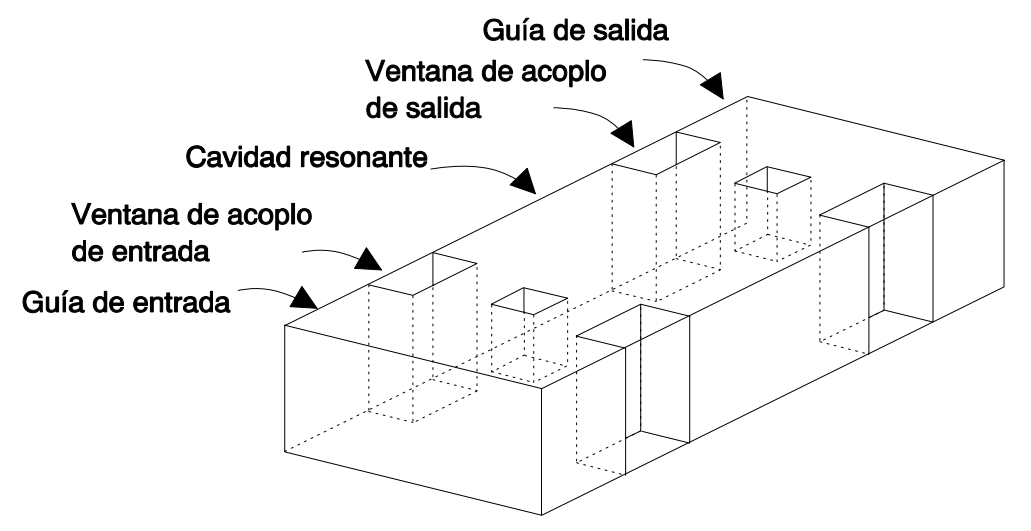

Figura 3.8: Estructura escogida para analizar la evolución del ancho frecuencial de la banda de paso de un filtro paso banda de un solo polo, con elementos de sintonía en las ventanas de acoplo de entrada y salida del filtro, en función de la penetración de dichos elementos.

nuevamente un elemento que tiene sección transversal cuadrada; aunque el lado de la sección presenta en este análisis un valor de $4.000 \mathrm{~mm}$.

Los resultados obtenidos al simular la estructura recogida en la figura 3.8 , variando lógicamente la penetración de los elementos de sintonía situados en las ventanas de acoplo de entrada y salida, se ofrecen en la figura 3.9; donde se muestra la evolución del ancho de banda relativo de la respuesta paso banda del filtro considerado. Este ancho de banda relativo se define como el cociente entre el ancho frecuencial a $3 \mathrm{~dB}$ de la mencionada respuesta paso banda del filtro y la frecuencia central de dicha banda de paso; que se representa además normalizado respecto del valor obtenido para este cociente cuando no existen elementos de sintonía en las ventanas de acoplo. Estos resultados recogidos en la figura 3.9, tal y como era de esperar, confirman que el ancho de banda de la respuesta electromagnética (parámetro $S_{21}$ ) del filtro paso banda aumenta cuando se incrementa la penetración del elemento de sintonía situado en sus ventanas de acoplo inductivas. De esta última gráfica (figura 3.9), considerando un mismo elemento de sintonía con sección transversal cuadrada (lado igual a $4.000 \mathrm{~mm}$ ) en las ventanas de acoplo inductivas de entrada y salida de la estructura simulada, puede deducirse con facilidad la penetración requerida en dichos tornillos, así como la variación necesaria de esta penetración, para una aplicación específica que requiera de la presencia de los mencionados elementos de sintonía.

Del estudio presentado sobre las posibles zonas de sintonía a conseguir, empleando para ello elementos de sintonía en las cavidades resonantes y en las ventanas de acoplo inductivas de un filtro paso banda de un solo polo, se concluye que dichos elementos pueden emplearse no sólo para compensar las degradaciones que experimentan las respuestas de los filtros debidas a tolerancias en su implementación; sino también para sintonizar los mencionados filtros paso banda en 


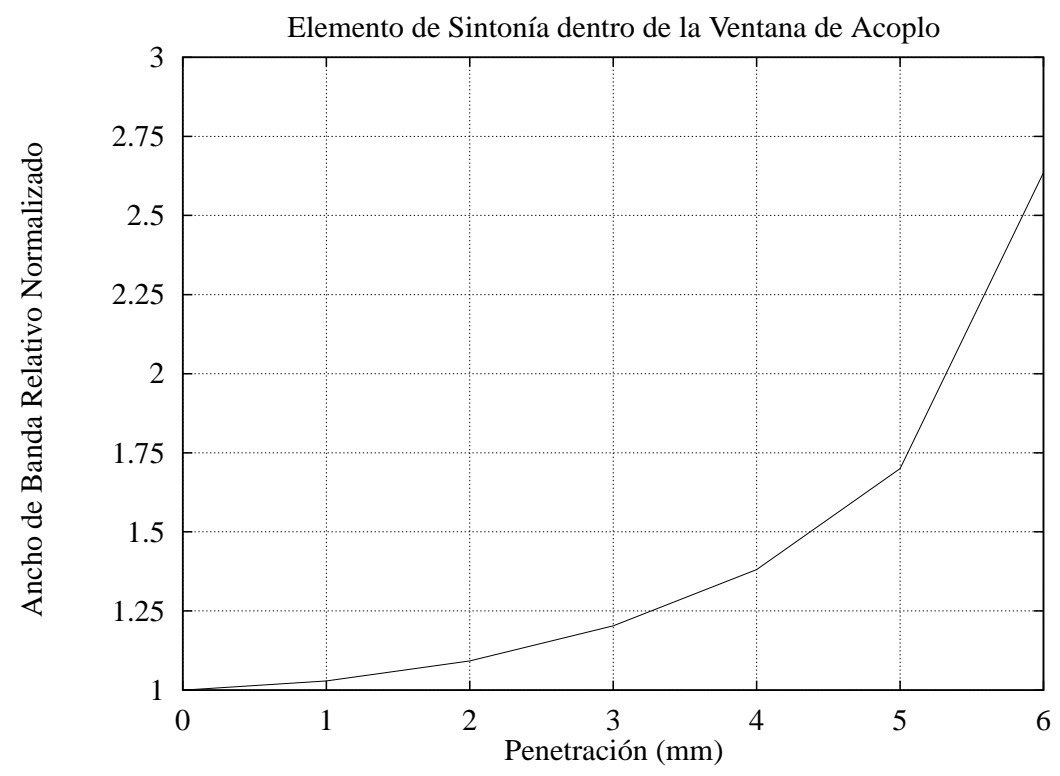

Figura 3.9: Evolución del ancho de banda relativo normalizado, que presenta la respuesta paso banda de la estructura mostrada en la figura 3.8, frente a la penetración del elemento de sintonía situado en las ventanas de acoplo de dicha estructura.

una determinada banda de frecuencias preservando un cierto ancho de banda. Concretamente, esta última posibilidad que ofrecen los mencionados elementos de sintonía, deducida de la enorme zona de frecuencias centrales de bandas de paso y anchos de banda relativos que pueden conseguirse con dichos elementos de sintonía (recordar figuras 3.7 y 3.9 ), se ha utilizado en el próximo apartado para presentar un procedimiento de diseño de estructuras básicas de filtros paso banda, implementadas con ventanas inductivas, que pueden sintonizarse dentro de una banda de frecuencias manteniendo su ancho de banda mediante el empleo de los correspondientes tornillos de sintonía.

\subsubsection{Procedimiento de Diseño de Filtros Paso Banda en Guía Rectangular con Ventanas de Acoplo Inducti- vas y Tornillos de Sintonía}

En el presente apartado, se describe un procedimiento general para diseñar una estructura básica de filtro paso banda en guía rectangular, con ventanas inductivas, cuya respuesta pueda sintonizarse en una determinada banda de frecuencias preservando un cierto ancho frecuencial mediante tornillos de sintonía. Este tipo de implementaciones pueden encontrar aplicación en el equipamiento utilizado en radioenlaces; en los cuales cada usuario individual utiliza una pequeña porción de la misma banda general de frecuencias. Así pues, en dicha aplicación puede 


\begin{tabular}{|c|c|c|}
\hline \hline Guía utilizada & \multicolumn{2}{|c|}{ Dimensiones de las guías ( $\mathbf{m m})$} \\
\cline { 2 - 3 } para implementar & Filtro a 11 GHz & Filtro a 13 $\mathbf{~ H H z}$ \\
\hline 1 a ventana (anchura) & 10.520 & 8.850 \\
\hline 1 a cavidad (longitud) & 15.680 & 11.972 \\
\hline 2 $^{\text {a }}$ ventana (anchura) & 7.098 & 5.658 \\
\hline 2 $^{\text {a }}$ cavidad (longitud) & 17.605 & 13.328 \\
\hline 3 $^{\text {a }}$ ventana (anchura) & 6.520 & 5.206 \\
\hline \hline
\end{tabular}

Tabla 3.3: Dimensiones optimizadas de las ventanas de acoplo inductivas y de las cavidades resonantes que integran los dos filtros paso banda en guía rectangular, sin elementos de sintonía, centrados respectivamente a $11 \mathrm{GHz}$ y $13 \mathrm{GHz}$ con un ancho de banda de $300 \mathrm{MHz}$ y respuesta tipo Chebyshev.

resultar interesante disponer por adelantado de la parte más costosa (la estructura básica recién comentada) de los filtros a utilizar por cada usuario; y en una etapa posterior seleccionar la frecuencia específica de operación de cada usuario sintonizando adecuadamente, mediante el empleo de una tapa superior con los elementos de sintonía apropiados, la estructura básica del mencionado filtro paso banda. Con el objeto de presentar esta técnica de diseño novedosa, a continuación se describe el proceso a seguir para diseñar una estructura básica de filtro paso banda simétrico de 4 polos, implementado en guía rectangular con ventanas de acoplo inductivas, cuya respuesta tipo Chebyshev pueda sintonizarse a cualquier frecuencia comprendida entre $11 \mathrm{GHz}$ y $13 \mathrm{GHz}$ preservando un mismo ancho de banda igual a $300 \mathrm{MHz}$.

Para obtener unas dimensiones óptimas de la estructura básica descrita previamente, deben diseñarse en primer lugar dos filtros paso banda simétricos de 4 polos, implementados en guía rectangular con ventanas de acoplo inductivas (ver figura 3.1), que presenten ambos una respuesta tipo Chebyshev con un ancho de banda de $300 \mathrm{MHz}$; y centrados respectivamente a la frecuencia inicial y final de la banda en la que se pretende desplazar la respuesta del filtro, en nuestro caso a $11 \mathrm{GHz}$ y $13 \mathrm{GHz}$. En el diseño de estos dos filtros, se ha seguido el procedimiento descrito en [40]; donde se propone una técnica alternativa para diseñar filtros de microondas basada en un simulador electromagnético como puede ser por ejemplo el programa DUMAS 3.0 (ver apéndice B), y que descompone la tarea compleja de diseño de filtros en un conjunto de tareas más simples con unos objetivos claramente identificados. Concretamente, este nuevo procedimiento propuesto en [40] implica la optimización de un número limitado de parámetros físicos del filtro en cada etapa del diseño; lo que resulta eficiente desde el punto de vista computacional, y a su vez provoca que el proceso de diseño converja rápidamente a la solución óptima. En el caso concreto de los dos diseños que nos ocupan, siguiendo el método propuesto en [40], se han escogido como 


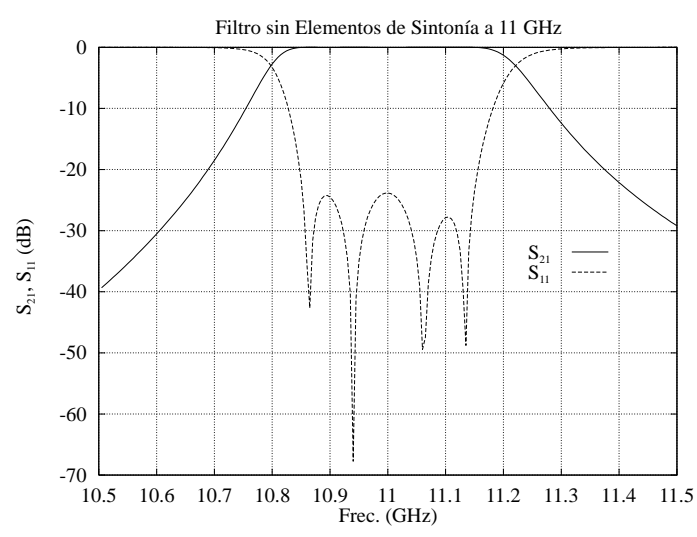

a)

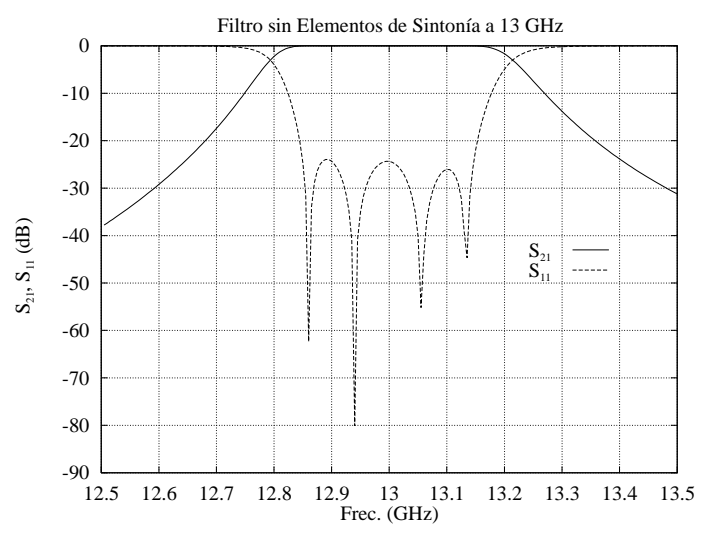

b)

Figura 3.10: Respuesta electromagnética (módulo de los parámetros $S_{21}$ y $S_{11}$ ) que presenta un filtro paso banda simétrico de 4 polos, implementado en guía rectangular con ventanas de acoplo inductivas y respuesta tipo Chebyshev, centrado a $11 \mathrm{GHz}$ y con un ancho de banda de $300 \mathrm{MHz}$ en a); y mismo tipo de filtro paso banda centrado a $13 \mathrm{GHz}$ en b).

parámetros físicos a optimizar las anchuras de las ventanas de acoplo inductivas y las longitudes de las cavidades resonantes de los dos filtros (recordar fig. 3.1). Las guías de entrada y salida de ambos filtros, así como las cavidades resonantes, se corresponden con guías normalizadas WR-75 $(a=19.050 \mathrm{~mm}, b=9.525 \mathrm{~mm})$; mientras que todas las ventanas de acoplo, al presentar carácter inductivo, tendrán una sección transversal rectangular de altura constante $b=9.525 \mathrm{~mm}$. Para la profundidad de todas las ventanas de acoplo, se ha escogido nuevamente un mismo valor; en este caso igual a $2.000 \mathrm{~mm}$. En cuanto a las anchuras de las diferentes ventanas de acoplo inductivas y las longitudes de las cavidades resonantes, parámetros escogidos para obtener las respuestas de los dos filtros paso banda sin elementos de sintonía, sus respectivos valores optimizados tras implementar el proceso de diseño seguido se encuentran resumidos en la tabla 3.3, recogida en la página 95; donde se observa que tan sólo se muestran las dimensiones de dichos parámetros para la mitad de las estructuras (tres ventanas de acoplo y dos cavidades resonantes), debido a que los dos filtros diseñados son simétricos. Las respuestas electromagnéticas (parámetros de dispersión $S_{21}$ y $S_{11}$ ) que presentan los dos filtros paso banda diseñados, centrados respectivamente a $11 \mathrm{GHz}$ y $13 \mathrm{GHz}$, se muestran en la figura 3.10 (apartados a y b); comprobando en dicha figura que ambas respuestas presentan el ancho de banda deseado (300 $\mathrm{MHz}$ ).

Las dimensiones finales de las ventanas de acoplo (anchuras) de la estructura básica de filtro paso banda, sintonizable con elementos de sintonía, quedan restringidas por los valores más pequeños de dichas dimensiones obtenidas en los diseños de los filtros paso banda sin elementos de sintonía; que corresponden al filtro cuya respuesta está centrada a $13 \mathrm{GHz}$ (consultar tabla 3.3). Esta 


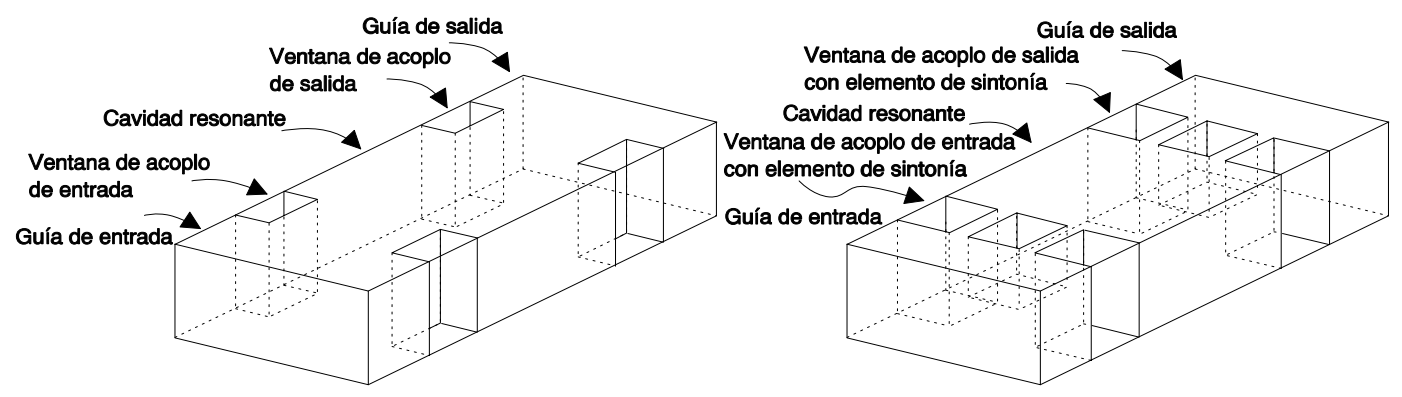

a)

b)

Figura 3.11: Estructura escogida para determinar el nivel de acoplamiento requerido por cada ventana de acoplo inductiva de los filtros sin elementos de sintonía en a), y estructura utilizada para obtener ese mismo nivel de acoplamiento empleando elementos de sintonía en la ventana correspondiente de la estructura básica en b).

restricción se debe a que el ancho de banda de las respuestas conseguidas en los filtros sin elementos de sintonía mediante las ventanas de acoplo (anchuras), se conseguirán en la estructura básica sintonizable introduciendo elementos de sintonía en las ventanas de acoplo de dicha estructura. Así pues, para la anchura de la primera ventana de acoplo de la citada estructura básica, se escoge un valor igual a $8.700 \mathrm{~mm}$; mientras para la segunda y tercera ventana de acoplo inductiva de dicha estructura se elige una misma anchura de valor $5.100 \mathrm{~mm}$. El nivel de acoplamiento requerido por los dos filtros diseñados previamente sin elementos de sintonía, centrados respectivamente a $11 \mathrm{GHz}$ y $13 \mathrm{GHz}$, es mayor que el proporcionado por las anchuras recién escogidas para las ventanas de acoplo de la estructura básica a diseñar (comparar con las respectivas anchuras de las ventanas de acoplo en la mencionada tabla 3.3); por lo que para conseguir esos niveles de acoplamiento, tal y como se termina de mencionar, se introducirán elementos de sintonía (en este caso con sección transversal cuadrada de lado igual a $2.000 \mathrm{~mm}$ ) situados respectivamente en cada una de las ventanas de acoplo inductivas de la estructura básica diseñada (recordar apartado 3.1.2). Para corroborar que las anchuras seleccionadas en las ventanas de acoplo inductivas de la estructura básica son apropiadas, resulta necesario determinar las penetraciones de los elementos de sintonía, situados respectivamente en las ventanas inductivas de la estructura básica, que permitan alcanzar los niveles de acoplamiento requeridos por los dos filtros sin elementos de sintonía. Los valores de estos niveles de acoplamiento se determinan simulando estructuras simétricas constituidas por una sola cavidad resonante $(a=19.050 \mathrm{~mm}, b=9.525 \mathrm{~mm})$; que se encuentra acoplada a las guías de entrada y salida (con iguales dimensiones que la cavidad) mediante sendas ventanas de acoplo inductivas, cuyas dimensiones corresponden en cada caso a las recogidas en la tabla 3.3. En la figura 3.11 (apartado a), se muestra el aspecto que presenta la estructura recién descrita para determinar los 


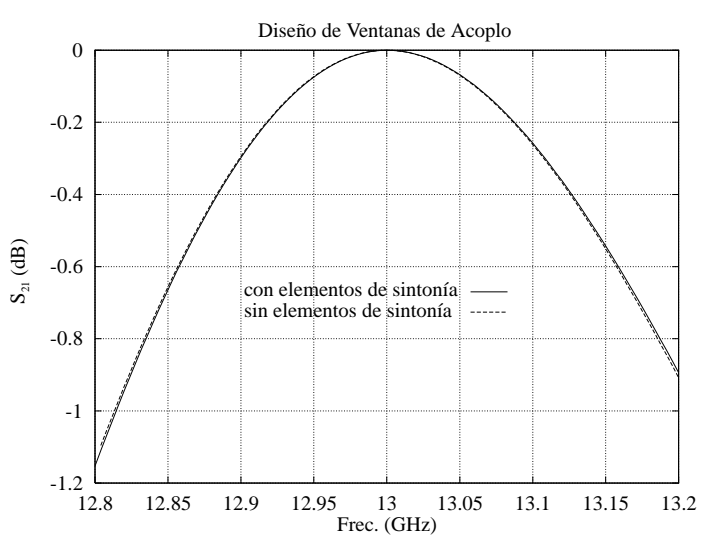

a)

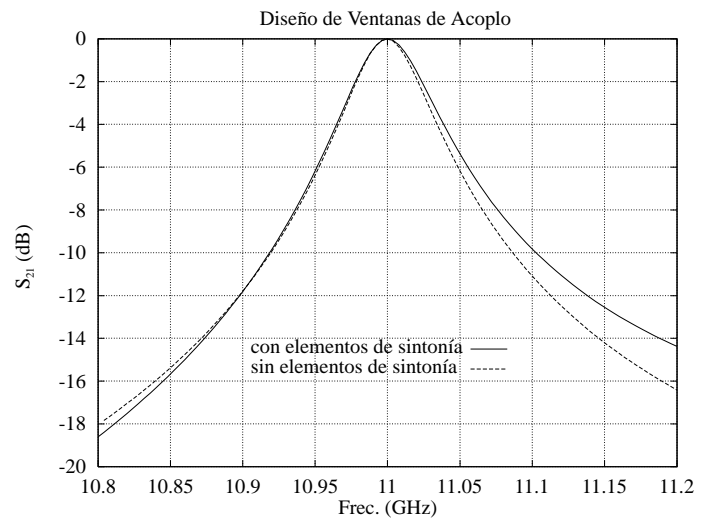

b)

Figura 3.12: Comparación entre las respuestas electromagnéticas (parámetros $S_{21}$ ) de las estructuras mostradas en la figura 3.11 (apartados a y b) para el caso menos crítico (primera ventana de acoplo de anchura $8.850 \mathrm{~mm}$ del filtro a $13 \mathrm{GHz}$ ) en a), e idéntica comparación para el caso más crítico (segunda ventana de acoplo de anchura $7.098 \mathrm{~mm}$ del filtro a $11 \mathrm{GHz}$ ) en b).

niveles de acoplamiento proporcionados por cada ventana inductiva en los filtros sin elementos de sintonía; observando además en dicha figura que las ventanas de acoplo de entrada y salida son idénticas (estructura simétrica). Las distintas respuestas electromagnéticas de la estructura propuesta, simuladas haciendo uso del programa DUMAS 3.0, se centran a la frecuencia correspondiente $(11 \mathrm{GHz}$ o $13 \mathrm{GHz}$ dependiendo de la ventana de acoplo que se considere); modificando para ello de manera adecuada la longitud de la cavidad resonante que integra la citada estructura (recordar apartado a en fig. 3.11). A continuación, usando las ventanas de acoplo inductivas elegidas para la estructura básica (la primera de ellas con anchura igual a $8.700 \mathrm{~mm}$, y la segunda y tercera con la misma anchura de valor $5.100 \mathrm{~mm}$ ), debe poderse alcanzar en cada caso el mismo nivel de acoplamiento obtenido con las ventanas inductivas de los diseños sin elementos de sintonía; para lo cual se emplea una estructura como la mostrada en la figura 3.11 (apartado b), que incorpora lógicamente elementos de sintonía en las dos ventanas de acoplo. Las respuestas electromagnéticas de esta nueva estructura, en cuya simulación se ha hecho uso de los programas DUMAS 3.0 y ANAPLAN-W, se han centrado nuevamente a la frecuencia correspondiente (11 GHz o $13 \mathrm{GHz}$ según el caso); con el objeto de poder comparar estas nuevas respuestas con aquéllas obtenidas anteriormente cuando no existían elementos de sintonía en las ventanas de acoplo, y determinar pues las penetraciones requeridas en los elementos de sintonía para superponer ambas respuestas en todos los casos considerados. Así por ejemplo, en la figura 3.12 (apartado a) se ofrece una comparación entre las respuestas electromagnéticas que presentan las dos estructuras mostradas en la figura 3.11 (apartados a y b) para el caso menos crítico; que corresponde a 


\begin{tabular}{|c|c|c|}
\hline \hline \multirow{2}{*}{$\begin{array}{c}\text { Elemento de Sintonía } \\
\text { situado en }\end{array}$} & \multicolumn{2}{|c|}{ Penetración (mm) } \\
\cline { 2 - 3 } & Filtro a 11 GHz & Filtro a 13 GHz \\
\hline 1 a ventana $($ anchura $=8.700 \mathrm{~mm})$ & 3.582 & 1.110 \\
\hline 2 $^{\text {a }}$ ventana $($ anchura $=5.100 \mathrm{~mm})$ & 4.477 & 2.414 \\
\hline 3 $^{\text {a }}$ ventana $($ anchura $=5.100 \mathrm{~mm})$ & 4.046 & 0.983 \\
\hline \hline
\end{tabular}

Tabla 3.4: Penetraciones de los elementos de sintonía situados en las ventanas de acoplo de la estrucutra básica para conseguir los niveles de acoplamiento requeridos por los diseños a $11 \mathrm{GHz}$ y $13 \mathrm{GHz}$.

la primera ventana de acoplo inductiva del filtro centrado a $13 \mathrm{GHz}$, de anchura $8.850 \mathrm{~mm}$, cuyo nivel de acoplamiento debe recuperarse insertando un elemento de sintonía en la primera ventana de acoplo de la estructura básica con anchura igual a $8.700 \mathrm{~mm}$. Como puede observarse en la figura 3.12 (apartado a), es posible conseguir que ambas respuestas se superpongan; habiendo empleado para ello un elemento de sintonía con sección cuadrada (de lado igual a $2.000 \mathrm{~mm}$ ) que penetra $1.110 \mathrm{~mm}$ en la ventana de acoplo. Por otra parte, en la misma figura 3.12 (apartado b) se recoge el mismo estudio comparativo recién descrito pero en esta ocasión para el caso más crítico; que se encuentra asociado a la segunda ventana inductiva del filtro centrado a $11 \mathrm{GHz}$, con anchura igual a $7.098 \mathrm{~mm}$, cuyo nivel de acoplamiento debe conseguirse introduciendo nuevamente un elemento de sintonía ahora en la segunda ventana de acoplo de la estructura básica con anchura $5.100 \mathrm{~mm}$. Los resultados mostrados en la figura 3.12 (apartado b) revelan que es posible conseguir el nivel de acoplamiento buscado; habiendo necesitado en este segundo ejemplo un elemento de sintonía con sección cuadrada (nuevamente de lado igual a $2.000 \mathrm{~mm}$ ) penetrando $4.477 \mathrm{~mm}$ en la ventana de acoplo. De la discusión realizada sobre estos dos últimos resultados, es posible concluir que los diferentes niveles de acoplamiento requeridos por las ventanas inductivas de los filtros centrados a $11 \mathrm{GHz}$ y $13 \mathrm{GHz}$ pueden conseguirse, empleando lógicamente los correspondientes elementos de sintonía, mediante las ventanas de acoplo escogidas para la estructura básica de filtro paso banda a diseñar. Un resumen completo de las penetraciones requeridas en estos elementos de sintonía, determinadas respectivamente para superponer en cada caso las respuestas asociadas a las dos estructuras consideradas en la figura 3.11 (apartados a y b), se encuentra recogido en la tabla 3.4 .

En cuanto a las dimensiones finales de las cavidades resonantes (longitudes) de la estructura básica de filtro paso banda a diseñar, quedan ahora restringidas por los valores más pequeños obtenidos para dichas longitudes en el proceso de sintonía (a $11 \mathrm{GHz}$ o a $13 \mathrm{GHz}$ según el caso) de la estructura constituida por una sola cavidad (recordar apartado b en fig. 3.11) considerada anteriormente; estructura que se ha utilizado en la obtención de las penetraciones de los elementos 


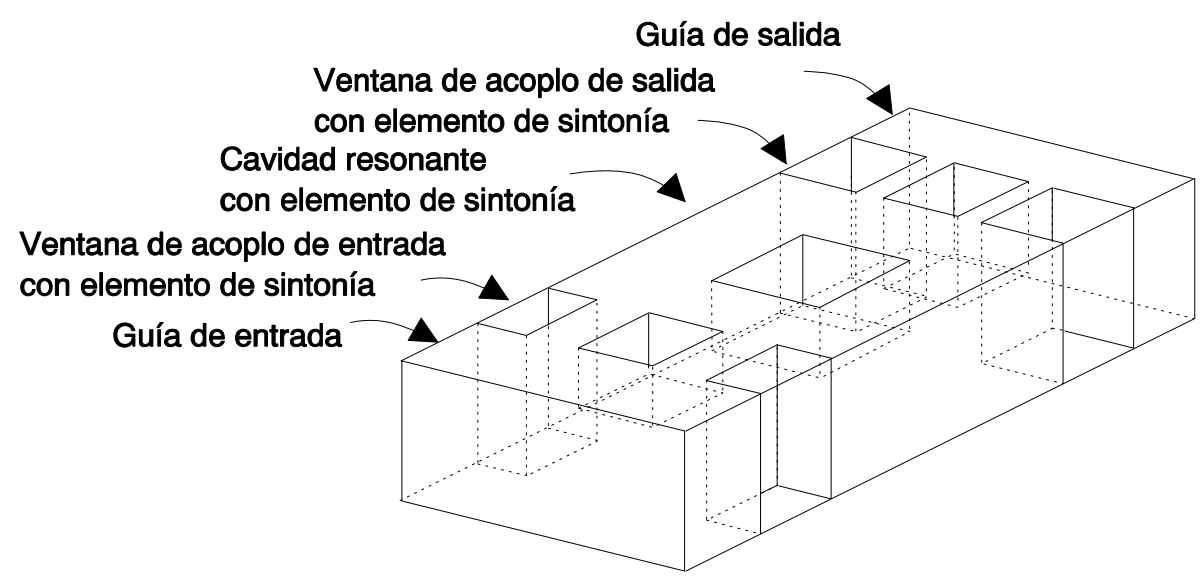

Figura 3.13: Estructura escogida para determinar las penetraciones del elemento de sintonía, a situar en la primera cavidad de la estructura básica, con el objeto de centrar la respuesta de esta primera cavidad a $11 \mathrm{GHz}$ y $13 \mathrm{GHz}$ respectivamente.

de sintonía, a situar en las respectivas ventanas de acoplo inductivas del modelo básico de filtro paso banda, que permiten obtener los niveles de acoplamiento requeridos. De esta manera, al sintonizar dicha estructura correspondiente a la primera ventana de acoplo de la implementación básica, resulta necesario escoger una cavidad en el esquema mostrado en la figura 3.11 (apartado b) de longitud igual a $13.886 \mathrm{~mm}$ para centrar su respuesta a $11 \mathrm{GHz}$; mientras que para centrar la respuesta de esa misma estructura con una sola cavidad a $13 \mathrm{GHz}$, se precisa una longitud para dicha cavidad de valor $10.814 \mathrm{~mm}$. Como consecuencia del comentario realizado previamente, se escoge un valor igual a $10.500 \mathrm{~mm}$ (menor que el más pequeño de los valores recién mencionados) para la longitud de la primera cavidad resonante de la estructura básica a diseñar; resultando preciso entonces situar en dicha cavidad un elemento de sintonía, nuevamente de sección transversal cuadrada (en esta ocasión con lado igual a $4.000 \mathrm{~mm}$ ), que permita sintonizar la respuesta de la citada estructura de una sola cavidad a $11 \mathrm{GHz}$ y $13 \mathrm{GHz}$ respectivamente. Para determinar las penetraciones de este nuevo elemento de sintonía, debe considerarse una estructura como aquélla mostrada en la figura 3.13; cuya única cavidad resonante corresponde a una guía normalizada WR-75 $(a=19.050 \mathrm{~mm}, b=9.525 \mathrm{~mm})$ de longitud $10.500 \mathrm{~mm}$, acoplada a las guías de entrada y salida (con sección transversal idéntica a la que presenta la cavidad) mediante las ventanas de acoplo inductivas escogidas para la estructura básica (la primera de anchura igual a $8.700 \mathrm{~mm}$ y la segunda con anchura de valor $5.100 \mathrm{~mm}$ ) que incorporan los elementos de sintonía apropiados (ver penetraciones en tabla 3.4) para conseguir en cada caso (filtro a $11 \mathrm{GHz}$ o a $13 \mathrm{GHz}$ ) los niveles de acoplamiento necesarios. Tras centrar pues las correspondientes simulaciones de la respuesta electromagnética (parámetros $S_{21}$ y $S_{11}$ ) de la estructura presentada en la figura 3.13 a $11 \mathrm{GHz}$ y $13 \mathrm{GHz}$, las penetraciones obtenidas en 


\begin{tabular}{|c|c|c|}
\hline \hline \multirow{2}{*}{$\begin{array}{c}\text { Elemento de Sintonía } \\
\text { situado en }\end{array}$} & \multicolumn{2}{|c|}{ Penetración (mm) } \\
\cline { 2 - 3 } & Filtro a 11 GHz & Filtro a 13 GHz \\
\hline $1^{\text {a }}$ cavidad (longitud $\left.=10.500 \mathrm{~mm}\right)$ & 3.229 & 1.825 \\
\hline $2^{\text {a }}$ cavidad (longitud $\left.=13.300 \mathrm{~mm}\right)$ & 2.920 & 0.470 \\
\hline \hline
\end{tabular}

Tabla 3.5: Penetraciones de los elementos de sintonía situados en las cavidades de la estructura básica para centrar su respuesta a $11 \mathrm{GHz}$ y $13 \mathrm{GHz}$ respectivamente.

cada caso para el elemento de sintonía situado en la única cavidad de dicha estructura se muestran en la tabla 3.5; donde, tal y como cabía esperar, la penetración del elemento de sintonía requerida para centrar la citada respuesta a $11 \mathrm{GHz}$ es bastante mayor que aquélla precisada por dicho elemento para posicionar la respuesta a $13 \mathrm{GHz}$, pues las dimensiones escogidas para la estructura básica de filtro paso banda se encuentran bastante próximas a las del diseño centrado a $13 \mathrm{GHz}$ sin emplear elementos de sintonía (recordar dimensiones en la tabla 3.3 mostrada en la página 95 ).

A continuación, para escoger la longitud de la segunda cavidad resonante de la estructura básica paso banda, debe seguirse el mismo proceso descrito anteriormente para obtener la longitud de la primera cavidad; aunque considerando ahora lógicamente la segunda cavidad resonante a diseñar. En esta ocasión, al sintonizar la estructura mostrada en la figura 3.11 (apartado b), correspondiente a la segunda ventana de acoplo de la implementación básica bajo diseño, se necesita que la longitud de la cavidad en la estructura considerada presente un valor igual a $17.860 \mathrm{~mm}$ para centrar la respuesta de esta estructura a $11 \mathrm{GHz}$; mientras que para situar la frecuencia central de la banda de paso de dicha respuesta en $13 \mathrm{GHz}$, es necesario considerar una cavidad en la mencionada estructura cuya longitud presente un valor de $13.427 \mathrm{~mm}$. En consecuencia, la longitud de la segunda cavidad de la estructura básica paso banda se escoge, al quedar restringida por el valor más pequeño de los dos recién presentados, con un valor de $13.300 \mathrm{~mm}$. Ahora bien, con el objeto de centrar la respuesta conjunta de las dos primeras cavidades de la estructura básica a $11 \mathrm{GHz}$ y $13 \mathrm{GHz}$ respectivamente, será necesario colocar en dicha cavidad un nuevo elemento de sintonía con sección transversal cuadrada (de lado otra vez igual a $4.000 \mathrm{~mm}$ ). Las penetraciones requeridas en este nuevo elemento de sintonía, presente en la segunda cavidad de la estructura básica diseñada, se determinan considerando una estructura como la que se muestra en la figura 3.14; constituida por dos cavidades resonantes representadas de nuevo mediante sendas guías normalizadas WR-75 ( $a=19.050 \mathrm{~mm}$, $b=9.525 \mathrm{~mm}$ ) de longitudes $10.500 \mathrm{~mm}$ (primera cavidad) y $13.300 \mathrm{~mm}$ (segunda cavidad), acopladas respectivamente a las guías de entrada y salida (de sección transversal idéntica a la de las dos cavidades) mediante la primera (ventana de acoplo de entrada) y tercera (ventana de acoplo de salida) ventana de acoplo 


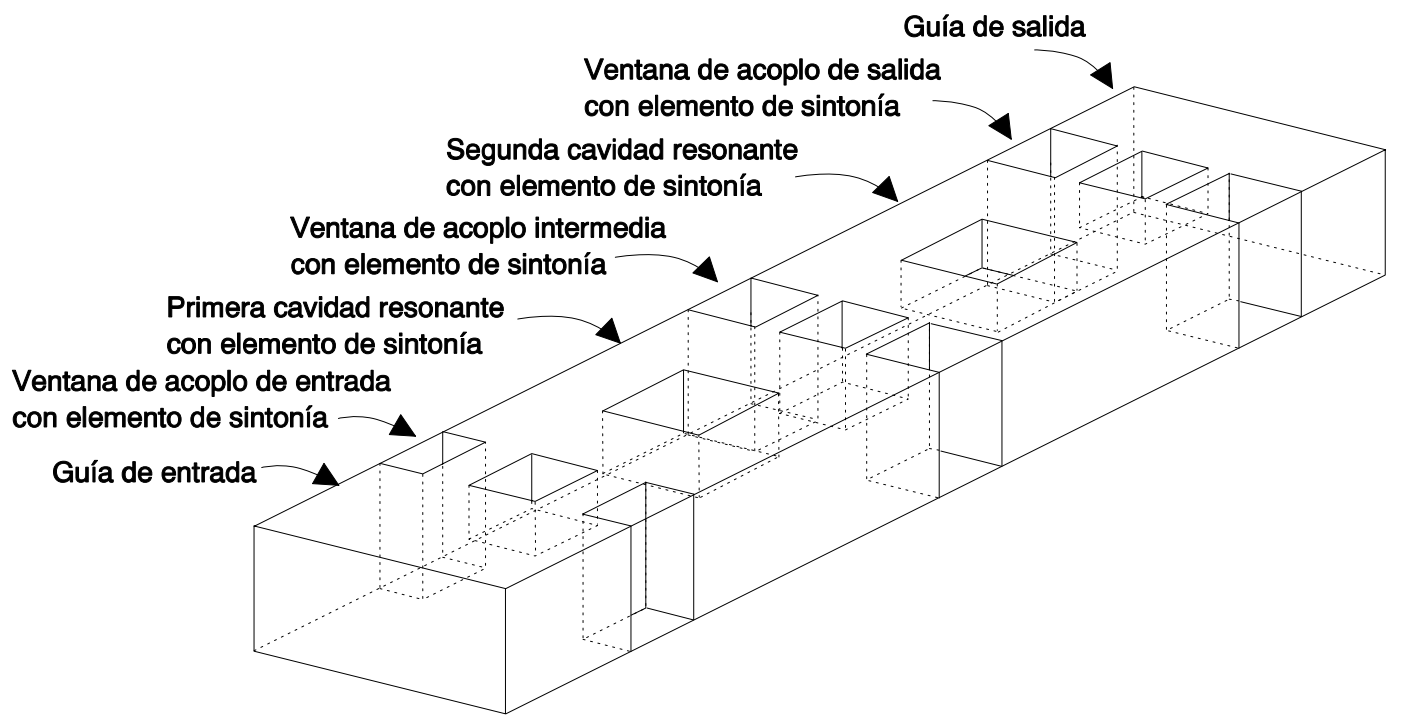

Figura 3.14: Estructura escogida para determinar las penetraciones del elemento de sintonía, a situar en la segunda cavidad de la estructura básica, con el objeto de centrar la respuesta conjunta de las dos primeras cavidades de la estructura básica a $11 \mathrm{GHz}$ y $13 \mathrm{GHz}$ respectivamente.

elegidas para la estructura básica (la primera de anchura igual a $8.700 \mathrm{~mm}$ y la tercera con anchura de valor $5.100 \mathrm{~mm}$ ), y acopladas entre sí (las dos cavidades de la estructura ofrecida en la figura 3.14) mediante una ventana de acoplo intermedia correspondiente a la segunda ventana de acoplo de la estructura básica (de anchura también igual a $5.100 \mathrm{~mm}$ ). En cada una de estas ventanas de acoplo, se incorporan (ver figura 3.14) los elementos de sintonía apropiados (cuyas penetraciones se resumen en la tabla 3.4) para obtener en cada caso (filtro con respuesta paso banda centrada a $11 \mathrm{GHz}$ o a $13 \mathrm{GHz}$ ) los niveles de acoplamiento requeridos por cada uno de estos dos diseños; mientras en la primera cavidad resonante de esta nueva estructura considerada (recordar fig. 3.14), se sitúa el elemento de sintonía seleccionado previamente para dicha cavidad con la penetración adecuada (consultar tabla 3.5) para cada uno de los dos posibles casos analizados (filtro a $11 \mathrm{GHz}$ o a $13 \mathrm{GHz}$ ). Centrando pues las correspondientes simulaciones de la respuesta electromagnética asociada a la estructura recogida en la figura 3.14 a $11 \mathrm{GHz}$ y $13 \mathrm{GHz}$ respectivamente, se obtienen las penetraciones requeridas en cada caso para el elemento de sintonía ubicado en la segunda cavidad de la citada estructura; penetraciones cuyos valores se encuentran resumidos nuevamente en la tabla 3.5 mostrada en la página anterior.

Una vez determinadas las anchuras de las ventanas de acoplo inductivas y las longitudes de las cavidades resonantes, siguiendo para ello los procesos descritos anteriormente, se ha completado el diseño de una estructura básica de filtro paso banda en guía rectangular con ventanas de acoplo inductivas; cuya respuesta 
electromagnética, del tipo Chebyshev y ancho frecuencial igual a $300 \mathrm{MHz}$, puede centrarse a cualquier frecuencia perteneciente a la banda comprendida entre $11 \mathrm{GHz}$ y $13 \mathrm{GHz}$ empleando los correspondientes elementos de sintonía. De manera adicional, con el objeto de comprobar la validez del diseño de la mencionada estructura básica de filtro paso banda, se determinan seguidamente las penetraciones de los elementos de sintonía (cilindros con sección transversal cuadrada), a situar en las ventanas de acoplo inductivas y en las cavidades resonantes de la estructura básica, para conseguir centrar la respuesta paso banda de dicha estructura básica a $11 \mathrm{GHz}$ y $13 \mathrm{GHz}$ preservando su ancho de banda de valor $300 \mathrm{MHz}$; en cuya obtención (valores finales de las penetraciones de los elementos de sintonía) pueden emplearse perfectamente como valores iniciales aquéllos determinados para dichos elementos durante el proceso de diseño de la estructura básica, que se encontrarán próximos a los valores finales requeridos en la estructura completa.

Así pues, para conseguir centrar la respuesta paso banda (tipo Chebyshev) de la estructura básica diseñada a $11 \mathrm{GHz}$ y $13 \mathrm{GHz}$, preservando su ancho de banda de valor igual a $300 \mathrm{MHz}$, se ha seguido el mismo procedimiento empleado anteriormente en el diseño de los dos filtros sin elementos de sintonía. En este método, cuya descripción se encuentra perfectamente detallada en [40], se diseña cavidad tras cavidad (junto con sus correspondientes ventanas de acoplo de entrada y salida) comparando la respuesta electromagnética de la estructura real con la que presenta una red ideal, cuya respuesta global coincide con aquélla deseada, en una configuración terminada de dicha red ideal correspondiente a la cavidad a diseñar en cada etapa (ver detalles en [40]). Al diseñar cada una de las cavidades de la estructura real, para conseguir que su respuesta electromagnética coincida con la asociada a la proporcionada por la configuración correspondiente de la red ideal, deben manejarse un número limitado de parámetros físicos a optimizar, tal y como se indica en [40]; habiendo escogido en este caso las penetraciones de los elementos de sintonía a situar en las ventanas de acoplo y en las cavidades resonantes de la estructura básica diseñada. En el proceso de diseño que se ha seguido, las respuestas electromagnéticas correspondientes a las diferentes estructuras reales consideradas se han simulado utilizando la herramienta de programación DUMAS 3.0 (ver apéndice B); empleando en dichas simulaciones 550 términos para implementar las diferentes series estáticas requeridas (recordar apartado 2.2.1 del capítulo 2), 450 sumandos en las diversas partes estáticas de las series dinámicas a evaluar (recordar apartado 2.2 .2 del capítulo 2), y 90 modos para describir los campos eléctrico y magnético en todas las guías integrantes de cada estructura debido a que las uniones entre dichas guías son bastante abruptas. Estos valores tan elevados necesarios para que las diferentes simulaciones de las estructuras reales sean correctas, y requeridos también en el proceso seguido anteriormente para diseñar la estructura básica de filtro paso banda, precisan de herramientas de programación optimizadas como DUMAS 3.0 con el objeto de 


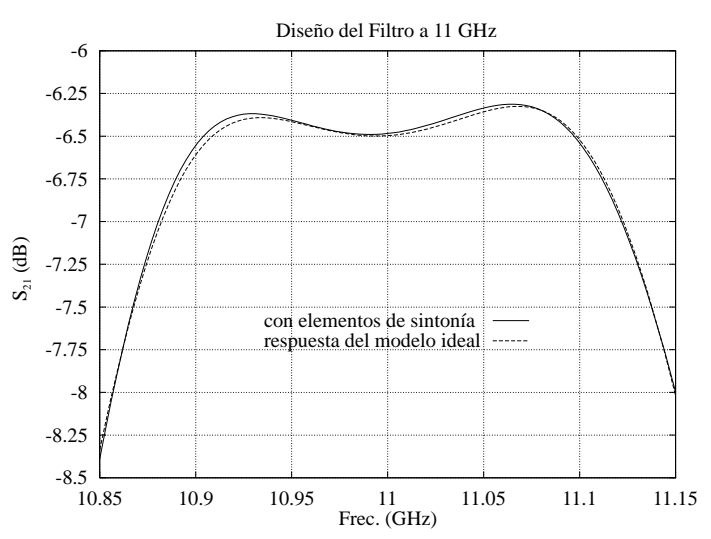

a)

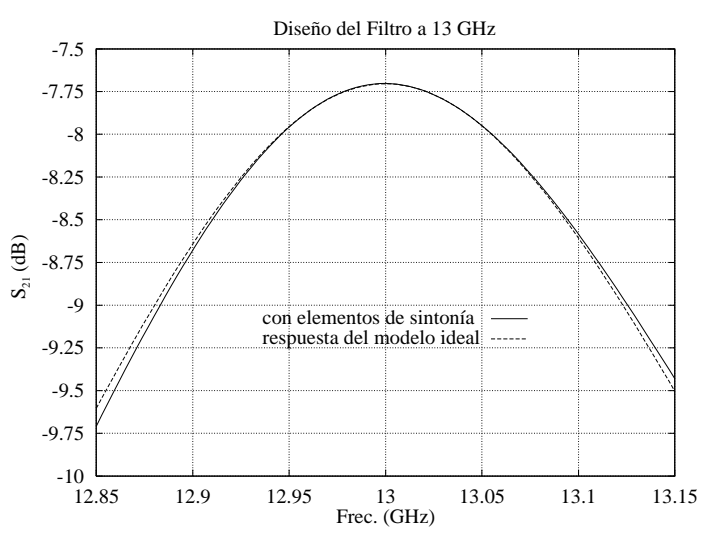

b)

Figura 3.15: Comparación entre las respuestas eléctromagnéticas (parámetros $S_{21}$ ) de la estructura real y de la red ideal tras finalizar el diseño de las dos primeras cavidades de la estructura básica, con elementos de sintonía, para centrar su respuesta paso banda a $11 \mathrm{GHz}$ en a); y misma comparación una vez culminado el proceso de diseño de la primera cavidad de la estructura básica, incluyendo también elementos de sintonía, para centrar su respuesta a $13 \mathrm{GHz}$ en b).

que los diseños puedan implementarse en tiempo real. Asimismo, como ya se ha comentado con anterioridad, las numerosas integrales de acoplamiento entre guías rectangulares y guías reentrantes, requeridas por la herramienta DUMAS 3.0 para analizar el efecto de los elementos de sintonía en la estructura básica diseñada, se han obtenido haciendo uso del mencionado programa ANAPLAN-W; que consta de un código eficiente para obtener todas las integrales necesarias de forma rápida y precisa. Debido a la simetría de las dos implementaciones consideradas (filtros paso banda con respuestas centradas respectivamente a $11 \mathrm{GHz}$ y $13 \mathrm{GHz}$ ), su diseño tan sólo requiere normalmente optimizar las dos primeras cavidades resonantes mediante el procedimiento descrito en [40]; pues una vez optimizado su comportamiento, la otra mitad del filtro se obtiene duplicando simétricamente las dimensiones optimizadas deducidas al diseñar las dos primeras cavidades. A modo de ejemplo, en la figura 3.15 (apartado a) se muestra una comparación entre las respuestas electromagnéticas (parámetros $S_{21}$ ) de la estructura real, incluyendo los elementos de sintonía apropiados, y de la red ideal tras finalizar el proceso de diseño de las dos primeras cavidades de la estructura básica cuya respuesta pretende centrarse a $11 \mathrm{GHz}$. Por otra parte, en dicha figura 3.15 (apartado b) se ofrece una comparación similar a la anterior; pero en este caso una vez concluido el proceso seguido en el diseño de la primera cavidad de la misma estructura básica con respuesta centrada ahora en $13 \mathrm{GHz}$.

Una vez finalizados los procedimientos de diseño seguidos para centrar la respuesta paso banda de la estructura básica considerada a $11 \mathrm{GHz}$ y $13 \mathrm{GHz}$, empleando para ello elementos de sintonía situados en las ventanas de acoplo y 


\begin{tabular}{|c|c|c|}
\hline \multirow{2}{*}{$\begin{array}{l}\text { Elemento de Sintonía } \\
\text { situado en }\end{array}$} & \multicolumn{2}{|c|}{ Penetración (mm) } \\
\hline & Filtro a $11 \mathrm{GHz}$ & Filtro a $13 \mathrm{GHz}$ \\
\hline 1으 ventana (anchura $=8.700 \mathrm{~mm})$ & 3.366 & 0.792 \\
\hline 1. cavidad (longitud $=10.500 \mathrm{~mm}$ ) & 3.298 & $\overline{1.816}$ \\
\hline $2^{\mathrm{a}}$ ventana $($ anchura $=5.100 \mathrm{~mm})$ & 4.078 & 2.141 \\
\hline $2^{\text {a }}$ cavidad (longitud $=13.300 \mathrm{~mm}$ ) & 2.972 & 0.456 \\
\hline $3^{\mathrm{a}}$ ventana (anchura $\left.=5.100 \mathrm{~mm}\right)$ & 3.570 & 0.986 \\
\hline
\end{tabular}

Tabla 3.6: Valores optimizados de las penetraciones de los elementos de sintonía situados en las ventanas de acoplo y en las cavidades de la estructura básica para centrar su respuesta a $11 \mathrm{GHz}$ y $13 \mathrm{GHz}$ respectivamente.

en las cavidades resonantes de la citada estructura básica, los valores optimizados de las penetraciones de dichos elementos pueden encontrase para cada caso (filtro paso banda con respuesta centrada a $11 \mathrm{GHz}$ o a $13 \mathrm{GHz}$ ) en la tabla 3.6; donde tan sólo se ofrecen las penetraciones de los elementos de sintonía situados en la primera mitad de la estructura básica, debido a la simetría física considerada en los diseños realizados. Observando las penetraciones optimizadas que se muestran en la tabla 3.6, se descubre que éstas presentan unos valores muy próximos a aquéllos recogidos en las tablas 3.4 y 3.5, obtenidos durante el diseño de la estructura básica; valores que se han escogido además, de manera acertada, como punto de partida en el proceso de diseño definitivo de los filtros con respuestas centradas respectivamente a $11 \mathrm{GHz}$ y $13 \mathrm{GHz}$. La elección de los mencionados valores para iniciar el diseño de los citados filtros provoca que el esfuerzo requerido para culminar dicha tarea no resulte especialmente costoso; pues los valores de las penetraciones de los elementos de sintonía con los que se inician los respectivos procesos de diseño están muy cercanos a la solución final buscada. Empleando pues las penetraciones optimizadas de los elementos de sintonía recogidas en la tabla 3.6, se han simulado las respuestas electromagnéticas (parámetros $S_{21} \mathrm{y}$ $\left.S_{11}\right)$ de la estructura básica diseñada situando los correspondientes elementos de sintonía en sus ventanas de acoplo y en sus cavidades resonantes; simulaciones que se muestran en la figura 3.16 (apartado a) para el caso en el que la respuesta de la estructura básica se encuentra centrada a $11 \mathrm{GHz}$, y en esa misma figura 3.16 (apartado b) cuando dicha respuesta está centrada a $13 \mathrm{GHz}$. En ambas respuestas, se observa como el ancho de banda requerido (de valor igual a $300 \mathrm{MHz}$ ) se ha conseguido preservar perfectamente a las frecuencias inicial y final (11 GHz y $13 \mathrm{GHz}$ respectivamente) de la banda de trabajo; por lo que se puede concluir que dicha respuesta Chebyshev con ancho de banda de $300 \mathrm{MHz}$, asociada a la estructura básica diseñada, podrá centrarse en cualquier frecuencia de la banda comprendida entre $11 \mathrm{GHz}$ y $13 \mathrm{GHz}$, mediante el empleo de los correspondientes elementos de sintonía con penetraciones adecuadas, preservan- 


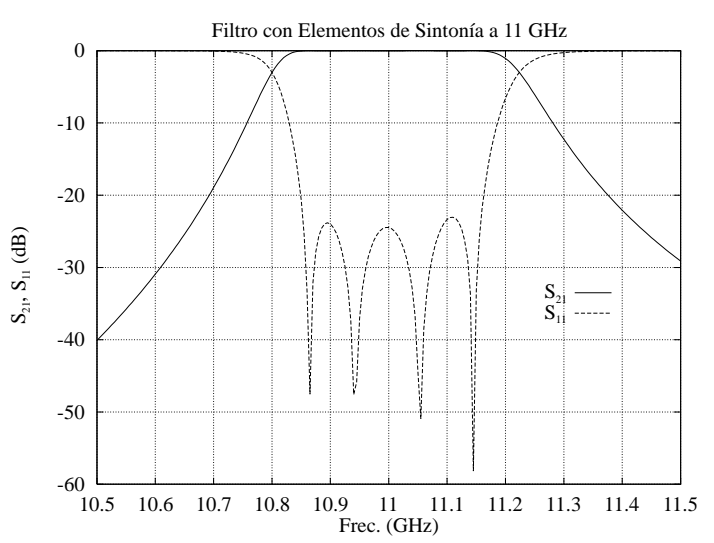

a)

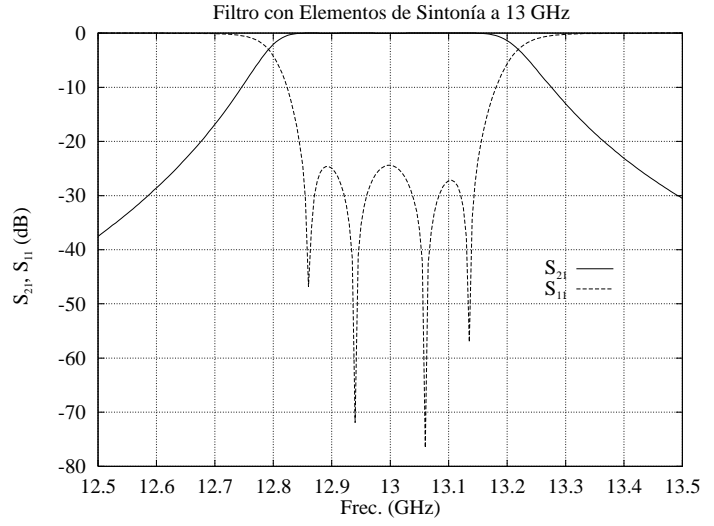

b)

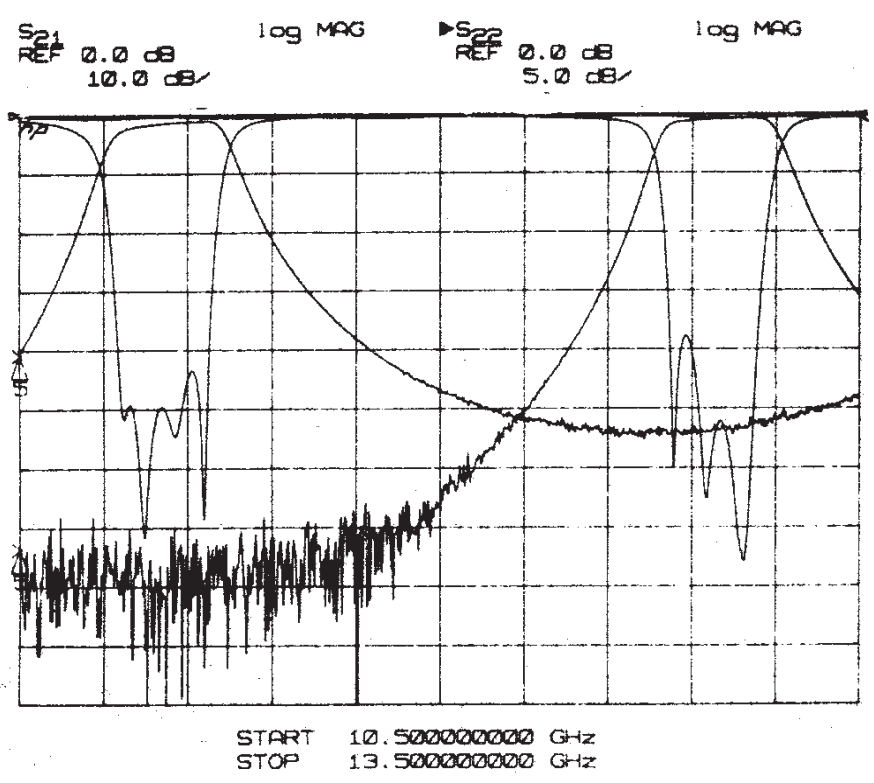

c)

Figura 3.16: Simulación de la respuesta electromagnética (módulo de los parámetros $S_{21}$ y $\left.S_{11}\right)$ que presenta la estructura básica diseñada empleando los elementos de sintonía apropiados para centrar la respuesta de la estructura a $11 \mathrm{GHz}$ en a), simulación de dicha respuesta cuando se utilizan los elementos de sintonía para centrar la respuesta de la estructura a $13 \mathrm{GHz}$ en b), y medidas de las respuestas que ofrece la implementación real de la estructura básica centrada a ambas frecuencias en $\mathbf{c}$ ).

do el citado ancho de banda. Asimismo, en la figura 3.16 (apartados a y b) se comprueba también que las pérdidas de retorno (parámetro $S_{11}$ ) se mantienen siempre por encima de $20 \mathrm{~dB}$ en toda la banda de paso; requisito que conviene observar en el diseño de estos filtros paso banda. Con el objeto de determinar el efecto que supone introducir los elementos de sintonía en la respuesta fuera 


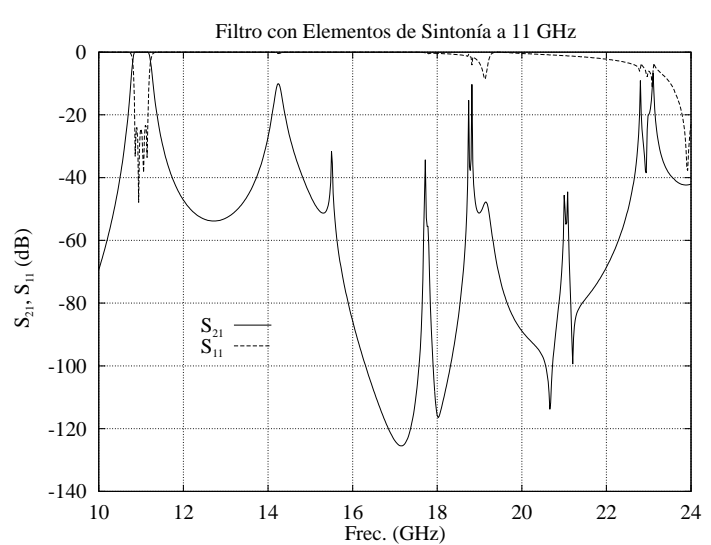

a)

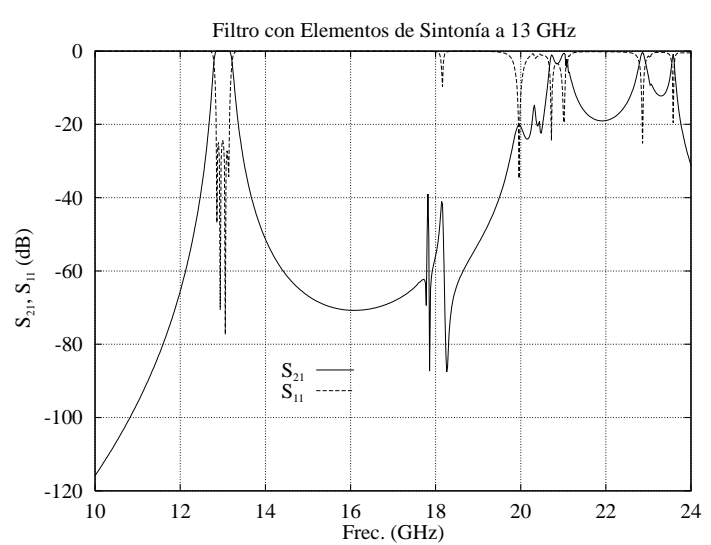

b)

Figura 3.17: Simulación de la respuesta electromagnética en banda y fuera de banda (módulo de los parámetros $S_{21}$ y $S_{11}$ ) que presenta la estructura básica diseñada empleando los elementos de sintonía apropiados para centrar la respuesta paso banda de la estructura a $11 \mathrm{GHz}$ en a), y misma simulación cuando los elementos de sintonía centran la respuesta paso banda de la estructura a $13 \mathrm{GHz}$ en b).

de banda de los dos filtros diseñados, se ha simulado nuevamente la respuesta electromagnética (parámetros $S_{21}$ y $S_{11}$ ) de los dos casos considerados anteriormente (filtro a $11 \mathrm{GHz}$ y $13 \mathrm{GHz}$ ) en una banda de frecuencias mayor que sus respectivas bandas de paso (en concreto entre $10 \mathrm{GHz}$ y $24 \mathrm{GHz}$ ); ofreciendo las dos respuestas obtenidas en la figura 3.17 (apartados a y b respectivamente). En ambos casos, se observa la existencia de ciertas frecuencias armónicas a las que los respectivos filtros permitirían el paso de señal; resultando pues necesario observar dicha circunstancia a priori por si alguna aplicación concreta requiriera de algún filtro paso bajo adicional, situado detrás del filtro paso banda diseñado. Además, conviene observar que la respuesta donde aparecen más nuevas zonas armónicas de paso a frecuencias más bajas corresponde al filtro centrado a $11 \mathrm{GHz}$; aquél en el que los elementos de sintonía, debido a razones de diseño, precisa de mayores penetraciones para centrar su respuesta paso banda a $11 \mathrm{GHz}$.

En el intento de validar la estructura básica diseñada, cuya respuesta paso banda puede centrarse a cualquier frecuencia dentro de la banda comprendida entre $11 \mathrm{GHz}$ y $13 \mathrm{GHz}$ preservando un ancho de banda de $300 \mathrm{MHz}$, se ha construido un modelo básico con las dimensiones obtenidas previamente en el proceso de diseño de la estructura básica. En la figura 3.18 (apartados a y b), se observa respectivamente una vista frontal y una vista de la parte superior de la estructura básica de filtro paso banda implementada; que está constituida, como ya se ha comentado con anterioridad, por ventanas de acoplo inductivas y por cavidades resonantes, cuyas dimensiones respectivas (anchuras y longitudes) corresponden a las mostradas entre paréntesis en la tabla 3.6. Para centrar la respuesta paso 


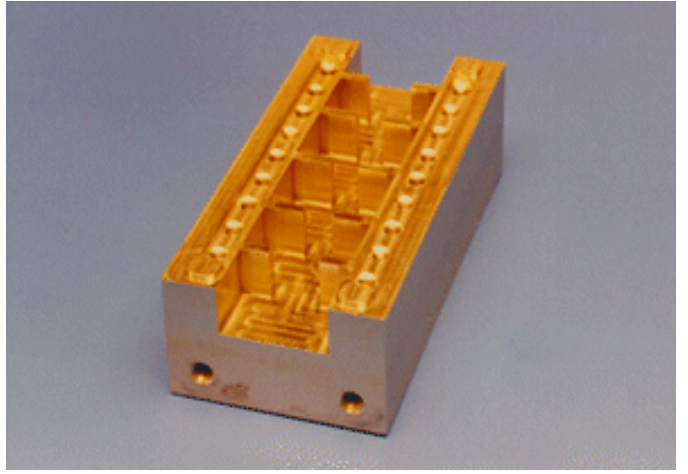

a)

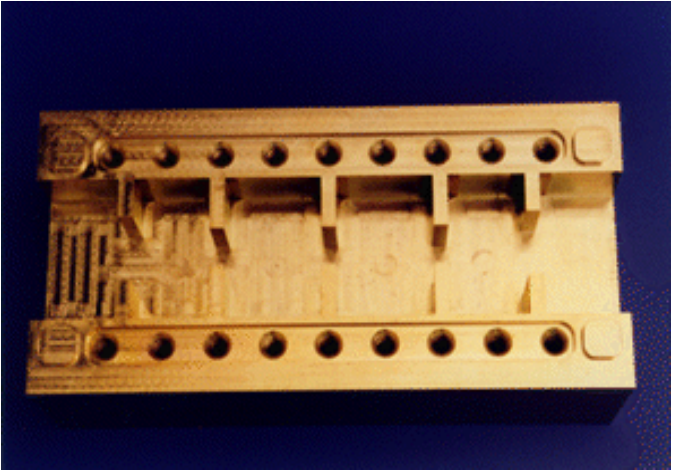

b)

Figura 3.18: Vista frontal de la estructura básica de filtro paso banda con ventanas inductivas en a), y vista de la parte superior de dicha estructura en b).

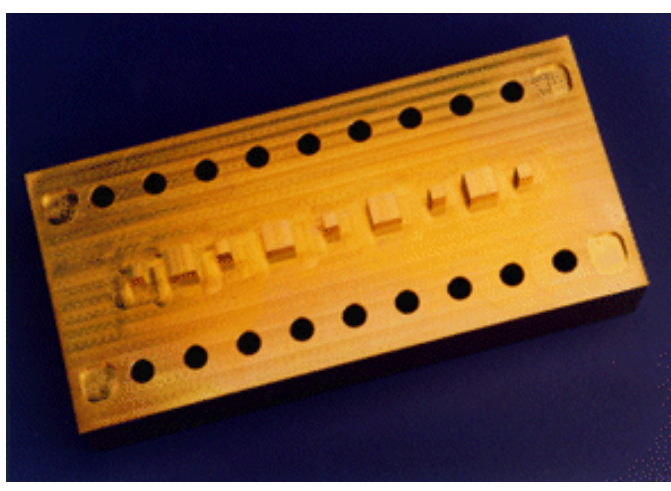

a)

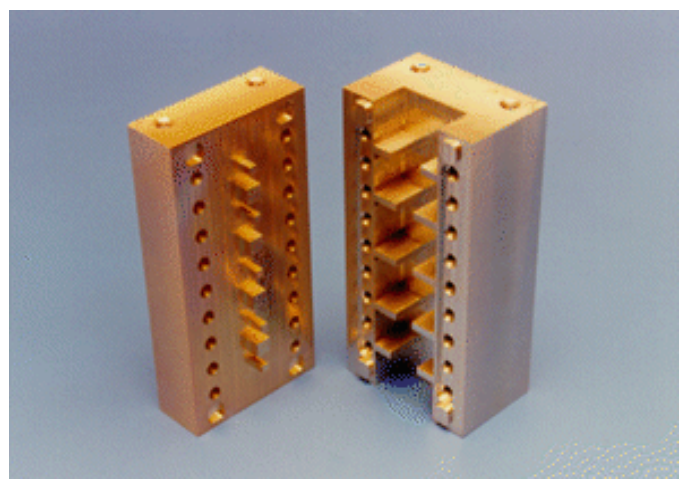

b)

Figura 3.19: Pieza con elementos de sintonía de sección transversal cuadrada (de lado igual a $2.000 \mathrm{~mm}$ o $4.000 \mathrm{~mm}$ ) cuyas penetraciones permiten centrar la respuesta paso banda de la estructura básica a $11 \mathrm{GHz}$ en a), y ensamblaje de esta pieza con la estructura básica para implementar el filtro con respuesta centrada a $11 \mathrm{GHz}$ en b).

banda de la estructura básica diseñada a la frecuencia inicial (11 GHz) y final $(13 \mathrm{GHz})$ de la banda de interés, se han implementado asimismo dos piezas con elementos de sintonía de sección transversal cuadrada (de lado igual a $2.000 \mathrm{~mm}$ o $4.000 \mathrm{~mm}$ según donde se sitúe cada elemento) y penetraciones correspondientes; tal y como puede observarse en la figura 3.19 (apartado a) para la pieza que centra la respuesta paso banda de la estructura básica a $11 \mathrm{GHz}$ y en la figura 3.20 (apartado a) cuando la pieza representa aquélla que centra la respuesta paso banda del modelo básico a $13 \mathrm{GHz}$. Los valores concretos de las penetraciones de los elementos de sintonía que integran dichas piezas se muestran para cada caso (filtro a $11 \mathrm{GHz}$ o a $13 \mathrm{GHz}$ ) en la tabla 3.6. La estructura final, cuya respuesta paso banda se encuentra centrada bien a $11 \mathrm{GHz}$ o bien a $13 \mathrm{GHz}$, se obtiene 


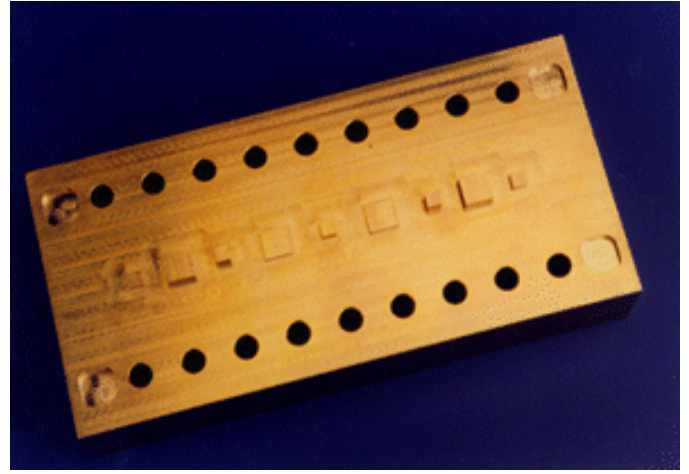

a)

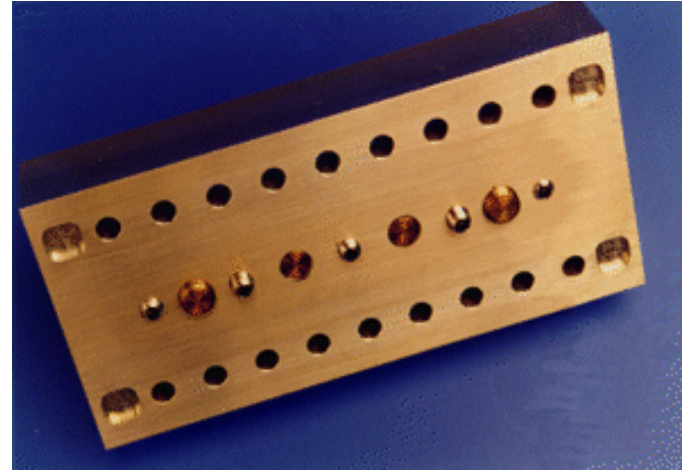

b)

Figura 3.20: Pieza con elementos de sintonía de sección transversal cuadrada (de lado igual a $2.000 \mathrm{~mm}$ o $4.000 \mathrm{~mm}$ ) cuyas penetraciones permiten centrar la respuesta paso banda de la estructura básica a $13 \mathrm{GHz}$ en a), y pieza con tornillos de sintonía de sección transversal circular (con diámetro $\phi=2 \mathrm{mmo} \phi=5 \mathrm{~mm}$ ) cuyas penetraciones pueden ajustarse manualmente para centrar la respuesta paso banda de la estructura básica a cualquier frecuencia comprendida entre $11 \mathrm{GHz}$ y $13 \mathrm{GHz}$ en b).

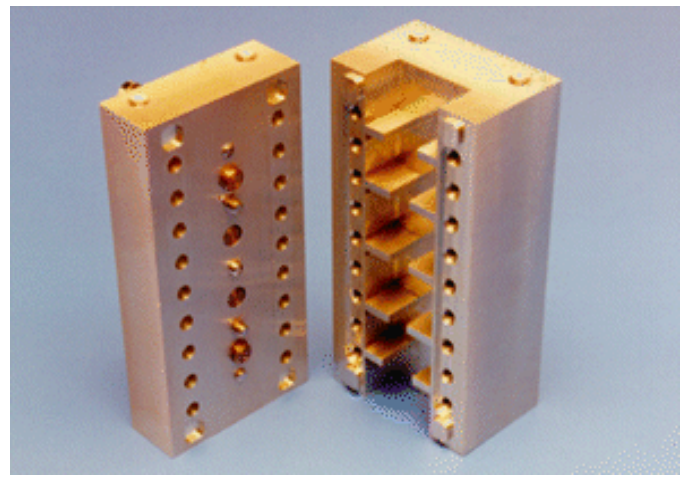

a)

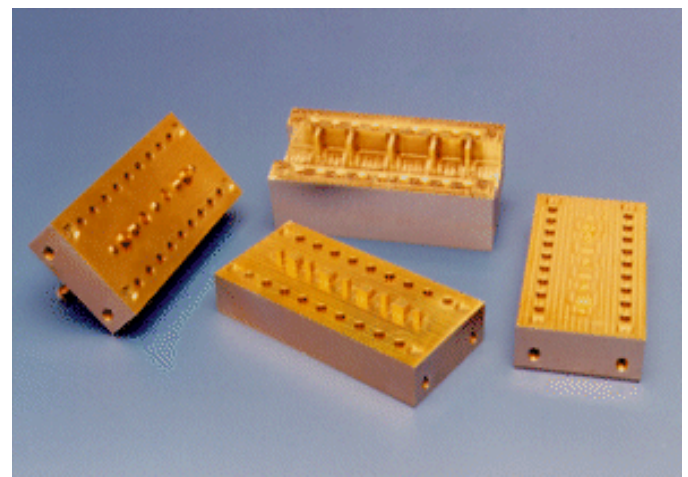

b)

Figura 3.21: Ensamblaje de la pieza que incluye tornillos de sintonía con la estructura básica construida para implementar un filtro paso banda cuya respuesta puede centrarse a cualquier frecuencia comprendida entre $11 \mathrm{GHz}$ y $13 \mathrm{GHz}$ en a), y vista conjunta de todas las piezas construidas en $b$ ).

finalmente ensamblando la estructura básica (ver fig. 3.18) con la pieza superior correspondiente (apartado a en fig. 3.19 o apartado a en fig. 3.20); ensamblaje a realizar como se indica en la figura 3.19 (apartado b).

Por otra parte, se ha construido también una pieza con tornillos de sintonía de sección transversal circular (con diámetro $\phi=2 \mathrm{~mm}$ o $\phi=5 \mathrm{~mm}$ según se sitúe el tornillo en una ventana de acoplo inductiva o en una cavidad resonante); que permita centrar la respuesta paso banda de la estructura básica a cualquier 
frecuencia comprendida entre $11 \mathrm{GHz}$ y $13 \mathrm{GHz}$, mediante el ajuste manual de la penetración de los diferentes tornillos de sintonía en el laboratorio, preservando un ancho de banda de $300 \mathrm{MHz}$. En la figura 3.20 (apartado b), se muestra el aspecto de esta pieza con tornillos de sintonía; cuyas penetraciones corresponden a las obtenidas en el laboratorio para sintonizar la respuesta paso banda de la estructura básica a $13 \mathrm{GHz}$. Comparando las dos imágenes mostradas en la figura 3.20 (apartados a y b), se observa una equivalencia (al menos una relación de aspecto) entre las penetraciones requeridas en los elementos de sintonía con sección transversal circular y cuadrada para centrar la respuesta de la estructura básica a $13 \mathrm{GHz}$; confirmando pues, en cierta manera, que la equivalencia propuesta entre tornillos de sintonía (sección transversal circular) y elementos de sintonía (sección transversal cuadrada) puede utilizarse para diseñar correctamente estructuras básicas de filtros paso banda, que posteriormente se sintonizan empleando tornillos de sintonía reales. En el caso que nos ocupa, la estructura paso banda final incluyendo elementos de sintonía se obtiene, al igual que en el caso anterior donde se utilizaban las piezas con elementos de sintonía de sección transversal cuadrada, ensamblando la estructura básica con la pieza que incorpora los tornillos de sintonía; ensamblaje que se ilustra gráficamente en la figura 3.21 (apartado a). Por último, en la figura 3.21 (apartado b) se ofrece un aspecto global de todas las piezas construidas para validar el procedimiento de diseño propuesto en este apartado; las cuales constituyen una estructura básica de filtro paso banda, común a todos los posibles diseños que pueden implementarse, una pieza con elementos de sintonía (sección transversal cuadrada) para centrar la respuesta de la citada estructura básica a $11 \mathrm{GHz}$, una pieza similar también integrada por elementos de sintonía (sección transversal cuadrada) que centran en esta ocasión la respuesta paso banda de la estructura básica a $13 \mathrm{GHz}$, y finalmente una pieza con tornillos de sintonía (sección transversal circular) que pueden ajustarse manualmente en el laboratorio para centrar la mencionada respuesta paso banda a cualquier frecuencia comprendida entre $11 \mathrm{GHz}$ y $13 \mathrm{GHz}$.

A continuación, con el objeto de corroborar las simulaciones de las respuestas electromagnéticas (parámetros $S_{21}$ y $S_{11}$ ) que presentan los diseños con elementos de sintonía (sección transversal cuadrada) mostradas en la figura 3.16 (apartados a y b), se ha realizado una campaña de medidas de las respuestas que ofrecen las implementaciones prácticas de los mencionados diseños (recordar apartado b en fig. 3.21); medidas que se han efectuado en el Área de Microondas del Laboratorio de Sistemas de Radiofrecuencia que la ESA (Agencia Espacial Europea) tiene en ESTEC (Centro Europeo de Tecnología e Investigación Espacial), empleando para ello un analizador de redes $\mathrm{HP} 8510 \mathrm{C}$ calibrado mediante el procedimiento normalizado TRL completo de dos puertos (en ingés full 2-port). Los resultados obtenidos tras realizar estas medidas (parámetros $S_{21}$ y $S_{11}$ ) se encuentran recogidos, por razones comparativas, en la figura 3.16 (apartado c) mostrada en la página 106; donde, tras comparar dichos resultados con las simulaciones de las 


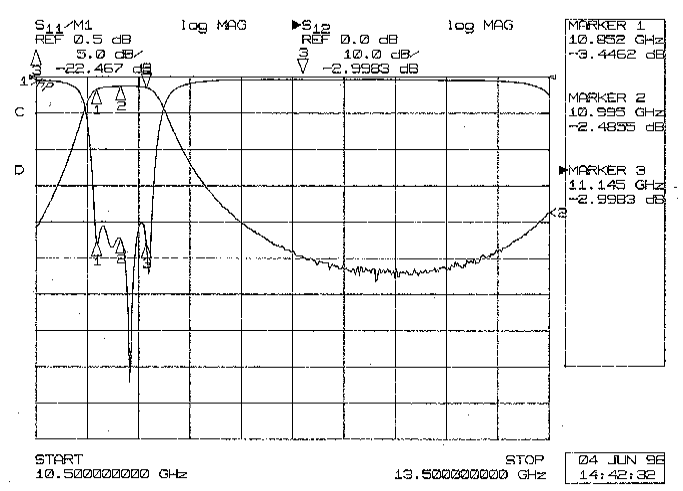

a)

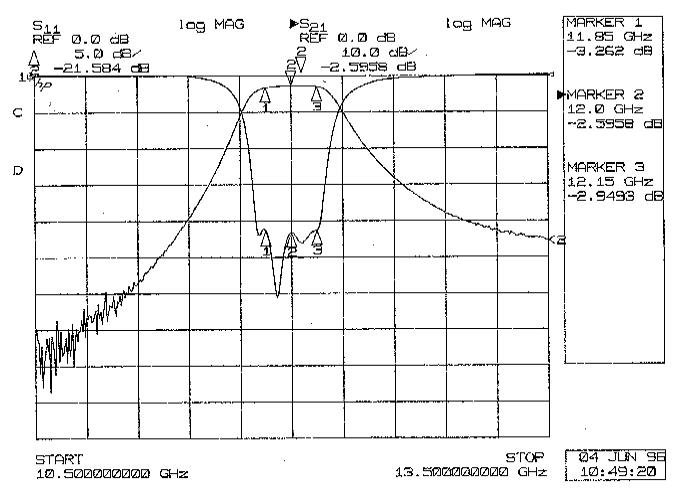

b)

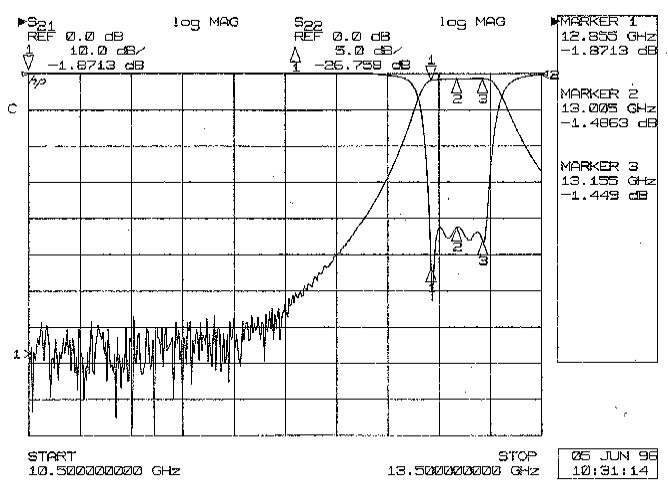

c)

Figura 3.22: Medidas de la respuesta electromagnética (módulo de los parámetros $S_{21}$ y $\left.S_{11}\right)$ que presenta la estructura básica construida empleando tornillos de sintonía para centrar la respuesta a $11 \mathrm{GHz}$ en a), a $12 \mathrm{GHz}$ en b), y a $13 \mathrm{GHz}$ en c).

respuestas de los mencionados filtros (apartados a y b), se concluye que existe un gran parecido entre las medidas de los diseños implementados y las respuestas previstas mediante las simulaciones. No obstante, observando con mayor detalle los resultados ofrecidos en la figura 3.16, se descubre que existe una cierta degradación en las pérdidas de retorno (parámetro $S_{11}$ ) que presenta la respuesta de la estructura básica sintonizada a $13 \mathrm{GHz}$ (comparar apartados b y c en fig. 3.16); debido muy probablemente a las tolerancias mecánicas (por encima de $10 \mu \mathrm{m}$ ) que se han producido en las implementaciones tanto de la estructura básica como de las piezas con elementos de sintonía. Aun así, los resultados obtenidos permiten confirmar en primer lugar la precisión con que la herramienta de programación DUMAS 3.0, en este caso junto con el programa comercial ANAPLAN-W, es capaz de analizar y diseñar (en tiempo real) estructuras complejas de microondas como son los filtros paso banda incluyendo el efecto de los elementos de sintonía; $y$, en sgundo lugar, dichos resultados validan completamente el procedimiento 
propuesto en el presente apartado para diseñar estructuras básicas paso banda, cuya respuesta puede sintonizarse dentro de una determinada banda de interés empleando elementos de sintonía.

Finalmente, con la intención de validar definitivamente las dimensiones obtenidas para la estructura básica de filtro paso banda (recordar implementación en fig. 3.18), se ha empleado la pieza construida con tornillos de sintonía de sección transversal circular (ver apartado b en fig. 3.20) para sintonizar experimentalmente la respuesta paso banda de la estructura básica, de ancho de banda igual a $300 \mathrm{MHz}$, a frecuencias comprendidas entre $11 \mathrm{GHz}$ y $13 \mathrm{GHz}$. Tras finalizar los correspondientes procesos de sintonía de la estructura ensamblada (recordar apartado a en fig. 3.21) en el laboratorio, se han medido unas respuestas electromagnéticas de dicha estructura global con el analizador de redes HP8510C mostradas en la figura 3.22 (apartados a, b y c); donde se observa que la respuesta paso banda de la estructura básica se encuentra centrada respectivamente a $11 \mathrm{GHz}, 12 \mathrm{GHz}$ y $13 \mathrm{GHz}$, tal y como se pretendía durante el proceso de ajuste de los tornillos de sintonía. De esta manera, se confirma que las dimensiones de la estructura básica diseñada, obtenidas siguiendo el procedimiento previamente descrito, son correctas en el sentido que permiten centrar la respuesta paso banda de la estructura básica a cualquier frecuencia perteneciente a la banda comprendida entre $11 \mathrm{GHz}$ y $13 \mathrm{GHz}$; empleando para centrar dicha respuesta tornillos de sintonía en las ventanas de acoplo y en las cavidades resonantes de la citada estructura básica. Asimismo, estos resultados validan plenamente el modelo utilizado para simular los efectos de los tornillos de sintonía en el interior de filtros paso banda; modelo consistente en representar los tornillos mediante secciones uniformes de guías reentrantes.

\subsection{Análisis y Diseño de Filtros Paso Banda de Modo Dual en Guía Circular}

Los filtros paso banda de modo dual se emplean asiduamente en la carga útil de los satélites de comunicaciones; debido principalmente, tal y como se recoge con detalle en [41-44], a la mayor selectividad, menor tamaño y por consiguiente menor masa, que dichos filtros presentan en comparación con aquéllos donde tan sólo se excita un único modo por cada cavidad resonante. En una configuración básica de un filtro paso banda de modo dual, cada una de las cavidades resonantes que integran dicho filtro debe soportar dos modos ortogonales y linealmente independientes excitados de manera simultánea. En tales circunstancias, el número de cavidades resonantes requeridas para implementar un filtro se reduce a la mitad del orden de su correspondiente función de transferencia; lo que reduce considerablemente el tamaño y el peso de la implementación práctica del filtro. Estas configuraciones de modo dual permiten adicionalmente implementar con suma fa- 
cilidad ceros de transmisión en la respuesta del filtro paso banda; facilitando por tanto, como se indica en [41,42], el diseño de filtros de modo dual con respuesta elíptica, cuya selectividad y buen comportamiento de las pérdidas de inserción permiten su empleo en aplicaciones que requieren de filtros y multiplexores de gran calidad.

Debido pues a la importancia que estos filtros de modo dual presentan en aplicaciones espaciales, en la presente sección se ofrecen dos implementaciones alternativas de filtros paso banda de modo dual en guía circular. La primera implementación se basa en la conexión en cascada de tramos uniformes de guías circulares y elípticas; calculando los modos de las guías elípticas mediante un procedimiento preciso y eficiente, basado en reescribir la ecuación de Helmholtz como un problema lineal de autovalores empleando para ello el Método de los Momentos (procedimiento Galerkin). La segunda de las implementaciones propuestas surge como consecuencia de que al construir los filtros de modo dual se incorporan en los diseños tornillos de sintonía; cuyo ajuste manual permite sintonizar la respuesta del filtro, y también compensar las posibles tolerancias producidas en el proceso de fabricación de los mencionados filtros. Esta segunda implementación se plantea para tener en cuenta el efecto de los citados tornillos de sintonía situados en los filtros de modo dual; para lo cual dichos tornillos se representan mediante un tramo uniforme de guía circular reentrante (en inglés ridge), cuyos modos e integrales de acoplamiento con los modos de las correspondientes guías circulares se determinan mediante el procedimiento expuesto en la sección 3.1 (apartado 3.1.1). Por lo que respecta al resto de elementos integrantes de esta segunda configuración alternativa, éstos continúan siendo tramos de guías uniformes circulares y elípticas. En cuanto al análisis y diseño de las dos estructuras propuestas, se ha realizado empleando básicamente la mencionada herramienta de programación DUMAS 3.0; a excepción claro está del cálculo de las integrales de acoplamiento entre los modos de las guías circulares y los modos de las guías reentrantes, en cuya deducción se ha empleado el programa comercial ANAPLAN-W. La utilización de DUMAS 3.0 para simular el comportamiento electromagnético de los filtros de modo dual, al caracterizar todas sus uniones planares empleando la técnica acelerada propuesta en el capítulo 2 (sección 2.1) y solucionar los sistemas en banda mediante el procedimiento recursivo planteado en el capítulo 2 (sección 2.3), garantiza el diseño de las estructuras de modo dual planteadas en tiempo real. Con el objeto de validar las dos estructuras de modo dual propuestas se construyen sendas implementaciones prácticas; que permiten confirmar, tras medir sus respectivas respuestas electromagnéticas, los resultados previstos en la etapa de diseño de los mencionados filtros. Antes de iniciar una descripción exhaustiva de los dos nuevos modelos propuestos de filtros de modo dual, conviene tener conocimiento de los elementos que integran este tipo de filtros, así como de las soluciones utilizadas hasta el momento en la implementación de estas estructuras; para de esta manera poder comprender mejor las novedades 
que introducen las implementaciones propuestas en esta sección. En consecuencia, se ha considerado oportuno ofrecer a continuación una breve, pero bastante completa, descripción de diferentes diseños escogidos para implementar los filtros de modo dual.

\subsubsection{Descripción de Soluciones para Implementar Filtros Paso Banda de Modo Dual}

La solución empleada convencionalmente para implementar filtros de modo dual utiliza iris rectangulares para conseguir los niveles de acoplo de entrada y salida requeridos, iris en forma de cruz para proporcionar niveles de acoplo desiguales entre las parejas de modos duales situados en cavidades adyacentes, y tornillos de sintonía para obtener los acoplos entre los modos duales dentro de cada cavidad. Además, para conseguir que los modos resuenen de manera síncrona (a la misma frecuencia de resonancia en todas las cavidades), se necesitan tornillos de sintonía adicionales que permitan controlar de manera independiente la frecuencia de resonancia asociada a cada modo. De esta manera, se concluye que un filtro paso banda de modo dual precisará de al menos tres tornillos de sintonía en cada cavidad; cuyo ajuste debe realizarse manualmente mediante un proceso de sintonización experimental bastante arduo y costoso. Adicionalmente, la presencia de los tornillos de sintonía en estas implementaciones reduce considerablemente la capacidad de potencia que pueden manejar los filtros de modo dual.

En primer lugar, se considerará el problema asociado al diseño preciso de los iris rectangulares e iris en forma de cruz; que proporcionan según se termina de explicar los acoplos de entrada y salida, así como los acoplos entre cavidades, requeridos en un filtro de modo dual. Con el objeto de que la respuesta del filtro de modo dual satisfaga los requerimientos impuestos, los valores de las dimensiones de los dos tipos de iris recién mencionados deben determinarse con una gran precisión. Los valores óptimos de las dimensiones de los iris de acoplo de entrada y salida pueden obtenerse usando un método propuesto en [32]; que se basa en la teoría de la difracción producida por pequeños orificios propuesta en [45], y en trabajos experimentales cuyos resultados se encuentran recogidos en [46-48]. Ahora bien, las fórmulas analíticas asociadas a este método proporcionan valores aproximados cuando las dimensiones de los iris resultan comparables al valor de la longitud de onda; debido principalmente a la introducción en el mencionado método de un coeficiente empírico que tiene en cuenta el grosor del iris. Aunque este problema, para el caso de guías circulares, se ha estudiado y se han ofrecido asimismo posibles soluciones en $[49,50]$; todavía no ha conseguido resolverse sin embargo para los iris rectangulares. Para el diseño riguroso de estos iris rectangulares, puede emplearse una técnica clásica de análisis de uniones planares denominada adaptación modal (en inglés mode matching), descrita con detalles por ejemplo en [5]; aunque por razones de eficiencia computacional, se recomien- 
da el uso de una técnica novedosa basada en la matriz de admitancias, propuesta inicialmente en [8], y posteriormente optimizada tal y como se ha descrito en el capítulo 2 (sección 2.2) o como se indica en [18]. En cuanto al diseño de los iris de acoplo entre cavidades adyacentes, que presentan forma de cruz, tradicionalmente ha resultado más complejo que el de los iris recién considerados. Una posible solución a este problema se presenta en [51]; donde se proponen implementaciones canónicas y longitudinales de filtros de modo dual, constituidas por cavidades cilíndricas dieléctricas, que no requieren la presencia de iris de acoplo entre las citadas cavidades. Concretamente, en las implementaciones canónicas propuestas en [51] los iris de acoplo vienen representados por simples iris circulares, o bien por tramos de guías circulares uniformes insertados entre los resonadores dieléctricos; proporcionando ambos tipos de iris el mismo nivel de acoplo para las dos parejas de modos duales situados en las cavidades adyacentes. Sin embargo, las implementaciones longitudinales requieren de acoplos desiguales para las parejas; por lo que en las soluciones propuestas en [51] se utilizan tornillos de sintonía para conseguir dichos niveles de acoplo diferentes. Ahora bien, estas últimas soluciones no resultan muy óptimas; pues sustituyen los iris de acoplo en forma de cruz, cuyo análisis resulta complejo, por unos tornillos que requieren de un ajuste manual bastante costoso de implementar. Por otra parte, la cantidad de acoplo que puede conseguirse empleando los mencionados tornillos resulta limitada; limitación puesta de manifiesto en [43], donde se propone utilizar un tramo uniforme de guía evanescente (ningún modo excitado) para conseguir los niveles de acoplo que necesitan las parejas de modos duales situados en cavidades adyacentes. Empleando este tipo de guías evanescentes, es posible conseguir los niveles de acoplo desiguales precisados para cada pareja sin utilizar los tradicionales iris en forma de cruz; habiendo analizado en [43] estas nuevas estructuras de modo dual, que incorporan guías evanescentes, mediante la técnica clásica de adaptación modal. Finalmente, en [52] se realiza un análisis riguroso de los iris en forma de cruz situados en guías rectangulares; análisis basado en la obtención de una matriz de dispersión generalizada, calculada mediante adaptación modal, que caracteriza la unión planar entre guía rectangular y guía en forma de cruz. La combinación de esta nueva unión planar con aquélla entre guías rectangulares, resuelta también mediante adaptación modal en [5], permite un análisis eficiente y preciso cuando los iris en forma de cruz se sitúan de forma arbitraria entre las cavidades adyacentes, cuando tienen secciones transversales diferentes en plano $\mathrm{E}$ y plano H, y cuando su grosor presenta cualquier valor. No obstante, el método de adaptación modal recién descrito, que emplea matrices de dispersión generalizadas, presenta varios inconvenientes de gran importancia; como por ejemplo el fenómeno de la convergencia relativa, mencionado en el capítulo 2 (apartado 2.2.4) y descrito detalladamente en [6,7], y el coste computacional que supone su implementación. Debido a estas razones, en [53] se propone un método variacional, basado en una expansión modal, para determinar con precisión los valores 
requeridos en las dimensiones de los iris de acoplo de entrada y salida y de acoplo entre cavidades adyacentes. Esta técnica, en comparación con la mencionada previamente, requiere de un menor esfuerzo computacional debido a que utiliza el concepto de los modos accesibles y localizados (ver su concepto en apéndice B); determinando, tras realizar las correspondientes pruebas de convergencia, cuántos modos de un tipo y del otro conviene utilizar en cada momento, lo que conduce a resultados precisos en tiempos de computación relativamente bajos. Como se desprende de esta breve revisión histórica, para implementar los iris de acoplo de entrada y salida, así como los iris entre parejas de modos duales situados en cavidades adyacentes, se han escogido opciones de diseño muy diferentes; y, asimismo, las técnicas de análisis electromagnético utilizadas para diseñar dichos iris han sido también múltiples y variadas.

Intentando ahora resolver el problema asociado a la obtención del acoplo requerido dentro de cada cavidad por los modos duales, así como el ajuste de sus respectivas frecuencias de resonancia, surge la necesidad de caracterizar completamente el comportamiento de los tornillos de sintonía empleados para conseguir dichos requerimientos; lo cual debe servir además para reducir de manera considerable el importante esfuerzo experimental dedicado tradicionalmente al ajuste de los mencionados tornillos. Aunque la caracterización de la respuesta de los tornillos es factible en un principio, empleando por ejemplo el método de los elementos finitos, la implementación práctica de este método requiere un esfuerzo computacional tan importante, debido básicamente a la complicada geometría de la estructura real con tornillos, que hace inviable el empleo de dicha técnica para diseñar en tiempo real los filtros de modo dual. Recientemente, con el objeto de reducir el esfuerzo computacional requerido por el análisis riguroso de los tornillos de sintonía empleados en los filtros de modo dual, se han propuesto diferentes soluciones alternativas; las cuales básicamente sustituyen los tornillos de sintonía por tramos de guías uniformes cuyas secciones transversales están perturbadas, para de esta forma conseguir los mismos efectos que producen los tornillos situados dentro de cada cavidad. Así por ejemplo, en [54] se propone sustituir los tornillos de sintonía por un tramo uniforme de guía circular reentrante, que presenta tres inserciones metálicas situadas en las mismas posiciones que ocupaban los tornillos de sintonía; en cuyo análisis se utiliza nuevamente el citado método de los elementos finitos, aunque en este caso la geometría de la estructura a caracterizar resulta más simple al no emplear tornillos de sintonía. Los resultados ofrecidos en [54] confirman que esta nueva estructura permite resolver el problema asociado al diseño de filtros de modo dual con tornillos de sintonía; pues el efecto de dichos tornillos puede recuperarse perfectamente empleando la mencionada guía circular reentrante. Posteriormente, las diversas soluciones propuestas para el diseño de filtros de modo dual, que siguen basadas en la filosofía de conseguir el efecto introducido por los tornillos de sintonía perturbando las secciones transversales de guías uniformes, no se preocupan de disponer de un 
control independiente de la frecuencia de resonancia asociada a cada uno de los modos duales; por lo que dichas soluciones tan sólo se centran en la sustitución adecuada del tornillo que implementa el acoplo requerido entre los modos duales de cada cavidad, no considerando en ninguno de estos diseños la existencia de tornillos que permitan ajustar las frecuencias de resonancia. De esta manera, en $[55,56]$ se propone conseguir el mencionado acoplo entre modos duales empleando uno o varios postes metálicos con bordes redondeados situados en direcciones oblicuas (guías circulares reentrantes con una sola inserción metálica); o bien mediante la distribución asimétrica de los iris rectangulares, también con esquinas redondeadas, empleados para obtener los acoplos de entrada y salida necesarios. En todas las estructuras de modo dual propuestas en $[55,56]$, basadas en las soluciones recién comentadas para conseguir el acoplo modal dentro de cada cavidad, los niveles de acoplo de entrada y salida precisados se han conseguido mediante iris rectangulares, siempre con esquinas redondeadas, y rotados $90^{\circ}$ uno respecto del otro; habiendo empleado en el análisis de la respuesta electromagnética de los citados filtros de modo dual una técnica híbrida que combina adaptación modal y elementos finitos, que tampoco resulta muy eficiente desde el punto de vista computacional. Por otra parte, en [57] el acoplo entre modos duales situados dentro de cavidades con sección transversal cuadrada se consigue suprimiendo una de sus esquinas; sustituyendo pues el tornillo tradicionalmente utilizado para conseguir el citado acoplo, y eliminando por tanto los esfuerzos requeridos en la sintonía de dicho tornillo. Asimismo, se comprueba que con la nueva estructura propuesta es posible obtener mayores niveles del mencionado acoplo que empleando tornillos de sintonía; y al suprimir los citados tornillos de los filtros de modo dual, se consigue que dichos filtros puedan manejar niveles más elevados de potencia. El método seguido en [57] para determinar el espectro modal de la nueva guía considerada, en la que se ha eliminado una de las esquinas de su sección transversal cuadrada original, se basa en resolver una ecuación matricial característica que se obtiene aplicando adaptación modal; mientras el análisis de las uniones planares entre dichas guías y las correspondientes guías rectangulares de las estructuras de modo dual, se resuelve empleando nuevamente la técnica de adaptación modal para calcular en este caso las matrices de dispersión generalizadas asociadas a cada una de las citadas uniones. Haciendo uso del nuevo concepto recién descrito para conseguir acoplar los modos duales dentro de cada cavidad, en [58,59] se ofrecen varios diseños de filtros de modo dual; en los que para obtener los acoplos necesarios para las parejas de modos duales situados en cavidades adyacentes, se utiliza la solución mencionada en el párrafo anterior consistente en el empleo de un tramo uniforme de guía evanescente. Tanto en [58] como en [59], las estructuras propuestas se analizan empleando las correspondientes matrices de dispersión generalizadas, cuya deducción se realiza mediante la conocida técnica de adaptación modal; ahora bien, mientras en [58] la guía de sección transversal cuadrada con una esquina suprimida se analiza empleando un procedimiento de 
resonancia transversal, en [59] dicha guía continúa caracterizándose mediante la resolución de una ecuación matricial característica. Aunque está comprobado que estas dos técnicas proporcionan resultados igualmente precisos, ambas requieren de un importante esfuerzo computacional en la obtención del espectro modal de la guías con sección transversal no normalizada; lo cual, unido al coste temporal que supone analizar la estructura completa empleando matrices de dispersión generalizadas, eleva considerablemente el tiempo total requerido para diseñar las diversas estructuras propuestas mediante las técnicas mencionadas.

Para finalizar, se describen dos nuevas geometrías alternativas de filtros de modo dual, propuestas muy recientemente, que pretenden eliminar en las implementaciones de dichos filtros cualquier tramo de guía con sección transversal perturbada; pues de esta forma, el análisis y diseño de los mencionados filtros puede realizarse en tiempos óptimos empleando métodos tradicionales (adaptación modal), sin necesidad de tener que acudir a métodos numéricos como los mencionados en el párrafo anterior que requieren de grandes esfuerzos computacionales. La primera de estas nuevas implementaciones, recogida en [60], propone un filtro de modo dual en guía circular de tan sólo 2 polos; en el cual, el acoplo entre los dos modos de la cavidad se consigue rotando el iris de acoplo de salida y la guía rectangular de salida un cierto ángulo respecto de la cavidad de la estructura, no siendo necesario incluir en la cavidad circular ningún tornillo de sintonía. En cuanto a los acoplos de entrada y salida precisados por el filtro de modo dual, se consiguen ajustando los grosores de los respectivos iris rectangulares de entrada y salida (rotado). Debido a la sencillez de esta nueva posible configuración, todas las guías integrantes presentan simples secciones transversales rectangulares o circulares, el diseño optimizado de dicha estructura se ha realizado en [60] empleando únicamente adaptación modal; sin haber requerido en dicho diseño el empleo de ningún método numérico, que resultan especialmente costosos de implementar desde el punto de vista computacional. La segunda configuración novedosa, propuesta en [61,62], corresponde a un filtro de modo dual implementado en guía rectangular; en el cual, nuevamente, no se altera en ningún momento la sección transversal de las cavidades resonantes. La filosofía de este nuevo diseño, basada en el concepto propuesto en [57] para conseguir el acoplo entre modos duales de una cavidad cortando una esquina de la misma, consiste en obtener el pretendido acoplo entre modos dentro de cada cavidad deformando su sección transversal cuadrada en una nueva sección ligeramente rectangular; pues de esta forma se consigue acoplar los dos modos diagonales de la cavidad cuya sección transversal es originalmente cuadrada. Como consecuencia de pretender acoplar los modos diagonales de las cavidades, las guías de entrada y salida del filtro deben estar rotadas $45^{\circ}$ respecto de la orientación de las cavidades, así como cada uno de los iris gruesos rectangulares empleados para conseguir respectivamente los acoplos precisados entre las diversas cavidades adyacentes. En dichas estructuras, los iris de acoplo de entrada y salida se implementan mediante una 
serie de discontinuidades capacitivas; que resultan necesarias para compensar desajustes de fase provocados al conseguir en el filtro los acoplos modales requeridos entre cavidades adyacentes. Con este nuevo tipo de estructuras de modo dual, resulta especialmente sencillo introducir en la respuesta de dichos filtros ceros de transmisión (respuesta elíptica); para lo cual, en el caso de un filtro de modo dual de 4 polos, tan sólo se requiere modificar adecuadamente las dimensiones del iris rectangular situado entre las dos cavidades adyacentes, y rotar la segunda cavidad $90^{\circ}$ respecto de la primera. La principal ventaja de esta segunda configuración alternativa radica nuevamente en su sencillez; ya que todas las guías integrantes presentan sección transversal rectangular, y no ha sido necesario incorporar ningún tornillo para obtener los acoplos necesarios. Así pues, en el procedimiento seguido tanto en [61] como en [62] para diseñar el tipo de filtro de modo dual recién descrito, se ha empleado tan sólo la técnica clásica de adaptación modal; que, debido a la simplicidad de la estructura considerada, no debe haber requerido de un importante esfuerzo computacional. A pesar de que estas dos últimas configuraciones de modo dual propuestas resultan atractivas por su sencillez, y en consecuencia por lo relativamente simple que resulta su análisis, conviene destacar que ninguna de ellas (al igual que gran parte de las estructuras descritas en el párrafo anterior) dispone de elementos en los diseños que permitan ajustar de manera independiente la frecuencia de resonancia asociada a cada uno de los modos duales; elementos no sólo interesantes por facilitar este ajuste frecuencial independiente para cada modo, sino también por permitir sintonizar de forma precisa la respuesta paso banda del filtro de modo dual en aplicaciones de banda estrecha, y por servir para compensar a posteriori las tolerancias que se producen en el proceso de construcción del filtro.

Frente a las diversas opciones existentes para implementar los filtros de modo dual, recogidas la mayoría de ellas en la revisión previa, en los dos próximos apartados se proponen dos nuevas configuraciones de filtros de modo dual implementados en guía circular; que resultan especialmente interesantes por diferentes razones. El primer diseño novedoso propuesto se basa en la conexión en cascada de tramos uniformes de guías circulares y guías elípticas; donde las guías circulares representan las cavidades resonantes de los filtros, y las guías elípticas se utilizan para implementar los iris de acoplo de entrada y salida, los iris de acoplo entre cavidades adyacentes, los iris de acoplo entre modos duales situados dentro de cada cavidad, y los iris que permiten el ajuste independiente de la frecuencia de resonancia asociada a cada modo. Hasta el momento, las guías elípticas no se han utilizado en filtros de modo dual en guía circular; debido básicamente a lo complejas que resultan las expresiones correspondientes a los modos de dichas guías elípticas, basadas en funciones de Mathieu [15]. En el diseño propuesto en el apartado 3.2.2, el análisis modal de las guías elípticas se realiza de manera rápida y eficaz sin emplear las mencionadas funciones de Mathieu; lo que permite un análisis eficiente y preciso de la estructura considerada. Este primer diseño 
de filtros de modo dual resulta bastante factible de implementar con la precisión requerida; ya que los iris elípticos pueden construirse fácilmente empleando máquinas fresadoras de gran precisión, o mediante electroerosión, eliminando pues la necesidad de utilizar tornillos de sintonía para ajustar a posteriori la respuesta del filtro. En cuanto a la segunda nueva configuración de filtro de modo dual en guía circular, nuevamente se emplean los iris elípticos para obtener los acoplos de entrada y salida precisados y el acoplo necesario entre cavidades adyacentes; sin embargo, la novedad de este segundo diseño radica en que el acoplo entre modos dentro de cada cavidad, así como el ajuste independiente de la frecuencia asociada a cada modo, se realiza mediante una guía circular reentrante constituida por tres inserciones metálicas con forma de cono cada una de ellas. Esta guía circular reentrante, similar a la empleada en la solución propuesta en [54], pretende simular también el efecto de los tornillos de sintonía reales que normalmente se incorporan en las implementaciones prácticas de los filtros de modo dual; aunque en la caracterización de este tipo de guías reentrantes necesaria para diseñar el filtro propuesto en el apartado 3.2.3, a diferencia del método de los elementos finitos empleado en [54], se utiliza la técnica eficiente descrita en la sección 3.1 (apartado 3.1.1). Esta segunda configuración de filtros de modo dual, al incorporar las mencionadas guías reentrantes, permite predecir de manera bastante precisa el efecto que supondría emplear en dichos filtros tornillos de sintonía reales en lugar de las inserciones cónicas consideradas; lo que puede utilizarse para iniciar el proceso de sintonía experimental de los tornillos reales con las penetraciones de las inserciones cónicas deducidas en las simulaciones, reduciendo pues de alguna manera el importante esfuerzo requerido en el proceso de ajuste manual de los tornillos. Por último, antes de iniciar la descripción de cada una de las nuevas implementaciones propuestas, conviene reseñar que el proceso de diseño de ambas estructuras se ha realizado utilizando el programa DUMAS 3.0; que, como es bien sabido, emplea técnicas muy eficientes al caracterizar dispositivos basadas en la caracterización de uniones planares mediante matrices de admitancias generalizadas, lo que sin duda ha permitido el diseño en tiempo real de las estructuras que se ofrecen seguidamente.

\subsubsection{Filtros Paso Banda de Modo dual en Guía Circular con Iris Elípticos}

En el presente apartado, se propone una nueva contribución en el tema de los filtros de modo dual en guía circular; pues se describe la configuración de un filtro paso banda de este tipo integrado por dos cavidades (4 polos), simétrico y con 2 ceros de transmisión (respuesta elíptica), en el que los diferentes niveles de acoplo requeridos por el filtro se obtienen empleando iris con sección transversal eliptica. Con el objeto de confirmar el comportamiento electromagnético previsto para esta estructura, se construye una implementación práctica de la misma; cuya 


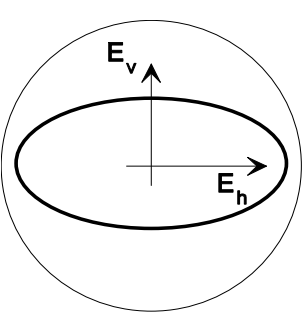

a)

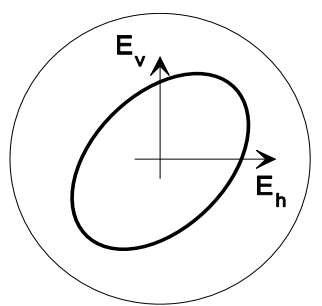

b)

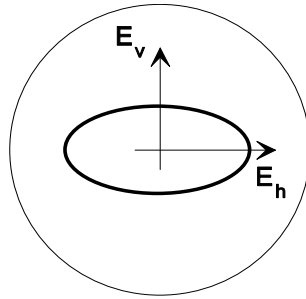

c)

Figura 3.23: Configuración de iris con sección transversal elíptica para implementar el ajuste de la frecuencia de resonacia asociada a cada modo en a), para obtener el nivel de acoplo entre modos duales dentro de cada cavidad en b), y para conseguir el acoplo entre cavidades adyacentes en $c$ ).

respuesta, una vez medida en el laboratorio, permite corroborar el funcionamiento del diseño presentado como filtro de modo dual en guía circular.

Para comprender mejor el funcionamiento de la nueva estructura propuesta de filtro de modo dual en guía circular, concretamente como se consiguen los diferentes niveles de acoplo precisados en este tipo de filtros mediante iris con sección transversal elíptica, en la figura 3.23 se muestran diversos iris elípticos con grosor insertados en guías circulares. Considerando por ejemplo el caso en el que uno de los ejes de la sección transversal elíptica del iris esté alineado con el campo eléctrico incidente que procede de la guía circular (ver apartado a en fig. 3.23), se constata que no se produce ningún tipo de acoplo entre los dos modos de la guía circular polarizados ortogonalmente; modos que en la figura 3.23 se designan mediante los símbolos $\mathrm{E}_{\mathrm{v}}$ y $\mathrm{E}_{\mathrm{h}}$. Sin embargo, para esta primera configuración del iris (apartado a en fig. 3.23), como consecuencia de la diferencia existente entre las dos dimensiones (mayor y menor) de la sección transversal elíptica del iris, los dos modos ortogonales $\left(\mathrm{E}_{\mathrm{v}} \mathrm{y} \mathrm{E}_{\mathrm{h}}\right)$ presentarán diferentes fases tras atravesar el mencionado iris; efecto que permite utilizar este tipo de iris (con la configuración descrita) para implementar el ajuste frecuencial independiente de cada modo, ajuste que debe realizarse dentro de cada cavidad del filtro de modo dual. Por contra, si el iris elíptico está rotado $45^{\circ}$ respecto del campo eléctrico incidente, tal y como se muestra en la figura 3.23 (apartado b), los dos modos incidentes con polarizaciones ortogonales $\left(\mathrm{E}_{\mathbf{v}}\right.$ y $\mathrm{E}_{\mathbf{h}}$ ) intercambian energía entre ellos mientran atraviesan el iris elíptico rotado, aunque a la salida de dicho iris presentan la misma fase; comportamiento que sugiere la posible utilización del mencionado iris rotado $45^{\circ}$ (ver apartado b en fig. 3.23) para obtener el acoplo requerido entre modos duales dentro de cada cavidad. Asimismo, el acoplo que necesita cada pareja de modos duales situados en cavidades adyacentes, implementado tradicionalmente mediante un iris en forma de cruz, puede obtenerse empleando un iris elíptico cuya configuración se recoge en la figura 3.23 (apartado c); en el que las dimensiones de los dos ejes de su sección transversal elíptica deben 


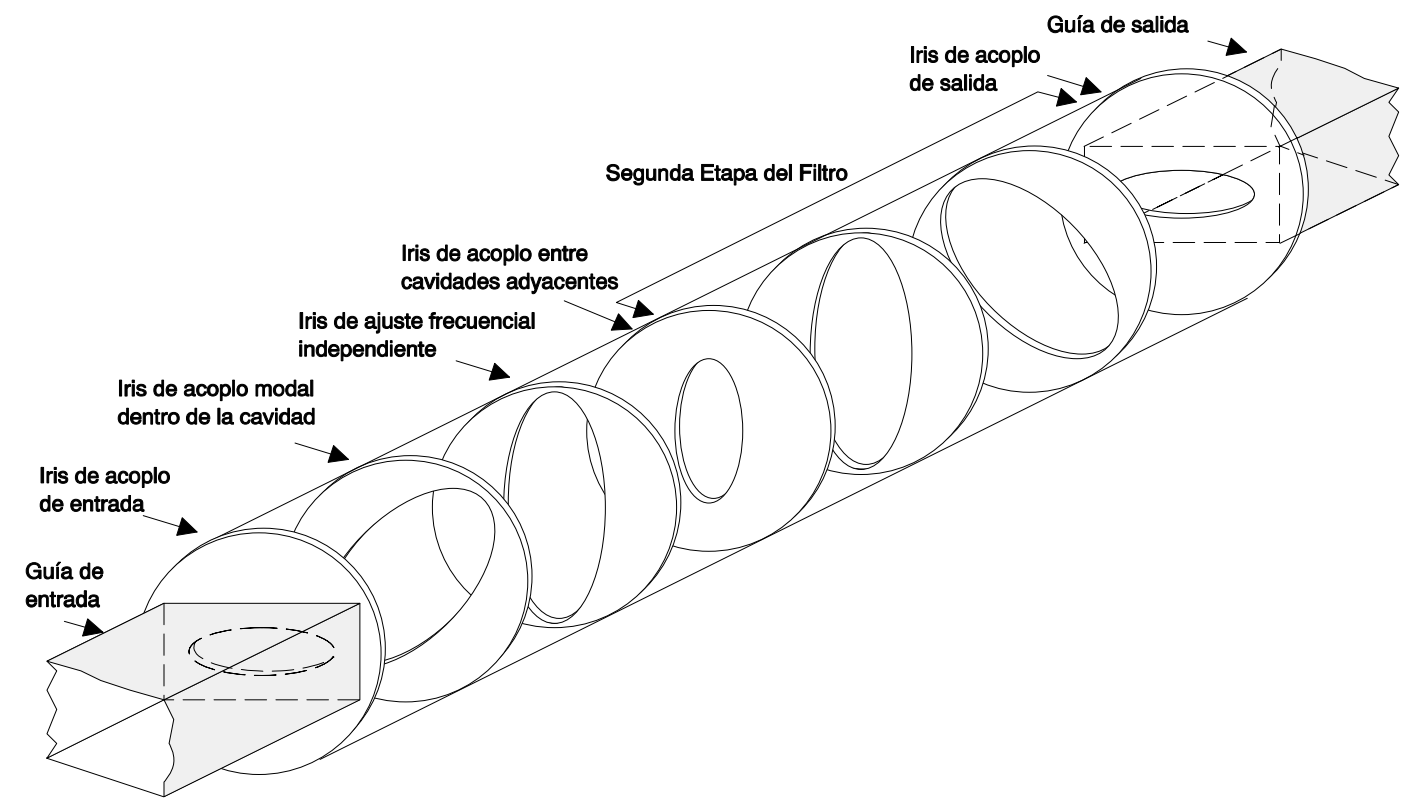

Figura 3.24: Configuración de un filtro de modo dual en guía circular de 4 polos, simétrico y con respuesta paso banda elíptica (2 ceros de transmisión), que emplea iris elípticos para conseguir los diferentes tipos de acoplo requeridos.

determinarse para conseguir respectivamente el nivel de acoplo precisado por cada pareja de modos duales. Por último, reseñar que los acoplos de entrada y salida que todo filtro de modo dual precisa, obtenidos de manera clásica empleando iris rectangulares, pueden conseguirse también mediante la utilización de iris elípticos orientados de igual manera que en la figura 3.23 (apartados a y c); tal y como se ha implementado en el diseño propuesto en este apartado.

Así pues, la estructura propuesta para implementar un filtro de modo dual en guía circular de 4 polos, simétrico y con respuesta paso banda elíptica (2 ceros de transmisión), se ofrece en la figura 3.24; estructura en la que tan sólo existen dos cavidades resonantes circulares, como corresponde a un filtro de modo dual de 4 polos, y en la que los diferentes tipos de acoplo requeridos por dicho filtro se obtienen mediante iris elípticos. En la figura 3.24, se observa que dentro de cada cavidad existen dos iris elípticos; uno de ellos (rotado) para acoplar los dos modos duales de la cavidad, y el otro (sin rotar) que permite ajustar de manera independiente la frecuencia de resonancia de cada uno de los citados modos duales. Los acoplos de entrada y salida entre las respectivas guías rectangulares y las cavidades, así como el acoplo entre cavidades adyacentes, se implementa nuevamente mediante tramos de guía elíptica con las dimensiones correspondientes; tal y como puede comprobarse en la figura 3.24. En cuanto a la simetría de la configuración propuesta, ésta hace referencia a que las dimensiones y la orientación de los iris elípticos presentan idénticos valores en las dos mitades 
en las que puede dividirse el filtro; a excepción claro está de la rotación $\left(45^{\circ}\right.$ y $-45^{\circ}$ ) de los iris elípticos de acoplo modal situados dentro de cada cavidad. Las orientaciones de estos dos iris, desplazadas $90^{\circ}$ una respecto de la otra, se escogen de esta manera para implementar una realimentación negativa entre las dos cavidades circulares adyacentes; que resulta necesaria, tal y como se indica por ejemplo en [43] y [57], para que la respuesta paso banda del filtro de modo dual sea elíptica ( 2 ceros de transmisión). El principal problema asociado al análisis y diseño del filtro de modo dual mostrado en la figura 3.24 consiste en la obtención de los modos de las diferentes guías elípticas que integran dicha estructura, y en la determinación de los valores que presentan las integrales de acoplamiento entre dichos modos y los modos de las correspondientes guías circulares o rectangulares; modos e integrales cuyo conocimiento resulta necesario para poder caracterizar, mediante matrices de admitancias generalizadas, las diferentes uniones planares entre guías elípticas y guías circulares o rectangulares que integran el filtro propuesto. Los modos de las guías elípticas se han descrito tradicionalmente (ver [15]) empleando funciones de Mathieu; cuya utilización en el cálculo de las mencionadas integrales de acoplamiento no resulta conveniente, pues debido a su complejidad las implementaciones computacionales que manejan dichas funciones resultan muy poco eficientes. Debido a esta razón, en el presente diseño se han obtenido los modos de las guías elípticas, así como las correspondientes integrales, utilizando un nuevo procedimiento muy eficiente descrito con detalle en [63]; que no utiliza en ningún momento las mencionadas funciones de Mathieu. De manera resumida, este nuevo procedimiento se basa en transformar la ecuación de Helmholtz, escrita en coordenadas elípticas, en un simple problema algebraico de autovalores; para lo cual se utiliza el conocido Método de los Momentos (procedimiento Galerkin), empleando como funciones base y funciones peso simples funciones trigonométricas que satisfacen en coordenadas elípticas las condiciones de contorno apropiadas. La solución del problema de autovalores que se obtiene puede implementarse fácilmente, de manera eficaz y generando resultados muy precisos, mediante la utilización de paquetes de programación especialmente diseñados para resolver problemas algebraicos lineales. Todo este procedimiento descrito para obtener el espectro modal de las guías elípticas, así como las integrales de acoplamiento entre los modos de dichas guías y los modos de guías circulares y rectangulares, se encuentra incorporado a la herramienta de programación DUMAS 3.0 (ver apéndice B); que hará uso del mismo para caracterizar las correspondientes uniones planares del filtro mostrado en la figura 3.24 en las que intervienen guías elípticas.

En el diseño del filtro de modo dual en guía circular mostrado en la figura 3.24, cuya respuesta paso banda con ancho de aproximadamente $200 \mathrm{MHz}$ pretende centrarse en torno a una frecuencia próxima a $11 \mathrm{GHz}$, se ha empleado un procedimiento que divide el diseño de la estructura global en sucesivas etapas con objetivos muy concretos; utilizando para conseguir dichos objetivos un 


\begin{tabular}{|l|c|c|c|}
\hline \hline \multirow{2}{*}{$\begin{array}{c}\text { Iris Elíptico } \\
\text { empleado para }\end{array}$} & \multicolumn{3}{c|}{ Dimensiones (mm) } \\
\cline { 2 - 4 } & Eje Mayor & Eje Menor & Grosor \\
\hline acoplo entrada/salida & 12.780 & 4.000 & 1.730 \\
\hline acoplo entre modos duales & 24.000 & 21.000 & 0.500 \\
\hline ajuste frecuencial & 24.000 & 20.414 & 0.600 \\
\hline acoplo entre cavidades & 8.700 & 4.000 & 1.515 \\
\hline \hline
\end{tabular}

Tabla 3.7: Valores optimizados de las dimensiones de los iris elípticos del filtro de modo dual mostrado en la figura 3.24 para centrar su respuesta con ancho de banda de $200 \mathrm{MHz}$ en torno a $11 \mathrm{GHz}$.

número limitado, y relativamente pequeño, de parámetros físicos a optimizar en cada etapa del proceso de diseño. En la estructura que se pretende diseñar, se han escogido como guías de entrada y salida sendos tramos uniformes de guía normalizada WR-75 $(a=19.050 \mathrm{~mm}, b=9.525 \mathrm{~mm})$, y como cavidades resonantes guías circulares de diámetro $\phi=24.000 \mathrm{~mm}$; mientras las dimensiones de todos los iris elípticos (dimensiones del eje mayor y del eje menor de la sección transversal elíptica y grosor del iris), así como las longitudes de los diferentes tramos de guías circulares en los que se divide cada cavidad (ver fig. 3.24), se han elegido como los parámetros físicos cuyos valores deben optimizarse en el proceso de diseño del filtro de modo dual mostrado en la figura 3.24. Durante el proceso de optimización de los mencionados parámetros físicos, siguiendo el procedimiento descrito en [40] que optimiza sucesivamente cavidad tras cavidad (junto con los correspondientes iris elípticos), se determinan en principio los valores necesarios en dichos parámetros para obtener la respuesta deseada del filtro considerando únicamente un solo modo dual (concretamente $\mathrm{E}_{\mathrm{v}}$ ) en cada cavidad; por lo que en esta etapa inicial no se consideran los acoplos requeridos entre los dos modos duales de cada cavidad. Posteriormente, considerando estos acoplos entre modos duales requeridos para obtener la respuesta final deseada del filtro de modo dual, se optimizan los valores obtenidos anteriormente para los parámetros físicos seleccionados; obteniendo pues de esta forma las dimensiones óptimas de la estructura considerada (recordar fig. 3.24). Tras implementar el proceso de diseño recién descrito, siempre con el objeto de obtener la respuesta paso banda deseada (ancho de banda de $200 \mathrm{MHz}$ centrada en torno a $11 \mathrm{GHz}$ ) para el filtro de modo dual propuesto, se deduce que todos los tramos de guía circular en los que se divide cada una de las dos cavidades resonantes deben presentar la misma longitud de valor $5.499 \mathrm{~mm}$; mientras los valores optimizados de las dimensiones de los diferentes iris elípticos se encuentran resumidos en la tabla 3.7, donde sólo se ofrecen las dimensiones de los iris situados en una cavidad al haber diseñado una estructura simétrica. Al diseñar el filtro de modo dual considerado, ha sido necesario realizar numerosas simulaciones de las respuestas electromagnéticas 


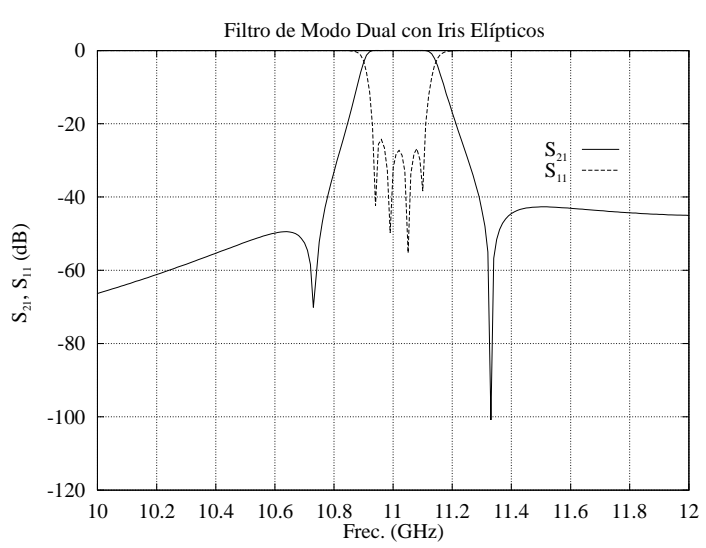

a)

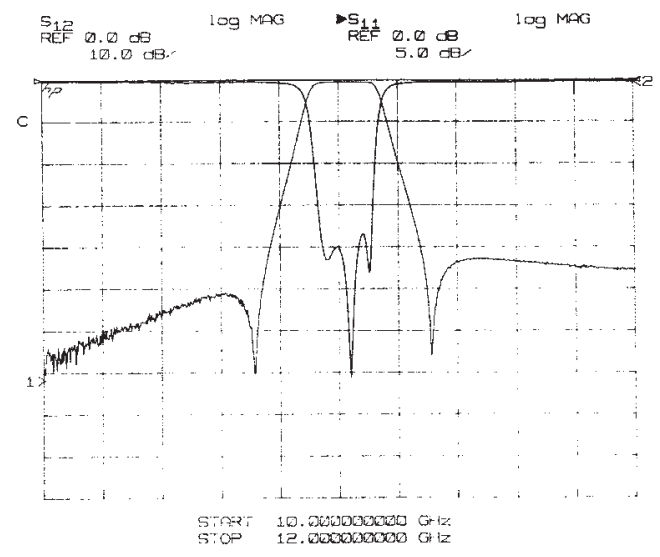

b)

Figura 3.25: Simulación de la respuesta electromagnética (módulo de los parámetros $S_{21}$ y $\left.S_{11}\right)$ que presenta el filtro de modo dual propuesto en la figura 3.24, diseñado para que su respuesta esté centrada en torno a $11 \mathrm{GHz}$ con un ancho de banda de $200 \mathrm{MHz}$, en a); y medidas de la respuesta que ofrece la implementación de dicho filtro en b).

correspondientes a diferentes estructuras intermedias; habiendo realizado todas estas simulaciones con el programa DUMAS 3.0 que, tal y como se ha comentado anteriormente, permite analizar estructuras con guías elípticas. En las mencionadas simulaciones se han utilizado 550 términos para determinar los valores de todas las series estáticas necesarias (recordar apartado 2.2.1 del capítulo 2), 450 términos al evaluar las diversas partes estáticas de las series dinámicas requeridas (recordar apartado 2.2.2 del capítulo 2), 110 modos en la descripción de los campos eléctrico y magnético de cada una de las guías que integran la estructura considerada, y aproximadamente unas 400 funciones base para aplicar el Método de los Momentos (procedimiento Galerkin) en la deducción de los modos de cada guía elíptica del filtro; valores todos ellos muy elevados que se requieren para obtener resultados convergentes y precisos al determinar las respuestas de las mencionadas estructuras, y que son causados por la enorme complejidad que supone su análisis. Lógicamente, el análisis y diseño de una estructura de filtro de modo dual como la propuesta, con los requerimientos tan elevados que precisa su análisis, no se habría podido realizar en tiempo real en el caso de no disponer de un programa como DUMAS 3.0; especialmente optimizado y preparado para diseñar estructuras de microondas complejas. Empleando pues los valores obtenidos en el proceso de diseño para las diferentes dimensiones escogidas como parámetros físicos a optimizar, así como los mismos valores utilizados en la etapa de diseño para los parámetros del programa DUMAS 3.0, se simula la respuesta electromagnética (parámetros $S_{21}$ y $S_{11}$ ) que presenta el filtro de modo dual con iris elípticos diseñado; respuesta ofrecida en la figura 3.25 (apartado a), donde 


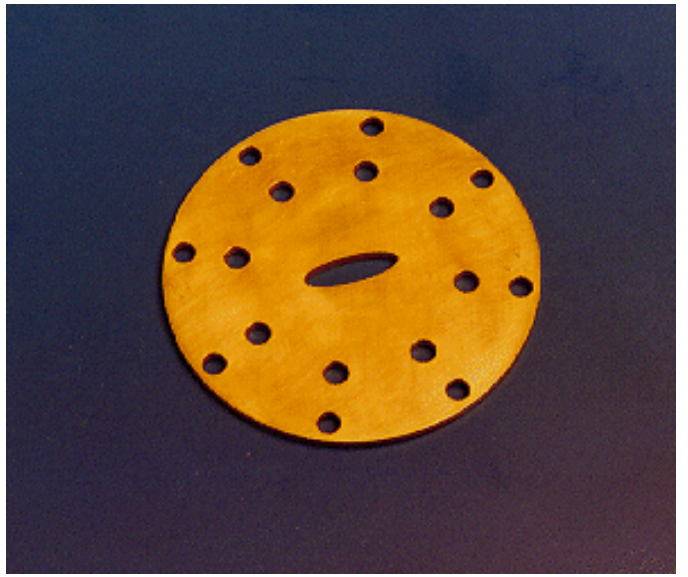

a)

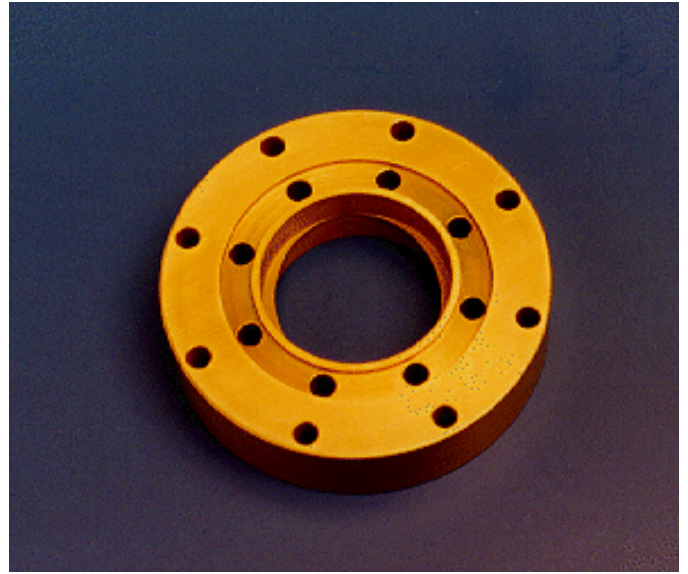

b)

Figura 3.26: Iris elíptico utilizado para conseguir implementar los acoplos de entrada y salida en a), e iris elíptico escogido para obtener el acoplo entre modos duales situados dentro de cada cavidad en b).

es posible observar como la banda de paso está centrada en una frecuencia muy próxima a $11 \mathrm{GHz}$ con un ancho frecuencial de aproximadamente $200 \mathrm{MHz}$. En la figura 3.25 (apartado a), se comprueba también la existencia de 4 polos en las pérdidas de retorno (parámetro $S_{11}$ ) habiendo empleado en el diseño tan sólo dos cavidades resonantes circulares, así como la presencia en la respuesta correspondiente al parámetro $S_{21}$ de 2 ceros de transmisión; tal y como corresponde al comportamiento de un filtro de modo dual en guía circular de 4 polos y respuesta elíptica. Asimismo, en dicha figura se descubre que la condición de igual nivel para todos los picos de las pérdidas de retorno (parámetro $S_{11}$ ) en la banda de paso está un poco degradada, debido a los efectos de dispersión que tienen lugar en las cavidades e iris del filtro; aunque los niveles de todos estos picos se mantienen siempre por encima de $20 \mathrm{~dB}$ en toda la banda de paso, condición que conviene satisfacer en este tipo de filtros.

Con el objeto de validar la estructura propuesta de filtro de modo dual en guía circular, así como el proceso de diseño seguido para centrar la respuesta paso banda de dicho filtro en torno a $11 \mathrm{GHz}$ con una ancho de banda igual a $200 \mathrm{MHz}$, se ha implementado una estructura real con las dimensiones correspondientes al filtro diseñado previamente. El iris elíptico que se ha utilizado para conseguir implementar los acoplos de entrada y salida requeridos se ofrece en la figura 3.26 (apartado a), cuya orientación en el filtro debe permanecer paralela al eje horizontal de cualquier sección transversal del filtro. En cuanto a los iris elípticos empleados para acoplar los dos modos duales en cada cavidad, ambos presentan un aspecto idéntico mostrado en la figura 3.26 (apartado b); aunque el iris situado en la primera cavidad debe orientarse formando $45^{\circ}$ respecto del eje 


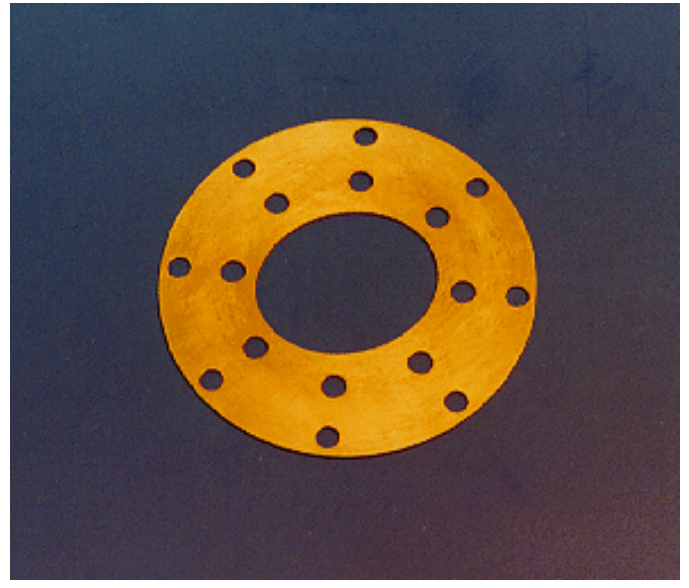

a)

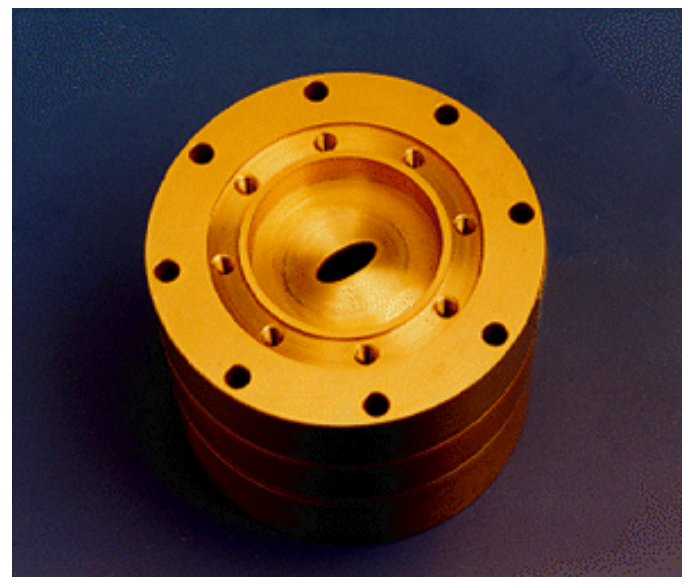

b)

Figura 3.27: Iris elíptico que permite realizar en cada cavidad el ajuste frecuencial independiente de cada modo dual en a), e iris elíptico elegido para conseguir el acoplo requerido entre cavidades adyacentes en $b$ ).

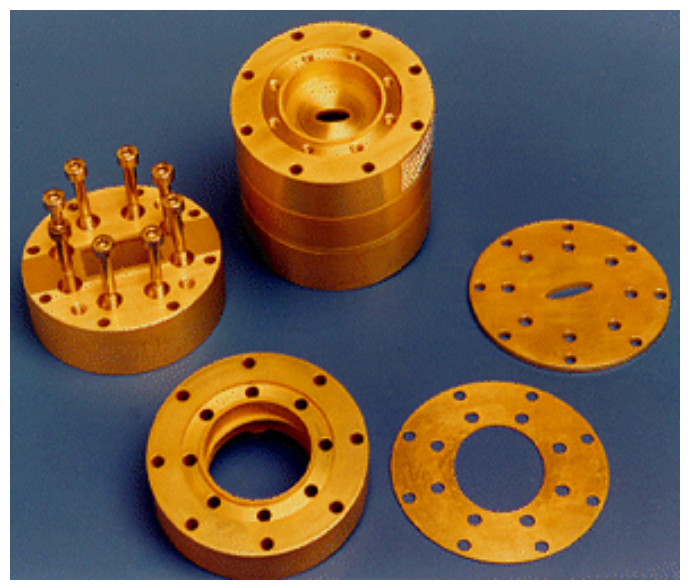

a)

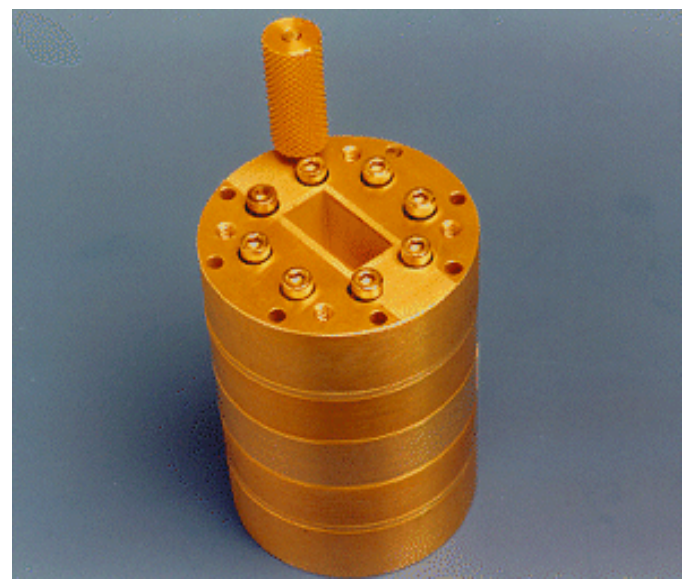

b)

Figura 3.28: Vista conjunta de todas las piezas construidas para implementar el filtro de modo dual diseñado en a), y aspecto final de dicho filtro una vez se ensamblan todas las piezas que lo integran en $b$ ).

vertical de cualquier sección transversal, y consecuentemente la orientación del iris ubicado en la segunda cavidad debe ser de $-45^{\circ}$ respecto del mencionado eje vertical. El ajuste de la frecuencia de resonancia de cada modo dual se consigue en cada cavidad mediante el iris elíptico recogido en la figura 3.27 (apartado a); que en este caso debe situarse en las dos cavidades alineado con el citado eje vertical, es decir rotado $90^{\circ}$ respecto del eje horizontal. Por último, en la figura 3.27 (apartado b) puede observarse el aspecto del iris elíptico que se emplea 
para obtener el acoplo entre cavidades adyacentes precisado; iris que debe orientarse nuevamente en la misma dirección del mencionado eje vertical. Todos estos iris elípticos construidos, cuyas dimensiones (eje mayor y menor de la sección transversal elíptica y grosor del iris) se encuentran resumidas en la tabla 3.7, se insertan entre tramos uniformes de guía circular con diámetro $\phi=24.000 \mathrm{~mm}$ y longitudes determinadas también anteriormente; a excepción de los iris de acoplo de entrada/salida, que se encuentran situados entre las guías de entrada/salida y uno de estos tramos de guía circular mencionados. Una vista conjunta de todos los iris recién descritos, así como de los diversos elementos que integran el filtro de modo dual implementado, puede encontrarse en la figura 3.28 (apartado a); mientras en la misma figura 3.28 (apartado b) se muestra el aspecto final que presenta el filtro construido, una vez que todos los iris y elementos integrantes del mismo se encuentran ensamblados de forma adecuada. La longitud total de este filtro de modo dual en guía circular de 4 polos, cuya vista en perspectiva se ofrece en la mencionada figura 3.28 (apartado b), presenta un valor aproximadamente igual a $40 \mathrm{~mm}$; longitud bastante reducida para un filtro con las características del que se ha diseñado, especialmente si dichas características se pretenden conseguir con un filtro en el que se excita un solo modo en cada cavidad.

Una vez construido el filtro de modo dual en guía circular diseñado, para validar las simulaciones de la respuesta de dicho dispositivo obtenidas con el programa DUMAS 3.0, se procede a medir la respuesta electromagnética (parámetros $S_{21}$ y $S_{11}$ ) de dicha estructura; cuyo aspecto global, tras ensamblar todas sus piezas, se muestra en la figura 3.28 (apartado b). Las medidas se han efectuado de nuevo en ESTEC (Centro Europeo de Tecnología e Investigación Espacial) que pertenece a la ESA (Agencia Espacial Europea), concretamente en el Área de Microondas del Laboratorio de Sistemas de Radiofrecuencia situado en el mencionado centro; habiendo empleado en la realización de las citadas medidas un analizador de redes HP8510C, calibrado previamente según el procedimiento normalizado TRL completo de dos puertos (en ingés full 2-port). Los resultados correspondientes a la medida realizada se encuentran recogidos, para poder compararlos con la simulación de la respuesta del dispositivo, en la figura 3.25 (apartado b) mostrada en la página 125; observando en dicha figura (apartados a y b) que los resultados obtenidos midiendo el dispositivo se asemejan bastante a la respuesta simulada del mismo. No obstante, en la medida ofrecida en la figura 3.25 (apartado b) es posible descubrir una cierta degradación en las pérdidas de retorno (parámetro $\left.S_{11}\right)$; consistente en la desaparición de uno de los 4 polos que debería presentar el mencionado parámetro $S_{11}$ del filtro construido, y en un incremento del valor de los picos de dichas pérdidas de retorno en la banda de paso del filtro cuyo valor se sitúa ligeramente por debajo del nivel de $20 \mathrm{~dB}$ recomendado. Pese a estas pequeñas divergencias entre las respuestas medida y simulada, atribuibles con toda probabilidad a las tolerancias (superiores al valor de $10 \mu \mathrm{m}$ requerido) producidas durante el proceso de implementación del filtro, es posible concluir de 
los resultados ofrecidos en la citada figura 3.25 que la estructura de filtro de modo dual en guía circular propuesta en el presente apartado se comporta realmente como tal; y que el programa DUMAS 3.0, empleado en el análisis y diseño de dicha estructura, es capaz de simular su comportamiento de manera muy precisa en tiempos de computación relativamente bajos en comparación a la complejidad de la estructura considerada.

\subsubsection{Filtros Paso Banda de Modo dual en Guía Circular con Iris Elípticos y Guías Circulares Reentrantes}

En este último apartado, se propone una segunda posible configuración de filtro paso banda de modo dual en guía circular; constituido de nuevo por dos cavidades resonantes circulares (4 polos), simétrico y con una respuesta que no presenta en este caso ningún cero de transmisión. Esta segunda configuración, descrita con detalle en [64], utiliza otra vez iris elípticos para implementar los acoplos de entrada y salida requeridos por el filtro, así como el acoplo modal necesario entre las dos cavidades adyacentes de la estructura propuesta; mientras la novedad de este diseño se centra en cómo se implementa el acoplo entre modos duales situados dentro de cada cavidad, y en cómo se ajusta la frecuencia de resonancia asociada a cada uno de los mencionados modos duales. Estos dos últimos efectos requeridos en todo filtro de modo dual se han obtenido tradicionalmente, tal y como se recoge en el apartado 3.2.1, introduciendo en cada cavidad resonante del filtro tres tornillos de sintonía; cuyas penetraciones deben determinarse mediante un proceso de ajuste experimental que resulta arduo y tedioso. Con el objeto de poder simular el comportamiento de estos filtros de modo dual, incluyendo el efecto de los mencionados tornillos de sintonía, éstos se sustituyeron en [54] por un tramo de guía circular reentrante con tres inserciones metálicas; en cuya caracterización se empleó el método de los elementos finitos, que como es bien sabido requiere en su aplicación de un importante esfuerzo computacional. Haciendo uso pues de esta idea, en el presente apartado se propone también emplear un tramo uniforme de guía circular reentrante para simular el comportamiento de los citados tornillos; guía reentrante constituida nuevamente por tres inserciones metálicas, pero en este caso con forma cónica cada una de ellas para simular mejor el efecto del correspondiente tornillo. Sin embargo, en la caracterización del comportamiento electromagnético que presenta esta guía circular reentrante, cuyo aspecto puede observarse en la figura 3.29 (apartado a), se empleará el método presentado en la sección 3.1 (apartado 3.1.1); que permite analizar y diseñar en tiempo real filtros de modo dual como los propuestos en este apartado, a diferencia del método de los elementos finitos utilizado en [54] que resulta bastante más ineficiente. Una vez culminado el proceso de diseño del filtro de modo dual de 4 polos propuesto, se ofrecen medidas de la respuesta electromagnética que presenta una implementación práctica de dicho filtro; confirmando pues de esta manera que la 


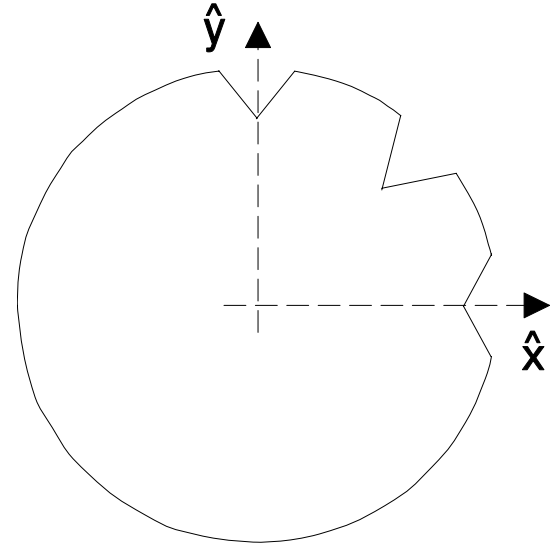

a)

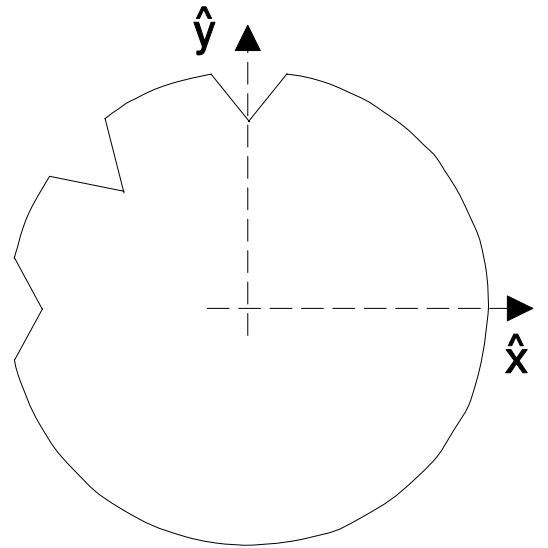

b)

Figura 3.29: Sección transversal de una guía circular reentrante, con tres inserciones metálicas en forma de cono, que pretende simular el efecto de los tornillos de sintonía tradicionalmente empleados en los filtros de modo dual en guía circular en a); y aspecto de la sección transversal de otra guía circular reentrante, obtenida a partir de la sección transversal de la guía original (apartado a) aplicando simetría respecto del eje $\hat{y}$, que se emplea junto a la guía original para implementar respuestas elípticas en b).

estructura diseñada se comporta efectivamente según lo previsto en las simulaciones. Asimismo, también se construyen sendos tramos de guías circulares con tres tornillos de sintonía, de diámetros iguales al grosor de las inserciones metálicas cónicas, para reemplazar respectivamente cada tramo uniforme de guía circular reentrante utilizado originalmente en la implementación práctica; ajustando en el laboratorio las penetraciones de dichos tornillos con el objeto de recuperar la respuesta simulada del filtro de modo dual. Con dicho proceso de ajuste experimental, que no resulta especialmente costoso escogiendo como valores iniciales de las penetraciones de los tornillos aquéllas determinadas en el proceso de diseño para las respectivas inserciones metálicas, se pretende demostrar que el filtro de modo dual diseñado utilizando guías circulares reentrantes, en lugar de los tradicionales tornillos de sintonía, seguirá comportándose como tal si en la práctica se requiere del uso de los mencionados tornillos; cuya utilización puede convertirse en indispensable si, por razones económicas, en algunas aplicaciones de bajo coste no es posible conseguir los niveles de precisión mecánica requeridos en el proceso de construcción del filtro para obtener la respuesta deseada.

Concretamente, la nueva estructura de filtro de modo dual en guía circular planteada en este apartado se muestra en la figura 3.30; estructura que corresponde a un filtro de 4 polos simétrico, cuya respuesta paso banda no presenta ningún cero de transmisión. Observando la figura 3.30, se comprueba que la mencionada estructura consta tan sólo de dos cavidades resonantes circulares, como debe ser en un filtro de modo dual de 4 polos, que se acoplan respectivamente a las guías de 


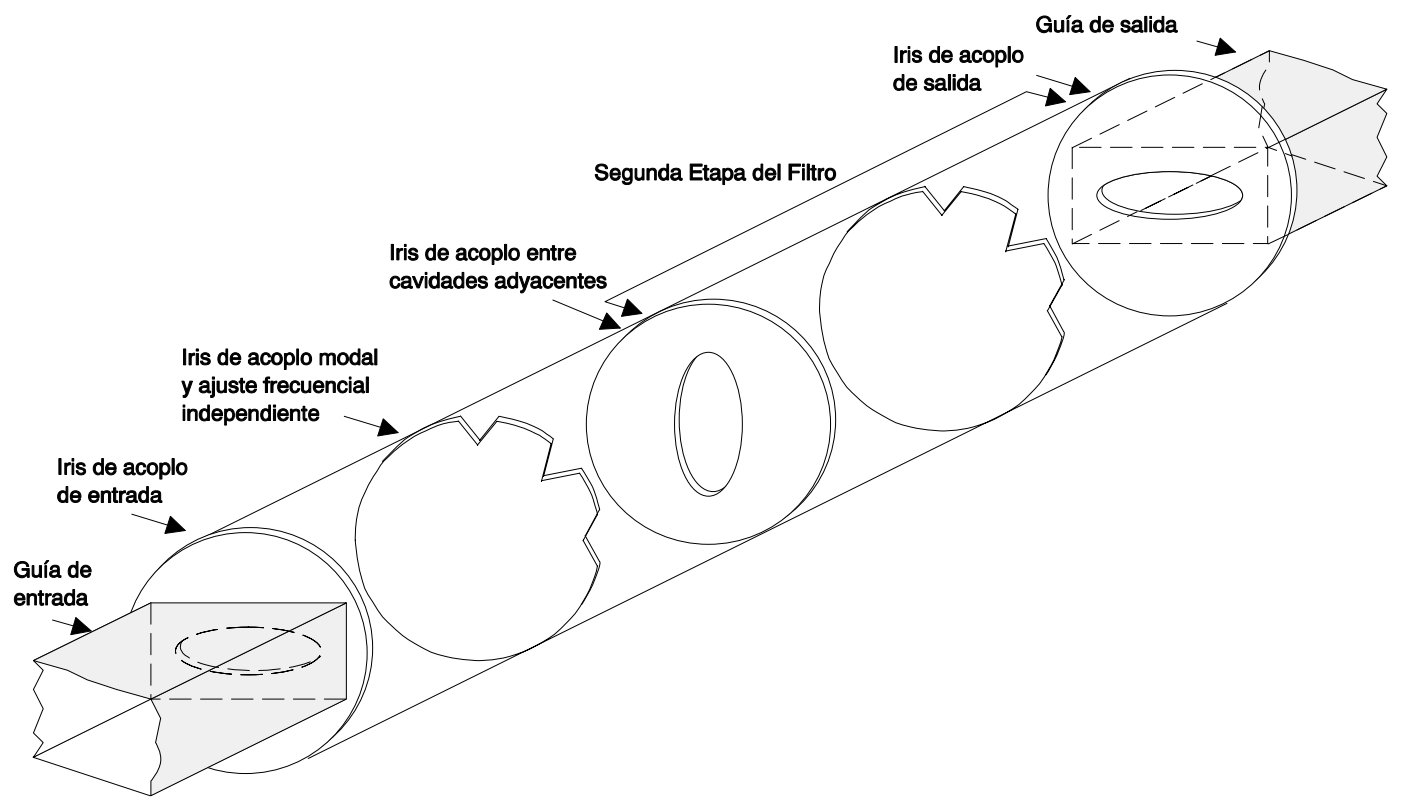

Figura 3.30: Configuración de un filtro de modo dual en guía circular de 4 polos, simétrico y con respuesta paso banda sin ceros de transmisión, que consta de iris elípticos y guías circulares reentrantes para implementar los diferentes requerimientos del filtro.

entrada y salida, así como entre ellas, mediante los correspondientes iris elípticos; cuya utilización para obtener los acoplos recién descritos se encuentra plenamente justificada en el apartado anterior, donde además se propone emplear también este mismo tipo de iris (elípticos) en la consecución del resto de efectos requeridos por los filtros de modo dual. Sin embargo, tal y como se ha mencionado en el párrafo anterior, en la nueva estructura propuesta (ver fig. 3.30) los dos requerimientos adicionales del filtro, el acoplo entre modos duales dentro de cada cavidad y el ajuste frecuencial independiente de cada uno de estos dos modos duales, se obtienen insertando en el centro de cada cavidad (lugar donde el campo eléctrico presenta un máximo) un tramo uniforme de guía circular reentrante cuya sección transversal se ofrece en la figura 3.29 (apartado a); que consta de tres inserciones metálicas, con forma de cono cada una de ellas, cuya misión respectiva es la de reemplazar al tornillo de sintonía correspondiente que se utiliza normalmente en este tipo de filtros. En particular, la inserción cónica dirigida según el eje $\hat{y}$ en la figura 3.29 (apartado a) se ocupa de ajustar la frecuencia de resonancia de uno de los dos modos duales presentes en la cavidad; mientras la inserción ortogonal a la anterior, aquélla que se encuentra orientada según el eje $\hat{x}$ en la misma figura 3.29 (apartado a), sirve para ajustar la frecuencia de resonancia asociada al segundo modo dual de la cavidad, cuya polarización es ortogonal a la del primer modo dual. Por su parte, la tercera inserción metálica posicionada formando un 
ángulo de $45^{\circ}$ respecto del eje $\hat{x}$ y respecto del eje $\hat{y}$ (ver de nuevo apartado a en fig. 3.29) permite obtener el acoplo modal requerido entre los dos modos duales de cada cavidad. En cuanto a la simetría física de la nueva configuración propuesta, en la figura 3.30 se comprueba que dicha simetría es total; de manera que las dimensiones y ubicación de los iris elípticos de acoplo de entrada y salida coinciden, así como las guías circulares reentrantes situadas dentro de cada cavidad resonante que se escogen idénticas (y con la misma orientación) para que la respuesta del filtro no presente ceros de transmisión. Si se pretendiera que la citada estructura presentase una respuesta elíptica ( 2 ceros de transmisión) sería necesario, tal y como se menciona en [43] y [57], que existiera una realimentación negativa entre las dos cavidades adyacentes del filtro; lo que en el caso que nos ocupa podría implementarse, de manera análoga a como se obtuvo dicho efecto en la estructura presentada en el apartado 3.2.2, desplazando $90^{\circ}$ las orientaciones de las inserciones metálicas responsables de conseguir en cada cavidad el acoplo requerido entre modos duales. Dicho desplazamiento se obtiene empleando una guía circular reentrante en la segunda cavidad cuya sección transversal se muestra en la figura 3.29 (apartado b); que se obtiene a partir de la sección transversal de la guía circular reentrante empleada en la primera cavidad (ver apartado a en fig. 3.29) aplicando simetría a dicha sección transversal respecto del eje vertical $\hat{y}$. Tanto en la configuración cuya respuesta no presenta ceros de transmisión (ver figura 3.30), como en aquélla modificada según se termina de describir para que la respuesta sea elíptica (2 ceros de transmisión), la principal dificultad asociada a su análisis y diseño estriba en la caracterización modal de las guías circulares reentrantes que integran ambas estructuras, así como en la obtención de las integrales de acoplamiento entre los modos de dichas guías y los modos de las correspondientes guías circulares; que se requieren para analizar, mediante el uso de matrices de admitancias generalizadas, aquellas uniones planares entre guías circulares y guías circulares reentrantes que integran la estructura considerada. En la determinación de las mencionadas integrales de acoplamiento, se ha seguido el método descrito anteriormente en la sección 3.1 (apartado 3.1.1) particularizado al caso que nos ocupa; es decir, a la evaluación de las mencionadas integrales entre los modos de una guía circular y una guía circular con su contorno básico modificado (guía reentrante). Dicho método, expuesto con todo detalle en [21] y [22], requiere obtener previamente los modos de la guía cuyo contorno básico circular se encuentra modificado (guía circular reentrante); para lo cual la correspondiente ecuación integral (asociada a los modos TE o TM) se transforma, tras aplicar de manera adecuada el Método de los Momentos (procedimiento Galerkin), en un simple problema matricial lineal de autovalores. La resolución del mencionado problema de autovalores puede implementarse de manera muy eficiente, y generando a su vez resultados muy precisos, empleando programas especialmente diseñados para resolver problemas matriciales lineales. Una vez resuelto el mencionado problema de autovalores, la obtención final de 
las integrales buscadas tan sólo requiere tratar adecuadamente, como se indica en la sección 3.1 (apartado 3.1.1), gran parte de las matrices obtenidas al resolver el espectro modal de la guía reentrante; por lo que el coste adicional que supone obtener dichas integrales es bastante reducido. El procedimiento general de cálculo de las integrales de acoplamiento entre los modos de guías rectangulares (o circulares) y guías con el mismo contorno básico rectangular (o circular) pero modificado se encuentra implementado en el programa comercial ANAPLAN-W; del que se ha hecho uso para obtener las integrales de acoplamiento necesarias para poder analizar la estructura propuesta en la figura 3.30, integrales que posteriormente han sido empleadas por el programa DUMAS 3.0 para analizar el comportamiento de la estructura global. En cuanto al análisis de las transiciones entre guías elípticas y guías circulares o rectangulares, presentes también en la estructura propuesta en la figura 3.30, éste se realiza completamente con el propio programa DUMAS 3.0; empleando para obtener los modos de las guías elípticas y las correspondientes integrales de acoplamiento el mismo método descrito en el apartado 3.2.2, que permite obtener asimismo resultados de gran precisión con un redudido coste computacional.

Considerando pues la estructura de filtro de modo dual propuesta en la figura 3.30, se pretende diseñar un filtro paso banda cuya respuesta con un ancho de valor aproximadamente igual a $200 \mathrm{MHz}$ esté centrada a $11 \mathrm{GHz}$; para lo cual se sigue un procedimiento de diseño idéntico al utilizado con la estructura propuesta en el apartado 3.2.2, procedimiento que consta de sucesivas etapas con objetivos muy concretos. En la estructura a diseñar (recordar fig. 3.30), las guías de entrada y salida corresponden a tramos uniformes de guía normalizada WR-75 ( $a=19.050 \mathrm{~mm}, b=9.525 \mathrm{~mm})$, las cavidades resonantes se implementan mediante guías de sección transversal circular con diámetro $\phi=24.000 \mathrm{~mm}$; mientras que las dimensiones de todos los iris elípticos empleados en la mencionada estructura (dimensiones del eje mayor y menor de la sección transversal de cada iris y su grosor), las dimensiones de las inserciones metálicas cónicas presentes en los tramos uniformes de guía circular reentrante situados dentro de cada cavidad (concretamente ancho de la base de la inserción y penetración de la misma), así como las longitudes de los dos tramos iguales en los que dichas guías circulares reentrantes dividen cada cavidad, se escogen como parámetros físicos que deben optimizarse durante el proceso de diseño del filtro para conseguir obtener la respuesta deseada. Asimismo, con la intención de que los tramos de guía circular reentrante puedan simular de manera aproximada el efecto de los tornillos de sintonía utilizados en el filtro propuesto para compensar tolerancias, se escoge un valor predeterminado para el grosor de las mencionadas guías reentrantes igual a $2.000 \mathrm{~mm}$; valor que se aproxima bastante al del diámetro real de los citados tornillos a utilizar en el filtro que se pretende diseñar. En cuanto al procedimiento seguido para obtener los valores finales de los parámetros físicos a optimizar, al igual que el método descrito en el apartado 3.2 .2 , se divide básicamente en dos 


\begin{tabular}{|c|c|c|c|}
\hline \hline \multirow{2}{*}{$\begin{array}{c}\text { Iris Elíptico } \\
\text { empleado para }\end{array}$} & \multicolumn{3}{|c|}{ Dimensiones (mm) } \\
\cline { 2 - 4 } & Eje Mayor & Eje Menor & Grosor \\
\hline acoplo entrada/salida & 12.780 & 4.000 & 1.660 \\
\hline acoplo entre cavidades & 8.700 & 4.000 & 1.793 \\
\hline \hline
\end{tabular}

Tabla 3.8: Valores optimizados de las dimensiones de los iris elípticos del filtro de modo dual mostrado en la figura 3.30 para centrar su respuesta con ancho de banda de aproximadamente $200 \mathrm{MHz}$ a $11 \mathrm{GHz}$.

etapas bien diferenciadas; en una primera se determinan para dichos parámetros unos valores iniciales, que posteriormente se optimizan en una segunda etapa. En la primera de estas dos etapas se obtienen pues los mencionados valores iniciales, empleando para ello el procedimiento expuesto en [40] que diseña cavidad tras cavidad (incluyendo sus correspondientes iris elípticos y guía circular reentrante), con el objeto de obtener la respuesta deseada cuando en la estructura real tan sólo se considera uno de los dos modos duales (pero siempre con la misma polarización) en cada cavidad; por lo que en dicha etapa no se considera el acoplo requerido entre modos duales dentro de cada cavidad. A continuación, considerando el citado acoplo y utilizando los valores de las dimensiones a optimizar determinados tras la etapa inicial, se determinan mediante la misma técnica propuesta en [40] los valores optimizados finales de las dimensiones escogidas para diseñar la estructura propuesta; siempre con el objetivo de que la respuesta paso banda de dicha estructura se encuentre centrada a $11 \mathrm{GHz}$ con un ancho de valor en torno a los $200 \mathrm{MHz}$. Así pues, tras implementar el proceso de optimización recién comentado, se concluye que los dos tramos de guía circular que integran cada una de las dos cavidades de la estructura propuesta (recordar fig. 3.30) deben presentar una misma longitud igual a $7.340 \mathrm{~mm}$; mientras que para las dimensiones optimizadas de los diferentes iris elípticos se obtienen unos valores recogidos en la tabla 3.8, y para las coordenadas de los puntos que definen las inserciones metálicas presentes en cada guía circular reentrante se deducen unos valores optimizados mostrados en la tabla 3.9. La obtención de todos estos valores se ha conseguido, mediante el proceso de diseño brevemente expuesto con anterioridad, simulando el comportamiento electromagnético de diferentes estructuras intermedias; habiendo realizado todas estas simulaciones con el programa DUMAS 3.0 utilizando resultados proporcionados por la herramienta ANAPLAN-W, que tal y como se ha comentado anteriormente proporciona las integrales de acoplamiento necesarias para caracterizar después uniones planares en las que intervienen guías circulares reentrantes. En todas las mencionadas simulaciones, debido a la complejidad de las estructuras consideradas constituidas por discontinuidades bastante abruptas, ha sido necesario emplear 550 sumandos para evaluar todas las series estáticas requeridas (recordar apartado 2.2.1 del capítulo 2), 450 términos 


\begin{tabular}{|c|r|r|}
\hline \hline Punto & $\mathbf{x}_{\mathbf{i}} \mathbf{( \mathbf { m m } )}$ & $\mathbf{y}_{\mathbf{i}} \mathbf{( \mathbf { m m } )}$ \\
\hline $\mathbf{i}=\mathbf{1}$ & 11.963 & -0.941 \\
\hline $\mathbf{i}=\mathbf{2}$ & 7.901 & 0.000 \\
\hline $\mathbf{i}=\mathbf{3}$ & 11.963 & 0.941 \\
\hline $\mathbf{i}=\mathbf{4}$ & 9.125 & 7.793 \\
\hline $\mathbf{i}=\mathbf{5}$ & 5.931 & 5.931 \\
\hline $\mathbf{i}=\mathbf{6}$ & 7.793 & 9.125 \\
\hline $\mathbf{i}=\mathbf{7}$ & 0.941 & 11.963 \\
\hline $\mathbf{i}=\mathbf{8}$ & 0.000 & 10.060 \\
\hline $\mathbf{i}=\mathbf{9}$ & -0.941 & 11.963 \\
\hline \hline
\end{tabular}

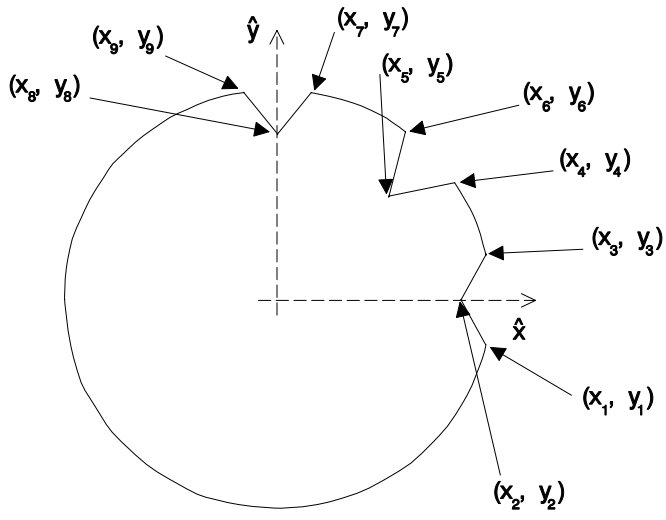

Tabla 3.9: Valores optimizados de las coordenadas de los puntos que definen tres inserciones metálicas, presentes en cada tramo de guía circular reentrante insertado dentro de cada cavidad resonante del filtro de modo dual mostrado en la figura 3.30, con el objeto de centrar la respuesta de dicha estructura con ancho de banda de aproximadamente $200 \mathrm{MHz}$ a $11 \mathrm{GHz}$.

al sumar las diversas partes estáticas de las series dinámicas precisadas (recordar apartado 2.2.2 del capítulo 2), y 110 modos para describir correctamente los campos eléctrico y magnético de cada una de las guías que integran cada estructura considerada. Estos requerimientos tan elevados, necesarios para obtener resultados convergentes y precisos en el análisis de la estructura propuesta, deben ser manejados mediante un programa especializado como DUMAS 3.0; con el cual es posible analizar y diseñar en tiempo real estructuras de microondas realmente complejas, debido a que el mencionado programa incorpora las técnicas eficientes descritas en el capítulo 2.

Tras finalizar pues el proceso de diseño de la estructura mostrada en la figura 3.30, haciendo uso de las dimensiones obtenidas en dicho proceso y de los valores precisados por los parámetros del programa DUMAS 3.0, se simula con esta herramienta la respuesta electromagnética (parámetros $S_{21}$ y $S_{11}$ ) del filtro de modo dual en guía circular diseñado; que emplea iris elípticos y tramos uniformes de guía circular reentrante para implementar los diferentes requerimientos de este tipo de filtros. Los resultados correspondientes a esta respuesta pueden observarse en la figura 3.31 (apartado a); donde es posible comprobar que la banda de paso presenta un ancho de valor en torno a los $200 \mathrm{MHz}$, y que dicha banda está centrada prácticamente a la frecuencia de $11 \mathrm{GHz}$. Asimismo, en la mencionada figura 3.31 (apartado a) se confirma la existencia de 4 polos en las pérdidas de retorno (parámetro $S_{11}$ ) y la ausencia de ceros de transmisión en la representación del parámetro $S_{21}$; comportamiento que corresponde plenamente al de un filtro de modo dual en guía circular constituido por dos cavidades resonantes (4 polos), que no presentan ningún tipo de realimentación negativa entre 


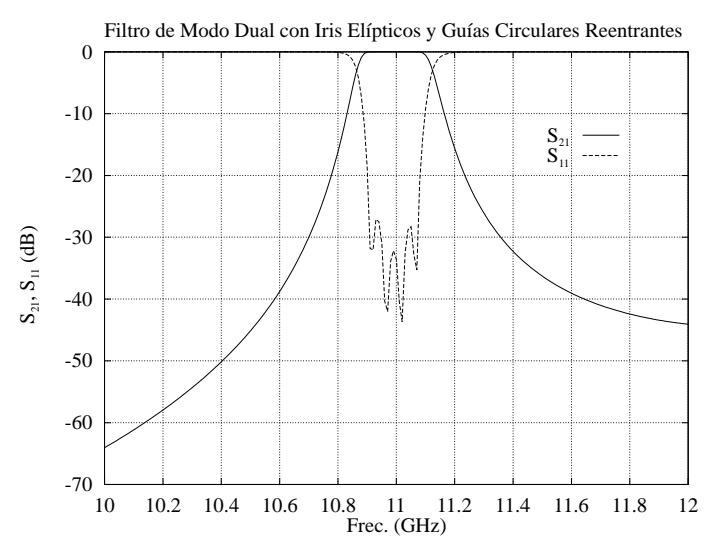

a)

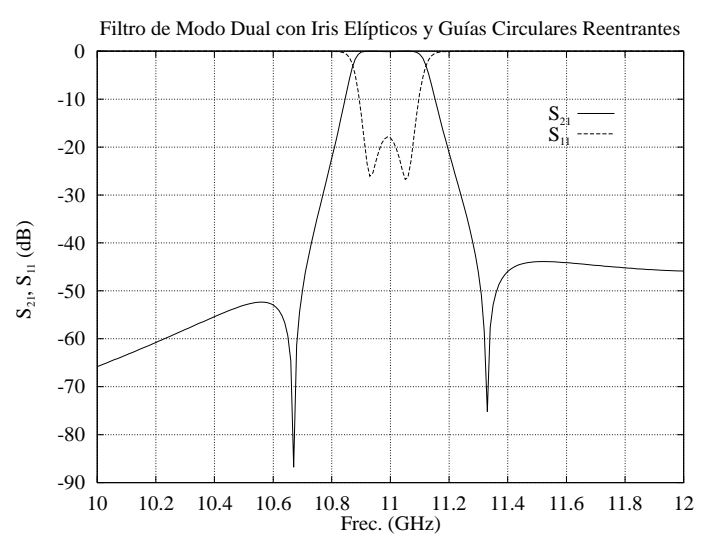

b)

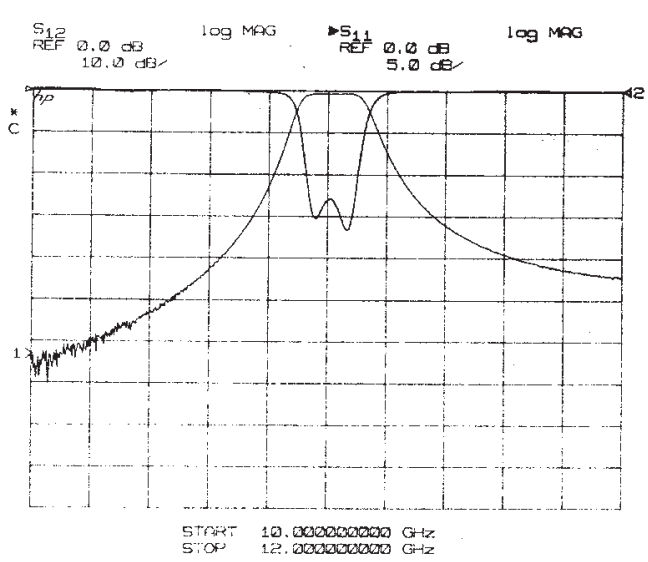

c)

Figura 3.31: Simulación de la respuesta electromagnética (módulo de los parámetros $S_{21}$ y $S_{11}$ ) que presenta el filtro de modo dual propuesto en la figura 3.30 diseñado para que su respuesta paso banda, sin ceros de transmisión, esté centrada a $11 \mathrm{GHz}$ con un ancho de banda aproximado de $200 \mathrm{MHz}$ en a), simulación de la respuesta electromagnética (módulo de los parámetros $S_{21}$ y $S_{11}$ ) de la misma estructura mostrada en la figura 3.30 pero modificada para conseguir que su respuesta presente 2 ceros de transmisión en b), y medidas de la respuesta que ofrece la implementación real de la estructura recogida en la figura 3.30 en c).

ellas (ausencia de ceros de transmisión) debido a que los tramos uniformes de guía circular reentrante situados en ambas cavidades son idénticos (dimensiones de las inserciones metálicas y orientación de las mismas). Por otra parte, en la figura 3.31 (apartado a) se observa como los picos que presenta las pérdidas de retorno (parámetro $S_{11}$ ) en la banda de paso del filtro no tienen todos ellos el mismo nivel; efecto causado, como ya se explicó para el otro filtro de modo dual en guía circular propuesto en el apartado 3.2.2, por la dispersión producida en las cavidades del filtro así como en sus respectivos iris de acoplo y tramos uniformes 
de guía circular reentrante. No obstante, el nivel de todos estos picos (ver de nuevo apartado a en fig. 3.31) se mantiene en toda la banda de paso del filtro siempre por encima de $20 \mathrm{~dB}$; condición cuyo cumplimiento resulta conveniente observar en este tipo de filtros. Si se pretendiera que la respuesta del filtro considerado fuera elíptica (presencia de dos ceros de transmisión), tal y como ya se ha comentado anteriormente, tan sólo sería necesario sustituir el tramo uniforme de guía circular reentrante situado en la segunda cavidad resonante del filtro (recordar fig. 3.30) por un tramo con las mismas inserciones metálicas pero situadas como se indica en la figura 3.29 (apartado b); ya que de esta manera se consigue implementar un nivel de acoplamiento negativo entre las dos cavidades adyacentes, requerido para conseguir la respuesta elíptica deseada en el filtro diseñado. Así pues, una vez simulada la respuesta electromagnética (parámetros $S_{21}$ y $S_{11}$ ) de esta nueva configuración alternativa, se obtienen los resultados ofrecidos en la figura 3.31 (apartado b); donde se observa una importante degradación en las pérdidas de retorno (parámetro $S_{11}$ ), traducida en la existencia de tan sólo 2 polos y en que el nivel del único pico en la banda de paso presenta un valor por debajo de $20 \mathrm{~dB}$. La razón básica de esta degradación observada consiste en haber utilizado en esta segunda simulación las mismas dimensiones para todos los elementos del filtro obtenidas en el proceso de diseño original (respuesta sin ceros de transmisión); incluso en las coordenadas de los puntos que definen las inserciones metálicas de las guías circulares reentrantes de cada cavidad recogidas previamente en la tabla 3.9. Con el objeto de recuperar los 4 polos del filtro en sus pérdidas de retorno, así como para conseguir que los picos de dichas pérdidas presenten en toda la banda de paso del filtro valores por encima de $20 \mathrm{~dB}$, es necesario optimizar ligeramente algunas de las dimensiones obtenidas en el diseño de la estructura original; resultando suficiente con toda probabilidad ajustar tan sólo las dimensiones de las inserciones metálicas de las guías circulares reentrantes, guías que deben situarse en ambas cavidades como se ha indicado (presentando simetría respecto del eje vertical) para conseguir que el filtro diseñado presente la deseada respuesta elíptica.

Con la intención de confirmar que la estructura propuesta en el presente apartado (recordar fig. 3.30) se comporta como un filtro de modo dual, así como para validar el proceso seguido en el diseño de dicha estructura de manera que su respuesta paso banda de ancho aproximadamente igual a $200 \mathrm{MHz}$ esté centrada a $11 \mathrm{GHz}$, se ha construido una implementación práctica del mencionado filtro empleando las dimensiones deducidas anteriormente. En dicha implementación, los acoplos de entrada y salida requeridos se obtienen mediante iris elípticos cuyo aspecto se recoge en la figura 3.32 (apartado a); iris que deben quedar orientados en dirección paralela a la del eje horizontal de cualquier sección transversal del filtro. Por lo que respecta al acoplo entre las dos cavidades adyacentes del filtro, su implementación se realiza mediante el iris elíptico mostrado en la figura 3.32 (apartado b); cuya orientación en este caso debe ser paralela al eje vertical de 


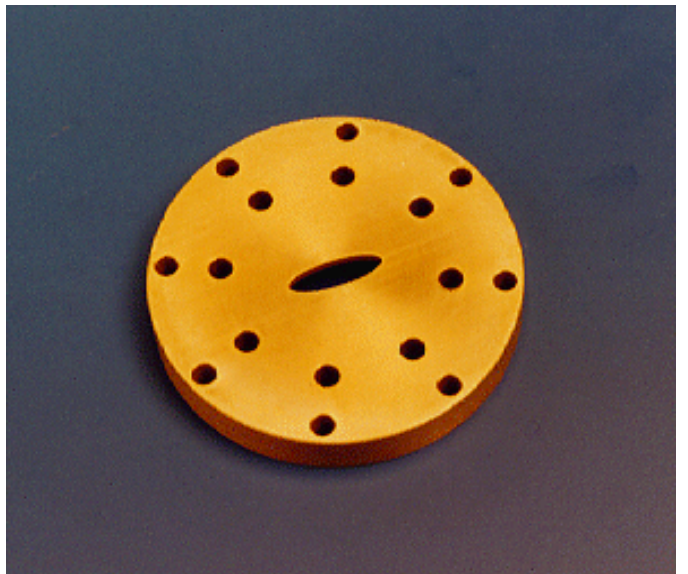

a)

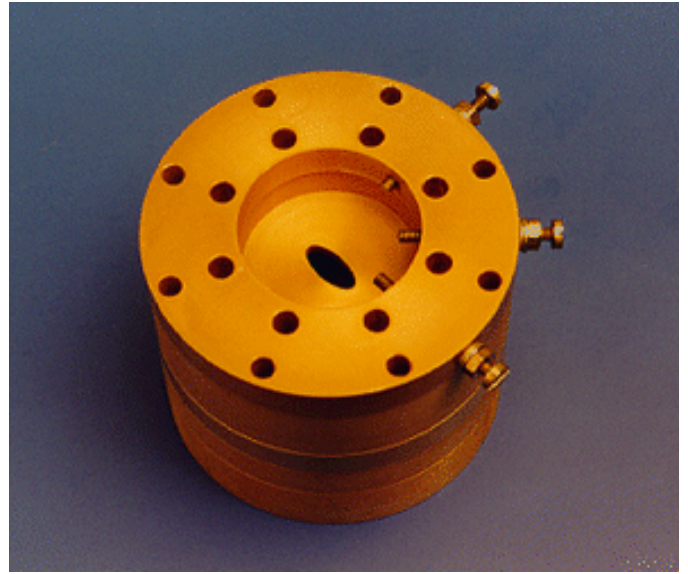

b)

Figura 3.32: Iris elíptico utilizado para conseguir implementar los acoplos de entrada y salida en a), e iris elíptico escogido para obtener el acoplo entre parejas de modos duales situados en cavidades adyacentes en b).

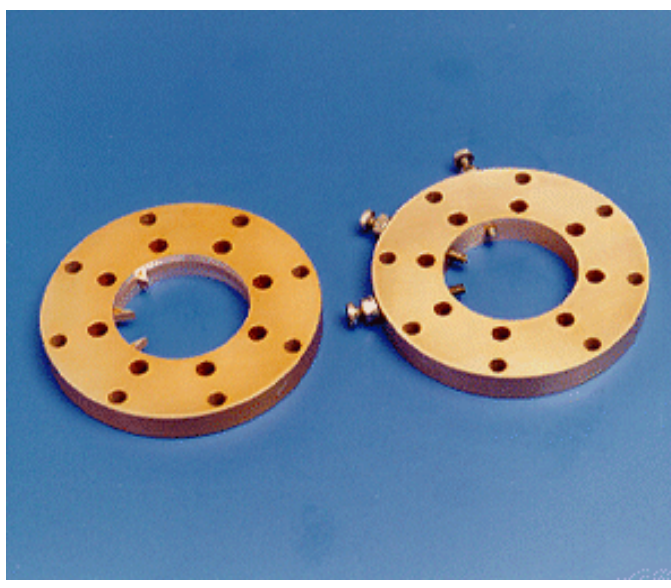

a)

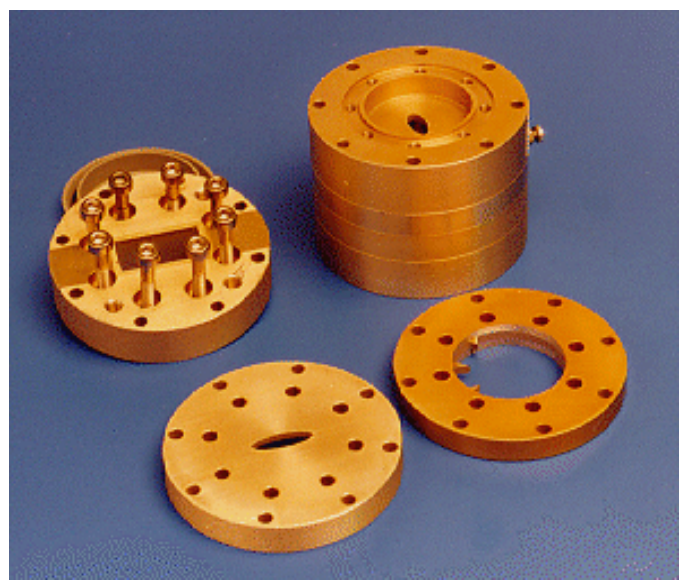

b)

Figura 3.33: Guía circular reentrante, con tres inserciones metálicas, que permite conseguir el acoplo requerido entre modos duales dentro de cada cavidad, así como ajustar independientemente la frecuencia de resonancia de cada uno de estos modos, junto con un tramo de guía circular equivalente en el que se insertan tres tornillos de sintonía en a); y vista conjunta de todas las piezas integrantes del filtro de modo dual diseñado, que emplea iris elípticos y guías circulares reentrantes, en b).

las secciones transversales, es decir el iris debe quedar rotado $90^{\circ}$ respecto de la orientación que presentan los iris de acoplo de entrada y salida. Los valores optimizados de las dimensiones físicas de estos dos iris elípticos, dimensiones del eje mayor y menor de sus respectivas secciones transversales y de los grosores de 
ambos iris, corresponden a los recogidos previamente en la tabla 3.8; valores que se han obtenido tras finalizar el proceso de diseño del filtro propuesto. En cuanto a los tramos uniformes de guía circular reentrante con tres inserciones metálicas en forma de cono, empleados para conseguir el acoplo entre modos duales dentro de cada cavidad y el ajuste independiente de la frecuencia de resonancia asociada a cada uno de dichos modos, se implementan de forma práctica tal y como se recoge en la figura 3.33 (apartado a); donde las dimensiones y ubicación de cada una de las inserciones metálicas mencionadas se deducen a partir de los valores mostrados en la tabla 3.9 para sus coordenadas respectivas, valores optimizados durante el proceso seguido en el diseño del filtro. Todas estas piezas recién descritas, recogidas junto al resto de elementos integrantes del filtro diseñado en la figura 3.33 (apartado b), deben insertarse adecuadamente entre tramos uniformes de guía circular con diámetro $\phi=24.000 \mathrm{~mm}$ y longitudes correspondientes deducidas también en la etapa de diseño; para de esta forma implementar el filtro de modo dual diseñado, en el que lógicamente los iris elípticos de acoplo de entrada y salida se insertarán entre las respectivas guías de entrada y salida y los mencionados tramos uniformes de guía circular.

Por otra parte, se han construido también tramos adicionales de guía circular con tres tornillos de sintonía, de diámetro igual al grosor de los tramos de guía circular reentrante $(\phi=2 \mathrm{~mm})$, con el objeto de que sustituyan respectivamente en el filtro de modo dual diseñado dichos tramos de guía circular reentrante con tres inserciones metálicas. De esta manera, ajustando experimentalmente en el laboratorio las penetraciones de cada uno de los mencionados tornillos, se pretende recuperar la respuesta paso banda (con ancho aproximado de $200 \mathrm{MHz}$ y centrada a $11 \mathrm{GHz}$ ) del filtro de modo dual diseñado originalmente empleando guías circulares reentrantes; demostrando pues que las dimensiones deducidas anteriormente para los restantes elementos del filtro de modo dual continúan siendo válidas en el caso, bastante usual por cierto en la práctica, de tener que utilizar tornillos de sintonía en el filtro para compensar defectos presentes en su implementación causados por tolerancias mecánicas. En la figura 3.34 (apartado a), se muestra pues este tramo uniforme de guía circular con tres tornillos de sintonía; cuyas penetraciones respectivas se han obtenido experimentalmente en el laboratorio para recuperar la respuesta paso banda deseada en el filtro construido. Con el objeto de comparar estas penetraciones de los tornillos de sintonía con aquéllas deducidas en el diseño para las correspondientes inserciones metálicas, los dos tramos de guía circular intercambiables entre sí se muestran conjuntamente en la figura 3.33 (apartado a); donde es posible comprobar que entre las penetraciones requeridas en un caso y en otro se preserva una relación de aspecto. Estos resultados confirman que es posible utilizar un tramo uniforme de guía circular reentrante, como el propuesto en este apartado, para simular el efecto real que producen los tornillos de sintonía tradicionalmente empleados en el tipo de filtros de modo dual considerados. Una visión conjunta de todas las piezas necesarias 


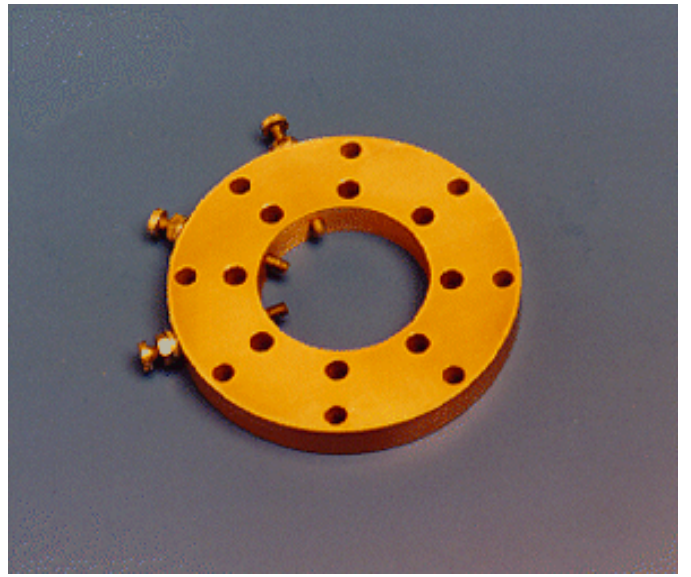

a)

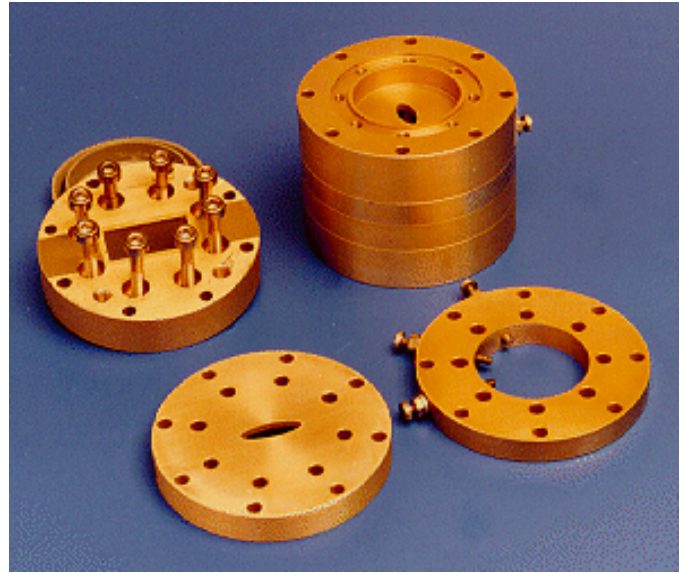

b)

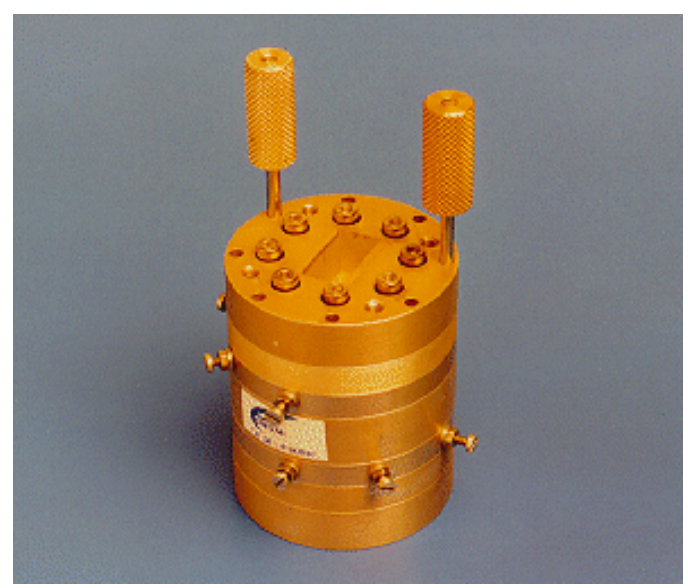

c)

Figura 3.34: Guía circular con tres tornillos de sintonía (diámetro $\phi=2 \mathrm{~mm}$ ), cuyas penetraciones pueden ajustarse manualmente para controlar de manera independiente la frecuencia de resonancia de cada modo y conseguir el acoplo entre modos requerido, en a), vista conjunta de todas las piezas construidas para implementar el filtro de modo dual diseñado, en el que se sustituyen las inserciones metálicas cónicas por tornillos de sintonía, en b), y aspecto final del filtro con tornillos de sintonía una vez ensambladas todas sus piezas en $c$ ).

para implementar este nuevo diseño, que incluye tornillos de sintonía, se ofrece en la figura 3.34 (apartado b); piezas que son las mismas utilizadas para implementar el filtro original (observar apartado b en fig. 3.33), a excepción del tramo uniforme de guía reentrante sustituida por el correspondiente tramo uniforme de guía circular con tornillos de sintonía. Después de ensamblar todas las piezas de esta segunda implementación práctica, que incorpora tornillos de sintonía, el fil- 
tro de modo dual construido presenta un aspecto global mostrado en perspectiva en la figura 3.34 (apartado c); cuya longitud total presenta un valor aproximadamente igual a $40 \mathrm{~mm}$, muy similar a la longitud del filtro de modo dual con sólo iris elípticos descrito en el apartado 3.2.2, y nuevamente bastante inferior a la longitud requerida por un filtro como el diseñado en el caso de implementarlo excitando un único modo en cada cavidad.

Una vez presentada la implementación práctica del filtro diseñado, con el objeto de corroborar la respuesta de dicho dispositivo simulada con el programa DUMAS 3.0, se mide el comportamiento electromagnético (parámetros $S_{21}$ y $\left.S_{11}\right)$ que presenta la estructura implementada; habiendo escogido en primer lugar la estructura constituida por las piezas y elementos mostrados en la figura 3.33 (apartado b), entre las que se encuentran tramos uniformes de guía circular reentrante insertados dentro de cada cavidad. La campaña de medidas realizada, al igual que el resto de medidas ofrecidas en el presente capítulo, se ha llevado a efecto en el Área de Microondas del Laboratorio de Sistemas de Radiofrecuencia que la ESA (Agencia Espacial Europea) tiene en ESTEC (Centro Europeo de Tecnología e Investigación Espacial); para lo cual se ha utilizado un analizador de redes de la serie $\mathrm{HP} 8510 \mathrm{C}$, en cuya calibración previa se ha empleado el procedimiento normalizado TRL completo de dos puertos (en inglés full 2-port). Las medidas obtenidas, correspondientes al caso en el que se pretende obtener una respuesta sin ceros de transmisión (ubicación idéntica de las inserciones metálicas situadas en cada cavidad resonante), se recogen por razones comparativas en la figura 3.31 (apartado c) mostrada en la página 136; donde se observa, tras comparar los resultados obtenidos mediante simulación y mediante medidas (apartados a y c en fig. 3.31), que el comportamiento del filtro construido es básicamente correcto (filtro de modo dual con respuesta sin ceros de transmisión). Sin embargo, la respuesta medida del dispositivo construido (apartado c en fig. 3.31) revela ciertas degradaciones en sus pérdidas de retorno (parámetro $S_{11}$ ); que consisten básicamente en la desaparición de 2 polos en dicho parámetro $S_{11}$, frente a los 4 polos que debieran estar presentes, y en que el nivel máximo de las citadas pérdidas de retorno se encuentra por debajo incluso de $15 \mathrm{~dB}$ (el nivel recomendado debería ser superior a $20 \mathrm{~dB}$ ). Las principales causas de estas degradaciones, más importantes que las observadas tras medir el filtro de modo dual con sólo iris elípticos propuesto en el apartado 3.2.2, son esencialmente las tolerancias mecánicas (superiores al valor de $10 \mu \mathrm{m}$ requerido) presentes durante el proceso de construcción del filtro propuesto en este apartado; las cuales, debido al modo particular en el que se han implementado físicamente las cavidades circulares del filtro (a base de tramos pertenecientes a diferentes piezas), han afectado de manera más intensa provocando una mayor degradación en la respuesta medida. Así y todo, pese a estas mayores degradaciones observadas, es posible concluir de dichas medidas que la estructura propuesta en el presente apartado se comporta como un filtro de modo dual en guía circular; y de manera adicional se comprueba 


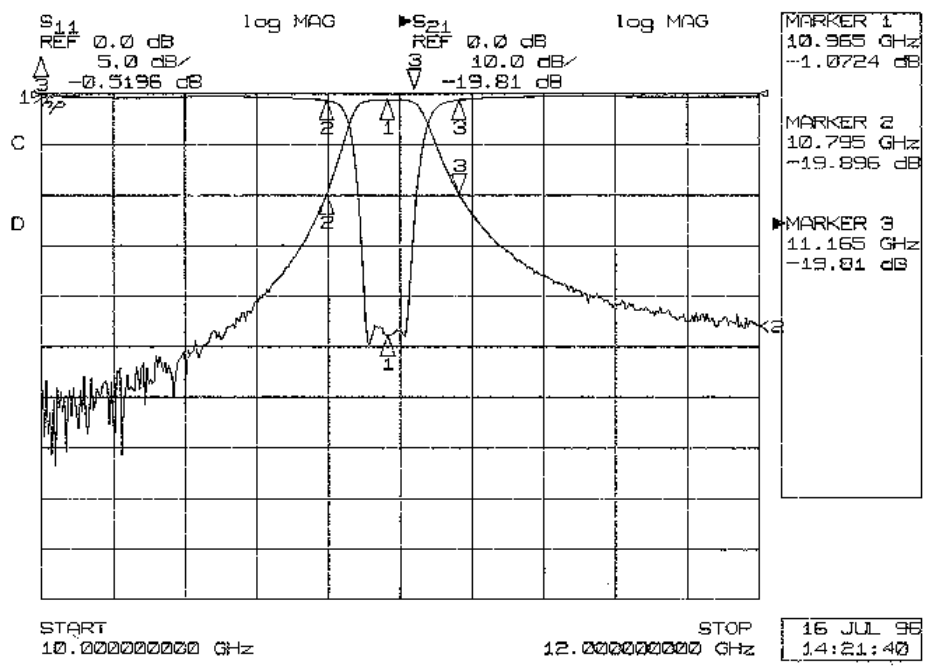

a)

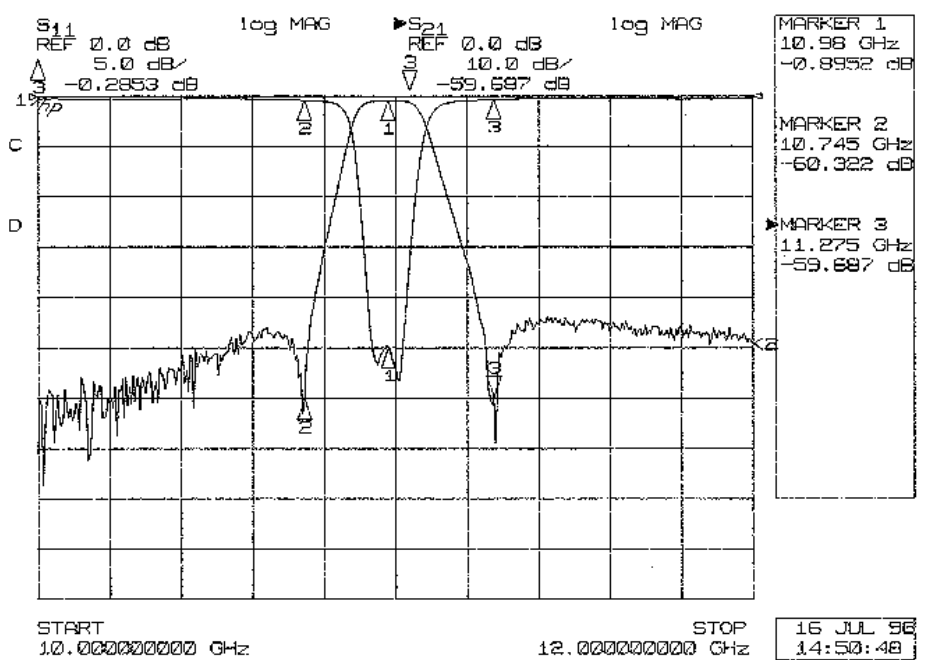

b)

Figura 3.35: Medidas de la respuesta electromagnética (módulo de los parámetros $S_{21}$ y $S_{11}$ ) del filtro de modo dual construido, con respuesta sin ceros de transmisión, empleando tornillos de sintonía en lugar de guías reentrantes en a); y medidas de la misma estructura, modificada para incorporar 2 ceros de transmisión en su respuesta, empleando nuevamente tornillos de sintonía en b).

nuevamente el buen funcionamiento del programa DUMAS 3.0, capaz de analizar y diseñar con precisión y en tiempo real estructuras tan complejas de microondas como el filtro de modo dual considerado.

Como ha podido comprobarse en la implementación práctica del filtro de modo 
dual propuesto, las tolerancias de fabricación provocan una importante degradación en la respuesta asociada a este tipo de filtros. Con el objeto de compensar los efectos negativos provocados por las mencionadas tolerancias, en la práctica se utilizan en este tipo de filtros tornillos de sintonía; cuyas penetraciones se ajustan experimentalmente para recuperar la respuesta deseada en el filtro implementado. Ahora bien, para dimensionar correctamente las diferentes partes del filtro de modo dual a construir, se requiere poder simular el comportamiento de dicho filtro incluyendo de alguna manera el efecto de los tornillos de sintonía; tal y como se ha realizado en el presente apartado empleando los mencionados tramos uniformes de guía circular reentrante. Así pues, para demostrar que el diseño del filtro de modo dual propuesto en este apartado es correcto, se ha sustituido en la implementación práctica del mismo cada guía circular reentrante por un tramo uniforme de guía circular con tres tornillos de sintonía (recordar apartado a en fig. 3.34), tramos situados en cada cavidad de manera que sus tornillos respectivos presentan las mismas orientaciones para que la respuesta del filtro no incluya ceros de transmisión; determinando las penetraciones de dichos tornillos para recuperar la respuesta deseada en el dispositivo construido. En la figura 3.35 (apartado a), se muestra la respuesta medida (parámetros $S_{21} \mathrm{y}$ $S_{11}$ ) del filtro construido empleando los mencionados tornillos; observando como efectivamente las pérdidas de retorno obtenidas presentan 4 polos en la banda de paso, y como el nivel máximo de dichas pérdidas se mantiene en toda la banda de paso por encima de $20 \mathrm{~dB}$ como era deseable. Las penetraciones que se requieren en los tornillos para medir esta respuesta (apartado a en fig. 3.35) se han determinado experimentalmente en el laboratorio; no habiendo requerido dicha determinación de un proceso extremadamente arduo o complejo, pues como penetraciones iniciales se escogieron aquéllas obtenidas en el proceso de diseño para las respectivas inserciones metálicas. Asimismo, como puede observarse en la figura 3.33 (apartado a), las penetraciones finales de los tornillos de sintonía una vez ajustadas guardan una relación de aspecto entre ellas bastante similar a la que presentan las penetraciones de las respectivas inserciones metálicas; lo que permite confirmar que el modelo escogido (inserciones metálicas con forma de cono) para representar el efecto de los tornillos reales resulta bastante apropiado, y por tanto puede emplearse para diseñar estructuras de modo dual con bajo coste económico (malas tolerancias) que requieran posteriormente el empleo de los mencionados tornillos. Por último, si el tramo de guía circular con tornillos presente en la segunda cavidad del filtro se sitúa adecuadamente, de manera simétrica a como se posiciona el mismo tramo con tornillos de la primera cavidad respecto del eje vertical de cualquier sección transversal, resulta posible obtener con la misma estructura empleada anteriormente una respuesta paso banda eliptica (2 ceros de transmisión); cuyo aspecto, tras haber ajustado nuevamente los diferentes tornillos de sintonía para satisfacer los requerimientos del filtro, se recoge en la figura 3.35 (apartado b). 



\section{Capítulo 4}

\section{Caracterización de Objetos Dispersores en Espacio Libre mediante Técnicas Espectrales}

El análisis de la dispersión electromagnética, que produce un objeto en espacio libre al incidirle una cierta excitación, ha sido estudiado utilizando técnicas clásicas; tales como Óptica Física, Óptica Geométrica, Teoría Física de la Difracción, Teoría Geométrica de la Difracción y Ecuaciones Integrales para el campo eléctrico y magnético entre otras posibles. Sin embargo, hasta el momento la literatura no ha recogido la caracterización de dichos objetos dispersores mediante una función de transferencia, definida en el dominio espectral, que permita determinar el comportamiento dispersor de dichos objetos ante cualquier incidencia.

En este capítulo se presenta el concepto de la función de transferencia de un objeto dispersor, representada en términos matemáticos por medio de una matriz que denominaremos matriz de caracterización. Dicha matriz relaciona espectro de campo dispersado por el objeto con espectro de campo incidente; y para su determinación se requiere el cálculo de dos matrices que llamaremos respectivamente matriz de corrientes y matriz de espectro. La matriz de corrientes permite obtener la distribución de corrientes inducidas sobre la superficie del objeto dispersor ante un cierto espectro de campo incidente. El cálculo de esta matriz se ha implementado haciendo uso de Óptica Física y de las Ecuaciones Integrales para el campo eléctrico y magnético. Finalmente, la matriz de espectro relaciona el espectro de campo dispersado por el objeto con las corrientes inducidas sobre su superficie. Para la determinación de esta matriz se exponen dos posibles técnicas, una de ellas basada en la Transformada de Fourier y la otra en el Teorema de Adición para las Funciones de Hankel. 


\subsection{Matriz de Caracterización}

La función de transferencia se ha utilizado tradicionalmente [65] en la caracterización de sistemas lineales e invariantes en el tiempo (LTI), representando la respuesta de dicho sistema en el dominio frecuencial ante una excitación constituida por un impulso definido en el dominio temporal. De esta manera, la respuesta del sistema ante cualquier excitación se obtiene, simplemente, multiplicando la función de transferencia del sistema por la excitación que se le aplica; tal y como se muestra en la siguiente expresión

$$
Y(f)=H(f) \cdot X(f)
$$

donde $X(f)$ representa cualquier posible excitación definida en el dominio frecuencial, $H(f)$ la función de transferencia que caracteriza al sistema e $Y(f)$ la respuesta del sistema, también definida en el dominio frecuencial, ante dicha excitación.

El concepto de la función de transferencia, que se termina de explicar para sistemas electrónicos LTI, es directamente trasladable al análisis de problemas electromagnéticos; pues éstos también presentan un comportamiento lineal e invariante en el tiempo. En [14], se plantea la posibilidad de caracterizar un objeto dispersor mediante una función de transferencia que relacione de alguna manera señales incidentes al objeto (excitación del sistema objeto dispersor) con las correspondientes señales dispersadas (respuesta de dicho sistema). Estas señales incidentes y dispersadas vienen representadas por campos electromagnéticos dependientes de la posición espacial $(\vec{E}(\rho, \phi, z)$ y $\vec{H}(\rho, \phi, z))$. De igual forma que las señales con dependencia temporal $(x(t)$ e $y(t))$ presentan espectros en el dominio transformado de la frecuencia temporal $(X(f)$ e $Y(f)$ ), las señales con dependencia de la posición espacial presentan igualmente espectros, aunque definidos en el domino transformado de la frecuencia espacial (ver apéndice C). Por tanto, así como en los sistemas electrónicos la función de transferencia relaciona las señales de entrada y salida en el dominio de la frecuencia $(X(f)$ e $Y(f)$, respectivamente), en la caracterización de objetos dispersores la función de transferencia relaciona espectros de campo incidente con espectros de campo dispersado. Este paralelismo entre la caracterización de sistemas electrónicos y problemas de dispersión electromagnética se refleja en la figura 4.1, donde $E I$ y $E D$ representan respectivamente los espectros de campo incidente y dispersado; y $D$ la función de transferencia que caracteriza al objeto dispersor.

La función de transferencia en problemas de dispersión electromagnética se expresa matemáticamente mediante una matriz, denominada matriz de caracterización, que permite obtener el espectro de campo dispersado por el objeto bajo análisis a partir del espectro de campo incidente. La principal ventaja asociada a este método para caracterizar la dispersión electromagnética radica en que, una vez obtenida la función de transferencia (o matriz de caracterización) de un obje- 


\section{SISTEMAS ELECTRÓNICOS}

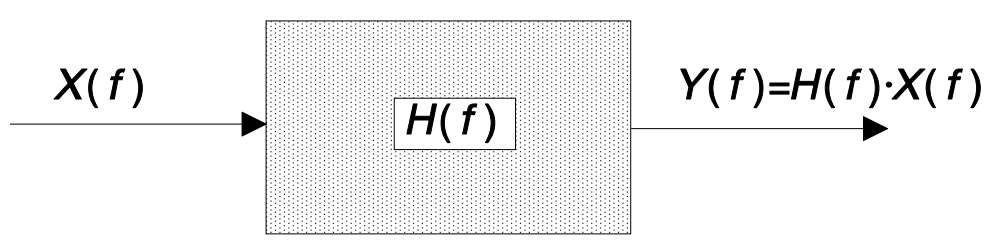

\section{PROBLEMAS ELECTROMAGNÉTICOS}

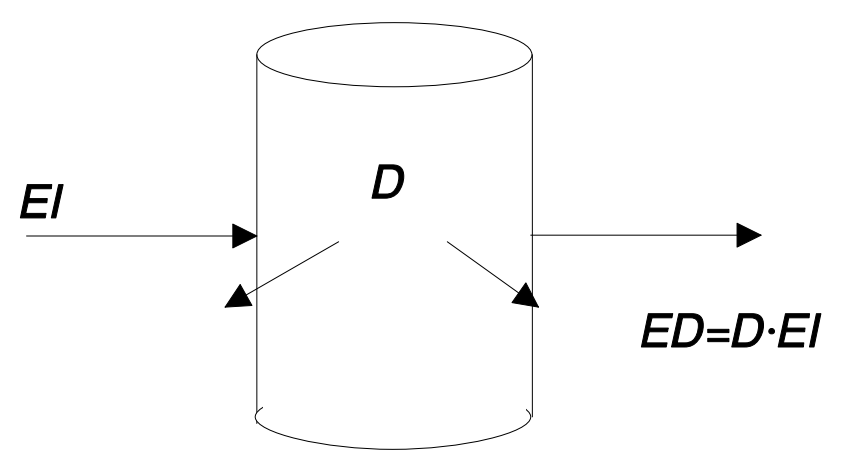

Figura 4.1: Función de transferencia en sistemas electrónicos y en problemas de dispersión electromagnética.

to dispersor, se puede prescindir de su geometría en el cálculo de la dispersión que produce dicho objeto ante cualquier incidencia; la cual se obtiene, simplemente, multiplicando su matriz de caracterización por el espectro asociado al campo incidente. Esta ventaja permite además, como se describirá en sucesivos capítulos, la caracterización del acoplo electromagnético que se produce entre varios objetos dispersores ante cualquier incidencia. Las matrices de caracterización permitirán estudiar, por tanto, el efecto de las interacciones múltiples que se originan entre diversos objetos sin necesidad de recurrir a la geometría de los mismos.

Un campo incidente arbitrario en una región homogénea y libre de fuentes se puede expresar como combinación de dos tipos de soluciones modales, que en [14] se denominan $\mathrm{TM}^{\mathrm{z}}$ (campo magnético contenido en el plano $X Y$ transversal al eje $\hat{z}$ ) y $\mathrm{TE}^{\mathrm{z}}$ (campo eléctrico contenido en el plano $X Y$ transversal al eje $\hat{z}$ ). En la caracterización completa de un objeto dispersor resultará necesario, pues, obtener dos funciones de transferencia (o matrices) que caractericen al objeto dispersor ante incidencia $\mathrm{TM}^{\mathrm{z}}$ y $\mathrm{TE}^{\mathrm{z}}$, respectivamente. De esta forma, para calcular la dispersión que produce un objeto ante un cierto campo incidente, éste debe descomponerse en un espectro incidente $\mathrm{TM}^{\mathrm{z}}$ (correspondiente a un desarrollo de modos con polarización $\mathrm{TM}^{\mathrm{z}}$ ) y en otro espectro incidente $\mathrm{TE}^{\mathrm{z}}$ (correspondiente 
en este caso a un desarrollo de modos $\mathrm{TE}^{\mathrm{z}}$ ). A continuación, cada uno de estos espectros incidentes debe multiplicarse por la correspondiente matriz de caracterización del objeto dispersor; dando lugar a los espectros de campo dispersado por el objeto bajo análisis ante incidencia $\mathrm{TM}^{\mathrm{z}}$ y $\mathrm{TE}^{\mathrm{z}}$. El campo total dispersado se define, aplicando superposición, como la suma de los campos dispersados por el objeto ante las dos polarizaciones incidentes consideradas.

La aplicación práctica de la matriz de caracterización recién presentada se resume perfectamente en [66] y en [67]; donde se caracterizan diversos objetos dispersores canónicos ante incidencia con polarización $\mathrm{TM}^{\mathrm{z}}$ y $\mathrm{TE}^{\mathrm{z}}$, respectivamente. Haciendo uso de dichas matrices de caracterización, así como de sus propiedades, se presenta en [68] un algoritmo recursivo generalizado que permite analizar problemas de acoplo electromagnético entre diversos objetos dispersores para cualquier campo incidente.

El concepto de la matriz de caracterización se ha particularizado en este trabajo al análisis de dispersores (conductores perfectos) cuya geometría es invariante en una dimensión, reduciéndose por tanto el problema a un caso bidimensional; lo cual simplifica considerablemente la formulación al tratar con espectros de campo unidimensionales. El sistema de referencia que se emplea es, por consiguiente, un sistema de coordenadas cilíndricas $(\rho, \phi)$; y el objeto dispersor se mantiene invariante a lo largo del eje $\hat{z}$, tal y como se muestra en la figura 4.2. El análisis de objetos cuya geometría no fuera invariante en ninguna dimensión (problemas tridimensionales) se realizaría de manera similar al caso bidimensional ofrecido a continuación; pero utilizando un sistema de coordenadas esféricas en lugar de cilíndricas, y expresando los campos en términos de sus correspondientes espectros en coordenadas esféricas.

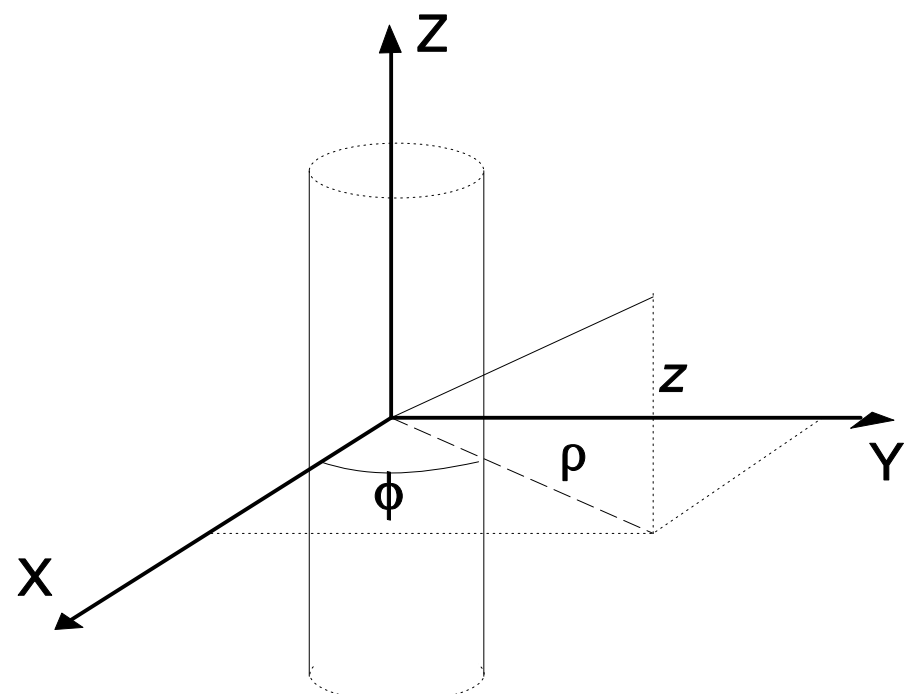

Figura 4.2: Sistema de coordenadas utilizado en la caracterización de dispersores bidimensionales. 
Como ya se ha mencionado anteriormente, el análisis de un objeto dispersor debe realizarse ante incidencia $\mathrm{TM}^{\mathrm{z}}$ y $\mathrm{TE}^{\mathrm{z}}$. Así pues, para el primer tipo de incidencia $\left(\mathrm{TM}^{\mathrm{z}}\right)$ y dado que el problema tiene carácter bidimensional, el campo eléctrico incidente tan sólo presenta componente en $\hat{z}\left(E_{z}^{i}\right)$. Consecuentemente, el campo eléctrico dispersado por dicho objeto tan sólo puede tener componente en $\hat{z}\left(E_{z}^{d}\right)$. El espectro del campo eléctrico $E_{z}^{d}$ quedará relacionado por tanto con el espectro del campo eléctrico $E_{z}^{i}$ a través de una matriz de caracterización, que llamaremos función de transferencia TM. De igual manera, para el segundo tipo de incidencia $\left(\mathrm{TE}^{\mathrm{z}}\right)$, se tiene ahora un campo magnético incidente que tan sólo presenta componente en $\hat{z}\left(H_{z}^{i}\right)$; y por consiguiente el campo magnético dispersado por el objeto tan sólo tiene componente según el eje $\hat{z}\left(H_{z}^{d}\right)$. En este segundo caso, el espectro del campo magnético $H_{z}^{d}$ se relaciona con el espectro del campo magnético $H_{z}^{i}$ a través de una segunda matriz de caracterización, que denominaremos función de transferencia TE.

Los campos indicente y dispersado, correspondientes a la polarización $\mathrm{TM}^{\mathrm{z}} \mathrm{y}$ $\mathrm{TE}^{\mathrm{z}}$, se expresan en términos de sus respectivos espectros cilíndricos [13]. En concreto, el campo eléctrico incidente $E_{z}^{i}$ (polarización $\mathrm{TM}^{\mathrm{z}}$ ) y el campo magnético incidente $H_{z}^{i}$ (polarización $\mathrm{TE}^{\mathrm{z}}$ ) se desarrollan en series de funciones de Bessel (ver apéndice $\mathrm{C}$ ), que presentan un valor finito en el origen; dando lugar a las siguientes expresiones

$$
\begin{aligned}
E_{z}^{i} & =\sum_{p=-N_{i}}^{N_{i}} i_{p}^{\mathrm{TM}} J_{p}(k \rho) e^{j p \phi} \\
H_{z}^{i} & =\sum_{p=-N_{i}}^{N_{i}} i_{p}^{\mathrm{TE}} J_{p}(k \rho) e^{j p \phi}
\end{aligned}
$$

donde $p$ representa el orden del p-ésimo modo incidente $J_{p}(k \rho) e^{j p \phi}$, y los coeficientes $i_{p}^{\mathrm{TM}}$ e $i_{p}^{\mathrm{TE}}$ las amplitudes de la p-ésima componente de los espectros cilíndricos asociados a los campos eléctrico y magnético incidentes. Aunque en principio los campos incidentes debieran expresarse mediante sumas de infinitos modos, a efectos prácticos estas series pueden truncarse en un número de modos tal, que permita reconstruir correctamente el campo incidente sobre la superficie del objeto bajo análisis. El criterio a seguir para seleccionar el número de modos a considerar en $(4.2)$ viene definido en $[1,12]$ como

$$
N_{i}>k a
$$

donde $k$ es el número de onda $(k=2 \pi / \lambda)$, y a representa la distancia del origen del sistema local de coordenadas al punto de la superficie del objeto más alejado de dicho origen. En los resultados prácticos, que se presentan en sucesivos capítulos, se ha escogido el criterio $N_{i}=1.5 \mathrm{ka}$; resultando adecuado para el tamaño de los 
objetos analizados. En el caso de caracterizar objetos cuya dimensión a resultara bastante menor que $\lambda$, podría escogerse un valor para $N_{i}$ mayor que el criterio recomendado de $1.5 \mathrm{ka}$; sin que ello supusiera un excesivo coste computacional. Por contra, si el objeto analizado es grande en términos de $\lambda$ el criterio propuesto puede disminuirse, ajustando más el valor seleccionado para $N_{i}$ al límite teórico definido en (4.3) como $k a$; con el objetivo de reducir el coste computacional. Persiguiendo esta misma finalidad, conviene escoger el centro del sistema local de coordenadas de manera que $a$, la distancia desde el origen al punto más lejano de la superficie del objeto, sea mínima; para de esta forma reducir las dimensiones de los espectros y las funciones de transferencia, y en consecuencia la complejidad de cálculo asociada al procedimiento en cuestión.

Los espectros de campo incidente, a efectos de implementación analítica, se expresan mediante vectores columna constituidos por $2 N_{i}+1$ elementos que representan las amplitudes $i_{p}^{\mathrm{TM}}$ e $i_{p}^{\mathrm{TE}}$ definidas en (4.2); los cuales presentan el siguiente aspecto

$$
E I^{\mathrm{TM}}=\left[\begin{array}{c}
i_{-N_{i}}^{\mathrm{TM}} \\
i_{-N_{i}+1}^{\mathrm{TM}} \\
\vdots \\
i_{N_{i}-1}^{\mathrm{TM}} \\
i_{N_{i}}^{\mathrm{TM}}
\end{array}\right]_{2 N_{i}+1 \times 1} E I^{\mathrm{TE}}=\left[\begin{array}{c}
i_{-N_{i}}^{\mathrm{TE}} \\
i_{-N_{i}+1}^{\mathrm{TE}} \\
\vdots \\
i_{N_{i}-1}^{\mathrm{TE}} \\
i_{N_{i}}^{\mathrm{TE}}
\end{array}\right]_{2 N_{i}+1 \times 1}
$$

Por otra parte, el campo eléctrico dispersado $E_{z}^{d}$ (polarización $\mathrm{TM}^{\mathrm{z}}$ ) y el campo magnético dispersado $H_{z}^{d}$ (polarización $\mathrm{TE}^{\mathrm{z}}$ ) se desarrollan en series de funciones de Hankel de segunda especie (ver apéndice $\mathrm{C}$ ), que presentan una singularidad en el origen y emergen hacia el infinito; dando lugar a las siguientes expresiones

$$
\begin{aligned}
& E_{z}^{d}=\sum_{q=-N_{d}}^{N_{d}} c_{q}^{\mathrm{TM}} H_{q}^{(2)}(k \rho) e^{j q \phi} \\
& H_{z}^{d}=\sum_{q=-N_{d}}^{N_{d}} c_{q}^{\mathrm{TE}} H_{q}^{(2)}(k \rho) e^{j q \phi}
\end{aligned}
$$

donde $q$ representa el orden del q-ésimo modo dispersado $H_{q}^{(2)}(k \rho) e^{j q \phi}$, y los coeficientes $c_{q}^{\mathrm{TM}}$ y $c_{q}^{\mathrm{TE}}$ son las amplitudes de la q-ésima componente de los espectros cilíndricos asociados a los campos eléctrico y magnético dispersados. De igual manera que para los campos incidentes, las series en (4.5) deben truncarse en un número de modos tal, que permita reconstruir el campo dispersado fuera de la circunferencia de radio a que contiene al objeto dispersor. Este número de modos 
emergentes, definido en (4.5) a partir de $N_{d}$, debe satisfacer la misma condición expresada anteriormente para $N_{i}$, es decir $N_{d}>k a$. En los casos prácticos analizados en este trabajo, se ha vuelto a escoger el mismo criterio que para $N_{i}$, es decir $N_{d}=1.5 \mathrm{ka}$; resultando apropiado para el tamaño de los objetos considerados. Ahora bien, como se indica en [1,12], este criterio puede resultar insuficiente para reconstruir el campo en las cercanías del elemento dispersor. En esta zona, debido a la existencia de campos evanescentes, se precisa de un mayor número de modos para reconstruir correctamente el campo dispersado; mientras que en la zona de campo lejano es suficiente con seguir el criterio expuesto. No obstante, adoptando el criterio recomendado $\left(N_{d}=1.5 \mathrm{ka}\right)$ para evaluar el campo dispersado en cualquier región del espacio (campo próximo y campo lejano), se han observado resultados bastante exactos (ver siguientes capítulos) en problemas de acoplo electromagnético entre diversos objetos que estén muy próximos o incluso en contacto.

De igual manera que para los espectros de campo incidente, a efectos de implementación analítica, los espectros de campo dispersado se expresan mediante vectores columna formados por $2 N_{d}+1$ elementos que representan las amplitudes $c_{q}^{\mathrm{TM}}$ y $c_{q}^{\mathrm{TE}}$ definidas en $(4.5)$; los cuales presentan el siguiente aspecto

$$
E D^{\mathrm{TM}}=\left[\begin{array}{c}
c_{-N_{d}}^{\mathrm{TM}} \\
c_{-N_{d}+1}^{\mathrm{TM}} \\
\vdots \\
c_{N_{d}-1}^{\mathrm{TM}} \\
c_{N_{d}}^{\mathrm{TM}}
\end{array}\right]_{2 N_{d}+1 \times 1} E D^{\mathrm{TE}}=\left[\begin{array}{c}
c_{-N_{d}}^{\mathrm{TE}} \\
c_{-N_{d}+1}^{\mathrm{TE}} \\
\vdots \\
c_{N_{d}-1}^{\mathrm{TE}} \\
c_{N_{d}}^{\mathrm{TE}}
\end{array}\right]_{2 N_{d}+1 \times 1}
$$

La caracterización del objeto dispersor supone calcular la respuesta de dicho objeto a cada posible modo incidente $J_{p}(k \rho) e^{j p \phi}$, tanto para polarización $\mathrm{TM}^{\mathrm{z}}$ como TEz . Esta respuesta se obtiene empleando técnicas clásicas de análisis de problemas electromagnéticos, en concreto en este trabajo se ha utilizado Óptica Física y Ecuación Integral; que, combinadas con métodos espectrales, permiten expresar los campos dispersados en términos de sus respectivos espectros a través de las siguientes expresiones

$$
\begin{aligned}
E_{z}^{i}=J_{p}(k \rho) e^{j p \phi} & \Longrightarrow E_{z}^{d}=\sum_{q=-N_{d}}^{N_{d}} d_{q p}^{\mathrm{TM}} H_{q}^{(2)}(k \rho) e^{j q \phi} \\
H_{z}^{i}=J_{p}(k \rho) e^{j p \phi} & \Longrightarrow H_{z}^{d}=\sum_{q=-N_{d}}^{N_{d}} d_{q p}^{\mathrm{TE}} H_{q}^{(2)}(k \rho) e^{j q \phi}
\end{aligned}
$$

donde los coeficientes $d_{q p}^{\mathrm{TM}}$ y $d_{q p}^{\mathrm{TE}}$ representan la contribución de la componente p-ésima del correspondiente espectro incidente (polarización $\mathrm{TM}^{\mathrm{z}}$ y $\mathrm{TE}^{\mathrm{z}}$, res- 
pectivamente) a la componente $q$-ésima del correspondiente espectro dispersado (polarización $\mathrm{TM}^{\mathrm{z}}$ y $\mathrm{TE}^{\mathrm{z}}$, respectivamente).

Por consiguiente, la respuesta global del objeto dispersor ante una incidencia definida por las amplitudes espectrales $i_{p}^{\mathrm{TM}}$ e $i_{p}^{\mathrm{TE}}$ (polarización $\mathrm{TM}^{\mathrm{z}}$ y TE $\mathrm{TE}^{\mathrm{z}}$, respectivamente) puede expresarse usando los coeficientes $d_{q p}^{\mathrm{TM}}$ y $d_{q p}^{\mathrm{TE}}$ de la siguiente manera

$$
\begin{aligned}
E_{z}^{i} & =\sum_{p=-N_{i}}^{N_{i}} i_{p}^{\mathrm{TM}} J_{p}(k \rho) e^{j p \phi} \\
& \Longrightarrow E_{z}^{d}=\sum_{p=-N_{i}}^{N_{i}} i_{p}^{\mathrm{TM}}\left(\sum_{q=-N_{d}}^{N_{d}} d_{q p}^{\mathrm{TM}} H_{q}^{(2)}(k \rho) e^{j q \phi}\right) \\
H_{z}^{i} & =\sum_{p=-N_{i}}^{N_{i}} i_{p}^{\mathrm{TE}} J_{p}(k \rho) e^{j p \phi} \\
& \Longrightarrow H_{z}^{d}=\sum_{p=-N_{i}}^{N_{i}} i_{p}^{\mathrm{TE}}\left(\sum_{q=-N_{d}}^{N_{d}} d_{q p}^{\mathrm{TE}} H_{q}^{(2)}(k \rho) e^{j q \phi}\right)
\end{aligned}
$$

Reordenando los sumatorios que aparecen en (4.8), se obtienen las siguientes expresiones para los campos dispersados por el objeto ante incidencia con polarización $\mathrm{TM}^{\mathrm{z}}$ y $\mathrm{TE}^{\mathrm{z}}$

$$
\begin{aligned}
& E_{z}^{d}=\sum_{q=-N_{d}}^{N_{d}}\left(\sum_{p=-N_{i}}^{N_{i}} i_{p}^{\mathrm{TM}} d_{q p}^{\mathrm{TM}}\right) H_{q}^{(2)}(k \rho) e^{j q \phi} \\
& H_{z}^{d}=\sum_{q=-N_{d}}^{N_{d}}\left(\sum_{p=-N_{i}}^{N_{i}} i_{p}^{\mathrm{TE}} d_{q p}^{\mathrm{TE}}\right) H_{q}^{(2)}(k \rho) e^{j q \phi}
\end{aligned}
$$

Comparando (4.9) con (4.5), es posible deducir una relación en términos matriciales entre los espectros de campo dispersado e incidente definida como

$$
\begin{aligned}
& E D^{\mathrm{TM}}=D^{\mathrm{TM}} \cdot E I^{\mathrm{TM}} \\
& E D^{\mathrm{TE}}=D^{\mathrm{TE}} \cdot E I^{\mathrm{TE}}
\end{aligned}
$$

donde $E D^{\mathrm{TM}}, E D^{\mathrm{TE}}$ y $E I^{\mathrm{TM}}, E I^{\mathrm{TE}}$ denotan respectivamente los vectores columna que representan los espectros de campo dispersado e incidente para polarización $\mathrm{TM}^{\mathrm{z}}$ y $\mathrm{TE}^{\mathrm{z}}$; mientras que $D^{\mathrm{TM}}$ y $D^{\mathrm{TE}}$ designan las matrices de caracterización que definen respectivamente las funciones de transferencia TM y TE del 
objeto analizado. Estas matrices se construyen a partir de los coeficientes $d_{q p}^{\mathrm{TM}} \mathrm{y}$ $d_{q p}^{\mathrm{TE}}$, previamente introducidos, de la siguiente forma

$$
\begin{aligned}
D^{\mathrm{TM}}= & {\left[\begin{array}{cccc}
d_{-N_{d}-N_{i}}^{\mathrm{TM}} & d_{-N_{d}-N_{i}+1}^{\mathrm{TM}} & \cdots & d_{-N_{d} N_{i}}^{\mathrm{TM}} \\
d_{-N_{d}+1-N_{i}}^{\mathrm{TM}} & d_{-N_{d}+1-N_{i}+1}^{\mathrm{TM}} & \cdots & d_{-N_{d}+1 N_{i}}^{\mathrm{TM}} \\
\vdots & \vdots & \ddots & \vdots \\
d_{N_{d}-N_{i}}^{\mathrm{TM}} & d_{N_{d}-N_{i}+1}^{\mathrm{TM}} & \cdots & d_{N_{d} N_{i}}^{\mathrm{TM}}
\end{array}\right]_{2 N_{d}+1 \times 2 N_{i}+1} } \\
D^{\mathrm{TE}}= & {\left[\begin{array}{cccc}
d_{-N_{d}-N_{i}}^{\mathrm{TE}} & d_{-N_{d}-N_{i}+1}^{\mathrm{TE}} & \cdots & d_{-N_{d} N_{i}}^{\mathrm{TE}} \\
d_{-N_{d}+1-N_{i}}^{\mathrm{TE}} & d_{-N_{d}+1-N_{i}+1}^{\mathrm{TE}} & \cdots & d_{-N_{d}+1 N_{i}}^{\mathrm{TE}} \\
\vdots & \vdots & \ddots & \vdots \\
d_{N_{d}-N_{i}}^{\mathrm{TE}} & d_{N_{d}-N_{i}+1}^{\mathrm{TE}} & \cdots & d_{N_{d} N_{i}}^{\mathrm{TE}}
\end{array}\right]_{2 N_{d}+1 \times 2 N_{i}+1} }
\end{aligned}
$$

Recordando (4.3), se puede observar en (4.11) que el tamaño de la matriz de caracterización, tanto para incidencia TM como para incidencia TE, aumenta a medida que las dimensiones eléctricas del objeto dispersor crecen; y por tanto, cuando éste sea muy grande en términos de $\lambda$ resultará más eficiente, desde el punto de vista computacional, segmentar el contorno del objeto en elementos más pequeños, caracterizarlos individualmente, y analizar entonces el acoplo electromagnético o realimentación múltiple entre dichos elementos, empleando para ello alguna de las técnicas descritas en el capítulo 6 .

La caracterización de un objeto dispersor se divide en dos fases. En un primer paso se calcula una matriz $D I$, denominada matriz de corrientes, que relaciona la distribución de corrientes inducidas sobre la superficie del objeto en cuestión con el espectro asociado al campo incidente. A continuación se construye una segunda matriz $D E$, denominada matriz de espectro, que relaciona el espectro asociado al campo dispersado por el objeto con la distribución de corrientes inducidas sobre su superficie. Los diversos métodos planteados en este trabajo para computar cada una de estas matrices se detallan en las próximas secciones. Finalmente, la matriz de caracterización o función de transferencia del objeto dispersor se obtiene simplemente multiplicando la matriz de espectro por la matriz de corrientes del siguiente modo

$$
D=D E \cdot D I
$$

Una vez calculada la matriz de caracterización, a efectos de cálculo del campo dispersado por el objeto bajo análisis, el espacio exterior al objeto dispersor se divide en dos regiones; tal y como se muestra en la figura 4.3. Esta división del espacio se debe a que tan sólo es posible reconstruir el campo dispersado a partir de su correspondiente espectro cilíndrico en la zona libre de fuentes; es decir, en la zona externa a la circunferencia que contiene al objeto (región R3 en fig. 4.3). 


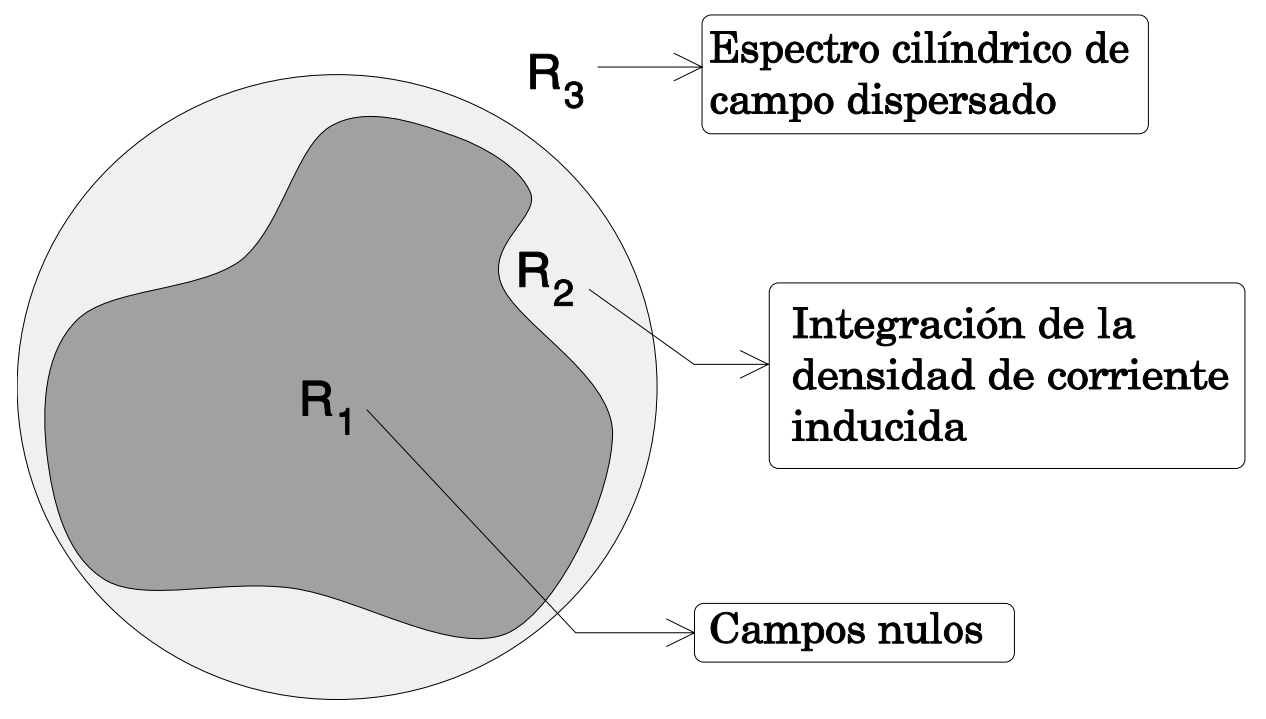

Figura 4.3: Regiones para calcular el campo dispersado por un conductor perfecto.

En la zona interna a dicha circunferencia pero externa al objeto (región $\mathbf{R} 2$ en fig. 4.3), el campo dispersado se obtiene a partir de la distribución de corrientes inducidas sobre la superficie del objeto dispersor. Para reconstruir el campo dispersado en la región libre de fuentes, a partir de su correspondiente espectro, se requiere multiplicar la matriz de caracterización (o función de transferencia) del objeto considerado por el espectro del campo incidente. La distribución de corrientes en la superficie del objeto se calcula multiplicando la matriz de corrientes por el espectro del campo incidente; y el campo dispersado en la zona interior de la circunferencia que contiene al objeto se obtiene integrando el campo dispersado por cada elemento de corriente inducida.

\subsection{Matriz de Corrientes}

En esta sección, se describen técnicas para construir la matriz de corrientes; que relaciona la distribución de corrientes inducidas en la superficie del objeto dispersor con el espectro asociado al campo incidente. La obtención de esta matriz requiere determinar en principio la distribución de la densidad de corriente inducida sobre la superficie del objeto por un cierto campo incidente.

El problema que se pretende resolver consiste por tanto en calcular la densidad de corriente inducida sobre un objeto metálico perfectamente conductor $(\sigma=\infty)$, cuya sección transversal (plano $X Y$ ) se muestra en la figura 4.4.

En la figura 4.4, se aprecia la existencia de unas fuentes $\vec{J}_{1}$ y $\vec{M}_{1}$ que producen unos campos $\vec{E}^{i}$ y $\vec{H}^{i}$, conocidos e incidentes sobre la superficie del objeto dispersor. Dicho objeto, como respuesta a estos campos incidentes, producirá unos 


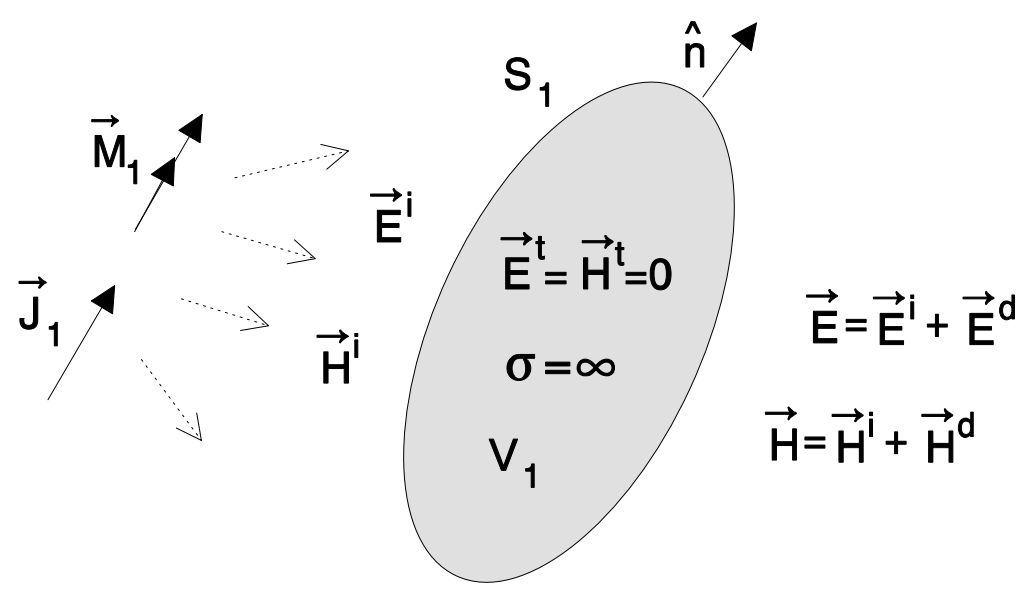

Figura 4.4: Sección transversal de un dispersor metálico perfectamente conductor.

campos dispersados $\vec{E}^{d}$ y $\vec{H}^{d}$; de modo que los campos totales en la zona externa al objeto metálico vendrán expresados como

$$
\begin{aligned}
\vec{E} & =\vec{E}^{i}+\vec{E}^{d} \\
\vec{H} & =\vec{H}^{i}+\vec{H}^{d}
\end{aligned}
$$

Los campos totales en el interior del objeto dispersor (volumen $V_{1}$ en fig. 4.4) son nulos $\left(\vec{E}^{t}=\vec{H}^{t}=0\right)$; mientras que sobre la superficie de dicho objeto (designada por $S_{1}$ en fig. 4.4) las componentes tangenciales de campo eléctrico deben anularse, al estar considerando un conductor eléctrico perfecto, y las componentes tangenciales de campo magnético deben igualar la densidad de corriente, que llamaremos $\vec{J}_{s}$, inducida sobre la superficie $S_{1}$ del objeto dispersor. Por tanto, sobre dicha superficie $S_{1}$ se satisfacen las siguientes ecuaciones

$$
\begin{gathered}
\vec{M}_{s}=-\hat{n} \times\left(\vec{E}-\vec{E}^{t}\right)=-\hat{n} \times(\vec{E})=-\hat{n} \times\left(\vec{E}^{i}+\vec{E}^{d}\right)=0 \\
\vec{J}_{s}=\hat{n} \times\left(\vec{H}-\vec{H}^{t}\right)=\hat{n} \times \vec{H}=\hat{n} \times\left(\vec{H}^{i}+\vec{H}^{d}\right)
\end{gathered}
$$

Haciendo uso del Método conocido como Equivalente Físico [69], el problema que estamos analizando puede sustituirse por otro problema donde las fuentes reales han sido reemplazadas por unas fuentes equivalentes; las cuales producen en la región externa al objeto dispersor los mismos campos dispersados por dicho objeto ante las fuentes reales en la situación original. Por tanto, es posible expresar el problema real mostrado en la figura 4.4 mediante otro problema, denominado equivalente físico, que se muestra a continuación en la figura 4.5. 


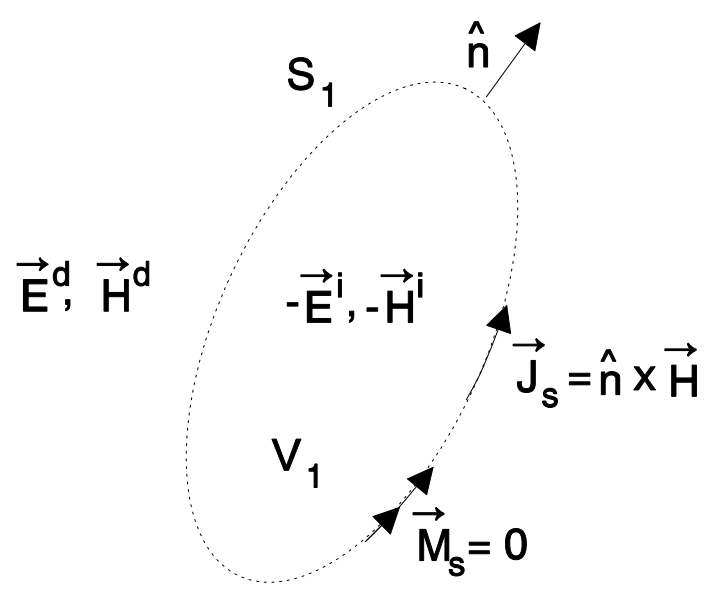

Figura 4.5: Equivalente físico de un dispersor metálico perfectamente conductor.

Como se puede apreciar en el equivalente físico de la figura 4.5, el objeto metálico del problema original se sustituye por la densidad de corriente superficial $\vec{J}_{s}\left(\vec{M}_{s}\right.$ es nula). Esta densidad de corriente produce en el exterior del objeto los mismos campos $\left(\vec{E}^{d}\right.$ y $\left.\vec{H}^{d}\right)$ que en la situación real (fig. 4.4). En el interior del objeto se asumen unos campos distintos (concretamente $-\vec{E}^{i}$ y $-\vec{H}^{i}$ ) a los que produciría $\vec{J}_{s}$, para que los campos totales en dicha región sean nulos al igual que en la situación real (fig. 4.4). Del equivalente físico se deduce que para resolver el problema de un dispersor metálico, tan sólo es necesario conocer la densidad de corriente superficial $\vec{J}_{s}$; que puede obtenerse según (4.14) o (4.15) a partir de las componentes tangenciales del campo eléctrico o magnético sobre $S_{1}$, donde los campos eléctrico $\left(\overrightarrow{E^{d}}\right)$ y magnético $\left(\vec{H}^{d}\right)$ dispersados son desconocidos.

Una técnica clásica, conocida como Óptica Física [69], permite determinar $\vec{J}_{s}$ mediante una aproximación a un valor conocido en problemas sujetos a determinadas condiciones.

Una posible alternativa a la aproximación de Óptica Física consiste en plantear, utilizando respectivamente (4.14) o (4.15), la correspondiente Ecuación Integral para el campo eléctrico o para el campo magnético [69]; donde la incógnita resulta ser la densidad de corriente $\vec{J}_{s}$ buscada. Estas dos Ecuaciones Integrales pueden resolverse aplicando un método numérico, conocido como el Método de los Momentos [70], que aproxima en este caso $\vec{J}_{s}$ por un conjunto de funciones conocidas con pesos incógnita; transformando la Ecuación Integral en un sistema de ecuaciones algebraicas de fácil resolución.

Ahora bien, en la construcción de la matriz de corrientes se requiere conocer la distribución de corrientes sobre la superficie del objeto dispersor; para lo cual 
se discretiza la superficie de dicho objeto en un número finito de segmentos $\left(N_{s}\right)$. Este número, lógicamente, debe crecer a medida que aumenta el tamaño eléctrico del objeto. En la figura 4.6, se muestra la discretización de un objeto dispersor en $N_{s}$ hilos de corriente paralelos al eje $\hat{z}$; debido a que la incidencia presenta polarización $\mathrm{TM}^{\mathrm{z}}$. En el caso de que la incidencia tuviera polarización $\mathrm{TE}^{\mathrm{z}}$, los hilos de corriente se orientarían en dirección tangente al contorno del objeto dispersor, y estarían contenidos en el plano transversal $X Y$.

a)

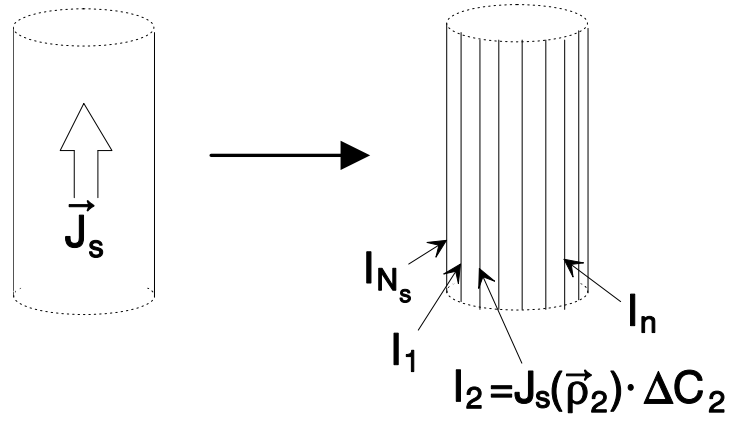

b)

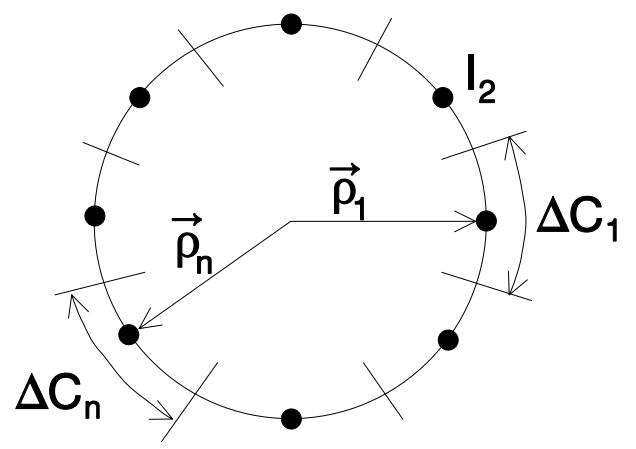

Figura 4.6: Discretización de un objeto dispersor para calcular la distribución de corrientes ante incidencia $\mathrm{TM}^{\mathrm{z}}$. Vista longitudinal en a) y transversal en b).

Por cada uno de los mencionados segmentos circulará una cierta intensidad de corriente $I_{n}$, que resulta de integrar la densidad de corriente $J_{s}\left(\overrightarrow{\rho^{\prime}}\right)$ a lo largo del correspondiente segmento de la superficie del objeto. Como los segmentos presentan una longitud $\left(\Delta C_{n}\right)$ lo suficientemente pequeña para preservar exactitud en los resultados, se puede considerar que la densidad de corriente superficial $J_{s}\left(\vec{\rho}^{\prime}\right)$ es constante a lo largo de cada segmento e igual a $J_{s}\left(\vec{\rho}_{n}\right)$; reduciéndose la integral al siguiente producto

$$
I_{n}=\int_{\Delta C_{n}} J_{s}\left(\overrightarrow{\rho^{\prime}}\right) d l^{\prime} \stackrel{\Delta C_{n} \rightarrow 0}{\simeq} J_{s}\left(\vec{\rho}_{n}\right) \Delta C_{n}
$$

donde el vector $\vec{\rho}_{n}$ indica un punto del $n$-ésimo segmento (normalmente el punto medio).

Tras esta discretización, es posible representar la distribución de corrientes inducidas en la superficie del objeto dispersor mediante el siguiente vector columna de $N_{s}$ elementos

$$
I=\left[\begin{array}{c}
I_{1} \\
I_{2} \\
\vdots \\
I_{N_{s}}
\end{array}\right]_{N_{s} \times 1}
$$


La matriz de corrientes de un objeto dispersor relacionará por tanto un vector de $N_{s}$ intensidades de corriente, como el mostrado en (4.17), con el espectro asociado al campo incidente. La determinación de los elementos de dicha matriz se lleva a cabo seleccionando en cada ocasión una componente distinta $\left(J_{p}(k \rho) e^{j p \phi}\right)$ del desarrollo modal incidente expresado en (4.2). A continuación, se determina la densidad de corriente que dicha componente modal incidente induce en la superficie del objeto dispersor, bien mediante Óptica Física o resolviendo la correspondiente Ecuación Integral. Finalmente, esta densidad de corriente se discretiza mediante (4.16) en un vector columna de intensidades de corriente; que constituye la p-ésima columna de la matriz de corrientes. Repitiendo este proceso para cada modo incidente $\left(p=-N_{i}, \ldots, N_{i}\right)$ en (4.2); se construye la matriz de corrientes, cuyas dimensiones son $N_{s} \times 2 N_{i}+1$. Cada elemento de la matriz de corrientes, denominado $I_{n p}$, representa por tanto la contribución del $p$-ésimo modo incidente a la corriente inducida en el $n$-ésimo segmento de la superficie del objeto; de modo que la relación entre la distribución de corrientes inducidas en el objeto y el espectro de campo incidente se expresa como

$$
I=D I \cdot E I
$$

donde $I$ es un vector columna (4.17) que contiene la corriente inducida en cada uno de $\operatorname{los} N_{s}$ segmentos del objeto dispersor, EI es el vector columna (4.4) con las componetes espectrales del campo incidente y $D I$ es la matriz de corrientes, cuyo aspecto se ofrece a continuación

$$
D I=\left[\begin{array}{cccc}
I_{1-N_{i}} & I_{1-N_{i}+1} & \cdots & I_{1 N_{i}} \\
I_{2-N_{i}} & I_{2-N_{i}+1} & \cdots & I_{2 N_{i}} \\
\vdots & \vdots & \ddots & \vdots \\
I_{N_{s}-N_{i}} & I_{N_{s}-N_{i}+1} & \cdots & I_{N_{s} N_{i}}
\end{array}\right]_{N_{s} \times 2 N_{i}+1}
$$

Es importante reseñar que esta matriz de corrientes resulta necesaria no sólo para obtener la matriz de caracterización de un objeto dispersor; sino también para determinar las corrientes inducidas sobre su superficie, a partir de las cuales se puede calcular el campo dispersado en el interior de la circunferencia que circunscribe a dicho objeto (región $\mathbf{R} 2$ en fig. 4.3). En el cálculo de este campo se necesita además conocer la posición de cada elemento de corriente, e incluso en el caso de incidencia con polarización $\mathrm{TE}^{\mathrm{z}}$ se requiere, como se verá posteriormente, tener conocimiento del vector unitario normal a la superficie del objeto dispersor en cada elemento de corriente.

Como se ha mencionado anteriormente existen dos técnicas clásicas, Óptica Física y Ecuación Integral resuelta por el Método de los Momentos, que permiten encontrar la densidad de corriente $\left(\vec{J}_{s}\right)$ inducida sobre la superficie de un objeto dispersor metálico. Las expresiones analíticas correspondientes a ambos métodos se detallan a continuación. 


\subsection{1 Óptica Física}

Cuando el objeto dispersor bajo análisis es un conductor perfecto, plano e infinito, las componentes tangenciales de campo magnético incidente y dispersado se encuentran en fase y presentan la misma amplitud [69]. En este caso, la densidad de corriente $\vec{J}_{s}$ puede calcularse fácilmente en función del campo magnético incidente como

$$
\vec{J}_{s}=\hat{n} \times \vec{H}=\hat{n} \times\left(\vec{H}^{i}+\vec{H}^{d}\right)=2 \hat{n} \times \vec{H}^{i}
$$

El equivalente físico, también conocido por equivalente de Óptica Física [69], para el caso de un conductor perfecto, plano e infinito se presenta en la figura 4.7. Si el objeto bajo análisis presenta una superficie plana no infinita, pero grande en términos eléctricos, puede considerarse localmente como plana e infinita, y se puede aplicar por tanto la aproximación de Óptica Física; que consiste en aproximar el campo magnético dispersado por el campo magnético incidente $\left(\vec{H}^{d}=\vec{H}^{i}\right)$.

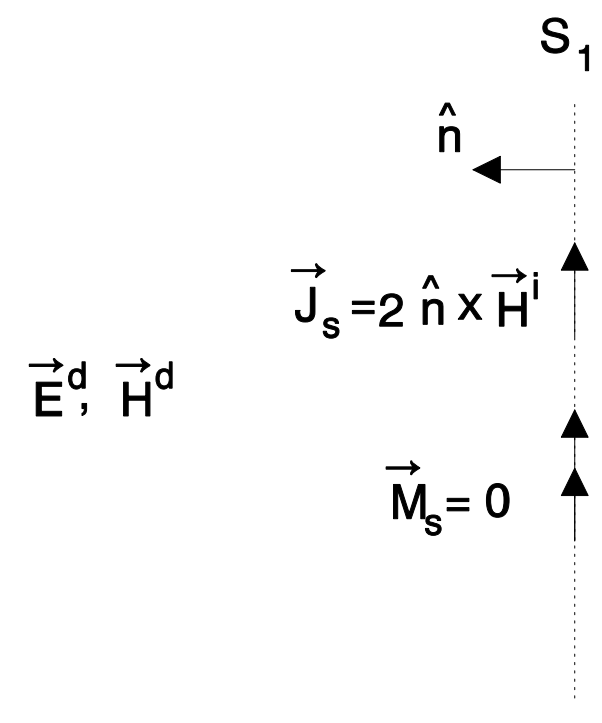

Figura 4.7: Equivalente físico de un conductor perfecto, plano e infinito.

Haciendo uso de la aproximación de Óptica Física, válida sólo si el objeto es grande eléctricamente, la densidad de corriente $\vec{J}_{s}$ se aproxima mediante la siguiente expresión

$$
\vec{J}_{s} \simeq 2 \hat{n} \times \vec{H}^{i}
$$

Como $\vec{H}^{i}$ es conocido, $\vec{J}_{s}$ queda perfectamente determinada utilizando (4.21). La aproximación de Óptica Física permite obtener la densidad de corriente inducida a partir del campo incidente, y por tanto abordar la construcción de la matriz de corrientes. No obstante, la aproximación de Óptica Física proporciona la densidad de corriente inducida por una onda plana incidente; mientras que la matriz de corrientes que se desea construir relaciona corrientes inducidas con 
espectro cilíndrico de campo incidente. Para construir esta matriz de corrientes se obtendrá en primer lugar una matriz $D I_{p}$, que relacione corrientes inducidas con espectro de ondas planas incidente (usando la aproximación de óptica física), y en segundo lugar una matriz $D_{p c}$ de transformación de espectro cilíndrico a plano (ver apéndice C). La matriz de corrientes deseada se obtiene multiplicando ambas matrices del siguiente modo

$$
D I=D I_{p} \cdot D_{p c}
$$

consiguiendo, de esta forma, que la matriz DI relacione corrientes inducidas con espectro cilíndrico incidente.

Como ha quedado demostrado, las corrientes inducidas por una onda plana incidente sobre un objeto metálico quedan perfectamente determinadas utilizando la aproximación de Óptica Física. Para construir una matriz que relacione corrientes inducidas con espectro plano incidente, se debe obtener la corriente inducida por cada una de las posibles ondas planas de dicho espectro plano incidente, que designaremos por $p(\beta)$. Este espectro contínuo, para poder implementar computacionalmente el problema, debe discretizarse; dando lugar a un vector de $N_{\beta}$ componentes espectrales de amplitud $p_{m}=p\left(\beta_{m}=2 \pi m / N_{\beta}\right)$, con $m$ variando desde 0 hasta $N_{\beta}-1$. Este vector permite reconstruir el campo incidente como una suma de $N_{\beta}$ ondas planas, cada una con una dirección de avance asociada de valor $\beta_{m}=2 \pi m / N_{\beta}$. Los pesos que ponderan cada una de estas ondas planas son precisamente los coeficientes $p_{m}$. Conociendo la respuesta del objeto metálico a cada una de las ondas planas por separado, se puede calcular la respuesta a cualquier campo como la suma de las respuestas a cada una de esas ondas, ponderadas por los correspondientes coeficientes del espectro de ondas planas asociado al campo incidente. Por lo tanto, para caracterizar al objeto dispersor ante cualquier incidencia es necesario calcular las corrientes inducidas por cada onda plana incidente según $\beta_{m}$; y posteriormente almacenarlas en la matriz $D I_{p}$, que relaciona intensidades de corriente inducidas en el objeto con espectro plano incidente de la siguiente manera

$$
\left[\begin{array}{c}
I_{1} \\
I_{2} \\
\vdots \\
I_{N_{s}}
\end{array}\right]_{N_{s} \times 1}=\left[\begin{array}{cccc}
A_{10} & A_{11} & \cdots & A_{1 N_{\beta}-1} \\
A_{20} & A_{21} & \cdots & A_{2 N_{\beta}-1} \\
\vdots & \vdots & \ddots & \vdots \\
A_{N_{s} 0} & A_{N_{s} 1} & \cdots & A_{N_{s} N_{\beta}-1}
\end{array}\right]_{N_{s} \times N_{\beta}} \cdot\left[\begin{array}{c}
p_{0} \\
p_{1} \\
\vdots \\
p_{N_{\beta}-1}
\end{array}\right]_{N_{\beta} \times 1}
$$

donde las expresiones analíticas para los coeficientes $A_{n m}$ dependen del tipo de incidencia (polarización $\mathrm{TM}^{\mathrm{z}}$ o $\mathrm{TE}^{\mathrm{z}}$ ) que se considere.

Para obtener la matriz de corrientes deseada $D I$, haciendo uso de la aproximación de Óptica Física, se requiere encontrar una matriz $D_{p c}$ que transforme el espectro cilíndrico asociado al campo incidente en un espectro de ondas planas. 
En el apéndice $\mathrm{C}$, se desarrolla el proceso seguido para obtener dicha matriz; que relaciona ambos espectros del siguiente modo

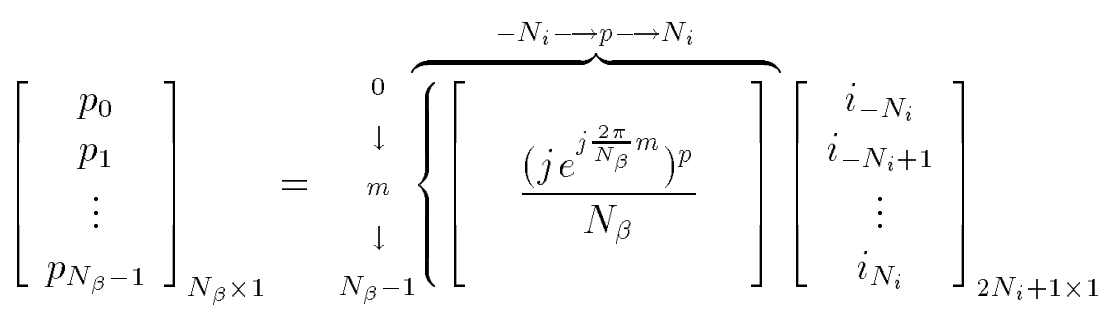

La matriz de corrientes $D I$, que relaciona la corriente inducida sobre la superficie del objeto con el espectro cilíndrico incidente, se expresa mediante el siguiente producto de las matrices $D I_{p}$ y $D_{p c}$

$$
D I=\left[\begin{array}{cccc}
A_{10} & A_{11} & \cdots & A_{1 N_{\beta}-1} \\
A_{20} & A_{21} & \cdots & A_{2 N_{\beta}-1} \\
\vdots & \vdots & \ddots & \vdots \\
A_{N_{s} 0} & A_{N_{s} 1} & \cdots & A_{N_{s} N_{\beta}-1}
\end{array}\right]_{N_{s} \times N_{\beta}} \quad \begin{gathered}
N_{\beta}-1 \\
\downarrow
\end{gathered}
$$

A continuación, se presentan las expresiones para los coeficientes $A_{n m}$ correspondientes a cada posible tipo de polarización $\left(\mathrm{TM}^{\mathrm{z}}\right.$ o $\left.\mathrm{TE}^{\mathrm{z}}\right)$ del campo incidente.

\section{Polarización $\mathrm{TM}^{\mathrm{Z}}$}

El campo eléctrico correspondiente a una onda plana incidente (polarización $\mathrm{TM}^{\mathrm{z}}$ ) con un ángulo de incidencia $\phi=\beta$ (ver figura C.1) viene definido por

$$
\overrightarrow{E^{i}}=e^{-j k \rho \cos (\phi-\beta)} \hat{z}
$$

y el campo magnético correspondiente se expresa como

$$
\vec{H}^{i}=\frac{1}{\eta}(\hat{x} \sin \beta-\hat{y} \cos \beta) e^{-j k \rho \cos (\phi-\beta)}
$$

donde $\eta$ es la impedancia característica en espacio libre $(\eta=\sqrt{\mu / \epsilon})$.

Haciendo uso de (4.27) y de la aproximación de Óptica Física (4.21), se obtiene la siguiente expresión para la densidad de corriente $\vec{J}_{s}$ que produce la onda plana (dirigida según $\phi=\beta$ ) en cualquier punto (definido por sus coordenadas cilíndricas $\left(\rho_{n}, \phi_{n}\right)$ y su vector director $\vec{\rho}_{n}=\rho_{n} \hat{\rho}$ ) de la superficie del objeto dispersor

$$
\begin{aligned}
\vec{J}_{s}\left(\overrightarrow{\rho_{n}}\right) & \simeq 2 \hat{n} \times \vec{H}^{i}\left(\vec{\rho}_{n}\right) \\
& =2 \hat{n} \times \frac{1}{\eta}(\hat{x} \sin \beta-\hat{y} \cos \beta) e^{-j k \rho_{n} \cos \left(\phi_{n}-\beta\right)}
\end{aligned}
$$


La expresión (4.28) para $\vec{J}_{s}$ es válida tan sólo en aquella región de la superficie del objeto que ilumina la onda plana, definida para el caso particular de un cilindro conductor perfecto como aquellos puntos de su contorno que satisfacen la condición $\phi \in[\beta+\pi / 2, \beta+3 \pi / 2]$ (ver figura 4.8). En el resto de la superficie del objeto dispersor la onda plana no incide; produciendo una zona de sombra donde la densidad de corriente inducida se considera nula, tal y como se muestra en la figura 4.8 para el caso del cilindro conductor.

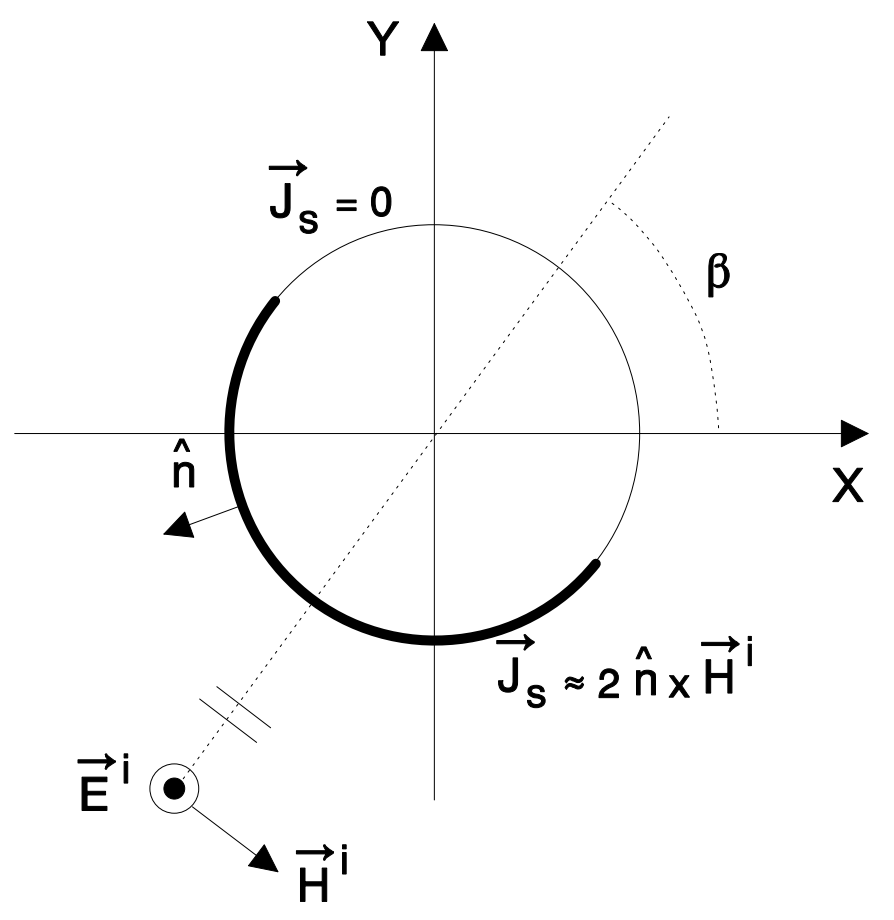

Figura 4.8: Incidencia de una onda plana sobre un cilindro infinito.

Como se ha explicado anteriormente, la densidad de corriente inducida $\vec{J}_{s}$ se muestrea a lo largo de la superficie del objeto dispersor; dando lugar a $N_{s}$ hilos de corriente (en este caso infinitos y dirigidos según el eje $\hat{z}$ ). La intensidad asociada a cada uno de estos hilos de corriente se aproxima, recordando (4.16), mediante el producto de la densidad de corriente en cada segmento, donde se supondrá constante, por la longitud de dicho segmento; tal y como se expresa a continuación

$$
I_{n}=\vec{J}_{s}\left(\vec{\rho}_{n}\right) \cdot \hat{z} \Delta C_{n}=J_{z}\left(\vec{\rho}_{n}\right) \Delta C_{n}
$$

donde $\vec{\rho}_{n}$ indica el $n$-ésimo elemento de corriente (normalmente el punto medio del correspondiente segmento) sobre la superficie del objeto dispersor. Al tratarse de incidencia con polarización $\mathrm{TM}^{\mathrm{z}}$, tanto el campo eléctrico incidente como la corriente presentan sólo componente en $\hat{z}$. Por esta razón, para obtener la corriente que circula por cada segmento se ha multiplicado escalarmente en (4.29) la densidad de corriente inducida $\vec{J}_{s}$ por el vector unitario $\hat{z}$. 
Finalmente, combinando (4.28) y (4.29) se obtiene la siguiente expresión para los coeficientes $A_{n m}$, presentes en (4.23), ante incidencia con polarización TM ${ }^{\mathrm{z}}$

$$
A_{n m}=\left\{\begin{array}{cl}
{\left[2 \hat{n} \times \frac{1}{\eta}\left(\hat{x} \sin \left(\beta_{m}\right)-\hat{y} \cos \left(\beta_{m}\right)\right)\right.} & \text { si } \phi_{n} \in\left[\beta_{m}+\frac{\pi}{2}, \beta_{m}+\frac{3 \pi}{2}\right] \\
\left.e^{-j k \rho_{n} \cos \left(\phi_{n}-\beta_{m}\right)} \Delta C_{n}\right] \cdot \hat{z} & \text { en caso contrario } \\
0 &
\end{array}\right.
$$

\section{Polarización $\mathrm{TE}^{\mathrm{Z}}$}

Cuando la onda plana incidente (con ángulo de incidencia $\phi=\beta$ ) presenta polarización $\mathrm{TE}^{\mathrm{z}}$, su campo magnético viene definido como

$$
\vec{H}^{i}=e^{-j k \rho \cos (\phi-\beta)} \hat{z}
$$

Usando de nuevo la aproximación de Óptica Física (4.21), se obtiene la siguiente expresión para la densidad de corriente $\vec{J}_{s}$ que induce la onda plana, con polarización $\mathrm{TE}^{\mathrm{z}} \mathrm{y}$ dirigida según $\phi=\beta$, en cualquier punto (definido de igual manera que en el apartado anterior por sus coordenadas cilíndricas y su vector director) de la superficie del objeto dispersor

$$
\begin{aligned}
\vec{J}_{s}\left(\vec{\rho}_{n}\right) & \simeq 2 \hat{n} \times \vec{H}^{i}\left(\vec{\rho}_{n}\right) \\
& =2 \hat{n} \times \hat{z} e^{-j k \rho_{n} \cos \left(\phi_{n}-\beta\right)}
\end{aligned}
$$

La validez de la expresión (4.32) para $\vec{J}_{s}$ se reduce, al igual que para la otra polarización, a la zona de la superficie del objeto que ilumina la onda plana; definida nuevamente, en el caso del cilindro, por aquellos puntos de su contorno que satisfacen la condición $\phi \in[\beta+\pi / 2, \beta+3 \pi / 2]$. En el resto de la superficie del objeto dispersor la onda plana no incide; produciendo de nuevo una zona de sombra donde la densidad de corriente inducida se considera nula.

Al igual que en el caso anterior, la densidad de corriente inducida $\vec{J}_{s}$ se muestrea a lo largo de la superficie del objeto dispersor; dando lugar a $N_{s}$ elementos de corriente (en este caso tangentes al contorno del objeto dispersor). De nuevo, la intensidad asociada a cada uno de estos elementos de corriente se aproxima, según (4.16), mediante el producto de la densidad de corriente en el segmento correspondiente, donde se supondrá constante, por la longitud de dicho segmento; dando lugar a la siguiente expresión

$$
I_{n}=\vec{J}_{s}\left(\vec{\rho}_{n}\right) \cdot \hat{l} \Delta C_{n}=J_{l}\left(\vec{\rho}_{n}\right) \Delta C_{n}
$$

donde $\vec{\rho}_{n}$ indica el $n$-ésimo elemento de corriente (normalmente el punto medio del correspondiente segmento) sobre la superficie del objeto dispersor. Al tratarse en esta ocasión de incidencia con polarización $\mathrm{TE}^{\mathrm{z}}$, el campo magnético incidente 
presenta tan sólo componente en $\hat{z}$, y por consiguiente la corriente inducida es tangente al contorno del objeto dispersor. Por esta razón, para obtener la corriente que circula por cada segmento se ha multiplicado escalarmente en (4.33) la densidad de corriente inducida $\vec{J}_{s}$ por el vector unitario tangente $\hat{l}$.

Finalmente, combinando (4.32) y (4.33) se obtiene la siguiente expresión para los coeficientes $A_{n m}$, presentes en (4.23), ante incidencia con polarización $\mathrm{TE}^{\mathrm{z}}$

$$
A_{n m}=\left\{\begin{array}{cl}
{\left[2 \hat{n} \times \hat{z} e^{-j k \rho_{n} \cos \left(\phi_{n}-\beta_{m}\right)} \Delta C_{n}\right] \cdot \hat{l}} & \text { si } \phi_{n} \in\left[\beta_{m}+\frac{\pi}{2}, \beta_{m}+\frac{3 \pi}{2}\right] \\
0 & \text { en caso contrario }
\end{array}\right.
$$

\subsubsection{Ecuación Integral}

El concepto del equivalente físico (recordar fig. 4.5) permite plantear [69] una Ecuación Integral para el campo eléctrico (EFIE) y otra para el campo magnético (MFIE); donde la incógnita es precisamente la densidad de corriente $\vec{J}_{s}$ inducida sobre la superficie del objeto dispersor.

En concreto, la Ecuación Integral para el campo eléctrico surge al forzar la continuidad de las componentes tangenciales de campo eléctrico sobre la superficie del objeto dispersor metálico; dando lugar a las ecuaciones

$$
\begin{aligned}
-\hat{n} \times{\overrightarrow{E^{i}}}^{i} & =\hat{n} \times{\overrightarrow{E^{d}}}^{\vec{E}^{d}}=\mathcal{F}\left(\vec{J}_{s}\right)
\end{aligned}
$$

donde el campo eléctrico dispersado $\overrightarrow{E^{d}}$ se encuentra relacionado con la densidad de corriente inducida $\vec{J}_{s}$ mediante una integral.

Forzando en la superficie del conductor la condición de contorno para las componentes tangenciales de campo magnético, surge la Ecuación Integral para el campo magnético; que se expresa mediante las siguientes ecuaciones

$$
\begin{aligned}
\vec{J}_{s} & =\hat{n} \times \vec{H}^{i}+\hat{n} \times \vec{H}^{d} \\
\vec{H}^{d} & =\mathcal{F}\left(\vec{J}_{s}\right)
\end{aligned}
$$

donde ahora el campo magnético dispersado $\overrightarrow{E^{d}}$ se encuentra relacionado con la densidad de corriente inducida $\vec{J}_{s}$ mediante otra expresión integral.

Estas ecuaciones integrales, recogidas en (4.35) y en (4.36), pueden resolverse utilizando el Método de los Momentos [70]; que reduce la correspondiente ecuación integral a un sistema de ecuaciones algebraicas. Este método permite, en general, resolver ecuaciones integrodiferenciales inhomogéneas del tipo $L(f)=g$; donde $L$ representa un operador integrodiferencial, $g$ la fuente o excitación conocida y $f$ la respuesta o incógnita a determinar. 
Para aplicar el Método de los Momentos resulta necesario definir un producto interior entre dos funciones $f$ y $g$, representado por $\langle f, g\rangle$, que satisfaga ciertas propiedades recogidas en [70]. Una vez definido el producto interior, la función incógnita $f$ a determinar se expresa como una expansión en serie de $N_{s}$ funciones base $\left(f_{1}, f_{2}, \ldots, f_{N_{s}}\right)$ de la forma

$$
f=\sum_{n=1}^{N_{s}} \alpha_{n} f_{n}
$$

convirtiendo el problema original $L(f)=g$ en la siguiente ecuación

$$
L(f)=L\left(\sum_{n=1}^{N_{s}} \alpha_{n} f_{n}\right)=\sum_{n=1}^{N_{s}} \alpha_{n} L\left(f_{n}\right)=g
$$

A continuación, se selecciona un conjunto de funciones test $\left(w_{1}, w_{2}, \ldots, w_{M}\right)$ definidas en el rango del operador $L$. Proyectando ambos lados de la ecuación inhomogénea (4.38) sobre estas funciones test, empleando para ello el producto interior definido previamente, se obtiene el siguiente sistema de $M$ ecuaciones con $N_{s}$ incógnitas

$$
\sum_{n=1}^{N_{s}} \alpha_{n}\left\langle w_{m}, L\left(f_{n}\right)\right\rangle=\left\langle w_{m}, g\right\rangle \quad m=1,2,3, \ldots, M
$$

que puede expresarse de forma matricial como

$$
\left[I_{m n}\right] \cdot\left[\alpha_{n}\right]=\left[g_{m}\right]
$$

donde $\left[I_{m n}\right]$ representa la siguiente matriz de coeficientes ${ }^{1}$

$$
\left[I_{m n}\right]=\left[\begin{array}{cccc}
\left\langle w_{1}, L\left(f_{1}\right)\right\rangle & \left\langle w_{1}, L\left(f_{2}\right)\right\rangle & \cdot & \left\langle w_{1}, L\left(f_{N_{s}}\right)\right\rangle \\
\left\langle w_{2}, L\left(f_{1}\right)\right\rangle & \left\langle w_{2}, L\left(f_{2}\right)\right\rangle & \cdot & \left\langle w_{2}, L\left(f_{N_{s}}\right)\right\rangle \\
\vdots & \vdots & \ddots & \vdots \\
\left\langle w_{M}, L\left(f_{1}\right)\right\rangle & \left\langle w_{M}, L\left(f_{2}\right)\right\rangle & \cdot & \left\langle w_{M}, L\left(f_{N_{s}}\right)\right\rangle
\end{array}\right]_{M \times N_{s}}
$$

y $\left[\alpha_{n}\right],\left[g_{m}\right]$ representan respectivamente las incógnitas y las excitaciones del sistema algebraico de ecuaciones mediante los siguientes vectores columna

$$
\left[\alpha_{n}\right]=\left[\begin{array}{c}
\alpha_{1} \\
\alpha_{2} \\
\vdots \\
\alpha_{N_{s}}
\end{array}\right]_{N_{s} \times 1}\left[g_{m}\right]=\left[\begin{array}{c}
\left\langle w_{1}, g\right\rangle \\
\left\langle w_{2}, g\right\rangle \\
\vdots \\
\left\langle w_{M}, g\right\rangle
\end{array}\right]_{M \times 1}
$$

\footnotetext{
${ }^{1}$ No confundir con la matriz de corrientes $D I$ definida en (4.19).
} 
El cálculo de las integrales que aparecen en la computación de los elementos de la matriz de coeficientes $\left[I_{m n}\right]$ resulta especialmente complejo en problemas electromagnéticos. Con el objeto de simplificar dicho cálculo, se introducen funciones delta de Dirac como funciones test $w_{m}$; dando lugar a una particularización del Método de los Momentos conocida como Point-Matching [70]. La introducción de estas funciones test implica exigir el cumplimiento de la mencionada ecuación $\sum_{n} \alpha_{n} L\left(f_{n}\right)=g$ en un conjunto discreto de puntos. Esta técnica se ha aplicado en la obtención de la matriz de corrientes para algunos de los resultados que se presentan en los siguientes capítulos; observándose en todos ellos la suficiente precisión requerida.

En los siguientes apartados, se aplica el Método de los Momentos (PointMatching) a la determinación de la matriz de corrientes; que debe relacionar la distribución de corrientes inducidas sobre la superficie de un objeto dispersor con el espectro asociado al campo incidente, tanto para polarización $\mathrm{TM}^{\mathrm{z}}$ como para $\mathrm{TE}^{\mathrm{z}}$. En cada posible tipo de polarización, se determina la Ecuación Integral que resulta más apropiada; identificando asimismo las funciones excitación e incógnita.

\section{Polarización $\mathrm{TM}^{\mathrm{z}}$}

En el caso de incidencia polarizada según $\mathrm{TM}^{\mathrm{z}}$, la matriz de corrientes $D I$ debe relacionar la distribución de corrientes inducidas con el espectro asociado al campo eléctrico incidente. Por tanto, se debe escoger la Ecuación Integral para el campo eléctrico definida en (4.35); que particularizada a una geometría bidimensional da lugar a

$$
\left.E_{z}^{i}\right|_{C}+\left.E_{z}^{d}\right|_{C}=0
$$

donde $\left.E_{z}^{i}\right|_{C}$ y $\left.\quad E_{z}^{d}\right|_{C}$ representan, respectivamente, las componentes según $\hat{z}$ del campo eléctrico incidente y dispersado evaluadas sobre el contorno $C$ del objeto dispersor.

La componente en $\hat{z}$ del campo eléctrico dispersado puede expresarse [70] en función de la densidad de corriente inducida (dirigida según el eje $\hat{z}$ ) a través de la siguiente expresión

$$
E_{z}^{d}(\vec{\rho})=-\frac{k \eta}{4} \int_{C} J_{z}\left(\overrightarrow{\rho^{\prime}}\right) H_{0}^{(2)}\left(k\left|\vec{\rho}-\overrightarrow{\rho^{\prime}}\right|\right) \mathrm{d} \rho^{\prime}
$$

donde $C$ representa el contorno del objeto dispersor en el que se induce la densidad de corriente. Por su parte, en (4.44) el vector $\overrightarrow{\rho^{\prime}}$ hace referencia a la posición de la fuente o densidad de corriente inducida; mientras que el vector $\vec{\rho}$ indica el punto de observación en el que se pretende calcular el campo eléctrico dispersado. El significado de ambas coordenadas puede observarse en la figura 4.9, donde se representa la sección de un objeto dispersor bidimensional. 


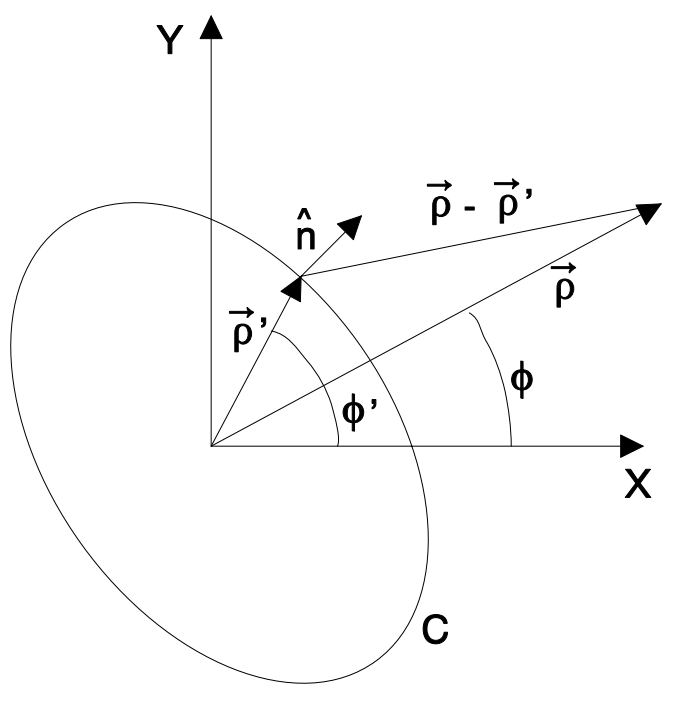

Figura 4.9: Sección de un dispersor infinito según $\hat{z}$. Coordenadas de la fuente y del punto de cálculo del campo dispersado.

Sustituyendo (4.44) en la condición de contorno expresada por (4.43), se obtiene la siguiente ecuación integral inhomogénea sobre la que aplicar el Método de los Momentos (Point-Matching)

$$
\left.E_{z}^{i}\right|_{C}=\frac{k \eta}{4} \int_{C} J_{z}\left(\overrightarrow{\rho^{\prime}}\right) H_{0}^{(2)}\left(k\left|\vec{\rho}-\overrightarrow{\rho^{\prime}}\right|\right) \mathrm{d} \rho^{\prime}
$$

donde $\vec{\rho}$ indica cualquier punto del contorno $C$ del objeto dispersor metálico. Fijándonos en (4.45) se identifica la excitación $\left(\left.E_{z}^{i}\right|_{C}\right)$ del problema, la incógnita $\left(J_{z}\left(\overrightarrow{\rho^{\prime}}\right)\right)$ a resolver, y el operador $L$ definido en este caso mediante la siguiente integral

$$
L\left(J_{z}\right)=\frac{k \eta}{4} \int_{C} J_{z}\left(\vec{\rho}^{\prime}\right) H_{0}^{(2)}\left(k\left|\vec{\rho}-\vec{\rho}^{\prime}\right|\right) \mathrm{d} \rho^{\prime}
$$

Las funciones base que se han escogido para expandir la función incógnita densidad de corriente son pulsos con el siguiente aspecto

$$
f_{n}\left(\rho^{\prime}\right)= \begin{cases}1 & \text { si } \overrightarrow{\rho^{\prime}} \in \Delta C_{n}=\prod\left(\frac{\overrightarrow{\rho^{\prime}}-\vec{\rho}_{n}}{\Delta C_{n}}\right) \\ 0 & \text { resto }\end{cases}
$$

donde $\Pi(\cdot)$ representa la función pulso, $\vec{\rho}_{n}$ el punto medio del n-ésimo segmento en el que $f_{n}$ es no nula, y $\Delta C_{n}$ la longitud de dicho segmento. Como se ha comentado anteriormente, el contorno del objeto dispersor se divide en $N_{s}$ segmentos; cada uno de ellos con una longitud $\Delta C_{n}$, tal y como se mostraba en la figura 4.6. Por tanto, la función pulso definida en (4.47) tiene valor no nulo en su correspondiente (n-ésimo) segmento. 
Para implementar el Método de los Momentos, se ha definido un producto interior entre dos funciones mediante la siguiente integral sobre el contorno del objeto dispersor

$$
\langle f, g\rangle=\int_{C} f \cdot g \mathrm{~d} \rho
$$

Al aplicar Point-Matching, se utilizan como funciones test $w_{m}$ las ya mencionadas funciones delta de Dirac

$$
w_{m}=\delta\left(\vec{\rho}-\vec{\rho}_{m}\right)
$$

posicionadas en los puntos $\vec{\rho}_{m}$, que se eligen en el centro de cada uno de los segmentos en los que se ha dividido el contorno del objeto dispersor; es decir, en el punto medio $\vec{\rho}_{n}$ de cada segmento donde la función base $f_{n}$ tiene valor no nulo. Por tanto, si $m=n$ se cumple que $\vec{\rho}_{m}=\vec{\rho}_{n}$. Para que la matriz de coeficientes $\left[I_{m n}\right]$ definida en (4.41) sea cuadrada, el número $N_{s}$ de funciones base $f_{n}$ debe ser igual al número $M$ de funciones test $w_{m}$; habiendo escogido pues $M=N_{s}$.

Los elementos del vector de excitaciones $\left[g_{m}\right]$ recogidos en $(4.42)$ se obtienen, tras aplicar Point-Matching, evaluando el campo eléctrico incidente en las posiciones $\vec{\rho}_{m}$ donde se ubican las funciones test; dando lugar a

$$
\begin{gathered}
g_{m}=E_{z}^{i}\left(\vec{\rho}_{m}\right)=E_{z}^{i}\left(x_{m}, y_{m}\right) \\
\vec{\rho}_{m}=x_{m} \hat{x}+y_{m} \hat{y}
\end{gathered}
$$

El cálculo de los elementos de la matriz de coeficientes $\left[I_{m n}\right]$, definidos según (4.41), requiere de varias simplificaciones. Así pues, asumiendo que la longitud de cada segmento $\left(\Delta C_{n}\right)$ es muy pequeña en términos eléctricos, y utilizando la aproximación de la función de Hankel de segunda especie y orden cero para argumento pequeño; se obtiene la siguiente expresión para los elementos $I_{n n}$ de la matriz de coeficientes

$$
I_{n n}=\frac{k \eta}{4} \Delta C_{n}\left[1-j \frac{2}{\pi} \ln \left(\frac{\gamma k \Delta C_{n}}{4 e}\right)\right]
$$

donde $e=2.718 \ldots$ y $\gamma$ representa la constante de Euler, cuyo valor se define en [71] del siguiente modo

$$
\begin{gathered}
\gamma=\lim _{m \rightarrow \infty}\left[1+\frac{1}{2}+\frac{1}{3}+\cdots+\frac{1}{m}-\ln m\right] \\
\gamma=0.5772156649 \ldots
\end{gathered}
$$

Para el cálculo de los elementos $I_{m n}$ se aproxima la densidad de corriente, presente en el n-ésimo segmento, por un hilo de corriente centrado en el punto medio $\vec{\rho}_{n}$ de dicho segmento. Así pues, el campo eléctrico producido por una 
función pulso (densidad de corriente) en el punto $\vec{\rho}_{m}$, significado del elemento $I_{m n}$, se evalúa como el campo que produce en dicho punto un hilo infinito de corriente, dirigido según el eje $\hat{z}$, y de intensidad $\Delta C_{n}$. Por tanto, la expresión para los elementos $I_{m n}(\operatorname{con} m \neq n)$ se resume a continuación como

$$
\begin{gathered}
I_{m n}=\frac{k \eta}{4} \Delta C_{n} H_{0}^{(2)}\left(k\left|\vec{\rho}_{m}-\vec{\rho}_{n}\right|\right)=\frac{k \eta}{4} \Delta C_{n} H_{0}^{(2)}\left(k \sqrt{\left(x_{m}-x_{n}\right)^{2}+\left(y_{m}-y_{n}\right)^{2}}\right) \\
\vec{\rho}_{m}=x_{m} \hat{x}+y_{m} \hat{y} \quad \vec{\rho}_{n}=x_{n} \hat{x}+y_{n} \hat{y}
\end{gathered}
$$

Al construir cada columna de la matriz de corrientes $D I$, se supone como campo incidente la componente apropiada del desarrollo modal recogido en (4.2) para $E_{z}^{i}$; dando lugar a la siguiente expresión para los elementos de la matriz de excitaciones $\left[g_{m p}\right]$

$$
g_{m p}=E_{z}^{i}\left(x_{m}, y_{m}\right)=J_{p}\left(k \rho_{m}\right) e^{j p \phi_{m}}
$$

Resolviendo, a continuación, los $2 N_{i}+1$ sistemas de ecuaciones algebraicas definidos mediante la relación $\left[I_{m n}\right] \cdot\left[\alpha_{n p}\right]=\left[g_{m p}\right]$, se obtiene la matriz $\left[\alpha_{n p}\right]$; que representa la densidad de corriente inducida sobre el objeto dispersor por cada modo incidente. La matriz de corrientes, que relaciona corrientes inducidas con el espectro asociado al campo incidente, se obtiene simplemente multiplicando cada elemento de la matriz $\left[\alpha_{n p}\right]$ por la longitud $\Delta C_{n}$ del segmento correspondiente; permitiendo definir pues la siguiente relación buscada

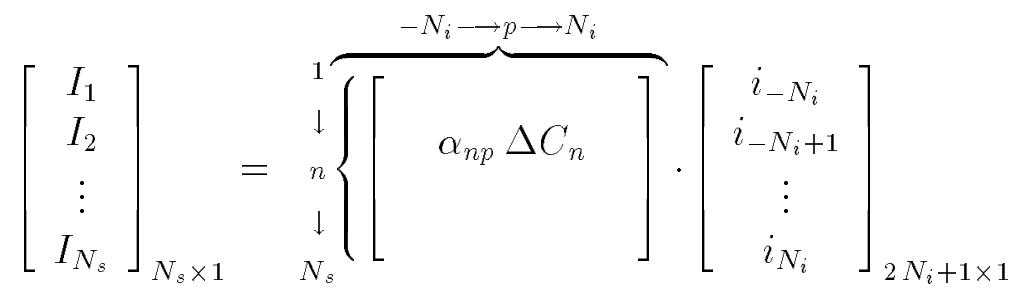

\section{Polarización TE $\mathrm{TE}^{\mathrm{z}}$}

En el caso de incidencia polarizada según $\mathrm{TE}^{\mathrm{z}}$, la matriz de corrientes $D I$ debe relacionar la distribución de corrientes inducidas con el espectro asociado al campo magnético incidente. Por tanto, en este caso se debe escoger la Ecuación Integral para el campo magnético definida en (4.36); que particularizada al problema de un objeto dispersor bidimensional presenta el siguiente aspecto

$$
J_{l^{\prime}}=-\left.H_{z}^{i}\right|_{C}-\left.H_{z}^{d}\right|_{C}
$$

donde $J_{l^{\prime}}$ representa la densidad de corriente inducida sobre el contorno $C$ del objeto dispersor, dirigida como se puede observar en la figura 4.10 en dirección tangente a dicho contorno; mientras $\left.H_{z}^{i}\right|_{C}$ y $\left.H_{z}^{d}\right|_{C}$ representan respectivamente 
las componentes según $\hat{z}$ del campo magnético incidente y dispersado, evaluadas también sobre el contorno del objeto. El sentido del vector unitario, tangente a dicho contorno, se escoge (ver fig. 4.10) siguiendo la regla de la mano derecha; de manera que dicho vector deja siempre a su izquierda la región interna del objeto dispersor.

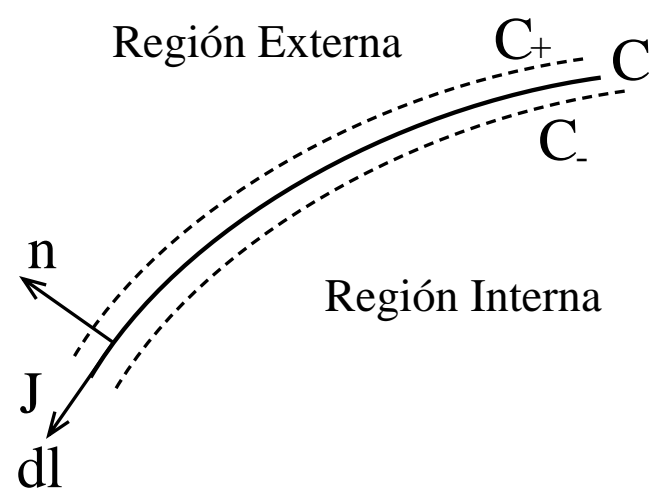

Figura 4.10: Sección de un dispersor infinito según $\hat{z}$. Densidad de corriente inducida por un campo incidente con polarización $\mathrm{TE}^{\mathrm{z}}$.

La componente en $\hat{z}$ del campo magnético dispersado por la densidad de corriente inducida $J_{l^{\prime}}$ (dirigida según el vector unitario tangente al contorno) viene expresada en [70] del siguiente modo

$$
H_{z}^{d}=\hat{z} \cdot \nabla \times \int_{C} J_{l^{\prime}}\left(\overrightarrow{\rho^{\prime}}\right) G\left(\vec{\rho}, \overrightarrow{\rho^{\prime}}\right) \mathrm{d} \vec{l}^{\prime}
$$

donde, de nuevo, $\vec{\rho}$ y $\overrightarrow{\rho^{\prime}}$ hacen referencia respectivamente al punto de observación en el que se pretende calcular el campo magnético dispersado, y a la posición de la fuente o densidad de corriente inducida sobre el objeto metálico. Por su parte, en (4.57) el vector $\mathrm{d} \overrightarrow{l^{\prime}}$ indica un elemento diferencial en la dirección de referencia de la densidad de corriente $J_{l^{\prime}}$ (ver fig. 4.10); mientras $G$ denota la función de Green bidimensional definida en [70] como

$$
G\left(\vec{\rho}, \overrightarrow{\rho^{\prime}}\right)=\frac{1}{4 j} H_{0}^{(2)}\left(k\left|\vec{\rho}-\overrightarrow{\rho^{\prime}}\right|\right)
$$

Sustituyendo ahora (4.57) en la Ecuación Integral para el campo magnético recogida en (4.56), se obtiene la siguiente ecuación integral inhomogénea sobre la que puede aplicarse de nuevo el Método de los Momentos (Point-Matching)

$$
-\left.H_{z}^{i}\right|_{C}=J_{l^{\prime}}\left(\overrightarrow{\rho^{\prime}}\right)+\hat{z} \cdot \nabla \times \int_{C} J_{l^{\prime}}\left(\overrightarrow{\rho^{\prime}}\right) G\left(\vec{\rho}, \overrightarrow{\rho^{\prime}}\right) \mathrm{d} \overrightarrow{l^{\prime}}
$$

donde $\vec{\rho}$ indica cualquier punto del contorno $C$ del objeto dispersor metálico. En (4.59) se puede identificar la excitación (en este caso $-\left.H_{z}^{i}\right|_{C}$ ) del problema, 
la nueva incógnita $\left(J_{l^{\prime}}\left(\overrightarrow{\rho^{\prime}}\right)\right)$ a resolver, y el operador $L$ definido en este caso del siguiente modo

$$
L\left(J_{l^{\prime}}\right)=J_{l^{\prime}}\left(\overrightarrow{\rho^{\prime}}\right)+\hat{z} \cdot \nabla \times \int_{C} J_{l^{\prime}}\left(\overrightarrow{\rho^{\prime}}\right) G\left(\vec{\rho}, \overrightarrow{\rho^{\prime}}\right) \mathrm{d} \overrightarrow{l^{\prime}}
$$

Como funciones base para expandir la función incógnita densidad de corriente se han escogido, al igual que en el caso anterior para polarización TM ${ }^{\mathrm{z}}$, funciones pulso con el siguiente aspecto

$$
f_{n}\left(\rho^{\prime}\right)= \begin{cases}1 & \text { si } \overrightarrow{\rho^{\prime}} \in \Delta C_{n}=\prod\left(\frac{\overrightarrow{\rho^{\prime}}-\vec{\rho}_{n}}{\Delta C_{n}}\right) \\ 0 & \text { resto }\end{cases}
$$

donde $\vec{\rho}_{n}$ representa el punto medio del n-ésimo segmento en el que $f_{n}$ es no nula, y $\Delta C_{n}$ la longitud de dicho segmento. Tal y como se ha comentado anteriormente, el contorno del objeto dispersor se divide en $N_{s}$ segmentos de longitud $\Delta C_{n}$.

El producto interior, que requiere el Método de los Momentos, se expresa a continuación en términos de una integral definida en el contorno del objeto dispersor como

$$
\langle f, g\rangle=\int_{C} f \cdot g \mathrm{~d} \rho
$$

Las funciones test, al aplicar Point-Matching, vienen expresadas mediante las siguientes funciones delta de Dirac

$$
w_{m}=\delta\left(\vec{\rho}-\vec{\rho}_{m}\right)
$$

donde los puntos $\vec{\rho}_{m}$ se sitúan en el centro de cada uno de los segmentos en los que se ha dividido el contorno del objeto dispersor. De nuevo, el número $N_{s}$ de funciones base $f_{n}$ se escoge igual al número $M$ de funciones test $w_{m}$; produciendo una matriz de coeficientes $\left[I_{m n}\right]$ que resulta cuadrada.

De esta forma, tras aplicar Point-Matching, los elementos del vector de excitaciones $\left[g_{m}\right]$, definidos según (4.42), se obtienen evaluando en esta ocasión el campo magnético incidente en las posiciones $\vec{\rho}_{m}$ donde se ubican las funciones delta; quedando expresados como

$$
\begin{gathered}
g_{m}=-H_{z}^{i}\left(\vec{\rho}_{m}\right)=-H_{z}^{i}\left(x_{m}, y_{m}\right) \\
\vec{\rho}_{m}=x_{m} \hat{x}+y_{m} \hat{y}
\end{gathered}
$$

El cálculo de los elementos de la matriz de coeficientes $\left[I_{m n}\right]$, definidos según (4.41), requiere de varias simplificaciones. Así pues, para los elementos $I_{n n}$ pertenecientes a la diagonal principal de la matriz de coeficientes, debido a la condición de simetría de este problema y a que la discontinuidad en $\left.H_{z}\right|_{C}$ es precisamente $J_{l^{\prime}}$, se deduce en [70] el siguiente valor

$$
I_{n n} \approx \frac{1}{2}
$$




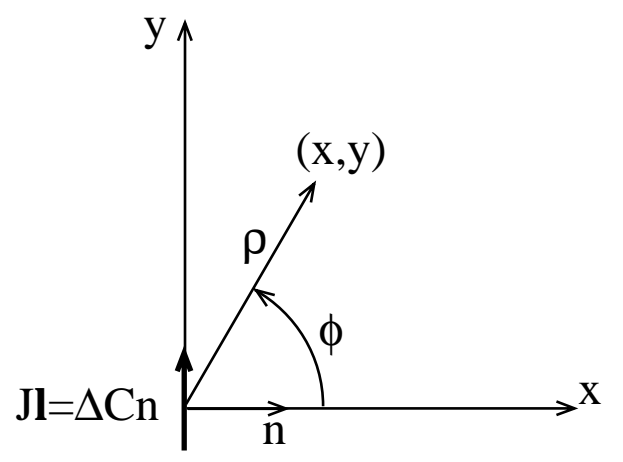

Figura 4.11: Fuente puntual de corriente y coordenadas locales.

En el cálculo de los elementos $I_{m n}$ se asume que la longitud de cada segmento es pequeña en términos eléctricos $\left(\Delta C_{n} \ll \lambda\right)$. De esta forma, el campo magnético producido por una función pulso (densidad de corriente) en el punto $\vec{\rho}_{m}$, significado del elemento $I_{m n}$, se evaluará como el campo que produce en dicho punto una fuente puntual de corriente, dirigida según el vector unitario $\hat{l}, \mathrm{y}$ de intensidad $\Delta C_{n}$. El campo magnético que produce dicha fuente puntual de corriente, situada en el origen de coordenadas tal y como se muestra en la figura 4.11 , en un punto de coordenadas $(\rho, \phi)$ suficientemente alejado de la fuente se recoge en $[70]$ a través de la expresión

$$
H_{z}=\frac{j}{4} k \Delta C_{n} \cos (\phi) H_{1}^{(2)}(k \rho)
$$

Trasladar la fuente puntual a un origen arbitrario $\vec{\rho}_{n}$ supone sustituir en (4.66) la expresión $\cos (\phi)$ por $(\hat{n} \cdot \vec{R})$, donde $\hat{n}$ representa el vector unitario normal a la dirección $(\hat{l})$ de la densidad de corriente $J_{l^{\prime}}$, y $\vec{R}$ se define del siguiente modo

$$
\begin{gathered}
\vec{R}=\frac{\vec{\rho}_{m}-\vec{\rho}_{n}}{\left|\vec{\rho}_{m}-\vec{\rho}_{n}\right|} \\
\vec{\rho}_{m}=x_{m} \hat{x}+y_{m} \hat{y} \quad \vec{\rho}_{n}=x_{n} \hat{x}+y_{n} \hat{y}
\end{gathered}
$$

De esta forma se obtiene, definitivamente, la siguiente expresión para los elementos $I_{m n}(\operatorname{con} m \neq n)$

$$
I_{m n}=\frac{j}{4} k \Delta C_{n}(\hat{n} \cdot \vec{R}) H_{1}^{(2)}\left(k \sqrt{\left(x_{m}-x_{n}\right)^{2}+\left(y_{m}-y_{n}\right)^{2}}\right)
$$

Para construir la matriz de corrientes $D I$ es necesario resolver los $2 N_{i}+1$ sistemas de ecuaciones algebraicas definidos matricialmente mediante la relación $\left[I_{m n}\right] \cdot\left[\alpha_{n p}\right]=\left[g_{m p}\right]$; donde en esta ocasión los elementos de la matriz de excitaciones $\left[g_{m p}\right]$ vienen definidos como

$$
g_{m p}=-H_{z}^{i}\left(x_{m}, y_{m}\right)=-J_{p}\left(k \rho_{m}\right) e^{j p \phi_{m}}
$$


Una vez obtenida la matriz $\left[\alpha_{n p}\right]$, se debe multiplicar cada uno de sus elementos por la longitud $\Delta C_{n}$ del segmento correspondiente; para obtener la matriz de corrientes $D I$ que relaciona, de igual manera que en (4.55), corrientes inducidas con espectro asociado al campo incidente, polarizado en este caso según $\mathrm{TE}^{\mathrm{z}}$.

\subsubsection{Estudio Comparativo entre Óptica Física y Método de los Momentos}

En esta sección, se comparan los dos métodos descritos para obtener la matriz de corrientes con el objeto de evaluar su exactitud. Se ha aplicado, por tanto, la aproximación de Óptica Física y el Método de los Momentos para encontrar, primero la matriz de corrientes de un cilindro metálico infinito, y después el campo que dispersa este cilindro ante una onda plana incidente con polarización $\mathrm{TM}^{\mathrm{z}}$, cuyo frente de fase avanza en la dirección del eje $\hat{x}$ y en el sentido creciente de la coordenada $x$ ( $\beta=0$ en figura C.1). Se ha escogido el cilindro porque presenta solución analítica conocida, que también se ha calculado para poder comparar con los resultados obtenidos siguiendo los métodos anteriormente propuestos; y de esta forma evaluar el grado de exactitud que presentan dichos métodos.

En primer lugar, se ha obtenido la densidad de corriente inducida $\left(\vec{J}_{s}\right)$ por la onda plana $(\beta=0)$ sobre la superficie del cilindro. Esta densidad de corriente se ha calculado para diferentes cilindros (de radio $0.2 \lambda, 0.5 \lambda, \lambda, 2 \lambda, 4 \lambda$ y $6 \lambda$ ). En la figura 4.12, se representa la evolución del error cuadrático medio normalizado de la densidad de corriente inducida con el radio del cilindro. La expresión analítica del parámetro representado se define como

$$
\varepsilon_{J_{s}}=\frac{\sqrt{\frac{1}{2 \pi} \int_{0}^{2 \pi}\left|\overrightarrow{J_{s}^{e}}(\phi)-\vec{J}_{s}^{\mathrm{m}}(\phi)\right|^{2} \mathrm{~d} \phi}}{\left|\overrightarrow{J_{s}^{\mathrm{e}}}\right|_{\max }}
$$

donde $\overrightarrow{J_{s}^{e}}(\phi)$ representa el valor exacto de la densidad de corriente inducida en función de la variable $\phi, \vec{J}_{s}^{\mathrm{m}}(\phi)$ el valor estimado de dicha densidad bien siguiendo la aproximación de Óptica Física o bien el Método de los Momentos, y $\left|\overrightarrow{J_{s}^{\mathrm{e}}}\right|_{\max }$ el máximo del valor absoluto exacto de la densidad de corriente inducida a lo largo de toda la superficie del cilindro.

En la figura 4.12, se observa que el error cuadrático medio asociado a Óptica Física es muy elevado para valores pequeños del radio (próximo al $64 \%$ para un cilindro de radio $0.2 \lambda$ ). Conforme el radio del cilindro aumenta, dicho error disminuye; llegando incluso a ser inferior al que introduce el Método de los Momentos (ambos métodos presentan un error similar, próximo al 16\%, para un cilindro de radio $6 \lambda$ ). Es interesante comentar que la evolución del error asociado al Método de los Momentos presenta un máximo (cilindro de radio $1 \lambda$ ); habiendo observado en dicho caso unas amplias fluctuaciones en la amplitud de la densidad de corriente inducida con respecto a su valor exacto. A partir de dicho máximo, este 


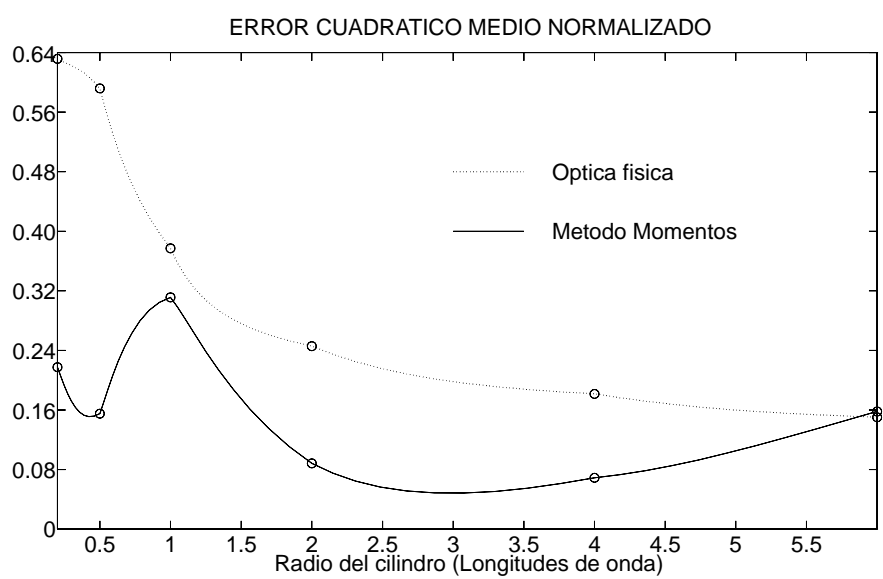

Figura 4.12: Evolución del error cuadrático medio de la densidad superficial de corriente, calculada mediante Óptica Física y Método de los Momentos, con el radio del cilindro infinito.

error disminuye para posteriormente (cilindros con radio mayor que $3 \lambda$ ) volver a aumentar; llegando incluso a alcanzar un valor ligeramente superior (cilindro de radio $6 \lambda$ ) al error introducido por Óptica Física.

Los resultados ofrecidos en la figura 4.12 confirman que Óptica Física ofrece resultados más exactos cuando las dimensiones del objeto aumentan; mientras que el Método de los Momentos resulta más inexacto. De cualquier forma, puede resultar sorprendente el elevado orden de error introducido por ambos métodos en el cálculo de la densidad de corriente inducida $\overrightarrow{J_{s}}$ (en ningún caso inferior al $5 \%$ ). Sin embargo, como se verá a continuación, estas fluctuaciones en la densidad de corriente inducida apenas afectan al cálculo del campo dispersado, que es función lineal de dicha densidad de corriente; y por tanto durante su computación se promedian las fluctuaciones detectadas en $\vec{J}_{s}$.

A continuación, para los mismos cilindros (de radio $0.2 \lambda, 0.5 \lambda, \lambda, 2 \lambda, 4 \lambda$ y $6 \lambda$ ) analizados anteriormente, se ha calculado el campo dispersado por la densidad de corriente inducida $\vec{J}_{s}$; que se ha obtenido siguiendo bien la aproximación de Óptica Física o bien el Método de los Momentos. En la figura 4.13, se representa la variación del error cuadrático medio normalizado del campo dispersado, calculado siguiendo ambos métodos, en función del radio del cilindro. La expresión que se ha utilizado para calcular dicho error, similar a la empleada anteriormente para la densidad de corriente, se define como

$$
\varepsilon_{E^{d}}=\frac{\sqrt{\frac{1}{2 \pi} \int_{0}^{2 \pi}\left|\vec{E}_{\mathrm{e}}^{d}(\phi)-\vec{E}_{\mathrm{m}}^{d}(\phi)\right|^{2} \mathrm{~d} \phi}}{\left|\vec{E}_{\mathrm{e}}^{d}\right|_{\max }}
$$

donde $\vec{E}_{\mathrm{e}}^{d}(\phi)$ representa el valor exacto del campo dispersado en función de la variable $\phi, \vec{E}_{\mathrm{m}}^{d}(\phi)$ el valor estimado de dicho campo bien siguiendo la aproxima- 
ción de Óptica Física o bien el Método de los Momentos para obtener la densidad de corriente inducida, y $\left|\vec{E}_{\mathrm{e}}^{d}\right|_{\max }$ el máximo del valor absoluto exacto de campo dispersado a lo largo de toda la superficie del cilindro.

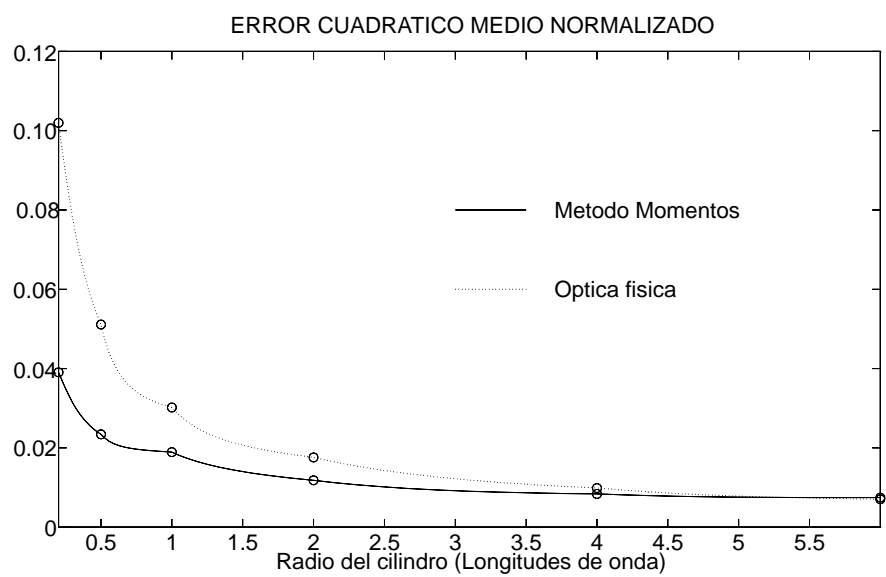

Figura 4.13: Evolución del error cuadrático medio del campo dispersado en la zona de campo lejano, calculado mediante Óptica Física y Método de los Momentos, con el radio del cilindro infinito.

En la figura 4.13 se puede observar, de nuevo, que la diferencia en el error asociado a Óptica Física y al Método de los Momentos se acorta a medida que crece el radio del cilindro; llegando incluso a ser menor el error introducido por Óptica Física para un cilindro de radio $6 \lambda$. Es interesante reseñar que el error del campo dispersado (fig. 4.13) presenta un nivel muy inferior al error de la densidad de corriente inducida (fig. 4.12), tanto para Óptica Física como para el Método de los Momentos; debido a que, como se ha comentado anteriormente, en el cálculo del campo dispersado se promedian las fluctuaciones observadas en la densidad de corriente inducida. Por esta razón, el máximo local del error asociado al Método de los Momentos (cilindro de radio $1 \lambda$ ), que resulta bastante pronunciado en la figura 4.12, se reduce considerablemente en la figura 4.13 donde resulta casi inapreciable.

El estudio comparativo que se ha presentado entre Óptica Física y Método de los Momentos permite concluir, como era de esperar, que el Método de los Momentos ofrece resultados más exactos para objetos dispersores pequeños en términos eléctricos. Sin embargo, a medida que las dimensiones del objeto crecen, el error que introduce la aproximación de Óptica Física en el cálculo del campo dispersado es prácticamente igual, o incluso inferior, al error introducido por el Método de los Momentos. Adicionalmente se ha comprobado que, para obtener resultados suficientemente precisos, el Método de los Momentos requiere de un mayor número de fuentes puntuales de corriente sobre la superficie del objeto dispersor que la aproximación de Óptica Física; lo cual supone un mayor esfuerzo 
computacional en el cálculo de la matriz de caracterización del objeto dispersor, pues en su construcción intervienen una matriz de corrientes y una matriz de espectro (ver sección 4.1) con dimensiones mayores. En conclusión, resulta más conveniente por cuestiones de exactitud utilizar el Método de los Momentos para caracterizar objetos dispersores cuyas dimensiones son pequeñas eléctricamente; mientras que para objetos grandes en términos eléctricos debe escogerse la aproximación de Óptica Física, que proporciona resultados más exactos que el Método de los Momentos con un menor coste computacional.

\subsection{Matriz de Espectro}

En esta sección, se describen dos técnicas que permiten obtener la matriz de espectro; que relaciona el espectro asociado al campo dispersado por un objeto con la distribución de corrientes inducidas en su superficie. La matriz de espectro debe relacionar, por tanto, un vector de $2 N_{d}+1$ amplitudes espectrales, tal y como se muestra en (4.6), con un vector de $N_{s}$ intensidades de corriente inducidas definido en (4.17).

En consecuencia, los elementos de dicha matriz se obtienen escogiendo en cada ocasión un elemento distinto (la n-ésima fuente puntual de corriente) del vector de corrientes. Posteriormente se calcula, en serie de ondas cilíndricas emergentes como se muestra en (4.5), el desarrollo espectral asociado al campo dispersado por dicha fuente puntual de corriente con amplitud unidad. Este contenido espectral constituye, por tanto, la n-ésima columna de la matriz de espectro. Repitiendo este proceso para cada fuente puntual $\left(n=1, \ldots, N_{s}\right)$ en $(4.17)$, se construye la matriz de espectro cuyas dimensiones son $2 N_{d}+1 \times N_{s}$. Cada elemento de dicha matriz, denominado $S_{q n}$, representa la contribución de la n-ésima fuente puntual de corriente con amplitud unidad a la q-ésima componente del desarrollo espectral asociado al campo dispersado; de modo que la relación entre el espectro de campo dispersado y la distribución de corrientes inducidas en la superficie del objeto se expresa en términos matriciales como

$$
E D=D E \cdot I
$$

donde ED representa el vector columna (4.6) con las amplitudes del espectro asociado al campo dispersado, I el vector columna (4.17) con las amplitudes de las corrientes inducidas sobre el objeto dispersor y $D E$ es la matriz de espectro, definida del siguiente modo

$$
D E=\left[\begin{array}{cccc}
S_{-N_{d} 1} & S_{-N_{d} 2} & \cdots & S_{-N_{d} N_{s}} \\
S_{-N_{d}+11} & S_{-N_{d}+12} & \cdots & S_{-N_{d}+1 N_{s}} \\
\vdots & \vdots & \ddots & \vdots \\
S_{N_{d} 1} & S_{N_{d} 2} & \cdots & S_{N_{d} N_{s}}
\end{array}\right]_{2 N_{d}+1 \times N_{s}}
$$


Existen dos técnicas, una de ellas utilizando la Transformada Discreta de Fourier y la otra empleando el Teorema de Adición para las Funciones de Hankel, que permiten (a priori) relacionar el espectro de campo dispersado con las corrientes inducidas en el objeto dispersor por un campo incidente con polarización $\mathrm{TM}^{\mathrm{z}} \mathrm{o}$ $\mathrm{TE}^{\mathrm{z}}$. Las expresiones analíticas correspondientes a los elementos $S_{q n}$ de la matriz de espectro, calculadas siguiendo ambos métodos, se presentan a continuación.

\subsubsection{Técnica basada en la Transformada Discreta de Fou- rier}

En el cálculo de la matriz de espectro, se requiere tener conocimiento del contenido espectral asociado al campo dispersado por cada fuente puntual de corriente inducida en la superficie del objeto dispersor. Como ya se ha comentado anteriormente, la n-ésima columna de dicha matriz representa el espectro asociado al campo dispersado por la n-ésima fuente puntual de corriente, de amplitud unidad y situada en $\vec{\rho}_{n}$ (recordar figura 4.6). Este espectro puede obtenerse haciendo uso de la Transformada Discreta de Fourier; para lo cual se necesita conocer previamente el campo dispersado ${ }^{2}$ por la n-ésima fuente puntual de corriente, que designaremos como $C_{n}^{d}$. La expresión analítica de este campo dispersado $\left(C_{n}^{d}\right)$ depende, lógicamente, del tipo de incidencia (polarización $\mathrm{TM}^{\mathrm{z}}$ o $\mathrm{TE}^{\mathrm{z}}$ ) que se esté considerando.

El campo dispersado por la n-ésima fuente puntual de corriente puede expresarse, recordando (4.5), mediante el siguiente desarrollo modal de funciones cilíndricas emergentes cuyos pesos constituyen precisamente el espectro buscado

$$
C_{n}^{d}(\vec{\rho})=\sum_{q=-N_{d}}^{N_{d}} S_{q n} H_{q}^{(2)}(k \rho) e^{j q \phi}
$$

Particularizando la ecuación (4.74) en una circunferencia de radio $a \lambda$ ( $a$ representa el radio normalizado respecto de $\lambda$ ) que contenga al objeto dispersor, tal y como se muestra en la figura 4.14, se obtiene la siguiente expresión

$$
\begin{gathered}
C_{n}^{d}(\vec{\rho})=\sum_{q=-N_{d}}^{N_{d}} S_{q n} H_{q}^{(2)}(2 \pi a) e^{j q \phi}=\sum_{q=-N_{d}}^{N_{d}} b_{q n} e^{j q \phi} \\
b_{q n}=S_{q n} H_{q}^{(2)}(2 \pi a)
\end{gathered}
$$

donde se han definido unos nuevos coeficientes $b_{q n}$ que incluyen las funciones de Hankel; las cuales presentan un argumento constante al haber evaluado el campo dispersado sobre una circunferencia.

\footnotetext{
${ }^{2}$ Este campo $\left(C_{n}^{d}\right)$ representa campo eléctrico $\left(E_{z n}^{d}\right)$ para incidencia polarizada según $\mathrm{TM}^{\mathrm{z}}$, y campo magnético $\left(H_{z n}^{d}\right)$ para incidencia polarizada según $\mathrm{TE}^{\mathrm{z}}$.
} 


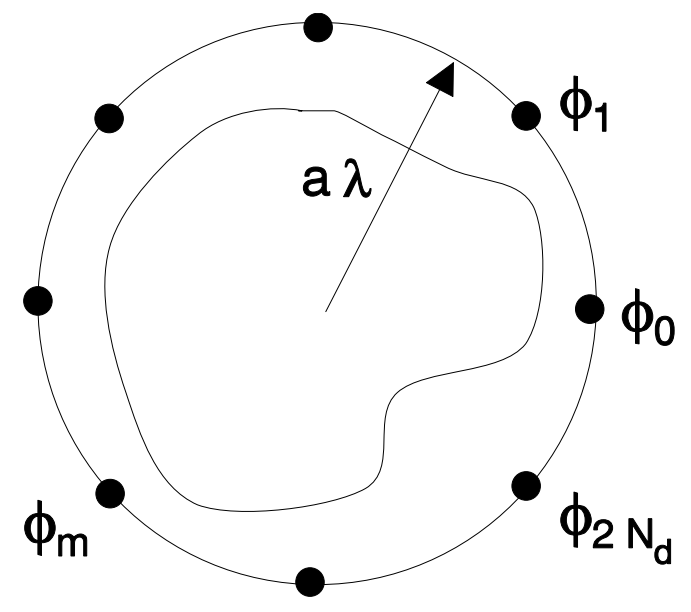

Figura 4.14: Circunferencia de radio $a \lambda$ y disposición de los puntos donde se calcula el campo dispersado; para obtener su espectro mediante la Transformada Discreta de Fourier.

Con el objeto de encontrar el valor de los coeficientes $S_{q n}$ del desarrollo modal presentado en (4.74), el campo dispersado se evalúa en $2 N_{d}+1$ puntos equiespaciados de la circunferencia (ver fig. 4.14). Las coordenadas cilíndricas $\left(\rho_{m}, \phi_{m}\right)$ de estos puntos se definen como

$$
\begin{aligned}
\rho_{m} & =a \lambda \\
\phi_{m} & =\frac{2 \pi}{\left(2 N_{d}+1\right)} m \quad m \in\left[0,1, \ldots, 2 N_{d}\right]
\end{aligned}
$$

Exigiendo en estos $2 N_{d}+1$ puntos de la circunferencia el cumplimiento de la ecuación (4.75), se obtiene la siguiente relación

$$
C_{n}^{d}\left(\vec{\rho}_{m}\right)=\sum_{q=-N_{d}}^{N_{d}} b_{q n} e^{j \frac{2 \pi}{\left(2 N_{d}+1\right)} q m}
$$

Para hacer coincidir la expresión que aparece en (4.77) con la definición de la Transformada Discreta de Fourier inversa [72], se requiere que el índice del sumatorio en (4.77) tenga un valor inicial $q=0 \mathrm{y}$ un valor final $q=2 N_{d}$. Para ello se define una función $\mathcal{G}(\cdot)$, que convierte la secuencia original de coeficientes $\left(b_{-N_{d} n}, b_{-N_{d}+1 n}, \ldots, b_{N_{d}-1 n}, b_{N_{d} n}\right)$, tal y como aparece en (4.77), en la secuencia $\left(b_{0 n}, b_{1 n}, \ldots, b_{N_{d} n}, b_{-N_{d} n}, b_{-N_{d}+1 n}, \ldots, b_{-1 n}\right)$ requerida en [72]. Esta redistribución de los coeficientes $b_{q n}$ permite reordenar el sumatorio del siguiente modo

$$
C_{n}^{d}\left(\vec{\rho}_{m}\right)=\frac{1}{\left(2 N_{d}+1\right)} \sum_{q=0}^{2 N_{d}}\left[\left(2 N_{d}+1\right) \mathcal{G}\left(b_{q n}\right)\right] e^{j \frac{2 \pi}{\left(2 N_{d}+1\right)} q m}
$$


En la expresión (4.78) se ha multiplicado y dividido por $\left(2 N_{d}+1\right)$; para de esta forma identificar en el término $\left[\left(2 N_{d}+1\right) \mathcal{G}\left(b_{q n}\right)\right]$ la Transformada Discreta de Fourier de la secuencia definida por $C_{n}^{d}\left(\vec{\rho}_{m}\right)$. Por tanto, entre ambos términos puede establecerse según [72] la siguiente relación

$$
\begin{aligned}
{\left[\left(2 N_{d}+1\right) \mathcal{G}\left(b_{q n}\right)\right] } & =\sum_{m=0}^{2 N_{d}} C_{n}^{d}\left(\vec{\rho}_{m}\right) e^{-j \frac{2 \pi}{\left(2 N_{d}+1\right)} q m} \\
& =\mathcal{F}\left(C_{n}^{d}\left(\vec{\rho}_{m}\right)\right)
\end{aligned}
$$

donde $\mathcal{F}(\cdot)$ es el símbolo que se ha escogido para representar la Transformada Discreta de Fourier.

Definiendo $\mathcal{G}^{-1}(x)$ como la operación inversa a la implementada por $\mathcal{G}(x)$, en (4.79) se pueden despejar fácilmente los coeficientes $b_{q n}$ como

$$
b_{q n}=\frac{1}{\left(2 N_{d}+1\right)} \mathcal{G}^{-1}\left(\mathcal{F}\left(C_{n}^{d}\left(\vec{\rho}_{m}\right)\right)\right)
$$

Recordando que los coeficientes $b_{q n}$ se encuentran directamente relacionados con las componentes espectrales $S_{q n}$ a través de (4.75), es posible despejar la siguiente expresión para las componentes de la matriz de espectro

$$
S_{q n}=\frac{\mathcal{G}^{-1}\left(\mathcal{F}\left(C_{n}^{d}\left(\vec{\rho}_{m}\right)\right)\right)}{\left(2 N_{d}+1\right) H_{q}^{(2)}(2 \pi a)}
$$

Repitiendo el proceso descrito para cada una de las $N_{s}$ fuentes puntuales de corriente, se completa la matriz de espectro $D E$; que relaciona el espectro asociado al campo dispersado por el objeto, coeficientes $c_{q}$ en (4.6), con la distribución de corrientes inducidas sobre la superficie del objeto, amplitudes $I_{n}$ en (4.17), de la siguiente forma

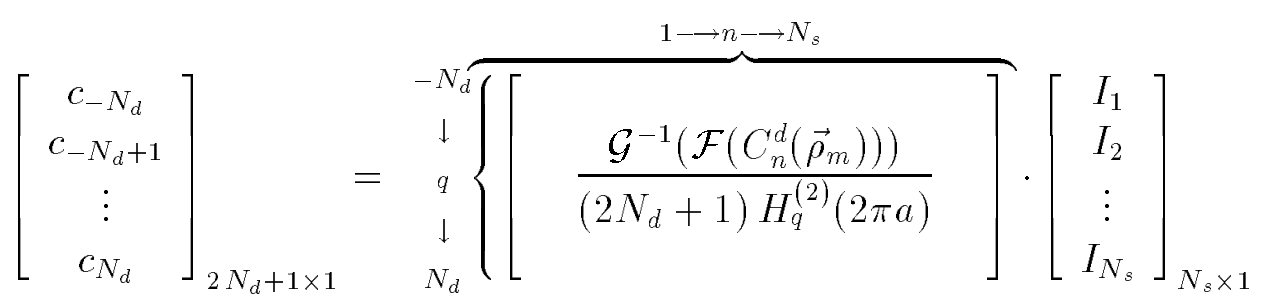

Como se ha mencionado anteriormente, la expresión a utilizar para el campo dispersado $\left(C_{n}^{d}\right)$ por una fuente puntual de corriente depende del tipo de polarización $\left(\mathrm{TM}^{\mathrm{z}}\right.$ o $\mathrm{TE}^{\mathrm{z}}$ ) del campo incidente. A continuación, se presentan las expresiones concretas que deben utilizarse en cada caso. 


\section{Polarización $T M^{\mathrm{z}}$}

Cuando el campo incidente está polarizado según $\mathrm{TM}^{\mathrm{z}}$, la matriz de espectro $D E$ debe relacionar el espectro asociado al campo eléctrico dispersado con la distribución de corrientes inducidas. En este caso, $C_{n}^{d}$ representa la componente en $\hat{z}$ del campo eléctrico dispersado por un hilo infinito de corriente, dirigido según el eje $\hat{z}$ y de amplitud unidad. La expresión para dicho campo eléctrico viene recogida en [69] como

$$
C_{n}^{d}(\vec{\rho})=E_{z n}^{d}(\vec{\rho})=-\frac{1}{4} \omega \mu H_{0}^{(2)}\left(k\left|\vec{\rho}-\vec{\rho}_{n}\right|\right)
$$

donde $\omega$ representa la pulsación asociada a la frecuencia de trabajo, y $\mu$ la permeabilidad magnética del medio.

\section{Polarización $\mathrm{TE}^{\mathrm{z}}$}

Cuando el campo incidente se encuentra polarizado según $\mathrm{TE}^{\mathrm{z}}$, la matriz de espectro $D E$ debe relacionar el espectro asociado al campo magnético dispersado con la distribución de corrientes inducidas. En este caso, $C_{n}^{d}$ representa la componente según $\hat{z}$ del campo magnético dispersado por una fuente puntual de corriente, dirigida según el vector unitario tangente al contorno del objeto y de amplitud unidad. El campo magnético que produce dicha fuente se define en [70] mediante la expresión

$$
C_{n}^{d}(\vec{\rho})=H_{z n}^{d}(\vec{\rho})=\frac{j}{4} k(\hat{n} \cdot \vec{R}) H_{1}^{(2)}\left(k\left|\vec{\rho}-\vec{\rho}_{n}\right|\right)
$$

donde $\hat{n}$ representa el vector unitario normal a la dirección de la fuente puntual de corriente (recordar fig. 4.11), y $\vec{R}$ se define mediante el siguiente vector

$$
\vec{R}=\frac{\vec{\rho}-\vec{\rho}_{n}}{\left|\vec{\rho}-\vec{\rho}_{n}\right|}
$$

\subsubsection{Técnica basada en el Teorema de Adición para las Funciones de Hankel}

El cálculo de los elementos $S_{q n}$, pertenecientes a la n-ésima columna de la matriz de espectro $D E$, se implementa igualando el campo dispersado $\left(C_{n}^{d}\right)$ por la n-ésima fuente puntual de corriente unitaria, situada en $\vec{\rho}_{n}$, con un desarrollo modal de funciones cilíndricas emergentes centradas en el origen de coordenadas; tal y como se ha expresado anteriormente en (4.74). Con el objeto de poder identificar una expresión para los coeficientes $S_{q n}$, se requiere expandir en (4.74) el campo dispersado $\left(C_{n}^{d}\right)$ en la citada serie de funciones cilíndricas emergentes; lo cual es posible (a priori) haciendo uso del Teorema de Adición para las Funciones de Hankel. A continuación, se discute como aplicar dicho Teorema para cada tipo de polarización $\left(\mathrm{TM}^{\mathrm{z}}\right.$ o $\left.\mathrm{TE}^{\mathrm{z}}\right)$ del campo incidente. 


\section{Polarización $\mathrm{TM}^{\mathrm{z}}$}

En este caso, $C_{n}^{d}$ representa de nuevo la componente en $\hat{z}$ del campo eléctrico dispersado por un hilo infinito de corriente, dirigido según el eje $\hat{z}$ y de amplitud unidad. La expresión para dicho campo eléctrico se recoge en (4.83); donde la función de Hankel puede expandirse, haciendo uso de la segunda versión del Teorema de Adición para las Funciones de Hankel (ver apéndice E), mediante el siguiente desarrollo en serie

$$
H_{0}^{(2)}\left(k\left|\vec{\rho}-\vec{\rho}_{n}\right|\right)=\sum_{q=-\infty}^{\infty} J_{q}\left(k \rho_{n}\right) e^{-j q \phi_{n}} H_{q}^{(2)}(k \rho) e^{j q \phi} \quad \rho>\rho_{n}
$$

Esta relación entre funciones de Hankel de segunda especie, recién presentada en (4.86) y recogida también en [69], puede utilizarse en (4.83); obteniendo la siguiente expresión para el campo eléctrico dispersado

$$
\begin{gathered}
C_{n}^{d}(\vec{\rho})=E_{z n}^{d}(\vec{\rho})=-\frac{1}{4} \omega \mu \sum_{q=-\infty}^{\infty} J_{q}\left(k \rho_{n}\right) e^{-j q \phi_{n}} H_{q}^{(2)}(k \rho) e^{j q \phi} \\
=\sum_{q=-\infty}^{\infty}\left[-\frac{1}{4} \omega \mu J_{q}\left(k \rho_{n}\right) e^{-j q \phi_{n}}\right] H_{q}^{(2)}(k \rho) e^{j q \phi} \quad \rho>\rho_{n}
\end{gathered}
$$

La restricción espacial presente en (4.86) y (4.87), procedente de la segunda versión del Teorema de Adición para las Funciones de Hankel (ver apéndice E), no representa problema alguno en la determinación de los elementos $S_{q n}$; pues la matriz de espectro debe proporcionar un espectro de campo dispersado que permita reconstruir dicho campo en la región libre de fuentes. Por tanto, igualando (4.87) con la expansión del campo dispersado $\left(C_{n}^{d}\right)$ recogida en (4.74), se despejan los coeficientes $S_{q n}$ como

$$
S_{q n}=-\frac{1}{4} \omega \mu J_{q}\left(k \rho_{n}\right) e^{-j q \phi_{n}}
$$

Una vez conocidos los coeficientes $S_{q n}$, se puede construir finalmente la matriz de espectro $D E$; que relaciona el espectro asociado en esta ocasión al campo eléctrico dispersado (vector de coeficientes $c_{q}$ ) con la distribución de corrientes inducidas (vector de amplitudes $I_{n}$ ) del siguiente modo

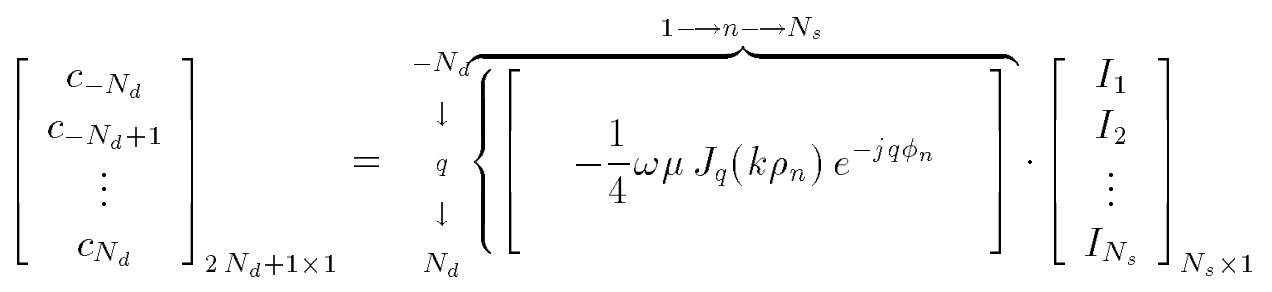




\section{Polarización $\mathrm{TE}^{\mathrm{z}}$}

En el caso de incidencia polarizada según $\mathrm{TE}^{\mathrm{z}}$, el campo $C_{n}^{d}$ representa la componente en $\hat{z}$ del campo magnético dispersado por una fuente puntual de corriente, dirigida según el vector unitario tangente al contorno del objeto y de amplitud unidad. La expresión para dicho campo magnético se recoge en (4.84); donde aparece un término constituido por el producto escalar $(\hat{n} \cdot \vec{R})$, y una función de Hankel de segunda especie y orden uno. Esta función podría desarrollarse (ver apéndice $\mathrm{E}$ ) en serie de funciones cilíndricas emergentes centradas en el origen de coordenadas; aunque, al desarrollar una función de Hankel de segunda especie y orden distinto de cero, el citado desarrollo incorpora una función exponencial de orden uno en este caso, que depende de la posición relativa del punto donde se calcula el campo a la fuente generadora. Asimismo, el producto en (4.84) de la función de Hankel de segunda especie y orden uno por el término $(\hat{n} \cdot \vec{R})$ no puede expresarse, empleando la segunda versión del Teorema de Adición para las Funciones de Hankel, mediante una serie de funciones cilíndricas emergentes centradas en el origen de coordenadas; por lo que resulta imposible identificar de esta manera los coeficientes $S_{q n}$ para incidencia polarizada según TE ${ }^{z}$.

En otras palabras, las fuentes puntuales de corriente inducidas por un campo incidente con polarización $\mathrm{TE}^{\mathrm{z}}$ generan un campo magnético con un cierto diagrama de radiación $(\hat{n} \cdot \vec{R})$; que además multiplica a una función de Hankel de segunda especie y orden uno. El producto de estos dos términos imposibilita, como se termina de explicar, la identificación de los coeficientes $S_{q n}$; que deben ser independientes de la posición en la que se calcula el campo dispersado. Por contra, en el caso de incidencia con polarización $\mathrm{TM}^{\mathrm{z}}$, las fuentes de corriente producen un campo eléctrico con un diagrama omnidireccional que multiplica a una función de Hankel de segunda especie y orden cero; permitido pues identificar fácilmente, haciendo uso de la segunda versión del Teorema de Adición para las Funciones de Hankel, los coeficientes $S_{q n}$ de la matriz de espectro.

\subsubsection{Estudio Comparativo entre Transformada Discreta de Fourier y Teorema de Adición para las Funciones de Hankel}

En el caso de incidencia con polarización $\mathrm{TM}^{\mathrm{z}}$, donde se pueden utilizar los dos métodos descritos para obtener la matriz de espectro, se ha comprobado que ambos presentan la misma exactitud. En concreto, la diferencia media entre los elementos de las matrices de espectro, obtenidas por uno y otro método, es del orden de $10^{-4}$; es decir, los elementos de dichas matrices son prácticamente idénticos. Como ambos métodos son igualmente exactos, es preferible utilizar el Teorema de Adición para las Funciones de Hankel; porque requiere un menor esfuerzo computacional que la Transformada Discreta de Fourier. 
En el caso de incidencia polarizada según $\mathrm{TE}^{\mathrm{z}}$, tal y como ha quedado demostrado en el apartado anterior, sólo es posible obtener la matriz de espectro mediante la Transformada Discreta de Fourier. Consecuentemente, la obtención de la matriz de caracterización de un objeto dispersor, para el caso de incidencia con polarización $\mathrm{TE}^{\mathrm{z}}$, resultará más costosa computacionalmente que para incidencia $\mathrm{TM}^{\mathrm{z}}$. 



\section{Capítulo 5}

\section{Caracterización de Objetos Dispersores Canónicos en Espacio Libre}

En el capítulo 4 se ha introducido el concepto de la función de transferencia o matriz de caracterización de un objeto dispersor; que permite analizar la dispersión electromagnética producida por dicho objeto en espacio libre ante cualquier posible incidencia. Asimismo, en dicho capítulo se han presentado distintas técnicas para obtener la matriz de corrientes y la matriz de espectro; requeridas en el cálculo de la matriz de caracterización. Estas técnicas se aplican en el presente capítulo al análisis del comportamiento dispersor de dos objetos canónicos, como son el cilindro y la tira, ambos metálicos e invariantes en una dimensión.

En primer lugar, debido a la geometría particular del cilindro infinito, se obtiene de forma analítica su correspondiente matriz de caracterización para incidencia polarizada según $\mathrm{TM}^{\mathrm{z}}$. Haciendo uso de esta matriz, se calcula el campo dispersado por dicho objeto ante distintas incidencias; corroborando de esta manera la principal ventaja asociada al nuevo método propuesto para analizar problemas de dispersión electromagnética. A continuación, aplicando las mencionadas técnicas propuestas en el capítulo 4, se determina de nuevo la matriz de caracterización del cilindro infinito para incidencia $\mathrm{TM}^{\mathrm{z}}$; y con ella se presentan resultados que, tras comparar con los obtenidos de forma analítica, garantizan la validez de las citadas técnicas. Finalmente, dichas técnicas se aplican a la caracterización completa de un objeto dispersor, en este caso se ha escogido la tira infinita, cuya geometría no presenta solución analítica. La tira se caracteriza tanto para incidencia con polarización $\mathrm{TM}^{\mathrm{z}}$ como $\mathrm{TE}^{\mathrm{z}}$; permitiendo de esta forma analizar la dispersión que produce este objeto ante cualquier posible campo incidente. 


\subsection{Cilindro Metálico Infinito ante Incidencia $\mathrm{TM}^{z}$. Solución Analítica}

La matriz de caracterización de un cilindro metálico infinito, debido a su geometría particular, presenta una solución analítica sencilla [69].

Particularizando la condición de contorno apropiada sobre la superficie del cilindro conductor, se encuentra una relación directa entre las amplitudes espectrales asociadas a los campos eléctricos incidente y dispersado; relación que define de forma precisa los elementos de la matriz de caracterización buscada. Además, los resultados obtenidos con dicha matriz se utilizarán como referencias con las que poder comparar los resultados de la siguiente sección (apartado 5.2.3); donde de nuevo se caracteriza la dispersión que produce un cilindro infinito, pero empleando las técnicas presentadas en el capítulo 4.

Tras obtener de forma analítica la matriz de caracterización de un cilindro metálico infinito, se ofrecen resultados correspondientes a la dispersión electromagnética que produce un cilindro concreto ante distintas incidencias.

\subsubsection{Cálculo Analítico de la Matriz de Caracterización}

En el cálculo de la matriz de caracterización de un cilindro metálico infinito no se requiere, como se demuestra a continuación, determinar previamente la matriz de corrientes y la matriz de espectro (ver capítulo 4); sino que sus elementos pueden deducirse fácilmente al imponer la correspondiente condición de contorno sobre la superficie del cilindro conductor.

Cuando se considera incidencia con polarización $\mathrm{TM}^{\mathrm{z}}$, como el caso que nos ocupa, la matriz de caracterización debe relacionar espectros asociados a los campos eléctricos incidente y dispersado. Debe escogerse pues la condición de continuidad para las componentes tangenciales del campo eléctrico definida en (4.35); que particularizada sobre la superficie del cilindro da lugar a la expresión

$$
E_{z}^{i}(\rho=a \lambda)+E_{z}^{d}(\rho=a \lambda)=0
$$

donde $a$ representa el radio normalizado (respecto de $\lambda$ ) del cilindro.

Recordando (4.2) y (4.5), los campos eléctricos incidente y dispersado se expresan mediante los siguientes desarrollos modales

$$
\begin{aligned}
& E_{z}^{i}(\rho)=\sum_{p=-\infty}^{\infty} i_{p}^{\mathrm{TM}} J_{p}(k \rho) e^{j p \phi} \\
& E_{z}^{d}(\rho)=\sum_{q=-\infty}^{\infty} c_{q}^{\mathrm{TM}} H_{q}^{(2)}(k \rho) e^{j q \phi}
\end{aligned}
$$


En (5.2), $i_{p}^{\mathrm{TM}}$ y $c_{q}^{\mathrm{TM}}$ representan respectivamente las amplitudes espectrales asociadas a los campos eléctricos incidente y dispersado. Tras sustituir en (5.1) dichos campos por sus respectivas expresiones modales recogidas en (5.2), se obtiene la siguiente ecuación

$$
\sum_{p=-\infty}^{\infty} i_{p}^{\mathrm{TM}} J_{p}(2 \pi a) e^{j p \phi}+\sum_{q=-\infty}^{\infty} c_{q}^{\mathrm{TM}} H_{q}^{(2)}(2 \pi a) e^{j q \phi}=0
$$

donde es posible agrupar ambos sumatorios del siguiente modo

$$
\sum_{n=-\infty}^{\infty}\left[i_{n}^{\mathrm{TM}} J_{n}(2 \pi a)+c_{n}^{\mathrm{TM}} H_{n}^{(2)}(2 \pi a)\right] e^{j n \phi}=0
$$

La ecuación (5.4) se cumple forzando simplemente la siguiente condición entre los coeficientes $i_{n}^{\mathrm{TM}}$ y $c_{n}^{\mathrm{TM}}$

$$
i_{n}^{\mathrm{TM}} J_{n}(2 \pi a)+c_{n}^{\mathrm{TM}} H_{n}^{(2)}(2 \pi a)=0
$$

que permite relacionarlos directamente de la siguiente manera

$$
c_{n}^{\mathrm{TM}}=-\frac{J_{n}(2 \pi a)}{H_{n}^{(2)}(2 \pi a)} i_{n}^{\mathrm{TM}}
$$

Haciendo uso de (5.6), se puede construir la matriz de caracterización de un cilindro metálico infinito para incidencia con polarización $\mathrm{TM}^{\mathrm{z}}$; cuyo aspecto se muestra a continuación

$$
D^{\mathrm{TM}}=\left[\begin{array}{cccc}
d_{-N-N}^{\mathrm{TM}} & 0 & \cdots & 0 \\
0 & d_{-N+1-N+1}^{\mathrm{TM}} & \cdots & 0 \\
\vdots & \vdots & \ddots & \vdots \\
0 & 0 & \cdots & d_{N N}^{\mathrm{TM}}
\end{array}\right]_{2 N+1 \times 2 N+1}
$$

y donde $2 N+1$, que determina el número de modos escogidos para representar tanto el campo eléctrico incidente como el dispersado, se selecciona para satisfacer el criterio $N>k a \lambda$ (ver sección 4.1); permitiendo de esta forma una perfecta reconstrucción de dichos campos.

Como puede observarse en (5.7), la matriz de caracterización $D^{\mathrm{TM}}$ es cuadrada y además diagonal; cuyos elementos no nulos se expresan como

$$
d_{i i}^{\mathrm{TM}}=-\frac{J_{i}(2 \pi a)}{H_{i}^{(2)}(2 \pi a)}
$$

Esta matriz de caracterización permite, por tanto, obtener el espectro asociado al campo que dispersa un cilindro ante cualquier incidencia $\mathrm{TM}^{\mathrm{z}}$; simplemente multiplicando para ello dicha matriz por el espectro asociado al campo incidente. A continuación, se ofrecen resultados de la dispersión que produce un cilindro, caracterizado como se termina de comentar, ante distintas incidencias polarizadas según $\mathrm{TM}^{\mathrm{z}}$. 


\subsubsection{Resultados}

Los resultados ofrecidos en este apartado corresponden al análisis de la dispersión electromagnética que produce un cilindro metálico infinito, de radio $0.5 \lambda$, ante incidencia con polarización $\mathrm{TM}^{\mathrm{z}}$. El comportamiento de dicho objeto dispersor se ha caracterizado mediante su función de transferencia o matriz de caracterización definida según (5.7) y (5.8).
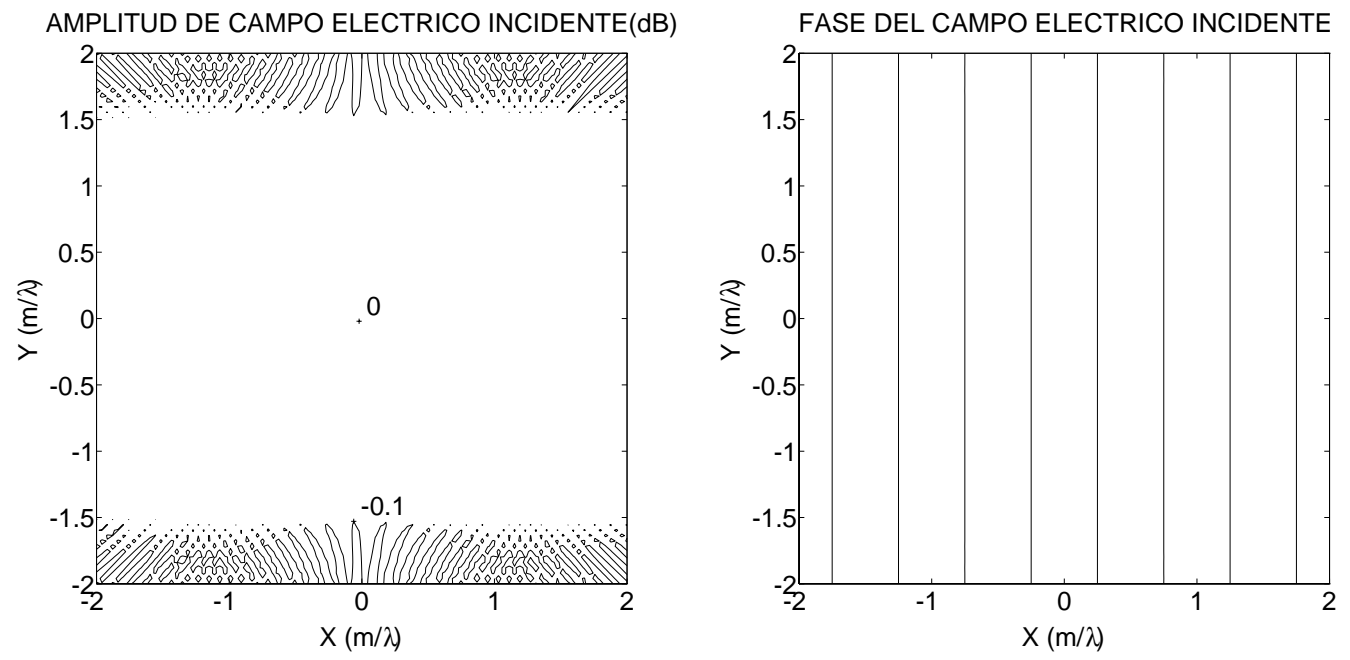

Figura 5.1: Amplitud y fase de una onda plana incidente con dirección de avance $\beta=0$; que se ha reconstruido a partir de su espectro cilíndrico.

Inicialmente, se pretende conocer la respuesta del cilindro en cuestión ante la incidencia de una onda plana con dirección de avance $\beta=0$ (ver figura C.1); es decir, ante una onda plana que avanza paralela al eje $\hat{x}$ y en el sentido creciente de la coordenada $x$. Esta onda plana puede observarse en la figura 5.1; donde se ha reconstruido a partir de su espectro cilíndrico equivalente (ver apéndice $\mathrm{C}$ ). En dicha figura, se comprueba que la onda plana presenta una amplitud constante y un frente de fase que avanza en la dirección $\beta=0$ dentro de una cierta zona de interés, zona donde lógicamente se posiciona el cilindro metálico infinito.

Como se ha comentado anteriormente, el espectro asociado al campo eléctrico dispersado por el cilindro, ante la onda plana incidente mostrada en la figura 5.1, se obtiene multiplicando la matriz de caracterización de dicho cilindro por el espectro asociado al campo eléctrico incidente. La reconstrucción del campo eléctrico dispersado, a partir de su espectro, se ofrece en la figura 5.2; donde se observa que el cilindro produce un máximo de campo dispersado en la zona contraria a la de procedencia de la onda plana incidente (región $x>0.5 \lambda$ ), región que se conoce como zona de sombra o cono de difracción. En dicha zona, la fase del campo dispersado se corresponde con la fase de una onda plana; por 

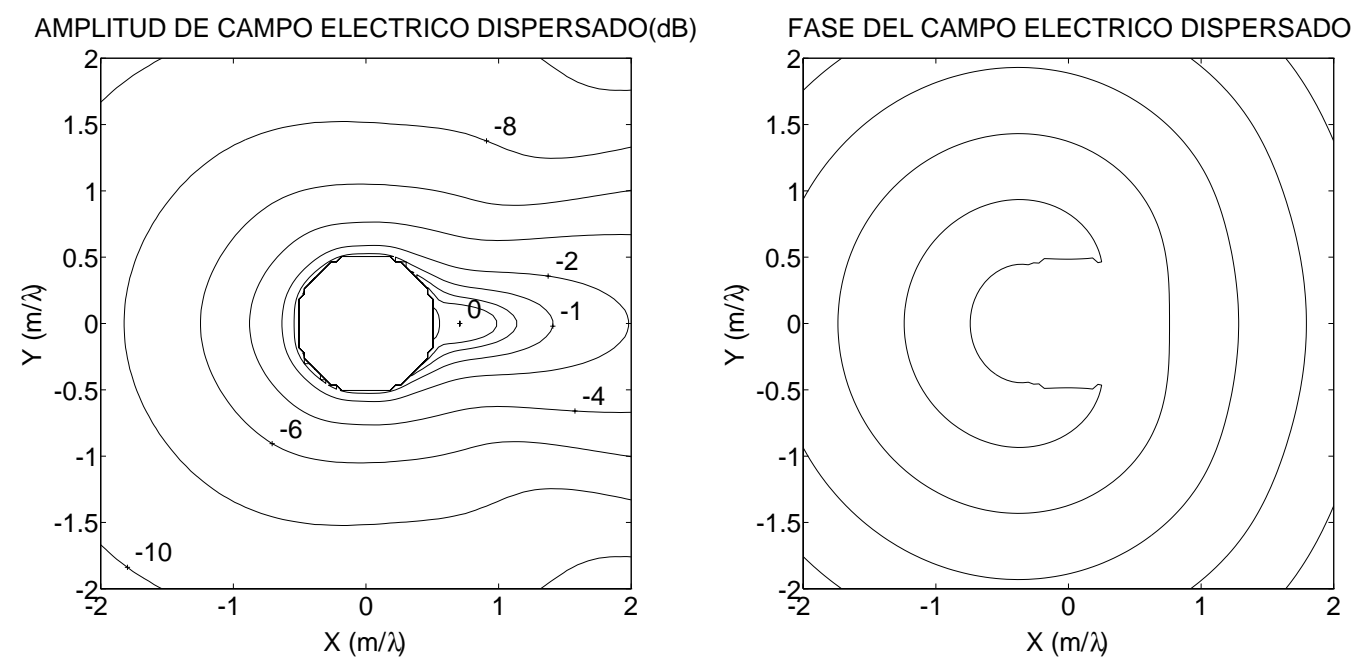

Figura 5.2: Amplitud y fase del campo eléctrico dispersado por un cilindro metálico infinito, de radio $0.5 \lambda$, ante la incidencia de una onda plana $(\beta=0)$.
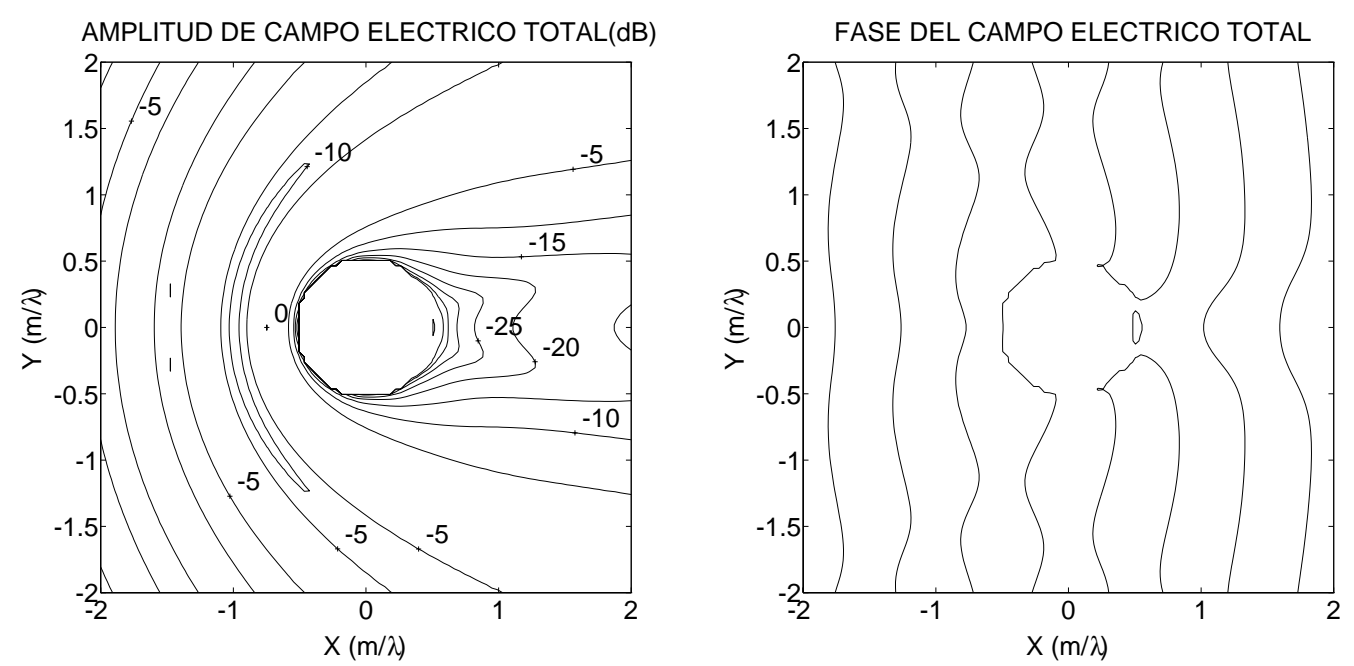

Figura 5.3: Amplitud y fase del campo eléctrico total; obtenido como la suma de la onda plana incidente $(\beta=0)$ y del campo eléctrico dispersado por un cilindro metálico infinito, de radio $0.5 \lambda$, ante dicha incidencia.

tanto, se puede decir que el cilindro dispersa en esa región una onda plana con la misma amplitud y dirección de avance del frente de fase que la onda plana incidente, aunque con un desfase de $180^{\circ}$. Esta circunstancia se ratifica en la figura 5.3; donde se representa el campo eléctrico total obtenido tras sumar los campos eléctricos incidente y dispersado. En la zona posterior al cilindro (región $x>0.5 \lambda$ ) se observa que estos campos eléctricos incidente y dispersado se suman 
en contrafase, produciendo la conocida zona de sombra o cono de difracción; mientras que en la zona anterior del cilindro (región $x<0.5 \lambda$ ) la suma de dichos campos corresponde a la suma de dos ondas planas cuyos frentes de fase avanzan en sentidos opuestos, originando en esta ocasión una onda estacionaria.
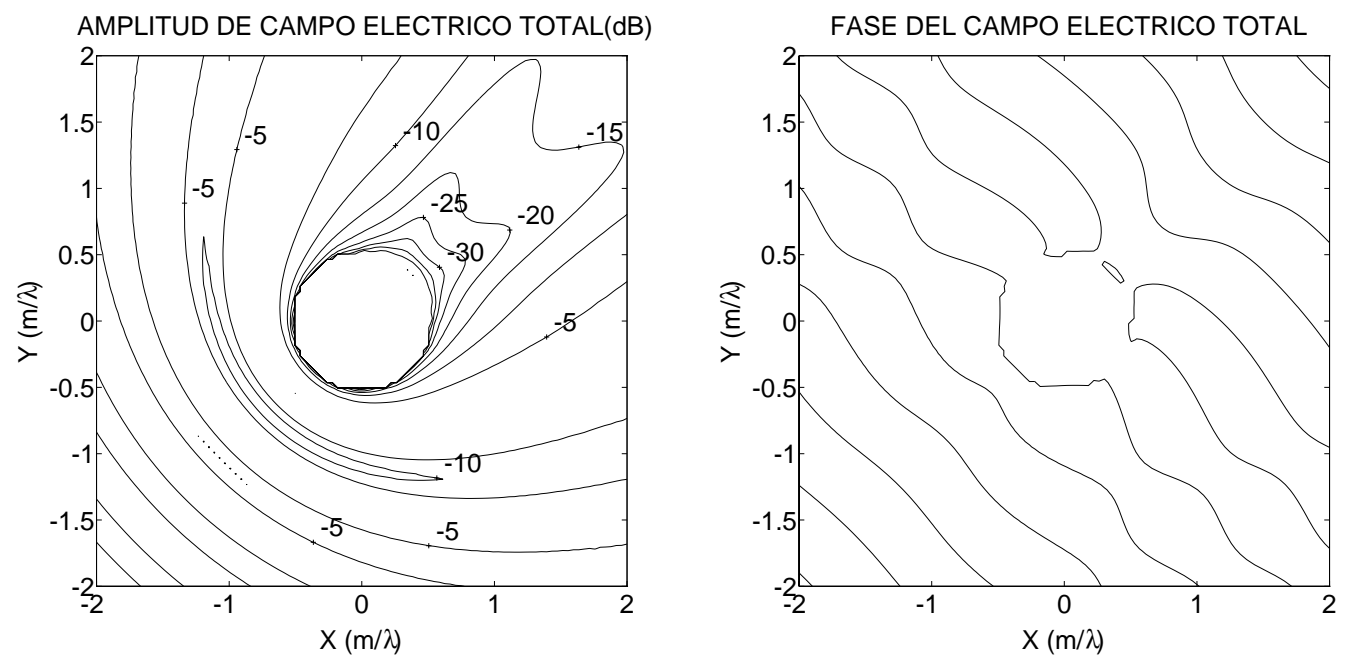

Figura 5.4: Amplitud y fase del campo eléctrico total; obtenido como la suma de la onda plana incidente $(\beta=\pi / 4)$ y del campo eléctrico dispersado por un cilindro metálico infinito, de radio $0.5 \lambda$, ante dicha incidencia.

Cuando se pretenda conocer la respuesta del mismo cilindro de radio $0.5 \lambda$ ante otra incidencia, tan sólo se requiere multiplicar la matriz de caracterización obtenida con anterioridad por el espectro asociado al nuevo campo incidente. Por ejemplo, se ha considerado la respuesta que produce dicho cilindro ante una onda plana incidente con un ángulo de incidencia $\beta=\pi / 4$. En la figura 5.4 , se representa la amplitud y la fase del campo eléctrico total ante la incidencia de dicha onda plana; observando que el cono de difracción y la onda estacionaria son acordes con la nueva dirección de la onda plana incidente.

Los ejemplos presentados sirven para ilustrar la utilidad de la función de transferencia o matriz de caracterización; pues a partir de ella, y de forma inmediata, se ha obtenido la respuesta del cilindro ante dos campos incidentes distintos.

\subsection{Cilindro Metálico Infinito ante Incidencia $\mathrm{TM}^{z}$. Solución No Analítica}

En esta sección, la matriz de caracterización de un cilindro metálico infinito se obtiene utilizando la matriz de corrientes y la matriz de espectro presentadas en el capítulo 4. En concreto, el cálculo de la matriz de corrientes se ha realizado 
mediante la aproximación de Óptica Física y el Método de los Momentos (PointMatching); particularizando las expresiones generales recogidas en la sección 4.2 al caso concreto que nos ocupa. En el cálculo de la matriz de espectro, tan sólo se ha considerado la técnica basada en el Teorema de Adición para las Funciones de Hankel; pues en la sección 4.3 se demuestra que mediante la Transformada Discreta de Fourier se obtiene exactamente la misma matriz, pero con mayor coste computacional.

Empleando la aproximación de Óptica Física y el Método de los Momentos (Point-Matching), se han caracterizado distintos cilindros metálicos infinitos; cuyos resultados se han comparado con aquellos obtenidos mediante la matriz de caracterización calculada de forma analítica, tal y como se describe en la sección anterior (apartado 5.1.1). De esta forma, se ha comprobado la validez de estas técnicas que permiten caracterizar cualquier objeto dispersor; y se ha comprobado, asimismo, su rango de validez atendiendo a las dimensiones de los objetos analizados.

\subsubsection{Cálculo de la Matriz de Corrientes mediante Óptica Física}

La matriz de corrientes, definida en la sección 4.2, relaciona la distribución de corrientes en la superficie del objeto dispersor con el espectro asociado al campo incidente. En primer lugar, se particulariza la aproximación de Óptica Física al cálculo de la matriz de corrientes para un cilindro metálico infinito. Aplicando los pasos descritos en el apartado 4.2.1, se alcanza la siguiente expresión general para la matriz de corrientes

$$
D I=\left[\begin{array}{cccc}
A_{10} & A_{11} & \cdots & A_{1 N_{\beta}-1} \\
A_{20} & A_{21} & \cdots & A_{2 N_{\beta}-1} \\
\vdots & \vdots & \ddots & \vdots \\
A_{N_{s} 0} & A_{N_{s} 1} & \cdots & A_{N_{s} N_{\beta}-1}
\end{array}\right]_{N_{s} \times N_{\beta}} \quad \begin{gathered}
N_{N_{\beta}-1} \\
\downarrow
\end{gathered}
$$

que consta del producto de dos matrices. La primera de ellas convierte el espectro cilíndrico del campo incidente, formado por $2 N_{i}+1$ componentes, en su correspondiente espectro de ondas planas, constituido por $N_{\beta}$ componentes o direcciones de avance. Esta primera matriz resulta necesaria para poder aplicar la aproximación de Óptica Física, que recordemos requiere de un espectro de ondas planas asociado al campo incidente. La segunda matriz relaciona este espectro de ondas planas con la amplitud de la corriente inducida en $N_{s}$ fuentes puntuales de corriente; obtenidas tras discretizar el contorno del objeto dispersor, y representadas en este caso por hilos infinitos de corriente. La expresión analítica de los elementos $A_{n m}$ para el caso concreto que nos ocupa de incidencia polarizada 
según $\mathrm{TM}^{\mathrm{z}}$, cuyo desarrollo se puede encontrar de nuevo en el apartado 4.2.1, se resume a continuación mediante

$$
A_{n m}=\left\{\begin{array}{cl}
{\left[2 \hat{n} \times \frac{1}{\eta}\left(\hat{x} \sin \left(\beta_{m}\right)-\hat{y} \cos \left(\beta_{m}\right)\right)\right.} & \text { si } \phi_{n} \in\left[\beta_{m}+\frac{\pi}{2}, \beta_{m}+\frac{3 \pi}{2}\right] \\
\left.e^{-j k \rho_{n} \cos \left(\phi_{n}-\beta_{m}\right)} \Delta C_{n}\right] \cdot \hat{z} & \text { en caso contrario } \\
0 &
\end{array}\right.
$$

donde, tal y como se representa en la figura $5.5, \beta_{m}$ indica la dirección de avance asociada a la $m$-ésima onda plana, que se expresa analíticamente como

$$
\beta_{m}=\frac{2 \pi}{N_{\beta}} m \quad m \in\left[0,1, \ldots, N_{\beta}-1\right]
$$

y donde las coordenadas cilíndricas $\left(\rho_{n}, \phi_{n}\right)$ definen la posición del $n$-ésimo hilo de corriente. En la figura 5.5, se observa que dichos hilos se distribuyen de forma equiespaciada sobre la superficie del cilindro conductor; de modo que para $\rho_{n} \mathrm{y}$ $\phi_{n}$ se eligen los valores

$$
\begin{aligned}
\rho_{n} & =a \lambda \\
\phi_{n} & =\frac{2 \pi}{N_{s}}(n-1) \quad n \in\left[1,2, \ldots, N_{s}\right]
\end{aligned}
$$

Además, en (5.10), $\hat{n}$ denota un vector unitario normal al contorno del cilindro en cada uno de los puntos donde se induce corriente; el cual viene descrito en función de los vectores directores $\hat{x}$ e $\hat{y}$ como

$$
\hat{n}=\hat{x} \cos \left(\phi_{n}\right)+\hat{y} \sin \left(\phi_{n}\right)
$$

y finalmente, $\Delta C_{n}$ es la longitud del $n$-ésimo segmento que contiene al correspondiente hilo de corriente. Al distribuir dichos hilos de forma equiespaciada sobre el contorno del objeto dispersor, la longitud $\Delta C_{n}$ presenta el siguiente valor constante e independiente del segmento considerado

$$
\Delta C_{n}=\frac{2 \pi a \lambda}{N_{s}} \quad \forall n, \quad n \in\left[1,2, \ldots, N_{s}\right]
$$

Sustituyendo en (5.10) el vector $\hat{n}$ y la longitud $\Delta C_{n}$ por sus respectivos valores, que se terminan de presentar en (5.13) y (5.14), se obtiene la siguiente expresión para los coeficientes $A_{n m}$ en el caso del cilindro conductor bajo análisis

$$
A_{n m}=\left\{\begin{array}{cl}
-\frac{4 \pi a \lambda}{\eta N_{s}} \cos \left(\phi_{n}-\beta_{m}\right) & \text { si } \phi_{n} \in\left[\beta_{m}+\frac{\pi}{2}, \beta_{m}+\frac{3 \pi}{2}\right] \\
e^{-j k \rho_{n} \cos \left(\phi_{n}-\beta_{m}\right)} & \text { en caso contrario } \\
0 &
\end{array}\right.
$$




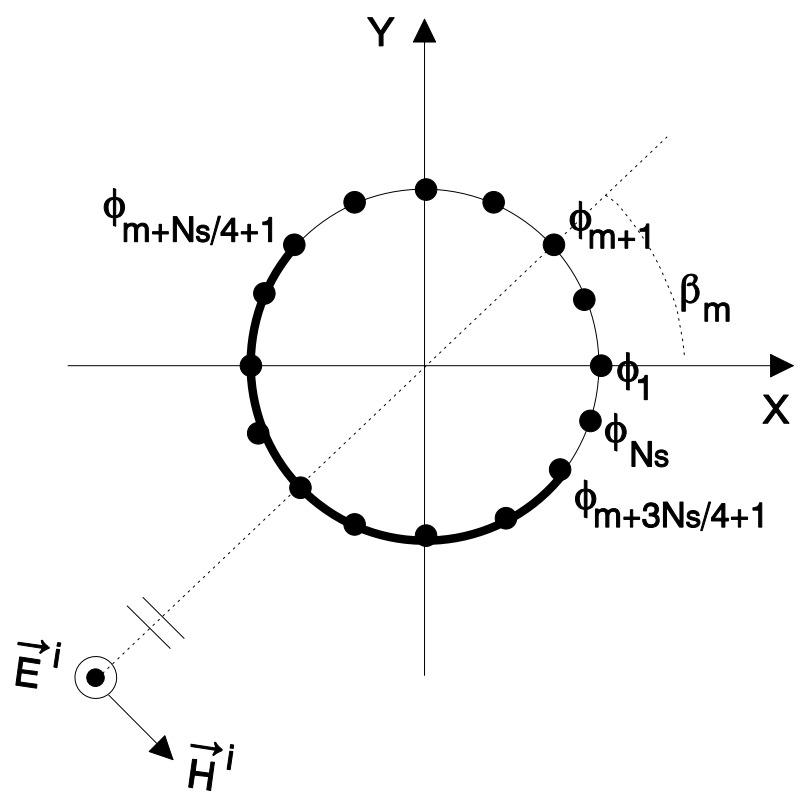

Figura 5.5: Posición de los hilos de corriente sobre la superficie del cilindro.

Los valores de $N_{s}$ y $N_{\beta}$ se escogen de modo que cada una de las ondas planas consideradas induzca hilos de corriente en los extremos de la zona que ilumina sobre la superficie del cilindro conductor. Por ejemplo, en la figura 5.5 se representa con trazo grueso la zona iluminada por una onda plana incidente con dirección de avance $\beta_{m}$; zona que se extiende sobre la superficie del cilindro desde $\phi=\beta_{m}+\pi / 2$ hasta $\phi=\beta_{m}+3 \pi / 2$. Forzando la condición de que en dichos extremos existan hilos de corriente inducida, se deduce que $N_{s}$ debe ser igual a $N_{\beta}$, y además múltiplo de 4 . De esta forma, cualquier onda plana incidente con dirección de avance $\beta_{m}$ induce $N_{s} / 2+1$ hilos infinitos de corriente, definidos en $(5.12)$ por sus coordenadas cilíndricas $\left(\rho_{n}, \phi_{n}\right)$ con $n$ variando entre un valor inicial $n_{i}=m+N_{s} / 4+1 \mathrm{y}$ un valor final $n_{f}=m+3 N_{s} / 4+1$. Estos valores para $n_{i}$ y $n_{f}$ hacen coincidir, tal y como se pretendía, las posiciones del primer y último hilo de corriente con los extremos de la zona iluminada (ver figura 5.5).

Como consecuencia de haber escogido el mismo valor para $N_{s}$ y $N_{\beta}$, la matriz que relaciona en (5.9) corrientes inducidas con espectro de ondas planas incidentes resulta cuadrada. Esta elección, que permite distribuir de forma adecuada los hilos de corriente sobre la superficie del cilindro, no perjudica en absoluto la precisión requerida en los resultados. En cuanto al valor concreto para $N_{s}$, el cual debe ser múltiplo de 4 , se ha comprobado experimentalmente que para obtener resultados suficientemente precisos, se requieren al menos 40 hilos de corriente por cada longitud de onda en el radio del cilindro analizado; es decir, $N_{s}$ debe satisfacer la relación

$$
N_{s}>40 a
$$


Finalmente, teniendo en cuenta el criterio expuesto para seleccionar los valores de $n_{i}$ y $n_{f}$; y sustituyendo en (5.15) tanto la dirección angular $\beta_{m}$ como las coordenadas cilíndricas $\left(\rho_{n}, \phi_{n}\right)$ por sus correspondientes valores, recogidos respectivamente en (5.11) y (5.12), se alcanza la siguiente expresión definitiva para los coeficientes $A_{n m}$

$$
A_{n m}=\left\{\begin{array}{cl}
-\frac{4 \pi a \lambda}{\eta N_{s}} \cos \left(\frac{2 \pi}{N_{s}}(n-1-m)\right) & \text { si } n \in\left[m+\frac{N_{s}}{4}+1, m+\frac{3 N_{s}}{4}+1\right] \\
e^{-j 2 \pi a \cos \left(\frac{2 \pi}{N_{s}}(n-1-m)\right)} & \text { resto } \\
0 &
\end{array}\right.
$$

que una vez sustituida en (5.9), permite obtener la matriz de corrientes para un cilindro metálico infinito ante incidencia con polarización $\mathrm{TM}^{\mathrm{z}}$.

\subsubsection{Cálculo de la Matriz de Corrientes mediante el Mé- todo de los Momentos}

La matriz de corrientes también se puede calcular (ver sección 4.2) resolviendo la correspondiente Ecuación Integral mediante el Método de los Momentos. A continuación se aplica dicha técnica, descrita en el apartado 4.2.2, a la obtención de la matriz de corrientes para el caso del cilindro metálico que nos ocupa.

Siguiendo los pasos propuestos en el apartado 4.2 .2 para el caso de incidencia con polarización $\mathrm{TM}^{\mathrm{z}}$ se obtiene, tras aplicar el Método de los Momentos (PointMatching) a la Ecuación Integral definida en (4.43), la siguiente expresión para la matriz de corrientes

$$
\left[\begin{array}{c}
I_{1} \\
I_{2} \\
\vdots \\
I_{N_{s}}
\end{array}\right]_{N_{s} \times 1}=\overbrace{N_{s}}=1 \overbrace{\left[\begin{array}{l}
\alpha_{n p} \Delta C_{n} \\
\end{array}\right]}^{-N_{i} \longrightarrow p-\rightarrow N_{i}} \cdot\left[\begin{array}{c}
i_{-N_{i}} \\
i_{-N_{i}+1} \\
\vdots \\
i_{N_{i}}
\end{array}\right]_{2 N_{i}+1 \times 1}
$$

donde $\Delta C_{n}$ representa la longitud del $n$-ésimo segmento; situado sobre el contorno del cilindro y en el que se ha definido la correspondiente función base $f_{n}$, representada en (4.47) por medio de una función pulso centrada en el punto medio de dicho segmento. De nuevo, el contorno del cilindro dispersor se divide en $N_{s}$ segmentos de igual longitud; cuyo valor se expresa fácilmente como

$$
\Delta C_{n}=\frac{2 \pi a \lambda}{N_{s}} \quad \forall n, n \in\left[1,2, \ldots, N_{s}\right]
$$

Por otro lado, los elementos $\alpha_{n p}$ presentes en (5.18) constituyen la solución al siguiente sistema matricial de ecuaciones algebraicas

$$
\left[I_{m n}\right] \cdot\left[\alpha_{n p}\right]=\left[g_{m p}\right]
$$


donde $\left[I_{m n}\right]$ representa la matriz de coeficientes definida en (4.41), cuyos elementos vienen recogidos en (4.51) y (4.53) como

$$
\begin{aligned}
I_{n n} & =\frac{k \eta}{4} \Delta C_{n}\left[1-j \frac{2}{\pi} \ln \left(\frac{\gamma k \Delta C_{n}}{4 e}\right)\right] \\
I_{m n} & =\frac{k \eta}{4} \Delta C_{n} H_{0}^{(2)}\left(k\left|\vec{\rho}_{m}-\vec{\rho}_{n}\right|\right)
\end{aligned}
$$

y $\left[g_{m p}\right]$ representa la matriz de excitaciones cuyos elementos, recordando (4.54), se expresan en función del campo eléctrico incidente mediante la relación

$$
g_{m p}=E_{z}^{i}\left(\vec{\rho}_{m}\right)=J_{p}\left(k \rho_{m}\right) e^{j p \phi_{m}}
$$

Por tanto, para construir la matriz de corrientes de un cilindro metálico infinito utilizando el Método de los Momentos, tan sólo se requiere particularizar las coordenadas que aparecen en $(5.21),(5.22)$ y $(5.23)$ a la geometría del cilindro bajo análisis.

En concreto, las coordenadas cilíndricas $\left(\rho_{n}, \phi_{n}\right)$ definen el punto medio del $n$-ésimo segmento sobre el contorno del cilindro; el cual, como se ha comentado anteriormente, se divide en $N_{s}$ segmentos de igual longitud. Consecuentemente, dichos puntos se distribuyen de forma equiespaciada a lo largo del contorno del siguiente modo

$$
\begin{aligned}
\rho_{n} & =a \lambda \\
\phi_{n} & =\frac{2 \pi}{N_{s}}(n-1) \quad n \in\left[1,2, \ldots, N_{s}\right]
\end{aligned}
$$

Por su parte, las coordenadas cilíndricas $\left(\rho_{m}, \phi_{m}\right)$ indican las posiciones sobre el contorno del cilindro donde se ubican las funciones delta de Dirac; que se escogen como funciones test en el Método de los Momentos (Point-Matching). Estas posiciones se eligen de nuevo equiespaciadas en el contorno del cilindro, y además se hacen coincidir con el punto medio $\left(\rho_{n}, \phi_{n}\right)$ de cada segmento; expresándose pues de la siguiente manera

$$
\begin{aligned}
\rho_{m} & =a \lambda \\
\phi_{m} & =\frac{2 \pi}{M}(m-1) \quad m \in[1,2, \ldots, M]
\end{aligned}
$$

Tal y como se indica en el apartado 4.2.2, el número $M$ de funciones test se escoge igual al número $N_{s}$ de funciones base; de modo que la matriz de coeficientes $\left[I_{m n}\right]$ en $(5.20)$ resulta cuadrada. De manera adicional, se ha comprobado experimentalmente que para obtener resultados con la suficiente precisión, hacen 
falta al menos 100 funciones base por cada longitud de onda en el radio del cilindro; por tanto, el criterio práctico que se ha seguido consiste en elegir un valor para $N_{s}$ que satisfaga la condición

$$
N_{s}>100 a
$$

Finalmente, tras sustituir en (5.21) y (5.22) la longitud $\Delta C_{n}$ de cada segmento por su valor recogido en (5.19), los elementos de la matriz de coeficientes $\left[I_{m n}\right]$ quedan expresados como

$$
\begin{aligned}
& I_{n n}=\frac{120 \pi^{3} a}{N_{s}}\left[1-j \frac{2}{\pi} \ln \left(\frac{\gamma \pi^{2} a}{N_{s} e}\right)\right] \\
& I_{m n}=\frac{120 \pi^{3} a}{N_{s}} H_{0}^{(2)}\left(k \sqrt{\left(x_{m}-x_{n}\right)^{2}+\left(y_{m}-y_{n}\right)^{2}}\right)
\end{aligned}
$$

donde las coordenadas cartesianas $\left(x_{n}, y_{n}\right)$ y $\left(x_{m}, y_{m}\right)$ se obtienen a partir de sus correspondientes coordenadas cilíndricas $\left(\rho_{n}, \phi_{n}\right)$ y $\left(\rho_{m}, \phi_{m}\right)$, definidas en $(5.24)$ y (5.25) respectivamente.

Una vez calculados los elementos de las matrices $\left[I_{m n}\right]$ y $\left[g_{m p}\right]$ como acabamos de indicar, se deben resolver los $2 N_{i}+1$ sistemas de ecuaciones algebraicas definidos matricialmente en (5.20); para de esta forma encontrar la matriz incógnita $\left[\alpha_{n p}\right]$. Sustituyendo los elementos de dicha matriz en (5.18), se obtiene definitivamente la matriz de corrientes para un cilindro metálico infinito ante incidencia con polarización $\mathrm{TM}^{\mathrm{z}}$.

\subsubsection{Resultados}

Los resultados que se presentan a continuación corresponden a la dispersión electromagnética producida por dos cilindros metálicos infinitos, de radios $0.5 \lambda$ y $6 \lambda$ respectivamente, ante incidencia con polarización $\mathrm{TM}^{\mathrm{z}}$. El comportamiento dispersor de cada uno de estos cilindros se ha caracterizado a través de su respectiva matriz de caracterización; la cual se ha obtenido tanto de forma analítica, siguiendo el desarrollo descrito en el apartado 5.1.1, como empleando para su cálculo la matriz de corrientes y la matriz de espectro. En ambos cilindros, la matriz de corrientes se ha determinado utilizando las expresiones correspondientes a la aproximación de Óptica Física y al Método de los Momentos, desarrolladas respectivamente en los apartados 5.2.1 y 5.2.2; mientras que la matriz de espectro se ha obtenido utilizando, únicamente, la técnica basada en el Teorema de Adición para las Funciones de Hankel descrita en el apartado 4.3.2, al resultar más eficiente computacionalmente que la técnica basada en la Transformada Discreta de Fourier (ver apartado 4.3.3).

De esta forma, comparando los resultados obtenidos mediante la aproximación de Óptica Física y el Método de los Momentos con aquéllos obtenidos de forma 


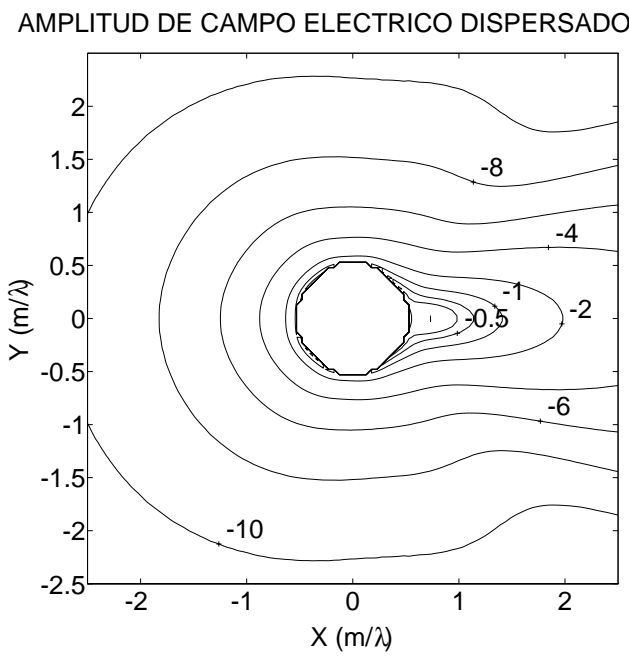

a)

AMPLITUD DE CAMPO ELECTRICO DISPERSADO (dB)

AMPLITUD DE CAMPO ELECTRICO DISPERSADO (dB)

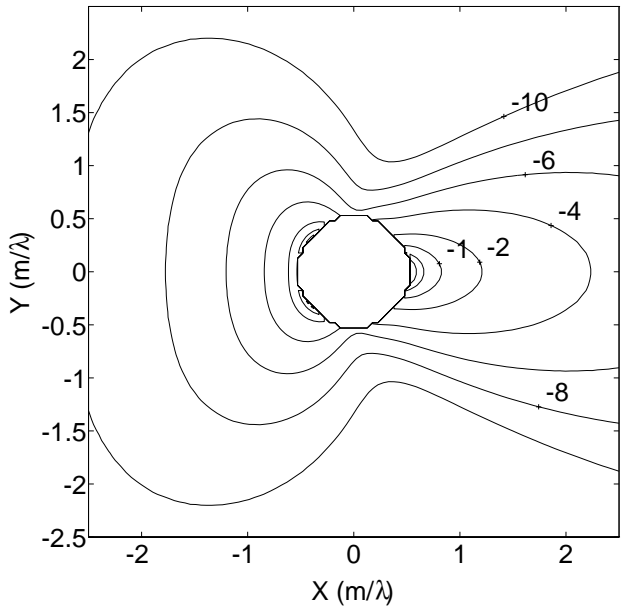

b)

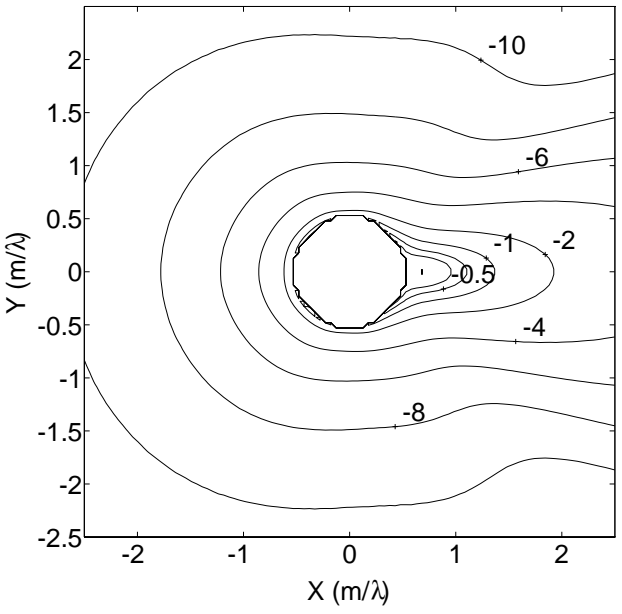

c)

Figura 5.6: Amplitud del campo eléctrico dispersado por un cilindro de radio $0.5 \lambda$ ante la incidencia de una onda plana $(\beta=0)$; calculado de forma analítica en a), mediante la aproximación de Óptica Física en b) y mediante el Método de los Momentos en c).

analítica, se puede garantizar la validez de las citadas técnicas; y, asimismo, determinar el rango de aplicación de cada una de ellas en función de las dimensiones eléctricas del objeto bajo análisis.

En primer lugar, empleando los tres métodos recién comentados, se ha calculado la respuesta del cilindro metálico infinito, de radio $0.5 \lambda$, ante una onda plana incidente con dirección de avance $\beta=0$ (ver figura C.1). En las figuras 5.6 y 5.7 se representa, respectivamente, la amplitud y la fase del campo eléctrico 


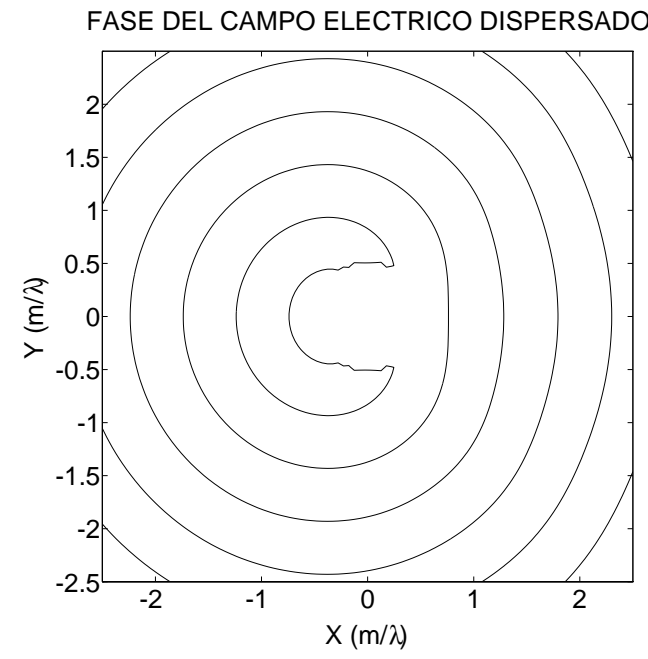

a)

FASE DEL CAMPO ELECTRICO DISPERSADO

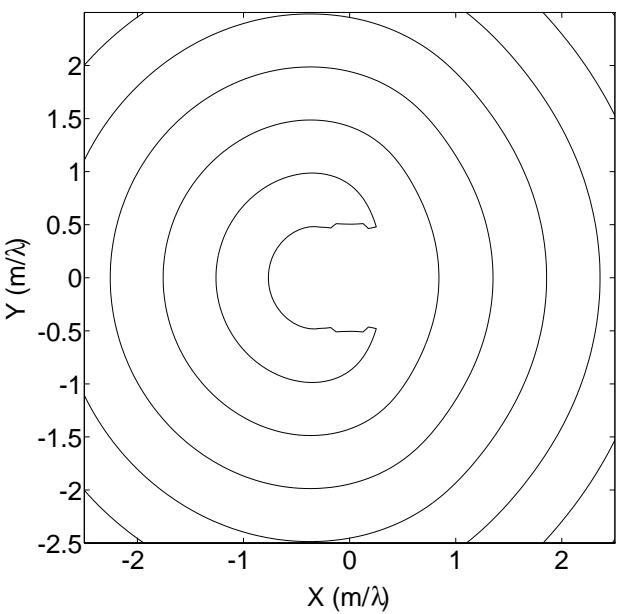

b)

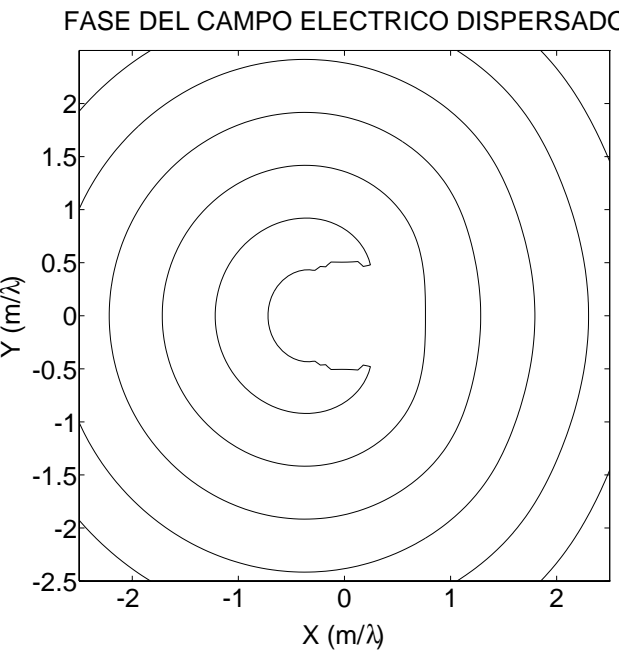

c)

Figura 5.7: Fase del campo eléctrico dispersado por un cilindro de radio $0.5 \lambda$ ante la incidencia de una onda plana $(\beta=0)$; calculado de forma analítica en a), mediante la aproximación de Óptica Física en b) y mediante el Método de los Momentos en c).

dispersado por dicho cilindro; donde se aprecia que el Método de los Momentos proporciona un campo eléctrico dispersado (apartado c en las figuras 5.6 y 5.7) muy próximo al exacto (solución analítica presentada en el apartado a de las figuras 5.6 y 5.7), mientras que la aproximación de Óptica Física (apartado b en las figuras 5.6 y 5.7) produce una solución que dista bastante de la obtenida analíticamente.

A continuación, utilizando de nuevo los tres métodos propuestos, se ha calcu- 


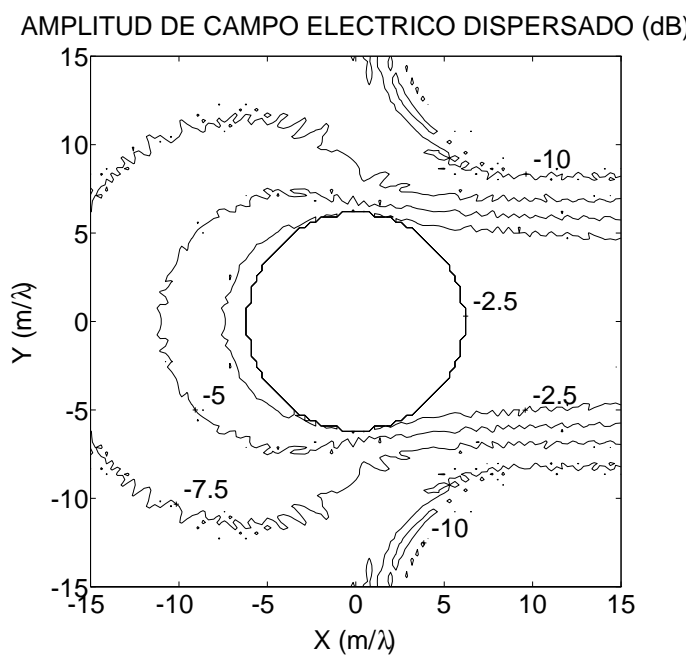

a)

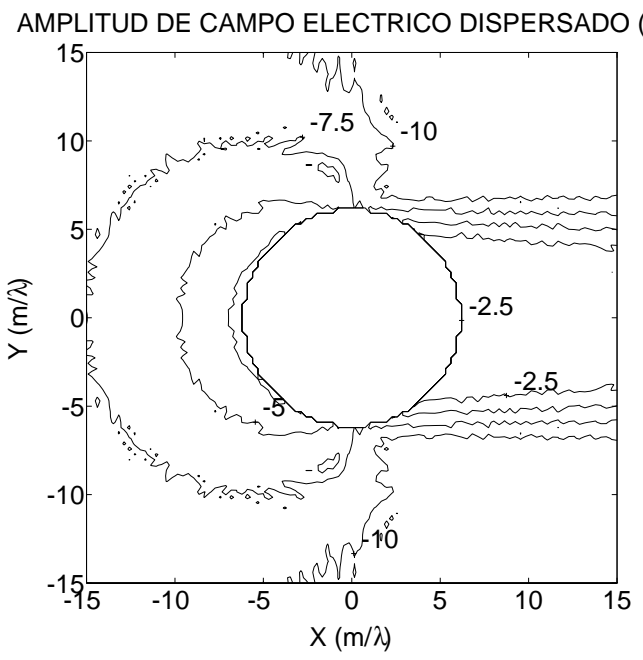

b)

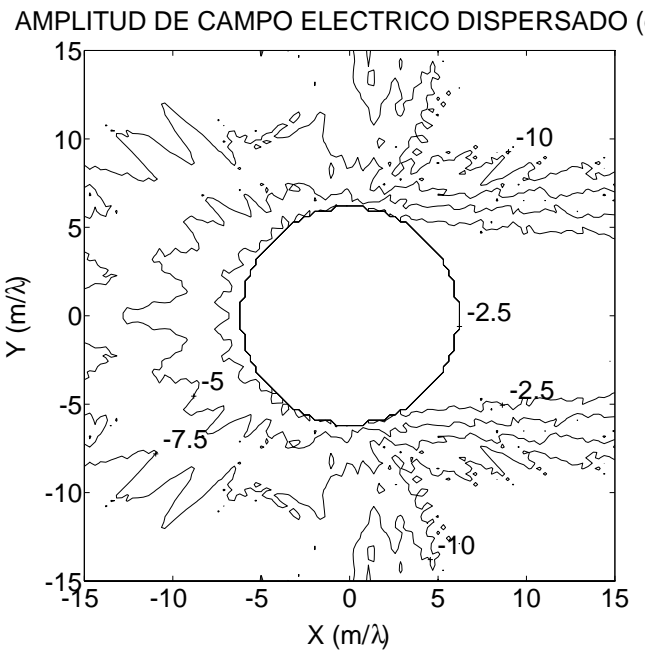

c)

Figura 5.8: Amplitud del campo eléctrico dispersado por un cilindro de radio $6 \lambda$ ante la incidencia de una onda plana $(\beta=0)$; calculado de forma analítica en a), mediante la aproximación de Óptica Física en b) y mediante el Método de los Momentos en c).

lado la respuesta del cilindro metálico infinito, de radio $6 \lambda$, ante la misma onda plana incidente con dirección de avance $\beta=0$. La amplitud y la fase del campo eléctrico dispersado se muestran, respectivamente, en las figuras 5.8 y 5.9; donde se observa que en esta ocasión la aproximación de Óptica Física proporciona un resultado (apartado b en las figuras 5.8 y 5.9) más parecido al exacto (solución analítica presentada en el apartado a de las figuras 5.8 y 5.9) que el obtenido con el Método de los Momentos (apartado c en las figuras 5.8 y 5.9). 


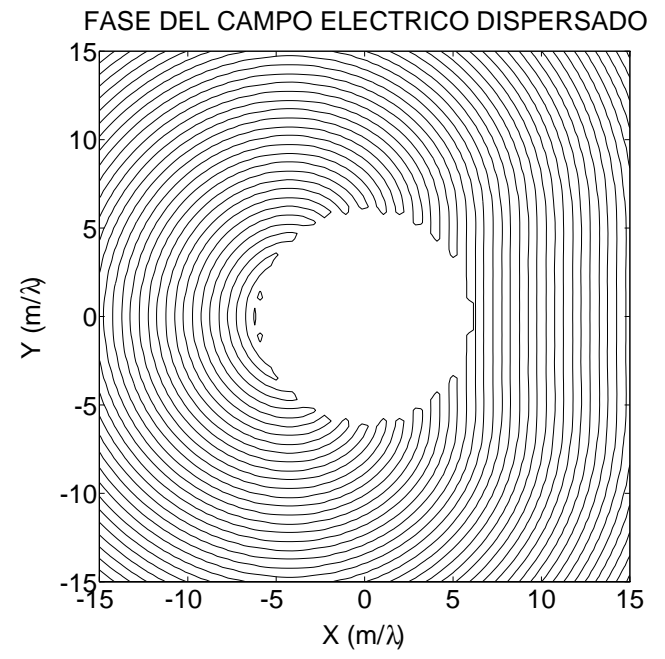

a)

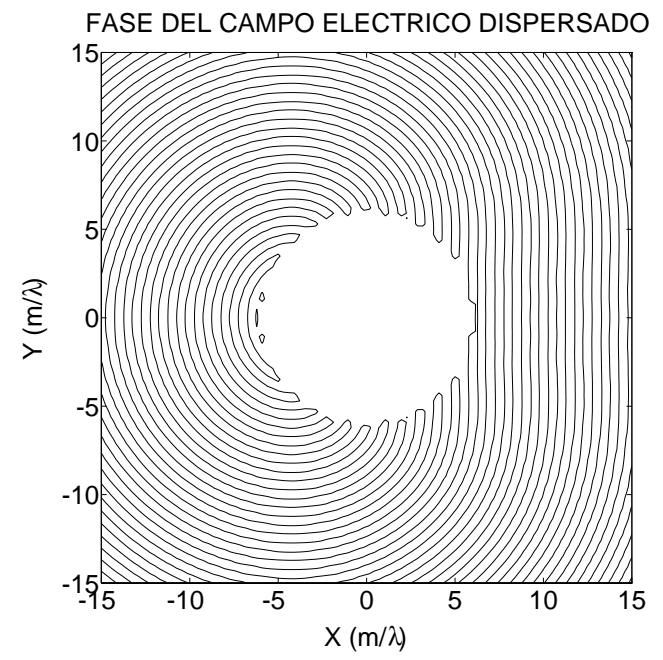

b)

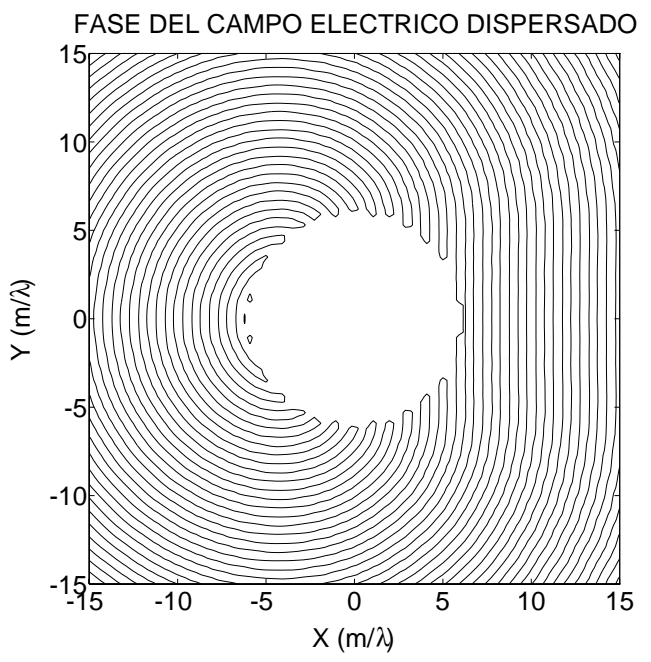

c)

Figura 5.9: Fase del campo eléctrico dispersado por un cilindro de radio $6 \lambda$ ante la incidencia de una onda plana $(\beta=0)$; calculado de forma analítica en a), mediante la aproximación de Óptica Física en b) y mediante el Método de los Momentos en c).

De estos resultados se concluye que las dos técnicas presentadas en el capítulo 4 para calcular la matriz de corrientes, son perfectamente utilizables en la obtención de la matriz de caracterización de un objeto dispersor. Además, dichos resultados corroboran el rango de aplicación, ya comentado en el capítulo 4, de cada una de las citadas técnicas en función de las dimensiones eléctricas del objeto a caracterizar. Así pues, el Método de los Momentos resulta más apropiado, a pesar de requerir un mayor esfuerzo computacional que la aproximación de 
Óptica Física, para objetos pequeños eléctricamente; al ofrecer en este caso resultados más exactos. Por su parte, la aproximación de Óptica Física se revela más conveniente conforme las dimensiones eléctricas del objeto aumentan; ya que proporciona resultados más precisos que el Método de los Momentos con un menor coste computacional.

\subsection{Tira Metálica Infinita ante Incidencia $\mathrm{TM}^{z}$}

La tira metálica infinita presenta una geometría que, a diferencia del cilindro conductor, no permite obtener de forma analítica su matriz de caracterización. Consecuentemente, en el análisis de la tira se requiere calcular la matriz de corrientes y la matriz de espectro; que se han presentado en el capítulo 4 y han sido validadas en la sección anterior (apartado 5.2.3).

En concreto se han caracterizado tiras metálicas de grosor infinitesimal, cuyas longitudes son pequeñas en términos eléctricos; obteniendo por tanto la matriz de corrientes mediante el Método de los Momentos. Caso de analizar tiras con dimensiones grandes eléctricamente, aunque no se ha contemplado dicha posibilidad en esta sección, resultaría más adecuado particularizar la aproximación de Óptica Física en el cálculo de la citada matriz de corrientes. Para obtener la matriz de espectro, al considerar incidencia con polarización $\mathrm{TM}^{\mathrm{z}}$, se utiliza de nuevo la técnica basada en el Teorema de Adición para las Funciones de Hankel; debido a las ya mencionadas razones de eficiencia computacional, descritas en el apartado 4.3.3, que esta técnica presenta en contraposición con aquélla basada en la Transformada Discreta de Fourier.

Finalmente, haciendo uso de estas matrices se presentan resultados de la dispersión electromagnética que produce una tira metálica infinita, de grosor despreciable, ante distintas incidencias con polarización $\mathrm{TM}^{\mathrm{z}}$.

\subsubsection{Cálculo de la Matriz de Corrientes mediante el Mé- todo de los Momentos}

Al caracterizar tiras infinitas de grosor despreciable, cuyas longitudes son pequeñas en términos eléctricos, se ha utilizado el Método de los Momentos en el cálculo de la matriz de corrientes $D I$; la cual, tras aplicar los pasos descritos en el apartado 4.2 .2 para polarización $\mathrm{TM}^{\mathrm{z}}$, presenta el siguiente aspecto

$$
D I=\left[\alpha_{n p} \Delta C_{n}\right]_{N_{s} \times 2 N_{i}+1}
$$

donde $\Delta C_{n}$ indica la longitud del $n$-ésimo segmento; situado sobre el contorno de la tira y en el que se ha definido la correspondiente función base $f_{n}$, en nuestro caso una función pulso centrada en el punto medio de dicho segmento, tal y como se representa en (4.47). Por tanto, la tira de longitud $w$ (dimensión normalizada 
respecto de $\lambda$ ) se divide en $N_{s}$ segmentos de igual tamaño, cuyo valor se expresa fácilmente del siguiente modo

$$
\Delta C_{n}=\frac{w \lambda}{N_{s}}
$$

Por su parte, los elementos $\alpha_{n p}$ presentes en (5.29) representan la solución a los conocidos sistemas de ecuaciones, expresados matricialmente como

$$
\left[I_{m n}\right] \cdot\left[\alpha_{n p}\right]=\left[g_{m p}\right]
$$

donde los elementos de las matrices $\left[I_{m n}\right]$ y $\left[g_{m p}\right]$, recordando $(4.51)$, (4.53) y (4.54), se resumen a continuación mediante las expresiones

$$
\begin{aligned}
I_{n n} & =\frac{k \eta}{4} \Delta C_{n}\left[1-j \frac{2}{\pi} \ln \left(\frac{\gamma k \Delta C_{n}}{4 e}\right)\right] \\
I_{m n} & =\frac{k \eta}{4} \Delta C_{n} H_{0}^{(2)}\left(k \sqrt{\left(x_{m}-x_{n}\right)^{2}+\left(y_{m}-y_{n}\right)^{2}}\right) \\
g_{m p} & =E_{z}^{i}\left(\vec{\rho}_{m}\right)=J_{p}\left(k \rho_{m}\right) e^{j p \phi_{m}}
\end{aligned}
$$

En (5.33), las coordenadas rectangulares $\left(x_{n}, y_{n}\right)$ definen el punto medio del $n$-ésimo segmento sobre el contorno de la tira. Por tanto, considerando que la longitud de la tira se extiende a lo largo del eje $\hat{x}$, las coordenadas $\left(x_{n}, y_{n}\right)$ se eligen de la siguiente manera

$$
\begin{array}{lll}
x_{n}=-\frac{w \lambda}{2}+\frac{w \lambda}{2 N_{s}}+\frac{w \lambda}{N_{s}}(n-1) & \\
y_{n}=0 & n \in\left[1,2, \ldots, N_{s}\right]
\end{array}
$$

Por otro lado, las coordenadas cilíndricas $\left(\rho_{m}, \phi_{m}\right)$ presentes en $(5.34)$, o sus equivalentes rectangulares $\left(x_{m}, y_{m}\right)$ en (5.33), determinan las posiciones donde se localizan las funciones delta, escogidas como funciones test al aplicar el Método de los Momentos (Point-Matching). Estas posiciones, que se hacen coincidir con el punto medio $\left(x_{n}, y_{n}\right)$ de cada segmento, se definen mediante las relaciones

$$
\begin{array}{ll}
x_{m}=-\frac{w \lambda}{2}+\frac{w \lambda}{2 N_{s}}+\frac{w \lambda}{N_{s}}(m-1) & \\
y_{m}=0 & \\
\rho_{m}=\sqrt{x_{m}^{2}+y_{m}^{2}} & m \in[1,2, \ldots, M] \\
\phi_{m}=\arctan \left(\frac{y_{m}}{x_{m}}\right) & m
\end{array}
$$


Como se puede observar en (5.35) y (5.36), los puntos definidos por las coordenadas rectangulares $\left(x_{n}, y_{n}\right)$ y $\left(x_{m}, y_{m}\right)$ se encuentran situados $\left(y_{n}=y_{m}=0\right)$ sobre el eje $\hat{x}$; lo que supone considerar en la práctica una tira metálica infinita de grosor nulo, y forzar que las componentes tangenciales de campo eléctrico se anulen, tan sólo, a lo largo del eje $\hat{x}$ desde $x=-w \lambda / 2$ hasta $x=w \lambda / 2$. En el caso de considerar que la tira presentase un cierto grosor (de valor $d \lambda \operatorname{con} d \rightarrow 0$ ), y por tanto forzar la condición de contorno para las componentes tangenciales de campo eléctrico sobre ambas caras de la tira, se ha comprobado que apenas se produce diferencia alguna en los resultados obtenidos con respecto a la situación anterior. Consecuentemente, se ha optado por forzar la correspondiente condición de contorno únicamente a lo largo del eje $\hat{x}$; lo cual requiere de un menor esfuerzo computacional al imponer dicha condición de contorno en un menor número de puntos.

Escogiendo un número $M$ de funciones test igual al número $N_{s}$ de funciones base; la matriz de coeficientes $\left[I_{m n}\right]$, que debe invertirse para poder resolver los sistemas de ecuaciones propuestos en (5.31), se hace cuadrada. Para obtener resultados suficientemente precisos, de nuevo se ha comprobado experimentalmente que se requieren al menos 100 funciones base por cada longitud de onda en la dimensión máxima de la tira; es decir, el valor a escoger para $N_{s}$ debe satisfacer el siguiente criterio

$$
N_{s}>100 \frac{w}{2}
$$

Finalmente, sustituyendo en (5.32) y (5.33) la longitud $\Delta C_{n}$ de cada segmento por su valor presentado en $(5.30)$, los elementos de la matriz de coeficientes $\left[I_{m n}\right]$ quedan expresados del siguiente modo

$$
\begin{aligned}
& I_{n n}=\frac{60 \pi^{2} w}{N_{s}}\left[1-j \frac{2}{\pi} \ln \left(\frac{\gamma \pi w}{N_{s} 2 e}\right)\right] \\
& I_{m n}=\frac{60 \pi^{2} w}{N_{s}} H_{0}^{(2)}\left(k \sqrt{\left(x_{m}-x_{n}\right)^{2}+\left(y_{m}-y_{n}\right)^{2}}\right)
\end{aligned}
$$

donde las coordenadas rectangulares $\left(x_{n}, y_{n}\right)$ y $\left(x_{m}, y_{m}\right)$ vienen recogidas en $(5.35)$ y (5.36), respectivamente.

Una vez calculados los elementos de las matrices $\left[I_{m n}\right]$ y $\left[g_{m p}\right]$ como se acaba de indicar, se deben resolver $\operatorname{los} 2 N_{i}+1$ sistemas de ecuaciones definidos matricialmente en (5.31); para de esta forma obtener los elementos de la matriz incógnita $\left[\alpha_{n p}\right]$. Sustituyendo dichos elementos en (5.29), se determina la matriz de corrientes de una tira metálica infinita, de grosor infinitesimal, ante incidencia con polarización $\mathrm{TM}^{\mathrm{z}}$. 


\subsubsection{Resultados}

A continuación, se presentan resultados de la dispersión electromagnética producida por una tira metálica de grosor infinitesimal, longitud $1 \lambda$ y orientada según el eje $\hat{y}$. La pequeña longitud en términos eléctricos $(w=1)$ de la tira permite emplear las expresiones deducidas en el apartado anterior, haciendo uso del Método de los Momentos, para calcular la matriz de corrientes de dicha tira; la cual se supondrá que está orientada según el eje $\hat{x}$. La matriz de espectro de esta misma tira paralela al eje $\hat{x}$, debido a las ya mencionadas razones de eficiencia computacional, se ha determinado utilizando el Teorema de Adición para las Funciones de Hankel (ver expresiones en el apartado 4.3.2). De esta forma, se obtiene la matriz de caracterización de una tira metálica infinita, de longitud $1 \lambda \mathrm{y}$ situada en la dirección del eje $\hat{x}$. A partir de dicha matriz, y utilizando las propiedades de transformación de espectros [14] particularizadas al caso que nos ocupa mediante las matrices de giro $G_{1}(\pi / 2)$ y $G_{2}(\pi / 2)$ definidas en el apéndice D, se obtiene la matriz de caracterización de la tira metálica infinita, de longitud $1 \lambda$ y paralela al eje $\hat{y}$. Esta matriz caracteriza perfectamente el comportamiento dispersor de la tira metálica; permitiendo conocer de manera rápida y sencilla la respuesta de dicha tira ante cualquier incidencia polarizada según $\mathrm{TM}^{\mathrm{z}}$.

Haciendo uso de la citada matriz de caracterización, se ha calculado inicialmente la respuesta de la tira ante una onda plana incidente con dirección de avance $\beta=0$ (ver figura C.1); cuya amplitud y fase, reconstruida a partir de su correspondiente espectro cilíndrico, puede observarse en la figura 5.1. El campo eléctrico dispersado por la tira, ante la incidencia de esta onda plana, se representa en la figura 5.10; donde se observa que la tira produce dos ondas planas que avanzan paralelas al eje $\hat{x}$, una de ellas en el sentido creciente de la coordenada $x$ y la otra en el sentido decreciente de dicha coordenada. El campo eléctrico total, obtenido como la suma del campo eléctrico incidente y del dispersado, se muestra en la figura 5.11; donde la onda plana dispersada hacia valores crecientes de la coordenada $x$ se suma en contrafase con la onda plana incidente, produciendo la conocida zona de sombra que aparece detrás del objeto dispersor. En cambio, la onda plana dispersada hacia valores decrecientes de la coordenada $x$, después de sumarse con la onda plana incidente que avanza en sentido contrario, origina una onda estacionaria.

Posteriormente, se ha obtenido la respuesta de la misma tira ante una onda plana incidente, pero en esta ocasión con dirección de avance $\beta=\pi / 6$ (ver figura C.1). En este caso, tal y como se observa en la figura 5.12 donde se representa la amplitud y la fase del campo eléctrico dispersado, la tira genera dos ondas planas que avanzan respectivamente en las direcciones angulares definidas según $\phi=\pi / 6$ y $\phi=(\pi-\pi / 6)$. La onda que avanza según $\phi=\pi / 6$ se suma de nuevo en contrafase con la onda plana incidente, produciendo una zona de sombra en dicha dirección angular (ver figura 5.13 donde se representa la amplitud y la 

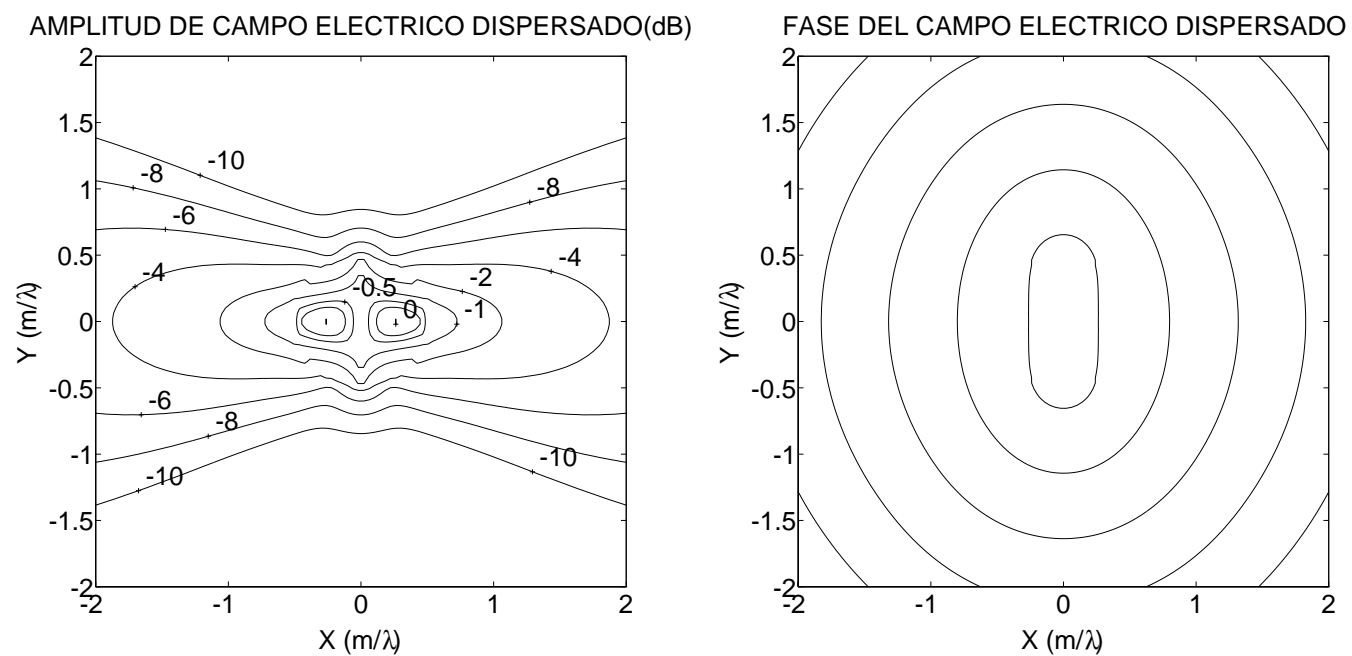

Figura 5.10: Amplitud y fase del campo eléctrico dispersado por una tira metálica infinita, de longitud $1 \lambda$ y orientada según el eje $\hat{y}$, anta la incidencia de una onda plana $(\beta=0)$.
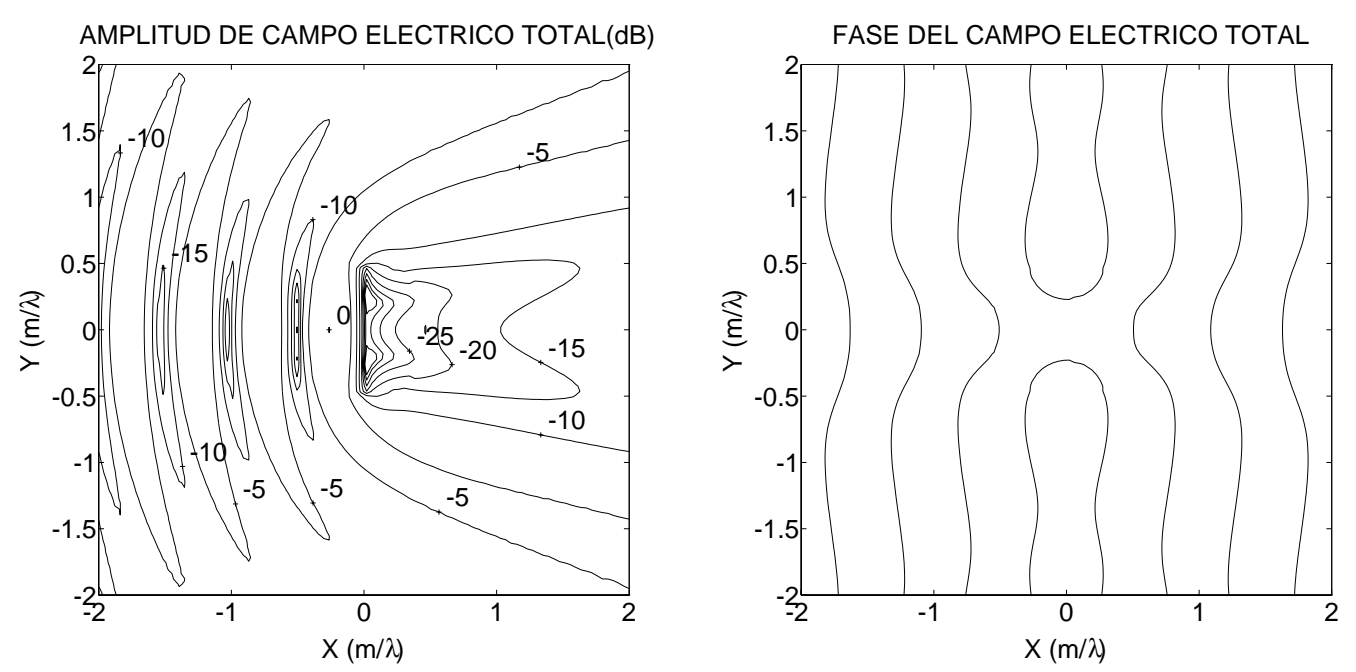

Figura 5.11: Amplitud y fase del campo eléctrico total; obtenido como la suma de la onda plana incidente $(\beta=0)$ y del campo eléctrico dispersado ante dicha incidencia por una tira metálica infinita, de longitud $1 \lambda$ y orientada según el eje $\hat{y}$.

fase del campo eléctrico total). Sin embargo, la onda que avanza en la dirección $\phi=(\pi-\pi / 6)$, tras sumarse con la onda plana incidente, no produce el mismo patrón de interferencias que en la situación anterior (comparar figuras 5.11 y 5.13); debido a que en este caso la onda dispersada no presenta la misma dirección de avance del frente de fase que la onda plana incidente. 

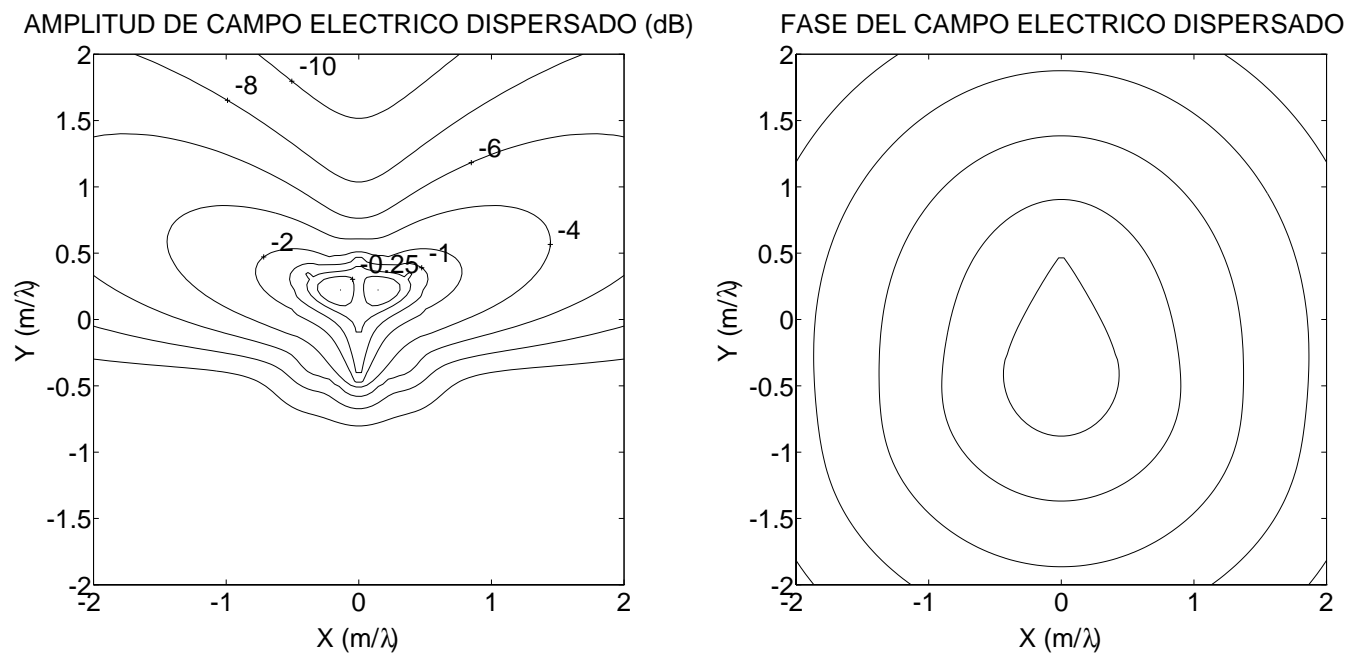

Figura 5.12: Amplitud y fase del campo eléctrico dispersado por una tira metálica infinita, de longitud $1 \lambda$ y orientada según el eje $\hat{y}$, anta la incidencia de una onda plana $(\beta=\pi / 6)$.
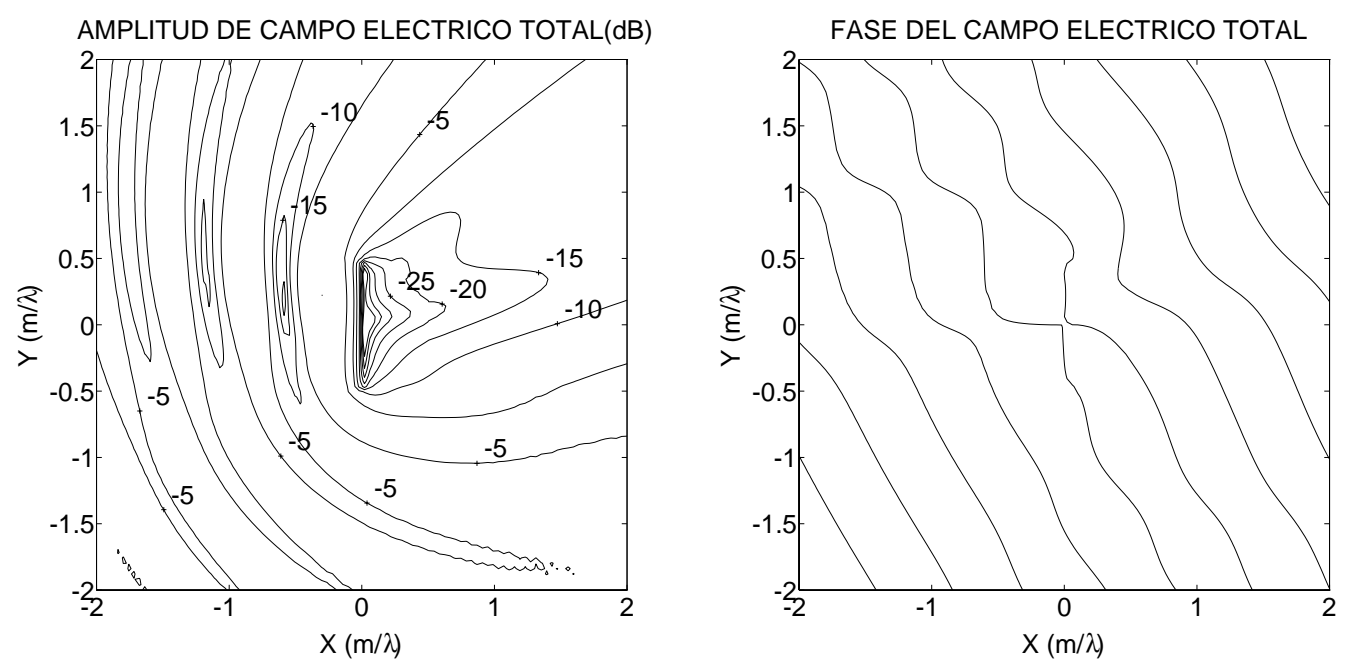

Figura 5.13: Amplitud y fase del campo eléctrico total; obtenido como la suma de la onda plana incidente $(\beta=\pi / 6)$ y del campo eléctrico dispersado ante dicha incidencia por una tira metálica infinita, de longitud $1 \lambda$ y orientada según el eje $\hat{y}$.

Estos resultados confirman que es posible caracterizar cualquier objeto dispersor, aunque su geometría no permita obtener una solución analítica, mediante una función de transferencia o matriz de caracterización. En estos casos, la obtención de dicha matriz debe llevarse a cabo calculando previamente una matriz de corrientes y una matriz de espectro; las cuales pueden determinarse de for- 
ma sencilla, tal y como ha quedado demostrado en este capítulo para distintas geometrías, haciendo uso de las diversas técnicas presentadas en el capítulo 4.

\subsection{Tira Metálica Infinita ante Incidencia $\mathrm{TE}^{\mathrm{z}}$}

En última instancia se pretende caracterizar, mediante las técnicas descritas en el capítulo 4, el comportamiento de un objeto dispersor ante incidencia con polarización $\mathrm{TE}^{\mathrm{z}}$. De nuevo se escoge la tira metálica infinita, que ya ha quedado analizada en la sección 5.3 ante incidencia polarizada según $\mathrm{TM}^{\mathrm{z}}$; para de esta forma tener completamente caracterizado dicho objeto ante cualquier posible incidencia (recordar sección 4.1).
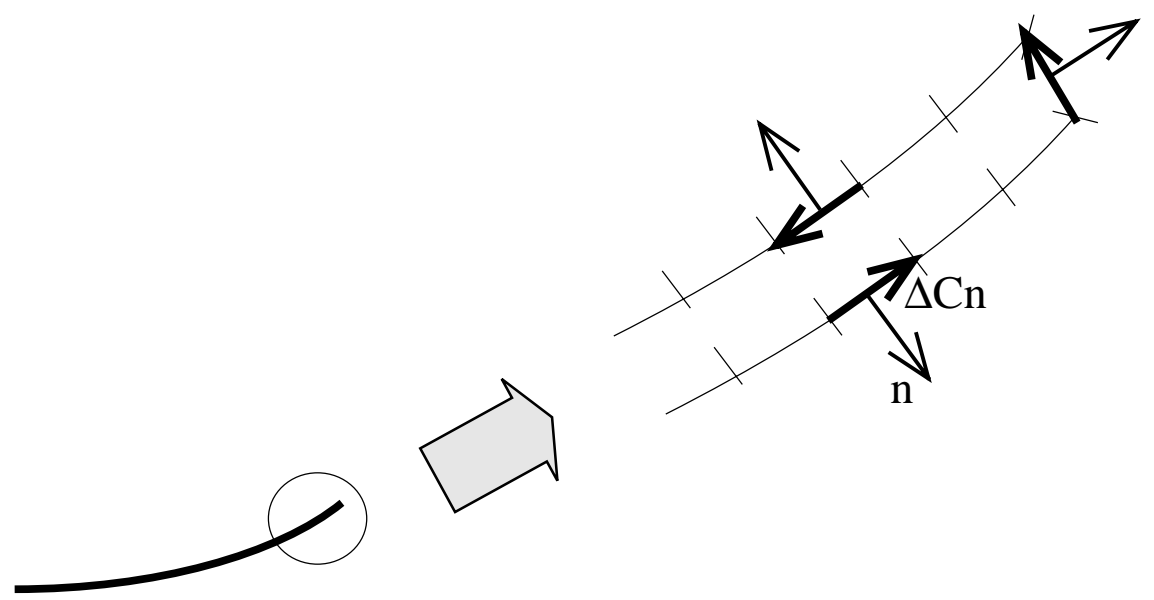

Figura 5.14: Segmentación del contorno de un objeto dispersor con grosor infinitesimal para incidencia con polarización $\mathrm{TE}^{\mathrm{z}}$.

Las tiras metálicas de grosor infinitesimal que se han considerado presentan longitudes pequeñas en términos eléctricos; habiéndose utilizado, por tanto, el Método de los Momentos en la obtención de la matriz de corrientes. Conviene reseñar, no obstante, que al aplicar dicho Método en la caracterización de un objeto dispersor ante incidencia con polarización $\mathrm{TE}^{\mathrm{z}}$, se debe considerar un cierto grosor no nulo en el objeto; para que la formulación desarrollada en el apartado 4.2.2 resulte válida [70]. Al analizar objetos con grosor infinitesimal, como el caso que nos ocupa, se debe asumir para dicho grosor un valor finito no nulo que tienda a cero. Por ejemplo, en la figura 5.14 se detalla como segmentar el contorno de un objeto dispersor cuyo grosor es infinitesimal. El valor límite del grosor viene impuesto por el condicionamiento de la matriz de coeficientes $\left[I_{m n}\right]$, que debe invertirse para resolver la correspondiente ecuación integral mediante el citado Método. En concreto, para la tira metálica se ha determinado a nivel práctico que un valor límite de $0.02 \lambda$ garantiza la inversión de dicha matriz. 
A pesar de no contemplar en esta sección el análisis de tiras con dimensiones grandes eléctricamente, éste simplemente requeriría utilizar la aproximación de Óptica Física en el cálculo de la matriz de corrientes; no resultando necesario en este caso el tener que considerar un grosor no nulo para la tira. En lo referente a la matriz de espectro, al considerar incidencia con polarización $\mathrm{TE}^{\mathrm{z}}$, tan sólo puede obtenerse haciendo uso de la Transformada Discreta de Fourier; lo que supone una mayor carga computacional (recordar apartado 4.3.3) frente al caso de incidencia polarizada según $\mathrm{TM}^{\mathrm{z}}$, donde se puede utilizar el Teorema de Adición para las Funciones de Hankel.

Utilizando la matriz de corrientes y la matriz de espectro, se construye la matriz de caracterización de la tira metálica infinita, de grosor despreciable, ante incidencia con polarización $\mathrm{TE}^{\mathrm{z}}$. Dicha matriz permite analizar la dispersión electromagnética producida por una tira metálica infinita; que tras comparar con algún otro resultado, presente en la literatura y obtenido siguiendo otros métodos a los aquí propuestos, permite validar las técnicas descritas en el capítulo 4 ante incidencia polarizada según $\mathrm{TE}^{\mathrm{z}}$.

\subsubsection{Cálculo de la Matriz de Corrientes mediante el Mé- todo de los Momentos}

Como las tiras a caracterizar presentan longitudes pequeñas en términos eléctricos, parece lógico escoger el Método de los Momentos para obtener la matriz de corrientes $D I$; cuyos elementos, tras aplicar los pasos propuestos en el apartado 4.2.2 para polarización $\mathrm{TE}^{\mathrm{z}}$, se obtienen mediante la expresión

$$
D I=\left[\alpha_{n p} \Delta C_{n}\right]_{2 N_{s}+2 \times 2 N_{i}+1}
$$

donde $\Delta C_{n}$ representa la longitud del $n$-ésimo segmento; situado sobre el contorno de la tira y en el que se define la correspondiente función pulso $f_{n}$ recogida en (4.47). Para poder aplicar el Método de los Momentos en el caso de incidencia con polarización $\mathrm{TE}^{\mathrm{z}}$, el objeto bajo análisis debe presentar un grosor $d$ (normalizado respecto de $\lambda$ ) no nulo, tal y como se indica en la figura 5.14. Por tanto, una tira de longitud $w$ (también normalizada respecto de $\lambda$ ) se divide en $2 N_{s}+2$ segmentos; de los cuales $2 N_{s}$ presentan la misma longitud y se distribuyen de forma simétrica en la cara superior e inferior de la tira, mientras que los 2 restantes tienen una longitud igual al grosor de la tira y se sitúan, respectivamente, en cada una de las dos caras laterales de la misma. De esta forma, la longitud $\Delta C_{n}$ de cada uno de estos segmentos presenta el siguiente aspecto

$$
\Delta C_{n}= \begin{cases}\frac{w \lambda}{N_{s}} & n \in \text { cara superior o inferior } \\ d & n \in \text { caras laterales }\end{cases}
$$


Por otra parte, en (5.40) los elementos $\alpha_{n p}$ representan la solución a los ya conocidos $2 N_{i}+1$ sistemas de ecuaciones algebraicas, expresados matricialmente del siguiente modo

$$
\left[I_{m n}\right] \cdot\left[\alpha_{n p}\right]=\left[g_{m p}\right]
$$

donde los elementos de la matriz de coeficientes $\left[I_{m n}\right]$ y de la matriz de excitaciones $\left[g_{m p}\right]$, recordando $(4.65),(4.68)$ y $(4.69)$, vienen definidos como

$$
\begin{aligned}
I_{n n} & =\frac{1}{2} \\
I_{m n} & =\frac{j}{4} k \Delta C_{n}(\hat{n} \cdot \vec{R}) H_{1}^{(2)}\left(k \sqrt{\left(x_{m}-x_{n}\right)^{2}+\left(y_{m}-y_{n}\right)^{2}}\right) \\
g_{m p} & =-H_{z}^{i}\left(x_{m}, y_{m}\right)=-J_{p}\left(k \rho_{m}\right) e^{j p \phi_{m}}
\end{aligned}
$$

En (5.44), las coordenadas rectangulares $\left(x_{n}, y_{n}\right)$ determinan el punto medio del $n$-ésimo segmento sobre el contorno de la tira. Dicho contorno, debido a la razón ya comentada anteriormente, se divide en $2 N_{s}+2$ segmentos. Considerando pues que la longitud de la tira se extiende a lo largo del eje $\hat{x}$, las coordenadas $\left(x_{n}, y_{n}\right)$ de los puntos situados en la cara superior de la tira se eligen de la siguiente manera

$$
\begin{array}{ll}
x_{n}=-\frac{w \lambda}{2}+\frac{w \lambda}{2 N_{s}}+\frac{w \lambda}{N_{s}}(n-1) & \\
y_{n}=\frac{d \lambda}{2} & n \in\left[1,2, \ldots, N_{s}\right]
\end{array}
$$

Los puntos situados en la cara inferior de la tira se distribuyen de forma simétrica con respecto a los puntos de la cara superior; por tanto, sus coordenadas $\left(x_{n}, y_{n}\right)$ pueden expresarse en función de (5.46) sustituyendo $y_{n}$ por su correspondiente valor para la cara inferior $\left(y_{n}=-d \lambda / 2\right)$. Los dos puntos restantes, situados en las caras laterales, se definen fácilmente mediante las coordenadas

$$
\begin{array}{ll}
x_{n}=-\frac{w \lambda}{2} & y_{n}=0 \\
x_{n}=\frac{w \lambda}{2} & y_{n}=0
\end{array}
$$

Por su parte, las coordenadas cilíndricas $\left(\rho_{m}, \phi_{m}\right)$ que aparecen en $(5.45)$, o sus equivalentes rectangulares $\left(x_{m}, y_{m}\right)$ presentes en (5.44), determinan las posiciones sobre el contorno de la tira donde se localizan las funciones delta, escogidas como funciones test al aplicar el Método de los Momentos (Point-Matching). El número $M$ de funciones delta se escoge igual al número $N_{s}$ de funciones base; produciendo 
de esta forma una matriz de coeficientes $\left[I_{m n}\right]$ que resulta cuadrada. Además, las posiciones de las funciones delta se hacen coincidir con el punto medio de cada segmento; de modo que los comentarios realizados para las coordenadas $\left(x_{n}, y_{n}\right)$, así como sus expresiones recogidas en (5.46), (5.47) y (5.48), aplican igualmente a las coordenadas rectangulares $\left(x_{m}, y_{m}\right)$. Las coordenadas cilindricas $\left(\rho_{m}, \phi_{m}\right)$ se obtienen directamente a partir de sus equivalentes rectangulares empleando las relaciones entre dichas coordenadas, recogidas por ejemplo en (5.36).

Tal y como ya se ha mencionado, resulta necesario considerar un cierto grosor en la tira metálica; para de esta forma obtener una matriz de coeficientes $\left[I_{m n}\right]$ invertible, y poder resolver la ecuación matricial presente en (5.42). Consecuentemente, para el caso que nos ocupa de incidencia $\mathrm{TE}^{\mathrm{z}}$, el número de puntos donde se impone el cumplimiento de la correspondiente Ecuación Integral resulta más del doble que el empleado para incidencia polarizada según $\mathrm{TM}^{\mathrm{z}}$ (ver apartado 5.3.1); lo que sin duda implica un mayor esfuerzo computacional en la determinación de la matriz de corrientes. Con la intención de reducir esta carga computacional podría pensarse en utilizar la aproximación de Óptica Física; que no requiere considerar, para su correcta aplicación, un cierto grosor en la tira metálica infinita. Ahora bien, los resultados proporcionados por dicha técnica no resultarían lo suficientemente precisos; ya que se están considerando tiras con dimensiones pequeñas en términos eléctricos.

De nuevo, se ha comprobado experimentalmente que para obtener resultados suficientemente precisos, se requieren al menos 100 funciones base por cada longitud de onda en la dirección máxima de la tira; es decir, el valor a escoger para $N_{s}$ debe satisfacer el criterio expuesto mediante la siguiente condición

$$
N_{s}>100 \frac{w}{2}
$$

Para finalizar, sustituyendo en (5.44) la longitud $\Delta C_{n}$ de cada segmento por su correspondiente valor recogido en (5.41), se obtienen los elementos de la matriz de coeficientes $\left[I_{m n}\right]$. Resolviendo a continuación los $2 N_{i}+1$ sistemas de ecuaciones algebraicas, definidos matricialmente en (5.42), se encuentran los elementos de la matriz incógnita $\left[\alpha_{n p}\right]$; que una vez sustituidos en (5.40) determinan la matriz de corrientes de una tira metálica infinita, con grosor infinitesimal, ante incidencia con polarización $\mathrm{TE}^{\mathrm{z}}$.

\subsubsection{Resultados}

Con el objeto de validar las expresiones propuestas en esta sección, que permiten analizar objetos dispersores ante incidencia polarizada según $\mathrm{TE}^{\mathrm{z}}$, se ha caracterizado la dispersión electromagnética producida ante dicha incidencia por una tira metálica de grosor infinitesimal, longitud $2 \lambda$ y orientada según el eje $\hat{x}$. La longitud $(w=2)$ de la tira impone la utilización de las expresiones deducidas 
en esta misma sección, empleando el Método de los Momentos, para obtener la matriz de corrientes. Por otra parte, el tipo de polarización $\left(\mathrm{TE}^{\mathrm{z}}\right)$ de la incidencia determina el uso de la Transformada Discreta de Fourier en el cálculo de la matriz de espectro. De esta forma, se obtiene la matriz de caracterización de la tira metálica bajo análisis.

Empleando esta matriz, se ha calculado la respuesta de la tira metálica ante un cierto campo magnético incidente, representado por medio de una onda plana con dirección de avance $\beta=-\pi / 3$ (ver figura C.1). En la figura 5.15, se representa el diagrama de radiación de la tira metálica ante dicha incidencia; obtenido a partir de la amplitud del campo magnético dispersado en la región de campo lejano. En dicha figura, se observa que se produce un máximo de radiación, lógicamente en la dirección angular $\phi=\pi / 3$. El resultado ofrecido en la figura 5.15 puede compararse con el presentado en [69, pág. 586]; donde se muestra la sección recta radar biestática por unidad de longitud para la misma tira e incidencia, pero calculada siguiendo otros métodos a los expuestos en este trabajo. Conviene destacar que entre ambos resultados existe una enorme coincidencia.

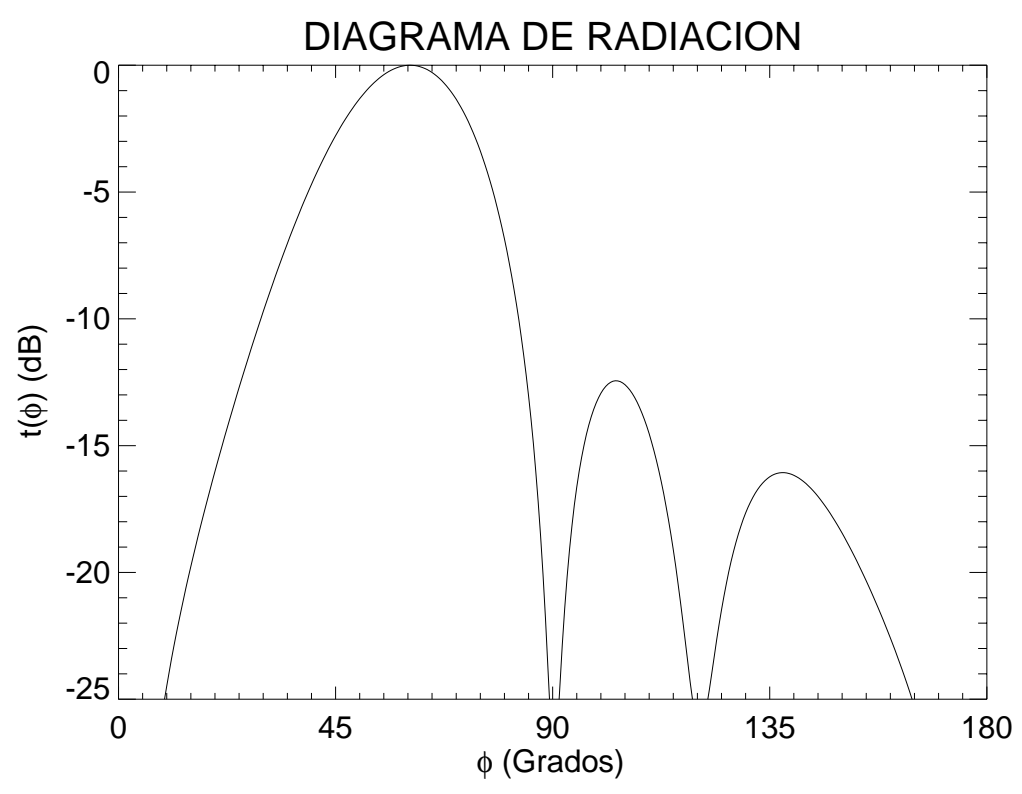

Figura 5.15: Diagrama de radiación (onda plana incidente con dirección de avance $\beta=-\pi / 3$ y polarizada según $\mathrm{TE}^{\mathrm{z}}$ ) de una tira metálica infinita, de longitud $2 \lambda \mathrm{y}$ orientada según el eje $\hat{x}$.

Estos resultados avalan por completo las técnicas presentadas en el capítulo 4 para caracterizar objetos dispersores ante incidencia con polarización $\mathrm{TE}^{\mathrm{z}}$. De esta forma, se está en condiciones de garantizar la caracterización de cualquier objeto dispersor ante cualquier posible incidencia; que, recordando la sección 4.1, puede siempre expresarse como combinación de soluciones modales $\mathrm{TM}^{\mathrm{z}}$ y TE${ }^{\mathrm{z}}$. 



\section{Capítulo 6}

\section{Caracterización Conjunta de Múltiples Objetos Dispersores en Espacio Libre mediante un Algoritmo Recursivo}

El análisis del acoplo electromagnético que se produce entre múltiples objetos dispersores en espacio libre, ante la presencia de un cierto campo incidente, constituye un tema de enorme interés y aplicación práctica. Las antenas multireflectoras representan un claro ejemplo; pues en su completa caracterización se requiere considerar las interacciones múltiples que tienen lugar entre sus dos elementos dispersores. Por otra parte, en el análisis de objetos individuales grandes en términos eléctricos resulta también interesante, tras segmentar el contorno del objeto dispersor en elementos más pequeños, el estudio del acoplo múltiple entre dichos elementos; para de esta forma caracterizar al objeto grande eléctricamente. Este procedimiento genera resultados más exactos que aquéllos obtenidos mediante las técnicas tradicionales de alta frecuencia, tales como Teoría Geométrica de la Difracción, Óptica Geométrica o la aproximación de Óptica Física; que en la caracterización de objetos grandes eléctricamente se prefieren a los métodos numéricos, como por ejemplo el Método de los Momentos o el Método de los Elementos Finitos, debido a las ya conocidas razones de eficiencia computacional.

En este capítulo, se expone un algoritmo recursivo generalizado que permite caracterizar de forma conjunta a múltiples objetos dispersores. Haciendo uso del concepto de la función de transferencia o matriz de caracterización, introducido en el capítulo 4 para describir el comportamiento dispersor de cada objeto individualmente, y tras aplicar el citado algoritmo recursivo; se presenta para cada objeto dispersor una matriz de caracterización conjunta, que define la dispersión producida por dicho objeto en presencia del resto. Dicho algoritmo, pensado inicialmente para incorporar un nuevo objeto en cada iteración, se refina para poder 
aplicarlo a la caracterización conjunta de agrupaciones de objetos dispersores. A su vez, la utilización del algoritmo revela la existencia de ciertas situaciones problemáticas; en las que, para aplicar correctamente el citado algoritmo, conviene tomar ciertas acciones preventivas que se describen detalladamente. Por otra parte, aunque el algoritmo se concibe inicialmente para determinar la dispersión que producen múltiples objetos cuando la fuente es externa al grupo; en el capítulo se describe cómo analizar el mismo problema cuando la fuente se sitúa entre los objetos. Finalmente, para cada una de las regiones en las que se divide el espacio, se explica como reconstruir el campo dispersado por múltiples objetos.

\subsection{Descripción de un Algoritmo Recursivo pa- ra la Caracterización Conjunta de Múltiples Objetos Dispersores}

La caracterización de la dispersión electromagnética producida por múltiples objetos dispersores es un tema que siempre ha despertado enorme interés en la literatura. Así pues, ya en [73] se encuentra resuelto el problema de dos cilindros conductores paralelos; mientras que en [74] se soluciona la dispersión que producen dos esferas conductoras. Estos dos problemas se analizan haciendo uso de transformaciones espectrales, en concreto se utilizan respectivamente las transformaciones cilíndrica-cilíndrica y esférica-esférica.

El análisis de antenas multireflectoras, integradas normalmente por dos elementos reflectores, constituye una de las posibles aplicaciones prácticas derivadas de la caracterización de múltiples objetos dispersores. Recientemente, se propone en [75] un método para analizar sistemas multireflectores que utiliza las funciones de transferencia o matrices de caracterización individual de cada uno de los elementos reflectores del sistema. El problema se resuelve multiplicando las matrices de caracterización individual de cada uno de los reflectores en el mismo orden con el que el campo incidente, tras experimentar diversas reflexiones, se encuentra a dichos elementos; para de esta forma obtener una matriz de caracterización global del sistema multireflector bajo análisis. Ahora bien, este procedimiento no tiene en cuenta las interacciones múltiples que se originan entre los diversos elementos reflectores; que si bien no son excesivamente importantes en el estudio de sistemas multireflectores, pues afectan especialmente en la zona de campo próximo y apenas en la zona de campo lejano, deben considerarse en un análisis general y completo de dichos sistemas.

Un primer algoritmo que permite solucionar la dispersión electromagnética producida por diversos objetos, teniendo presente las interacciones múltiples existentes entre ellos, puede encontrarse en [76]; donde se aplica el principio Poincaré-Huygens para obtener una matriz de caracterización global, a partir de 
las matrices de caracterización individual de cada uno de los objetos dispersores. Siguiendo este método, en dicha publicación se analiza con éxito la dispersión que producen dos esferas conductoras. Ahora bien, la principal desventaja del método se basa en el hecho de analizar conjuntamente, en una sola iteración, el comportamiento dispersor de todos los objetos; lo que supone un incremento considerable en la complejidad de las expresiones deducidas cuando crece el número de objetos dispersores bajo análisis.

Posteriormente, en [77-79] se presenta un nuevo algoritmo recursivo para resolver los problemas de interacción múltiple entre diversos objetos dispersores. En cada iteración, este algoritmo incorpora un nuevo objeto al grupo analizado en la iteración anterior; calculando de forma recursiva una nueva matriz de caracterización global. Precisamente, el hecho de añadir un nuevo objeto en cada iteración puede causar, en el caso de tener varios objetos dispersores equidistantes del origen de coordenadas, que se incumpla la restricción del Teorema de Adición para las Funciones de Hankel (ver apéndice E); originando singularidades en el cálculo de la dispersión electromagnética. Debido a esta razón, en [80] se introduce una versión modificada del algoritmo recién descrito, que permite incorporar en cada iteración un número genérico de objetos dispersores; evitando de esta forma violar la citada restricción del Teorema de Adición cuando existen varios objetos equidistantes. Entre los pocos inconvenientes asociados a este método, únicamente destaca la necesidad de utilizar nuevamente fórmulas recursivas, aplicadas en sentido inverso al seguido en la implementación del algoritmo, que permiten calcular el campo dispersado por todos los objetos en el interior de la mínima circunferencia que los contiene.

Un algoritmo iterativo que permite resolver el acoplo electromagnético entre múltiples objetos dispersores se recoge en [81]; donde se aplica al análisis de las interacciones múltiples que se producen entre varios cilindros conductores o dieléctricos. Este nuevo algoritmo resuelve el problema de forma iterativa siguiendo el progreso físico de las ondas; pues en cada iteración el campo dispersado por cada uno de los objetos se calcula a partir del campo que dispersa el resto de objetos en la iteración anterior. Entre los principales inconvenientes de este algoritmo, uno de ellos hace referencia al grado de complejidad que supone verificar en cada iteración si se han generado resultados convergentes. Asimismo, el método presenta problemas de convergencia cuando se pretende analizar la dispersión producida por objetos grandes en términos eléctricos.

Por otra parte, el análisis de las interacciones múltiples entre varios objetos dispersores se resuelve en [13] mediante un método basado en teoría de grafos; el cual utiliza también matrices de caracterización individual asociadas a cada uno de los objetos, junto con unas matrices de transformación de espectros que simplemente permiten trasladarlos a distintas posiciones. La principal desventaja de este método radica en que su complejidad se incrementa considerablemente a medida que crece el número de elementos dispersores bajo análisis; llegando 
incluso a convertirse en un problema irresoluble cuando dicho número es elevado. A su vez, la totalidad de los cálculos que se realizan durante la computación del campo dispersado por el conjunto de objetos debe repetirse para cada incidencia.

En esta sección, se describe un nuevo algoritmo recursivo que permite resolver la dispersión electromagnética producida por múltiples objetos dispersores. Este nuevo algoritmo utiliza teoría de grafos, recogida por ejemplo en [13], para analizar la presencia de un nuevo objeto dispersor en cada iteración. Consecuentemente, la complejidad del método no crece con el número de objetos dispersores analizados; pues añadir un nuevo objeto al grupo tan sólo supone realizar una simple iteración adicional. Además, el método ofrece resultados precisos en cada iteración sin necesidad de comprobar su grado de convergencia; siendo estos resultados igualmente válidos en el análisis de objetos grandes y pequeños en términos eléctricos. Tras aplicar el algoritmo, el comportamiento dispersor de cada objeto queda representado por una función de transferencia o matriz de caracterización conjunta; que permite obtener el campo dispersado por cada objeto, considerando la presencia del resto, ante cualquier posible incidencia. De esta manera, no resulta necesario tener que repetir cálculos para cada posible incidencia. En realidad, cada objeto queda completamente caracterizado, al igual que cuando se analizan individualmente, mediante dos funciones de transferencia o matrices de caracterización conjunta; determinando una de ellas el comportamiento dispersor del objeto en presencia del resto ante incidencia con polarización $\mathrm{TM}^{\mathrm{z}}$, y la otra ante incidencia polarizada según $\mathrm{TE}^{\mathrm{z}}$. Asimismo, dichas matrices de caracterización permiten obtener, de manera sencilla, el campo dispersado por todos los objetos en la región interna a la mínima circunferencia que los contiene.

A continuación, se determina inicialmente el cálculo de las funciones de transferencia o matrices de caracterización conjunta, mediante teoría de grafos [13], para un problema de dispersión múltiple constituido por dos objetos dispersores. Posteriormente, se aplica el método recursivo al análisis de la interacción electromagnética que se produce entre los dos primeros objetos dispersores y un nuevo tercer objeto dispersor. Finalmente, el algoritmo se generaliza para caracterizar de forma conjunta el comportamiento dispersor de $N$ objetos; analizados mediante una última iteración en la que se resuelve el acoplo entre $N-1$ objetos dispersores, caracterizados previamente con el mismo algoritmo recursivo, y el $N$-ésimo objeto dispersor.

\subsubsection{Matrices de Caracterización Conjunta de Dos Ob- jetos Dispersores}

Inicialmente, se presentan las expresiones correspondientes a la dispersión electromagnética producida por dos objetos dispersores ante un cierto campo incidente; teniendo en cuenta las interacciones múltiples que se producen entre dichos objetos mediante la teoría de grafos descrita en [13]. Las expresiones deducidas se 


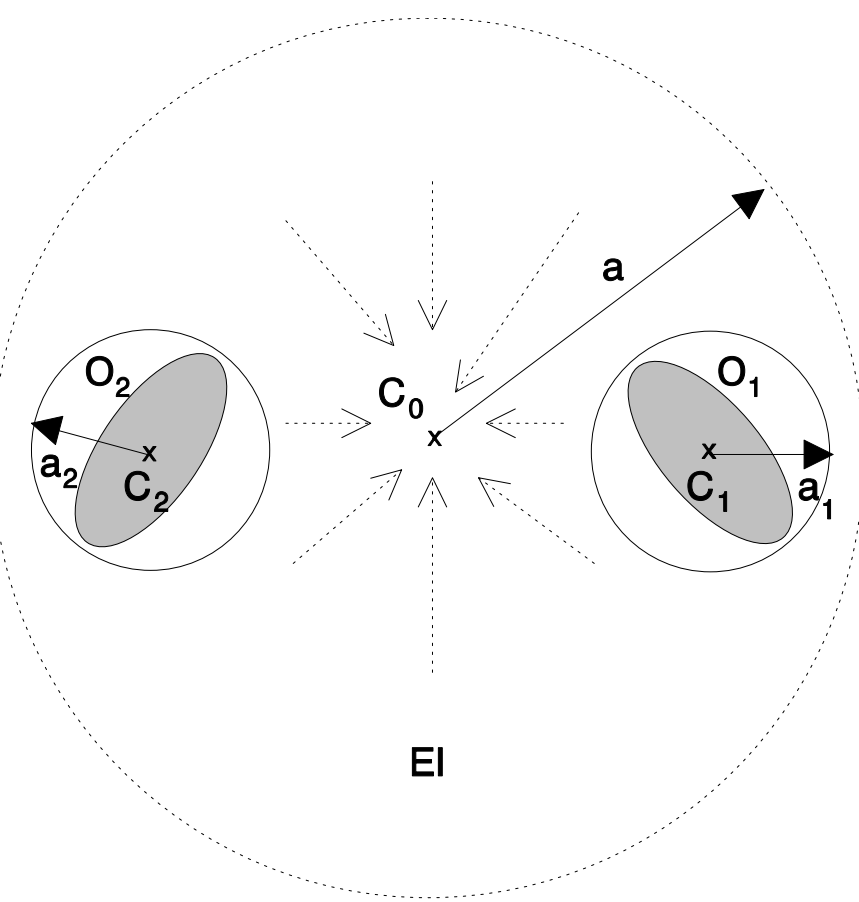

Figura 6.1: Esquema general de un problema formado por dos dispersores $O_{1}$ y $O_{2}$.

pueden aplicar igualmente al caso de incidencia con polarización $\mathrm{TM}^{\mathrm{z}}$ y al de incidencia polarizada según $\mathrm{TE}^{\mathrm{z}}$.

El problema a resolver se esquematiza en la figura 6.1 ; donde puede observarse la existencia de dos objetos dispersores, que llamaremos $O_{1}$ y $O_{2}$, sobre los que incide un cierto campo del que se conoce su espectro de ondas cilíndricas, representado por el vector columna $E I$. Los objetos dispersores se encuentran centrados respectivamente en los puntos $C_{1}$ y $C_{2}$, tal y como puede apreciarse de nuevo en la figura 6.1 ; mientras que los modos cilíndricos correspondientes al desarrollo espectral del campo incidente se refieren al punto $C_{0}$, definido por sus coordenadas rectangulares $\left(x_{0}, y_{0}\right)$. Por tanto, recordando (4.2), el campo incidente $^{1}$ sobre ambos objetos dispersores $O_{1}$ y $O_{2}$, que designaremos por $C_{z}^{i}$, se expresa como

$$
C_{z}^{i}=\sum_{p=-N_{i}}^{N_{i}} i_{p} J_{p}\left(k \rho_{0}\right) e^{j p \phi_{0}}
$$

donde $\left(\rho_{0}, \phi_{0}\right)$ son las coordenadas, referidas al origen local situado en $C_{0}$, del punto donde se pretende evaluar el campo incidente; mientras los coeficientes $i_{p}$, que constituyen los elementos del vector columna $E I$, representan las amplitudes del espectro asociado a dicho campo incidente. Por otra parte, el límite $N_{i}$ del

\footnotetext{
${ }^{1}$ Este campo $\left(C_{z}^{i}\right)$ representa campo eléctrico $\left(E_{z}^{i}\right)$ para incidencia polarizada según $\mathrm{TM}^{\mathrm{z}}, \mathrm{y}$ campo magnético $\left(H_{z}^{i}\right)$ para incidencia polarizada según $\mathrm{TE}^{\mathrm{Z}}$.
} 
sumatorio se escoge siguiendo el criterio $N_{i}>k a$, expuesto en el capítulo 4 (ver sección 4.1); de manera que el campo incidente se puede reconstruir con total exactitud en una circunferencia, de radio $a$, con centro en el origen local de coordenadas $C_{0}$. Lógicamente, el radio $a$ debe ser tal que la circunferencia contenga a los dos objetos dispersores $O_{1}$ y $O_{2}$ bajo análisis (ver figura 6.1). Además, la elección del punto $C_{0}$ conviene realizarla con el objetivo de minimizar el valor obtenido para $N_{i}$; lo cual se consigue, normalmente, escogiendo como $C_{0}$ el punto medio de las posiciones $C_{1}$ y $C_{2}$, donde se centran respectivamente los objetos $\mathrm{O}_{1}$ y $\mathrm{O}_{2}$.

La aplicación de la teoría de grafos [13] al problema que nos ocupa requiere caracterizar previamente, de manera aislada, a cada uno de los objetos dispersores $O_{1}$ y $O_{2}$ mediante sendas matrices de caracterización individual $D_{1}$ y $D_{2}$; cuyo cálculo se detalla en el capítulo 4 . Las matrices $D_{1}$ y $D_{2}$ a utilizar, bien las obtenidas para polarización $\mathrm{TM}^{\mathrm{z}}$ o las calculadas para polarización $\mathrm{TE}^{\mathrm{z}}$, dependen del tipo de incidencia que se considere sobre el problema de dispersión múltiple constituido por los dos objetos $\mathrm{O}_{1}$ y $\mathrm{O}_{2}$.

La respuesta producida por estos dos objetos dispersores ante un cierto campo incidente, representado por su correspondiente espectro cilíndrico, se obtiene resolviendo dos problemas complementarios. En una primera situación, se asume que el campo incidente afecta tan sólo al primer objeto $O_{1}$, y no al segundo objeto $\mathrm{O}_{2}$. Como consecuencia de las interacciones múltiples que tienen lugar entre ambos objetos, la resolución completa de este primer supuesto requiere calcular la dispersión que producen tanto $O_{1}$ como $O_{2}$. A continuación, se asume que el mismo campo incidente original afecta tan sólo al segundo objeto $O_{2}$, y no al primer objeto $O_{1}$. De igual manera, el cálculo del acoplo electromagnético existente en este caso entre los dos objetos precisa, para un completo análisis de este segundo problema, conocer de nuevo los campos dispersados por los dos objetos $O_{1}$ y $O_{2}$. Finalmente, la solución al problema original se obtiene, aplicando superposición, como la suma de las respuestas a cada uno de los dos problemas considerados.

En primer lugar, se debe resolver el problema en el que el campo incidente, definido por medio de su espectro $E I$, afecta tan sólo al objeto $O_{1}$; el cual produce, ante dicha incidencia, un campo dispersado que puede obtenerse utilizando su función de transferencia o matriz de caracterización individual $D_{1}$. Ahora bien, la matriz $D_{1}$ (recordar capítulo 4) permite relacionar el espectro de campo dispersado por $O_{1}$ con el espectro de campo incidente sobre $O_{1}$ cuando ambos espectros se refieren a un mismo punto; que normalmente se escoge centrado en el objeto (ver sección 4.1), y en este caso se corresponde con $C_{1}$. Sin embargo, el espectro $E I$ asociado al campo incidente se refiere, tal y como se ha indicado anteriormente, al punto $C_{0}$. Por tanto, resulta necesario trasladar el espectro $E I$ centrado en $C_{0}$ al punto $C_{1}$; para de esta forma obtener un espectro $E I_{1}$, correspondiente al mismo campo incidente original, sobre el que pueda utilizarse la matriz $D_{1}$. Esta operación se define en términos matriciales mediante una matriz de traslación 
de espectro incidente a incidente, cuyo concepto se detalla perfectamente en el apéndice E; obteniendo el nuevo espectro buscado $E I_{1}$ del siguiente modo

$$
E I_{1}=T J_{10} \cdot E I
$$

donde $T J_{10}$ representa la matriz de traslación de espectro incidente referido al punto $C_{0}$ a espectro incidente referido al punto $C_{1}$. Los elementos de esta matriz de traslación, cuyo cálculo se desarrolla por completo en el apéndice E, presentan el siguiente aspecto

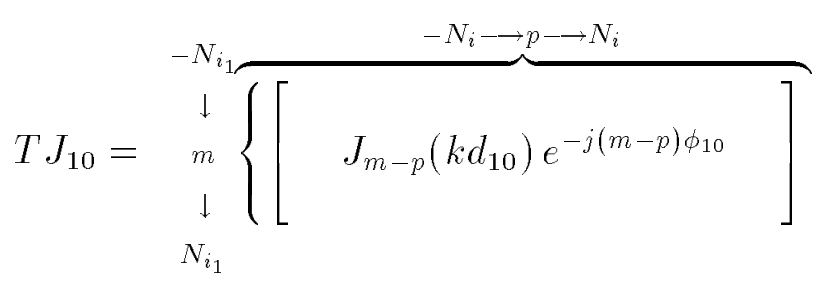

Los parámetros $d_{10} \mathrm{y} \phi_{10}$, presentes en (6.3), se obtienen a partir de las coordenadas rectangulares $\left(x_{0}, y_{0}\right)$ y $\left(x_{1}, y_{1}\right)$, correspondientes respectivamente a los puntos $C_{0}$ y $C_{1}$, mediante las expresiones

$$
\begin{aligned}
& d_{10}=\sqrt{\left(x_{0}-x_{1}\right)^{2}+\left(y_{0}-y_{1}\right)^{2}} \\
& \phi_{10}=\arctan \left(\frac{y_{0}-y_{1}}{x_{0}-x_{1}}\right)
\end{aligned}
$$

Es interesante reseñar que la matriz de traslación $T J_{10}$, definida según (6.3), convierte el espectro de campo incidente, referido al punto $C_{0}$ y con $2 N_{i}+1 \mathrm{com}-$ ponentes espectrales, en un nuevo espectro asociado al mismo campo incidente; aunque referido ahora al punto $C_{1}$ y con $2 N_{i_{1}}+1$ componentes. El valor a escoger para $N_{i_{1}}$ debe satisfacer el criterio $N_{i_{1}}>k a_{1}$; donde $a_{1}$ representa el radio de la mínima circunferencia, centrada en $C_{1}$, que contiene al objeto dispersor $O_{1}$. De esta manera, el campo incidente original se reconstruye perfectamente sobre el contorno del objeto $O_{1}$ utilizando el nuevo espectro $E I_{1}$.

Una vez referido el espectro de campo incidente al centro del primer objeto dispersor $O_{1}$, es posible utilizar la matriz $D_{1}$ para obtener el espectro asociado al campo que dispersa $O_{1}$ ante dicha incidencia. Este espectro de campo dispersado, el cual se refiere también al punto $C_{1}$, se calcula pues mediante el producto $D_{1} \cdot T J_{10} \cdot E I$; tal y como se ilustra en la figura 6.2 .

A su vez, el campo dispersado por este primer objeto $O_{1}$ se propaga hasta convertirse en campo incidente sobre el segundo objeto $\mathrm{O}_{2}$; el cual genera, ante dicha incidencia, un campo dispersado que puede obtenerse mediante su función de transferencia o matriz de caracterización individual $D_{2}$. Ahora bien, la matriz $D_{2}$ relaciona el espectro de campo dispersado por $O_{2}$ con el espectro asociado 

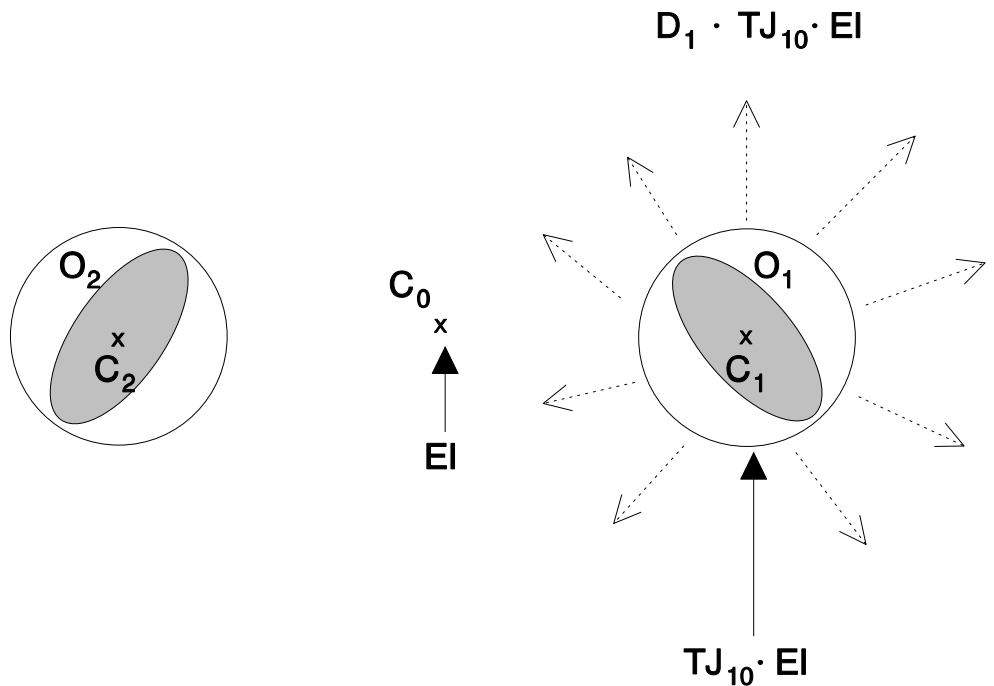

Figura 6.2: Campo dispersado por $O_{1}$ ante el campo incidente original.

al campo incidente sobre $\mathrm{O}_{2}$ cuando ambos espectros se refieren al centro de dicho objeto; representado en esta ocasión por el punto $C_{2}$. Estos espectros de campo dispersado e incidente se definen, recordando la sección 4.1, en términos de las amplitudes correspondientes a los desarrollos en serie de modos cilíndricos emergentes desde $\mathrm{O}_{2}$ e incidentes sobre $\mathrm{O}_{2}$, respectivamente. Sin embargo, el campo incidente sobre $\mathrm{O}_{2}$ viene expresado en serie de modos cilíndricos emergentes referidos al centro $C_{1}$ del primer objeto dispersor. Por tanto, el espectro de campo dispersado referido al punto $C_{1}$ debe transformarse en espectro de campo incidente centrado en el punto $C_{2}$. Esta operación se implementa matricialmente mediante una matriz de traslación de espectro emergente a incidente, cuyo concepto se recoge de nuevo en el apéndice E. Consecuentemente, el campo incidente sobre $\mathrm{O}_{2}$, expresado en serie de modos cilíndricos incidentes referidos al punto $C_{2}$, presenta un espectro asociado que se obtiene a través del producto matricial $T_{21} \cdot D_{1} \cdot T J_{10} \cdot E I$; donde $T_{21}$ representa la matriz de traslación de modos emergentes centrados en $C_{1}$ a modos incidentes centrados en $C_{2}$. Los elementos de dicha matriz, cuyo cálculo se detalla completamente en el apéndice E, se resumen a continuación como

$$
T_{21}=\begin{gathered}
p \\
\downarrow \\
\downarrow \\
N_{i_{2}}
\end{gathered}
$$

donde los parámetros $d_{21}$ y $\phi_{21}$ se determinan a partir de las coordenadas rectangulares $\left(x_{1}, y_{1}\right)$ y $\left(x_{2}, y_{2}\right)$, correspondientes respectivamente a los puntos $C_{1}$ y $C_{2}$, 
mediante las expresiones

$$
\begin{aligned}
& d_{21}=\sqrt{\left(x_{1}-x_{2}\right)^{2}+\left(y_{1}-y_{2}\right)^{2}} \\
& \phi_{21}=\arctan \left(\frac{y_{1}-y_{2}}{x_{1}-x_{2}}\right)
\end{aligned}
$$

Es interesante observar que la matriz de traslación $T_{21}$, definida según (6.5), convierte un desarrollo en serie de $2 N_{d_{1}}+1$ modos cilíndricos emergentes, referidos al centro $C_{1}$ del primer objeto dispersor, en un nuevo desarrollo en serie constituido por $2 \mathrm{~N}_{i_{2}}+1$ modos cilíndricos incidentes, referidos ahora al centro $C_{2}$ del segundo objeto dispersor. El valor escogido para $N_{i_{2}}$ debe ser tal que satisfaga el criterio $N_{i_{2}}>k a_{2}$; donde $a_{2}$ representa el radio de la mínima circunferencia, centrada en $C_{2}$, que contiene al objeto dispersor $O_{2}$. Siguiendo este criterio, el campo dispersado por $O_{1}$ e incidente sobre $O_{2}$ se reconstruye perfectamente sobre el contorno de este segundo objeto dispersor. En cuanto a los valores para $N_{d_{1}} \mathrm{y}$ $N_{d_{2}}$, que permiten reconstruir el campo dispersado por cada uno de los objetos $O_{1}$ y $O_{2}$, se escogen igual a los valores seleccionados con anterioridad para $N_{i_{1}}$ y $N_{i_{2}}$, respectivamente; tal y como se indica en el capítulo 4 (sección 4.1).

En resumen, el campo dispersado por el objeto $O_{1}$ se convierte en un campo incidente sobre el objeto $O_{2}$; cuyo espectro, referido al punto $C_{2}$, se calcula mediante el producto $T_{21} \cdot D_{1} \cdot T J_{10} \cdot E I$. De esta forma, el espectro asociado al campo que dispersa $\mathrm{O}_{2}$ ante dicha incidencia se obtiene multiplicando la matriz $D_{2}$ por el anterior espectro de campo incidente. Por tanto, este espectro de campo dispersado por $O_{2}$, referido también al punto $C_{2}$, se expresa a través del producto matricial $D_{2} \cdot T_{21} \cdot D_{1} \cdot T J_{10} \cdot E I$; tal y como se recoge gráficamente en la figura 6.3.

De nuevo, el campo dispersado por este segundo objeto $O_{2}$ se propaga hasta convertirse en campo incidente sobre el primer objeto $O_{1}$. Con la intención de calcular el campo que dispersa $O_{1}$ ante dicha incidencia, resulta necesario expresar la serie de modos cilíndricos dispersados por $\mathrm{O}_{2}$, referidos al punto $C_{2}$, en serie de modos cilíndricos incidentes sobre $O_{1}$ centrados en el punto $C_{1}$. Esta operación se implementa, como se ha comentado anteriormente, mediante una matriz de traslación de espectro emergente a incidente; que se designa en esta ocasión por $T_{12}$, al transformar modos cilíndricos emergentes referidos al punto $C_{2}$ en modos cilíndricos incidentes referidos al punto $C_{1}$. Consecuentemente, sobre $O_{1}$ incide un nuevo campo, cuyo espectro se obtiene aplicando la matriz de traslación $T_{12}$ al espectro de campo previamente dispersado por $O_{2}$; lo cual da lugar al producto matricial $T_{12} \cdot D_{2} \cdot T_{21} \cdot D_{1} \cdot T J_{10} \cdot E I$. Esta nueva incidencia sobre $O_{1}$ se suma al campo incidente original, referido en términos espectrales al punto $C_{1}$ mediante el producto $T J_{10} \cdot E I$. Ambos campos incidentes sobre $O_{1}$ causan que dicho objeto disperse otro campo, que tras propagarse incide sobre $O_{2}$. Ante dicha incidencia, $O_{2}$ genera un nuevo campo que retorna a $O_{1}$; para sumarse de esta forma a 


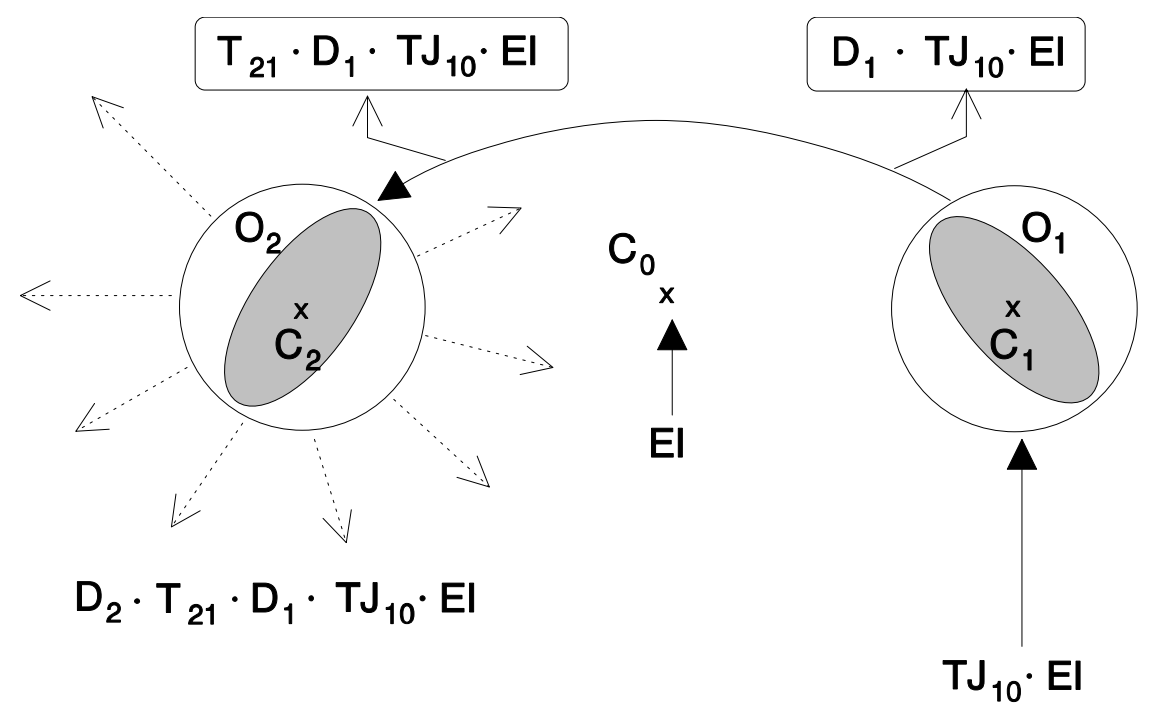

Figura 6.3: Campo dispersado por $O_{2}$ ante un campo incidente que procede de $O_{1}$.

los dos campos incidentes considerados inicialmente. En el límite, el proceso descrito alcanza una situación estable; en la cual el campo incidente sobre el primer objeto dispersor se obtiene como la suma del campo incidente original, representado espectralmente por el producto $T J_{10} \cdot E I$, y del campo incidente sobre $O_{1}$ como consecuencia de la realimentación que se produce entre $O_{1}$ y $O_{2}$, cuyo espectro referido al punto $C_{1}$ se denominará $E A$.

Resumiendo, tras producirse infinitas realimentaciones entre $O_{1}$ y $O_{2}$, se alcanza un régimen permanente en el que sobre $O_{1}$ inciden tan sólo dos campos; los cuales se expresan en términos espectrales como $T J_{10} \cdot E I$ y $E A$, respectivamente. La suma de estos dos campos incidentes sobre $O_{1}$ causa que dicho objeto disperse un campo, con un espectro asociado de valor $D_{1} \cdot\left(T J_{10} \cdot E I+E A\right)$. Este espectro de campo dispersado por $O_{1}$ se traslada del punto $C_{1}$ al punto $C_{2}$ utilizando la correspondiente matriz de traslación de espectro emergente a incidente, en este caso la matriz $T_{21}$ descrita anteriormente, que permite obtener el espectro asociado al campo incidente sobre $O_{2}$ mediante el producto $T_{21} \cdot D_{1} \cdot\left(T J_{10} \cdot E I+E A\right)$. Ante dicha incidencia, el espectro de campo dispersado por el objeto $O_{2}$ se calcula multiplicando la matriz de caracterización individual $D_{2}$ por el anterior espectro de campo incidente; lo que da lugar a la expresión $D_{2} \cdot T_{21} \cdot D_{1} \cdot\left(T J_{10} \cdot E I+E A\right)$. A continuación, aplicando una nueva matriz de traslación de espectro emergente a incidente, designada en este caso como $T_{12}$, el espectro de campo dispersado por $O_{2}$ se traslada del punto $C_{2}$ al punto $C_{1}$; convirtiéndose de esta forma en un espectro de campo incidente sobre $O_{1}$ de valor $T_{12} \cdot D_{2} \cdot T_{21} \cdot D_{1} \cdot\left(T J_{10} \cdot E I+E A\right)$. En la figura 6.4, se representa gráficamente la secuencia de todas las operaciones recién descritas. Finalmente, recordando que $E A$ representa precisamente el espectro de campo incidente sobre $O_{1}$ debido a las múltiples interacciones entre los 


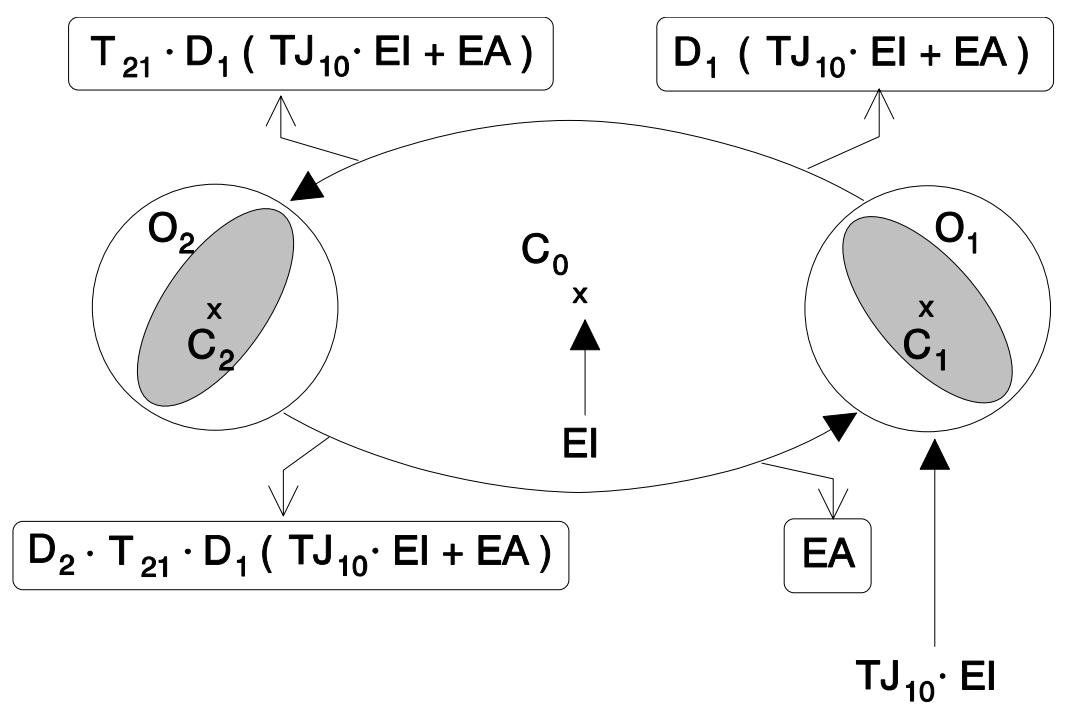

Figura 6.4: Diagrama completo de la realimentación entre $O_{1}$ y $O_{2}$ cuando el campo incidente original, de espectro $E I$, afecta tan sólo a $O_{1}$.

dos objetos dispersores, se debe exigir el cumplimiento de la siguiente igualdad

$$
E A=T_{12} \cdot D_{2} \cdot T_{21} \cdot D_{1} \cdot\left(T J_{10} \cdot E I+E A\right)
$$

que permite plantear el siguiente sistema de ecuaciones para encontrar el espectro incógnita $E A$

$$
\left(I_{1}-T_{12} \cdot D_{2} \cdot T_{21} \cdot D_{1}\right) \cdot E A=T_{12} \cdot D_{2} \cdot T_{21} \cdot D_{1} \cdot T J_{10} \cdot E I
$$

donde $I_{1}$ representa la matriz identidad de tamaño $2 N_{i_{1}}+1$. Resolviendo pues el sistema de ecuaciones expresado matricialmente en (6.8), se obtienen las $2 N_{i_{1}}+1$ componentes (incógnitas) del vector columna $E A$; a partir del cual es posible encontrar los espectros de los campos dispersados por $O_{1}$ y $O_{2}$ en este primer problema. Estos espectros, representados respectivamente por $E D_{1}^{(1)}$ y $E D_{2}^{(1)}$, se calculan fácilmente del siguiente modo

$$
\begin{aligned}
& E D_{1}^{(1)}=D_{1} \cdot\left(T J_{10} \cdot E I+E A\right) \\
& E D_{2}^{(1)}=D_{2} \cdot T_{21} \cdot D_{1} \cdot\left(T J_{10} \cdot E I+E A\right)
\end{aligned}
$$

A continuación, siguiendo el mismo procedimiento descrito anteriormente para resolver la primera situación, se debe solucionar el segundo problema en el que el campo incidente, definido mediante su espectro $E I$, afecta tan sólo al segundo objeto dispersor. En este caso, tras producirse múltiples interacciones entre los dos objetos dispersores, se alcanza un régimen estable en el cual sobre $O_{2}$ existe 
un campo incidente desconocido, cuyo espectro referido al punto $C_{2}$ se designa como EB. Adicionalmente, sobre dicho objeto $O_{2}$ se tiene el campo incidente original, cuyo espectro debe referirse también al punto $C_{2}$; para lo cual se utiliza una matriz de traslación de espectro incidente a incidente, denominada en esta ocasión $T J_{20}$. Los elementos de dicha matriz se calculan de forma análoga a los de la matriz $T J_{10}$; es decir, utilizando las expresiones (6.3) y (6.4) una vez sustituidos los parámetros $d_{10}$ y $\phi_{10}$ por sus equivalentes $d_{20}$ y $\phi_{20}$, en los que deben emplearse las coordenadas $\left(x_{2}, y_{2}\right)$ del punto $C_{2}$. De esta forma, la incidencia que existe en régimen permanente sobre el segundo objeto dispersor se expresa, en términos espectrales, mediante la suma del espectro incógnita $E B$ y el espectro asociado al campo incidente original $T J_{20} \cdot E I$, ambos referidos al punto $C_{2}$. Ante dicha incidencia, el objeto $O_{2}$ dispersa un campo con un espectro asociado de valor $D_{2} \cdot\left(T J_{20} \cdot E I+E B\right)$. Este campo dispersado se traslada del punto $C_{2}$ al punto $C_{1}$ aplicando la correspondiente matriz de traslación $T_{12}$; que origina un campo incidente sobre el objeto $O_{1}$, cuyo espectro se obtiene mediante el producto $T_{12} \cdot D_{2} \cdot\left(T J_{20} \cdot E I+E B\right)$. Debido a esta incidencia, el objeto $O_{1}$ genera un campo dispersado de espectro $D_{1} \cdot T_{12} \cdot D_{2} \cdot\left(T J_{20} \cdot E I+E B\right)$; en cuyo cálculo se ha utilizado la matriz de caracterización individual $D_{1}$ de dicho objeto dispersor. Inmediatamente después, este espectro de campo dispersado por $O_{1}$, que se refiere al punto $C_{1}$, se transforma mediante la matriz de traslación $T_{21}$ en un espectro de campo incidente sobre $O_{2}$, referido al punto $C_{2}$, y de valor $T_{21} \cdot D_{1} \cdot T_{12} \cdot D_{2} \cdot\left(T J_{20} \cdot E I+E B\right)$. Un diagrama con todas las operaciones recién descritas se recoge gráficamente en la figura 6.5. Por último, este espectro de campo incidente sobre $\mathrm{O}_{2}$, que se ha obtenido estudiando las interacciones múltiples producidas entre $O_{1}$ y $O_{2}$, coincide con la definición del espectro $E B$; por lo que debe cumplirse la siguiente igualdad

$$
E B=T_{21} \cdot D_{1} \cdot T_{12} \cdot D_{2} \cdot\left(T J_{20} \cdot E I+E B\right)
$$

que permite plantear el siguiente sistema de ecuaciones para obtener en esta ocasión el espectro incógnita $E B$

$$
\left(I_{2}-T_{21} \cdot D_{1} \cdot T_{12} \cdot D_{2}\right) \cdot E B=T_{21} \cdot D_{1} \cdot T_{12} \cdot D_{2} \cdot T J_{20} \cdot E I
$$

donde $I_{2}$ representa en este caso la matriz identidad de tamaño $2 N_{i_{2}}+1$. El sistema de ecuaciones expresado matricialmente en $(6.11)$ se resuelve para obtener las $2 N_{i_{2}}+1$ componentes (incógnitas) del vector $E B$; el cual se utiliza a su vez para encontrar los espectros correspondientes a los campos dispersados por $O_{1}$ y $\mathrm{O}_{2}$ en este segundo problema. Estos espectros, denominados respectivamente $E D_{1}^{(2)}$ y $E D_{2}^{(2)}$, se calculan fácilmente mediante las siguientes expresiones

$$
\begin{aligned}
& E D_{1}^{(2)}=D_{1} \cdot T_{12} \cdot D_{2} \cdot\left(T J_{20} \cdot E I+E B\right) \\
& E D_{2}^{(2)}=D_{2} \cdot\left(T J_{20} \cdot E I+E B\right)
\end{aligned}
$$




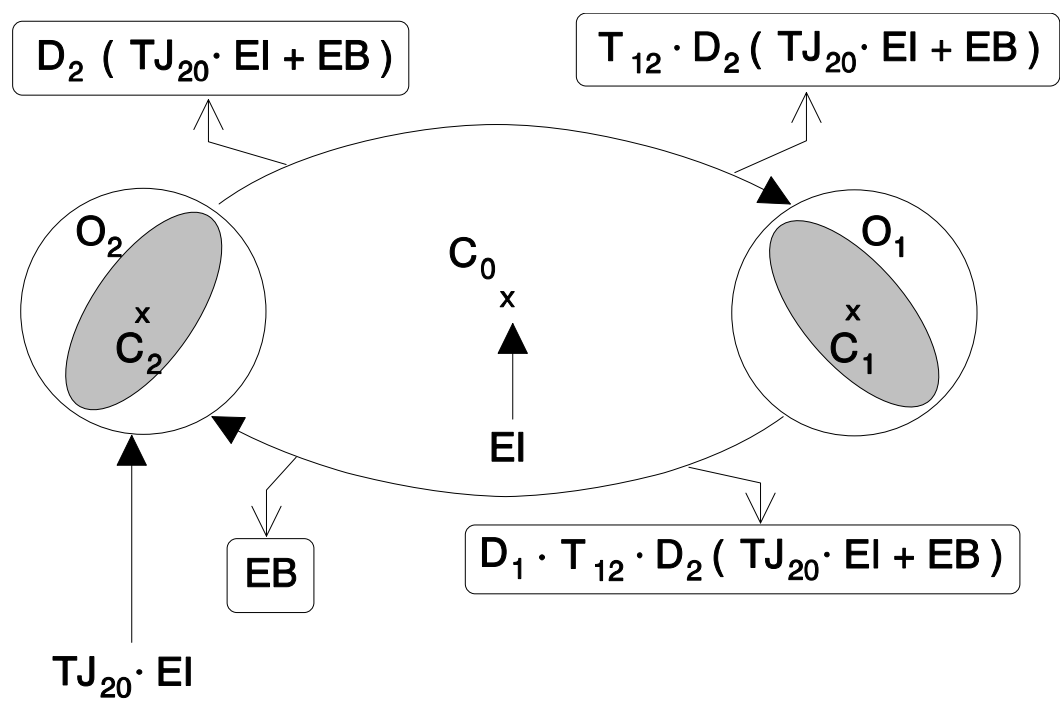

Figura 6.5: Diagrama completo de la realimentación entre $O_{1}$ y $O_{2}$ cuando el campo incidente original, de espectro $E I$, afecta tan sólo a $\mathrm{O}_{2}$.

Una vez resueltos los dos problemas complementarios, cuyas soluciones se recogen respectivamente en (6.9) y (6.12), se debe aplicar superposición para obtener la respuesta al problema original; en el que el campo incidente afecta a los dos objetos dispersores. Por tanto, los espectros $E D_{1}$ y $E D_{2}$ correspondientes a los campos dispersados por cada uno de los dos objetos se calculan, teniendo en cuenta las interacciones múltiples que se producen entre ambos objetos, del siguiente modo

$$
\begin{aligned}
E D_{1} & =E D_{1}^{(1)}+E D_{1}^{(2)} \\
& =D_{1} \cdot\left(T J_{10} \cdot E I+E A+T_{12} \cdot D_{2} \cdot\left(T J_{20} \cdot E I+E B\right)\right) \\
E D_{2} & =E D_{2}^{(1)}+E D_{2}^{(2)} \\
& =D_{2} \cdot\left(T J_{20} \cdot E I+E B+T_{21} \cdot D_{1} \cdot\left(T J_{10} \cdot E I+E A\right)\right)
\end{aligned}
$$

Ahora bien, la solución recogida en (6.13) determina la dispersión electromagnética producida por dos objetos dispersores ante un campo incidente concreto, representado por su correspondiente espectro EI. Con el objeto de no tener que repetir para cada posible incidencia los cálculos descritos anteriormente, se plantea la caracterización de cada objeto dispersor por medio de una función de transferencia o matriz de caracterización conjunta; que permita obtener el espectro de campo dispersado por dicho objeto, considerando las interacciones múltiples que se producen entre ambos objetos, mediante el producto de la citada matriz por el espectro asociado al campo incidente. El concepto de la matriz 
de caracterización conjunta, análogo al introducido en el capítulo 4 para caracterizar individualmente el comportamiento de un objeto dispersor, permite a su vez analizar fácilmente el acoplo que se produce entre los dos objetos considerados inicialmente y un tercer objeto dispersor. De esta forma, la dispersión que produce un sistema constituido por $N$ objetos queda perfectamente determinada mediante $N$ matrices de caracterización conjunta; que permiten obtener respectivamente el campo dispersado por cada uno de los objetos, teniendo en cuenta la presencia del resto, ante cualquier posible incidencia.

Retornando al problema constituido por dos objetos dispersores, el cálculo de los elementos de sus respectivas matrices de caracterización conjunta se implementa seleccionando, en cada ocasión, una componente distinta $\left(J_{p}(k \rho) e^{j p \phi}\right)$ del desarrollo modal incidente recogido en (6.1); para posteriormente, siguiendo el método basado en teoría de grafos, obtener los desarrollos espectrales de los campos dispersados por cada uno de los objetos ante dicha incidencia, desarrollos espectrales que constituyen respectivamente la p-ésima columna de las correspondientes matrices de caracterización conjunta. Con el objeto de poder utilizar el método descrito anteriormente, la $p$-ésima componente modal incidente debe expresarse en términos de su espectro asociado; el cual se representa mediante el siguiente vector columna designado como $E I_{p}$

$$
\left.E I_{p}=\begin{array}{c}
-N_{i} \\
\downarrow \\
\downarrow \\
N_{i}
\end{array}\right\}\left[\begin{array}{c}
0 \\
0 \\
\vdots \\
1 \\
\vdots \\
0
\end{array}\right]
$$

donde, lógicamente, todas las componentes de dicho vector son nulas excepto la $p$-ésima que presenta amplitud unidad.

En primer lugar, para cada objeto dispersor se debe encontrar el espectro incógnita asociado a un campo que incide sobre dicho objeto; que aparece como consecuencia de las múltiples interacciones entre ambos objetos dispersores. Dichos espectros, denominados respectivamente $E A_{p}$ y $E B_{p}$, se obtienen tras resolver los correspondientes sistemas de ecuaciones; los cuales, recordando (6.8) y (6.11), se expresan del siguiente modo

$$
\begin{aligned}
& \left(I_{1}-T_{12} \cdot D_{2} \cdot T_{21} \cdot D_{1}\right) \cdot E A_{p}=T_{12} \cdot D_{2} \cdot T_{21} \cdot D_{1} \cdot T J_{10} \cdot E I_{p} \\
& \left(I_{2}-T_{21} \cdot D_{1} \cdot T_{12} \cdot D_{2}\right) \cdot E B_{p}=T_{21} \cdot D_{1} \cdot T_{12} \cdot D_{2} \cdot T J_{20} \cdot E I_{p}
\end{aligned}
$$

Una vez se conocen los vectores $E A_{p}$ y $E B_{p}$; es posible calcular, utilizando (6.13), los espectros correspondientes a los campos dispersados por ambos objetos 
mediante las siguientes operaciones matriciales

$$
\begin{aligned}
& E D_{1}^{(p)}=D_{1} \cdot\left(T J_{10} \cdot E I_{p}+E A_{p}+T_{12} \cdot D_{2} \cdot\left(T J_{20} \cdot E I_{p}+E B_{p}\right)\right) \\
& E D_{2}^{(p)}=D_{2} \cdot\left(T J_{20} \cdot E I_{p}+E B_{p}+T_{21} \cdot D_{1} \cdot\left(T J_{10} \cdot E I_{p}+E A_{p}\right)\right)
\end{aligned}
$$

donde $E D_{1}^{(p)}$ y $E D_{2}^{(p)}$ constituyen respectivamente la $p$-ésima columna de las matrices de caracterización conjunta, designadas como $D_{1}^{(1)}$ y $D_{2}^{(1)}$, de ambos objetos dispersores. Repitiendo el proceso anterior para cada posible modo incidente $\left(p=-N_{i}, \ldots, N_{i}\right)$ en $(6.1)$, se construyen las matrices de caracterización conjunta de la siguiente manera

$$
\begin{aligned}
& D_{1}^{(1)}=\left[\begin{array}{llll}
E D_{1}^{\left(-N_{i}\right)} & E D_{1}^{\left(-N_{i}+1\right)} & \cdots & E D_{1}^{\left(N_{i}\right)}
\end{array}\right]_{2 N_{d_{1}}+1 \times 2 N_{i}+1} \\
& D_{2}^{(1)}=\left[\begin{array}{llll}
E D_{2}^{\left(-N_{i}\right)} & E D_{2}^{\left(-N_{i}+1\right)} & \cdots & E D_{2}^{\left(N_{i}\right)}
\end{array}\right]_{2 N_{d_{2}}+1 \times 2 N_{i}+1}
\end{aligned}
$$

Cada una de las columnas pertenecientes a las matrices de caracterización conjunta $D_{1}^{(1)}$ y $D_{2}^{(1)}$ requiere la resolución de dos sistemas de ecuaciones; que se recogen en (6.15), y están constituidos respectivamente por $2 N_{i_{1}}+1$ y $2 N_{i_{2}}+1$ incógnitas. La obtención de dichas matrices supone, por tanto, repetir el proceso descrito anteriormente en $2 N_{i}+1$ ocasiones. Con la intención de reducir cálculos, sustituyendo en (6.15) el espectro de campo incidente $E I_{p}$ por una matriz identidad $I$ de tamaño $2 N_{i}+1$, resulta posible expresar el proceso completo en términos matriciales; originando los siguientes sistemas de ecuaciones

$$
\begin{aligned}
& \left(I_{1}-T_{12} \cdot D_{2} \cdot T_{21} \cdot D_{1}\right) \cdot E A=T_{12} \cdot D_{2} \cdot T_{21} \cdot D_{1} \cdot T J_{10} \cdot I \\
& \left(I_{2}-T_{21} \cdot D_{1} \cdot T_{12} \cdot D_{2}\right) \cdot E B=T_{21} \cdot D_{1} \cdot T_{12} \cdot D_{2} \cdot T J_{20} \cdot I
\end{aligned}
$$

donde cada una de estas ecuaciones representa $2 N_{i}+1$ sistemas de ecuaciones con $2 N_{i_{1}}+1$ y $2 N_{i_{2}}+1$ incógnitas, respectivamente. Como puede observarse a continuación, cada columna de la matriz I representa una componente distinta $\left(E I_{p}\right)$ del desarrollo modal incidente; mientras que cada columna de las matrices $E A$ y $E B$, tras resolver los sistemas de ecuaciones planteados en (6.18), representa la solución parcial ( $E A_{p}$ y $E B_{p}$ ) asociada a la correspondiente incidencia

$$
\begin{aligned}
I & =\left[\begin{array}{llll}
E I_{-N_{i}} & E I_{-N_{i}+1} & \cdots & E I_{N_{i}}
\end{array}\right]_{2 N_{i}+1 \times 2 N_{i}+1} \\
E A & =\left[\begin{array}{llll}
E A_{-N_{i}} & E A_{-N_{i}+1} & \cdots & E A_{N_{i}}
\end{array}\right]_{2 N_{i_{1}}+1 \times 2 N_{i}+1} \\
E B & =\left[\begin{array}{llll}
E B_{-N_{i}} & E B_{-N_{i}+1} & \cdots & E B_{N_{i}}
\end{array}\right]_{2 N_{i_{2}}+1 \times 2 N_{i}+1}
\end{aligned}
$$


Tras haber obtenido las matrices $E A$ y $E B$, deben aplicarse las expresiones deducidas en (6.16) al cálculo de las matrices de caracterización conjunta $D_{1}^{(1)} \mathrm{y}$ $D_{2}^{(1)}$ de los dos objetos dispersores; que se obtienen finalmente implementando las siguientes operaciones matriciales

$$
\begin{aligned}
& D_{1}^{(1)}=D_{1} \cdot\left(T J_{10} \cdot I+E A+T_{12} \cdot D_{2} \cdot\left(T J_{20} \cdot I+E B\right)\right) \\
& D_{2}^{(1)}=D_{2} \cdot\left(T J_{20} \cdot I+E B+T_{21} \cdot D_{1} \cdot\left(T J_{10} \cdot I+E A\right)\right)
\end{aligned}
$$

De esta forma, la dispersión electromagnética producida por los dos objetos dispersores queda perfectamente caracterizada; permitiendo obtener la respuesta de dicho sistema, ante cualquier campo incidente, de manera sencilla e inmediata. Así pues, ante una incidencia concreta definida por las amplitudes espectrales $i_{p}$ presentes en (6.1), los coeficientes $c_{q}^{(1)}$ y $c_{q}^{(2)}$ de los desarrollos modales correspondientes a los campos dispersados por cada uno de los objetos se obtienen, teniendo en cuenta las interacciones múltiples que se producen entre dichos objetos, multiplicando respectivamente las matrices $D_{1}^{(1)}$ y $D_{2}^{(1)}$ por el espectro de campo incidente $E I$ del siguiente modo

$$
\begin{aligned}
& {\left[\begin{array}{c}
c_{-N_{d_{1}}}^{(1)} \\
c_{-N_{d_{1}}+1}^{(1)} \\
\vdots \\
c_{N_{d_{1}}}^{(1)}
\end{array}\right]=D_{1}^{(1)} \cdot\left[\begin{array}{c}
i_{-N_{i}} \\
i_{-N_{i}+1} \\
\vdots \\
i_{N_{i}}
\end{array}\right]} \\
& {\left[\begin{array}{c}
c_{-N_{d_{2}}}^{(2)} \\
c_{-N_{d_{2}}+1}^{(2)} \\
\vdots \\
c_{N_{d_{2}}}^{(2)}
\end{array}\right]=D_{2}^{(1)} \cdot\left[\begin{array}{c}
i_{-N_{i}} \\
i_{-N_{i}+1} \\
\vdots \\
i_{N_{i}}
\end{array}\right]}
\end{aligned}
$$

donde se recuerda que el espectro de campo incidente $E I$ se refiere al punto $C_{0}$ (ver figura 6.1); mientras los desarrollos modales de los campos dispersados, que se expresan mediante los coeficientes $c_{q}^{(1)}$ y $c_{q}^{(2)}$, se encuentran referidos respectivamente a los centros $C_{1}$ y $C_{2}$ de cada uno de los objetos dispersores. Por lo que respecta al punto $C_{0}$, tal y como se ha indicado anteriormente, se suele escoger como el punto medio de las posiciones $C_{1}$ y $C_{2}$; para de esta forma minimizar el número de modos $\left(2 N_{i}+1\right)$ con el que se reconstruye el campo incidente sobre la superficie de ambos objetos dispersores, y reducir asimismo las dimensiones de las matrices de caracterización conjunta $D_{1}^{(1)}$ y $D_{2}^{(1)}$.

A partir de los coeficientes $c_{q}^{(1)}$ y $c_{q}^{(2)}$, calculados previamente según (6.21), el campo total dispersado por los dos objetos bajo análisis se expresa, considerando 
el acoplo electromagnético que se produce entre dichos objetos, mediante la suma de los siguientes desarrollos en serie de modos cilíndricos emergentes

$$
E_{z}^{d}=\sum_{q=-N_{d_{1}}}^{N_{d_{1}}} c_{q}^{(1)} H_{q}^{(2)}\left(k \rho_{1}\right) e^{j q \phi_{1}}+\sum_{q=-N_{d_{2}}}^{N_{d_{2}}} c_{q}^{(2)} H_{q}^{(2)}\left(k \rho_{2}\right) e^{j q \phi_{2}}
$$

donde $\left(\rho_{1}, \phi_{1}\right)$ y $\left(\rho_{2}, \phi_{2}\right)$ son las coordenadas del punto donde se pretende evaluar el campo dispersado por los dos objetos considerados; las cuales se refieren a sendos orígenes locales situados en los puntos $C_{1}$ y $C_{2}$, respectivamente.

Resumiendo, en este apartado se ha resuelto la dispersión electromagnética que produce un sistema constituido por dos objetos dispersores; habiendo utilizado al resolver dicho problema la teoría de grafos recogida en [13]. El comportamiento de cada uno de los dos objetos dispersores ha quedado definido en términos de una matriz de caracterización conjunta; que tiene en cuenta el efecto del acoplo electromagnético entre ambos objetos. El concepto de la matriz de caracterización conjunta se puede generalizar, como se verá a continuación, a problemas de interacción electromagnética entre múltiples objetos dispersores.

\subsubsection{Introducción de un Tercer Objeto Dispersor}

Tras caracterizar la dispersión electromagnética producida por dos objetos dispersores mediante sendas matrices de caracterización conjunta, obtenidas mediante teoría de grafos, se pretende aplicar el mismo método para analizar las interacciones múltiples que tienen lugar al introducir en dicho grupo un tercer objeto dispersor. Para ello, se debe considerar a los dos objetos caracterizados inicialmente como un único elemento dispersor equivalente; cuyo comportamiento queda perfectamente definido (ver apartado 6.1.1) por las matrices de caracterización conjunta $D_{1}^{(1)}$ y $D_{2}^{(1)}$. De esta forma, se puede utilizar nuevamente el método basado en teoría de grafos [13]; para analizar en esta ocasión el sistema constituido por el elemento dispersor equivalente y el tercer objeto recién introducido. La aplicación de dicho método produce tres nuevas matrices de caracterización conjunta; las cuales permiten calcular fácilmente los campos dispersados por cada uno de los tres objetos considerando la presencia del resto.

En la figura 6.6, se representa el problema bajo análisis; constituido por tres objetos dispersores, que llamaremos $O_{1}, O_{2}$ y $O_{3}$, centrados respectivamente en los puntos $C_{1}, C_{2}$ y $C_{3}$. Sobre estos tres objetos incide un cierto campo cuyo desarrollo modal, definido según (6.1), se refiere al punto $C_{0}$. La elección de dicho punto, al igual que cuando se analizan dos objetos dispersores, pretende minimizar el número de modos $\left(2 N_{i}+1\right)$ necesarios para reconstruir, con total exactitud, el campo incidente sobre el contorno de todos los objetos. Este objetivo se cumple normalmente situando $C_{0}$ en el punto medio de los tres centros $C_{1}, C_{2} \mathrm{y}$ $C_{3}$; pues la reconstrucción correcta del campo incidente requiere que se satisfaga 


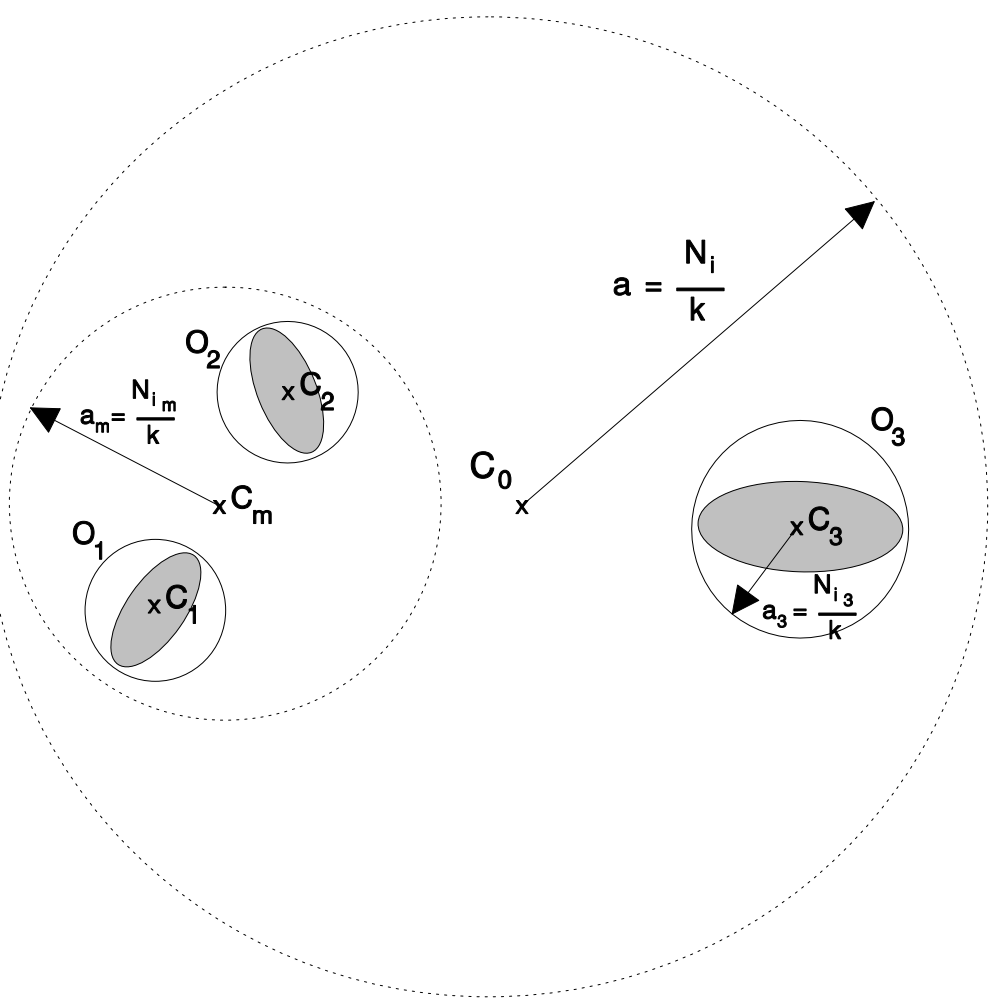

Figura 6.6: Esquema general de un problema de realimentación múltiple entre tres dispersores $\mathrm{O}_{1}, \mathrm{O}_{2}$ y $\mathrm{O}_{3}$.

el criterio $N_{i}>k \cdot a$, siendo $a$ el radio de la mínima circunferencia con centro en $C_{0}$ que contiene a los tres objetos dispersores (ver figura 6.6).

La aplicación del método basado en teoría de grafos requiere la caracterización por separado de los dos objetos bajo análisis; en este caso del elemento dispersor equivalente, integrado por los objetos $O_{1}$ y $O_{2}$, y del tercer objeto dispersor. Para caracterizar al elemento dispersor equivalente, se utilizan las matrices de caracterización conjunta $D_{1}^{(1)}$ y $D_{2}^{(1)}$; que relacionan respectivamente los espectros de campo dispersado por los objetos $O_{1}$ y $O_{2}$, emergentes cada uno de ellos desde los centros $C_{1}$ y $C_{2}$, con un espectro de campo incidente referido al punto $C_{m}$. Este campo incidente se reconstruye correctamente sobre ambos objetos empleando $2 N_{i_{m}}+1$ modos; donde $N_{i_{m}}$ debe satisfacer el conocido criterio $N_{i_{m}}>k \cdot a_{m}$, representando $a_{m}$ el radio de la mínima circunferencia con centro en $C_{m}$ que contiene a $O_{1}$ y $O_{2}$ (ver figura 6.6). Tal y como se indica en el apartado 6.1.1, para minimizar el número de modos $\left(2 N_{i_{m}}+1\right)$ requeridos, el punto $C_{m}$ se suele elegir como el punto medio de los centros $C_{1}$ y $C_{2}$. Por su parte, el comportamiento dispersor del tercer objeto $\mathrm{O}_{3}$ se representa por medio de su matriz de caracterización individual $D_{3}$; la cual relaciona el espectro de campo dispersado por dicho objeto con el espectro de campo incidente cuando ambos espectros se refieren al 
mismo punto, en este caso al centro $C_{3}$ del objeto. En esta ocasión, la reconstrucción exacta del campo incidente sobre el contorno del objeto $O_{3}$ requiere utilizar $2 N_{i_{3}}+1$ modos; con $N_{i_{3}}$ cumpliendo la condición $N_{i_{3}}>k \cdot a_{3}$, donde $a_{3}$ es el radio de la mínima circunferencia con centro en $C_{3}$ que contiene completamente al tercer objeto dispersor. En cuanto a la correcta reconstrucción de los campos que dispersan los objetos $O_{1}, O_{2}$ y $O_{3}$, recordando el capítulo 4, se requiere utilizar respectivamente $2 N_{d_{1}}+1,2 N_{d_{2}}+1$ y $2 N_{d_{3}}+1$ modos; debiéndose satisfacer los criterios $N_{d_{1}}>k \cdot a_{1}, N_{d_{2}}>k \cdot a_{2}$ y $N_{d_{3}}>k \cdot a_{3}$, donde los parámetros $a_{1}, a_{2}$ y $a_{3}$ representan los radios de dichos objetos (ver figuras 6.1 y 6.6 ).

De este modo, cumpliendo todos los criterios anteriormente expuestos para la correcta reconstrucción de los campos incidentes y dispersados, se consigue minimizar las dimensiones de todas las matrices que intervienen en el proceso de caracterización conjunta de los tres objetos dispersores.

Al igual que en el análisis de la dispersión electromagnética producida por dos objetos (recordar apartado 6.1.1), la solución al problema que nos ocupa (ver figura 6.6) se obtiene resolviendo dos situaciones complementarias. Inicialmente, se asume que la incidencia afecta tan sólo al elemento dispersor equivalente constituido por $O_{1}$ y $O_{2}$, y no al tercer objeto $O_{3}$. Tras las múltiples interacciones que tienen lugar entre todos los objetos dispersores, para resolver completamente esta primera situación deben calcularse los campos dispersados por el elemento equivalente y por el objeto $O_{3}$. A continuación, se supone que el campo incidente afecta tan sólo al tercer objeto $O_{3}$, y no al elemento dispersor equivalente. Como en la situación anterior, debido al acoplo electromagnético que se produce entre todos los objetos dispersores, la respuesta a este segundo supuesto requiere determinar los campos dispersados tanto por el elemento equivalente como por el objeto $\mathrm{O}_{3}$. La solución al problema original formado por los tres objetos dispersores se obtiene, aplicando superposición, mediante la suma de las respuestas correspondientes a las dos situaciones complementarias analizadas.

Con la intención de reducir cálculos, el espectro asociado al campo incidente se expresa de nuevo mediante una matriz identidad $I$ de tamaño $2 N_{i}+1$; en la cual cada columna, definida como el vector $E I_{p}$ en (6.14), representa una componente distinta del desarrollo modal incidente recogido en (6.1). Por tanto, tras resolver ante dicha incidencia el problema en cuestión, se obtienen tres matrices de caracterización conjunta; que representan el comportamiento dispersor de los objetos $O_{1}, O_{2}$ y $O_{3}$ teniendo en cuenta las interacciones múltiples que se producen entre todos ellos. Estas matrices, designadas respectivamente como $D_{1}^{(2)}, D_{2}^{(2)}$ y $D_{3}^{(2)}$, se construyen de igual manera que en $(6.17)$ con $2 N_{i}+1$ vectores columna; representando cada uno de ellos el espectro asociado al campo que dispersa el correspondiente objeto ante una incidencia de espectro $E I_{p}$. Es interesante reseñar que en la notación empleada para designar las matrices de caracterización, el subíndice determina el objeto caracterizado; mientras el superíndice indica el número de iteraciones requeridas en la obtención de la matriz. Así pues, en el 
ejemplo que nos ocupa, las matrices $D_{1}, D_{2}$ y $D_{3}$ designan las matrices de caracterización individual de cada uno de los tres objetos dispersores; no llevando superíndice debido a que se obtienen sin realizar ninguna iteración. Tras analizar el elemento equivalente, constituido por $O_{1}$ y $O_{2}$, el comportamiento dispersor de cada uno de estos objetos se representa respectivamente por su correspondiente matriz de caracterización conjunta $D_{1}^{(1)}$ y $D_{2}^{(1)}$; donde el valor 1 en el superíndice indica que se ha implementado una primera iteración, usando teoría de grafos [13], para evaluar el acoplo electromagnético entre dichos objetos. Finalmente, realizando una segunda iteración entre el elemento dispersor equivalente y el tercer objeto $O_{3}$, se calculan las ya mencionadas matrices de caracterización conjunta $D_{1}^{(2)}, D_{2}^{(2)}$ y $D_{3}^{(2)}$; las cuales definen el comportamiento dispersor de cada uno de los tres objetos considerados en el problema bajo análisis.

En primer lugar, se debe analizar pues el problema en el que el campo incidente, definido a través de su espectro $I$, afecta tan sólo al elemento dispersor equivalente. Ante dicha incidencia, se producen múltiples interacciones entre este elemento equivalente y el tercer objeto dispersor $O_{3}$; que en el límite, tras alcanzar una situación estable, quedan representadas por un campo incidente desconocido sobre el elemento integrado por los objetos $O_{1}$ y $O_{2}$. El espectro asociado a dicha incidencia, referido al punto $C_{m}$, se expresa mediante la matriz $E A$; que constituye precisamente la incógnita a resolver en este primer problema. Por tanto, sobre $O_{1}$ y $O_{2}$ inciden en régimen permanente dos campos con espectros asociados $E A$ e $I$; que se refieren respectivamente a los puntos $C_{m}$ y $C_{0}$. Con el objeto de poder utilizar las matrices de caracterización conjunta $D_{1}^{(1)}$ y $D_{2}^{(1)}$, que definen el comportamiento dispersor de los objetos $O_{1}$ y $O_{2}$, el espectro $I$ del campo incidente original debe referirse al centro $C_{m}$ del elemento equivalente. Para ello, debe emplearse una matriz de traslación de espectro incidente a incidente, denominada $T J_{m 0}$, cuyos elementos se calculan utilizando las coordenadas del punto $C_{m}$ en las expresiones $(6.3)$ y (6.4). De esta forma, el espectro de campo incidente original $T J_{m 0} \cdot I$ y el espectro incógnita $E A$, al quedar referidos ambos al punto $C_{m}$, se suman para expresar en términos espectrales la incidencia que existe sobre el elemento dispersor equivalente; ante la cual $O_{1}$ dispersa un campo de espectro $D_{1}^{(1)} \cdot\left(T J_{m 0} \cdot I+E A\right)$, y $O_{2}$ genera otro campo dispersado de espectro $D_{2}^{(1)} \cdot\left(T J_{m 0} \cdot I+E A\right)$. Ambos espectros de campo dispersado, centrados respectivamente en $C_{1}$ y $C_{2}$, se transforman haciendo uso de las correspondientes matrices de traslación de espectro emergente a incidente, designadas como $T_{31} \mathrm{y}$ $T_{32}$, en sendos espectros de campo incidente sobre $O_{3}$ referidos al centro $C_{3}$ de dicho objeto dispersor. Estos espectros se representan en términos matriciales mediante los productos $T_{31} \cdot D_{1}^{(1)} \cdot\left(T J_{m 0} \cdot I+E A\right)$ y $T_{32} \cdot D_{2}^{(1)} \cdot\left(T J_{m 0} \cdot I+E A\right)$. Por su parte, los elementos de las matrices $T_{31}$ y $T_{32}$ se calculan de forma análoga a los de la matriz $T_{21}$ obtenida en el apartado 6.1.1; es decir, utilizando las expresiones (6.5) y (6.6) con las coordenadas adecuadas en cada caso. Ante la suma de estos dos campos incidentes sobre $O_{3}$, dicho objeto genera un campo dispersado 


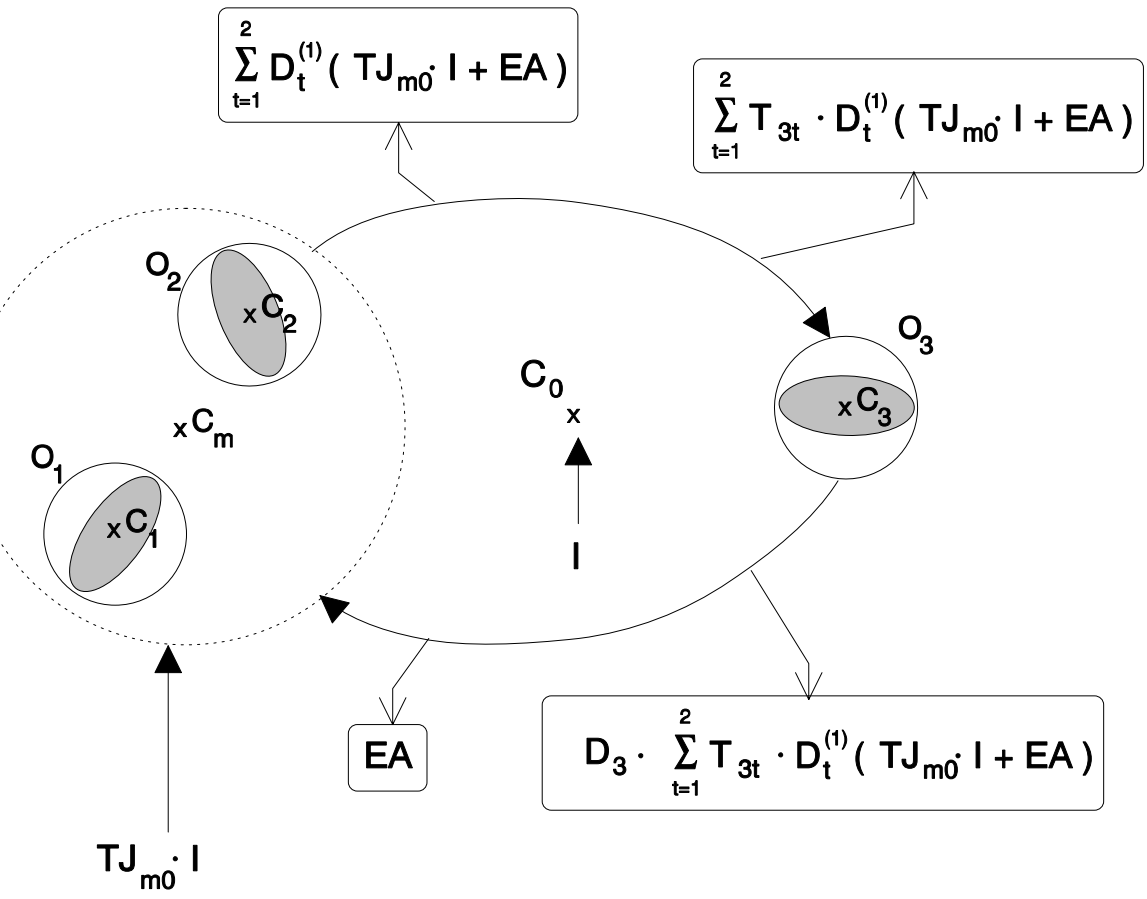

Figura 6.7: Diagrama completo de la realimentación entre $O_{1}, O_{2}$ y $O_{3}$ cuando el campo incidente original, de espectro $I$, afecta tan sólo a $O_{1}$ y $O_{2}$.

de espectro $D_{3} \cdot\left(\sum_{t=1}^{2} T_{3 t} \cdot D_{t}^{(1)}\right) \cdot\left(T J_{m 0} \cdot I+E A\right)$; que se ha obtenido utilizando lógicamente la matriz de caracterización individual $D_{3}$ del tercer objeto dispersor. A continuación, este espectro asociado al campo que dispersa $O_{3}$, referido al centro $C_{3}$ de dicho objeto, se transforma mediante la correspondiente matriz de traslación $T_{m 3}$ en un espectro de campo incidente sobre los objetos $O_{1}$ y $O_{2}$, centrado en el punto $C_{m}$, y de valor $T_{m 3} \cdot D_{3} \cdot\left(\sum_{t=1}^{2} T_{3 t} \cdot D_{t}^{(1)}\right) \cdot\left(T J_{m 0} \cdot I+E A\right)$. De nuevo, los elementos de la matriz $T_{m 3}$ se calculan, empleando las coordenadas apropiadas, según las expresiones (6.5) y (6.6). La totalidad de las operaciones descritas hasta este punto se recogen gráficamente en la figura 6.7. Finalmente, este último espectro de campo incidente sobre los objetos $O_{1}$ y $O_{2}$, referido al punto $C_{m}$, coincide con la definición del espectro incógnita $E A$; por lo que debe cumplirse la siguiente igualdad

$$
E A=T_{m 3} \cdot D_{3} \cdot\left(\sum_{t=1}^{2} T_{3 t} \cdot D_{t}^{(1)}\right) \cdot\left(T J_{m 0} \cdot I+E A\right)
$$

que permite encontrar el espectro incógnita $E A$ resolviendo conjuntamente una serie de sistemas de ecuaciones, expresados matricialmente del siguiente modo

$$
\left(I_{1}-T_{m 3} \cdot D_{3} \cdot\left(\sum_{t=1}^{2} T_{3 t} \cdot D_{t}^{(1)}\right)\right) \cdot E A=T_{m 3} \cdot D_{3} \cdot\left(\sum_{t=1}^{2} T_{3 t} \cdot D_{t}^{(1)}\right) \cdot T J_{m 0} \cdot I
$$


En concreto, la ecuación (6.24) representa $2 N_{i}+1$ sistemas de ecuaciones, tantos como posibles espectros de campo incidente se recogen en la matriz $I$; teniendo cada uno de los sistemas $2 N_{i_{m}}+1$ incógnitas. Resolviendo pues la ecuación matricial presente en (6.24), donde $I_{1}$ es la matriz identidad de tamaño $2 N_{i_{m}}+1$, se obtiene la solución al primero de los dos problemas complementarios.

Posteriormente, siguiendo el mismo razonamiento empleado en el análisis del caso anterior, se debe estudiar el segundo problema en el que el campo incidente, definido en términos espectrales por la matriz $I$, afecta tan sólo al tercer objeto dispersor. En dicha situación, se produce un acoplo electromagnético entre el objeto $O_{3}$ y el elemento dispersor equivalente constituido por $O_{1}$ y $O_{2}$; que se expresa en régimen estable como un campo incidente sobre $O_{3}$. El espectro asociado a esta incidencia, representado por la matriz $E B$, constituye la incógnita de este segundo problema. Implementando el conjunto de operaciones que se indican en la figura 6.8, análogas a las recogidas en la figura 6.7 para el supuesto anterior; y recordando la definición del espectro incógnita $E B$, se puede forzar el cumplimiento de la siguiente igualdad

$$
E B=\left(\sum_{t=1}^{2} T_{3 t} \cdot D_{t}^{(1)}\right) \cdot T_{m 3} \cdot D_{3} \cdot\left(T J_{30} \cdot I+E B\right)
$$

que permite obtener el espectro incógnita $E B$ tras resolver de forma conjunta una serie de sistemas de ecuaciones, expresados matricialmente como

$$
\left(I_{2}-\left(\sum_{t=1}^{2} T_{3 t} \cdot D_{t}^{(1)}\right) \cdot T_{m 3} \cdot D_{3}\right) \cdot E B=\left(\sum_{t=1}^{2} T_{3 t} \cdot D_{t}^{(1)}\right) \cdot T_{m 3} \cdot D_{3} \cdot T J_{30} \cdot I
$$

donde $I_{2}$ representa en este caso una matriz identidad de tamaño $2 N_{i_{3}}+1$. Concretamente, la ecuación matricial en (6.26) representa $2 N_{i}+1$ sistemas de ecuaciones, tantos como espectros de campo incidente considerados en la matriz $I$; presentando cada uno de dichos sistemas $2 N_{i_{3}}+1$ incógnitas. Resolviendo pues los sistemas de ecuaciones presentes en (6.26), se encuentra la solución al segundo de los dos problemas complementarios.

Utilizando los espectros $E A$ y $E B$, obtenidos tras resolver las ecuaciones (6.24) y (6.26); y aplicando superposición de las soluciones a los dos problemas complementarios, como en (6.13), se encuentran las siguientes expresiones para las matrices de caracterización conjunta de cada uno de los tres objetos dispersores

$$
\begin{aligned}
& D_{t}^{(2)}=D_{t}^{(1)} \cdot\left(T_{m 3} \cdot D_{3} \cdot\left(T J_{30} \cdot I+E B\right)+T J_{m 0} \cdot I+E A\right) \quad t \in[1,2] \\
& D_{3}^{(2)}=D_{3} \cdot\left(\left(\sum_{t=1}^{2} T_{3 t} \cdot D_{t}^{(1)}\right) \cdot\left(T J_{m 0} \cdot I+E A\right)+T J_{30} \cdot I+E B\right)
\end{aligned}
$$




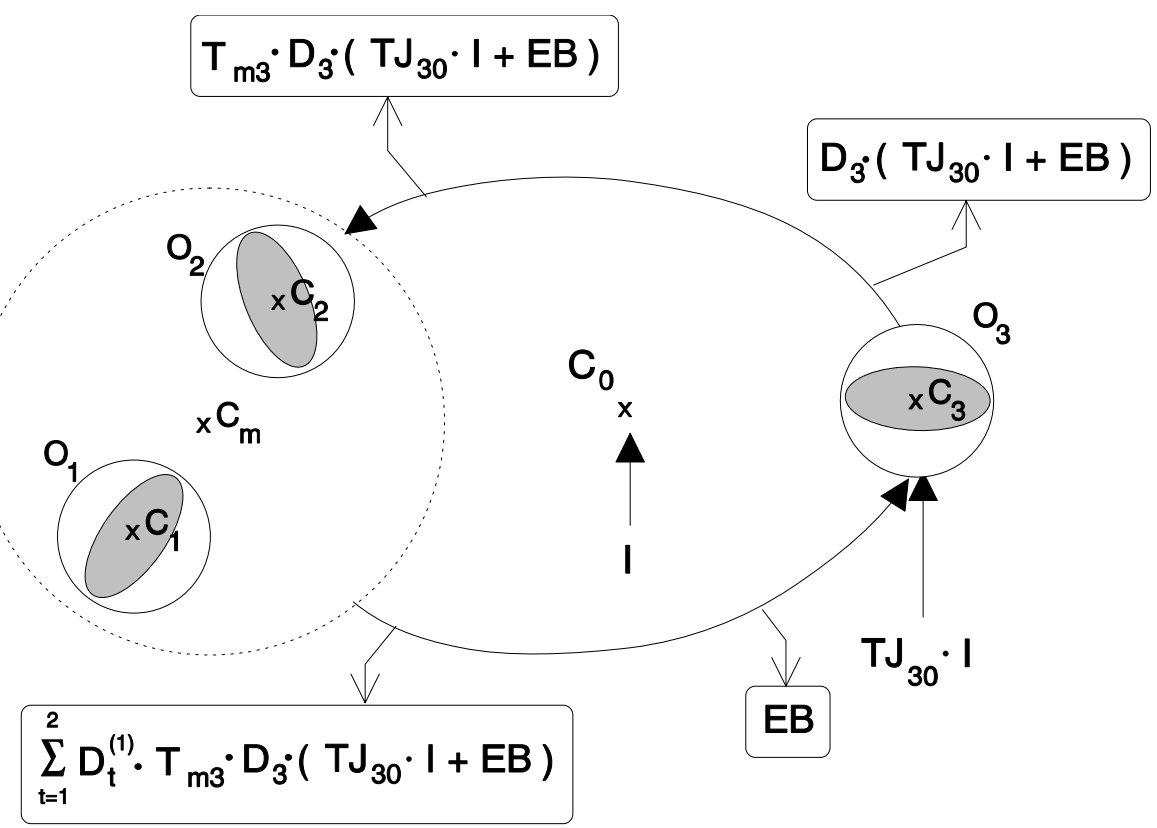

Figura 6.8: Diagrama completo de la realimentación entre $O_{1}, O_{2}$ y $O_{3}$ cuando el campo incidente original, de espectro $I$, afecta tan sólo a $\mathrm{O}_{3}$.

En resumen, la dispersión electromagnética producida por un sistema constituido por tres objetos dispersores, teniendo en cuenta las interacciones múltiples, se ha analizado en este apartado aplicando el mismo método descrito anteriormente para caracterizar la dispersión que producen dos objetos. Concretamente, el método basado en teoría de grafos [13] se ha particularizado al análisis del acoplo electromagnético que se origina entre un elemento dispersor equivalente, constituido por los dos primeros objetos caracterizados previamente empleando la misma técnica, y el tercer objeto dispersor. Utilizando este mismo algoritmo en sucesivas iteraciones, tal y como se describe a continuación, resulta posible caracterizar conjuntamente la dispersión que produce un número genérico $(N)$ de objetos dispersores.

\subsubsection{Generalización del Algoritmo a $N$ Objetos Disper- sores}

El algoritmo recién descrito para caracterizar de forma conjunta el comportamiento dispersor de tres objetos, el cual utiliza los resultados obtenidos previamente en el análisis del acoplo entre dos objetos, se puede generalizar al estudio de la dispersión electromagnética que produce un sistema formado por $N$ objetos dispersores. La aplicación de dicho algoritmo requiere, en principio, caracterizar individualmente cada uno de los $N$ objetos mediante sus respectivas matrices de 
caracterización $D_{1}, \ldots, D_{N}$; cuyo cálculo se recoge en el capítulo 4 . La estrategia a seguir en la caracterización conjunta de los $N$ objetos dispersores se basa en realizar sucesivas iteraciones; añadiendo en cada una de ellas un nuevo objeto y analizando a su vez, mediante el método basado en teoría de grafos [13], el acoplo producido entre dicho objeto y el grupo analizado en la iteración anterior. Por tanto, antes de abordar las distintas iteraciones se requiere una ordenación adecuada, tal y como se discute en la sección 6.3 (apartado 6.3.4), de los objetos bajo análisis; determinando de esta forma en que iteración resulta más adecuado incorporar cada nuevo objeto. Así pues, en la primera iteración se caracteriza el comportamiento dispersor de los dos primeros objetos mediante sus matrices de caracterización conjunta $D_{1}^{(1)}$ y $D_{2}^{(1)}$; obtenidas empleando el método descrito en el apartado 6.1.1. En una segunda iteración, haciendo uso de los resultados obtenidos en la iteración anterior, se resuelve el acoplo electromagnético que se produce entre los dos primeros objetos y el tercer objeto dispersor; obteniendo mediante el método deducido en el apartado 6.1.2 las matrices $D_{1}^{(2)}, D_{2}^{(2)}$ y $D_{3}^{(2)}$. Dichas matrices caracterizan de forma conjunta la dispersión producida por cada uno de los tres objetos. De igual manera, en la tercera iteración se obtienen las matrices de caracterización conjunta de los cuatro primeros objetos dispersores. Y así sucesivamente hasta completar $N-1$ iteraciones; tras las cuales, el acoplo electromagnético que se origina entre los $N$ objetos dispersores queda completamente analizado. Como consecuencia de la última iteración, se obtienen las matrices de caracterización conjunta $D_{t}^{(N-1)}$, con $t \in[1, \ldots, N]$; las cuales definen respectivamente el comportamiento dispersor de cada uno de los $N$ objetos en presencia del resto.

A continuación, se presentan los resultados obtenidos tras realizar la $i$-ésima iteración; en la que se caracteriza conjuntamente la dispersión producida por los $i+1$ primeros objetos. El método basado en teoría de grafos [13] se aplica en este caso, de igual manera que en el apartado 6.1 .2 para caracterizar el comportamiento dispersor de tres objetos, al análisis de las interacciones múltiples que se producen entre un elemento dispersor equivalente, constituido en el problema que nos ocupa por los $i$ primeros objetos, y el objeto $O_{i+1}$ incorporado en esta última iteración. El comportamiento dispersor del elemento equivalente se define mediante las matrices de caracterización conjunta $D_{t}^{(i-1)}$, con $t \in[1, \ldots, i]$; cuyo cálculo se realiza en la iteración anterior. Dichas matrices relacionan respectivamente el espectro de campo dispersado por el t-ésimo objeto, referido a su centro $C_{t}$, con el espectro de campo incidente referido al centro $C_{m}$ del elemento dispersor equivalente. De nuevo, el punto $C_{m}$ se escoge con la intención de minimizar el número de modos requeridos para reconstruir, con total exactitud, el campo incidente sobre todos y cada uno de los $i$ primeros objetos. Por su parte, la dispersión que origina el objeto $O_{i+1}$ se obtiene a partir de su matriz de caracterización individual $D_{i+1}$; que relaciona el espectro de campo dispersado por $O_{i+1}$ con el espectro de campo incidente cuando ambos espectros se refieren 
a un mismo punto, en esta ocasión al centro $C_{i+1}$ de dicho objeto. El análisis de la $i$-ésima iteración requiere descomponer el problema asociado en dos situaciones complementarias. En un primer supuesto, se asume que el campo incidente original afecta tan sólo al elemento dispersor equivalente, constituido por los $i$ primeros objetos dispersores; mientras que en un segundo caso, se asume que dicho campo incide tan sólo sobre el último objeto incorporado $O_{i+1}$. El espectro de este campo incidente, referido al centro $C_{0}$ del sistema formado por los $i+1$ objetos dispersores, se expresa mediante la matriz identidad $I$ de tamaño $2 N_{i}+1$; ya que de esta forma se consideran todas las posibles componentes del desarrollo modal incidente recogido en (6.1). En cuanto a la elección del punto $C_{0}$, al igual que en los apartados anteriores, el criterio seguido pretende minimizar el número de modos necesarios para reconstruir, de manera exacta, el campo incidente sobre el contorno de todos los objetos. La incógnita a resolver en cada uno de los dos problemas complementarios se expresa respectivamente mediante la matriz $E A$ y EB; cuyo significado es análogo al expuesto en el apartado 6.1.2 para el caso de tres objetos dispersores. Considerando pues las interacciones múltiples que se producen entre todos los objetos en ambas situaciones complementarias, tal y como se describe gráficamente en las figuras 6.7 y 6.8 para un caso más simple, es posible obtener las matrices incógnita $E A$ y $E B$ resolviendo los siguientes sistemas de ecuaciones; que se expresan matricialmente como

$$
\begin{gathered}
\left(I_{1}-T_{m i+1} \cdot D_{i+1} \cdot\left(\sum_{t=1}^{i} T_{i+1 t} \cdot D_{t}^{(i-1)}\right)\right) \cdot E A= \\
T_{m i+1} \cdot D_{i+1} \cdot\left(\sum_{t=1}^{i} T_{i+1 t} \cdot D_{t}^{(i-1)}\right) \cdot T J_{m 0} \cdot I \\
\left(I_{2}-\left(\sum_{t=1}^{i} T_{i+1 t} \cdot D_{t}^{(i-1)}\right) \cdot T_{m i+1} \cdot D_{i+1}\right) \cdot E B= \\
\left(\sum_{t=1}^{i} T_{i+1 t} \cdot D_{t}^{(i-1)}\right) \cdot T_{m i+1} \cdot D_{i+1} \cdot T J_{i+10} \cdot I
\end{gathered}
$$

donde $I_{1}$ e $I_{2}$ representan sendas matrices identidad con los tamaños correspondientes; mientras $T J_{m 0}$ y $T J_{i+10}$ son las matrices de traslación de espectro incidente centrado en $C_{0}$ a espectro incidente centrado respectivamente en $C_{m} \mathrm{y}$ $C_{i+1}$. Por su parte, $T_{i+1 t}$ designa una matriz de traslación de espectro emergente centrado en $C_{t}$ a espectro incidente centrado en $C_{i+1}$; y la matriz $T_{m i+1}$ permite transformar modos cilíndricos emergentes referidos al punto $C_{i+1}$ en modos cilíndricos incidentes referidos al punto $C_{m}$.

Una vez conocidas las matrices $E A$ y $E B$, tras resolver los sistemas de ecuaciones recogidos en (6.28), se debe aplicar superposición de las soluciones a los dos problemas complementarios analizados; para de esta forma encontrar la respuesta al problema original, en el que el campo incidente afecta a todos los objetos 
dispersores. Por tanto, las matrices de caracterización conjunta que definen el comportamiento dispersor de los $i+1$ primeros objetos, obtenidas tras implementar la $i$-ésima iteración, presentan el siguiente aspecto

$$
\begin{aligned}
D_{t}^{(i)}=D_{t}^{(i-1)} \cdot( & T_{m i+1} \cdot D_{i+1} \cdot\left(T J_{i+10} \cdot I+E B\right) \\
& \left.+T J_{m 0} \cdot I+E A\right) \quad t \in[1, \ldots, i] \\
D_{i+1}^{(i)}=D_{i+1} \cdot( & \left(\sum_{t=1}^{i} T_{i+1 t} \cdot D_{t}^{(i-1)}\right) \cdot\left(T J_{m 0} \cdot I+E A\right) \\
& \left.+T J_{i+10} \cdot I+E B\right)
\end{aligned}
$$

Es interesante reseñar que estos resultados son consecuentes con los deducidos en el apartado 6.1.2, presentados en (6.27), para el problema constituido por tres objetos dispersores. Por lo que respecta al tamaño de las matrices $D_{t}^{(i)}$, con $t \in[1, \ldots, i+1]$, sus dimensiones son respectivamente $2 N_{d_{t}}+1 \times 2 N_{i}+1$; donde $2 N_{d_{t}}+1$ representa el número de modos a utilizar en la reconstrucción del campo dispersado por el $t$-ésimo objeto, y $2 N_{i}+1$ el número de modos necesarios para reconstruir el campo incidente sobre los $i+1$ objetos analizados. Debido al criterio seguido en la elección de los puntos a los que se refieren los distintos espectros utilizados, las dimensiones de todas las matrices involucradas en las iteraciones presentan el mínimo valor posible en cada caso; lo que reduce considerablemente el esfuerzo computacional asociado al método propuesto.

En conclusión, este último apartado introduce un nuevo algoritmo recursivo, basado en teoría de grafos [13], que permite analizar completamente la dispersión electromagnética producida por un número genérico $(N)$ de objetos dispersores. Una de las principales ventajas asociadas a este método consiste en que su complejidad no crece con el número de objetos dispersores presentes. Además, una vez resuelto el problema bajo análisis se tiene para cada objeto dispersor una matriz de caracterización conjunta; la cual permite obtener fácilmente la dispersión que produce dicho objeto, teniendo en cuenta la presencia del resto, ante cualquier posible incidencia.

\subsection{Refinamiento del Algoritmo Recursivo para la Caracterización Conjunta de Grupos de Objetos Dispersores}

El algoritmo recursivo descrito en la sección 6.1 permite analizar numerosos problemas de dispersión electromagnética producida por múltiples objetos dispersores. Dicho algoritmo incorpora en cada iteración un nuevo objeto dispersor 
al grupo caracterizado de igual manera en la iteración anterior; analizando mediante teoría de grafos [13] la presencia del nuevo objeto. Ahora bien, existen problemas constituidos por múltiples objetos dispersores que se encuentran agrupados; en los que cada grupo consta de varios objetos próximos entre sí, y a su vez los grupos se encuentran alejados unos de otros. Por ejemplo, éste es el caso de las antenas multireflectoras integradas por dos reflectores grandes en términos eléctricos. Un análisis preciso y eficiente de dichos sistemas requiere segmentar previamente el contorno de cada reflector en objetos más pequeños; estudiando a continuación las interacciones múltiples que se originan entre dichos objetos. Lógicamente, los objetos que constituyen cada reflector están próximos entre sí, y a su vez alejados de aquellos pertenecientes al otro reflector. En este tipo de situaciones, no resulta posible aplicar el citado algoritmo recursivo; pues algunas de las operaciones de traslación de espectro emergente a incidente, requeridas en ciertas iteraciones del algoritmo, incumplen la restricción asociada a la primera versión del Teorema de Adición para las Funciones de Hankel (ver apéndice E). Una discusión detallada de esta limitación del algoritmo recursivo se presenta en la sección 6.3 (apartado 6.3.1).

La solución a este tipo de problemas, constituidos por agrupaciones de objetos, se obtiene aplicando un nuevo algoritmo recursivo; que permite caracterizar conjuntamente grupos de objetos dispersores. Este nuevo algoritmo, como se verá a continuación, no es mas que un simple refinamiento de aquél presentado en la sección 6.1 para caracterizar conjuntamente un número genérico de objetos dispersores. La aplicación del nuevo algoritmo requiere previamente caracterizar de forma conjunta, siguiendo el algoritmo recursivo descrito en la sección 6.1 (apartado 6.1.3), el comportamiento dispersor de los objetos pertenecientes a cada grupo. Esta operación debe realizarse, por separado, para cada uno de los grupos que integran el problema bajo análisis. La estrategia del nuevo algoritmo recursivo, a diferencia del anterior, consiste en incorporar en cada iteración un nuevo grupo de objetos dispersores; y a su vez analizar, mediante el método basado en teoría de grafos [13], las interacciones múltiples que se producen entre el nuevo grupo y el resto de grupos caracterizados en la iteración anterior. Al igual que el anterior algoritmo requiere una ordenación adecuada de los objetos bajo análisis, el nuevo algoritmo recursivo precisa también de una correcta ordenación de todas las agrupaciones de objetos; la cual determina en qué iteración debe incorporarse cada nuevo grupo de objetos. Por tanto, en una primera iteración se caracteriza conjuntamente el comportamiento dispersor de los dos primeros grupos de objetos. El método utilizado en dicha caracterización, basado en teoría de grafos [13], es el mismo empleado en el análisis de la $i$-ésima iteración del anterior algoritmo (ver apartado 6.1.3); salvo que el segundo elemento dispersor está constituido en este caso por un grupo de objetos, y no por un solo objeto dispersor. En una segunda iteración, usando de nuevo el mismo método basado en teoría de grafos [13], se analizan las interacciones múltiples que se producen entre los 
dos primeros grupos y el tercer grupo de objetos dispersores. Este análisis asume que las dos primeras agrupaciones de objetos constituyen un elemento dispersor equivalente; cuyo comportamiento se define mediante los resultados obtenidos en la iteración anterior. Aplicando sucesivamente este nuevo algoritmo recursivo, se alcanza una última iteración; en la cual, tras incorporar el último grupo de objetos dispersores, se caracteriza conjuntamente la dispersión electromagnética producida por todos los grupos en los que se distribuyen, de forma adecuada, los objetos del problema bajo estudio.

A continuación, se presentan los resultados correspondientes al análisis de las interacciones múltiples que se producen entre dos grupos de objetos dispersores, constituidos respectivamente por $M_{1}$ y $M_{2}$ objetos. Dicha situación, recogida gráficamente en la figura 6.9, representa una iteración genérica del nuevo algoritmo recursivo. La utilización en dicho problema del método basado en teoría de grafos [13] requiere caracterizar previamente, de forma conjunta, los objetos de cada grupo. Así pues, tras aplicar las correspondientes iteraciones, el comportamiento dispersor de cada uno de los objetos del primer grupo se representa respectivamente por las matrices de caracterización conjunta $D_{t}^{1}$, con $t \in\left[1, \ldots, M_{1}\right]$; donde el valor 1 en el superíndice indica que dichas matrices corresponden a los objetos del primer grupo. Cada una de las matrices $D_{t}^{1}$ relaciona pues el espectro de campo dispersado por el $t$-ésimo objeto, referido al centro $C_{t}^{1}$ de dicho objeto (ver figura 6.9), con el espectro de campo incidente centrado en el punto $C_{m_{1}}$. La elección del punto $C_{m_{1}}$ se escoge normalmente, como puede apreciarse en la figura 6.9 , en el punto medio de los centros $C_{t}^{1}$ de todos los objetos pertenecientes al primer grupo; para de esta forma minimizar el número de modos $\left(2 N_{i_{1}}+1\right)$ necesarios en la correcta reconstrucción del campo incidente sobre los $M_{1}$ objetos de dicho grupo. Por su parte, los objetos del segundo grupo quedan caracterizados mediante sus respectivas matrices de caracterización conjunta $D_{t}^{2}$, con $t \in\left[1, \ldots, M_{2}\right]$; donde el valor 2 en el superíndice hace referencia en este caso al segundo grupo de objetos. En lo referente a las matrices $D_{t}^{2}$, éstas permiten obtener respectivamente el espectro de campo dispersado por el $t$-ésimo objeto del segundo grupo, referido al centro $C_{t}^{2}$ de dicho objeto (ver figura 6.9 ), a partir del espectro de campo incidente centrado en el punto $C_{m_{2}}$. El punto $C_{m_{2}}$ se posiciona de manera usual, tal y como se observa en la figura 6.9, en el punto medio de los centros $C_{t}^{2}$ de todos los objetos que integran el segundo grupo; con el objeto de minimizar nuevamente el número de modos $\left(2 N_{i_{2}}+1\right)$ requeridos para reconstruir, con total exactitud, la incidencia existente sobre los $M_{2}$ objetos de este segundo grupo.

Una vez caracterizados los dos elementos dispersores equivalentes, el método basado en teoría de grafos [13] se utiliza para resolver dos problemas complementarios. En uno de ellos se asume que el campo incidente afecta tan sólo al primer grupo de objetos, y en el otro se supone que la incidencia afecta únicamente al segundo grupo. En realidad, el campo incidente está constituido por todas las 


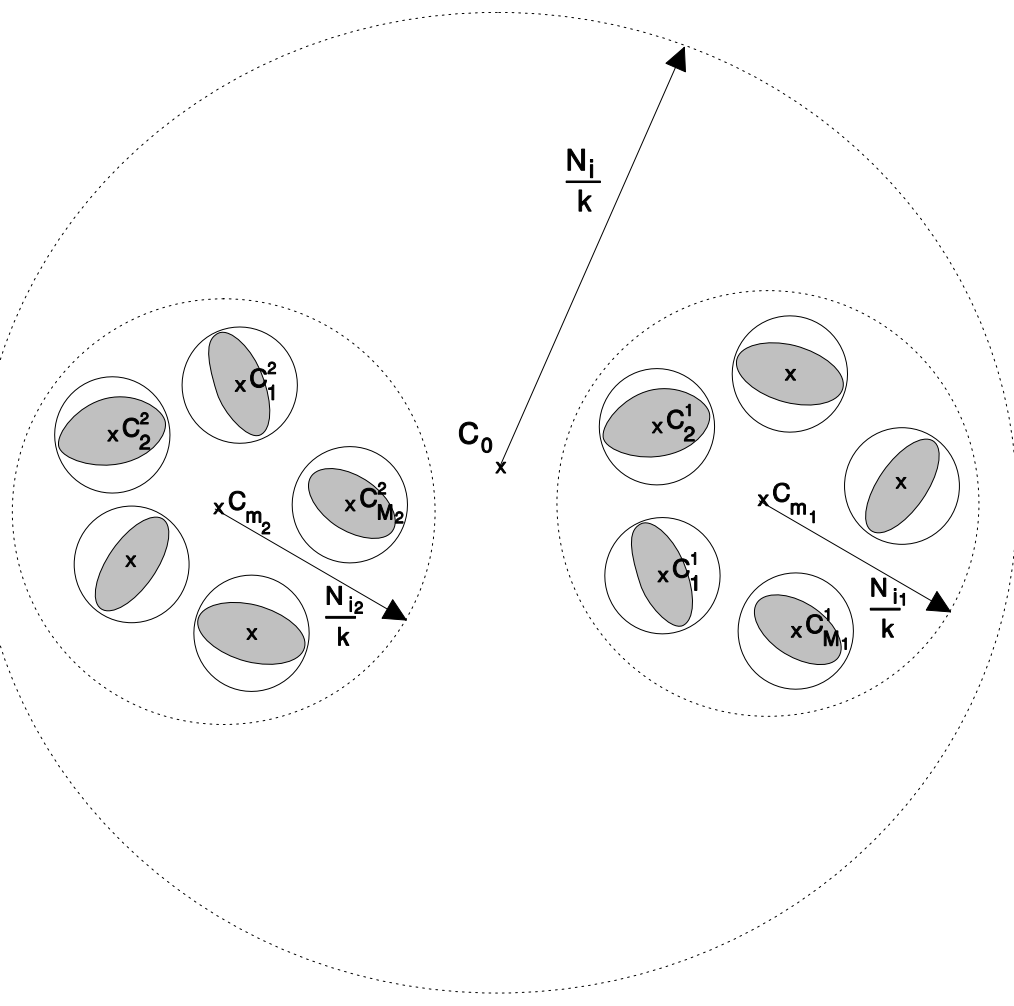

Figura 6.9: Esquema general de un problema de acoplo electromagnético entre dos grupos de dispersores.

posibles componentes, con amplitud unidad, del desarrollo modal incidente recogido en (6.1); lo cual se expresa en términos espectrales mediante una matriz identidad $I$ de tamaño $2 N_{i}+1$. Todos estos espectros se refieren al punto $C_{0}$ (ver figura 6.9); cuya posición se elige normalmente en el punto medio de los centros de todos los objetos analizados en la presente iteración. De esta forma, se pretende minimizar el número de modos $\left(2 N_{i}+1\right)$ requeridos para reconstruir, de manera exacta, el campo incidente sobre todos los objetos. La resolución de cada uno de los dos problemas complementarios requiere determinar una matriz incógnita, designada respectivamente como $E A$ y $E B$. En cada problema, dicha matriz representa en términos espectrales la incidencia sobre el correspondiente grupo de objetos; que se origina debido a las múltiples interacciones entre todos los objetos. Al haber caracterizado por un lado los $M_{1}$ objetos del primer grupo, y por otro los $M_{2}$ objetos del segundo grupo, el acoplo que se produce en cada problema entre ambos grupos puede resolverse mediante teoría de grafos [13], de manera similar a como se recoge gráficamente en las figuras 6.4 y 6.5 para el caso de dos objetos dispersores, recordando que el comportamiento dispersor de cada grupo se define en términos de las correspondientes matrices de caracterización conjunta. Consecuentemente, las matrices incógnita $E A$ y $E B$ se obtienen 
resolviendo los siguientes sistemas de ecuaciones, análogos a los presentados en (6.28) para cuando se incorporaba un solo objeto en cada iteración; y expresados matricialmente como

$$
\begin{gathered}
\left(I_{1}-\left(\sum_{t=1}^{M_{2}} T_{m_{1} t} \cdot D_{t}^{2}\right) \cdot\left(\sum_{t=1}^{M_{1}} T_{m_{2} t} \cdot D_{t}^{1}\right)\right) \cdot E A= \\
\left(\sum_{t=1}^{M_{2}} T_{m_{1} t} \cdot D_{t}^{2}\right) \cdot\left(\sum_{t=1}^{M_{1}} T_{m_{2} t} \cdot D_{t}^{1}\right) \cdot T J_{m_{1} 0} \cdot I \\
\left(I_{2}-\left(\sum_{t=1}^{M_{1}} T_{m_{2} t} \cdot D_{t}^{1}\right) \cdot\left(\sum_{t=1}^{M_{2}} T_{m_{1} t} \cdot D_{t}^{2}\right)\right) \cdot E B= \\
\left(\sum_{t=1}^{M_{1}} T_{m_{2} t} \cdot D_{t}^{1}\right) \cdot\left(\sum_{t=1}^{M_{2}} T_{m_{1} t} \cdot D_{t}^{2}\right) \cdot T J_{m_{2} 0} \cdot I
\end{gathered}
$$

donde $I_{1}$ e $I_{2}$ designan sendas matrices identidad de tamaños $2 N_{i_{1}}+1$ y $2 N_{i_{2}}+1$; mientras $T J_{m_{1} 0}$ y $T J_{m_{2} 0}$ son las matrices de traslación de espectro incidente centrado en $C_{0}$ a espectro incidente centrado respectivamente en $C_{m_{1}}$ y $C_{m_{2}}$. Por su parte, $T_{m_{1} t}$ representa una matriz de traslación de espectro emergente centrado en $C_{t}^{2}$ a espectro incidente centrado en $C_{m_{1}}$; y $T_{m_{2} t}$ define una matriz que transforma modos cilíndricos emergentes referidos al punto $C_{t}^{1}$ en modos cilíndricos incidentes referidos al punto $C_{m_{2}}$.

Una vez conocidas las matrices $E A$ y $E B$, tras resolver los sistemas de ecuaciones recogidos en (6.30), se calcula la solución al problema original aplicando superposición de las respuestas obtenidas en cada una de las dos situaciones consideradas. De esta forma, se determinan las siguientes expresiones para las matrices de caracterización conjunta $D_{i}$, con $i \in\left[1, \ldots, M_{1}+M_{2}\right]$; que definen respectivamente el comportamiento dispersor de los objetos del primer y segundo grupo considerando las interacciones múltiples entre todos los objetos

$$
\begin{aligned}
D_{i}= & D_{i}^{1} \cdot\left(\left(\sum_{t=1}^{M_{2}} T_{m_{1} t} \cdot D_{t}^{2}\right) \cdot\left(T J_{m_{2} 0} \cdot I+E B\right)\right. \\
& \left.+T J_{m_{1} 0} \cdot I+E A\right) \quad i \in\left[1, \ldots, M_{1}\right] \\
D_{i}= & D_{i-M_{1}}^{2} \cdot\left(\left(\sum_{t=1}^{M_{1}} T_{m_{2} t} \cdot D_{t}^{1}\right) \cdot\left(T J_{m_{1} 0} \cdot I+E A\right)\right. \\
& \left.+T J_{m_{2} 0} \cdot I+E B\right) \quad i \in\left[M_{1}+1, \ldots, M_{1}+M_{2}\right]
\end{aligned}
$$

donde las dimensiones de las matrices $D_{i}$ son $2 N_{d_{i}}+1 \times 2 N_{i}+1$; representando $2 N_{d_{i}}+1$ el número de modos con los que se reconstruye correctamente, fuera de la región con fuentes asociada al i-ésimo objeto, el campo que dispersa precisamente 
dicho objeto. Como se ha comentado anteriormente, $2 N_{i}+1$ indica el número de modos necesarios en la correcta reconstrucción del campo incidente sobre los $M_{1}+M_{2}$ objetos caracterizados.

Lógicamente, si el segundo grupo de objetos está constituido por un único objeto dispersor $\left(M_{2}=1\right)$, las expresiones recién presentadas en (6.31) se transforman directamente en aquéllas recogidas en (6.29); que se obtuvieron empleando el algoritmo descrito en la sección 6.1 (apartado 6.1.3) para incorporar un nuevo objeto dispersor en cada iteración.

Tras caracterizar conjuntamente a los dos grupos de objetos dispersores, se puede considerar que los $M_{1}+M_{2}$ objetos forman parte de un mismo grupo; al cual se le puede añadir en la siguiente iteración un solo objeto, cuyo análisis debiera realizarse siguiendo el algoritmo descrito en la sección 6.1 (apartado 6.1.3), o bien se le puede incorporar un nuevo grupo de objetos dispersores, debiendo emplear entonces la versión refinada del algoritmo recursivo descrita en esta sección.

A modo de síntesis, en la presente sección se ha introducido un nuevo algoritmo recursivo; que, a diferencia del presentado en la sección 6.1, permite incorporar más de un objeto dispersor en cada iteración. Este nuevo algoritmo, pensado realmente para añadir en cada iteración un nuevo grupo constituido por un número genérico de objetos dispersores, resuelve algunos problemas en los que el algoritmo recursivo original incumple la restricción asociada a la primera versión del Teorema de Adición para las Funciones de Hankel; por ejemplo, en el análisis de sistemas multireflectores y de otras situaciones problemáticas que se mencionan en la próxima sección. Finalmente, tal y como se ha podido comprobar durante la presentación del nuevo algoritmo recursivo para grupos de objetos, éste no es más que una simple versión refinada de aquél descrito originalmente en la sección 6.1; recogiendo por tanto esta nueva versión todas las ventajas comentadas anteriormente para el algoritmo recursivo original.

\subsection{Limitaciones en la Caracterización Conjun- ta de Múltiples Objetos Dispersores y Solu- ciones}

El algoritmo recursivo descrito inicialmente en la sección 6.1, junto con su versión refinada ofrecida en la sección 6.2 , permiten obtener la dispersión electromagnética que producen múltiples objetos dispersores en la gran mayoría de situaciones. No obstante, la propia estrategia de análisis seguida por la correspondiente versión del algoritmo recursivo puede provocar errores, caso de no tomar las debidas precauciones, en la resolución de ciertos problemas de dispersión múltiple.

Básicamente, se han identificado tres posibles situaciones en las que una aplicación indebida del algoritmo recursivo conduce a resultados erróneos. En los 
tres primeros apartados de esta sección, se plantean respectivamente estas limitaciones del algoritmo recursivo; y a su vez, se describen las acciones que deben adoptarse para que el algoritmo genere resultados correctos. En cada apartado, tras describir de forma genérica la correspondiente limitación del algoritmo recursivo y su posible solución, se resuelven casos prácticos en los que se presenta la problemática previamente estudiada.

Las limitaciones del algoritmo recursivo, así como las correspondientes soluciones, son igualmente válidas para cualquiera de las dos posibles versiones del algoritmo recursivo descritas en el presente capítulo. Con el objeto de no perder generalidad, las citadas situaciones problemáticas y las acciones preventivas a tomar para evitarlas se describen en el ámbito de un mismo problema, recogido gráficamente en la figura 6.9 y constituido por dos grupos de objetos dispersores. Ambos grupos han sido caracterizados previamente por separado, utilizando la versión del algoritmo recursivo presentada en la sección 6.1 (apartado 6.1.3), mediante las matrices de caracterización conjunta de sus respectivos objetos. La problemática estudiada en los próximos apartados surge al analizar las interacciones múltiples que se producen entre los dos grupos; para lo cual se utiliza la última versión del algoritmo recursivo recogida en la sección 6.2. Ahora bien, aunque la discusión de la problemática detectada se centra en la versión del algoritmo recursivo que incorpora un grupo de objetos en cada iteración; los resultados de dicha discusión son directamente trasladables a la versión original del algoritmo recursivo, que añade un solo objeto en cada iteración, asumiendo simplemente que el segundo grupo de objetos consta de un único objeto dispersor $\left(M_{2}=1\right)$.

Tras plantear las distintas situaciones críticas con las que puede encontrarse el algoritmo recursivo descrito en este capítulo, y encontrar las posibles soluciones que deben adoptarse para obtener resultados correctos en su aplicación, se discute en un último apartado la posibilidad de automatizar las decisiones a tomar en la aplicación del citado algoritmo recursivo. A su vez, en dicho apartado se presentan diversos criterios prácticos que resultan útiles para poder resolver correctamente, de forma computacional, un gran número de situaciones; entre las que se incluyen algunas de las descritas en esta sección, cuyo análisis correcto requiere observar ciertas acciones preventivas.

\subsubsection{Agrupación de Objetos Dispersores}

La primera situación problemática, que provoca errores al aplicar el algoritmo recursivo, se produce cuando existe algún objeto de un grupo con su centro situado dentro de la mínima circunferencia que contiene a todos los objetos del otro grupo. En este caso, debido a la restricción que presenta la primera versión del Teorema de Adición para las Funciones de Hankel (ver apéndice E), el campo dispersado por el anterior objeto no se reconstruye correctamente en todo el contorno de algunos objetos del otro grupo; produciéndose, a partir de ese mo- 


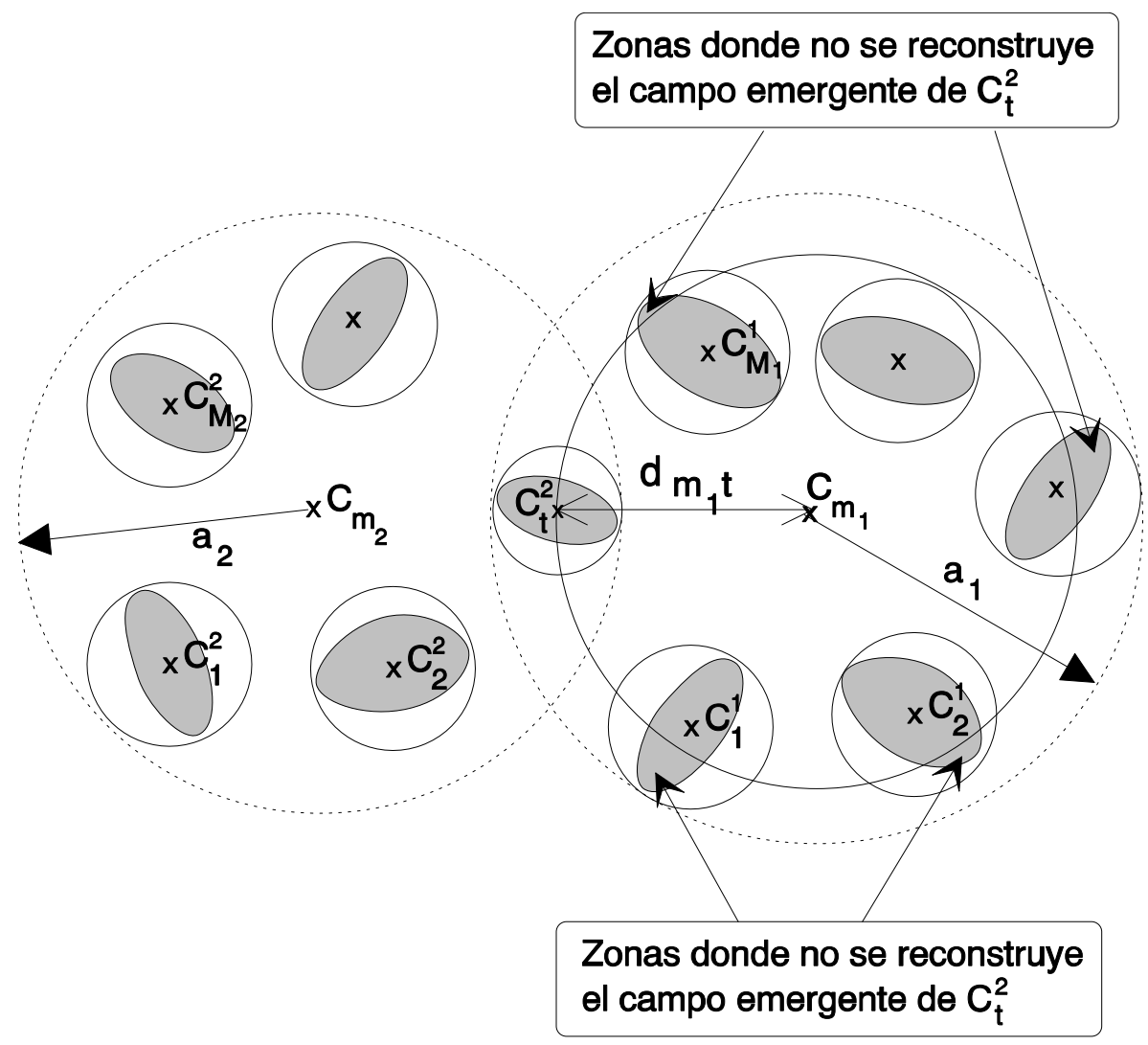

Figura 6.10: Diagrama explicativo de la primera situación problemática que provoca errores en la aplicación del algoritmo recursivo.

mento, errores en los resultados obtenidos mediante la aplicación del mencionado algoritmo recursivo.

La situación recién descrita se esquematiza gráficamente en la figura 6.10; donde se observa que el centro $C_{t}^{2}$ del tésimo objeto del segundo grupo se halla dentro de la mínima circunferencia, con centro en el punto $C_{m_{1}}$, que contiene a todos los objetos del primer grupo. La elección del punto $C_{m_{1}}$, al que se refiere el espectro de campo incidente al primer grupo, se realiza con la intención de minimizar el número de modos necesarios en la correcta reconstrucción del campo incidente sobre el contorno de todos los objetos del primer grupo; tal y como se ha explicado anteriormente en la sección 6.2. La razón por la que debe evitarse esta primera situación se basa en la reconstrucción incorrecta, sobre algunas partes del contorno de ciertos objetos del primer grupo, del campo dispersado por el t-ésimo objeto del segundo grupo. En el método basado en teoría de grafos [13], aplicado en la sección 6.2 a la caracterización conjunta de grupos de objetos dispersores, es necesario en un cierto momento transformar el espectro de campo emergente, referido al punto $C_{t}^{2}$, en espectro de campo incidente centrado en el 
punto $C_{m_{1}}$; para lo cual se utiliza la matriz de traslación de espectro emergente a incidente denominada $T_{m_{1} t}$. Ahora bien, dicha traslación (recordar apéndice $\mathrm{E}$ ) tan sólo permite reconstruir correctamente el campo incidente al primer grupo de objetos en el interior de una circunferencia, con centro en el punto $C_{m_{1}}, y$ de radio $d_{m_{1} t}$; representando $d_{m_{1} t}$ la distancia que existe entre los centros $C_{m_{1}}$ y $C_{t}^{2}$ (ver figura 6.10). En estas circunstancias, tal y como puede observarse en la figura 6.10 , siempre existen partes del contorno de ciertos objetos del primer grupo que están situadas fuera de la circunferencia límite de radio $d_{m_{1} t}$; razón por la cual, el campo incidente no se reconstruye correctamente en el contorno completo de todos los objetos del primer grupo. A partir de este instante, los resultados que proporciona el algoritmo recursivo son incorrectos; resultando por tanto imposible la caracterización conjunta de los objetos pertenecientes a los dos grupos considerados.

Antes de abordar la solución al problema genérico descrito en la figura 6.10 , conviene considerar una situación problemática más simple que aparece con gran frecuencia. Dicha situación surge cuando se intenta caracterizar agrupaciones de objetos, lo bastante separadas como para no incurrir en la anterior situación, mediante el algoritmo recursivo descrito en la sección 6.1 (apartado 6.1.3); que incorpora un solo objeto en cada iteración. Un ejemplo de este posible problema se recoge en la figura 6.11 (apartado a); donde se observa la existencia de dos grupos, ampliamente separados el uno del otro, constituidos respectivamente por tres objetos dispersores. La caracterización conjunta de los tres primeros objetos, centrados en los puntos $C_{1}, C_{2}$ y $C_{3}$ de la figura 6.11 (apartado a), mediante la incorporación al grupo de un nuevo objeto en cada iteración no incumple, en ningún momento, la mencionada restricción de la primera versión del Teorema de Adición para las Funciones de Hankel. A su vez, el análisis del acoplo que se produce en la siguiente iteración entre el cuarto objeto dispersor, centrado en el punto $C_{4}$, y el grupo de tres objetos previamente caracterizados resulta igualmente correcto. Sin embargo, al introducir posteriormente el quinto objeto dispersor, con centro en el punto $C_{5}$, e intentar estudiar el acoplo con el grupo de cuatro objetos analizado anteriormente, se observa que el centro $\left(C_{5}\right)$ del quinto objeto dispersor se encuentra situado dentro de la mínima circunferencia, centrada en el punto $C_{m}$, que contiene a los cuatro primeros objetos dispersores (ver apartado a en la figura 6.11). Por tanto, procediendo de esta forma, se incurre en el mismo tipo de error discutido en este primer apartado. La solución, en el caso concreto que nos ocupa, requiere analizar separadamente los dos grupos de tres objetos dispersores; aplicando en el análisis conjunto de los objetos pertenecientes a cada grupo el algoritmo recursivo presentado en la sección 6.1 (apartado 6.1.3). A continuación, empleando la versión del algoritmo introducida en la sección 6.2, se debe caracterizar de forma conjunta en una última iteración los dos grupos considerados. Esta estrategia, como se desprende de la figura 6.11 (apartado b), no conduce a ninguna situación problemática similar a la descrita gráficamente en 


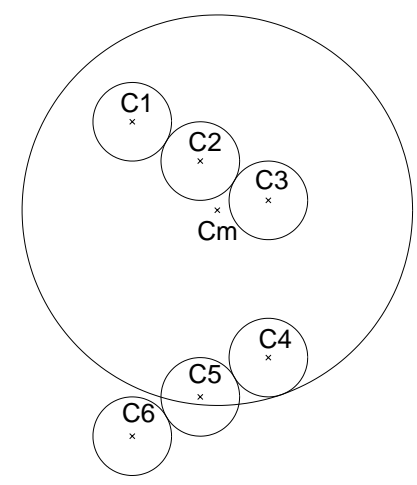

a)

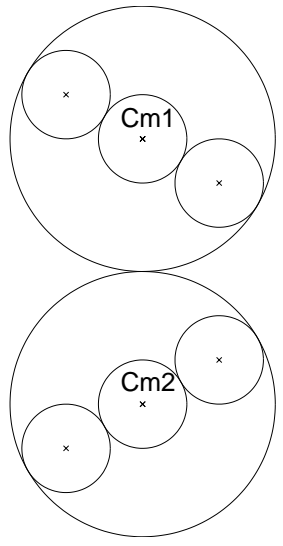

b)

Figura 6.11: Ejemplo práctico de la primera limitación del algoritmo recursivo que incorpora un solo objeto en cada iteración. Presentación del problema en a), y posible solución al mismo en b).

la figura 6.10. En general, aquellos problemas constituidos por agrupaciones de objetos dispersores que estén suficientemente alejadas entre sí, siempre y cuando no se incurra en la limitación recogida en la figura 6.10, deben analizarse siguiendo la misma estrategia utilizada en la resolución del problema mostrado en la figura 6.11; es decir, caracterizando por separado cada grupo de objetos mediante la versión original del algoritmo recursivo (ver apartado 6.1.3), y finalmente utilizando la versión refinada de dicho algoritmo para analizar conjuntamente la dispersión producida por agrupaciones de objetos (ver sección 6.2).

Retornando al problema genérico descrito en la figura 6.10 , se observa que el grado de proximidad entre los dos grupos de objetos dispersores impide una correcta aplicación del algoritmo presentado en la sección 6.2. Dicha razón, unida a la elección del punto $C_{m_{1}}$ al que se refiere el espectro de campo incidente al primer grupo de objetos, causan que el centro de algún objeto del segundo grupo se encuentre situado dentro de la mínima circunferencia, centrada en el punto $C_{m_{1}}$, que contiene a todos los objetos del primer grupo; tal y como se recoge en la figura 6.10. Concretamente, el punto $C_{m_{1}}$ se escoge (recordar sección 6.2) con el objeto de minimizar el radio de la citada circunferencia con centro en dicho punto; y, por consiguiente, reducir el número de modos requeridos en la correcta reconstrucción del campo incidente al primer grupo de objetos. Ahora bien, sacrificando este criterio, en el ejemplo mostrado en la figura 6.10 es posible situar el punto $C_{m_{1}}$ en una posición adecuada; de manera que exista una circunferencia, con centro en el punto $C_{m_{1}}$, que contenga a todos los objetos del 


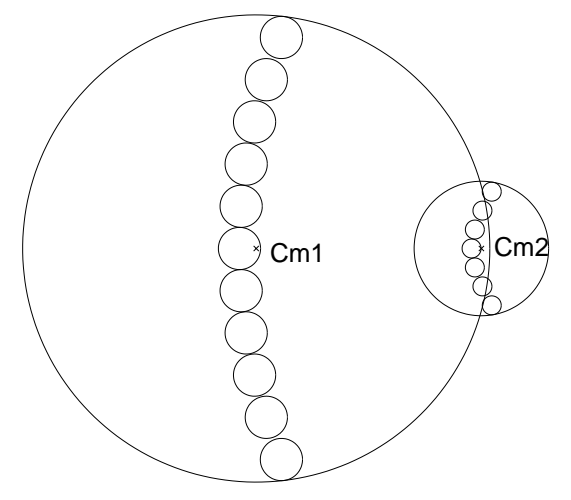

a)

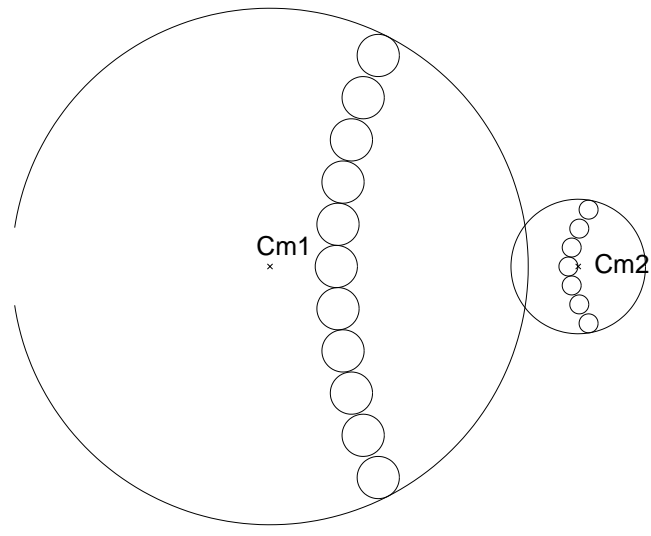

b)

Figura 6.12: Ejemplo práctico de la primera limitación del algoritmo recursivo que permite analizar agrupaciones de objetos dispersores. Presentación del problema en a), y posible solución al mismo en b).

primer grupo sin incluir el centro de ningún objeto perteneciente al segundo grupo. De esta forma, el problema de la figura 6.10 puede resolverse correctamente siguiendo el algoritmo descrito en la sección 6.2 ; aunque, debido al mayor número de modos requeridos en la correcta reconstrucción del campo incidente sobre los objetos del primer grupo, el esfuerzo computacional que supone la aplicación del citado algoritmo será lógicamente mayor. Un ejemplo práctico de la situación problemática comentada en este apartado se muestra en la figura 6.12; donde se tienen dos grupos de objetos dispersores, que conforman respectivamente un reflector parabólico y un reflector hiperbólico, integrantes ambos de una estructura multireflectora conocida como antena Cassegrain. Los objetos dispersores, debido a su proximidad espacial, se han separado en dos grupos; tal y como se indica gráficamente en la figura 6.12 (apartado a). A su vez, en dicho apartado de la figura 6.12 se observa también que los puntos $C_{m_{1}}$ y $C_{m_{2}}$, a los que se refieren respectivamente los espectros de campo incidente a cada grupo, se eligen como la media aritmética de los centros de los objetos dispersores del grupo correspondiente; con la intención de minimizar el número de modos necesarios para reconstruir, con total exactitud, el campo incidente a cada grupo. Como consecuencia de esta elección, y debido al grado de proximidad entre los dos grupos, existen centros de algunos elementos del reflector hiperbólico que están situados en el interior de la mínima circunferencia, con centro en el punto $C_{m_{1}}$, que contiene a todos los objetos pertenecientes al reflector parabólico (ver apartado a en la figura 6.12); incumpliéndose por tanto la restricción espacial impuesta por la 
primera versión del Teorema de Adición para las Funciones de Hankel (recordar apéndice $\mathrm{E}$ ). Sin embargo, desplazando de forma adecuada el punto $C_{m_{1}}$ hacia la izquierda (ver apartado b en la figura 6.12), se consigue que exista una circunferencia, centrada en dicho punto, que contiene a todos los elementos de la parábola sin incluir ningún centro de los elementos pertenecientes a la hipérbola; permitiendo pues analizar correctamente el problema de acoplo electromagnético entre ambos reflectores. Consecuentemente, esta nueva solución al problema precisa de un mayor esfuerzo computacional; pues supone un incremento del número de modos con los que se reconstruye, de forma precisa, el campo incidente sobre la parábola.

\subsubsection{Segmentación del Contorno de los Objetos Disper- sores}

Al utilizar el algoritmo recursivo se produce una segunda limitación cuando, debido a la proximidad espacial de dos objetos dispersores cualesquiera, parte del contorno de uno de ellos se encuentra dentro de la mínima circunferencia que contiene al otro. Bajo dicha circunstancia, el campo dispersado por el segundo objeto no se reconstruye correctamente en todo el contorno del primer objeto; por lo que, a partir de ese preciso instante, los resultados generados por el algoritmo recursivo son incorrectos.

Un ejemplo de esta segunda situación problemática se recoge gráficamente en la figura 6.13; donde se observa que parte del contorno del t-ésimo objeto perteneciente al segundo grupo está situada dentro de la circunferencia, con centro en el punto $C_{t}^{1}$, que contiene al $t$-ésimo objeto del primer grupo. Recordando el capítulo 4 (sección 4.1), el campo dispersado por el t-ésimo objeto del primer grupo, reconstruido a partir de su espectro de campo dispersado, tan sólo es válido en la región libre de fuentes; es decir, fuera de la citada circunferencia que contiene a dicho objeto. Por tanto, en el caso que nos ocupa existe una parte del contorno perteneciente al t-ésimo objeto del segundo grupo, situada dentro de la circunferencia mencionada anteriormente, en la que se desconoce el valor del campo dispersado por el $t$-ésimo objeto del primer grupo; resultando pues imposible caracterizar conjuntamente, mediante alguna de las versiones del conocido algoritmo recursivo, el comportamiento dispersor de los dos objetos considerados en la situación problemática recién expuesta.

La solución al problema recogido en este apartado consiste en evitar que la circunferencia asociada a todo objeto dispersor, que encierra a la correspondiente región con fuentes, contenga alguna porción de los contornos asociados respectivamente al resto de objetos dispersores; existiendo, tal y como se describe a continuación, dos posibles alternativas para implementar la solución buscada.

La primera de las dos posibles alternativas, al igual que la solución adoptada para resolver la primera limitación descrita en el apartado 6.3.1, se basa 


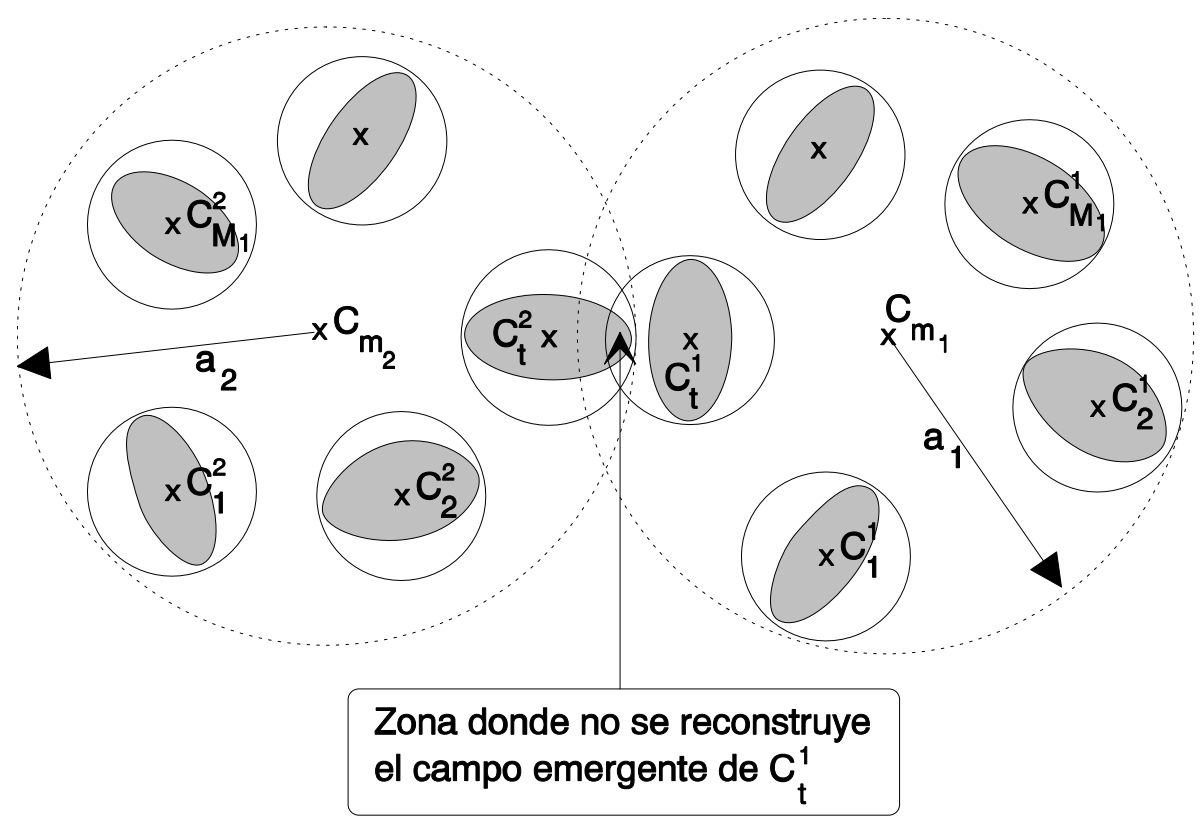

Figura 6.13: Diagrama explicativo de la segunda situación problemática que provoca errores en la aplicación del algoritmo recursivo.

en desplazar adecuadamente el centro de aquel objeto dispersor cuya mínima circunferencia invada el contorno de algún otro objeto; de manera que en esta ocasión la circunferencia centrada en el nuevo punto, encerrando a la región con fuentes del correspondiente objeto, no contenga ninguna porción de los contornos asociados al resto de objetos. El desplazamiento del centro de la citada circunferencia respecto de su posición natural, escogida normalmente en el punto medio geométrico del objeto, supone un aumento en el valor del radio asociado a dicha circunferencia; lo que, a su vez, repercute en un incremento del número de modos necesarios en la reconstrucción del campo incidente, y del campo dispersado, relativos ambos al objeto problemático en cuestión. Consecuentemente, esta primera solución implica una mayor carga computacional en la resolución del problema considerado. Por ejemplo, la situación problemática planteada en la figura 6.13 se resuelve, siguiendo esta primera estrategia, mediante un ligero desplazamiento del punto $C_{t}^{1}$ a la derecha de su actual posición; para que la nueva circunferencia asociada al $t$-ésimo objeto del primer grupo, de mayor radio que aquélla mostrada en la figura 6.13 , contenga todo el contorno de dicho objeto sin invadir ninguna porción del contorno perteneciente al t-ésimo objeto del otro grupo.

La segunda alternativa propuesta para resolver la problemática bajo estudio consiste, al igual que cuando se analizan objetos dispersores con dimensiones grandes en términos eléctricos, en segmentar en elementos más pequeños el contorno del objeto dispersor cuya circunferecia contiene porciones del contorno de otros objetos; con la intención última de que las nuevas circunferencias, que contienen 


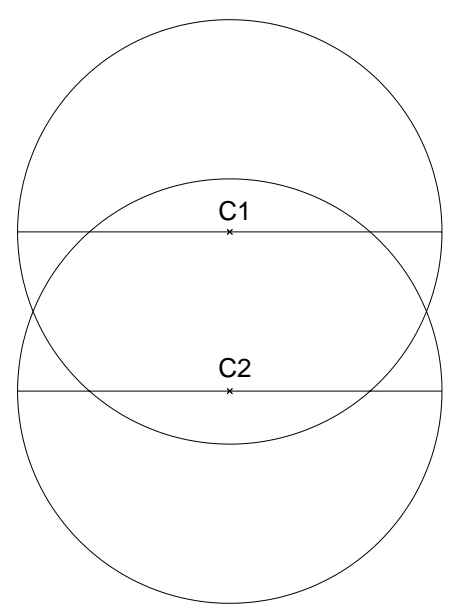

a)

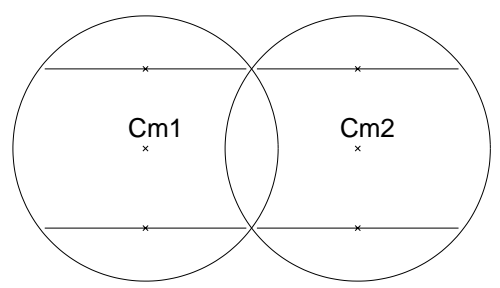

b)

Figura 6.14: Ejemplo práctico de la segunda limitación del algoritmo recursivo. Presentación del problema en a), y posible solución al mismo en b).

a los elementos recién creados, no invadan nuevamente el contorno de ningún otro objeto dispersor. Al reducir el tamaño de los nuevos objetos recién creados, lógicamente disminuyen los radios de las mínimas circunferencias que los contienen; lo cual, sin duda, implica un descenso del número de modos requeridos en la caracterización del comportamiento dispersor de dichos objetos. Por otra parte, al aumentar el número inicial de objetos a considerar, se produce un crecimiento en el número de iteraciones del algoritmo recursivo. En definitiva, resulta difícil evaluar si esta segunda solución implica un incremento o un descenso de la carga computacional asociada al algoritmo recursivo; que finalmente dependerá, como se desprende de los resultados ofrecidos en el capítulo 7 (sección 7.1), tanto del número final de objetos a caracterizar conjuntamente como de su tamaño. Un ejemplo práctico donde puede aplicarse la solución recién descrita se presenta en la figura 6.14 ; en la que se tiene un problema de dispersión electromagnética producida por dos tiras metálicas paralelas, situadas de forma muy próxima la una a la otra. Esta proximidad provoca que la circunferencia correspondiente a cada tira contenga una porción de la tira opuesta, e incluso el centro de la circunferencia asociada a dicha tira (ver apartado a en la figura 6.14). En la citada figura, puede observarse el incumplimiento de las dos limitaciones del algoritmo recursivo descritas hasta este momento. Para evitar la segunda limitación del método, cada una de las tiras se fragmenta en dos subtiras de igual longitud (ver apartado b en la figura 6.14); de manera que la nueva circunferencia asociada a cada subtira no contiene ninguna porción del resto de subtiras. De esta forma, el problema original de las dos tiras se ha convertido en un nuevo problema constituido por cuatro 
tiras, de menor longitud, enfrentadas dos a dos. Si a continuación se agrupan por un lado las dos tiras superiores, y por otro las dos tiras inferiores, en el análisis final de ambos grupos se incurrirá en la primera de las limitaciones del algoritmo recursivo (recordar apartado 6.3.1). De manera definitiva, la solución al problema se obtiene agrupando las dos tiras de la izquierda y las dos de la derecha por separado, tal y como se indica gráficamente en la figura 6.14 (apartado b); para finalmente, en una última iteración, analizar conjuntamente ambos grupos sin incurrir en ninguna de las dos limitaciones del algoritmo recursivo planteadas en un principio.

Conviene, llegados a este punto, realizar un pequeño comentario sobre cierta estrategia de análisis de objetos dispersores; que consiste en segmentar inicialmente el contorno de dichos objetos en elementos con dimensiones más reducidas, y luego aplicar el algoritmo recursivo presentado en este capítulo para analizar de forma conjunta su comportamiento dispersor. Esta estrategia, que se utiliza no sólo al resolver la limitación descrita en el presente apartado sino también, tal y como se recoge en la introducción de este capítulo, en el análisis de objetos dispersores grandes en términos eléctricos, al ser implementada presenta efectos desiguales; que dependen del tipo de polarización del campo incidente sobre el objeto dispersor bajo análisis. Así pues, la segmentación del contorno de un objeto en unidades más pequeñas ante incidencia con polarización $\mathrm{TM}^{\mathrm{z}}$ no interrumpe, de manera física, ningún elemento de corriente inducida que exista sobre el contorno del citado objeto; pues dicha corriente circula paralela al eje $\hat{z}$ (recordar sección 4.2 del capítulo 4). En este caso, consecuentemente, la técnica de la segmentación no introduce ningún error adicional. Por contra, ante incidencia polarizada según $\mathrm{TE}^{\mathrm{z}}$, la corriente inducida sobre el objeto dispersor es tangente a su contorno (recordar nuevamente la sección 4.2 del capítulo 4); razón por la cual, al segmentar el contorno del objeto dispersor en elementos más pequeños, se interrumpe físicamente la continuidad de la corriente entre dichos elementos. Este error, aunque pequeño ${ }^{2}$, supone una pérdida de precisión en los resultados obtenidos, empleando la estrategia de la segmentación, en problemas de dispersión electromagnética ante incidencia $\mathrm{TE}^{\mathrm{z}}$. Por tanto, si ante este tipo de polarización se desean obtener resultados tan precisos como aquéllos obtenidos para incidencia polarizada según $\mathrm{TM}^{\mathrm{z}}$, en la resolución de la problemática analizada en el presente apartado debe emplearse la primera de las dos soluciones alternativas propuestas; mientras que en la caracterización de objetos grandes en términos eléctricos, debiera emplearse la técnica basada en el Método de los Momentos sin previa segmentación del contorno del objeto. Indudablemente, conviene evaluar si este grado de mejora en la precisión compensa el esfuerzo computacional que supone analizar los objetos sin segmentación.

\footnotetext{
${ }^{2}$ En el capítulo 7 se presentan resultados, empleando segmentación, de objetos grandes en términos eléctricos ante incidencia polarizada según $\mathrm{TE}^{\mathrm{z}}$; los cuales hacen presagiar que el error introducido por la segmentación del contorno de los objetos es ciertamente pequeño.
} 


\subsubsection{Reconstrucción del Campo Incidente sobre Grupos de Objetos Dispersores}

En este apartado, se describe finalmente la tercera limitación del algoritmo recursivo; que se encuentra asociada al número de modos utilizados en la reconstrucción del campo incidente sobre cada grupo de objetos dispersores. Concretamente, dicha limitación se produce al elegir un número de modos tal que la circunferencia correspondiente, centrada normalmente en el punto medio del grupo, incluya no sólo a todos los objetos de dicho grupo; sino también al centro de algún objeto perteneciente a otro grupo. Bajo este supuesto, las matrices de traslación de espectro emergente a incidente presentan elementos con valores muy grandes (ver apéndice $\mathrm{E}$ ); los cuales pueden provocar que los sistemas matriciales de ecuaciones del algoritmo recursivo estén mal condicionados, y por consiguiente que no tengan solución.

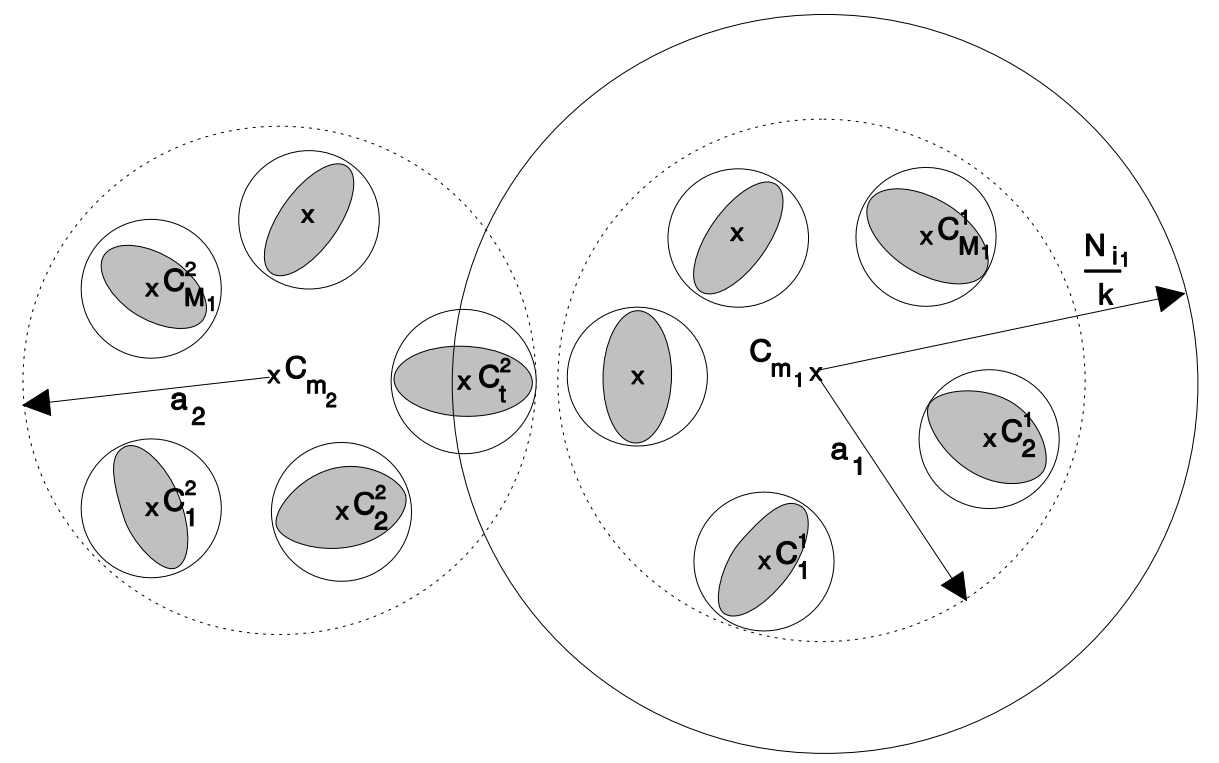

Figura 6.15: Diagrama explicativo de la tercera situación problemática que provoca errores en la aplicación del algoritmo recursivo.

Esta última limitación se recoge gráficamente en la figura 6.15; donde nuevamente se pretende analizar la dispersión producida por dos grupos de objetos dispersores, cada uno de los cuales ha sido caracterizado previamente. Como puede apreciarse en la citada figura, los puntos $C_{m_{1}}$ y $C_{m_{2}}$ se eligen para minimizar los radios (denominados $a_{1}$ y $a_{2}$ ) de las dos circunferencias, centradas en dichos puntos, que contienen respectivamente a todos los objetos de cada grupo (ver circunferencias con trazo punteado en la figura 6.15). De esta forma, se minimiza también el número de modos requeridos en la correcta reconstrucción del campo incidente sobre cada grupo, de valor $2 N_{i_{1}}+1$ y $2 N_{i_{2}}+1$ respectivamente, pues 
se satisfacen las relaciones $N_{i_{1}}=k a_{1} \mathrm{y} \quad N_{i_{2}}=k a_{2}$. En esta ocasión, el problema surge al intentar reconstruir el campo incidente sobre los objetos del primer grupo con un número de modos $\left(2 N_{i_{1}}+1\right)$ mayor que el valor mínimo recomendado previamente. Este nuevo número de modos permite realmente reconstruir el campo incidente dentro de una circunferencia, centrada en el punto $C_{m_{1}}$ y de radio $N_{i_{1}} / k$, que contiene el centro del $t$-ésimo objeto del segundo grupo (ver circunferencia con trazo continuo en la figura 6.15). En esta situación, tal y como se indica en el apéndice E (apartado E.2.1), la matriz que transforma el espectro de campo dispersado por el $t$-ésimo objeto del segundo grupo, referido al punto $C_{t}^{2}$, en espectro de campo incidente sobre el primer grupo de objetos, centrado en el punto $C_{m_{1}}$, contiene algunos elementos con amplitudes muy elevadas. Al utilizar esta matriz en la aplicación del algoritmo recursivo, los sistemas matriciales asociados a dicho algoritmo se vuelven mal condicionados; lo que dificulta, e incluso imposibilita en ocasiones, su resolución computacional.

En principio, la solución al tipo de problema recién descrito consiste en no escoger, en la reconstrucción del campo incidente sobre un grupo de objetos, un número de modos superior al valor mínimo recomendado anteriormente; es decir, si se elige un número de modos igual al producto $k a$, siendo a el radio de la mínima circunferencia que contiene a todos los objetos del grupo, no se incurrirá en la situación presentada anteriormente siempre y cuando no se incumpla la primera de las limitaciones comentadas en esta sección (recordar apartado 6.3.1). Ahora bien, este criterio resulta excesivamente estricto; pues apenas deja margen a errores en la reconstrucción del campo incidente en ciertas porciones del contorno de algunos objetos, concretamente de aquellos objetos más próximos a la citada circunferencia límite. De manera experimental, se ha comprobado que siguiendo el criterio de escoger un número de modos igual al producto $1.5 \mathrm{ka}$; se consigue un cierto margen que permite reconstruir con éxito el campo incidente sobre el contorno de todos los objetos del grupo. Por tanto, la solución óptima debe seguir este último criterio para determinar el número de modos con el que debe reconstruirse el campo incidente; excepto cuando ello suponga incurrir en la situación problemática descrita en este apartado. En dicho caso, se debe disminuir el número de modos considerado inicialmente; aun a riesgo de no poder reconstruir correctamente el campo incidente sobre todos los objetos del grupo.

\subsubsection{Conclusiones. Automatización del Algoritmo Re- cursivo}

En los apartados anteriores de esta sección, se han detectado ciertas limitaciones a la aplicación directa del algoritmo recursivo; que se ha descrito inicialmente en la sección 6.1, y se ha refinado posteriormente en la sección 6.2. En realidad, los apartados de esta sección recogen diversas situaciones; cuyo análisis mediante el citado algoritmo recursivo, sin tomar ciertas acciones preventivas, conduce a 
resultados erróneos. A su vez, en dichos apartados se plantean diversas estrategias para evitar los errores previamente detectados; con el objeto de garantizar que el algoritmo recursivo descrito en el presente capítulo, mediante cualquiera de sus dos versiones o combinándolas adecuadamente, sea capaz de proporcionar resultados correctos.

La principal y más importante conclusión, que puede extraerse de las soluciones aportadas en esta sección a cada una de las situaciones problemáticas planteadas, es que se puede resolver prácticamente cualquier problema de acoplo electromagnético entre múltiples objetos dispersores empleando el algoritmo recursivo presentado en este capítulo. Lógicamente, tal y como se comenta a continuación con más detalle, la obtención de la solución correcta al problema bajo análisis requiere, en ciertas ocasiones, tomar determinadas acciones preventivas; las cuales han quedado perfectamente descritas en los tres primeros apartados de esta sección.

Así pues, de los tres primeros apartados de esta sección se concluye que, para aplicar con garantías de éxito el algoritmo recursivo a la resolución de un problema de dispersión electromagnética producida por múltiples objetos, es necesario implementar inicialmente las siguientes acciones preparatorias. En primer lugar, los objetos que constituyen el problema bajo análisis deben agruparse por proximidad espacial; con la intención de evitar que se produzca la primera de las situaciones problemáticas, descrita en el apartado 6.3.1. Posteriormente, los objetos que integran cada grupo deben ordenarse de forma adecuada; de manera que al caracterizar conjuntamente su comportamiento dispersor, mediante el algoritmo deducido en la sección 6.1 (apartado 6.1.3), el objeto que se incorpore en cada nueva iteración sea el más próximo a aquéllos analizados anteriormente. De esta forma, se evita incurrir de nuevo en la primera de las limitaciones del algoritmo recursivo. Asimismo, las agrupaciones de objetos también deben ordenarse; para que en su caracterización conjunta, siguiendo la versión del algoritmo recursivo presentada en la sección 6.2, también se incorpore en cada nueva iteración el grupo de objetos más próximo a aquellos otros grupos caracterizados previamente. A pesar de estos esfuerzos por evitar la primera situación problemática, puede producirse algún caso, como por ejemplo cuando dos grupos de objetos están muy próximos entre sí, que esté relacionado con dicha limitación. La solución en estos casos, tal y como se recoge en el apartado 6.3.1, consiste en desplazar el punto al cual se refiere el espectro de campo incidente sobre el grupo causante del problema. Tras evitar la primera de las tres posibles situaciones problemáticas, debe comprobarse que ninguna de las otras dos pueden producirse durante el análisis del problema. En el supuesto de que aparezca algún conflicto entre objetos asociado a la segunda limitación del método, la solución consiste simplemente en desplazar el centro del objeto problemático; o bien en realizar una segmentación adecuada del contorno de dicho objeto. Para no incurrir en la tercera de las limitaciones, tan sólo debe tenerse precaución al elegir el número de modos que se 
emplean en la reconstrucción del campo incidente sobre cada grupo de objetos. Una vez se satisfacen todos estos criterios, se puede asegurar que la aplicación del algoritmo recursivo al problema bajo análisis genera resultados correctos.

La resolución de cualquier problema de dispersión electromagnética producida por múltiples objetos, mediante la aplicación del conocido algoritmo recursivo, requiere pues observar los distintos factores resumidos en el párrafo anterior. Una automatización de dicho algoritmo recursivo, que permitiera resolver prácticamente cualquier problema de dispersión múltiple, supondría considerar en su implementación todas las posibles situaciones problemáticas que pudieran producirse, así como decidir correctamente la mejor estrategia a seguir para evitarlas. Lógicamente, dicha tarea resultaría ardua, por no decir imposible, de llevar a la práctica; pues siempre existirán situaciones nuevas no contempladas en la versión automatizada del algoritmo recursivo. De hecho, la resolución de ciertos problemas complejos de acoplo electromagnético requiere de una persona experta, conocedora del algoritmo y de sus posibles limitaciones, que decida como solucionar correctamente dichos problemas aplicando de la forma más adecuada el algoritmo recursivo. No obstante, un gran número de problemas no presentan soluciones tan complicadas de calcular; las cuales se obtienen observando simplemente algunas precauciones básicas descritas en los apartados anteriores. Este tipo de problemas sí que pueden resolverse aplicando una versión automatizada del algoritmo recursivo; que se ocupe de realizar, en cada caso, las acciones previas necesarias para obtener la solución correcta al problema concreto bajo análisis. Por ejemplo, una posible versión simplificada del algoritmo recursivo debiera observar, previamente a su aplicación, las acciones preventivas que se enumeran a continuación: una correcta agrupación de objetos dispersores, la elección del punto al que se refiere el espectro de campo incidente sobre cada grupo, una ordenación apropiada de objetos dispersores y de agrupaciones, y finalmente la determinación del número de modos que requiere la reconstrucción del campo incidente sobre cada grupo. A continuación, y de manera indicativa, se ofrecen algunos criterios prácticos a seguir en la implementación computacional de dichas acciones preventivas.

Atendiendo en primer lugar a la agrupación de objetos, un posible criterio consiste en considerar que un grupo se constituye a partir de objetos cuando la distancia entre sus centros es inferior a un valor umbral. De acuerdo con este criterio, si el centro de un objeto está separado de todos los centros de los objetos pertenecientes a un grupo por una distancia superior al valor umbral; dicho objeto no forma parte del grupo, y debe pertenecer por tanto a un grupo nuevo. La elección del valor para la distancia umbral determina el grado en que se desea separar a los objetos en grupos. Eligiendo, por ejemplo, un valor umbral igual a la media aritmética de la dimensión máxima de todos los objetos que integran el problema bajo análisis, se garantiza que sólo aquellos objetos muy próximos entre si formarán parte de un mismo grupo. La correcta agrupación de objetos, como 
bien es sabido, pretende evitar que durante la aplicación del algoritmo recursivo se produzca la primera de las situaciones problemáticas (recordar apartado 6.3.1).

Una vez se han agrupado debidamente los objetos, para cada grupo debe escogerse el punto al que referir el espectro asociado al campo incidente. Con la intención de simplificar al máximo la implementación del algoritmo recursivo, se propone elegir las coordenadas de dicho punto como la media aritmética de las coordenadas de los centros de los objetos pertenecientes al grupo. Esta elección reduce el número de componentes del espectro asociado al campo incidente; y por tanto disminuye la carga computacional que implica la ejecución del algoritmo recursivo. Sin embargo, la elección de este criterio impide resolver problemas como los mostrados en el apartado 6.3.1; en concreto aquéllos donde se requería desplazar ligeramente el centro de algún grupo respecto de su punto medio.

En cuanto a la ordenación de los objetos dispersores pertenecientes a un grupo, ésta puede realizarse siguiendo el orden en sentido creciente de la distancia euclídea entre los centros de los objetos y el centro del grupo. De esta forma, en primer lugar se caracterizan conjuntamente los dos objetos más próximos al punto medio del grupo. Posteriormente, se analizan las interacciones múltiples entre dichos objetos y el siguiente objeto más próximo al punto medio, y así sucesivamente; minimizando por tanto el riesgo de incurrir nuevamente en la primera situación problemática (ver apartado 6.3.1). Este mismo criterio puede aplicarse igualmente a la ordenación de las agrupaciones de objetos dispersores; ordenando los grupos en función de las distancias entre sus respectivos centros y el punto medio de todos ellos. El orden de los grupos determina en que iteración del algoritmo, descrito en la sección 6.2, debe incorporarse cada grupo de objetos; intentando evitar, en la medida de lo posible, la primera limitación del algoritmo recursivo (recordar apartado 6.3.1).

Por último, debe elegirse un número de modos $\left(2 N_{i}+1\right)$ con el que reconstruir el campo incidente sobre cada grupo de objetos; para lo cual se propone seguir en todo momento el criterio $N_{i}=k a$, siendo a el radio de la mínima circunferencia que contiene a todos los objetos del grupo correspondiente. A pesar de la rigurosidad excesiva de este criterio, que puede ocasionar una reconstrucción incorrecta del campo incidente sobre ciertos objetos (recordar apartado 6.3.3), su seguimiento garantiza que no se produzca la tercera situación problemática, enunciada de forma detallada en el apartado 6.3.3, siempre y cuando no esté presente la primera de dichas situaciones problemáticas.

Tal y como se ha comentado anteriormente, la implementación de los criterios recién descritos conduce a una versión computacional simplificada del algoritmo recursivo; que permite resolver numerosos problemas de dispersión electromagnética producida por múltiples objetos, tales como sistemas multireflectores cuyos reflectores primario y secundario se hallan alejados el uno del otro. Por contra, estas mismas acciones preventivas no permiten resolver, entre otras posibles situaciones, todas aquellas relacionadas con la segunda limitación del algoritmo 
recursivo (recordar apartado 6.3.2); ni tampoco aquellos problemas que requieren desplazar el centro de algún grupo respecto de su punto medio, como cuando los reflectores que integran una antena multireflectora se encuentran muy próximos entre sí. Concluyendo, cualquier versión automatizada del algoritmo recursivo, por muy completa que pueda ser, siempre resolverá ciertos problemas de forma errónea; a no ser que en dichos casos se actúe externamente indicando la estrategia a seguir.

\subsection{Iteración Especial para Fuente Interna al Problema de Múltiples Objetos Dispersores}

El algoritmo recursivo, descrito en las secciones 6.1 y 6.2 del presente capítulo, permite calcular la dispersión electromagnética producida por múltiples objetos ante cualquier excitación; siempre y cuando dicha excitación esté generada por una fuente situada fuera de la mínima circunferencia que contiene a todos los objetos analizados. No obstante, existen situaciones en las que la excitación sobre los objetos dispersores está generada por una fuente situada dentro de la citada circunferencia. En esta sección, se presenta una nueva versión del algoritmo recursivo; que permite caracterizar conjuntamente el comportamiento dispersor de los múltiples objetos ante este nuevo tipo de incidencia.

Al situar la fuente en el interior de la circunferencia que contiene a todos los objetos dispersores, el campo incidente generado por dicha fuente se expresa en serie de modos cilíndricos emergentes; es decir, utilizando funciones de Hankel de segunda especie centradas en la posición que ocupa la fuente. Estas funciones se escogen, recordando el capítulo 4 (sección 4.1), porque permiten recoger la singularidad que se produce en el punto donde se sitúa la fuente. Por tanto, el problema bajo análisis es similar al planteado en las secciones 6.1 y 6.2 de este capítulo; excepto que en esta ocasión el campo incidente sobre todos los objetos no se expresa mediante funciones de Bessel, sino como se termina de explicar mediante funciones de Hankel de segunda especie.

La resolución del problema planteado en esta sección se realiza separando los objetos a caracterizar en dos grupos; siempre y cuando dicha separación sea posible de implementar. La dispersión electromagnética producida por cada uno de estos grupos se caracteriza por separado, mediante la aplicación del algoritmo recursivo descrito en la sección 6.1 (apartado 6.1.3), considerando como campo incidente un desarrollo en serie de funciones de Bessel. Finalmente, en una última iteración, se caracteriza conjuntamente el comportamiento dispersor de los objetos pertenecientes a ambos grupos ante una incidencia constituida por funciones de Hankel de segunda especie, que se centran en el punto donde se encuentra situada la fuente interna. El análisis de esta última iteración se implementa, tal y como se detalla a continuación, mediante una versión del algoritmo recursivo 

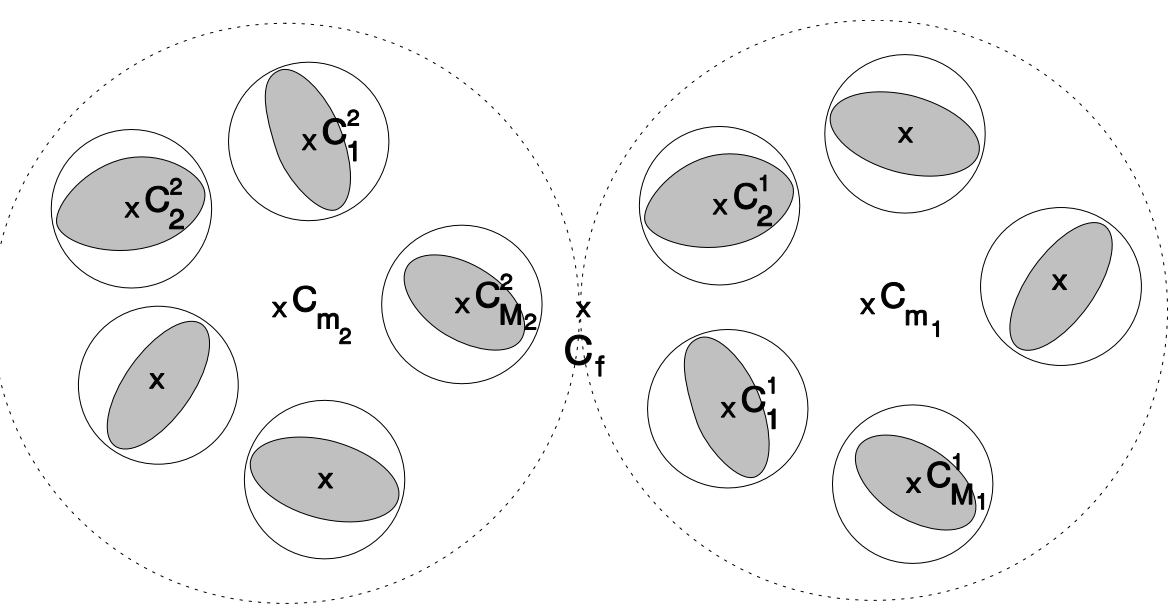

Figura 6.16: Esquema general de un problema de acoplo electromagnético entre dos grupos de dispersores con fuente interna situada en $C_{f}$.

muy similar a la descrita en la sección 6.2 ; salvo que como espectro de campo incidente se considera el espectro asociado al campo que genera la fuente interna al problema. Como consecuencia de este análisis, el comportamiento dispersor de cada objeto se define en términos de una matriz de caracterización conjunta; que relaciona el espectro de campo dispersado por el correspondiente objeto con el espectro asociado al campo emergente producido por la fuente interna.

El problema a caracterizar en la última iteración se recoge gráficamente en la figura 6.16; donde se tienen dos grupos constituidos respectivamente por $M_{1}$ y $M_{2}$ objetos dispersores, sobre los cuales incide un campo producido por una fuente situada en el punto $C_{f}$ (ver figura 6.16). Tras caracterizar dichos grupos por separado, aplicando la versión del algoritmo recogida en la sección 6.1 (apartado 6.1.3), el comportamiento dispersor de los objetos del primer grupo se expresa en función de las matrices $D_{t}^{1}$, con $t \in\left[1, \ldots, M_{1}\right]$; mientras que para los objetos del segundo grupo se tienen las matrices $D_{t}^{2}$, con $t \in\left[1, \ldots, M_{2}\right]$. Como bien es sabido, cada una de las matrices $D_{t}^{1}$ relaciona el espectro de campo dispersado por el t-ésimo objeto del primer grupo, referido al centro $C_{t}^{1}$ de dicho objeto, con el espectro de campo incidente referido al punto $C_{m_{1}}$. Por su parte, la matriz $D_{t}^{2}$ caracteriza el comportamiento dispersor del $t$-ésimo objeto del segundo grupo; relacionando en esta ocasión el espectro asociado al campo que dispersa dicho objeto, referido a su centro $C_{t}^{2}$, con el espectro asociado al campo incidente centrado en el punto $C_{m_{2}}$. Las posiciones de los puntos $C_{m_{1}}$ y $C_{m_{2}}$, al igual que en la sección 6.2 , se eligen para minimizar el radio de las circunferencias que contienen respectivamente a todos los objetos de cada grupo; y reducir por tanto el esfuerzo computacional que supone reconstruir correctamente el campo incidente sobre dichos grupos. Seguidamente, la caracterización conjunta de ambos grupos ante una incidencia constituida por modos cilíndricos emergentes, centrados en 
el punto $C_{f}$, se realiza siguiendo el mismo método deducido anteriormente en la sección 6.2. La única diferencia al aplicar dicho método, basado en teoría de grafos [13], consiste en considerar que la incidencia al problema debe representar en este caso todas las posibles componentes, con amplitud unidad, de un desarrollo modal emergente; lo cual se expresa en términos espectrales mediante una matriz identidad $I$ de tamaño $2 N_{f}+1$, representando $2 N_{f}+1$ el número de componentes modales que requiere la representación del campo generado por la fuente interna. Recordando pues el método expuesto en la sección 6.2, el problema original se descompone en dos situaciones complementarias; en cada una de las cuales se asume que el campo incidente afecta tan sólo a uno de los dos grupos considerados, y no al otro. Como consecuencia de las múltiples interacciones entre todos los objetos, en cada problema complementario aparece un nuevo campo incidente sobre el correspondiente grupo de objetos; cuyo espectro asociado constituye precisamente la incógnita del problema. Dichos espectros incógnita se definen respectivamente mediante las matrices $E A$ y $E B$; planteando para su obtención unos sistemas de ecuaciones similares a los recogidos en (6.30), donde el campo incidente se expresaba en serie de modos cilíndricos incidentes. En esta ocasión, los nuevos sistemas de ecuaciones deben tener presente el nuevo tipo de incidencia; por lo que se expresan matricialmente como

$$
\begin{gathered}
\left(I_{1}-\left(\sum_{t=1}^{M_{2}} T_{m_{1} t} \cdot D_{t}^{2}\right) \cdot\left(\sum_{t=1}^{M_{1}} T_{m_{2} t} \cdot D_{t}^{1}\right)\right) \cdot E A= \\
\left(\sum_{t=1}^{M_{2}} T_{m_{1} t} \cdot D_{t}^{2}\right) \cdot\left(\sum_{t=1}^{M_{1}} T_{m_{2} t} \cdot D_{t}^{1}\right) \cdot T_{m_{1} f} \cdot I \\
\left(I_{2}-\left(\sum_{t=1}^{M_{1}} T_{m_{2} t} \cdot D_{t}^{1}\right) \cdot\left(\sum_{t=1}^{M_{2}} T_{m_{1} t} \cdot D_{t}^{2}\right)\right) \cdot E B= \\
\left(\sum_{t=1}^{M_{1}} T_{m_{2} t} \cdot D_{t}^{1}\right) \cdot\left(\sum_{t=1}^{M_{2}} T_{m_{1} t} \cdot D_{t}^{2}\right) \cdot T_{m_{2} f} \cdot I
\end{gathered}
$$

donde se observa que las matrices $T J_{m_{1} 0}$ y $T J_{m_{2} 0}$, presentes en los sistemas de ecuaciones definidos según (6.30), han sido sustituidas de forma consecuente por las matrices $T_{m_{1} f}$ y $T_{m_{2} f}$; que representan las matrices de traslación de espectro emergente centrado en $C_{f}$ a espectro incidente centrado respectivamente en $C_{m_{1}}$ y $C_{m_{2}}$. Por su parte, las matrices $I_{1}$ e $I_{2}$ designan sendas matrices identidad de tamaños $2 N_{i_{1}}+1$ y $2 N_{i_{2}}+1$; donde dichos números indican respectivamente cuántos modos se utilizan en la reconstrucción del campo incidente sobre los $M_{1}$ objetos del primer grupo, y cuántos se utilizan al reconstruir el campo incidente sobre los $M_{2}$ objetos del segundo grupo. Finalmente, el significado de las matrices $T_{m_{1} t}$ y $T_{m_{2} t}$ es idéntico al ofrecido en la sección 6.2 ; es decir, $T_{m_{1} t}$ es una matriz de traslación de espectro emergente centrado en $C_{t}^{2}$ a espectro incidente centrado 
en $C_{m_{1}}$, y $T_{m_{2} t}$ es una matriz de traslación de espectro emergente centrado en $C_{t}^{1}$ a espectro incidente centrado en $C_{m_{2}}$.

De igual manera que en la sección 6.2 , tras obtener las matrices $E A$ y $E B$ resolviendo en este caso los sistemas de ecuaciones recogidos en (6.32), se debe aplicar superposición de las respuestas obtenidas en cada uno de los dos problemas complementarios. De este modo, el comportamiento dispersor de todos los objetos analizados se define respectivamente a partir de las matrices de caracterización conjunta $D_{i}$, con $i \in\left[1, \ldots, M_{1}+M_{2}\right]$; que presentan el siguiente aspecto

$$
\begin{aligned}
D_{i}= & D_{i}^{1} \cdot\left(\left(\sum_{t=1}^{M_{2}} T_{m_{1} t} \cdot D_{t}^{2}\right) \cdot\left(T_{m_{2} f} \cdot I+E B\right)\right. \\
& \left.+T_{m_{1} f} \cdot I+E A\right) \quad i \in\left[1, \ldots, M_{1}\right] \\
D_{i}= & D_{i-M_{1}}^{2} \cdot\left(\left(\sum_{t=1}^{M_{1}} T_{m_{2} t} \cdot D_{t}^{1}\right) \cdot\left(T_{m_{1} f} \cdot I+E A\right)\right. \\
& \left.+T_{m_{2} f} \cdot I+E B\right) \quad i \in\left[M_{1}+1, \ldots, M_{1}+M_{2}\right]
\end{aligned}
$$

De nuevo, es interesante observar como las expresiones en (6.33) son análogas a las recogidas en (6.31) para incidencia desarrollada en serie de funciones de Bessel; exceptuando la sustitución de las matrices $T J_{m_{1} 0}$ y $T J_{m_{2} 0}$ por sus equivalentes $T_{m_{1} f}$ y $T_{m_{2} f}$, las cuales resultan apropiadas al caso que nos ocupa. Por lo que respecta a las dimensiones de las matrices $D_{i}$, éstas son $2 N_{d_{i}}+1 \times 2 N_{f}+1$; donde $2 N_{d_{i}}+1$ es el número de modos utilizados en la reconstrucción del campo dispersado por el $i$-ésimo objeto, y $2 N_{f}+1$ es el número de modos cilíndricos emergentes con los que se expresa el campo generado por la fuente interna al problema. Definitivamente, las matrices de caracterización conjunta $D_{i}$ relacionan, tal y como se pretendía, el espectro de campo dispersado por el correspondiente objeto con el espectro asociado al campo generado por una fuente; el cual se expresa de igual manera que el espectro de campo dispersado por un objeto.

Las matrices de caracterización conjunta recién obtenidas determinan el campo dispersado por cada objeto, teniendo en cuenta las interacciones múltiples entre todos ellos, cuando la fuente que genera el campo incidente se encuentra situada en el punto $C_{f}$. En el caso de que la fuente se desplace de posición a un nuevo punto $C_{f}^{\prime}$, existen dos posibles estrategias para calcular la nueva dispersión electromagnética producida por el mismo grupo de objetos dispersores. La primera estrategia consiste en utilizar las mismas matrices de caracterización conjunta, obtenidas previamente cuando la fuente estaba situada en $C_{f}$; para lo cual debe transformarse el espectro emergente asociado al campo producido por la fuente situada en $C_{f}^{\prime}$, referido precisamente a dicho punto, en un nuevo espectro emergente asociado al mismo campo pero referido al punto $C_{f}$. Esta operación recién descrita se implementa mediante una matriz de traslación de espectro emergente 
a emergente; cuyos elementos se calculan como se indica en el apéndice E. Ahora bien, la utilización correcta de esta matriz de traslación requiere que la circunferencia centrada en $C_{f}$, y de radio la distancia entre $C_{f}$ y $C_{f}^{\prime}$, no contenga ninguna porción del contorno de los objetos dispersores pertenecientes al problema bajo análisis; pues el campo generado por la fuente situada en $C_{f}^{\prime}$ no se reconstruye correctamente, a partir del espectro emergente referido al punto $C_{f}$, en el interior de la citada circunferencia (ver apartado E.2.2 del apéndice E). Suponiendo que se produzca dicha situación, el cambio de ubicación de la fuente interna puede resolverse mediante una segunda estrategia alternativa; consistente en repetir el algoritmo descrito en esta sección cuando la fuente se sitúa en el punto $C_{f}^{\prime}$. De esta forma, se obtienen unas nuevas matrices de caracterización conjunta; que permiten obtener el campo dispersado por el mismo grupo de objetos para la nueva posición de la fuente.

La correcta aplicación del método descrito en la presente sección requiere evitar, en principio, todas aquellas situaciones problemáticas presentadas en la sección 6.3; que lógicamente pueden producirse cuando el nuevo método, siguiendo el algoritmo recursivo deducido en la sección 6.1 (apartado 6.1.3), caracteriza el comportamiento dispersor de cada uno de los dos grupos en los que se dividen todos los objetos. Las posibles acciones preventivas que deben observarse para evitar dichas situaciones se recogen en la mencionada sección 6.3. Adicionalmente, existe una nueva situación problemática asociada a la última iteración del método propuesto; que aparece cuando se pretende analizar el acoplo entre dos grupos de objetos ante una excitación definida en serie de modos cilíndricos emergentes. Esta nueva situación problemática surge, bien cuando parte del contorno de algún objeto del primer grupo esta fuera de la circunferencia con centro en el punto $C_{m_{1}}$, y de radio la distancia entre los puntos $C_{f}$ y $C_{m_{1}}$; o bien cuando parte del contorno de algún objeto del segundo grupo está fuera de la circunferencia con centro en el punto $C_{m_{2}}$, y de radio la distancia entre los puntos $C_{f}$ y $C_{m_{2}}$. En realidad, el problema se origina al transformar el espectro de campo emergente, referido al punto $C_{f}$, en espectros de campo incidente sobre ambos grupos centrados respectivamente en los puntos $C_{m_{1}}$ y $C_{m_{2}}$; utilizando para ello las matrices de traslación de espectros $T_{m_{1} f}$ y $T_{m_{2} f}$. Dichas matrices, debido a la conocida restricción de la primera versión del Teorema de Adición para las Funciones de Hankel (recordar apartado E.2.1 del apéndice E), permiten reconstruir correctamente el campo incidente sobre el primer y el segundo grupo de objetos dentro de sendas circunferencias, centradas en los puntos $C_{m_{1}}$ y $C_{m_{2}}$; y cuyos radios son la distancia entre $\operatorname{los}$ puntos $C_{f}$ y $C_{m_{1}}$ para la primera circunferencia, y la distancia entre los puntos $C_{f}$ y $C_{m_{2}}$ para la segunda circunferencia. Por tanto, suponiendo que parte del contorno de algún objeto del primer o segundo grupo quede fuera de la correspondiente circunferencia; el campo incidente no se reconstruirá de forma correcta, debido a la razón anteriormente expuesta, en dicha parte del contorno del objeto. A partir de este instante, los resultados 
que proporciona el método presentado en esta sección para la última iteración son incorrectos; lo que imposibilita la caracterización conjunta de los dos grupos de objetos dispersores ante una fuente interna. Por ejemplo, éste no es el caso de la situación mostrada previamente en la figura 6.16; donde las mencionadas circunferencias, dibujadas con trazo punteado en dicha figura, no contienen respectivamente ninguna porción del contorno de los objetos del primer y segundo grupo. Sin embargo, existen ciertos problemas reales en los que, al separar los objetos en dos grupos, resulta prácticamente imposible que no se produzca la anterior situación problemática; imposibilitando por tanto el análisis de dichos problemas mediante el método deducido en esta sección. Una posible alternativa para resolver estos problemas consiste en separar los objetos en más de dos grupos; concretamente en tres grupos, o incluso en cuatro cuando la situación lo requiera. Finalmente, en una última iteración, se analiza conjuntamente el acoplo electromagnético entre esos tres o cuatro grupos cuando la fuente es interna al problema. Las expresiones a utilizar en estos casos son lógicamente diferentes de las presentadas en esta sección para el caso de dos grupos; y su deducción se puede obtener, tal y como se expone detalladamente en [13], empleando el conocido método basado en teoría de grafos. Con la intención de no extender en demasía los resultados ofrecidos en la presente sección, dichas expresiones no se recogen en este trabajo.

Resumiendo, en esta sección se ha abordado el análisis de la dispersión electromagnética producida por múltiples objetos cuando la fuente, que genera el campo incidente sobre dichos objetos, es interna al problema bajo análisis. El método planteado para solucionar este tipo de problemas se basa en dividir inicialmente los objetos en dos grupos, que se caracterizan por separado utilizando la primera versión del algoritmo recursivo descrita en este capítulo (recordar sección 6.1); para a continuación analizar en una última iteración, mediante una modificación de la versión del algoritmo recursivo presentada en la sección 6.2, el acoplo entre ambos grupos ante un espectro de campo emergente asociado a la fuente interna al problema. Tras aplicar este método, el comportamiento dispersor de cada objeto queda definido en términos de una matriz de caracterización conjunta; cuyo concepto es ciertamente novedoso, pues relaciona en este caso espectro de campo dispersado con espectro de campo emergente referido al punto donde se sitúa la fuente. Ante un cambio en la ubicación de dicha fuente, se han presentado dos posibles acciones para resolver este nuevo problema constituido por los mismos objetos dispersores. Una de ellas utiliza, mediante una traslación previa de espectros emergentes, las mismas matrices de caracterización conjunta obtenidas para la posición inicial de la fuente; mientras la otra recalcula dichas matrices para la nueva posición de la fuente. Finalmente, se ha expuesto una nueva situación problemática asociada a la estrategia seguida por el método descrito; la cual provoca que los resultados generados sean incorrectos. Asimismo, se ha planteado una posible solución para evitar esta nueva limitación. 


\subsection{Reconstrucción del Campo Dispersado por Múltiples Objetos Dispersores}

Tras haber analizado distintos problemas de dispersión electromagnética producida por múltiples objetos dispersores, haciendo uso de los distintos métodos descritos en el presente capítulo; en esta última sección, se muestran las distintas regiones en las que se divide el espacio libre para reconstruir correctamente el campo dispersado por todos los objetos, así como las expresiones concretas a utilizar para dicha reconstrucción en cada una de las citadas regiones.

Los resultados que se ofrecen a continuación corresponden a la reconstrucción del campo dispersado por $N$ objetos; cuyo comportamiento dispersor se define mediante las matrices de caracterización conjunta $D_{i}$, con $i \in[1, \ldots, N]$. Dichas matrices se obtienen aplicando alguno de los métodos presentados en este capítulo; y permiten obtener fácilmente el espectro de campo dispersado por el correspondiente objeto, referido a su centro y expresado mediante el vector columna $\left[c_{q}^{i}\right]$, a través del producto matricial

$$
\left[c_{q}^{i}\right]=D_{i} \cdot\left[i_{p}\right]
$$

donde $\left[i_{p}\right]$ es un vector columna que representa el espectro asociado al campo incidente sobre todos los objetos dispersores. Con el objeto de no perder generalidad, los elementos del vector $\left[i_{p}\right]$ representan tanto las amplitudes de un desarrollo modal incidente, cuando la fuente es externa al problema bajo análisis; como un desarrollo en serie de modos cilíndricos emergentes, que se encuentra asociado a una fuente interna al problema considerado. En cada caso, el método utilizado en la deducción de las matrices de caracterización conjunta $D_{i}$ es lógicamente distinto; tal y como se recoge en las secciones 6.1 y 6.2 por una parte, y en la sección 6.4 por otra.

Por lo que respecta a la reconstrucción del campo dispersado por los $N$ objetos, el espacio libre se divide en tres regiones; tal y como se recoge gráficamente en la figura 6.17. Seguidamente, se exponen las diferentes expresiones correspondientes al campo dispersado por todos los objetos en cada una de las mencionadas regiones. Recordando el capítulo 4 (sección 4.1), el espectro asociado al campo que dispersa un objeto permite reconstruir dicho campo en la región libre de fuentes de corriente inducidas; es decir, fuera de la mínima circunferencia que contiene al objeto en cuestión. Por tanto, en el problema de dispersión que nos atañe, constituido por $N$ objetos dispersores, el campo dispersado ${ }^{3}$ por todos los objetos en la región libre de fuentes, representada en la figura 6.17 por las

\footnotetext{
${ }^{3}$ Este campo dispersado, denominado $C_{z}^{d}$, representa campo eléctrico dispersado $\left(E_{z}^{d}\right)$ para incidencia polarizada según $\mathrm{TM}^{\mathrm{z}}$, y campo magnético dispersado $\left(H_{z}^{d}\right)$ para incidencia polarizada según $\mathrm{TE}^{\mathrm{z}}$.
} 


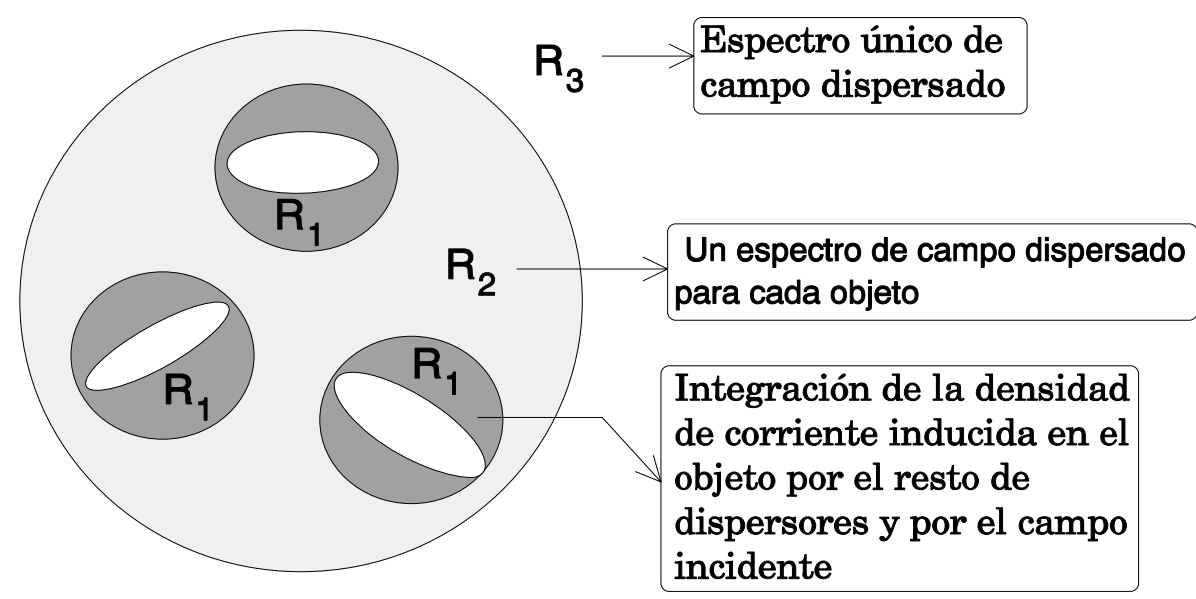

Figura 6.17: División del espacio libre en tres regiones para la reconstrucción del campo dispersado por múltiples objetos.

regiones $R_{2}$ y $R_{3}$, se obtiene implementando la siguiente expresión

$$
C_{z}^{d}(\vec{\rho})=\sum_{i=1}^{N}\left(\sum_{q=-N_{d_{i}}}^{N_{d_{i}}} c_{q}^{i} H_{q}^{(2)}\left(k \rho_{i}\right) e^{j q \phi_{i}}\right)
$$

donde $\left(\rho_{i}, \phi_{i}\right)$ son las coordenadas cilíndricas, referidas a un origen local situado normalmente en el centro del $i$-ésimo objeto, del punto $\vec{\rho}$ en el que se pretende evaluar el campo dispersado por todos los objetos. En cuanto a los límites del sumatorio interno en (6.35), éstos determinan el número de modos $\left(2 N_{d_{i}}+1\right)$ que se utilizan en la reconstrucción del campo dispersado por el $i$-ésimo objeto.

La división de la zona libre de fuentes en dos regiones ( $R_{2}$ y $R_{3}$ en fig. 6.17) se realiza porque en la región $R_{3}$, situada fuera de la circunferencia que contiene a los $N$ objetos (ver fig. 6.17), es posible reconstruir el campo dispersado por todos los objetos a partir de un único espectro de campo emergente. Este único espectro se obtiene transformando los espectros de campo dispersado por cada uno de $\operatorname{los} N$ objetos, que se refieren a sus respectivos centros, en espectros de campo emergente referidos a un mismo punto; el cual se designa como $C_{0}$, se sitúa normalmente en el punto medio de los centros de todos los objetos, y es asimismo el centro de la anterior circunferencia que separa las regiones $R_{2}$ y $R_{3}$. Estas operaciones de transformación de espectros se implementan mediante las correspondientes matrices de traslación de espectro emergente a emergente; cuyos elementos se calculan como se indica en el apéndice E (apartado E.2.2). La suma de los $N$ espectros trasladados proporciona un único espectro de campo emergente, referido por supuesto al punto $C_{0}$, que permite expresar el campo 
dispersado por todos los objetos en la región $R_{3}$ del siguiente modo

$$
C_{z}^{d}(\vec{\rho})=\sum_{q=-N_{d}}^{N_{d}}\left(\sum_{i=1}^{N} T H_{0 i} \cdot\left[c_{q}^{i}\right]\right) H_{q}^{(2)}\left(k \rho_{0}\right) e^{j q \phi_{0}}
$$

donde la matriz $T H_{0 i}$ designa en este caso la matriz de traslación de espectro emergente, referido al centro $C_{i}$ del $i$-ésimo objeto, a espectro emergente centrado en el punto $C_{0}$. Es interesante reseñar además que la reconstrucción del campo dispersado por el $i$-ésimo objeto, a partir de su nuevo espectro emergente centrado en $C_{0}$, tan sólo es válida fuera de la circunferencia con centro en dicho punto y radio la distancia entre $C_{0}$ y $C_{i}$. Por otra parte, las coordenadas cilíndricas $\left(\rho_{0}, \phi_{0}\right)$ determinan nuevamente la posición del punto $\vec{\rho}$ donde se pretende evaluar el campo dispersado por todos los objetos; aunque, en esta ocasión, dichas coordenadas se refieren a un origen global situado en el punto $C_{0}$. En cuanto al número de modos $\left(2 N_{d}+1\right)$ utilizados en (6.36) para reconstruir el campo dispersado, se satisface el criterio $N_{d}>k a$; donde $a$ representa el radio de la mínima circunferencia, centrada en el punto $C_{0}$, que contiene a $\operatorname{los} N$ objetos dispersores. La posición del punto $C_{0}$ se elige normalmente, como ya se ha comentado con anterioridad, en el punto medio de los centros de todos los objetos; con la conocida intención de minimizar el valor del radio a de la citada circunferencia, y reducir asimismo el número de modos $\left(2 N_{d}+1\right)$ requeridos.

Para finalizar, tan sólo falta por calcular el campo dispersado en unas zonas libres de fuentes; que se encuentran situadas dentro de las mínimas circunferencias que contienen a los objetos dispersores. En cada una de estas zonas, representadas en su conjunto por la región $R_{1}$ (ver fig. 6.17), no se puede utilizar el espectro de campo dispersado por el correspondiente objeto asociado a dicha zona; pues la reconstrucción de este campo dispersado a partir de su espectro sólo es válida fuera de la circunferencia que encierra al objeto. Así pues, el campo dispersado por todos los objetos en la parte de la región $R_{1}$ asociada a uno de los objetos, por ejemplo al $t$-ésimo, se obtiene superponiendo dos campos. El primero de ellos procede de la dispersión producida por el resto de objetos, exceptuando al $t$-ésimo, en la mencionada porción de la región $R_{1}$; el cual se puede calcular a partir de los espectros asociados a los campos que dispersan cada uno de estos $N-1$ objetos. El segundo de los campos a considerar procede lógicamente de la dispersión que produce el t-ésimo objeto; y su cálculo, en la región $R_{1}$, se realiza a partir de las corrientes inducidas sobre el contorno de dicho objeto. En principio, será necesario pues calcular esta distribución de corrientes inducidas; que aparecen como consecuencia del campo incidente sobre el objeto. La incidencia a considerar sobre el t-ésimo objeto se debe tanto al campo incidente original sobre todos los objetos, como a los campos dispersados por el resto de objetos ante dicho campo incidente original. La distribución de corrientes inducidas sobre el contorno del $t$-ésimo objeto, representada por el vector columna $\left[I_{n}\right]$, se obtiene multiplicando la matriz de corrientes $D I_{t}$ asociada a dicho objeto, cuyo cálculo se describe de 
forma detallada en el capítulo 4 (sección 4.2), por el correspondiente espectro de campo incidente al objeto; lo cual se resume en la siguiente expresión

$$
\left[I_{n}\right]=D I_{t} \cdot\left(M T_{t 0} \cdot\left[i_{p}\right]+\sum_{\substack{i=1 \\ i \neq t}}^{N} T_{t i} \cdot\left[c_{q}^{i}\right]\right)
$$

donde $M T_{t 0}$ designa la matriz de traslación $T J_{t 0}$, que traslada el espectro de campo incidente centrado en el punto $C_{0}$ a espectro de campo incidente centrado en el punto $C_{t}$, cuando el campo incidente está generado por una fuente externa; o bien designa la matriz de traslación $T_{t 0}$, que transforma el espectro de campo emergente referido al punto $C_{0}$ en espectro de campo incidente referido al punto $C_{t}$, cuando el campo incidente está producido por una fuente interna al grupo de objetos. En la primera situación, el punto $C_{0}$ define la posición a la que se refiere el espectro asociado al campo incidente sobre todos los objetos; mientras en el segundo caso, dicho punto $C_{0}$ indica donde se encuentra situada la fuente interna al problema bajo análisis.

Una vez obtenida la distribución de corrientes inducidas sobre el contorno del t-ésimo objeto, tal y como se recoge en (6.37), es posible expresar el campo dispersado por todos los objetos, en la porción de $R_{1}$ contenida dentro de la circunferencia asociada al t-ésimo objeto, mediante la siguiente suma

$$
C_{z}^{d}(\vec{\rho})=\sum_{\substack{i=1 \\ i \neq t}}^{N}\left(\sum_{q=-N_{d_{i}}}^{N_{d_{i}}} c_{q}^{i} H_{q}^{(2)}\left(k \rho_{i}\right) e^{j q \phi_{i}}\right)+\sum_{n=1}^{N_{s}} I_{n} C_{n}^{d}\left(\vec{\rho}_{t}\right)
$$

donde se observa que el primer sumando es idéntico a la expresión (6.35); y representa la suma de los campos dispersados por todos los objetos, exceptuando al t-ésimo, en la parte de la región $R_{1}$ mencionada anteriormente. En dicho sumando, las coordenadas cilíndricas $\left(\rho_{i}, \phi_{i}\right)$ definen la posición del punto $\vec{\rho}$, perteneciente a la citada porción de la región $R_{1}$, en el que se pretende evaluar el campo dispersado; refiriéndose dichas coordenadas a un origen local situado normalmente en el centro del $i$-ésimo objeto. En cuanto a los límites del sumatorio interno en el primer término de la expresión mostrada en (6.38), nuevamente hacen referencia al número de modos $\left(2 N_{d_{i}}+1\right)$ que se requieren en la reconstrucción del campo dispersado por el correspondiente objeto. Por otra parte, el segundo sumando en (6.38) representa la suma de los campos que generan los diferentes elementos de corriente inducidos sobre el contorno del $t$-ésimo objeto. En este sumando, para incidencia polarizada según $\mathrm{TM}^{\mathrm{z}}, C_{n}^{d}$ representa la componente en $\hat{z}$ del campo eléctrico dispersado por un hilo infinito de corriente, dirigido según 
el eje $\hat{z}$ y de amplitud unidad. Dicho campo eléctrico, recordando (4.83), se define del siguiente modo

$$
C_{n}^{d}\left(\vec{\rho}_{t}\right)=E_{z n}^{d}\left(\vec{\rho}_{t}\right)=-\frac{1}{4} \omega \mu H_{0}^{(2)}\left(k\left|\vec{\rho}_{t}-\vec{\rho}_{n}\right|\right)
$$

Sin embargo, para incidencia polarizada según $\mathrm{TE}^{\mathrm{z}}, C_{n}^{d}$ representa la componente en $\hat{z}$ del campo magnético dispersado por una fuente puntual de corriente, dirigida según el vector unitario tangente al contorno del objeto y de amplitud unidad. Recordando ahora la expresión (4.84), este campo magnético se obtiene como se indica a continuación

$$
C_{n}^{d}\left(\vec{\rho}_{t}\right)=H_{z n}^{d}\left(\vec{\rho}_{t}\right)=\frac{j}{4} k(\hat{n} \cdot \vec{R}) H_{1}^{(2)}\left(k\left|\vec{\rho}_{t}-\vec{\rho}_{n}\right|\right)
$$

donde $\hat{n}$ representa el vector unitario normal a la dirección de la fuente puntual de corriente; y $\vec{R}$ se define, recordando (4.85), de la siguiente manera

$$
\vec{R}=\frac{\vec{\rho}_{t}-\vec{\rho}_{n}}{\left|\vec{\rho}_{t}-\vec{\rho}_{n}\right|}
$$

En las expresiones $(6.39),(6.40)$ y $(6.41)$, utilizadas para calcular $C_{n}^{d}$, el vector $\overrightarrow{\rho_{t}}$ define la posición, referida al origen local del tésimo objeto, en la que se pretende evaluar el campo dispersado por los distintos elementos de corriente; mientras $\vec{\rho}_{n}$ determina la posición, referida también al citado origen local, de la $n$-ésima fuente puntual de corriente. Finalmente, $N_{s}$ representa el número total de fuentes de corriente inducidas sobre el contorno del t-ésimo objeto.

En la presente sección, a efectos de cálculo de dispersión electromagnética, se ha dividido el espacio libre en tres regiones; donde se emplean distintas expresiones para evaluar el campo dispersado por múltiples objetos. En aquella región externa a la mínima circunferencia que contiene a todos los objetos, es posible calcular el campo dispersado por dichos objetos a partir de un único espectro; el cual se obtiene sumando los espectros de campo dispersado por cada uno de los objetos, una vez que dichos espectros han sido trasladados al centro de la citada circunferencia. En la región situada dentro de la anterior circunferencia, pero fuera de las mínimas circunferencias que contienen respectivamente a los distintos objetos, el campo dispersado tan sólo se puede obtener sumando los campos que dispersan todos los objetos; que se deducen de los correspondientes espectros referidos precisamente a los centros de los objetos. Por último, en las zonas situadas dentro de las mínimas circunferencias que contienen a los objetos dispersores, el campo dispersado se obtiene sumando dos contribuciones diferentes. Por un lado, se tiene el campo dispersado por todos los objetos a excepción de uno de ellos, concretamente aquél contenido dentro de la circunferencia donde se pretende evaluar el campo dispersado. El valor de esta primera contribución se obtiene nuevamente a partir de los espectros de campo dispersado referidos a 
los centros de los correspondientes objetos. La segunda contribución se debe al propio objeto contenido en su mínima circunferencia; que se obtiene integrando los campos dispersados por los elementos de corriente inducidos sobre el contorno del citado objeto. Estos elementos de corriente son inducidos por el campo incidente original, y por los campos que dispersan los restantes objetos.

En definitiva, con las expresiones ofrecidas en esta sección se completa el presente capítulo; en el que inicialmente se han presentado varios métodos para resolver distintos problemas de dispersión electromagnética producida por múltiples objetos en espacio libre, posteriormente se han descrito tanto las limitaciones asociadas a dichos métodos como diversas acciones para evitarlas, y tras resolver los problemas se ha determinado el valor del campo dispersado por todos los objetos en cualquier región del espacio. 



\section{Capítulo 7}

\section{Ejemplos de la Dispersión Electromagnética producida por Múltiples Objetos Dispersores en Espacio Libre}

El algoritmo recursivo descrito en el capítulo 6 permite resolver, utilizando la estrategia adecuada, prácticamente cualquier problema de dispersión electromagnética producida por múltiples objetos en espacio libre. Asimismo, dicho algoritmo puede emplearse en una caracterización eficiente del comportamiento dispersor de objetos grandes en términos eléctricos; para lo cual se requiere una previa segmentación del contorno de dichos objetos en elementos más pequeños, y un análisis posterior del acoplo entre estos elementos mediante el citado algoritmo recursivo. En este capítulo, se plantean diversas situaciones con múltiples objetos dispersores; que se resuelven aplicando algunas de las estrategias planteadas en el capítulo 6 . La validez de los resultados obtenidos se confirma contrastándolos con resultados calculados mediante otras técnicas clásicas de análisis, o bien comparándolos con resultados presentes en la literatura.

En primer lugar, se ofrece un estudio de la eficiencia computacional que supone el análisis de un objeto grande en términos eléctricos, tras segmentar el contorno de dicho objeto en elementos más pequeños, mediante el citado algoritmo recursivo; con el objeto de optimizar esta técnica de análisis. Una vez realizado este estudio, dicha técnica se emplea en el análisis de tiras metálicas infinitas, con grosor despreciable, y longitud relativamente grande en términos eléctricos; garantizando la validez del análisis realizado mediante una comparación de los resultados obtenidos con aquéllos determinados mediante otras técnicas. Posteriormente, utilizando nuevamente segmentación, se analizan elementos reflectores cuyas dimensiones son grandes en términos eléctricos. Entre los reflectores analizados, se encuentran los reflectores hiperbólico y parabólico; elementos ambos 
integrantes de diversos sistemas multireflectores. En estos dispositivos, tras caracterizar su comportamiento dispersor, se verifican algunas de sus propiedades más conocidas mediante los diferentes resultados ofrecidos; demostrando pues el buen funcionamiento de la técnica escogida en su caracterización. A su vez, en el caso de los reflectores parabólicos, se ofrecen diversos diagramas de la amplitud del campo dispersado en zona de campo lejano; cuya validez se demuestra al compararlos con los mismos diagramas obtenidos mediante el Método de la Distribución en la Apertura. A continuación, después de validar completamente la técnica de análisis basada en segmentación, se ofrecen resultados correspondientes al análisis de sistemas multireflectores, concretamente antenas Cassegrain; que están constituidas, como bien es sabido, por los reflectores parabólico e hiperbólico caracterizados previamente. Finalmente, se analiza también el comportamiento dispersor de una antena tipo bocina; cuya caracterización requiere, al igual que en el análisis de los sistemas multireflectores, emplear la iteración especial del algoritmo recursivo para fuente interna al problema.

\subsection{Eficiencia Computacional de la Técnica de Análisis basada en la Segmentación del Con- torno de un Objeto Dispersor}

La segmentación del contorno de un objeto dispersor en elementos de menor tamaño, y su posterior análisis mediante el algoritmo recursivo descrito en el capítulo 6 , constituye una técnica que permite caracterizar de manera eficiente y precisa el comportamiento dispersor de objetos grandes en términos eléctricos. A su vez, esta técnica se recomienda también en el capítulo 6 (apartado 6.3.2) para evitar la segunda de las limitaciones del algoritmo recursivo presentado en dicho capítulo; debiendo segmentar en este caso el contorno del objeto dispersor cuya mínima circunferencia contiene porciones del contorno de otro objeto. En la presente sección, se ofrece un estudio de la eficiencia computacional asociada a la citada técnica de la segmentación; que, como parece lógico de antemano, dependerá del coste temporal asociado a la caracterización individual de los distintos elementos en los que se ha segmentado el contorno del objeto, y del tiempo empleado por el algoritmo recursivo en el análisis del acoplo electromagnético entre dichos elementos. A raíz de este estudio, se establece un compromiso entre ambos tiempos para reducir, al máximo posible, el coste computacional que supone el análisis completo del objeto original.

La evolución de los dos tiempos mencionados anteriormente, que determinan la eficiencia computacional de la técnica basada en la segmentación, en función del número de elementos en los que se segmenta el contorno de un objeto dispersor es contrapuesta; es decir, un aumento del número de estos segmentos reduce 
su tamaño, y por consiguiente el tiempo dedicado a su caracterización individual, aunque supone un incremento del tiempo empleado por el algoritmo recursivo. En el caso de reducir el número de dichos segmentos, se produce la situación contraria a la recién descrita. Por tanto, parece necesario buscar un compromiso entre número de segmentos y tamaño de los mismos; para de esta forma equilibrar los dos costes temporales que se terminan de mencionar, y reducir al mínimo el coste total del proceso. De cualquier manera, se consigue una mayor eficiencia computacional segmentando el contorno del objeto en un número de elementos simples idénticos, como por ejemplo tiras metálicas de grosor infinitesimal, que se ajusten bien al contorno del objeto bajo análisis; pues de esta forma sólo es necesario caracterizar de manera precisa a uno solo de estos elementos, al ser válida su matriz de caracterización individual para el resto de elementos tras implementar sobre dicha matriz las correspondientes operaciones de giro (ver apéndice D). La segmentación del contorno de cualquier objeto en tiras metálicas iguales, que definan con precisión el contorno original del objeto, requiere en algunas ocasiones escoger un número elevado de tiras que permita ajustar localmente la forma original del contorno; lo que sin duda dilatará un poco más el tiempo empleado por el algoritmo recursivo, al tener que analizar un número elevado de elementos individuales. No obstante, se prefiere disponer de elementos idénticos; dado el enorme ahorro temporal que supone caracterizar individualmente a uno solo de dichos elementos.

Con el objeto de evaluar la eficiencia computacional de la técnica basada en segmentación, se ha analizado un ejemplo bastante sencillo; consistente en caracterizar el comportamiento dispersor de una tira metálica infinita, de grosor despreciable y longitud $4 \lambda$, ante incidencia polarizada según $\mathrm{TM}^{\mathrm{z}}$ y según $\mathrm{TE}^{\mathrm{z}}$. En dicho análisis, la tira metálica original se ha segmentado en un número $(N)$ de subtiras (elementos) de igual tamaño; variando $N$ desde 1 hasta 30 para incidencia $\mathrm{TM}^{\mathrm{z}}$, y desde 1 hasta 40 para incidencia TE ${ }^{\mathrm{z}}$. Para cada uno de estos valores de la variable $N$, en la figura 7.1 (apartados a y b) se representan los tiempos de computación asociados a la caracterización individual de las subtiras, y a la resolución del problema de acoplo electromagnético entre dichos elementos; así como la suma de ambos tiempos, que determina el tiempo total empleado en la caracterización de la tira mediante la técnica de la segmentación. Todos estos tiempos se han determinado ejecutando en un $\mathrm{PC}$, con procesador 486-DX4 a $100 \mathrm{MHz}$ y $8 \mathrm{MB}$ de RAM, programas escritos en lenguaje $\mathrm{C}++$ compilados con GNU C ++ . En cuanto al coste computacional relacionado con la caracterización individual de las subtiras, al ser todas de igual tamaño, dicho coste representa únicamente el asociado al cálculo de la matriz de caracterización individual de una sola subtira; ya que en el presente caso esta matriz es la misma para el resto de subtiras. Asimismo, en la obtención de la matriz de caracterización individual de la subtira, debido a la precisión numérica que se desea obtener en los resultados, se ha utilizado el Método de los Momentos para calcular la matriz 


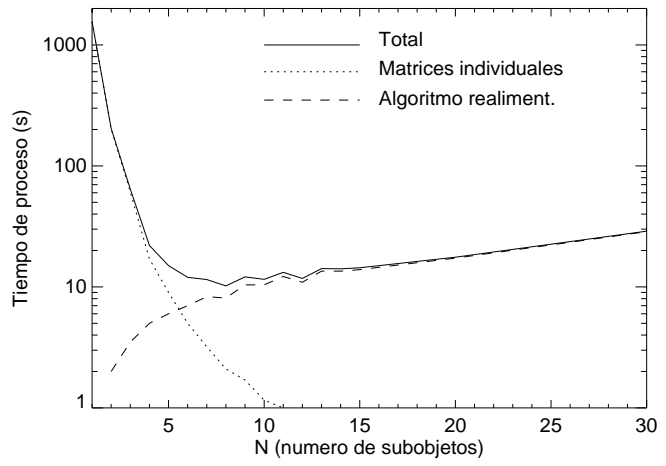

a)

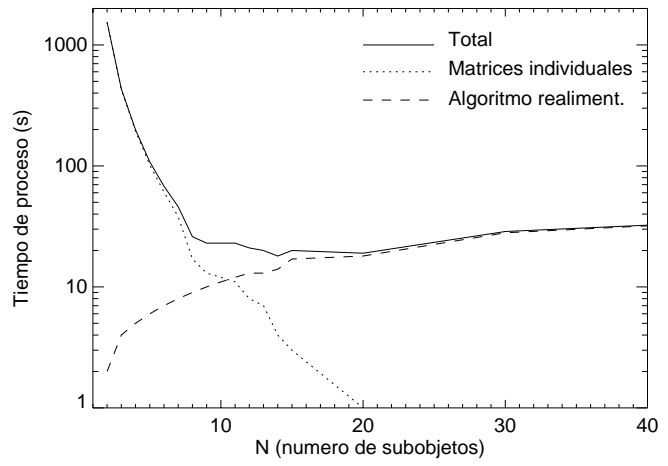

b)

Figura 7.1: Tiempos de cálculo en la caracterización de una tira metálica infinita, de longitud $4 \lambda$, ante incidencia polarizada según $\mathrm{TM}^{\mathrm{z}}$ en a); y ante incidencia polarizada según $\mathrm{TE}^{\mathrm{z}}$ en $\mathrm{b}$ ).

de corrientes asociada a dicha matriz de caracterización individual de la subtira (recordar apartado 4.2 del capítulo 4).

De manera general, tanto para incidencia $\mathrm{TM}^{\mathrm{z}}$ como $\mathrm{TE}^{\mathrm{z}}$, se observa en la figura 7.1 que el tiempo de computación asociado a la caracterización individual de las subtiras decrece, como cabía esperar, a medida que aumenta el número $N$ de subtiras en que se divide la tira original; pues el tamaño de la subtira sobre la que aplicar el Método de los Momentos es cada vez menor. El comportamiento de este tiempo de computación evoluciona, en función de $N$, de la forma $O\left(N^{-3}\right)$; ya que la complejidad del Método de los Momentos empleado en la caracterización individual de la subtira es proporcional, tal y como se recoge en [80], al cubo del número de incógnitas a resolver por dicho método, y por tanto inversamente proporcional al número de subtiras elevado al cubo. Sin embargo, el tiempo de cálculo que emplea el algoritmo recursivo en resolver el acoplo electromagnético entre $N$ subtiras es directamente proporcional, como se desprende de los resultados mostrados en la figura 7.1, a dicho número $N$ de elementos individuales a caracterizar conjuntamente. De los resultados ofrecidos en la figura 7.1 también se concluye, para ambos tipos de polarización $\left(\mathrm{TM}^{\mathrm{z}}\right.$ y $\mathrm{TE}^{\mathrm{z}}$ ) en la incidencia, el enorme ahorro en tiempo de computación que supone el empleo de la técnica de análisis de objetos grandes basada en la segmentación del contorno de dichos objetos; puesto que, en ambos casos, se observa un factor de mejora mayor que 100 entre los tiempos totales empleados en la caracterización de la tira mediante segmentación y sin ella.

Observando el caso de incidencia polarizada según TM (apartado a en fig. 7.1), se descubre que para un número $N$ de subtiras inferior a 5 , es decir subtiras de longitud mayor que $0.8 \lambda$, el efecto predominante en el tiempo total de proceso está relacionado con la caracterización individual de cada subtira; pues la dimen- 
sión de la subtira es bastante grande, y se requiere la resolución de un número elevado de incógnitas mediante el Método de los Momentos. Por contra, cuando el número $N$ de subtiras a caracterizar de forma conjunta es mayor que 12, debido a que su tamaño es inferior a $0.33 \lambda$, se constata que la eficiencia computacional de la técnica basada en segmentación está dominada por el tiempo que emplea el algoritmo recursivo en resolver las interacciones múltiples entre las diversas subtiras; ya que el número de elementos a caracterizar conjuntamente empieza a ser considerable, mientras el tamaño de cada objeto individual determina un número de incógnitas a resolver mediante el Método de los Momentos que es realmente bajo. En resumen, segmentando el contorno original de la tira en un número $N$ de subtiras comprendido entre 5 y 12 , por ejemplo en subtiras de longitud $0.5 \lambda$, se obtiene un mínimo (ver apartado a en fig. 7.1) en el tiempo total empleado en la caracterización del comportamiento dispersor de la tira metálica original empleando segmentación. Eligiendo pues el número óptimo de subtiras, el tiempo total de cálculo se reduce de un valor ligeramente superior a $1000 \mathrm{seg}$, obtenido caracterizando a la tira original sin segmentación mediante el Método de los Momentos, a aproximadamente un valor de $10 \mathrm{seg}$ mediante una segmentación de la tira original en 8 subtiras; sin que ello suponga, tal y como se comprueba en la próxima sección de este capítulo, apenas pérdida alguna de precisión en los resultados finales obtenidos.

A continuación, considerando el caso de incidencia polarizada según $\mathrm{TE}^{\mathrm{z}}$ (apartado b en fig. 7.1), se comprueba que la evolución de los tiempos de computación con el número $N$ de subtiras es similar a la deducida para el otro tipo de polarización; aunque, tras comparar los dos apartados de la figura 7.1, se descubre que el tiempo dedicado a la caracterización individual de una subtira ante incidencia $\mathrm{TE}^{\mathrm{z}}$ es bastante mayor que ante incidencia $T \mathrm{M}^{\mathrm{z}}$. No obstante, esta diferencia de tiempos era de esperar; puesto que al caracterizar una tira metálica de grosor infinitesimal ante incidencia $\mathrm{TE}^{\mathrm{z}}$, utilizando el Método de los Momentos, es necesario considerar un cierto grosor en la tira (recordar sección 5.4 del capítulo 5), lo que implica duplicar al menos el número de incógnitas a resolver por el citado método. En general, este incremento en el tiempo de caracterización individual de una elemento ante incidencia $\mathrm{TE}^{\mathrm{z}}$ se traduce, de manera consecuente, en un mayor coste computacional de la técnica de análisis basada en segmentación para este tipo de incidencia; lo cual ya ocurría en la caracterización completa de un objeto dispersor, mediante el Método de los Momentos, sin realizar una previa segmentación del contorno de dicho objeto. En el caso concreto que nos ocupa, dicho incremento temporal supone un cambio en el valor óptimo del número de subtiras en las que debe dividirse la tira original; tal y como puede observarse en la figura 7.1 (apartado b). Así pues, dividiendo el contorno de la tira en un número $N$ de subtiras comprendido entre 10 y 20, por ejemplo en 14 subtiras, se alcanza un valor mínimo para el tiempo total empleado en la caracterización de la tira original, ante incidencia $\mathrm{TE}^{\mathrm{z}}$, empleando segmentación. Este valor mínimo 
corresponde a un tiempo de aproximadamente $18 \mathrm{seg}$; que comparado con el tiempo de caracterización de la tira original utilizando el Método de los Momentos sin segmentación, de valor mayor que $2000 \mathrm{seg}$, supone un factor de mejora por encima de 100 en la eficiencia computacional que se consigue con la técnica basada en segmentación. No obstante, la aplicación de esta técnica basada en segmentación a problemas de dispersión electromagnética, cuando la incidencia es del tipo $\mathrm{TE}^{\mathrm{z}}$, puede suscitar dudas sobre la precisión de los resultados obtenidos; pues, ante este tipo de incidencia, los elementos de corriente inducidos sobre el contorno del objeto son tangentes al mismo, y por tanto al segmentar dicho contorno se interrumpe físicamente la continuidad de la corriente. Los resultados ofrecidos en la próxima sección para una tira metálica segmentada ante incidencia $\mathrm{TE}^{\mathrm{z}}$, así como los recogidos en las siguientes secciones para otros objetos también segmentados y ante el mismo tipo de incidencia, revelan que la interrupción física de la corriente producida por la segmentación del contorno del objeto apenas influye en la precisión de los resultados obtenidos; ya que éstos son muy parecidos a los deducidos mediante otras técnicas clásicas de análisis.

En la presente sección, se ha estudiado la eficiencia computacional de la técnica de análisis de objetos dispersores grandes en términos eléctricos basada en la segmentación del contorno de dichos objetos. Este estudio, realizado para un caso sencillo constituido por una tira metálica infinita de grosor despreciable, ha revelado que la técnica basada en segmentación reduce enormemente el tiempo empleado en la caracterización del comportamiento dispersor del objeto; tanto para incidencia con polarización $\mathrm{TM}^{\mathrm{z}}$ como $\mathrm{TE}^{\mathrm{z}}$. Debido a esta razón, en los problemas constituidos por múltiples objetos dispersores, cuyos resultados se ofrecen en las próximas secciones, se ha empleado en todos ellos una adecuada segmentación en tiras metálicas del contorno de los objetos que integran dichos problemas. Asimismo, en el ejemplo de la tira metálica considerado en esta sección, se ha determinado para ambos tipos de incidencia el número óptimo de subtiras, y por tanto la longitud de las mismas, en las que debe dividirse la tira original para reducir al mínimo el esfuerzo computacional en la caracterización de dicha tira mediante segmentación. Estos resultados pueden exportarse también, con ciertas precauciones, al análisis de otros problemas constituidos por múltiples objetos dispersores; pues la dimensión óptima de las tiras en las que debiera segmentarse el contorno de los objetos estará próxima, con toda probabilidad, al valor deducido para el caso de la tira analizada en esta sección.

\subsection{Múltiples Tiras Metálicas Infinitas ante In- cidencia $\mathrm{TM}^{z}$ y $\mathrm{TE}^{z}$}

Con el objeto de validar la técnica basada en segmentación para analizar objetos grandes en términos eléctricos, así como las diferentes versiones del algoritmo re- 
cursivo mostradas en el capítulo 6 para analizar el acoplo electromagnético entre múltiples objetos dispesores, en esta sección se presentan resultados correspondientes al análisis de la dispersión que producen múltiples tiras metálicas infinitas, de grosor despreciable, ante incidencia $\mathrm{TM}^{\mathrm{z}}$ y $\mathrm{TE}^{\mathrm{z}}$.

Así pues, en primer lugar se ha caracterizado el comportamiento dispersor de una tira metálica infinita, de grosor despreciable y longitud grande en términos eléctricos, ante incidencia polarizada según $\mathrm{TM}^{\mathrm{z}}$ y según $\mathrm{TE}^{\mathrm{z}}$. Para caracterizar este objeto dispersor, se ha empleado la técnica cuya eficiencia computacional ha sido examinada en la sección anterior; que en este caso consiste en segmentar la tira en elementos de menor dimensión, y posteriormente aplicar la versión original del algoritmo recursivo (recordar sección 6.1 del capítulo 6) para caracterizar las interacciones entre dichos elementos. Una vez se obtienen los resultados de este primer ejemplo, éstos se comparan con los recogidos en la literatura; en cuyo cálculo se utilizan otras técnicas clásicas que también permiten analizar problemas de dispersión electromagnética. Por otra parte, en segundo lugar se ofrecen resultados correspondientes a la caracterización del comportamiento dispersor, únicamente ante incidencia con polarización $\mathrm{TM}^{\mathrm{z}}$, de dos tiras metálicas infinitas, de grosor despreciable y con longitudes relativamente grandes, que se encuentran bastante separadas entre sí. En el análisis de esta situación, se ha empleado nuevamente segmentación de cada tira en subtiras más pequeñas; pero en esta ocasión, debido a que los dos grupos de subtiras se encuentran alejados entre sí, se ha utilizado la versión refinada del algoritmo recursivo para análisis de grupos de objetos dispersores (recordar sección 6.2 del capítulo 6). En este caso, el mismo problema se analiza también sin segmentar las tiras originales, empleando pues la versión original del algoritmo recursivo aplicada a dos objetos dispersores; cuyos resultados sirven como referencia para comparar los obtenidos anteriormente, y confirmar pues la validez de la versión del algoritmo recursivo para grupos de objetos dispersores.

\subsubsection{Una Tira Metálica Infinita Grande en Términos Eléc- tricos ante Incidencia $\mathrm{TM}^{\mathrm{z}}$ y $\mathrm{TE}^{\mathrm{z}}$}

En el presente apartado, se ofrecen resultados correspondientes al análisis de tiras metálicas infinitas, de grosor despreciable, cuyas dimensiones son grandes en términos eléctricos. La técnica empleada para caracterizar el comportamiento dispersor de cada tira, debido a su dimensión, se basa en la segmentación del contorno de la tira en elementos más pequeños; y en el posterior análisis del acoplo electromagnético entre estos nuevos elementos, que definen la tira, mediante la versión original del algoritmo recursivo descrito en el capítulo 6 (sección 6.1 ). Esta técnica basada en segmentación, cuya eficiencia computacional ha quedado demostrada en la sección 7.1, se ha aplicado a la caracterización de las mencionadas tiras tanto para incidencia $\mathrm{TM}^{\mathrm{z}}$ como $\mathrm{TE}^{\mathrm{z}}$; con la intención final de poder 


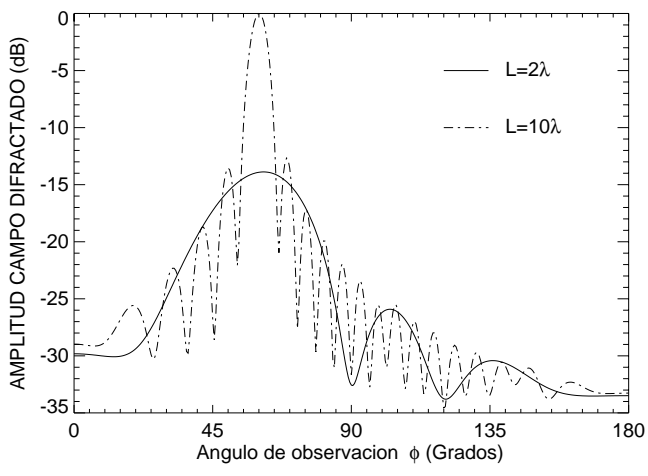

a)

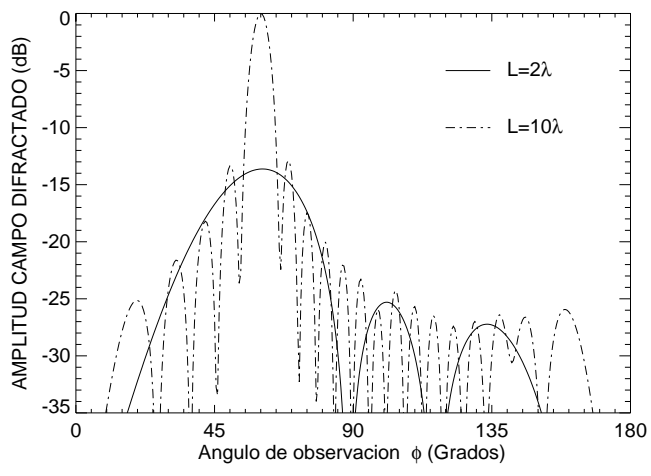

b)

Figura 7.2: Amplitud del campo eléctrico dispersado en zona de campo lejano por dos tiras metálicas infinitas, de grosor despreciable y longitudes $2 \lambda$ y $10 \lambda$ respectivamente, ante la incidencia de una onda plana $(\beta=-\pi / 3)$ polarizada según $\mathrm{TM}^{\mathrm{z}}$ en a); y polarizada según $\mathrm{TE}^{\mathrm{z}}$ en $\mathrm{b}$ ).

validar dicha técnica, para ambos tipos de incidencia, mediante una comparación cualitativa de los resultados obtenidos con aquéllos mostrados en la literatura.

Así pues, se ha caracterizado el comportamiento dispersor de dos tiras metálicas infinitas, de grosor despreciable y longitudes $2 \lambda$ y $10 \lambda$ respectivamente, ante incidencia polarizada según $\mathrm{TM}^{\mathrm{z}}$ y según $\mathrm{TE}^{\mathrm{z}}$. Para ambos tipos de polarización, las tiras se han segmentado en idénticas subtiras de longitud $\lambda / 2$; en cuya caracterización individual se ha utilizado el Método de los Momentos. La caracterización conjunta de todas las subtiras se ha realizado, tal y como se describe en la sección 6.1 del capítulo 6 , incorporando una nueva subtira en cada iteración del algoritmo basado en teoría de grafos. Una vez caracterizado el comportamiento dispersor de ambas tiras, orientadas en la dirección del eje $\hat{x}$, se ha determinado la amplitud del campo dispersado por cada una de las tiras, en zona de campo lejano, ante la incidencia de una onda plana $(\beta=-\pi / 3)$ polarizada según $\mathrm{TM}^{\mathrm{z}}$ y según $\mathrm{TE}^{\mathrm{z}}$. Los resultados correspondientes a ambas polarizaciones se ofrecen en la figura 7.2 (apartados a y b respectivamente); donde se observa en todos los casos, como era previsible, un máximo en la amplitud del campo dispersado para la dirección especular $(\beta=\pi / 3)$. Los resultados mostrados en la figura 7.2 se encuentran normalizados, para cada tipo de polarización de la onda incidente, al máximo de amplitud del campo dispersado en zona de campo lejano; que corresponde, en ambos casos, al valor de la amplitud asociada al campo dispersado en la mencionada dirección especular por la tira de mayor longitud.

Para validar la técnica de análisis empleada al obtener los resultados de la figura 7.2, éstos pueden compararse en términos cualitativos con los recogidos en [69, pp. 583 y 586]; donde se representa el ancho de dispersión biestático o sección recta radar biestática por unidad de longitud, magnitud correspondiente 
al concepto de la sección recta radar (RCS) en problemas bidimensionales [69], para las mismas tiras e incidencias consideradas en este apartado. Estos resultados, ofrecidos en [69, pp. 583 y 586], se calculan a partir del campo dispersado por cada tira en zona de campo lejano; el cual se determina integrando los campos que generan las corrientes inducidas por la onda plana incidente sobre el contorno de la tira, deducidas mediante la aproximación de Óptica Física. Comparando pues, en términos cualitativos, estos resultados con los mostrados en la figura 7.2, se observa una gran similitud entre ellos; tanto en la forma de las gráficas, como en el número de lobulaciones que presenta cada una de ellas y en los niveles relativos entre los distintos lóbulos. Por tanto, se puede concluir que la segmentación del contorno de un objeto en elementos de menor tamaño, junto con la versión original del algoritmo recursivo presentado en el capítulo 3 , constituyen una técnica que permite analizar correctamente objetos grandes en términos eléctricos; resultando válida tanto para incidencia $\mathrm{TM}^{\mathrm{z}}$ como $\mathrm{TE}^{\mathrm{z}}$.

\subsubsection{Dos Tiras Metálicas Infinitas Alejadas ante Inciden- cia $\mathrm{TM}^{\mathrm{z}}$}

Una vez se ha comprobado en el apartado anterior que la técnica basada en segmentación, haciendo uso de la versión original del algoritmo recursivo descrita en el capítulo 6 (sección 6.1), proporciona resultados correctos; en este apartado, se pretende demostrar la validez de los resultados generados mediante la versión refinada del citado algoritmo, recogida en la sección 6.2 del capítulo 6 , que permite analizar conjuntamente agrupaciones de objetos dispersores. Para ello, se ha escogido un problema sencillo; constituido por dos tiras metálicas infinitas bastante alejadas entre sí, de grosor despreciable, e inclinadas $-30^{\circ}$ y $30^{\circ}$ respecto del eje $\hat{x}$ como se indica en la figura 7.3. En un primer análisis, el comportamiento dispersor de ambas tiras se pretende caracterizar conjuntamente, ante incidencia polarizada según $\mathrm{TM}^{\mathrm{z}}$, mediante una previa segmentación de cada tira en tres subtiras (ver apartado a en fig. 7.3); para a continuación aplicar la versión del algoritmo recursivo que permite caracterizar conjuntamente grupos de objetos dispersores, en este caso los dos grupos constituidos respectivamente por tres subtiras. Posteriormente, el mismo problema se resuelve sin segmentación de las tiras; aplicando únicamente la versión original del algoritmo recursivo, validada en el apartado anterior, a la caracterización conjunta de las dos tiras. De esta forma, se dispone de unos resultados con los que poder comparar aquéllos obtenidos mediante segmentación; y poder confirmar pues que la versión del algoritmo recursivo para agrupaciones de objetos genera también resultados correctos.

La estrategia de análisis del problema constituido por las dos tiras, mediante una segmentación de cada una de ellas en tres subtiras, se recoge gráficamente en la figura 7.3 (apartado a); donde se observa claramente la formación de dos grupos de objetos dispersores, integrados respectivamente por las tres subtiras 


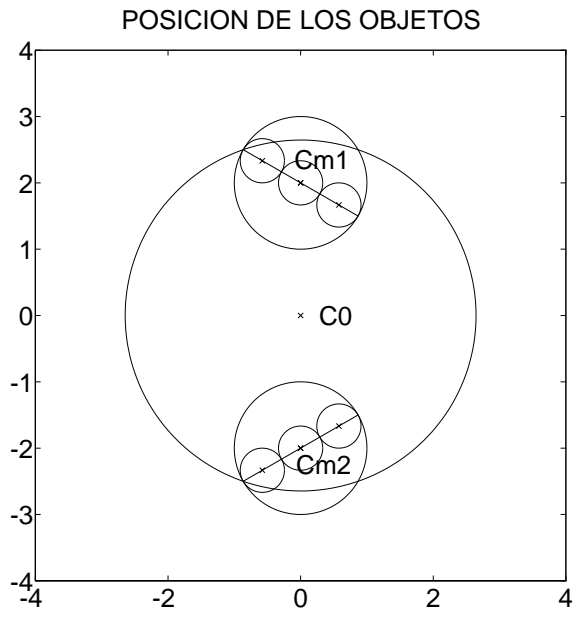

a)

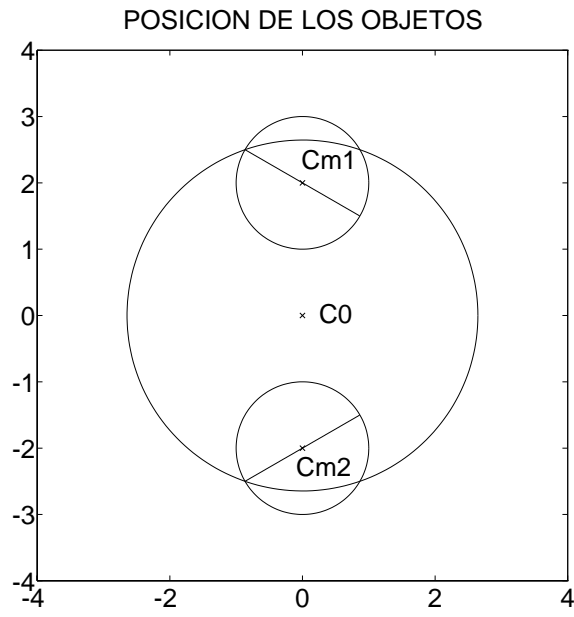

b)

Figura 7.3: Estrategia de análisis de la dispersión producida por dos tiras metálicas, alejadas entre sí, mediante su previa segmentación en dos grupos de objetos dispersores en a); y sin segmentación en b).

que definen cada tira. La razón de este agrupamiento de objetos obedece a la separación entre las dos tiras metálicas; que impide aplicar correctamente la versión original del algoritmo recursivo a la caracterización conjunta de las seis subtiras, pues dicha versión incurre en la primera de las limitaciones del algoritmo (recordar apartado 6.3.1). Así pues, la solución a este problema consiste en utilizar la versión del algoritmo recursivo para caracterización conjunta de agrupaciones de objetos dispersores; que en este caso requiere caracterizar previamente, mediante la versión original del algoritmo recursivo, el comportamiento dispersor conjunto de las tres subtiras integrantes de cada grupo, y analizar finalmente el acoplo electromagnético entre dichos grupos. La elección de los centros de cada grupo, denominados $C_{m_{1}}$ y $C_{m_{2}}$, así como la elección del centro de todos los objetos, designado como $C_{0}$, se realiza con la intención de minimizar los radios de las circunferencias, centradas en dichos puntos, que contienen a los correspondientes objetos; tal y como se muestra en la figura 7.3 (apartado a). De esta manera, se minimiza el número de modos a emplear en la correcta reconstrucción del campo incidente sobre los dos grupos, y sobre todos los objetos del problema bajo análisis; reduciendo el esfuerzo computacional del método de análisis empleado. Asimismo, como puede observarse en la figura 7.3, ninguna de las circunferencias que contienen a los objetos de cada grupo incluye el centro de algún objeto del otro grupo; por lo que no se incumple la primera de las limitaciones del algoritmo recursivo. Por otra parte, se ha escogido el mismo tamaño para todas las subtiras que integran el problema bajo análisis; pues de esta forma sólo es necesario 


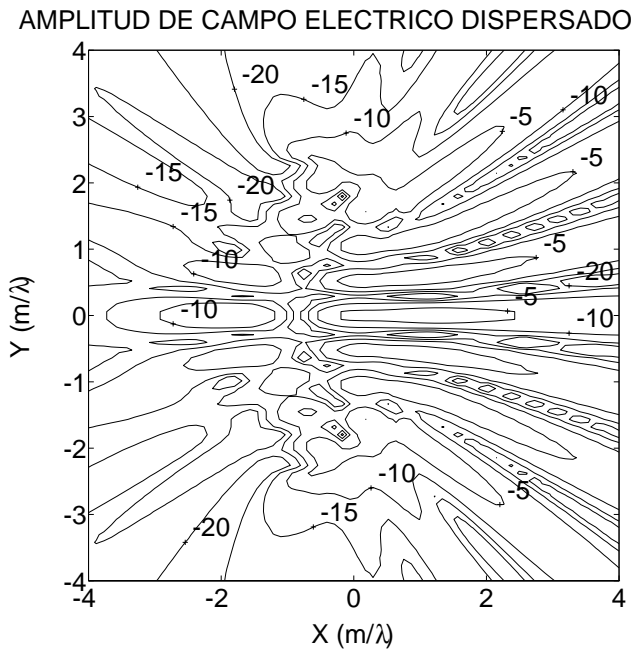

a)

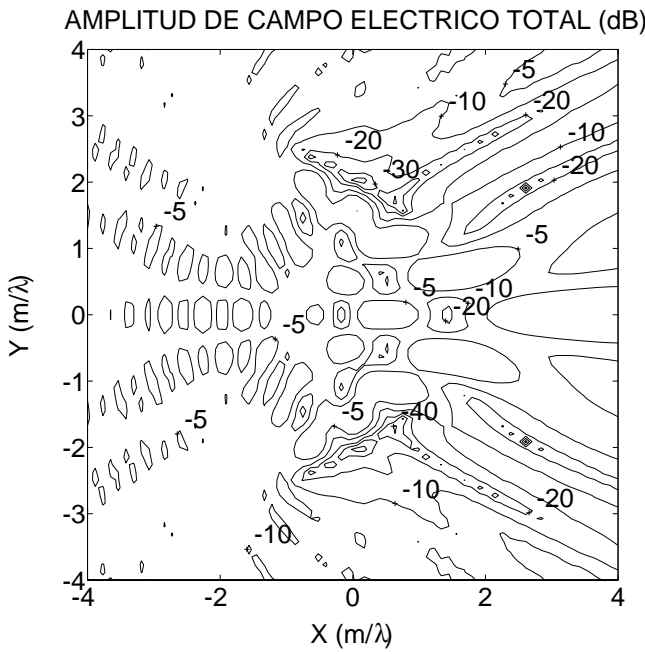

c)

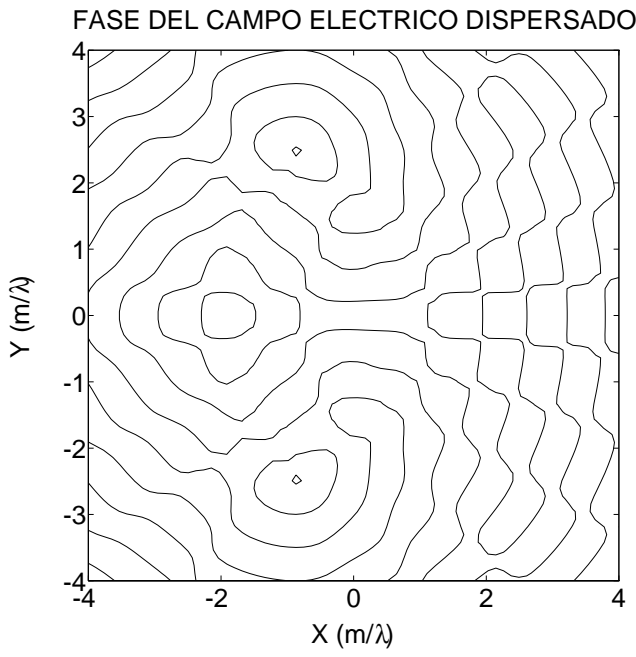

b)

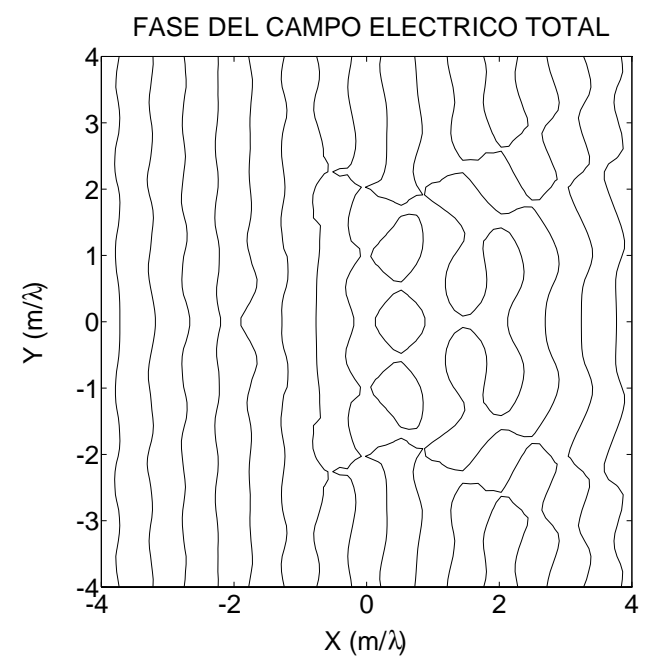

d)

Figura 7.4: Amplitud y fase del campo eléctrico dispersado por dos tiras metálicas, obtenido mediante segmentación de cada tira en tres subtiras, ante la incidencia de una onda plana $(\beta=0)$ en a) y en b); y amplitud y fase del campo eléctrico total, definido como la suma de los anteriores campos eléctricos incidente y dispersado, en c) y en d).

calcular la matriz de caracterización individual de una subtira, y obtener las del resto implementando sobre dicha matriz las correspondientes operaciones de giro.

Tras determinar las matrices de caracterización conjunta de las seis subtiras que integran el problema bajo análisis, mediante la estrategia descrita en el párrafo anterior; se ha calculado la dispersión que producen las dos tiras metálicas ante la incidencia de una onda plana, polarizada según $\mathrm{TM}^{\mathrm{z}}$, con dirección de 


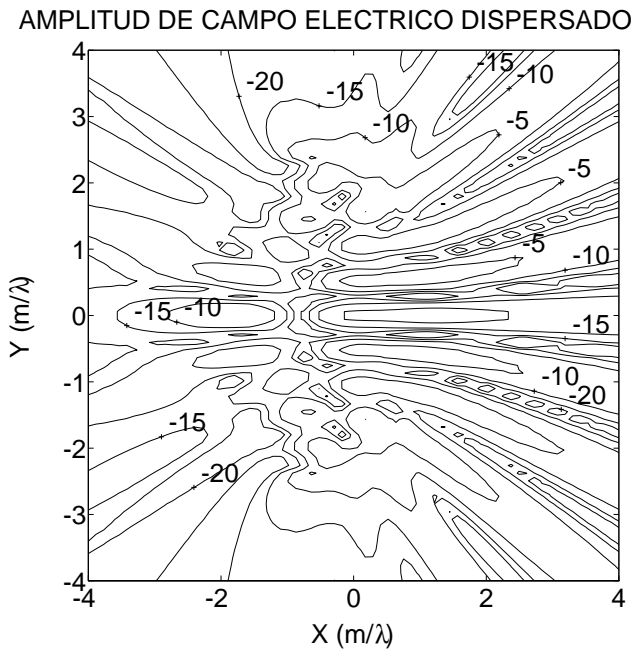

a)

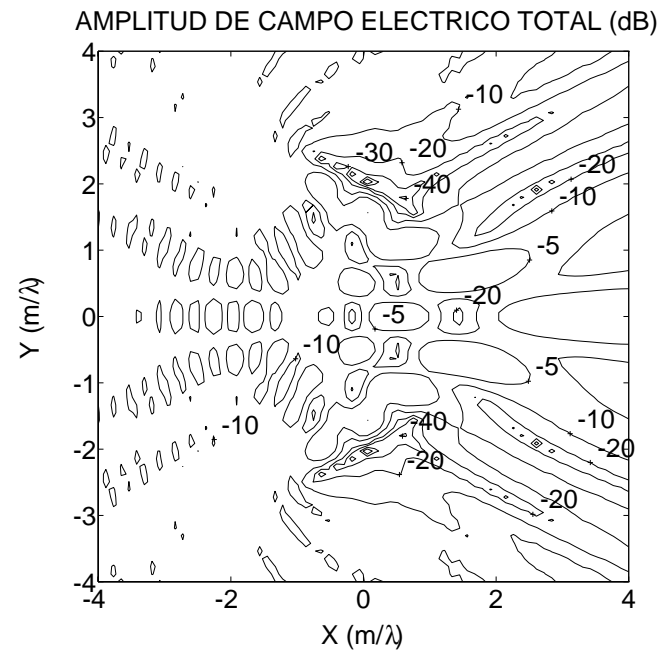

b)

Figura 7.5: Amplitud del campo eléctrico dispersado por dos tiras metálicas, obtenido sin segmentación, ante la incidencia de una onda plana $(\beta=0)$ en a); y amplitud del campo eléctrico total, definido como la suma de los anteriores campos eléctricos incidente y dispersado, en b).

avance $\beta=0$. La amplitud y la fase del campo eléctrico dispersado por las dos tiras, ante la citada incidencia, se representan en la figura 7.4 (apartados a y b); donde se observa, en el caso de la amplitud, la existencia de una onda estacionaria entre las dos tiras. Por otra parte, ante la misma onda plana incidente, nuevamente en la figura 7.4 (apartados c y d) se ofrece la amplitud y la fase del campo eléctrico total; definido como la suma del campo eléctrico incidente y del campo eléctrico dispersado. En este caso, en la gráfica correspondiente a la amplitud del campo eléctrico total se descubren dos conos de difracción, situados tal y como cabía esperar detrás de las dos tiras. Además, en dicha figura 7.4 (apartado c) se detecta también un máximo de campo eléctrico total en el punto medio de la zona comprendida entre las dos tiras; zona donde se produce una concentración de campo eléctrico total.

Para confirmar la validez de los resultados mostrados en la figura 7.4, se ha resuelto el mismo problema de las dos tiras metálicas sin realizar ningún tipo de segmentación; siguiendo en este caso una estrategia de análisis, recogida gráficamente en la figura 7.3 (apartado b), que se basa en la caracterización conjunta de las dos tiras haciendo uso de la versión original del algoritmo recursivo. Nuevamente, la elección de los centros $C_{m_{1}}, C_{m_{2}}$ y $C_{0}$, con diferentes significados a los ofrecidos en el análisis anterior, se realiza con la intención de minimizar los diferentes números de modos que requiere la correcta reconstrucción, en este nuevo análisis, de los distintos campos incidentes. De igual manera, observando 
todas las circunferencias dibujadas en la figura 7.3 (apartado b), se comprueba que la aplicación de la citada versión original del algoritmo recursivo no incurre en ninguna de sus posibles limitaciones (recordar sección 6.3). Así pues, tras caracterizar las dos tiras metálicas mediante el método recién expuesto, se ha determinado la respuesta de dichas tiras ante la misma incidencia considerada anteriormente; es decir, ante la incidencia de una onda plana, con dirección de avance $\beta=0$, y polarizada según $\mathrm{TM}^{\mathrm{z}}$. La amplitud asociada al campo eléctrico dispersado en dicha situación, así como la amplitud correspondiente al campo eléctrico total, se muestran en la figura 7.5 (apartados a y b); que resultan muy parecidas, prácticamente idénticas, a las respectivas amplitudes representadas en la figura 7.4 (apartados a y c). De esta comparación, al haber comprobado en el apartado anterior la validez de los resultados generados por la versión original del algoritmo recursivo, es posible concluir que la versión refinada de dicho algoritmo para agrupaciones de objetos dispersores es igualmente válida; y que por tanto proporciona también resultados correctos.

\subsection{Reflectores ante Incidencia $\mathrm{TM}^{z}$ y $\mathrm{TE}^{z}$}

Tras comprobar en la sección 7.1 la eficiencia computacional asociada a la técnica de análisis basada en segmentación, y haber confirmado en la sección 7.2 la validez de los resultados obtenidos con dicha técnica; en la presente sección, utilizando la citada técnica, se ofrecen resultados de la dispersión electromagnética producida por reflectores cuyas dimensiones son grandes en términos eléctricos. Concretamente, se ha caracterizado el comportamiento dispersor de un reflector hiperbólico y de dos reflectores parabólicos; habiendo empleado en su caracterización, una vez segmentado el contorno de los reflectores en tiras de igual longitud, la versión original del algoritmo recursivo descrita en el capítulo 6 (sección 6.1).

En primer lugar, se ha analizado la dispersión electromagnética producida por un reflector hiperbólico, grande en términos eléctricos, ante incidencia con polarización $\mathrm{TM}^{\mathrm{z}}$; ofreciendo resultados que concuerdan con aquéllos previstos a priori para la incidencia escogida. Posteriormente, se ha caracterizado un reflector parabólico, de dimensión también grande desde el punto de vista eléctrico, ante incidencia polarizada según $\mathrm{TM}^{\mathrm{z}}$. En este caso, se muestran diversos resultados ante distintos campos eléctricos incidentes; permitiendo corroborar algunas de las propiedades dispersoras más interesantes de los reflectores parabólicos. Por último, se ha estudiado la dispersión producida por un nuevo reflector parabólico, cuya dimensión sigue siendo grande eléctricamente, pero ante incidencia polarizada según $\mathrm{TE}^{\mathrm{z}}$. Asimismo, para los dos reflectores parabólicos considerados, se ha determinado la amplitud de los campos dispersados por estos dos reflectores en zona de campo lejano, y se han comparado dichos resultados con los obtenidos mediante el conocido Método de la Distribución en la Apertura; validando nueva- 
mente, tanto para incidencia $\mathrm{TM}^{\mathrm{z}}$ como $\mathrm{TE}^{\mathrm{z}}$, la técnica empleada al caracterizar el comportamiento dispersor de ambos reflectores parabólicos.

\subsubsection{Reflector Hiperbólico ante Incidencia $\mathrm{TM}^{\mathrm{z}}$}

En este primer apartado, se analiza la dispersión electromagnética producida por un reflector hiperbólico; elemento que constituye el reflector secundario del tipo de antenas Cassegrain estudiadas en la sección 7.4. Concretamente, se ha caracterizado el comportamiento dispersor, ante incidencia polarizada según $\mathrm{TM}^{\mathrm{z}}$, de un reflector hiperbólico con un diámetro $D$ igual a $4 \lambda$, una excentricidad $e$ de valor 2 , y una distancia focal $c$ con valor igual a $\lambda$. En la caracterización de este reflector, debido a las razones de eficiencia computacional expuestas en la sección 7.1, se aproxima el contorno de la hipérbola por una serie de tiras metálicas infinitas, de grosor despreciable e igual longitud, cuya caracterización conjunta se realiza mediante el algoritmo recursivo descrito en el capítulo 6.

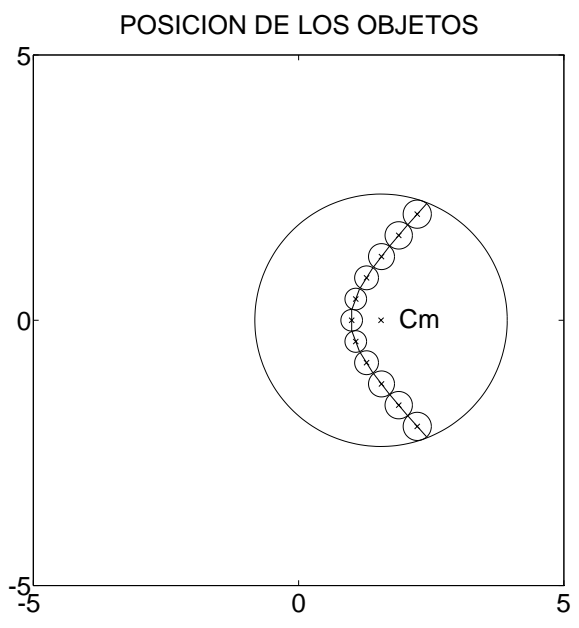

Figura 7.6: Estrategia de análisis de la dispersión producida por un reflector hiperbólico $(c=\lambda, e=2, D=4 \lambda)$, aproximando su contorno mediante 11 tiras de igual longitud.

Así pues, la estrategia de análisis empleada en la caracterización del reflector hiperbólico, recogida gráficamente en la figura 7.6, consiste en una aproximación del contorno de dicho reflector mediante 11 tiras metálicas de igual longitud; cuyo comportamiento dispersor conjunto puede analizarse, observando la figura 7.6, mediante la versión original del algoritmo recursivo recogida en la sección 6.1 del capítulo 6. Para aplicar correctamente esta versión del algoritmo recursivo, que incorpora un nuevo objeto en cada iteración, tan sólo resulta necesario determinar de forma adecuada el orden de incorporación de cada tira al grupo previamente 


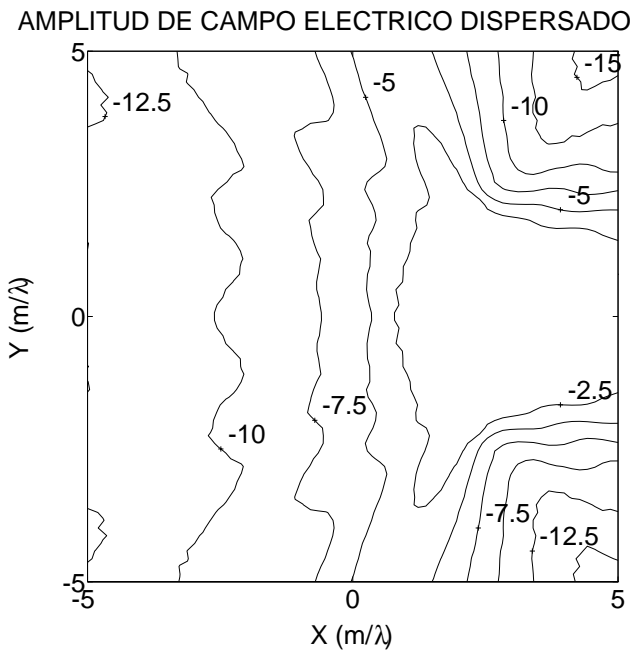

a)

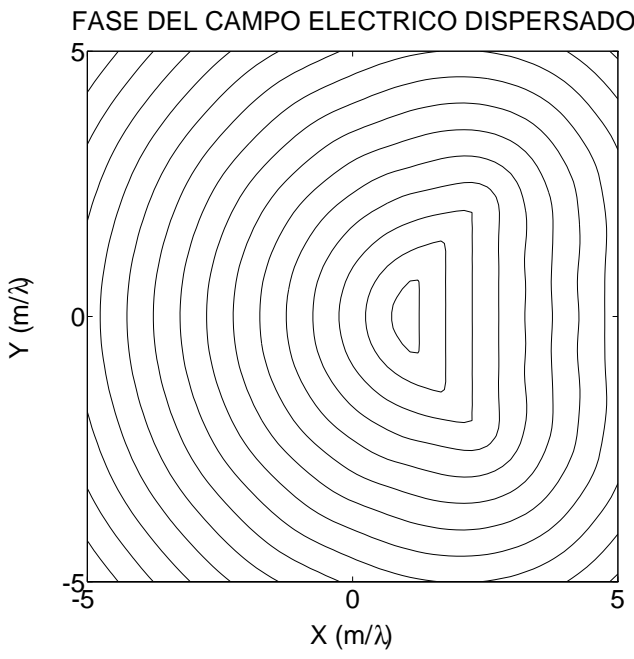

b)

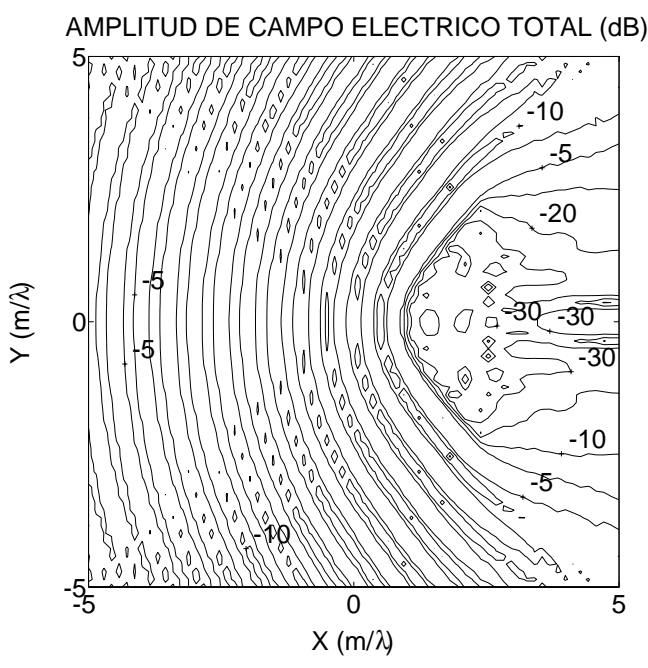

c)

Figura 7.7: Amplitud y fase del campo eléctrico dispersado por un reflector hiperbólico $(c=\lambda, e=2, D=4 \lambda)$, ante la incidencia de una onda plana $(\beta=0)$, en a) y en $b)$; y amplitud del campo eléctrico total, definido como la suma de los anteriores campos eléctricos incidente y dispersado, en c).

caracterizado; con el objeto de no incurrir en la primera de las limitaciones del algoritmo recursivo (recordar apartado 6.3.1). Una posible ordenación de las tiras estaría basada en la distancia entre el centro de cada tira y el centro de todas las tiras a caracterizar, denominado $C_{m}$ en la figura 7.6. Por otra parte, la elección de $C_{m}$ como el punto al que referir el espectro de campo incidente sobre todos los objetos se debe a que minimiza, como puede observarse en la figura 7.6, el radio 
de la mínima circunferencia centrada en dicho punto y que contiene a todas las tiras; reduciendo consecuentemente el número de modos necesarios en la correcta reconstrucción del citado campo incidente.

Después de caracterizar conjuntamente el comportamiento dispersor de las 11 tiras metálicas, que definen el contorno del reflector hiperbólico bajo análisis, se ha determinado la respuesta de las mismas ante la incidencia de una onda plana, polarizada según $\mathrm{TM}^{\mathrm{z}}$, con un frente de fase que avanza en la dirección $\beta=0$. Los resultados correspondientes a la amplitud y a la fase del campo eléctrico dispersado por el reflector hiperbólico, ante la citada onda plana incidente, se recogen en la figura 7.7 (apartados a y b); mientras la amplitud del campo eléctrico total, definido mediante la suma de los campos eléctricos incidente y dispersado, se representa en la figura 7.7 (apartado c). Observando la amplitud y la fase del campo eléctrico dispersado (apartados a y b en fig. 7.7), se descubre que el reflector hiperbólico genera en su zona posterior una onda plana; cuyo frente de fase avanza en la misma dirección $(\beta=0)$ que la onda incidente, aunque dichas ondas se encuentran desfasadas $180^{\circ}$. Como consecuencia de este desfase, al sumar los campos eléctricos incidente y dispersado, detrás del reflector hiperbólico se produce una zona de sombra o cono de difracción; tal y como puede observarse en la amplitud del campo eléctrico total (apartado $c$ en fig. 7.7). Por otra parte, en la zona frontal del reflector se genera un campo eléctrico dispersado cuya fase se corresponde, como era de esperar, con la de una onda cilíndrica con origen en el foco del reflector hiperbólico (ver apartado b en fig. 7.7); mientras la amplitud del campo eléctrico dispersado en dicha zona se va atenuando progresivamente (ver apartado a en fig. 7.7), de manera acorde también con el comportamiento esperado. Así pues, al sumar esta onda cilíndrica con la onda plana incidente, se produce un denso patrón de interferencias en la zona frontal del reflector; que puede observarse con detalle en la amplitud del campo eléctrico total representada en la figura 7.7 (apartado c). De estos resultados, coincidentes con los esperados, se concluye la validez del método seguido en este apartado para caracterizar el comportamiento dispersor del reflector hiperbólico; que constituye un elemento integrante de las antenas Cassegrain caracterizadas en la sección 7.4 empleando también segmentación.

\subsubsection{Reflector Parabólico ante Incidencia $\mathrm{TM}^{\mathrm{Z}}$}

En este segundo apartado, se analiza el comportamiento dispersor de un reflector parabólico, grande en términos eléctricos, que constituye el reflector principal del tipo de antenas Cassegrain consideradas en la sección 7.4. En el presente apartado, se ha caracterizado en particular el comportamiento dispersor, ante incidencia polarizada según $T \mathrm{~T}^{\mathrm{z}}$, de un reflector parabólico con un diámetro $D$ igual a $10 \lambda$ y una distancia focal $f$ de valor $8 \lambda$; empleando pues en la caracterización del reflector, debido al valor del diámetro, una aproximación de su contorno 
mediante tiras metálicas infinitas, de grosor despreciable e igual longitud, cuyo comportamiento dispersor conjunto se determina mediante el algoritmo recursivo descrito en el capítulo 6. Una vez caracterizado el anterior reflector parabólico, se ofrecen resultados correspondientes a la dispersión producida por dicho reflector ante distintas incidencias, todas ellas con polarización $\mathrm{TM}^{\mathrm{z}}$, pero generadas por distintas fuentes; permitiendo corroborar con estos resultados algunas de las propiedades dispersoras más típicas de los reflectores parabólicos. Por último, ante una incidencia concreta, se calcula la amplitud del campo dispersado por este reflector en zona de campo lejano mediante la técnica recién descrita, y empleando el conocido Método de la Distribución en la Apertura [82]; para de esta forma, comparando ambos resultados, comprobar la validez de aquéllos obtenidos aproximando el contorno del reflector parabólico por un conjunto de tiras.

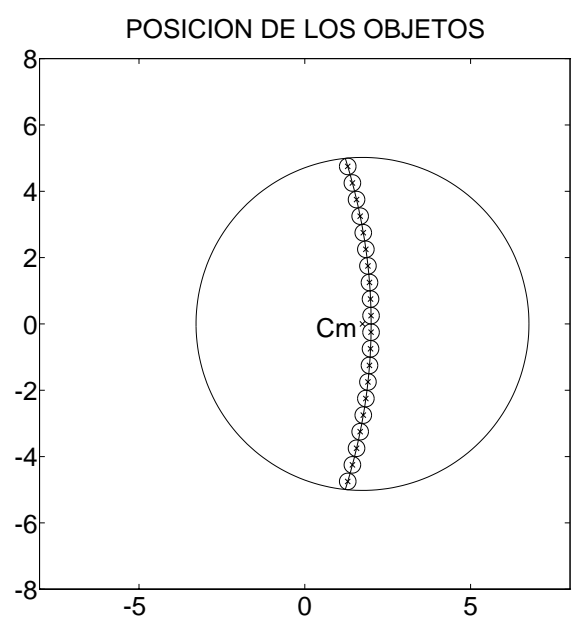

Figura 7.8: Estrategia de análisis de la dispersión producida por un reflector parabólico ( $D=10 \lambda, f=8 \lambda$ ), aproximando su contorno mediante 20 tiras de igual longitud.

La estrategia seguida en la caracterización del comportamiento dispersor del reflector parabólico considerado se recoge gráficamente en la figura 7.8; donde se observa que su contorno ha sido aproximado por 20 tiras metálicas, nuevamente de igual longitud debido a las razones de eficiencia computacional mencionadas en la sección 7.1. La caracterización conjunta de las 20 tiras puede realizarse, observando la figura 7.8, mediante la versión original del algoritmo recursivo (ver sección 6.1); que incorpora en cada iteración una nueva tira al grupo previamente caracterizado. La única precaución que debe observarse al aplicar dicho método, para evitar la primera limitación del algoritmo recursivo (recordar apartado 6.3.1), consiste en determinar de forma adecuada la iteración en la que debe incorporarse cada nueva tira; por ejemplo, en función de la distancia que existe entre el centro de cada tira y el centro de todos los objetos, representado por $C_{m}$ 


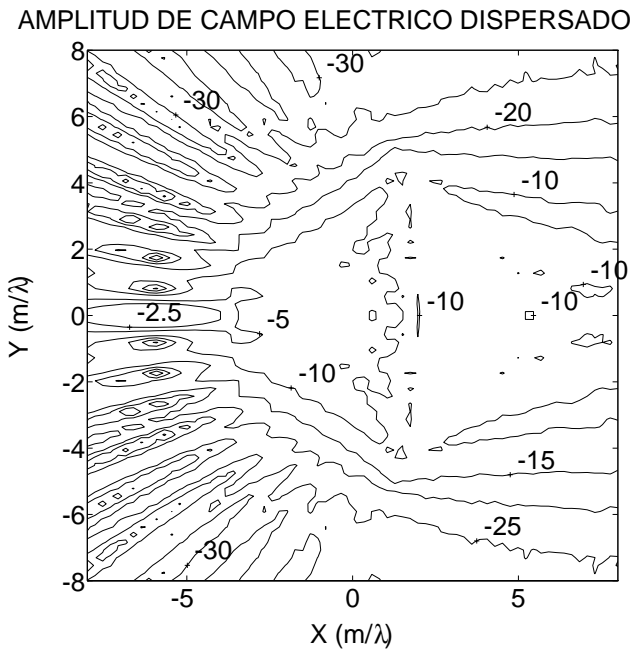

a)

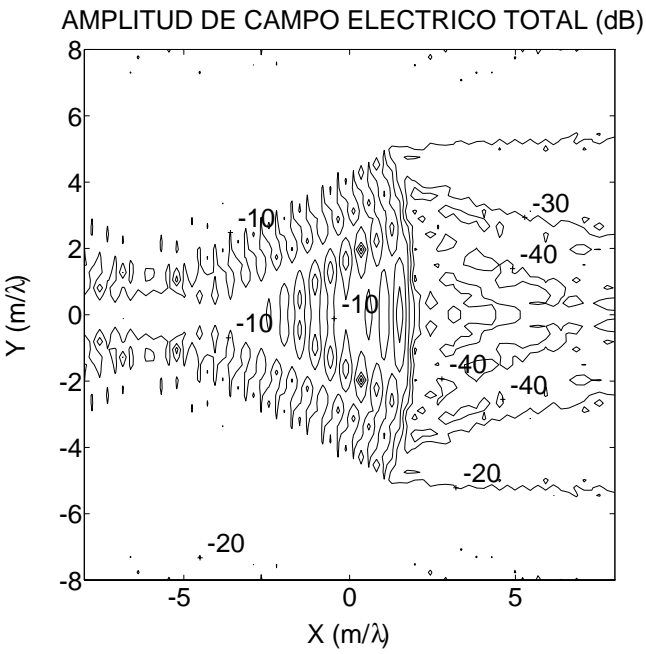

c)

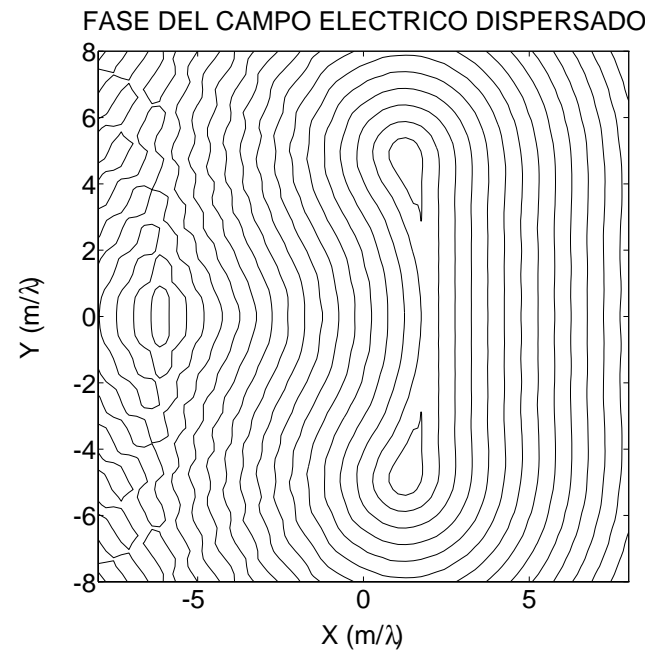

b)

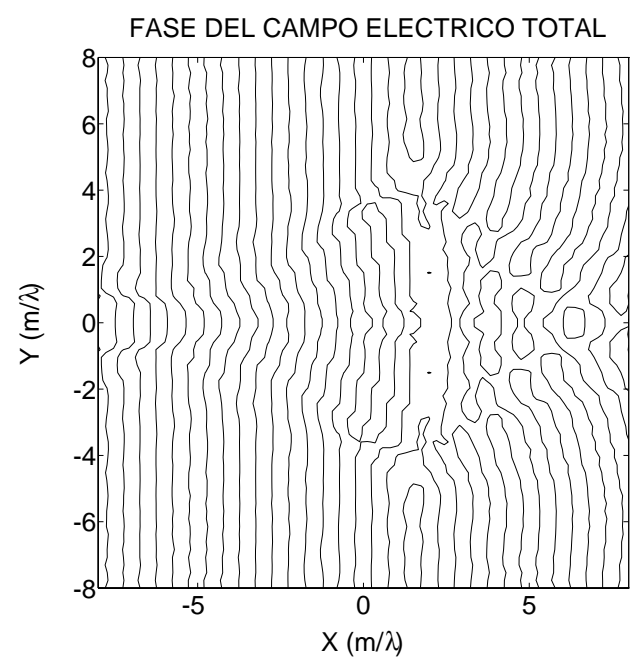

d)

Figura 7.9: Amplitud y fase del campo eléctrico dispersado por un reflector parabólico $(D=10 \lambda, f=8 \lambda)$, ante la incidencia de una onda plana $(\beta=0)$, en a) y en b); y amplitud y fase del campo eléctrico total, definido como la suma de los anteriores campos eléctricos incidente y dispersado, en c) y en $d$ ).

en la figura 7.8. De nuevo, dicho punto $C_{m}$ se ha escogido como el punto al que referir el espectro asociado al campo indicente sobre el reflector parabólico; pues esta elección reduce, como bien es sabido, el número de modos necesarios en la correcta reconstrucción del mencionado campo incidente.

Así pues, tras haber caracterizado de forma conjunta el comportamiento dispersor de las 20 tiras metálicas mostradas en la figura 7.8, que definen el con- 
torno del reflector parabólico bajo estudio; se ha determinado la respuesta de dicho reflector ante distintas incidencias, polarizadas todas ellas según $\mathrm{TM}^{\mathrm{z}}$, pero generadas por diferentes tipos de fuentes. En principio, se ha analizado la dispersión electromagnética que produce el mencionado reflector parabólico ante la incidencia de una onda plana, cuyo frente de fase avanza en la dirección $\beta=0$; representando en la figura 7.9 (apartados a y b) la amplitud del campo dispersado por el reflector ante la citada incidencia, y en la figura 7.9 (apartados c y d) la amplitud y la fase de la suma de los campos eléctricos incidente y dispersado. Es interesante observar en la figura 7.9 (apartados a y b) que el reflector parabólico genera, en su zona posterior, una onda plana con dirección de avance $\beta=0$; que sumada a la onda plana incidente, al encontrarse dichas ondas desfasadas $180^{\circ}$, produce una zona de sombra o cono de difracción en la amplitud del campo eléctrico total (ver apartado $c$ en fig. 7.9). De manera adicional, observando la fase del campo eléctrico dispersado en la figura 7.9 (apartado b) se descubre que los extremos del reflector parabólico, como consecuencia de la onda plana incidente, generan dos ondas difractadas con frentes de fase cilíndricos; las cuales determinan una forma de cono para la mencionada zona de sombra (ver apartado $d$ en fig. 7.9), razón por la que a esta zona de sombra también se la conoce como cono de difracción. Estudiando a continuación la dispersión producida por el reflector parabólico en su zona frontal, se descubre que la onda dispersada presenta un frente de fase correspondiente a una onda cilíndrica centrada en el foco del reflector (ver apartado b en fig. 7.9); que avanza desde la superficie del reflector hacia el foco. Por otra parte, en dicha zona, la amplitud del campo dispersado presenta un máximo de amplitud en el foco del reflector; que, junto con el resultado recién comentado para la fase, permiten corroborar la conocida propiedad de los reflectores parabólicos sobre la concentración en su foco de la radiación recibida en forma de onda plana. En esta misma zona frontal, al sumar los campos eléctricos incidente y dispersado, se produce un denso patrón de interferencias en la zona comprendida entre el reflector y su foco, que se prolonga a lo largo del eje $\hat{x}$ tras superar al foco (ver apartado c en fig. 7.9); mientras la fase del campo eléctrico total revela que tras el foco del reflector predomina la onda plana incidente (ver apartado d en fig. 7.9).

A continuación, se pretende determinar la dispersión electromagnética que produce el mismo reflector parabólico considerado en el ejemplo anterior; pero en esta ocasión ante un campo incidente, con una cierta directividad, generado por una fuente situada en el foco. En esta situación, recogida de manera gráfica en la figura 7.10, el campo incidente se expresa en principio mediante una serie de modos cilíndricos emergentes centrados en el foco del reflector parabólico, representado por el punto $C_{f}$ en la mencionada figura. Con el objeto de reutilizar, ante esta nueva incidencia, las mismas matrices de caracterización conjunta empleadas al resolver el ejemplo anterior; resulta necesario expresar dicha incidencia en términos de una serie de modos cilíndricos incidentes centrados en el punto $C_{m}$. 


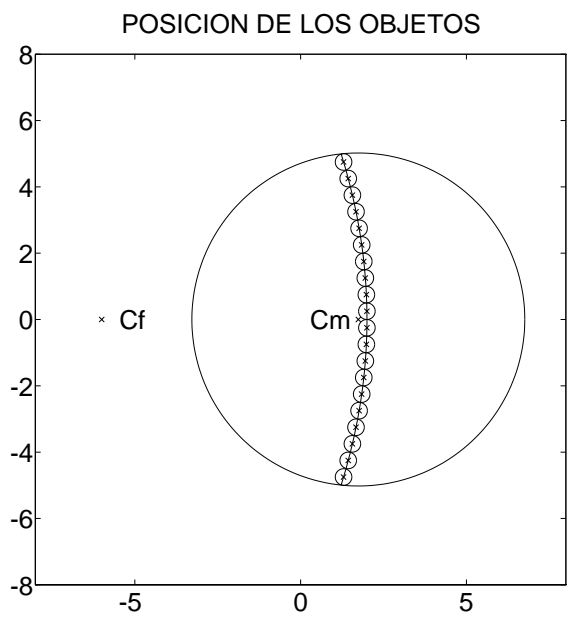

Figura 7.10: Estrategia de análisis de la dispersión producida por un reflector parabólico $(D=10 \lambda, f=8 \lambda)$, aproximando su contorno mediante 20 tiras de igual longitud, cuando la fuente que genera el campo incidente está situada en el foco (punto $C_{f}$ ) del reflector parabólico.

Para ello, los modos cilíndricos emergentes centrados en el punto $C_{f}$ deben transformarse, mediante la correspondiente matriz de traslación de espectro emergente a incidente (recordar apéndice $\mathrm{E}$ ), en los mencionados modos cilíndricos incidentes referidos al punto $C_{m}$. Ahora bien, esta transformación garantiza una correcta reconstrucción del campo emergente original, mediante un desarrollo en serie de modos cilíndricos incidentes centrados en el punto $C_{m}$, en el interior de una circunferencia con centro en dicho punto y radio la distancia entre los puntos $C_{m}$ y $C_{f}$ (recordar apartado E.2.1); región que contiene en este caso (ver figura 7.10) a todas las tiras que definen el contorno del reflector parabólico analizado. Por tanto, empleando la citada transformación de espectros, es posible resolver este nuevo problema utilizando las matrices de caracterización conjunta empleadas en el ejemplo anterior.

El campo generado por una fuente situada en el foco, con una cierta directividad, se expresa mediante un desarrollo en serie de modos cilíndricos emergentes centrados en dicho punto (recordar apartado C.1.2). El número de modos cilíndricos escogidos depende de la resolución espacial con la que se define la directividad del campo incidente; mientras las amplitudes espectrales, que acompañan a los respectivos modos cilíndricos emergentes, se determinan con la intención de reconstruir la mencionada directividad del campo incidente. En el presente ejemplo, se ha escogido una fuente situada en el foco del reflector parabólico, de coordenadas cartesianas normalizadas $(-6,0)$, que genera un campo eléctrico incidente sobre el reflector parabólico representado en la figura 7.11; donde se ob- 

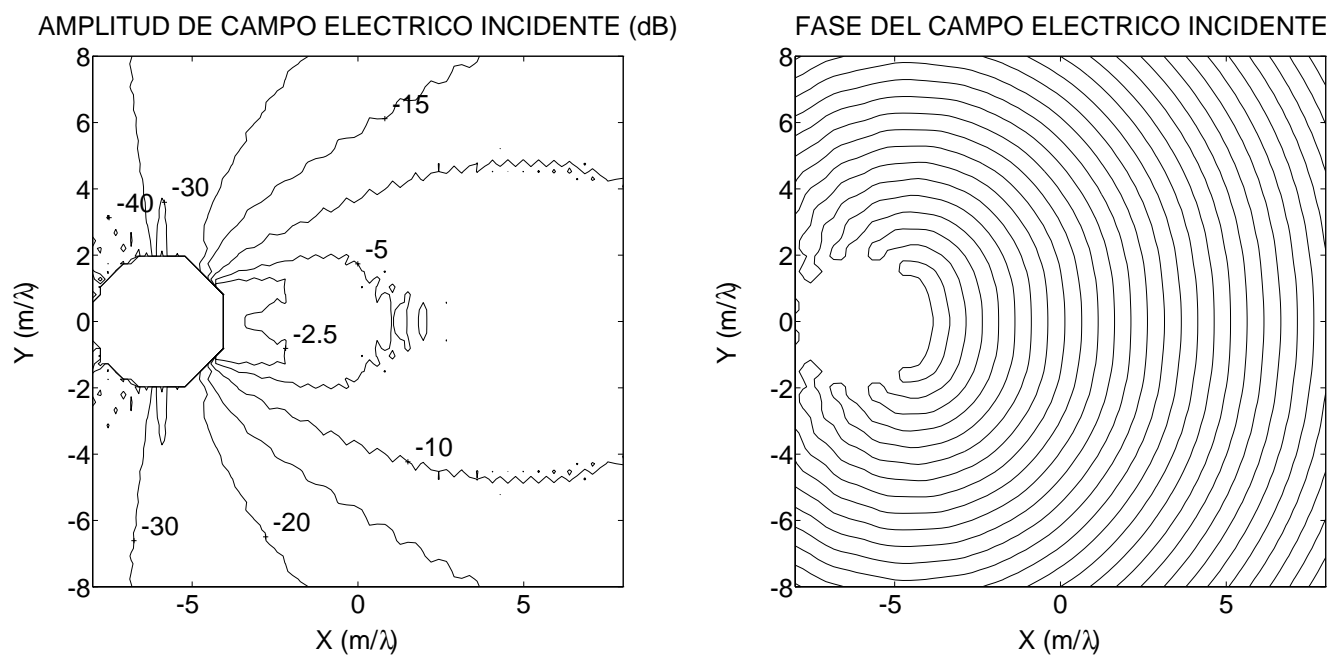

Figura 7.11: Amplitud y fase del campo eléctrico incidente sobre un reflector parabólico $(D=10 \lambda, f=8 \lambda)$, generado por una fuente situada en el foco $C_{f}$ de coordenadas cartesianas normalizadas $(-6,0)$.

serva una cierta directividad en la amplitud del campo eléctrico, y una evolución de la fase en forma de onda cilíndrica centrada en el foco.

Utilizando pues las matrices de caracterización conjunta empleadas en el ejemplo anterior, se ha determinado el campo eléctrico dispersado por el reflector parabólico mostrado en la figura 7.10; habiendo transformado previamente el espectro asociado al campo emergente, mostrado en la figura 7.11, en espectro incidente centrado en el punto $C_{m}$. La reconstrucción de la amplitud y la fase del campo eléctrico dispersado por el citado reflector, ante la mencionada incidencia, se recoge en la figura 7.12 (apartados a y b); donde se observa, en la parte frontal del reflector, una amplitud de campo eléctrico dispersado de valor prácticamente constante (ver apartado a en fig. 7.12), y una fase de campo eléctrico dispersado cuyo frente de fase corresponde al de una onda plana (ver apartado b en fig. 7.12). Estos resultados para el campo eléctrico dispersado, en la zona frontal del reflector parabólico, permiten confirmar otra conocida propiedad de este tipo de reflectores; según la cual, enfocan en una dirección preferente el campo que dispersan ante la incidencia de un campo generado por una fuente situada en su foco. Debido a esta interesante propiedad, el sistema integrado por la fuente y el reflector parabólico presenta una gran directividad. Por otra parte, en la zona posterior del reflector, la amplitud del campo eléctrico dispersado presenta valores similares a los que tiene en dicha zona la amplitud del campo eléctrico incidente (comparar apartado a en fig. 7.12 con apartado a en fig. 7.11); mientras la fase del campo eléctrico dispersado corresponde a la que presenta una onda cilíndrica centrada en el foco (ver apartado b en fig. 7.12), idéntica a la recogi- 


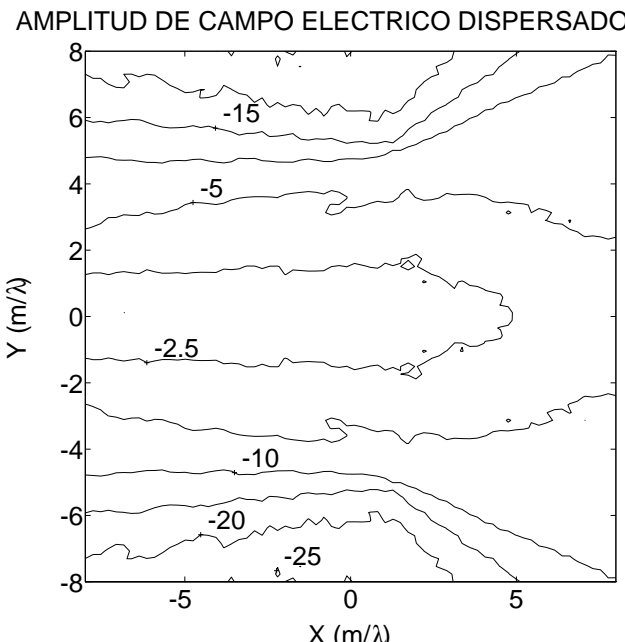

a)

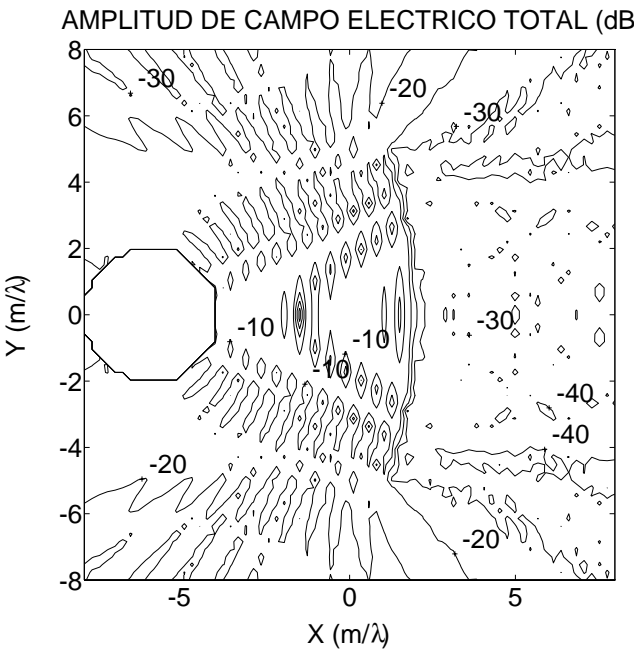

c)

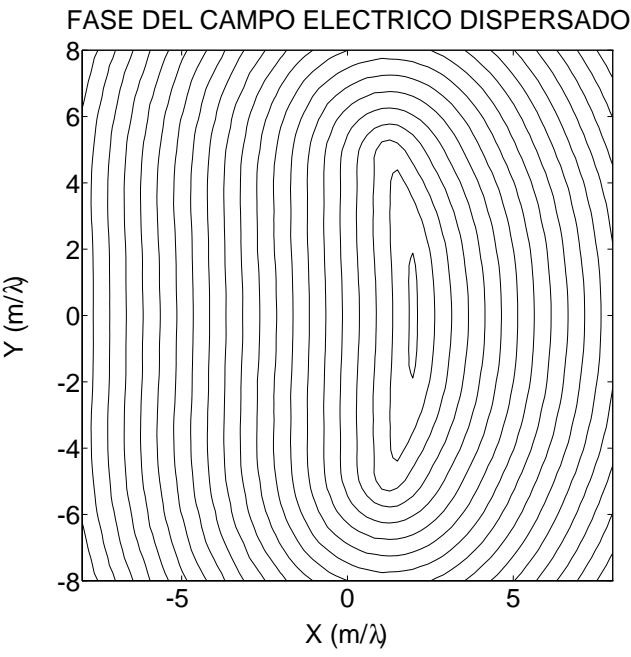

b)

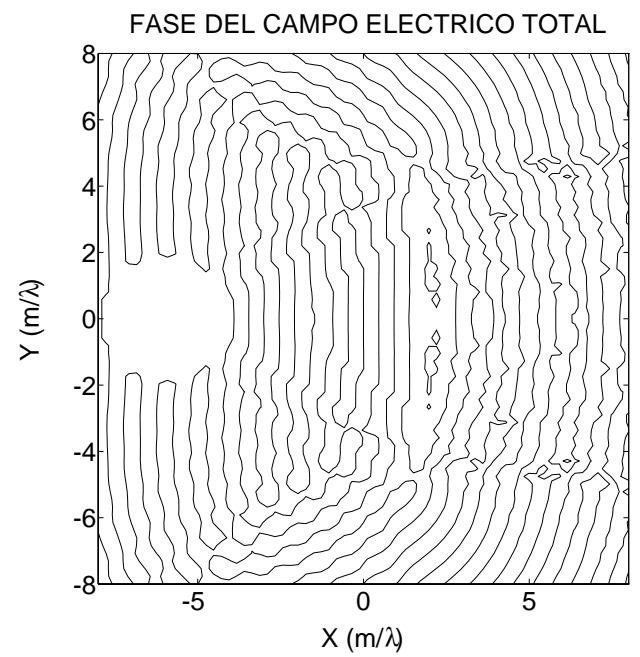

d)

Figura 7.12: Amplitud y fase del campo eléctrico dispersado por un reflector parabólico $(D=10 \lambda, f=8 \lambda)$, ante la incidencia de un campo generado por una fuente situada en el foco del reflector, en a) y en b); y amplitud y fase del campo eléctrico total, definido como la suma de los anteriores campos eléctricos incidente y dispersado, en c) y en d).

da en la figura 7.11 (apartado b) pero con un desfase de $180^{\circ}$. De esta forma, al sumar los campos eléctricos incidente y dispersado, en la parte posterior del reflector parabólico se produce una zona de sombra; tal y como se recoge en la figura 7.12 (apartados c y d), donde se representa la amplitud y la fase del campo eléctrico total. En este caso, a pesar de las ondas con frentes de fase cilíndricos que difractan los extremos del reflector (ver apartado b en fig. 7.12), éstas no de- 
terminan una forma de cono para la zona de sombra; de manera contraria a lo que ocurría en el ejemplo anterior para la incidencia de una onda plana. Por último, en la zona frontal del reflector parabólico, se observa en la amplitud del campo eléctrico total un denso patrón de interferencias entre el reflector y el foco (ver apartado c en fig. 7.12); que, una vez superado el foco, desaparece al predominar la onda plana dispersada (ver apartados c y d en fig. 7.12).

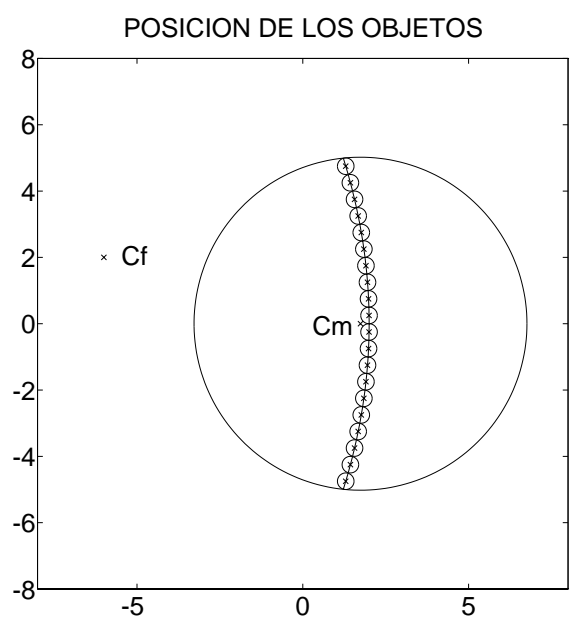

Figura 7.13: Estrategia de análisis de la dispersión producida por un reflector parabólico $(D=10 \lambda, f=8 \lambda)$, aproximando su contorno mediante 20 tiras de igual longitud, cuando la fuente que genera el campo incidente está situada $2 \lambda$ por encima del foco del reflector parabólico.

Con el objeto de estudiar el efecto que supone desplazar verticalmente, respecto del foco de un reflector parabólico, la fuente generadora del campo incidente sobre dicho reflector, seguidamente se ofrecen resultados de la dispersión electromagnética producida por un reflector parabólico, idéntico al considerado en los ejemplos anteriores, cuando el campo incidente está generado nuevamente por una fuente directiva; que, tal y como se recoge gráficamente en la figura 7.13, se encuentra situada en esta ocasión $2 \lambda$ por encima del foco del reflector parabólico. Esta nueva posición de la fuente respecto del reflector parabólico se encuentra representada en la figura 7.13; donde se denomina $C_{f}$ al punto en el que se sitúa la fuente. Para analizar esta nueva situación, al igual que en el ejemplo anterior con la fuente situada en el foco del reflector, es posible utilizar las mismas matrices de caracterización conjunta obtenidas en el primer ejemplo para las 20 tiras que definen el contorno del reflector considerado. Ahora bien, su utilización requiere que la incidencia sobre el reflector se exprese, como se ha mencionado anteriormente, en serie de modos cilíndricos incidentes centrados en el punto medio de todas las tiras, denominado $C_{m}$ en la figura 7.13, que constituye el punto 

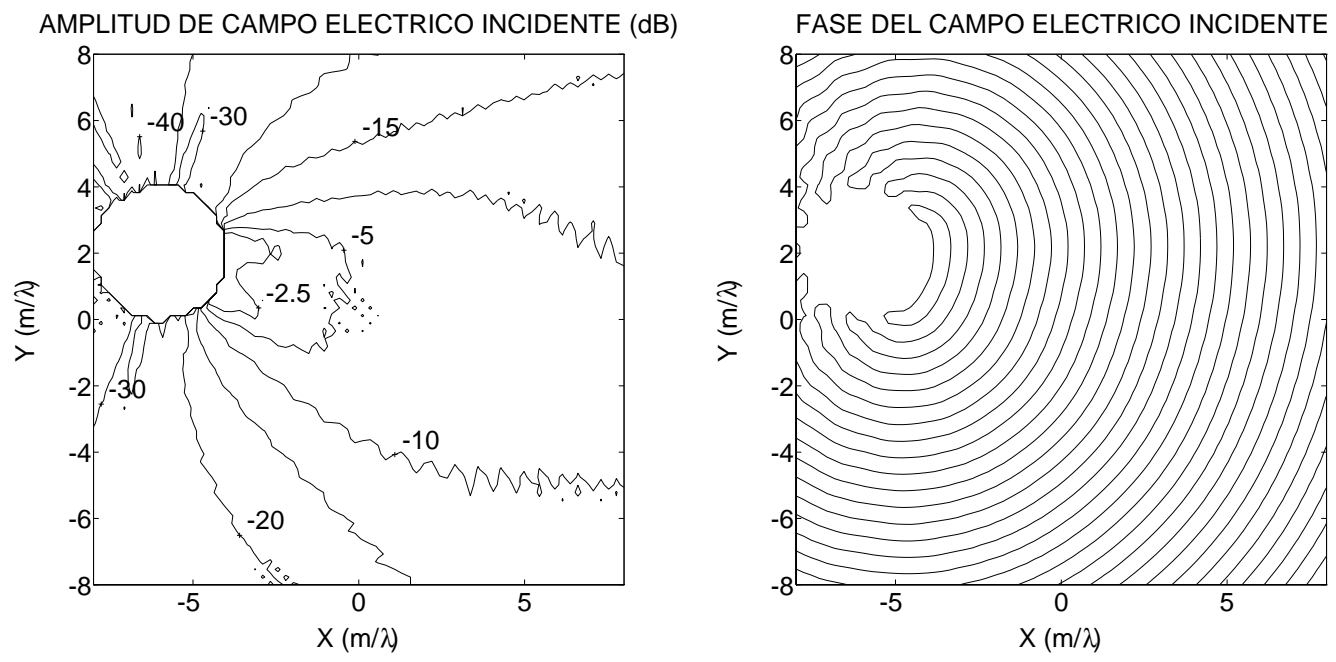

Figura 7.14: Amplitud y fase del campo eléctrico incidente sobre un reflector parabólico ( $D=10 \lambda, f=8 \lambda$ ), generado por una fuente situada $2 \lambda$ por encima del foco en un punto $C_{f}$ de coordenadas cartesianas normalizadas $(-6,2)$.

elegido para referir el campo incidente sobre el reflector parabólico; pues permite minimizar el radio de la mínima circunferencia con centro en dicho punto y que contiene a todas las tiras (ver fig. 7.13). Debido a que el punto $C_{f}$, generador del campo incidente sobre el reflector parabólico, se encuentra situado fuera de la mínima circunferencia recién descrita (ver fig. 7.13); es posible utilizar una matriz de traslación de espectro emergente a incidente, como la empleada en el ejemplo anterior, que transforme en este caso modos cilíndricos emergentes, centrados en la nueva posición de la fuente (punto $C_{f}$ en fig. 7.13), en modos cilíndricos incidentes centrados en el citado punto $C_{m}$. Realizada esta operación de traslación de espectros, será posible reutilizar ante la nueva incidencia las mismas matrices de caracterización conjunta empleadas en los dos ejemplos anteriores.

El aspecto que ofrece en este caso el campo generado por la fuente situada en el nuevo punto $C_{f}$, reconstruido a partir del correspondiente desarrollo modal emergente (recordar apartado C.1.2), se recoge en la figura 7.14; donde se observa un cierto comportamiento directivo en la amplitud del campo eléctrico incidente, y una evolución de la fase asociada a dicho campo que se corresponde con la de una onda cilíndrica centrada en $C_{f}$. Por otra parte, en la figura 7.14 se detecta que la dirección de apuntamiento del lóbulo principal de la amplitud, así como la dirección de avance del frente de fase, se encuentran desviadas respecto de la horizontal; de manera que la fuente situada en $C_{f}$ genera un campo incidente que ilumina la superficie del reflector parabólico bajo análisis.

Así pues, empleando las matrices de caracterización conjunta de las 20 tiras mostradas en la figura 7.13, se ha determinado la respuesta del reflector parabólico 


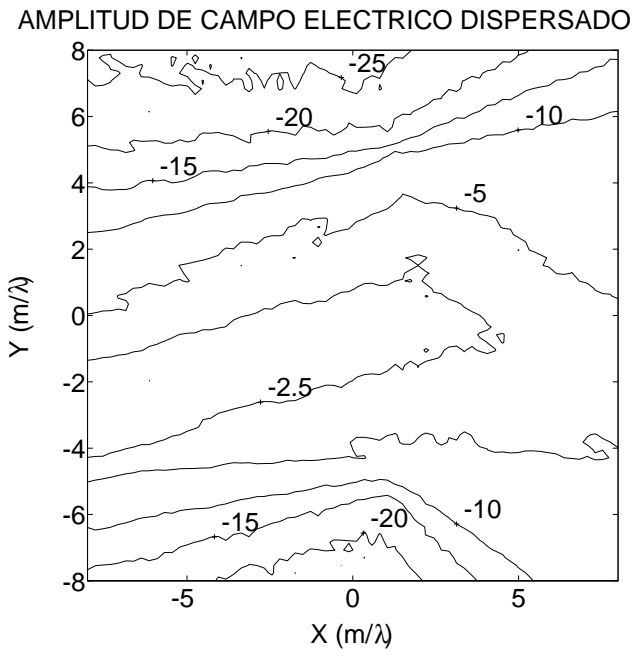

a)

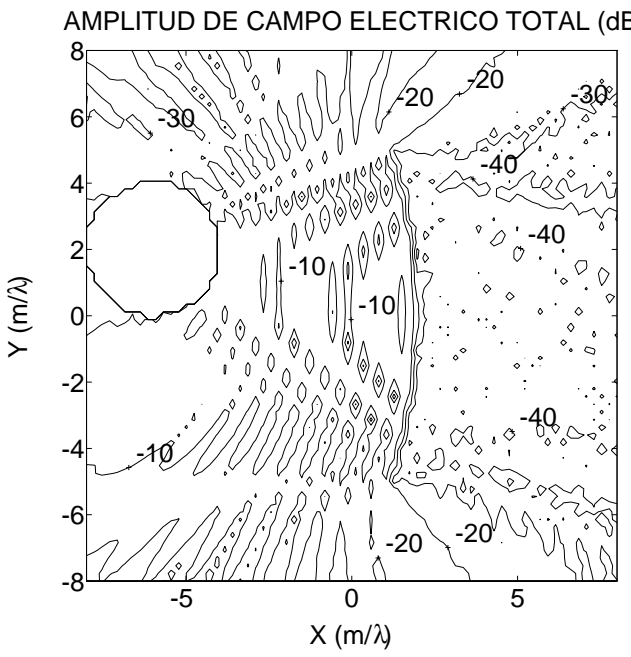

c)

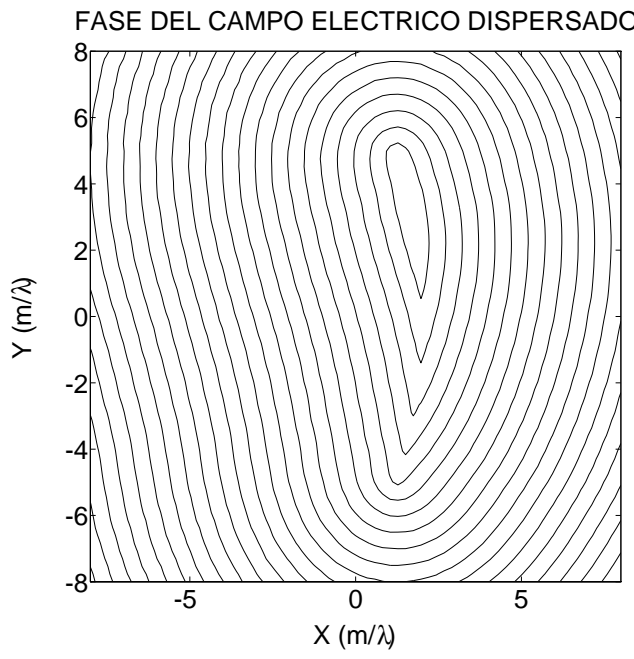

b)

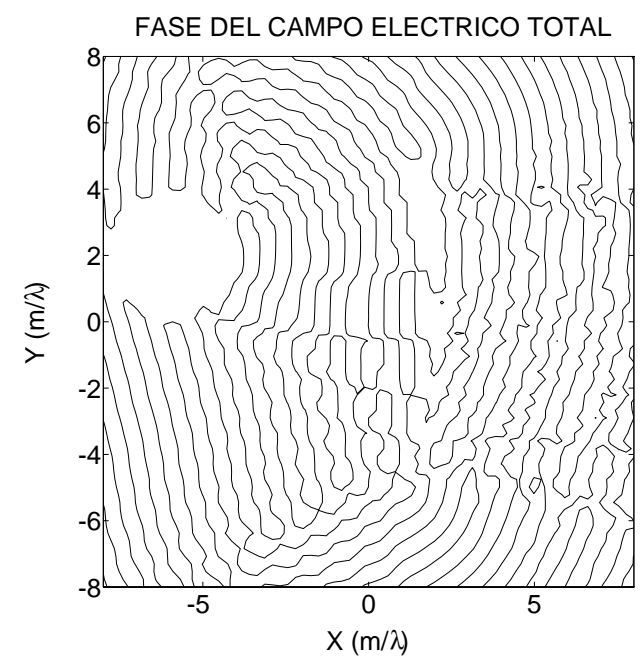

d)

Figura 7.15: Amplitud y fase del campo eléctrico dispersado por un reflector parabólico $(D=10 \lambda, f=8 \lambda)$, ante un campo incidente generado por una fuente situada $2 \lambda$ por encima del foco del reflector, en a) y en b); y amplitud y fase del campo eléctrico total, definido como la suma de los anteriores campos eléctricos incidente y dispersado, en $\mathbf{c}$ ) $y$ en $d)$.

cuyo contorno está definido por dichas tiras; en esta ocasión ante una incidencia generada por una fuente directiva situada $2 \lambda$ por encima del foco del citado reflector parabólico. La reconstrucción de la amplitud y la fase del campo eléctrico dispersado por dicho reflector, ante la mencionada incidencia, se recoge en la figura 7.15 (apartados a y b); mientras la reconstrucción de la amplitud y la fase 


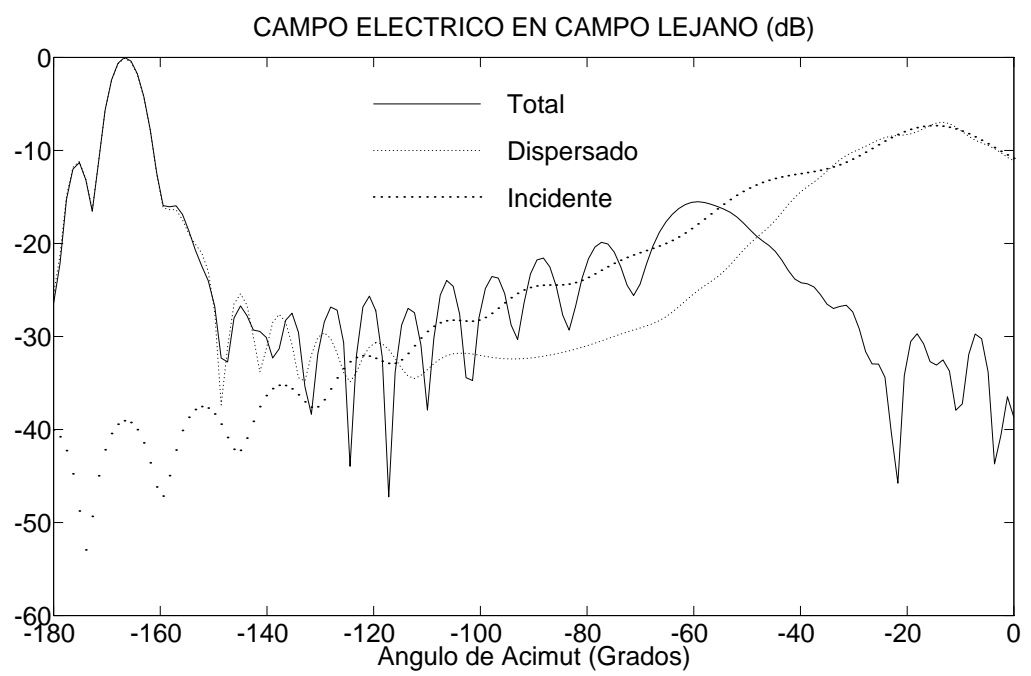

Figura 7.16: Amplitud del campo eléctrico incidente, dispersado y total en zona de campo lejano correspondiente al análisis de un reflector parabólico $(D=10 \lambda, f=8 \lambda)$, con fuente situada $2 \lambda$ por encima del foco.

del campo eléctrico total, definido como la suma de los campos eléctricos incidente y dispersado, se ofrece en la figura 7.15 (apartados c y d). En la zona posterior del reflector, se observa un campo eléctrico dispersado correspondiente a una onda cilíndrica generada desde el punto $C_{f}$, idéntica en amplitud a la onda cilíndrica asociada al campo eléctrico incidente, y desfasada $180^{\circ}$ respecto de dicha onda incidente (comparar apartados a y b en fig. 7.15 con la figura 7.14 ); de manera que, tras sumar ambas ondas cilíndricas, se produce una zona de sombra detrás del reflector (ver apartados c y d en fig. 7.15). Esta zona de sombra, a pesar de que nuevamente los extremos del reflector generan sendas ondas difractadas (ver apartado b en fig. 7.15), no presenta forma de cono al igual que ocurría cuando la fuente estaba situada en el foco del reflector; sin embargo, esta zona de sombra se encuentra desviada respecto de la horizontal, contrariamente a lo que sucedía en el ejemplo anterior, debido a la nueva posición de la fuente en el presente ejemplo. Por contra, en la parte frontal del reflector se genera una onda plana dispersada (ver apartados a y b en fig. 7.15); cuyo frente de fase avanza en la dirección especular correspondiente a la dirección de avance de la onda cilíndrica incidente sobre el reflector (ver apartado b en fig. 7.15). Este comportamiento del reflector parabólico corrobora una conocida propiedad de este tipo de reflectores; los cuales, ante una onda incidente generada por una fuente desplazada ligeramente del foco en el eje vertical, desvían la dirección de avance de la onda plana dispersada respecto de la horizontal. Sumando pues en la parte frontal del reflector parabólico el campo dispersado recién descrito y el campo incidente, se obtiene un denso patrón de interferencias entre el reflector 
y la posición donde se encuentra la fuente (ver apartado c en fig. 7.15); que desaparece tras superar a la fuente, donde predomina la onda plana dispersada por el reflector parabólico (ver apartados c y d en fig. 7.15).

Para finalizar este ejemplo, con el objeto de confirmar el efecto que supone el desplazamiento vertical, respecto del foco del reflector parabólico, de la fuente que genera el campo incidente sobre dicho reflector; se ha representado en la figura 7.16 la amplitud del campo eléctrico incidente, dispersado y total en zona de campo lejano correspondientes al mismo problema recién analizado. En dicha figura, se observa que el máximo del lóbulo principal del campo eléctrico dispersado y total (representados respectivamente con línea discontinua y continua) se produce a $15^{\circ}$ del valor angular acimutal asociado a la dirección horizontal mencionada anteriormente. En concreto, el valor acimutal donde el lóbulo principal de ambos campos eléctricos presenta su valor máximo (ver fig. 7.16) corresponde a la dirección de avance de la onda plana dispersada por el citado reflector parabólico (ver apartados b y d en fig. 7.15).

En todos los ejemplos recogidos en este apartado, ante distintos tipos de incidencia, se ha caracterizado un mismo reflector parabólico empleando una aproximación de su contorno mediante tiras metálicas infinitas, de grosor despreciable e igual longitud, cuya caracterización conjunta se ha realizado empleando la versión original del algoritmo recursivo (recordar sección 6.1). La aplicación de dicha técnica, debido a la dimensión del reflector parabólico analizado y a razones de eficiencia computacional (recordar sección 7.1), ha proporcionado los resultados mostrados en el presente apartado; que han permitido corroborar algunas de las propiedades más conocidas de los reflectores parabólicos, validando pues en cierta medida el método de análisis empleado. Ahora bien, para validar con mayores garantías los resultados obtenidos mediante la técnica recién descrita, a continuación se ofrece una comparación entre los resultados que ofrecen dicha técnica y el Método de la Distribución en la Apertura, tradicionalmente empleado en el análisis de reflectores parabólicos, al resolver la dispersión electromagnética producida por un cierto reflector parabólico ante una determinada incidencia.

Concretamente, se ha analizado el mismo reflector parabólico considerado en los ejemplos anteriores de este apartado; aunque, en esta ocasión, se ha supuesto que el campo incidente está generado por una fuente omnidireccional situada en el foco del reflector. Esta situación es idéntica a la recogida gráficamente en la figura 7.10; excepto que la incidencia se expresa mediante una sola componente modal emergente, aquélla que incluye a la función de Hankel de segunda especie y orden cero (recordar apartado C.1.2), debido al carácter omnidireccional de la fuente. En principio, el comportamiento dispersor del reflector parabólico se ha caracterizado utilizando las matrices de caracterización conjunta de las 20 tiras que definen el contorno de dicho reflector (ver figura 7.10); determinando, ante la mencionada incidencia, la amplitud del campo eléctrico dispersado por el reflector en zona de campo lejano. La utilización de las matrices de caracterización previa- 


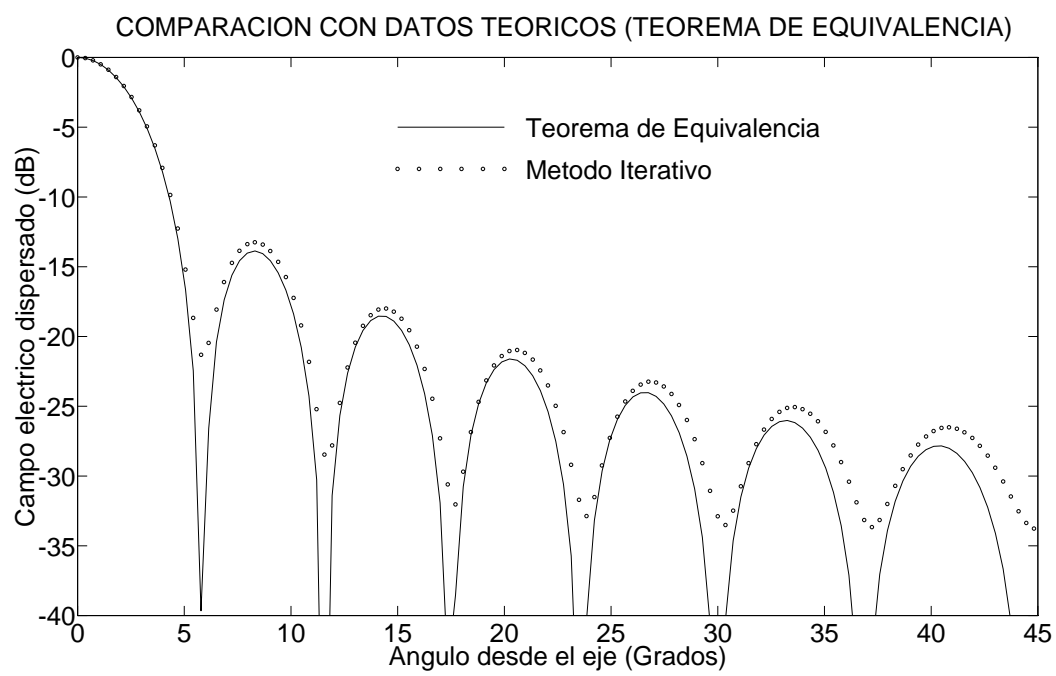

Figura 7.17: Amplitud del campo eléctrico dispersado en zona de campo lejano por un reflector parabólico ( $D=10 \lambda, f=8 \lambda)$, calculada empleando el algoritmo recursivo y mediante el Método de la Distribución en la Apertura, ante un campo incidente (TM ${ }^{\mathrm{z}}$ ) generado por una fuente omnidireccional situada en el foco del reflector.

mente calculadas ha sido posible, de igual manera que en los ejemplos anteriores, porque la fuente se encuentra situada fuera de la mínima circunferencia, centrada en el punto medio de todas las tiras, que las contiene a todas ellas (recordar figura 7.10). Por otra parte, se ha resuelto el mismo problema mediante el conocido Método de la Distribución en la Apertura [82]; que, utilizando el Teorema de Equivalencia Superficial [69], determina la amplitud del campo eléctrico dispersado en zona de campo lejano. Este método clásico, empleado tradicionalmente con reflectores parabólicos, permite obtener el campo dispersado por el reflector en zona de campo lejano, mediante el mencionado Teorema de Equivalencia Superficial, a partir del conocimiento del campo dispersado por el reflector en un plano denominado plano de apertura; que es perpendicular al eje del reflector parabólico y normalmente se sitúa en su foco. Este campo en el plano de la apertura se obtiene, a su vez, empleando Óptica Geométrica en la superficie del reflector para obtener el campo dispersado ante la incidencia conocida; y asumiendo posteriormente que dicho campo dispersado se propaga hasta el plano de apertura como una onda plana. Los resultados correspondientes a la amplitud del campo eléctrico que dispersa el reflector parabólico bajo estudio, ante la incidencia omnidireccional considerada, se recogen en la figura 7.17; donde con línea continua se representa dicha amplitud obtenida mediante el Método de la Distribución en la Apertura, y con línea punteada se dibuja la misma amplitud calculada tras aplicar el conocido algoritmo recursivo al conjunto de tiras que definen el contorno del reflector analizado. En la citada figura, se observa que ambos resultados son 
bastante coincidentes; aunque los nulos asociados a la solución obtenida mediante el Método de la Distribución en la Apertura son absolutos, a diferencia de los nulos correspondientes a la solución calculada empleando el algoritmo recursivo descrito en el capítulo 6 . Estos resultados confirman la validez de la técnica de análisis de objetos grandes en términos eléctricos, ante incidencia $\mathrm{TM}^{\mathrm{z}}$, mediante la aproximación de su contorno por objetos iguales de menor tamaño, y su posterior caracterización conjunta mediante la versión original del algoritmo recursivo recogida en la sección 6.1 ; llegando incluso a concluir que su precisión puede ser mayor que la obtenida mediante el Método de la Distribución en la Apertura, debido a las diversas aproximaciones realizadas siguiendo dicho Método.

\subsubsection{Reflector Parabólico ante Incidencia $\mathrm{TE}^{\mathrm{z}}$}

En este último apartado, tras haber analizado en el anterior un reflector parabólico ante incidencia polarizada según $\mathrm{TM}^{\mathrm{z}}$, se caracteriza el comportamiento dispersor de otro reflector parabólico, grande también en términos eléctricos, pero ante incidencia con polarización $\mathrm{TE}^{\mathrm{z}}$. Concretamente, en el presente apartado se estudia la dispersión electromagnética producida por un reflector parabólico de diámetro $D$ igual a $15 \lambda$, y distancia focal $f$ de valor $20 \lambda$; en cuya caracterización, debido al valor del diámetro de dicho reflector, se aproxima su contorno mediante un conjunto de tiras metálicas infinitas, de grosor despreciable e igual longitud, caracterizadas mediante el algoritmo recursivo descrito en el capítulo 6 . Con el objeto de confirmar la validez de la técnica de análisis recién descrita, se compara la amplitud del campo magnético dispersado por el citado reflector en zona de campo lejano, obtenida empleando la mencionada técnica, con la misma amplitud calculada haciendo uso del conocido Método de la Distribución en la Apertura; concluyendo de esta forma, al igual que se hizo en el apartado anterior para incidencia polarizada según $\mathrm{TM}^{\mathrm{z}}$, que la técnica basada en aproximar el contorno de un objeto mediante elementos idénticos de menor tamaño, caracterizados posteriormente de forma conjunta, proporciona también resultados correctos ante incidencia con polarización $\mathrm{TE}^{\mathrm{z}}$.

La estrategia seguida en la caracterización del reflector parabólico considerado en el presente apartado, que se muestra en la figura 7.18, se basa en una aproximación del contorno de dicho reflector mediante 30 tiras metálicas de igual longitud y grosor infinitesimal; cuya caracterización conjunta puede realizarse, como en el caso de los reflectores analizados en los dos apartados anteriores de esta sección, mediante la versión original del algoritmo recursivo (ver sección 6.1). Dicha versión incorpora, en cada iteración, una nueva tira al grupo caracterizado de forma conjunta en la iteración anterior; resultando necesario determinar, de manera precisa, la iteración en la que debe incorporarse cada tira. Este orden debe establecerse con sumo cuidado, por ejemplo siguiendo un criterio basado en la distancia de los centros de cada tira al punto medio de dichos centros, para 

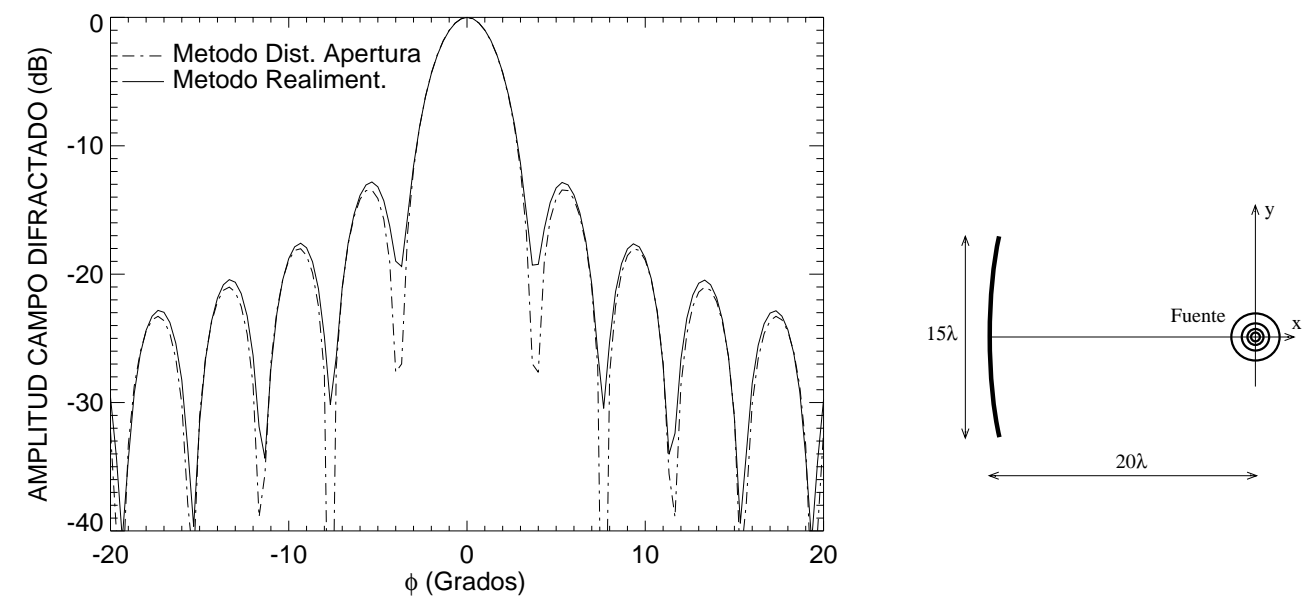

Figura 7.18: Amplitud del campo magnético dispersado en zona de campo lejano por un reflector parabólico $(D=15 \lambda, f=20 \lambda)$, calculada empleando el algoritmo recursivo y mediante el Método de la Distribución en la Apertura, ante un campo incidente ( $\mathrm{TE}^{\mathrm{z}}$ ) generado por una fuente omnidireccional situada en el foco del reflector.

evitar que se incurra en la primera de las limitaciones del algoritmo recursivo (recordar apartado 6.3.1). De igual manera que para los reflectores analizados anteriormente, en este caso se ha escogido también como punto al que referir el espectro de campo incidente sobre el reflector parabólico el punto medio de los centros de todas sus tiras; pues de esta forma se minimiza el radio de la mínima circunferencia centrada en dicho punto que contiene a todas las tiras, y se reduce por tanto el número de modos requeridos en la correcta reconstrucción del campo incidente sobre el reflector parabólico considerado.

Utilizando pues las matrices de caracterización conjunta obtenidas para las 30 tiras metálicas que definen el contorno del reflector parabólico bajo análisis; se ha determinado, ante un campo incidente $\mathrm{TE}^{\mathrm{z}}$ generado por una fuente omnidireccional situada en el foco del citado reflector, la amplitud del campo magnético dispersado en zona de campo lejano por dicho reflector. La reconstrucción del campo magnético incidente, debido a su carácter omnidireccional, se obtiene empleando un solo modo cilíndrico emergente, aquél que contiene a la función de Hankel de segunda especie y orden cero centrada en el foco del reflector; el cual, para poder utilizar las matrices de caracterización conjunta previamente deducidas, debe referise al punto medio de los centros de todas las tiras. Esta operación se implementa mediante la correspondiente matriz de traslación de espectro emergente a incidente; quedando garantizada la validez de la reconstrucción del campo emergente original sobre el contorno del reflector, a partir del espectro cilíndrico incidente trasladado, debido a la distancia que existe entre el foco y el punto medio de los centros de todas las tiras (ver figura 7.18). El mismo problema recién 
expuesto se ha resuelto también a partir del conocido Método de la Distribución en la Apertura [82]; empleado tradicionalmente en el análisis de la dispersión electromagnética producida por reflectores parabólicos. Este Método determina la amplitud del campo magnético dispersado por el reflector parabólico, haciendo uso del Teorema de Equivalencia Superficial [69], a partir del conocimiento del campo magnético dispersado por el reflector en un plano denominado plano de apertura; que es perpendicular al eje del reflector y se sitúa normalmente en su foco. La determinación del campo magnético sobre el plano de apertura se realiza encontrando en primer lugar, mediante la aplicación de Óptica Geométrica sobre la superficie del reflector, el campo que dicho dispersor genera en sus inmediaciones como consecuencia del campo que incide sobre él; y propagando posteriormente dicho campo dispersado hasta el plano de apertura como si se tratara de una onda plana. Los resultados correspondientes a la amplitud del campo magnético dispersado por el reflector parabólico escogido en zona de campo lejano, ante la mencionada incidencia, se recogen ambos en la figura 7.18; donde, con línea continua, se representa la citada amplitud obtenida aplicando el conocido algoritmo recursivo a un conjunto de tiras que aproximan el contorno del reflector parabólico, y con trazo de línea y punto se muestra la misma amplitud calculada mediante el Método de la Distribución en la Apertura. En esta figura, se observa que ambos resultados son prácticamente coincidentes; aunque, tal y como ocurría en el caso del reflector parabólico ante incidencia $\mathrm{TM}^{\mathrm{z}}$, los nulos de la solución calculada mediante el Método de la Distribución en la Apertura son absolutos, de manera contraria a lo que ocurre con los nulos de la solución deducida siguiendo la estrategia de análisis propuesta en el presente capítulo. Al igual que para incidencia $\mathrm{TM}^{\mathrm{z}}$, los resultados mostrados en la figura 7.18 confirman la plena validez, en este caso para incidencia $\mathrm{TE}^{\mathrm{z}}$, de la técnica de análisis de objetos grandes en términos eléctricos basada en la aproximación de su contorno mediante tiras metálicas infinitas, de grosor despreciable e igual longitud, y la posterior caracterización conjunta del comportamiento dispersor de dichas tiras mediante el algoritmo recursivo propuesto en el capítulo 6; llegando incluso a concluir, debido a las diversas aproximaciones empleadas en el Método de la Distribución en la Apertura, que la técnica propuesta en este capítulo proporciona resultados más exactos que los obtenidos con el citado Método.

\subsection{Antenas Cassegrain ante Incidencia $\mathrm{TM}^{z}$ y $\mathrm{TE}^{\mathrm{z}}$}

Tras haber caracterizado en la sección anterior el comportamiento dispersor de reflectores hiperbólicos y parabólicos, elementos ambos integrantes de diversos sistemas multireflectores, en esta sección se analiza la dispersión electromagnética producida por una posible configuración de las antenas Cassegrain; que consta 
de un reflector parabólico como elemento principal de la antena multireflectora, y de un reflector hiperbólico que ejerce el papel de reflector secundario. Debido a las dimensiones de ambos reflectores, tal y como se indica en la sección anterior, el comportamiento dispersor de cada reflector se determina por separado aproximando su contorno mediante un conjunto de tiras metálicas infinitas, de grosor despreciable e igual longitud, caracterizadas de forma conjunta empleando la versión original del algoritmo recursivo (ver sección 6.1); para finalmente, en una última iteración especial, analizar el comportamiento dispersor de la estructura Cassegrain cuando la fuente generadora del campo incidente sobre la antena es interna al problema (ver sección 6.4).

Concretamente, se ha caracterizado en primer lugar el comportamiento dispersor de una antena Cassegarin simétrica ante incidencia polarizada según $\mathrm{TM}^{\mathrm{z}}$; y en segundo lugar se ha estudiado la dispersión electromagnética producida por una antena Cassegrain offset ante incidencia con polarización TE ${ }^{z}$. En ambos casos, los resultados ofrecidos concuerdan plenamente con los esperados para cada tipo de antena, y permiten asimismo corroborar las principales propiedades dispersoras de dichas antenas; confirmando pues, de alguna manera, la validez de la técnica escogida en su caracterización.

\subsubsection{Antena Cassegrain Simétrica ante Incidencia $T M^{Z}$}

En este apartado, se analiza el comportamiento dispersor de una antena Cassegrain simétrica, cuyos elementos integrantes son grandes en términos eléctricos, ante incidencia polarizada según $\mathrm{TM}^{\mathrm{z}}$. Concretamente, la antena Cassegrain considerada consta de un reflector hiperbólico con un diámetro $D$ igual a $4 \lambda$, una excentricidad $e$ de valor 2 , y una distancia focal $c$ con valor igual a $\lambda$; y de un reflector parabólico cuyo diámetro $D$ es igual a $14 \lambda$, y que presenta una distancia focal $f$ de valor $8 \lambda$. En el análisis de esta antena, debido a las grandes dimensiones eléctricas de los reflectores que la integran, se aproxima el contorno de cada reflector por un conjunto de tiras metálicas infinitas; las cuales se caracterizan de forma conjunta, mediante el algoritmo recursivo descrito en el capítulo 6, para definir por separado el comportamiento dispersor de cada uno de los reflectores mencionados. Posteriormente, la caracterización definitiva de la antena Cassegrain se realiza, utilizando las matrices que definen el comportamiento dispersor de los reflectores que la integran, mediante una iteración especial para fuente interna al problema recogida también en el capítulo 6 . Una vez definido el comportamiento dispersor de la antena Cassegrain, se determina la respuesta de dicha antena ante un campo incidente, polarizado según $\mathrm{TM}^{\mathrm{z}}$, que genera una fuente directiva situada en el foco del sistema multireflector analizado; comprobando de esta forma algunas de las propiedades más típicas de estas antenas.

La estrategia concreta de análisis seguida en la caracterización de la antena Cassegrain se recoge gráficamente en la figura 7.19; donde se observa la formación 


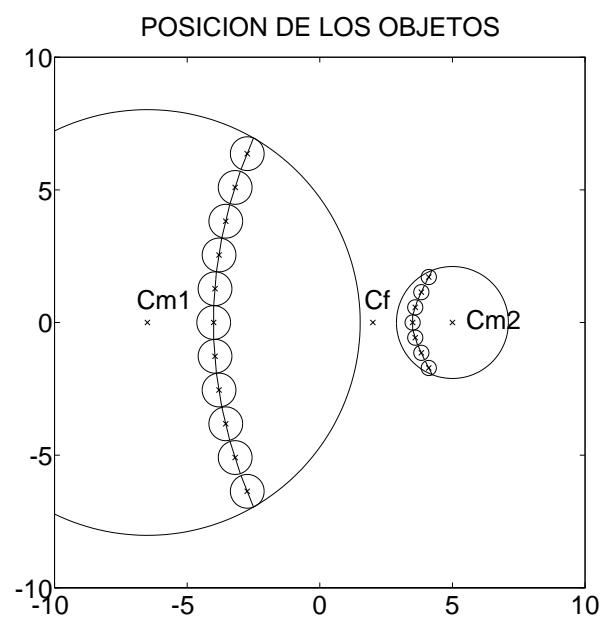

Figura 7.19: Estrategia de análisis de la dispersión electromagnética producida por una antena Cassegrain simétrica, cuando la fuente que genera el campo incidente está situada en el foco (punto $C_{f}$ ) del sistema multireflector, aproximando los contornos del reflector hiperbólico $(c=\lambda, e=2, D=4 \lambda)$ y del reflector parabólico $(D=14 \lambda, f=8 \lambda)$ que integran dicha antena mediante 7 y 11 tiras respectivamente.

de dos grupos de objetos dispersores constituidos respectivamente por las 7 tiras que aproximan el contorno del reflector hiperbólico, y por las 11 tiras que aproximan el contorno del reflector parabólico. La caracterización conjunta de cada uno de estos dos grupos se realiza, por separado, utilizando la versión original del algoritmo recursivo expuesta en la sección 6.1 ; aunque, en este caso, la elección de los puntos a los que referir los espectros de campo incidente sobre cada grupo, denominados $C_{m_{1}}$ y $C_{m_{2}}$ en la figura 7.19 , no coinciden con los puntos medios de los centros de las tiras que integran cada grupo. La razón de las nuevas posiciones escogidas para los puntos $C_{m_{1}}$ y $C_{m_{2}}$, mostradas en la figura 7.19, obedece al hecho de que la caracterización definitiva de la antena Cassegrain, cuando la fuente es interna al problema bajo análisis (ver punto $C_{f}$ en fig. 7.19), se realiza aplicando una iteración especial para fuente interna descrita en la sección 6.4; cuya correcta aplicación requiere que el punto donde se encuentra la fuente esté situado fuera de las mínimas circunferencias, centradas en los puntos $C_{m_{1}}$ y $C_{m_{2}}$, que contienen respectivamente a todas las tiras de cada grupo. La elección de las posiciones para los puntos $C_{m_{1}}$ y $C_{m_{2}}$ garantiza, como puede observarse en la figura 7.19, el cumplimiento de la mencionada condición; aunque esto supone que las mínimas circunferencias centradas en los puntos $C_{m_{1}}$ y $C_{m_{2}}$, y que contienen a todas las tiras de cada grupo, presentan radios mayores a los que tendrían en el caso de que los puntos $C_{m_{1}}$ y $C_{m_{2}}$ estuvieran situados en los puntos medios de los centros de las tiras de cada grupo, y por consiguiente se produce un incremento 

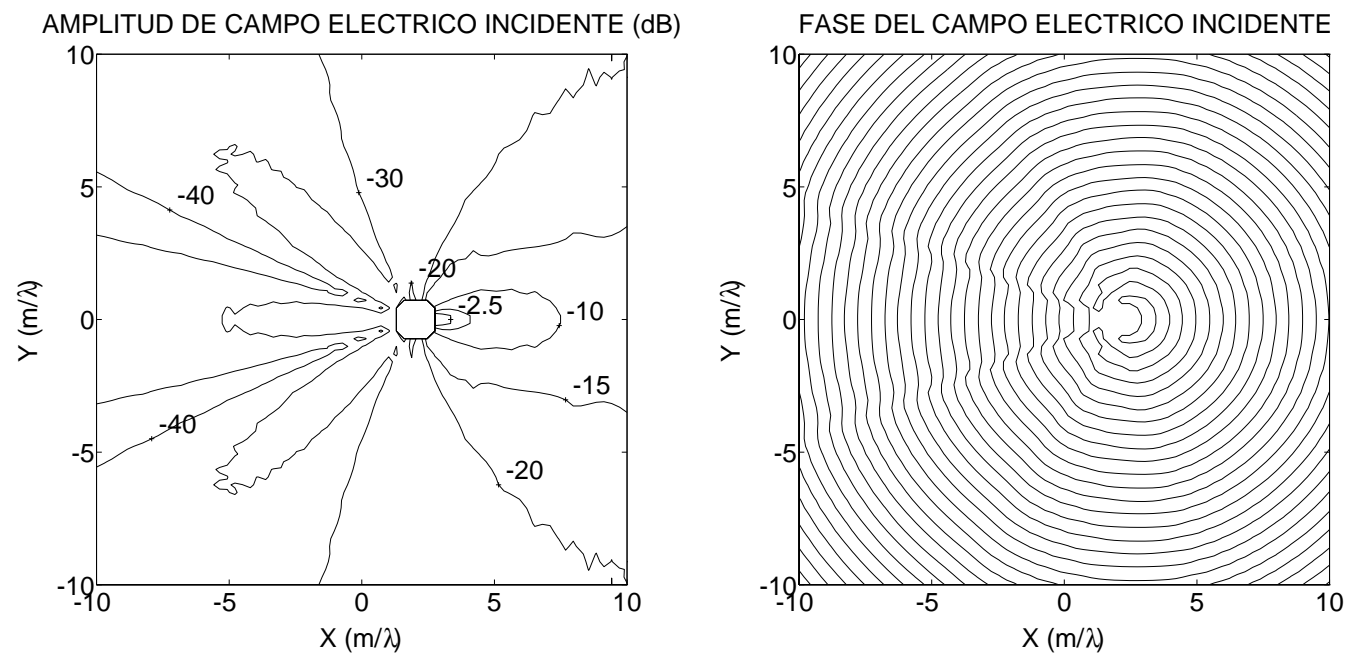

Figura 7.20: Amplitud y fase del campo eléctrico incidente sobre una antena Cassegrain simétrica, constituida por un reflector hiperbólico $(c=\lambda, e=2, D=4 \lambda)$ y un reflector parabólico $(D=14 \lambda, f=8 \lambda)$, que está generado por una fuente situada en el foco del sistema multireflector.

del número de modos necesarios en la correcta reconstrucción del campo incidente sobre cada grupo de tiras.

El campo eléctrico incidente sobre la antena Cassegrain está generado en esta ocasión por una fuente, situada en el foco del sistema multireflector (recordar punto $C_{f}$ en fig. 7.19), que presenta un determinado comportamiento directivo. La reconstrucción de este campo eléctrico incidente, recordando el apartado C.1.2, se implementa mediante el correspondiente desarrollo en serie de modos cilíndricos emergentes; que determina una amplitud y una fase para la incidencia sobre la antena Cassegrain recogidas en la figura 7.20. En dicha figura, se observa que la amplitud presenta un lóbulo principal apuntando en la dirección del reflector hiperbólico de la antena Cassegrain; mientras el frente de fase evoluciona, también en la dirección del citado reflector hiperbólico, de igual manera que una onda cilíndrica centrada en el punto $C_{f}$.

Una vez calculadas las matrices de caracterización conjunta de las tiras que definen los contornos del reflector hiperbólico y parabólico de la antena Cassegrain, se ha obtenido la respuesta de dicha antena ante la incidencia $\mathrm{TM}^{\mathrm{z}}$ descrita en el párrafo anterior. La amplitud y la fase del campo eléctrico dispersado por la citada antena se encuentran representadas en la figura 7.21 (apartados a y b); mientras la amplitud y la fase del campo eléctrico total, definido como la suma de los anteriores campos eléctricos incidente y dispersado, se muestran en la misma figura 7.21 (apartados c y d). En las regiones situadas detrás de cada uno de los reflectores integrantes de la antena Cassegrain, se observa que el campo 


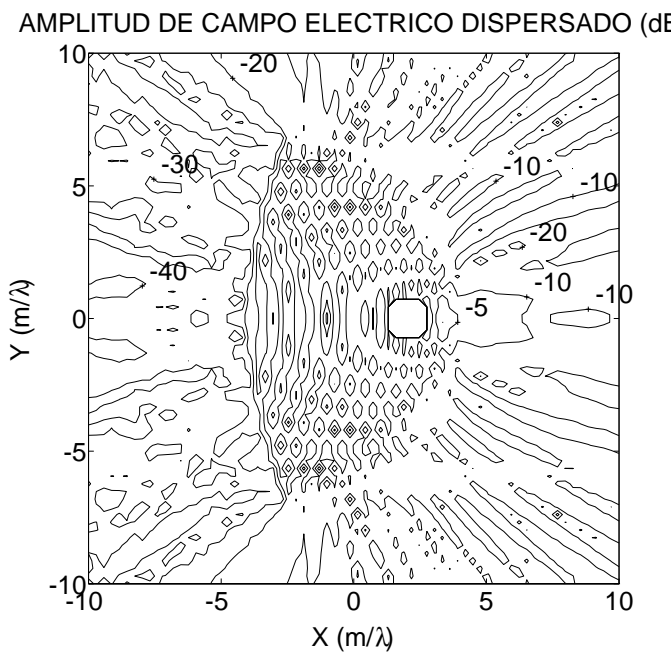

a)

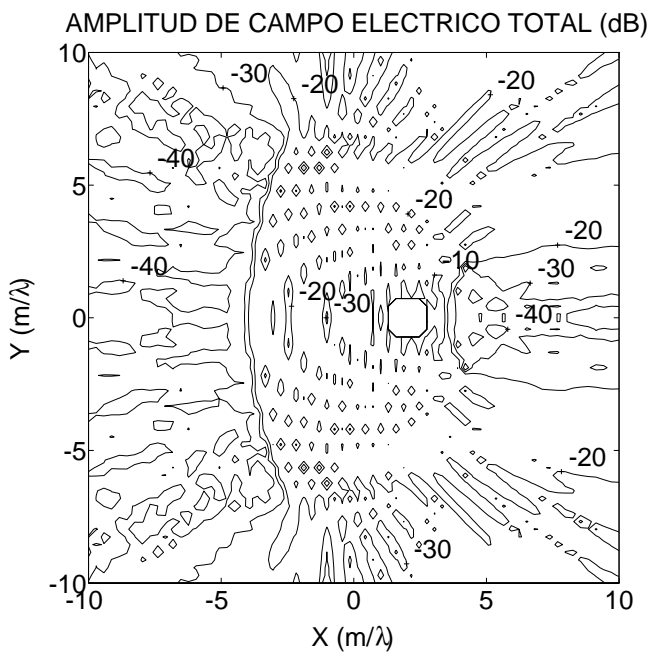

c)

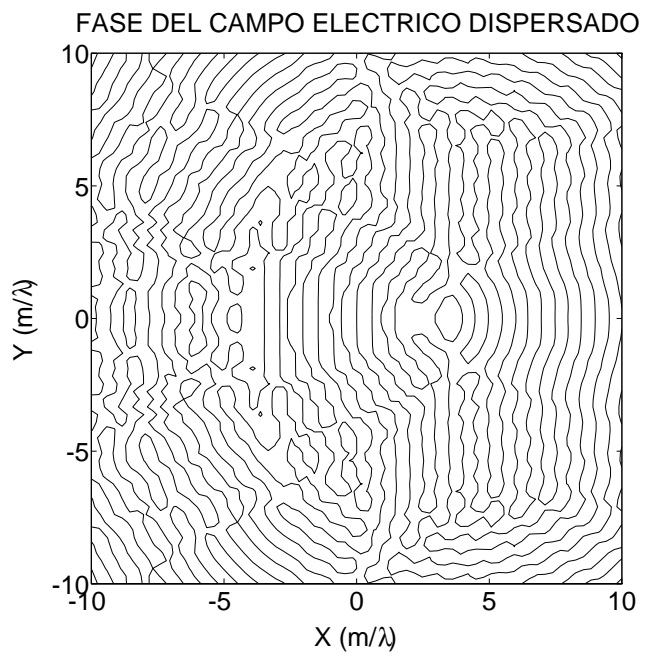

b)

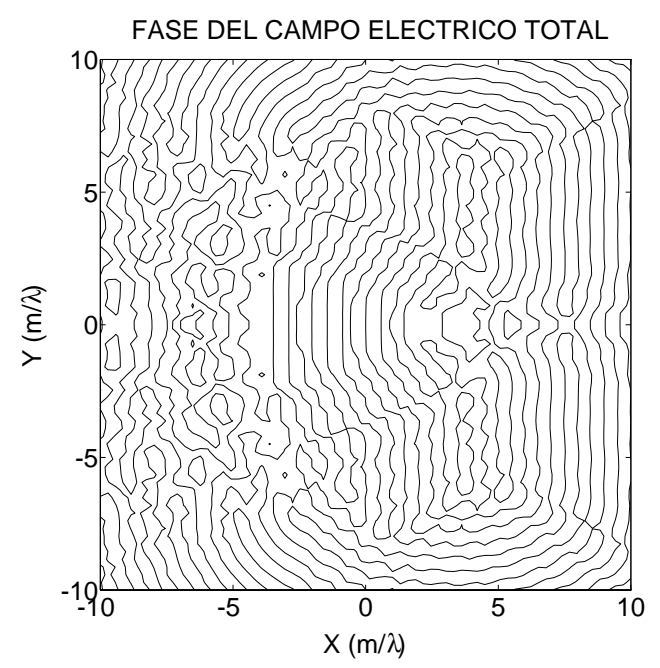

d)

Figura 7.21: Amplitud y fase del campo eléctrico dispersado por una antena Cassegrain simétrica, constituida por un reflector hiperbólico $(c=\lambda, e=2, D=4 \lambda)$ y un reflector parabólico $(D=14 \lambda, f=8 \lambda)$, ante una incidencia generada por una fuente situada en el foco en a) y en b); y amplitud y fase del campo eléctrico total, definido como la suma de los anteriores campos eléctricos incidente y dispersado, en c) y en d).

eléctrico dispersado presenta un comportamiento similar al del campo eléctrico incidente (comparar apartados a y b en fig. 7.21 con la figura 7.20), aunque las fases de estos dos campos difieren $180^{\circ}$; de manera que al sumar dichos campos se originan sendas zonas de sombra en las mencionadas regiones (ver apartado c en fig. 7.21). Por otra parte, el campo eléctrico dispersado se comporta como 


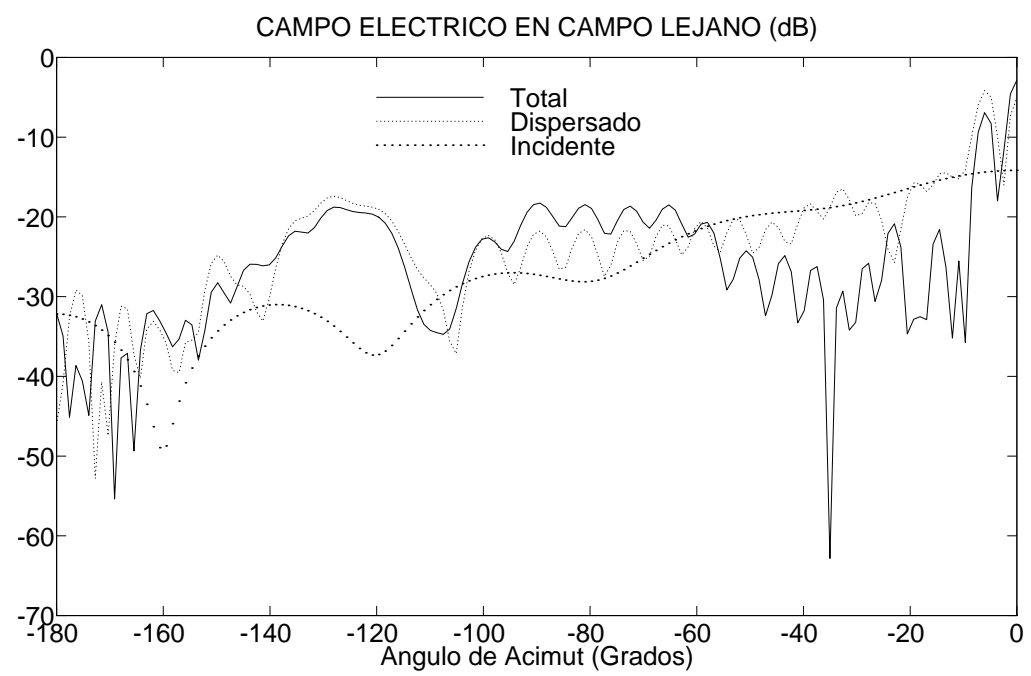

Figura 7.22: Amplitud del campo eléctrico incidente, dispersado y total en zona de campo lejano correspondiente al análisis de una antena Cassegrain simétrica, constituida por un reflector hiperbólico $(c=\lambda, e=2, D=4 \lambda)$ y un reflector parabólico $(D=14 \lambda, f=8 \lambda)$, con fuente situada en el foco del sistema multireflector.

una onda plana, cuyo frente de fase avanza en la dirección $\beta=0$, a partir de la apertura del reflector parabólico (ver apartado b en fig. 7.21); comportamiento que se repite en la evolución de la fase del campo eléctrico total (ver apartado d en fig. 7.21). Este último resultado confirma una de las propiedades más conocidas de la antena Cassegrain; según la cual, dichas antenas enfocan el campo eléctrico incidente en una dirección de radiación preferente. En el análisis tradicional de las antenas Cassegrain, dicha propiedad se constata utilizando el concepto de la parábola equivalente [82]; que permite sustituir los dos reflectores que integran la antena Cassegrain por un reflector parabólico equivalente, cuyo comportamiento dispersor (recordar los resultados de la sección 7.3) confirma la generación de la mencionada onda plana por parte de la antena Cassegrain original. Ahora bien, esta técnica clásica de análisis no tiene en cuenta el efecto de bloqueo que introduce el reflector hiperbólico en el comportamiento de la antena Cassegrain; efecto que, sin embargo, sí se considera al utilizar el método de análisis empleado en esta sección para caracterizar el comportamiento dispersor de la antena Cassegrain (ver apartado c en fig. 7.21). Por último, observando de nuevo la figura 7.21 (apartado c), se descubre la existencia de un denso patrón de interferencias en la región comprendida entre el reflector hiperbólico y parabólico de la antena Cassegrain; que, fuera de dicha zona, desparece al predominar la onda plana dispersada por el sistema multireflector (ver apartados c y d en fig. 7.21).

Para finalizar con este ejemplo, en la figura 7.22 se representa la amplitud del campo eléctrico incidente, dispersado y total en zona de campo lejano corres- 
pondientes al problema de la antena Cassegrain recién analizado. Como era de esperar, en dicha figura se observa un máximo de radiación para la amplitud del campo eléctrico total en la dirección del eje del reflector; existiendo, sin embargo, una relación entre el lóbulo principal y el lóbulo secundario de esta misma gráfica que es bastante baja (aproximadamente de $5 \mathrm{~dB}$ ), debido básicamente al efecto de bloqueo introducido por el reflector hiperbólico cuya dimensión es grande en comparación con la del reflector parabólico. De manera adicional, en la citada amplitud del campo eléctrico total, se descubren unos niveles altos de radiación en la zona comprendida entre valores acimutales de $-120^{\circ}$ y $-140^{\circ}$; atribuibles al campo generado por la fuente que desborda directamente al reflector parabólico, y al campo dispersado por el reflector hiperbólico que también desborda posteriormente al citado reflector parabólico. El estudio de todos estos fenómenos, tales como el bloqueo que ejerce el reflector hiperbólico o el desbordamiento del reflector parabólico, así como su posible corrección pueden realizarse gracias a la utilización de la técnica de análisis descrita en esta sección; que genera resultados más exactos a los proporcionados por otros métodos clásicos de análisis como el Método de la Parábola Equivalente, el Método del Alimentador Virtual o el Método de la Distribución en la Apertura, recogidos todos ellos en [82], y cuyos resultados no permiten detectar los efectos mencionados al ser tan sólo válidos dichos resultados en el semiespacio situado delante del reflector parabólico.

\subsubsection{Antena Cassegrain Offset ante Incidencia $\mathrm{TE}^{\mathrm{z}}$}

Tras haber analizado en el apartado anterior una antena Cassegrain simétrica, con elementos reflectores grandes en términos eléctricos, ante incidencia $\mathrm{TM}^{\mathrm{z}}$; en este apartado se caracteriza el comportamiento dispersor de una antena Cassegrain offset, con unas dimensiones grandes también eléctricamente, pero ante incidencia con polarización $\mathrm{TE}^{\mathrm{z}}$. Concretamente, la antena Cassegrain considerada consta de un reflector hiperbólico con una excentricidad $e$ de valor 1.66, una distancia focal $c$ igual a $10 \lambda$, y una apertura cuyo diámetro $D$ presenta un valor igual a $3 \lambda$; y de un reflector parabólico con una distancia focal $f$ de valor $20 \lambda$, y una apertura cuyo diámetro $D$ es igual a $15 \lambda$. Debido a los elevados valores de estas dimensiones de los reflectores integrantes de la antena Cassegrain offset, la citada antena se ha caracterizado siguiendo la misma estrategia utilizada en el apartado anterior; que consiste en aproximar inicialmente el contorno de cada reflector de la antena Cassegrain offset por un conjunto de tiras metálicas infinitas, cuya caracterización conjunta mediante el algoritmo recursivo descrito en el capítulo 6 permite definir por separado el comportamiento dispersor de los citados reflectores. Posteriormente, como la fuente generadora del campo incidente $\left(\mathrm{TE}^{\mathrm{z}}\right)$ es interna al problema bajo análisis, la antena Cassegrain offset se caracteriza de forma definitiva aplicando una última iteración especial para fuente interna, recogida también en el capítulo 6 . Por último, después de caracterizar el 

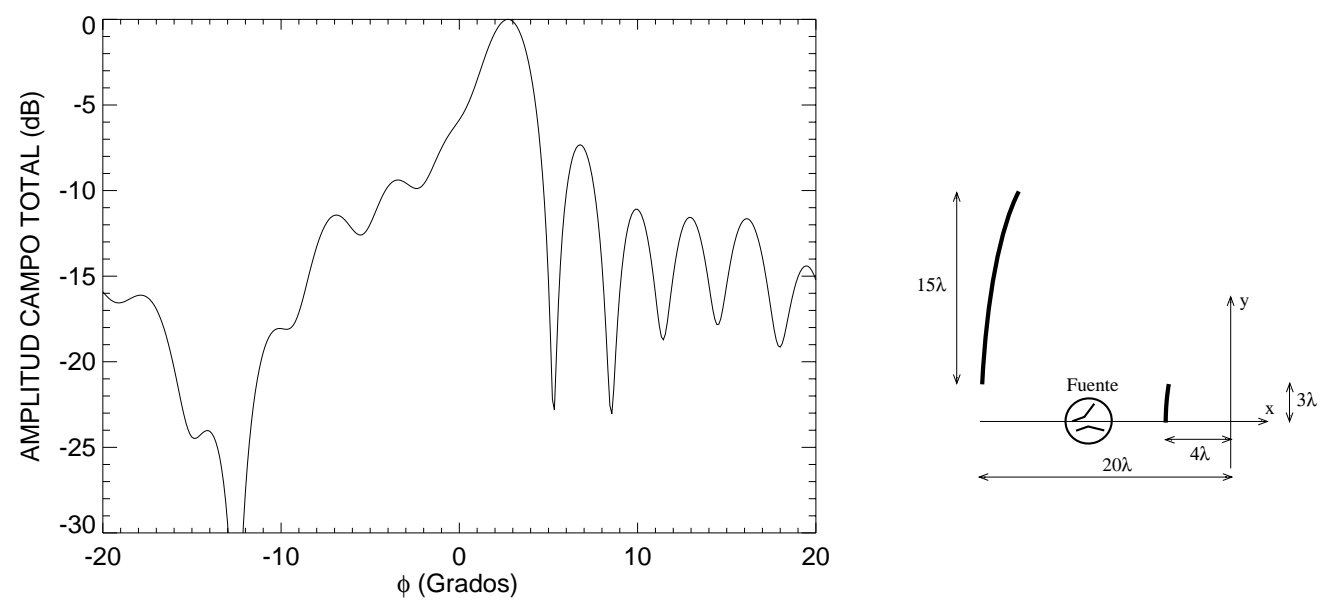

Figura 7.23: Amplitud del campo magnético total en zona de campo lejano correspondiente al análisis de una antena Cassegrain offset, constituida por un reflector hiperbólico $(c=10 \lambda, e=1.66, D=3 \lambda)$ y un reflector parabólico $(D=15 \lambda, f=20 \lambda)$, ante un campo incidente $\left(\mathrm{TE}^{\mathrm{z}}\right)$ generado por una fuente directiva situada entre los dos reflectores que integran la antena Cassegrain.

comportamiento dispersor de la antena Cassegrain offset, se ofrecen resultados de la dispersión producida por dicha antena cuando el campo incidente, polarizado según $\mathrm{TE}^{\mathrm{z}}$, está generado por una fuente directiva situada entre los dos reflectores que definen la antena Cassegrain offset; comprobando que el bloqueo generado por el reflector hiperbólico se reduce en comparación al originado en el caso de la antena Cassegrain simétrica.

En el análisis del comportamiento dispersor de la antena Cassegrain offset, cuya configuración se muestra gráficamente en la figura 7.23 , se ha seguido la misma estrategia de análisis empleada en el apartado anterior; que inicialmente requiere aproximar el contorno de los reflectores de la antena Cassegrain por un conjunto de tiras metálicas infinitas, habiendo escogido en este caso 6 tiras para el reflector hiperbólico y 30 para el reflector parabólico. A continuación, empleando la versión original del algoritmo recursivo (ver sección 6.1), se caracteriza por separado el comportamiento dispersor conjunto de las tiras que constituyen el reflector hiperbólico, y el de las tiras que definen el reflector parabólico. Finalmente, debido a la ubicación de la fuente generadora del campo incidente sobre la antena Cassegrain offset, el comportamiento dispersor de la citada antena se obtiene en una última iteración especial para fuente interna (ver sección 6.4). En cuanto a la elección de los puntos a los que referir respectivamente los espectros de campo incidente sobre cada uno de los dos grupos de tiras considerados, contrariamente a lo que ocurría en el ejemplo del apartado anterior, es posible escoger en este caso los puntos medios de los centros de todas las tiras que integran 


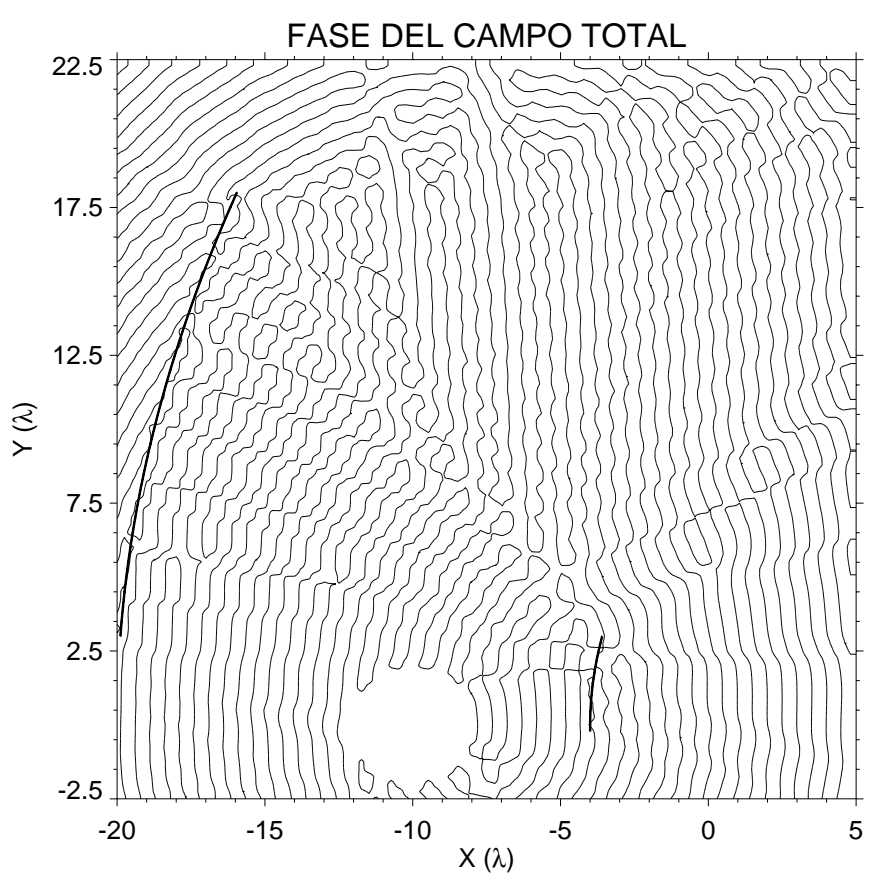

Figura 7.24: Fase del campo magnético total; obtenido como la suma del campo magnético incidente y del campo magnético dispersado ante dicha incidencia por una antena Cassegrain offset, que está constituida por un reflector hiperbólico $(c=10 \lambda$, $e=1.66, D=3 \lambda)$ y un reflector parabólico $(D=15 \lambda, f=20 \lambda)$.

cada grupo; ya que con dicha elección, como puede intuirse en la figura 7.23, las mínimas circunferencias centradas en dichos puntos, que además contienen a los respectivos reflectores, no incluyen a la fuente. De esta manera, no sólo se cumple la restricción espacial introducida por la iteración para fuente interna (recordar sección 6.4); sino que se minimiza además el número de componentes modales necesarias para reconstruir correctamente el campo incidente sobre cada uno de los dos reflectores de la antena Cassegrain.

Una vez caracterizado el comportamiento dispersor de la antena Cassegrain offset, se ha determinado la respuesta de esta antena frente a un campo incidente, con polarización $\mathrm{TE}^{\mathrm{z}}$, generado por una fuente directiva situada entre los dos reflectores de la antena Cassegrain. En la figura 7.23, se muestra la amplitud del campo magnético total en zona de campo lejano correspondiente al problema analizado; campo que se obtiene sumando los campos magnéticos incidente y dispersado por la antena Cassegrain en la mencionada zona de campo lejano. En dicha figura, se observa que el máximo del lóbulo principal se produce en un valor acimutal ligeramente desplazado de la dirección del eje de la antena Cassegrain; observación que se confirma en la representación de la fase del mismo campo magnético total en zona de campo próximo, mostrada en la figura 7.24, donde se 
constata que el frente de fase asociado a la onda plana producida en la apertura del reflector parabólico no avanza exactamente en la dirección angular $\beta=0$. Esta desviación en la dirección de avance del frente de fase de la onda plana puede estar produciéndose, entre otras posibles causas, debido a que el campo generado por la fuente desborda al reflector hiperbólico; fenómeno que puede corregirse modificando de forma adecuada el comportamiento directivo de la fuente generadora del campo incidente sobre la antena Cassegrain. Para concluir con este ejemplo, retornando de nuevo a la amplitud del campo magnético total en zona de campo lejano mostrada en la figura 7.23, se observa que el nivel de lóbulo principal a secundario presenta un valor (aproximadamente de $8 \mathrm{~dB}$ ) mayor que el obtenido en el ejemplo del apartado anterior (de valor aproximadamente $5 \mathrm{~dB}$ ) con una antena Cassegrain simétrica; debido a que la antena Cassegrain offset, por su configuración, elimina parte del bloqueo que introduce el reflector hiperbólico sobre el reflector parabólico. De hecho, en el caso analizado en este apartado, el nivel de lóbulo principal a secundario obtenido es todavía relativamente bajo; siendo posible mejorarlo mediante un diseño más optimizado de la estructura Cassegrain que permita reducir el efecto del bloqueo. Estas operaciones recién descritas, tales como la elección adecuada del comportamiento directivo de la fuente o el diseño de la configuración óptima de la antena Cassegrain, se pueden implementar fácilmente utilizando la estrategia de análisis descrita en esta sección; que genera resultados precisos en tiempos de computación bajos.

\subsection{Antena Bocina ante Incidencia $\mathrm{TM}^{z}$}

En esta última sección, con el objeto de mostrar la potencia del método de análisis de objetos grandes en términos eléctricos propuesto en el presente capítulo, se caracteriza el comportamiento de una antena bocina cuando la excitación de dicha antena, situada en su interior, presenta polarización $\mathrm{TM}^{\mathrm{z}}$. La antena bocina considerada en esta sección, cuya apertura tiene un diámetro $D$ igual a $4 \lambda$, se muestra en la figura 7.25. Debido a las dimensiones de la citada antena, su contorno se segmenta en 10 tiras; que se dividen en dos grupos para caracterizar el comportamiento de la citada antena con excitación interna, en este caso situada en el punto $C_{f}$ (ver figura 7.25). De esta manera, empleando la técnica basada en la iteración especial para fuente interna al problema, recogida en el capítulo 6 y empleada con éxito en los ejemplos de la sección anterior; se determina el comportamiento de la antena bocina. Finalmente, para validar el método de análisis utilizado, se muestran resultados correspondientes al campo generado por la bocina cuando la excitación $\left(\mathrm{TM}^{\mathrm{z}}\right)$ de la antena, generada por una fuente situada en su interior, tiene carácter omnidireccional.

La estrategia seguida en el análisis de la bocina considerada se muestra en la figura 7.25; donde se observa la segmentación del contorno de la bocina en 10 


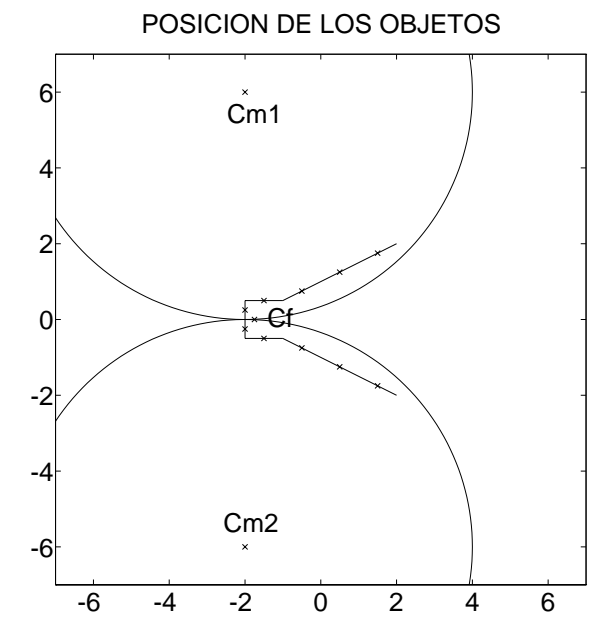

Figura 7.25: Estrategia de análisis del campo generado por una antena del tipo bocina $(D=4 \lambda)$, aproximando su contorno mediante 10 tiras metálicas, cuando la fuente que excita la bocina está situada en el punto $C_{f}$.

tiras metálicas infinitas, de grosor despreciable, divididas en dos agrupaciones. La razón de este agrupamiento de las tiras obedece a que la fuente generadora de la excitación se encuentra situada en el punto $C_{f}$ (ver figura 7.25), situado en el interior del problema de dispersión electromagnética a caracterizar; por lo que debe emplearse la iteración especial para fuente interna al problema, cuya aplicación requiere (recordar sección 6.4) la mencionada división de todos los objetos en dos grupos. Asimismo, dicha iteración especial requiere que el punto donde se posiciona la fuente ( $C_{f}$ en este caso) se encuentre situado fuera de las mínimas circunferencias, centradas en los puntos a los que se refieren los espectros de campo incidente sobre cada grupo de tiras (puntos $C_{m_{1}}$ y $C_{m_{2}}$ en fig. 7.25 ), y que contienen respectivamente a las tiras que integran cada uno de los dos grupos considerados. El cumplimiento de esta restricción adicional supone desplazar los puntos $C_{m_{1}}$ y $C_{m_{2}}$ de los puntos medios de los centros de todas las tiras integrantes de cada grupo (ver figura 7.25); lo que representa finalmente un incremento del número de modos a emplear en la correcta reconstrucción del campo incidente sobre cada grupo de tiras, y por tanto un mayor coste computacional en la obtención de resultados mediante el método escogido.

En cuanto a la excitación ( $\mathrm{TM}^{\mathrm{z}}$ ) generada por la fuente, situada en un punto interno de la antena bocina (punto $C_{f}$ en fig. 7.25), se escoge un comportamiento omnidireccional; que se consigue expresando dicha excitación mediante un solo modo cilíndrico emergente, aquél definido en términos de la función de Hankel de segunda especie y orden cero (recordar apartado C.1.2). La amplitud y la fase del campo eléctrico asociado a la excitación, que constituye el campo incidente 


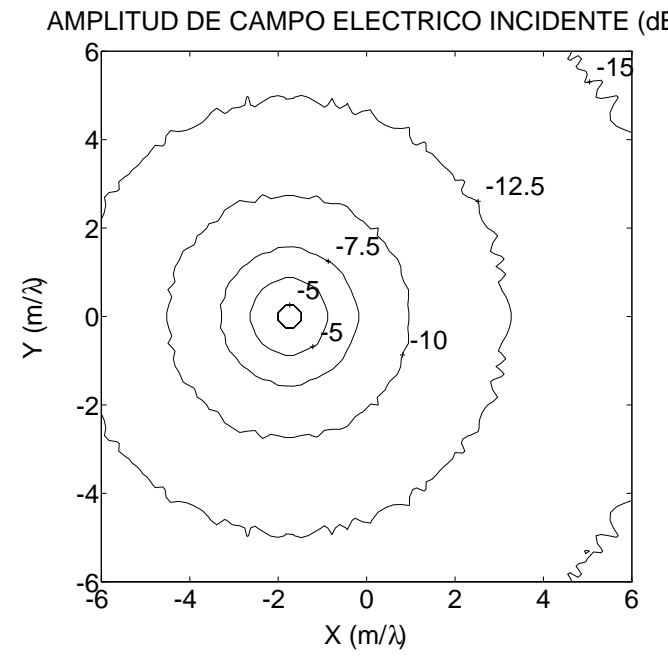

a)

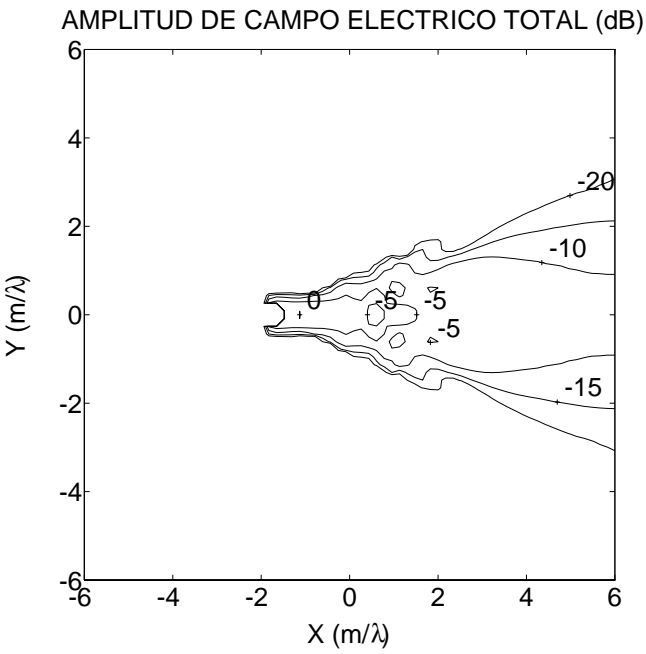

c)

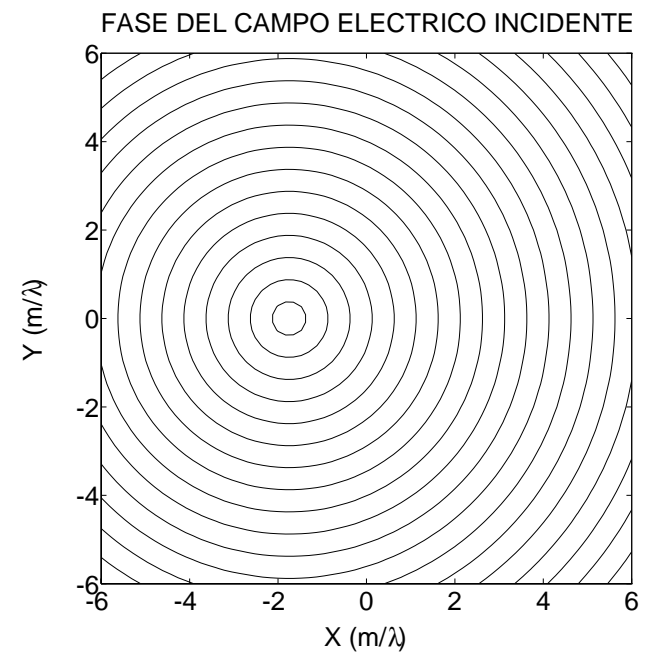

b)

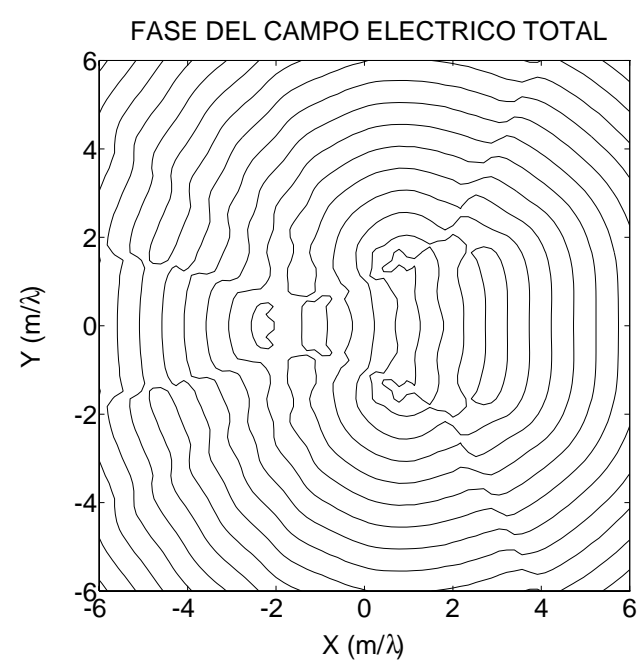

d)

Figura 7.26: Amplitud y fase de la excitación $\left(\mathrm{TM}^{\mathrm{z}}\right)$ de la antena bocina $(D=4 \lambda)$, generada por una fuente omnidireccional situada en el punto $C_{f}$, en a) y en b); y amplitud y fase del campo eléctrico generado por la bocina, definido como la suma de la excitación y el campo eléctrico dispersado por las tiras que definen la bocina ante dicha excitación, en $c$ ) y en $\mathrm{d}$ ).

sobre el problema de dispersión electromagnética producida por las 10 tiras mencionadas anteriormente, se representan en la figura 7.26 (apartados a y b); donde se observa un comportamiento omnidireccional en la amplitud (ver apartado a), y una evolución del frente de fase correspondiente a una onda cilíndrica generada en el punto $C_{f}$ (ver apartado b). Ante dicha incidencia, utilizando las matrices 


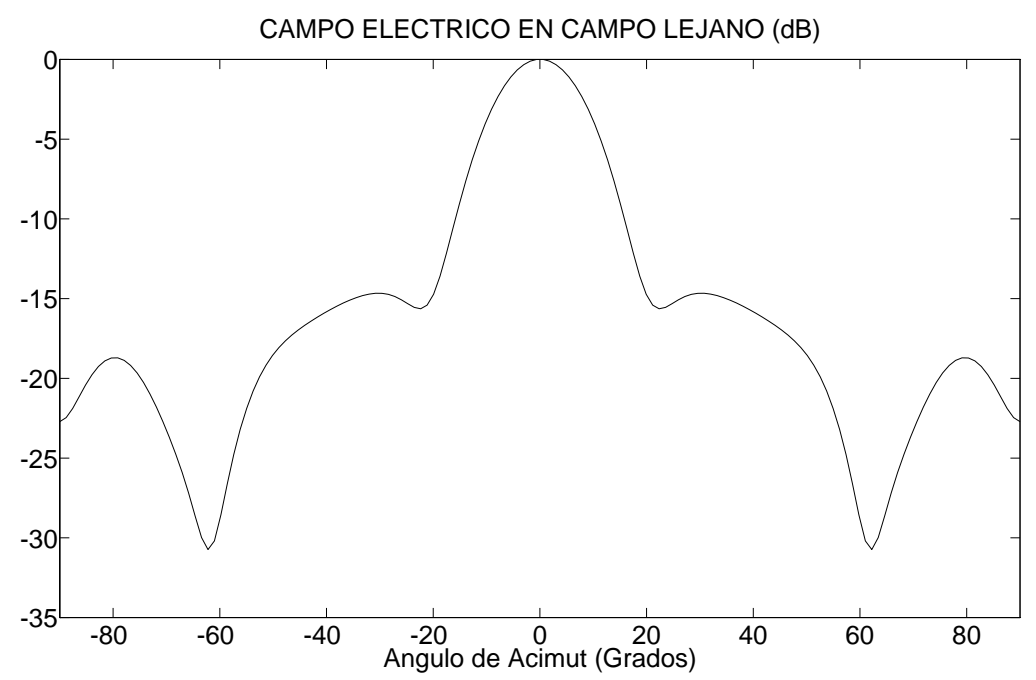

Figura 7.27: Amplitud del campo eléctrico dispersado en zona de campo lejano por las tiras metálicas que definen una antena bocina $(D=4 \lambda)$, ante una excitación $\left(\mathrm{TM}^{\mathrm{z}}\right)$ generada por una fuente situada en el punto $C_{f}$ de la bocina.

de caracterización conjunta de las 10 tiras que definen el contorno de la bocina, se obtiene el campo eléctrico dispersado por las mismas; que, sumado al campo eléctrico incidente, constituye el campo generado por la bocina cuya amplitud y fase se recogen en la figura 7.26 (apartados c y d). La amplitud y la fase de este campo eléctrico total revelan que la bocina, ante la excitación considerada, genera una onda plana cuyo frente de fase avanza en la dirección $\beta=0$ (ver apartados c y d en fig. 7.26).

Por último, para conocer el comportamiento de la antena bocina en zona de campo lejano, a partir de los resultados anteriores se ha determinado en dicha zona la amplitud del campo eléctrico dispersado por las 10 tiras que definen la bocina; representando la citada amplitud en la figura 7.27. En esta figura, se confirma la dirección de avance $(\beta=0)$ de la onda plana generada por la bocina; pues el máximo del lóbulo principal se produce en el valor acimutal asociado a la mencionada dirección de propagación. Asimismo, en la figura 7.27 se comprueba que el nivel de lóbulo principal a secundario en la amplitud del campo generado por la bocina es de aproximadamente $15 \mathrm{~dB}$. Los resultados ofrecidos en esta sección confirman la gran utilidad del método presentado en este capítulo; que permite analizar, además de los problemas de dispersión electromagnética considerados anteriormente, la radiación que generan estructuras semicerradas como las antenas tipo bocina. 



\section{Capítulo 8}

\section{Conclusiones}

En la presente tesis, se ha abordado con éxito el análisis de problemas de dispersión electromagnética, producida tanto en sistemas guiados (problemas cerrados) como en espacio libre (problemas abiertos), empleando para ello métodos modales basados en matrices generalizadas. Estos métodos se han aplicado al estudio de los fenómenos de dispersión presentes en diversas situaciones prácticas, tales como por ejemplo en los dispositivos de microondas integrados por diferentes guías, o en aquellas situaciones donde existe un cierto campo incidente sobre elementos dispersores situados en espacio libre; habiendo permitido dicho estudio el análisis de numerosos elementos radiantes, e incluso el diseño de algunas estructuras pasivas de microondas complejas.

Para analizar los problemas de dispersión electromagnética en sistemas guiados, mediante matrices de inmitancias (admitancias o impedancias según el caso), se ha propuesto una técnica que acelera el cálculo de los elementos de dichas matrices cuya obtención requiere evaluar una serie con infinitos términos; habiendo validado con un estudio comparativo la mejora que supone, en términos de eficiencia computacional, calcular los citados elementos mediante la nueva técnica descrita. De igual manera, tras acelerar la caracterización individual de las discontinuidades integrantes de un dispositivo pasivo de microondas, se plantea una nueva estrategia para resolver el sistema de ecuaciones lineales asociado al citado dispositivo; estrategia basada en un nuevo algoritmo recursivo que explota la naturaleza en banda del citado sistema, que se revela enormemente eficiente una vez realizado el correspondiente estudio comparativo.

Estas dos técnicas propuestas, que reducen considerablemente el coste asociado al análisis de dispositivos complejos de microondas, se han incorporado a una herramienta de programación pensada para el análisis y el diseño de estructuras pasivas de microondas implementadas en tecnología guiada; generando pues un programa muy completo y optimizado, de nombre comercial DUMAS 3.0, que está siendo distribuido por la Agencia Espacial Europea (ESA), en el marco de los acuerdos que mantiene dicha institución con sus países miembros, a las empresas 
del sector aeroespacial de estos países que diseñan filtros de microondas. Con el citado programa, se han diseñado algunas estructuras pasivas realmente complejas; tales como filtros paso banda en guía rectangular con tornillos de sintonía, filtros paso banda de modo dual en guía circular con iris elípticos, y filtros paso banda de modo dual en guía circular con iris elípticos y tornillos de sintonía. Asimismo, se han planteado para cada uno de estos filtros unas estrategias de diseño ciertamente novedosas; que han quedado completamente validadas, junto con el funcionamiento del mencionado programa DUMAS 3.0 que incorpora las nuevas técnicas propuestas en la tesis, mediante las medidas de las respuestas asociadas a unas implementaciones prácticas de los filtros diseñados.

Entre las posibles líneas futuras de trabajo, relacionadas con el análisis eficiente de la dispersión originada en sistemas guiados, puede resultar interesante esforzarse en intentar reducir el esfuerzo computacional requerido en la evaluación de las series estáticas (recordar concepto en el apartado 2.2.1 del capítulo 2), y de las partes estáticas de las series dinámicas (recordar concepto en el apartado 2.2.2 del capítulo 2), que aparecen al aplicar la técnica de aceleración descrita en la tesis. Aunque dichas series sólo se calculan una vez en el análisis en frecuencia del comportamiento del dispositivo, pues son independientes de la frecuencia, en determinadas aplicaciones con discontinuidades muy abruptas su computación puede resultar costosa; por lo que sería de gran interés, para acelerar todavía más el método propuesto, identificar alguna estrategia de suma de dichas series que tuviera en cuenta las contribuciones modales más significativas. Otra posible aplicación de la técnica propuesta, que en esencia permite evaluar de manera óptima unas determinadas series, consiste en su traslación a otros métodos de análisis de problemas cerrados donde aparecen este mismo tipo de series. Concretamente, estas series aparecen también durante el análisis de problemas de dispersión mediante el planteamiento de una ecuación integral, que surge tras forzar las correspondientes condiciones de contorno. La técnica de análisis basada en Ecuación Integral constituye un método que suele emplearse con geometrías complejas, como por ejemplo en la caracterización de codos en guía; tema en el que ya se está empezando a trabajar para acelerar la evaluación de ciertas series. Una nueva línea de trabajo asociada a la técnica de aceleración propuesta, que puede resultar especialmente interesante, consiste en la utilización combinada de dicha técnica con un método basado en teoría de cavidades para caracterizar ciertos dispositivos de microondas; concretamente aquéllos que presentan a la vez uniones entre guías diferentes en planos transversales y longitudinales (respecto de un eje del sistema), tales como pueden ser por ejemplo los dispositivos conocidos como T mágicas. De hecho, en la actualidad ya se dispone de resultados para estas estructuras que garantizan la validez de esta nueva técnica de análisis combinado; no habiendo incluido dichos resultados en el presente trabajo por no extender en demasía su contenido. A su vez, empleando la mencionada combinación de técnicas de análisis, se está diseñando en la actualidad un trans- 
ductor ortomodal; que está constituido por un cuerpo principal definido mediante una cavidad triangular, a cuyos tres puertos acceden respectivamente una guía circular y sendas guías rectangulares.

En cuanto a la futura utilización del programa comercial DUMAS 3.0, éste puede emplearse en el diseño de nuevas estructuras pasivas de microondas que incorporen algunos de los elementos novedosos susceptibles de ser analizados con dicho programa; tales como son los tornillos de sintonía modelados con guías reentrantes, o los iris con sección transversal elíptica. En este sentido, se propone el diseño con dicho programa de filtros paso banda implementados con cavidades evanescentes y elementos de sintonía; filtros cuya excitación, tanto a la entrada como a la salida, puede conseguirse bien con guías rectangulares (filtros evanescentes clásicos) o con guías coaxiales (filtros interdigitales). Adicionalmente, se plantea el diseño con DUMAS 3.0 de dispositivos giradores de polarización; implementados en guía circular, con iris cuadrados y elípticos, cuya configuración es similar a la de los filtros de modo dual propuestos en la tesis.

Por otra parte, para estudiar la dispersión electromagnética producida por objetos en espacio libre, se ha definido un nuevo tipo de matriz generalizada, denominada matriz de caracterización individual, que caracteriza el comportamiento dispersor de cada uno de estos objetos; habiendo determinado asimismo una estrategia genérica que permite obtener dicha matriz para cualquier posible objeto. Dicha estrategia combina métodos clásicos de análisis, ya sean de alta frecuencia o numéricos, con técnicas de obtención de espectros asociados a campos electromagnéticos; pues la citada matriz de caracterización individual se define en el dominio espectral. En concreto, como técnicas clásicas para determinar las corrientes inducidas sobre la superficie del objeto dispersor, se han considerado en este trabajo la conocida aproximación de Óptica Física y el tradicional Método de los Momentos; que han sido estudiadas con detalle, determinando unos criterios prácticos para decidir qué técnica conviene emplear en cada problema concreto. En cuanto a la obtención de los espectros correspondientes a los campos dispersados, se han propuesto nuevamente otras dos posibles técnicas, una de ellas basada en la Transformada Discreta de Fourier y la otra en el Teorema de Adición para las Funciones de Hankel; presentando también una discusión detallada acerca del posible ámbito de aplicación de cada una de dichas técnicas. Este nuevo concepto de la matriz de caracterización individual, y su estrategia de cálculo, se ha utilizado para analizar la dispersión producida por objetos dispersores canónicos, como son la tira y el cilindro, ambos metálicos y bidimensionales; concluyendo de los resultados obtenidos la validez del nuevo método propuesto para analizar fenómenos de dispersión en espacio libre, así como también algunas de las principales ventajas asociadas a dicho método.

Una vez definido el concepto de la matriz de caracterización individual, asociada al comportamiento dispersor aislado de cada objeto en espacio libre, se ha presentado un nuevo algoritmo recursivo para analizar la dispersión electro- 
magnética producida por múltiples objetos; cuya aplicación genera unas nuevas matrices asociadas respectivamente a los objetos del problema bajo análisis, denominadas ahora matrices de caracterización conjunta, que determinan el comportamiento dispersor de cada objeto teniendo en cuenta la presencia del resto. A su vez, se plantea el análisis de objetos dispersores grandes en términos eléctricos, tradicionalmente realizado con métodos de alta frecuencia, mediante una segmentación de su contorno en elementos de menor dimensión, y su posterior análisis conjunto mediante el algoritmo recursivo propuesto en la tesis. Esta estrategia de análisis se aplica de hecho a la resolución de algunos problemas de dispersión constituidos por varias tiras metálicas; cuyos resultados se comparan con los obtenidos mediante otras técnicas clásicas de análisis, confirmando pues la validez tanto de la técnica de segmentación descrita como del algoritmo recursivo propuesto. La misma estrategia de análisis recién mencionada, basada en segmentación, se ha empleado para caracterizar la dispersión producida por reflectores hiperbólicos y parabólicos grandes eléctricamente; habiendo comparado los diagramas de radiación obtenidos para el reflector parabólico con los deducidos mediante el conocido Método de la Distribución en la Apertura, de donde se concluye que la técnica de segmentación junto con el algoritmo recursivo proporciona resultados muy exactos. De igual manera, empleando la versión adecuada del algoritmo recursivo, se ofrecen resultados correspondientes a la dispersión producida por antenas $\mathrm{Ca}$ ssegrain, integradas por los citados reflectores hiperbólico y parabólico; habiendo observado en los resultados obtenidos algunos fenómenos propios de este tipo de antenas, que empleando otros métodos clásicos de análisis (como por ejemplo el Método de la Distribución en la Apertura, el Método de la Parábola Equivalente o el Método del Alimentador Virtual) no habrían podido detectarse. Parece lógico pensar pues que la nueva técnica de análisis de dispersión múltiple propuesta en esta tesis, basada en segmentación y en el nuevo alogoritmo recursivo expuesto, debe utilizarse en el diseño eficiente y preciso de estructuras radiantes en espacio libre, tales como por ejemplo las mencionadas antenas Cassegrain. Finalmente, la potencia de esta nueva técnica se pone de manifiesto analizando el comportamiento de una antena tipo bocina; observando como la distribución de campos generada por dicho elemento radiante coincide con el comportamiento previsto para este tipo de estructuras.

Una primera línea futura de trabajo en problemas abiertos, relacionada con la caracterización individual del comportamiento dispersor de cada objeto y con el algoritmo recursivo para analizar el acoplo entre múltiples objetos, consiste en agrupar los diversos programas que implementan dichos métodos de caracterización (individual y conjunta) en un mismo entorno; de manera que el usuario pudiera escoger de entre una serie de objetos canónicos (tira y cilindro en estos momentos) aquéllos que integran el problema a analizar, así como su distribución en el espacio libre, y que el programa se encargara de resolver de forma automática dicho problema de dispersión múltiple. Lógicamente, con la intención de ampliar 
el número de objetos dispersores canónicos ofrecidos por el mencionado programa, resultaría interesante implementar la caracterización individual de nuevos objetos metálicos, como por ejemplo reflectores individuales hiperbólicos y parabólicos, e incluso de objetos no metálicos constituidos por materiales dieléctricos, no homogéneos, o anisótropos. Asimismo, debería crearse una librería con matrices de caracterización individual de objetos previamente caracterizados; para de esta forma, en aquellos problemas donde intervinieran estos elementos, no tener que repetir el proceso de cálculo de sus respectivas matrices.

Como segunda línea futura de investigación, utilizando el nuevo algoritmo recursivo propuesto para resolver problemas de dispersión múltiple, se plantea realizar una extensión de dicha técnica recursiva al análisis de nuevas aplicaciones prácticas; como pueden ser el estudio de fenómenos de propagación en entornos urbanos (comunicaciones móviles), y el análisis de problemas de dispersión en sistemas guiados (como diafragmas inductivos en guías de placas paralelas) tradicionalmente resueltos con otras técnicas. En la actualidad, ambas líneas se encuentran en sus inicios; debiendo explorarlas todavía con un mayor detalle para garantizar sus posibilidades de éxito, y para extender posteriormente el método de análisis a la resolución de problemas más complejos.

Una tercera y última línea de investigación, de gran interés por cierto, se encuentra relacionada con una lógica extensión de los conceptos asociados al análisis de problemas de dispersión en espacio libre al caso de objetos tridimensionales; lo que no supone en absoluto ninguna complejidad conceptual añadida, pues la filosofía del método es la misma, aunque requiere el manejo de espectros de ondas esféricas en lugar de espectros cilíndricos (problemas bidimensionales).

De los comentarios realizados en los párrafos anteriores, es posible concluir de forma definitiva que con el trabajo presentado se han alcanzado todos los objetivos propuestos al inicio de la tesis; habiendo sido capaces de analizar con éxito, mediante métodos modales y matrices generalizadas, problemas de dispersión electromagnética tanto en sistemas guiados como en espacio libre con las peculiaridades que cada uno de dichos problemas conlleva. Por último, mencionar simplemente que el trabajo realizado ha despertado interés en la comunidad científica internacional; interés avalado por la publicación de artículos en revistas internacionales, referenciados en la bibliografía mediante las citas [18], [66], [67] y [68], y por las comunicaciones presentadas en congresos internacionales, recogidas también en la bibliografía a través de las citas [28], [64] y [75]. 



\section{Apéndice A}

\section{Desarrollos en Serie de Taylor de la función $f_{r}(k)$}

La técnica que permite acelerar el cálculo de los elementos $Y_{m, n}^{(2,2)}$ de la matriz de admitancias generalizada de uniones planares entre guías arbitrarias, descrita de forma detallada en el capítulo 2, se basa en extraer la dependencia frecuencial que presentan las series infinitas a evaluar durante el cálculo de dichos elementos. Para ello, esta nueva técnica descompone en principio la serie asociada originalmente al elemento $Y_{m, n}^{(2,2)}$ en una serie estática, que resulta independiente de la frecuencia, y en una serie dinámica que debe calcularse para cada frecuencia. A continuación, con el objeto de continuar reduciendo el esfuerzo computacional dedicado a la evaluación de los elementos $Y_{m, n}^{(2,2)}$, la mencionada técnica trunca la serie dinámica en una contribución finita de términos, a evaluar en cada frecuencia, y en una serie infinita de la que es posible extraer su dependencia con la frecuencia. Esta última extracción frecuencial puede realizarse gracias al desarrollo en serie de Taylor de una función, denominada $f_{r}(k)$, que forma parte de los elementos integrantes de la citada serie infinita asociada a la serie dinámica.

En este apéndice, se presentan las expresiones analíticas explícitas de los coeficientes que forman parte de los desarrollos en serie de Taylor de la función $f_{r}(k)$; tanto para el caso en el que el índice $r$ de la serie infinita asociada a la serie dinámica designa un modo TE, como para el caso en el que dicho índice $r$ determina un modo TM.

\section{A.1 Modos TE}

La función $f_{r}(k)$, recordando $(2.20)$, queda definida del siguiente modo

$$
f_{r}(k)=1-\frac{Y_{0 r}^{(1)} \cdot \cot \left(\beta_{r}^{(1)} \cdot l_{\mathrm{ref}}\right)}{\hat{Y}_{0 r}^{(1)} \cdot \cot \left(\hat{\beta}_{r}^{(1)} \cdot l_{\mathrm{ref}}\right)}
$$


La expresión correspondiente a $f_{r}(k)$, para el caso en el que el índice $r$ hace referencia a un modo TE, se obtiene tras sustituir en (A.1) $Y_{0 r}^{(1)}, \beta_{r}^{(1)}, \hat{Y}_{0 r}^{(1)}$ y $\hat{\beta}_{r}^{(1)}$ por sus correspondientes valores; lo que origina que dicha función $f_{r}(k)$, tal y como se recoge en (2.23) para modos TE, presente el siguiente aspecto

$$
f_{r}(k)=1-\frac{\sqrt{1-\left(k / k_{t, r}^{(1)}\right)^{2}} \cdot \operatorname{coth}\left(c \cdot \sqrt{1-\left(k / k_{t, r}^{(1)}\right)^{2}}\right)}{\operatorname{coth}(c)}
$$

Debido a que esta función $f_{r}(k)$ presenta un comportamiento suave en la zona asociada a valores de la variable normalizada $k / k_{t, r}^{(1)}$ próximos a 0 (recordar apartado a en fig. 2.8), resulta posible aproximar en dicha zona la citada función $f_{r}(k)$ mediante un desarrollo en serie de Taylor centrado en $k / k_{t, r}^{(1)}=0$; cuyo aspecto se recoge a continuación

$$
f_{r}(k)=\sum_{p=0}^{\infty} b_{p, r} \cdot\left(\frac{k}{k_{t, r}^{(1)}}\right)^{p}
$$

Las expresiones explícitas de los coeficientes $b_{p, r}$ no nulos correspondientes al mencionado desarrollo en serie de Taylor de la función $f_{r}(k)$ se definen, para el caso en el que el índice $r$ designa un modo TE y hasta un valor máximo del índice $p$ igual a 8 , de la siguiente manera

$$
\begin{aligned}
b_{2, r}= & \frac{1}{2}+\frac{A_{1}}{\left(e^{2 c}-1\right)} \\
b_{4, r}= & \frac{1}{8}+\frac{A_{4}}{\left(e^{2 c}-1\right)}+\frac{A_{1} \cdot A_{2}}{2 \cdot\left(e^{2 c}-1\right)^{2} \cdot\left(e^{2 c}+1\right)} \\
b_{6, r}= & \frac{1}{16}+\frac{2 \cdot A_{7} \cdot e^{2 c}}{\left(e^{2 c}-1\right) \cdot\left(e^{2 c}+1\right)}+\frac{2 \cdot c \cdot A_{3} \cdot e^{4 c}}{\left(e^{2 c}-1\right) \cdot\left(e^{2 c}+1\right)^{2}} \\
& -\frac{c^{2} \cdot A_{6} \cdot e^{4 c}}{2 \cdot\left(e^{2 c}-1\right) \cdot\left(e^{2 c}+1\right)^{3}}+\frac{A_{2} \cdot A_{4}}{2 \cdot\left(e^{2 c}-1\right)^{2} \cdot\left(e^{2 c}+1\right)} \\
& +\frac{\left[-e^{6 c}+\left(8 \cdot c^{2}+1\right) \cdot e^{4 c}+\left(8 \cdot c^{2}+4 \cdot c+1\right) \cdot e^{2 c}\right] \cdot A_{1}}{8 \cdot\left(e^{2 c}-1\right)^{3} \cdot\left(e^{2 c}+1\right)} \\
& -\frac{\left(1+4 \cdot A_{5}\right) \cdot A_{1}}{8 \cdot\left(e^{2 c}-1\right)^{3} \cdot\left(e^{2 c}+1\right)} \\
b_{8, r}= & \frac{5}{128}+\frac{2 \cdot\left(\frac{1}{24} \cdot c^{4}-\frac{1}{8} \cdot c^{3}+\frac{5}{32} \cdot c^{2}-\frac{5}{64} \cdot c\right) \cdot e^{2 c}}{\left(e^{2 c}-1\right) \cdot\left(e^{2 c}+1\right)} \\
& +\frac{2 \cdot c \cdot A_{7} \cdot e^{4 c}}{\left(e^{2 c}-1\right) \cdot\left(e^{2 c}+1\right)^{2}}+\frac{c \cdot A_{3} \cdot A_{6} \cdot e^{4 c}}{2 \cdot\left(e^{2 c}-1\right) \cdot\left(e^{2 c}+1\right)^{3}}
\end{aligned}
$$




$$
\begin{aligned}
& +\frac{A_{2} \cdot A_{7} \cdot e^{2 c}}{\left(e^{2 c}-1\right)^{2} \cdot\left(e^{2 c}+1\right)^{2}}+\frac{c \cdot A_{2} \cdot A_{3} \cdot e^{4 c}}{\left(e^{2 c}-1\right)^{2} \cdot\left(e^{2 c}+1\right)^{3}} \\
& -\frac{c^{2} \cdot A_{2} \cdot A_{6} \cdot e^{4 c}}{4 \cdot\left(e^{2 c}-1\right)^{2} \cdot\left(e^{2 c}+1\right)^{4}}+\frac{\left[-e^{6 c}+\left(8 \cdot c^{2}+1\right) \cdot e^{4 c}\right] \cdot A_{4}}{8 \cdot\left(e^{2 c}-1\right)^{3} \cdot\left(e^{2 c}+1\right)} \\
& -\frac{\left[\left(4 \cdot c^{2}+3\right) \cdot e^{4 c}+\left(-16 \cdot c^{2}+6\right) \cdot e^{2 c}\right] \cdot c^{2} \cdot e^{4 c}}{12 \cdot\left(e^{2 c}-1\right) \cdot\left(e^{2 c}+1\right)^{4}} \\
& -\frac{\left(4 \cdot c^{2}-6 \cdot c+3+6 \cdot A_{5}\right) \cdot c^{2} \cdot e^{4 c}}{12 \cdot\left(e^{2 c}-1\right) \cdot\left(e^{2 c}+1\right)^{4}} \\
& +\frac{\left[\left(8 \cdot c^{2}+4 \cdot c+1\right) \cdot e^{2 c}-\left(1+4 \cdot A_{5}\right)\right] \cdot A_{4}}{8 \cdot\left(e^{2 c}-1\right)^{3} \cdot\left(e^{2 c}+1\right)} \\
& +\frac{\left[-3 \cdot e^{8 c}+\left(16 \cdot c^{3}-12 \cdot c+6\right) \cdot e^{6 c}+64 \cdot c^{3} \cdot e^{4 c}\right] \cdot A_{1}}{48 \cdot\left(e^{2 c}-1\right)^{4} \cdot\left(e^{2 c}+1\right)} \\
& +\frac{\left[\left(16 \cdot c^{3}-12 \cdot c-6\right) \cdot e^{2 c}+\left(3+24 \cdot A_{5}\right)\right] \cdot A_{1}}{48 \cdot\left(e^{2 c}-1\right)^{4} \cdot\left(e^{2 c}+1\right)}
\end{aligned}
$$

donde las constantes $c, A_{1}, A_{2}, A_{3}, A_{4}, A_{5}, A_{6}$ y $A_{7}$ se definen como

$$
\begin{aligned}
c & =k_{t, r}^{(1)} \cdot l_{\mathrm{ref}} \\
A_{1} & =-\frac{2 \cdot c \cdot e^{2 c}}{\left(e^{2 c}+1\right)} \\
A_{2} & =-e^{4 c}+4 \cdot c \cdot e^{2 c}+1 \\
A_{3} & =\frac{c^{2}}{2}-\frac{c}{4} \\
A_{4} & =\frac{2 \cdot\left[\left(A_{3}-c^{2}\right) \cdot e^{2 c}+A_{3}\right] \cdot e^{2 c}}{\left(e^{2 c}+1\right)^{2}} \\
A_{5} & =c \cdot e^{4 c} \\
A_{6} & =(1+2 \cdot c) \cdot e^{2 c}+(1-2 \cdot c) \\
A_{7} & =-\frac{c^{3}}{6}+\frac{c^{2}}{4}-\frac{c}{8}
\end{aligned}
$$

\section{A.2 Modos TM}

La función $f_{r}(k)$, recordando nuevamente (2.20), se define como

$$
f_{r}(k)=1-\frac{Y_{0 r}^{(1)} \cdot \cot \left(\beta_{r}^{(1)} \cdot l_{\mathrm{ref}}\right)}{\hat{Y}_{0 r}^{(1)} \cdot \cot \left(\hat{\beta}_{r}^{(1)} \cdot l_{\mathrm{ref}}\right)}
$$


La expresión correspondiente a $f_{r}(k)$, para el caso en el que el índice $r$ hace referencia a un modo TM, se obtiene tras sustituir en (A.16) $Y_{0 r}^{(1)}, \beta_{r}^{(1)}, \hat{Y}_{0 r}^{(1)} \mathrm{y}$ $\hat{\beta}_{r}^{(1)}$ por sus correspondientes valores; lo que origina que dicha función $f_{r}(k)$, tal y como se recoge en (2.23) para modos TM, presente el siguiente aspecto

$$
f_{r}(k)=1-\frac{\operatorname{coth}\left(c \cdot \sqrt{1-\left(k / k_{t, r}^{(1)}\right)^{2}}\right)}{\sqrt{1-\left(k / k_{t, r}^{(1)}\right)^{2}} \cdot \operatorname{coth}(c)}
$$

Debido a que esta función $f_{r}(k)$ también presenta una evolución suave en la zona asociada a valores de la variable normalizada $k / k_{t, r}^{(1)}$ próximos a 0 (recordar apartado b en fig. 2.8), resulta posible aproximar de nuevo en dicha zona la citada función $f_{r}(k)$ mediante un desarrollo en serie de Taylor centrado en $k / k_{t, r}^{(1)}=0$; cuyo aspecto se recoge a continuación

$$
f_{r}(k)=\sum_{p=0}^{\infty} b_{p, r} \cdot\left(\frac{k}{k_{t, r}^{(1)}}\right)^{p}
$$

Las expresiones analíticas de los coeficientes $b_{p, r}$ no nulos correspondientes al mencionado desarrollo en serie de Taylor de la función $f_{r}(k)$ se definen, para el caso en el que el índice $r$ designa un modo TM y hasta un valor máximo del índice $p$ igual a 8 , de la siguiente manera

$$
\begin{aligned}
b_{2, r}= & -\frac{1}{2}+\frac{A_{1}}{\left(e^{2 c}-1\right)} \\
b_{4, r}= & -\frac{3}{8}+\frac{A_{4}}{\left(e^{2 c}-1\right)}+\frac{A_{1} \cdot A_{2}}{2 \cdot\left(e^{2 c}-1\right)^{2} \cdot\left(e^{2 c}+1\right)} \\
b_{6, r}= & -\frac{5}{16}+\frac{2 \cdot A_{7} \cdot e^{2 c}}{\left(e^{2 c}-1\right) \cdot\left(e^{2 c}+1\right)}+\frac{2 \cdot c \cdot A_{3} \cdot e^{4 c}}{\left(e^{2 c}-1\right) \cdot\left(e^{2 c}+1\right)^{2}} \\
& -\frac{c^{2} \cdot A_{6} \cdot e^{4 c}}{2 \cdot\left(e^{2 c}-1\right) \cdot\left(e^{2 c}+1\right)^{3}}+\frac{A_{2} \cdot A_{4}}{2 \cdot\left(e^{2 c}-1\right)^{2} \cdot\left(e^{2 c}+1\right)} \\
& +\frac{\left[3 \cdot e^{6 c}+\left(8 \cdot c^{2}-3\right) \cdot e^{4 c}+\left(8 \cdot c^{2}-12 \cdot c-3\right) \cdot e^{2 c}\right] \cdot A_{1}}{8 \cdot\left(e^{2 c}-1\right)^{3} \cdot\left(e^{2 c}+1\right)} \\
& +\frac{\left(3+12 \cdot A_{5}\right) \cdot A_{1}}{8 \cdot\left(e^{2 c}-1\right)^{3} \cdot\left(e^{2 c}+1\right)} \\
b_{8, r}= & -\frac{35}{128}+\frac{2 \cdot\left(\frac{1}{24} \cdot c^{4}-\frac{1}{8} \cdot c^{3}+\frac{5}{32} \cdot c^{2}-\frac{5}{64} \cdot c\right) \cdot e^{2 c}}{\left(e^{2 c}-1\right) \cdot\left(e^{2 c}+1\right)} \\
& +\frac{2 \cdot c \cdot A_{7} \cdot e^{4 c}}{\left(e^{2 c}-1\right) \cdot\left(e^{2 c}+1\right)^{2}}+\frac{c \cdot A_{3} \cdot A_{6} \cdot e^{4 c}}{2 \cdot\left(e^{2 c}-1\right) \cdot\left(e^{2 c}+1\right)^{3}}
\end{aligned}
$$




$$
\begin{aligned}
& +\frac{A_{2} \cdot A_{7} \cdot e^{2 c}}{\left(e^{2 c}-1\right)^{2} \cdot\left(e^{2 c}+1\right)^{2}}+\frac{c \cdot A_{2} \cdot A_{3} \cdot e^{4 c}}{\left(e^{2 c}-1\right)^{2} \cdot\left(e^{2 c}+1\right)^{3}} \\
& -\frac{c^{2} \cdot A_{2} \cdot A_{6} \cdot e^{4 c}}{4 \cdot\left(e^{2 c}-1\right)^{2} \cdot\left(e^{2 c}+1\right)^{4}}+\frac{\left[3 \cdot e^{6 c}+\left(8 \cdot c^{2}-3\right) \cdot e^{4 c}\right] \cdot A_{4}}{8 \cdot\left(e^{2 c}-1\right)^{3} \cdot\left(e^{2 c}+1\right)} \\
& -\frac{\left[\left(4 \cdot c^{2}+3\right) \cdot e^{4 c}+\left(-16 \cdot c^{2}+6\right) \cdot e^{2 c}\right] \cdot c^{2} \cdot e^{4 c}}{12 \cdot\left(e^{2 c}-1\right) \cdot\left(e^{2 c}+1\right)^{4}} \\
& -\frac{\left(4 \cdot c^{2}-6 \cdot c+3+6 \cdot A_{5}\right) \cdot c^{2} \cdot e^{4 c}}{12 \cdot\left(e^{2 c}-1\right) \cdot\left(e^{2 c}+1\right)^{4}} \\
& +\frac{\left[\left(8 \cdot c^{2}-12 \cdot c-3\right) \cdot e^{2 c}+\left(3+12 \cdot A_{5}\right)\right] \cdot A_{4}}{8 \cdot\left(e^{2 c}-1\right)^{3} \cdot\left(e^{2 c}+1\right)} \\
& +\frac{\left[15 \cdot e^{8 c}+\left(16 \cdot c^{3}+48 \cdot c^{2}+60 \cdot c-30\right) \cdot e^{6 c}+64 \cdot c^{3} \cdot e^{4 c}\right] \cdot A_{1}}{48 \cdot\left(e^{2 c}-1\right)^{4} \cdot\left(e^{2 c}+1\right)} \\
& +\frac{\left[\left(16 \cdot c^{3}-48 \cdot c^{2}+60 \cdot c+30\right) \cdot e^{2 c}-\left(15+120 \cdot A_{5}\right)\right] \cdot A_{1}}{48 \cdot\left(e^{2 c}-1\right)^{4} \cdot\left(e^{2 c}+1\right)}
\end{aligned}
$$

donde las constantes $c, A_{1}, A_{2}, A_{3}, A_{4}, A_{5}, A_{6}$ y $A_{7}$ se definen en este caso mediante las siguientes expresiones

$$
\begin{aligned}
c & =k_{t, r}^{(1)} \cdot l_{\mathrm{ref}} \\
A_{1} & =-\frac{2 \cdot c \cdot e^{2 c}}{\left(e^{2 c}+1\right)} \\
A_{2} & =e^{4 c}+4 \cdot c \cdot e^{2 c}-1 \\
A_{3} & =\frac{c^{2}}{2}-\frac{c}{4} \\
A_{4} & =\frac{2 \cdot\left[\left(A_{3}-c^{2}\right) \cdot e^{2 c}+A_{3}\right] \cdot e^{2 c}}{\left(e^{2 c}+1\right)^{2}} \\
A_{5} & =c \cdot e^{4 c} \\
A_{6} & =(1+2 \cdot c) \cdot e^{2 c}+(1-2 \cdot c) \\
A_{7} & =-\frac{c^{3}}{6}+\frac{c^{2}}{4}-\frac{c}{8}
\end{aligned}
$$





\section{Apéndice B}

\section{DUMAS 3.0: Un Programa para el Análisis y el Diseño Eficiente de Estructuras Pasivas de Microondas}

El análisis y el diseño de estructuras pasivas de microondas, a medida que se incrementa su complejidad, requiere de programas especialmente optimizados que generen de manera eficiente resultados muy precisos; tal y como se ha puesto de manifiesto en los ejemplos de diseño recogidos en el capítulo 3. Con el objeto de acelerar la caracterización electromagnética de las diferentes uniones planares que integran las estructuras pasivas de microondas, empleando en dicha caracterización matrices de admitancias generalizadas, en el capítulo 2 (sección 2.2) se describe una técnica novedosa para calcular eficientemente aquellos elementos de las mencionadas matrices cuya evaluación tradicional resulta más costosa. Por otra parte, en el mismo capítulo 2 (sección 2.3) se propone un método recursivo para resolver con eficiencia el sistema de ecuaciones lineales, cuya matriz de coeficientes presenta una estructura en banda al utilizar matrices de admitancias generalizadas, que surge al analizar el comportamiento electromagnético del dispositivo considerado. Estas dos nuevas técnicas, que permiten reducir sustancialmente el coste temporal asociado al análisis de dispositivos complejos (recordar resultados ofrecidos en el capítulo 2), se han incorporado a un programa de análisis y diseño de estructuras pasivas de microondas; generando una versión de dicho programa denominada DUMAS 3.0, cuyos rasgos más destacados pretenden describirse en el presente apéndice.

Junto a las dos técnicas recién comentadas que incorpora el citado programa DUMAS 3.0, éste ofrece también la posibilidad de analizar y diseñar dispositivos de microondas empleando modos accesibles y localizados; cuyos respectivos conceptos se exponen inicialmente en este apéndice. Como uno de los principales 
objetivos del mencionado programa es el diseño de filtros paso banda, a continuación se describen dos redes ideales que incorpora dicho programa para poder diseñar los citados filtros paso banda, implementados con cavidades resonantes en las que tan sólo se excita el modo fundamental, siguiendo el procedimiento expuesto en [40]. Asimismo, se presenta una tercera red ideal pensada para poder diseñar con ella nuevamente filtros paso banda; pero implementados en esta ocasión con cavidades evanescentes (no se excita ningún modo) y elementos de sintonía, lo que a su vez requiere modificar ligeramente el procedimiento de diseño planteado en [40]. Por último, reseñar que DUMAS 3.0 está preparado para trabajar conjuntamente con el programa comercial ANAPLAN-W; permitiendo pues la incorporación a los diseños de tramos uniformes de guías reentrantes con contornos básicos rectangulares y circulares, que pretenden simular el efecto de los tornillos de sintonía utilizados tradicionalmente en numerosos dispositivos por diversas razones (ver capítulo 3). Con la intención pues de mostrar esta opción adicional, así como para describir brevemente el modo de funcionamiento básico del programa DUMAS 3.0, se ofrece finalmente un ejemplo práctico de manejo del mismo; correspondiente al análisis de la respuesta electromagnética que presenta el filtro de modo dual en guía circular, con iris elípticos y guías circulares reentrantes, propuesto en el capítulo 3 (apartado 3.2.3).

\section{B.1 Modos Accesibles y Localizados}

Los modos accesibles y localizados, introducidos de alguna manera en [9], se definen por primera vez empleando dichas acepciones en [83]; habiendo sido utilizados posteriormente en numerosas ocasiones, tal y como puede comprobarse por ejemplo en $[53,84,85]$. Estos tipos de modos surgen al caracterizar uniones planares entre guías arbitrarias mediante redes equivalentes multimodales, en el caso que nos ocupa basadas en matrices de admitancias generalizadas, que en principio requieren un número infinito de modos para describir los campos eléctrico y magnético existentes cerca de la unión planar en cada una de las guías que definen dicha unión; aunque en la práctica, para poder implementar computacionalmente las mencionadas matrices, el número teóricamente infinito de modos a utilizar se trunca en un valor finito que garantiza resultados convergentes. Este valor finito dependerá lógicamente del tipo de discontinuidad considerada; que excitará un mayor o un menor número de modos en sus proximidades. Ahora bien, cuando se pretende analizar dispositivos de microondas constituidos por la conexión en cascada de diferentes tramos de guías uniformes, si la longitud de dichos tramos es suficientemente grande, tan sólo una pequeña parte de los modos excitados en las proximidades de cada unión (aquéllos con números de onda de corte de valor más bajo) afectan a las discontinuidades vecinas; por lo que en la conexión de las redes equivalentes multimodales asociadas a cada unión, haciendo uso de las 
correspondientes matrices de admitancias generalizadas asociadas a los tramos de guía uniforme que las unen, deben considerarse únicamente esos pocos modos mencionados que constituyen los llamados modos accesibles. En cuanto al resto de modos excitados en las proximidades de cada discontinuidad (aquéllos con números de onda de corte de valor elevado), que se denominan modos localizados, deben tenerse en cuenta únicamente en la caracterización de cada unión planar; sin embargo, como estos modos se encuentran al corte en los respectivos tramos de guía uniforme a los que pertenecen, cada uno de los accesos asociados a dichos modos en las correspondientes matrices de admitancias generalizadas se cargarán con la admitancia característica del modo al que representan.

Según se termina de explicar, cuando se caracteriza una unión planar empleando modos accesibles y localizados, debe determinarse una nueva matriz de admitancias generalizada que relaciona únicamente modos accesibles; cuya deducción, basada en la matriz de admitancias generalizada asociada originalmente a la mencionada unión, requiere imponer sobre esta última matriz la citada condición de carga de los modos localizados que se consideran en cada puerto de la unión. Seguidamente, se ofrecen las expresiones analíticas que permiten relacionar las mencionadas matrices; para lo cual se escoge una unión planar genérica entre dos guías arbitrarias, de la que se determina inicialmente su red equivalente multimodal calculando la correspondiente matriz de admitancias generalizada. Haciendo uso pues de dicha matriz, que denominaremos $Y$, es posible relacionar los vectores columna $I$ y $V$, constituidos respectivamente por corrientes y voltajes modales, de la siguiente manera

$$
I=Y \cdot V
$$

En (B.1), los dos vectores columna $I$ y $V$ están constituidos respectivamente por dos subvectores; cada uno de los cuales representa a su vez las amplitudes de las funciones vectoriales modales normalizadas, asociadas bien al campo eléctrico o magnético transversal, que permiten evaluar dichos campos en cada uno de los dos puertos que definen la unión. Las dimensiones de estos subvectores se corresponden respectivamente con el número de modos totales escogidos para representar los campos eléctrico y magnético en cada puerto; números que en el caso considerado, tal y como puede observarse en la figura B.1 (apartado a), se designan mediante los parámetros $\mathrm{NM}(1)$ para el puerto 1 y $\mathrm{NM}(2)$ para el puerto 2. A continuación, debido a que la discontinuidad caracterizada se piensa conectar a las uniones vecinas mediante tramos de guía uniforme suficientemente largos, los modos considerados en cada puerto se dividen en accesibles y localizados; representando ahora en la figura B.1 (apartados a y b) el número de modos accesibles escogido en cada puerto mediante los parámetros $\mathrm{NMa}(1)$ y $\mathrm{NMa}(2)$ respectivamente. Con el objeto de obtener una nueva matriz de admitancias equivalente para la misma unión planar considerada, que ahora relacione tan sólo corrientes y voltajes asociados a los modos accesibles presentes en cada puerto 

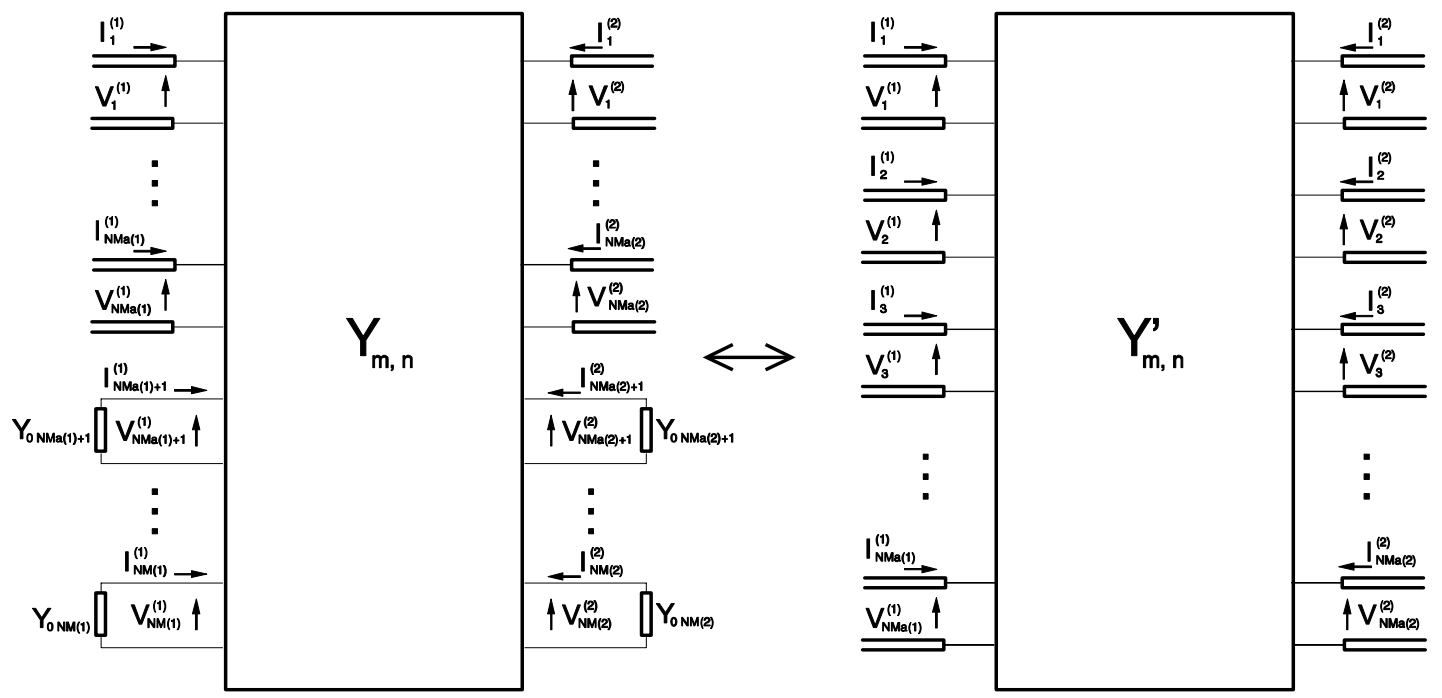

Figura B.1: Representación de una unión planar entre dos guías arbitrarias mediante una red equivalente multimodal, basada en la matriz de admitancias generalizada original cuyos modos localizados están cargados con sus respectivas admitancias características, en a); y representación de la misma unión planar mediante otra red equivalente multimodal, basada en una matriz de admitancias equivalente a la original que sólo relaciona modos accesibles, en b).

de la unión, resulta necesario como es sabido cargar los accesos de la matriz original correspondientes a los modos localizados con sus respectivas admitancias características; presentando pues dicha matriz original cargada el aspecto que se ofrece en la figura B.1 (apartado a). Para trasladar dicha condición de carga a la relación matricial definida anteriormente en (B.1) por la matriz $Y$, conviene considerar dos matrices diagonales denominadas $A$ y $B$; cuyas diagonales principales están constituidas por tantos elementos como la suma de los números de modos totales asociados respectivamente a cada puerto, presentando cada uno de dichos elementos un valor igual a 1 o igual a 0 . En concreto, la matriz $A$ contiene elementos unitarios en aquellas posiciones de la diagonal principal correspondientes a modos localizados definidos en ambos puertos de la unión, y valores nulos en el resto de elementos; mientras la matriz $B$ es complementaria de la matriz $A$ recién descrita (elementos unitarios en las posiciones de la diagonal principal asociadas a los modos accesibles y valores nulos en los restantes elementos). Así pues, como la suma de las matrices $A$ y $B$ es igual a la matriz identidad $U$, la relación expuesta previamente en (B.1) puede expresarse también como

$$
I=Y \cdot A \cdot V+Y \cdot B \cdot V
$$

Para definir la condición de carga de los accesos de la matriz de admitancias generalizada correspondientes a los modos localizados, condición recogida 
gráficamente en la figura B.1 (apartado a), es necesario definir una matriz de impedancias de carga también diagonal; en la que los elementos de su diagonal principal asociados a los modos localizados son iguales respectivamente a las impedancias características de dichos modos con signo negativo, y los elementos restantes correspondientes a los modos accesibles presentan todos ellos valores nulos. El signo negativo que presentan todos los elementos no nulos de la mencionada matriz se debe a la elección de los sentidos de las corrientes modales en la red equivalente multimodal; los cuales, tal y como puede apreciarse en la figura B.1 (apartado a), se escogen siempre para que la potencia transportada por cada modo fluya siempre hacia la unión planar caracterizada. Haciendo uso pues de la matriz de impedancias de carga recién descrita, denominada $Z_{0}$, la condición de adaptación impuesta sobre los modos localizados puede expresarse matricialmente mediante la siguiente relación

$$
A \cdot V=Z_{0} \cdot I
$$

Sustituyendo en (B.2) la condición de carga recién expuesta en (B.3), y tras manipular algebraicamente la expresión resultante, se obtiene la siguiente nueva relación matricial entre los vectores columna $I$ y $V$

$$
\left(U-Y \cdot Z_{0}\right) \cdot I=Y \cdot B \cdot V
$$

donde $U$ designa de nuevo la matriz identidad ya descrita anteriormente. Premultiplicando a continuación ambos lados de la igualdad recogida en (B.4) por el producto de matrices $B \cdot\left(U-Y \cdot Z_{0}\right)^{-1}$, es posible despejar el vector columna $B \cdot I$ como se indica seguidamente

$$
B \cdot I=B \cdot\left(U-Y \cdot Z_{0}\right)^{-1} \cdot Y \cdot B \cdot V
$$

En esta última expresión recogida en (B.5), el vector columna $B \cdot I$ contiene en las posiciones correspondientes a los modos accesibles sus respectivas corrientes modales, y valores nulos en el resto de los elementos; mientras el vector columna $B \cdot V$ presenta el mismo tipo de comportamiento pero en esta ocasión para los voltajes modales. Con el objeto de identificar pues la nueva matriz de admitancias generalizada asociada a la unión planar considerada, que relaciona únicamente modos accesibles (ver apartado b en fig. B.1), resulta conveniente definir unos nuevos vectores columna $I^{\prime}$ y $V^{\prime}$; que están integrados respectivamente por los elementos no nulos de los mencionados vectores columna $B \cdot I$ y $B \cdot V$. Adicionalmente, denominando a la nueva matriz de admitancias buscada como $Y^{\prime}$, debe satisfacerse la siguiente relación entre los vectores columna $I^{\prime}$ y $V^{\prime}$

$$
I^{\prime}=Y^{\prime} \cdot V^{\prime}
$$

Recordando ahora las respectivas relaciones expuestas en el párrafo anterior entre los vectores columna $I^{\prime}, V^{\prime}$ y los productos $B \cdot I, B \cdot V$, así como la relación 
entre estos dos productos definida según (B.5), es posible concluir definitivamente que la matriz $Y^{\prime}$ está constituida por subbloques de la matriz definida por el producto $B \cdot\left(U-Y \cdot Z_{0}\right)^{-1} \cdot Y$. Esta nueva matriz $Y^{\prime}$, equivalente a la matriz original $Y$, relaciona únicamente los modos accesibles considerados en cada puerto de la unión planar caracterizada; representando a partir de este instante la red equivalente multimodal correspondiente a la mencionada unión.

El programa DUMAS 3.0 contempla los dos tipos de modos recién descritos; permitiendo al usuario definir para cada guía integrante de la estructura bajo análisis el número de modos accesibles, aquéllos que integran las redes equivalentes multimodales a conectar entre sí para analizar la estructura global, así como el número de modos totales (accesibles más localizados), los cuales se utilizan únicamente al caracterizar las uniones planares en las que intervienen dichas guías. Para utilizar de manera adecuada estos dos tipos de modos, es recomendable realizar estudios previos de convergencia; que determinen en ambos casos el número mínimo de modos requeridos para obtener resultados convergentes al analizar la estructura considerada. El empleo de modos accesibles y localizados representa, como se desprende del desarrollo ofrecido anteriormente, un mayor esfuerzo computacional en la obtención de la red equivalente multimodal asociada a cada discontinuidad de la estructura global; puesto que primero debe calcularse la matriz de admitancias original correpondiente a cada discontinuidad, y posteriormente debe procesarse dicha matriz para obtener la nueva matriz de admitancias equivalente buscada que relaciona tan sólo modos accesibles. Ahora bien, este mayor coste computacional recién expuesto puede quedar sobradamente compensado durante la resolución del sistema de ecuaciones lineales en banda asociado a la estructura global bajo análisis; sistema cuya resolución, más o menos rápida, depende de las dimensiones de las matrices que integran la red global construida, es decir del número de modos accesibles escogido para cada guía de dicha estructura. En consecuencia, la posibilidad que ofrece DUMAS 3.0 de utilizar modos accesibles y localizados permite diseñar con dicho programa, de una forma más optimizada, ciertas estructuras pasivas de microondas; concretamente aquellas estructuras que, debido al tipo de discontinuidades que la integran y a los largos tramos de guía uniforme que unen dichas discontinuidades, requieren considerar un número relativamente bajo de modos accesibles en cada una de sus guías.

\section{B.2 Redes Ideales para el Diseño de Filtros}

Entre las diferentes opciones que ofrece el programa DUMAS 3.0, una de las más interesantes consiste en la posibilidad de diseñar con dicho programa, siguiendo el procedimiento descrito en [40], filtros paso banda con cavidades resonantes en las que tan sólo se propagan sus respectivos modos fundamentales; para lo cual el citado programa incorpora dos redes ideales, cuya respuesta puede analizarse 
por tramos para determinar según se indica en [40] las dimensiones finales de la estructura real a diseñar. A continuación, se mencionan las peculiaridades más importantes que presentan estos dos tipos de redes ideales incorporadas a DUMAS $3.0 ; y$ posteriormente se muestra con un ejemplo práctico cómo hacer uso de una de ellas para diseñar un filtro.

La primera de estas dos redes ideales constituye un modelo paso banda propuesto en [32]; que está integrado por simples inversores de impedancia y por resonadores serie; escogiendo para implementar dichos resonadores serie en el modelo ideal tramos de guía uniforme de longitud $\lambda_{g 0} / 2$, donde $\lambda_{g 0}$ representa la longitud de onda en cada una de las citadas guías correspondiente a la frecuencia central de la banda de paso del filtro a sintetizar. La elección de guías reales en el modelo ideal, que se escogen idénticas a las utilizadas posteriormente en la estructura real a diseñar, permite predecir con bastante precisión en el modelo ideal el efecto dispersivo que en la respuesta real del dispositivo introducen las guías empleadas como resonadores; y asimismo, al emplear tanto en el modelo ideal como en el real el mismo tipo de guías, se garantiza casi con total seguridad que la respuesta ofrecida por el modelo ideal podrá recuperarse empleando la estructura real. Ahora bien, el principal inconveniente de esta primera red ideal se encuentra asociado precisamente al hecho de utilizar guías reales como resonadores serie del modelo; inconveniente que se pone de manifiesto al intentar diseñar filtros cuya banda de paso presenta un gran ancho, debido a que el propio comportamiento dispersivo de las guías reales utilizadas en el modelo afecta negativamente a su respuesta. Concretamente, este efecto dispersivo repercute en la respuesta ofrecida por el modelo ideal dentro de la banda de paso; provocando que los picos asociados a las pérdidas de retorno (parámetro $S_{11}$ ) en dicha banda de paso no presenten todos ellos el mismo nivel. Con el objeto de poder diseñar correctamente filtros paso banda cuyas respuestas sean muy anchas resulta necesario pues, debido a la limitación recién expuesta que presenta este primer modelo, establecer una segunda red ideal cuyo comportamiento resulte adecuado para las mencionadas aplicaciones de grandes anchos de banda; red ideal que se describe básicamente a continuación.

La segunda red ideal, a diferencia del primer modelo ideal, se basa en un prototipo paso bajo recogido también en [32]; que está constituido nuevamente por inversores de impedancia, y en esta ocasión por inductancias serie. Como resulta que independientemente del valor que presenten las citadas inductancias, la respuesta del filtro siempre puede recuperarse eligiendo inversores de impedancia adecuados (ver detalles en [32]); para dichas inductancias serie se eligen unos valores que tienen en cuenta el comportamiento dispersivo de las guías utilizadas en las estructuras reales como resonadores, aunque la manera de considerar el comportamiento dispersivo es diferente a la implementada en el primer tipo de red ideal. Adicionalmente, debido a que la respuesta a sintetizar es paso banda y el prototipo escogido es paso bajo, resulta necesario implementar una transforma- 
ción paso bajo a paso banda que traslade la respuesta ideal a la banda de interés; transformación escogida de manera que el citado comportamiento dispersivo de las guías empleadas como resonadores en la estructura real vuelve a introducirse en la red ideal. Con este segundo tipo de red ideal, al no utilizar directamente guías reales en el prototipo paso bajo, se resuelve el problema planteado por la primera red ideal para diseñar filtros con respuesta paso banda muy ancha; puesto que, independientemente de dicho ancho de banda, la respuesta ideal obtenida con este segundo tipo de red no presenta ningun tipo de degradación, a diferencia de lo que ocurría en las pérdidas de retorno (parámetro $S_{11}$ ) con el primer tipo de red. Ahora bien, al manejar el segundo tipo de red ideal se debe ser extremadamente cuidadoso; ya que el hecho de poder obtener con una red ideal cualquier tipo de respuesta, no supone en modo alguno poder conseguir esa misma respuesta empleando una estructura real de filtro paso banda. De hecho, pueden darse casos prácticos en los que las respuestas deseadas se obtengan perfectamente empleando el segundo tipo de red ideal; que, sin embargo, no puedan conseguirse en la práctica al utilizar guías reales tanto para implementar las ventanas de acoplo como las cavidades resonantes del filtro a diseñar.

De los comentarios realizados en los dos párrafos anteriores, a la hora de elegir el tipo de red ideal a emplear en el diseño de un filtro paso banda, se deduce que el parámetro más importante a considerar es el ancho de banda relativo que se desea obtener en la respuesta a implementar; ancho de banda relativo que se define, como es bien sabido, mediante el cociente entre el ancho de banda absoluto y la frecuencia central de la banda de paso de la respuesta del filtro. Para elegir pues entre un tipo u otro de red ideal, se recomienda seguir el siguiente criterio deducido empíricamente; que consiste en elegir el primer tipo de red ideal para diseñar filtros cuya respuesta presente un ancho de banda relativo inferior al $3 \%$, y el segundo tipo de red ideal cuando se pretende que la respuesta paso banda del filtro a diseñar tenga un ancho de banda relativo superior o igual al citado valor umbral del $3 \%$. La elección del valor crítico se ha realizado observando que el primer modelo ideal, para filtros con anchos de banda relativos ligeramente superiores o incluso iguales al $3 \%$, comienza a producir respuestas paso banda con degradaciones en las pérdidas de retorno (parámetro $S_{11}$ ); habiendo comprobado que dichas degradaciones desaparecen al utilizar el segundo modelo de red ideal, y que es posible además implementar la respuesta ideal con elementos reales. No obstante, tal y como se ha comentado con anterioridad, si el ancho de banda relativo de la respuesta a obtener crece bastante, aun cuando la respuesta obtenida con el modelo ideal no presente ninguna degradación, no resultará posible sintetizar en la práctica dicha respuesta ideal; debido básicamente al comportamiento dispersivo de las guías utilizadas en la implementación real del filtro.

A modo de ejemplo, seguidamente se expone cómo utilizar la opción de diseño que ofrece el programa DUMAS 3.0 empleando las redes ideales recién descritas; para lo cual se ha escogido uno de los filtros paso banda en guía rectangular 


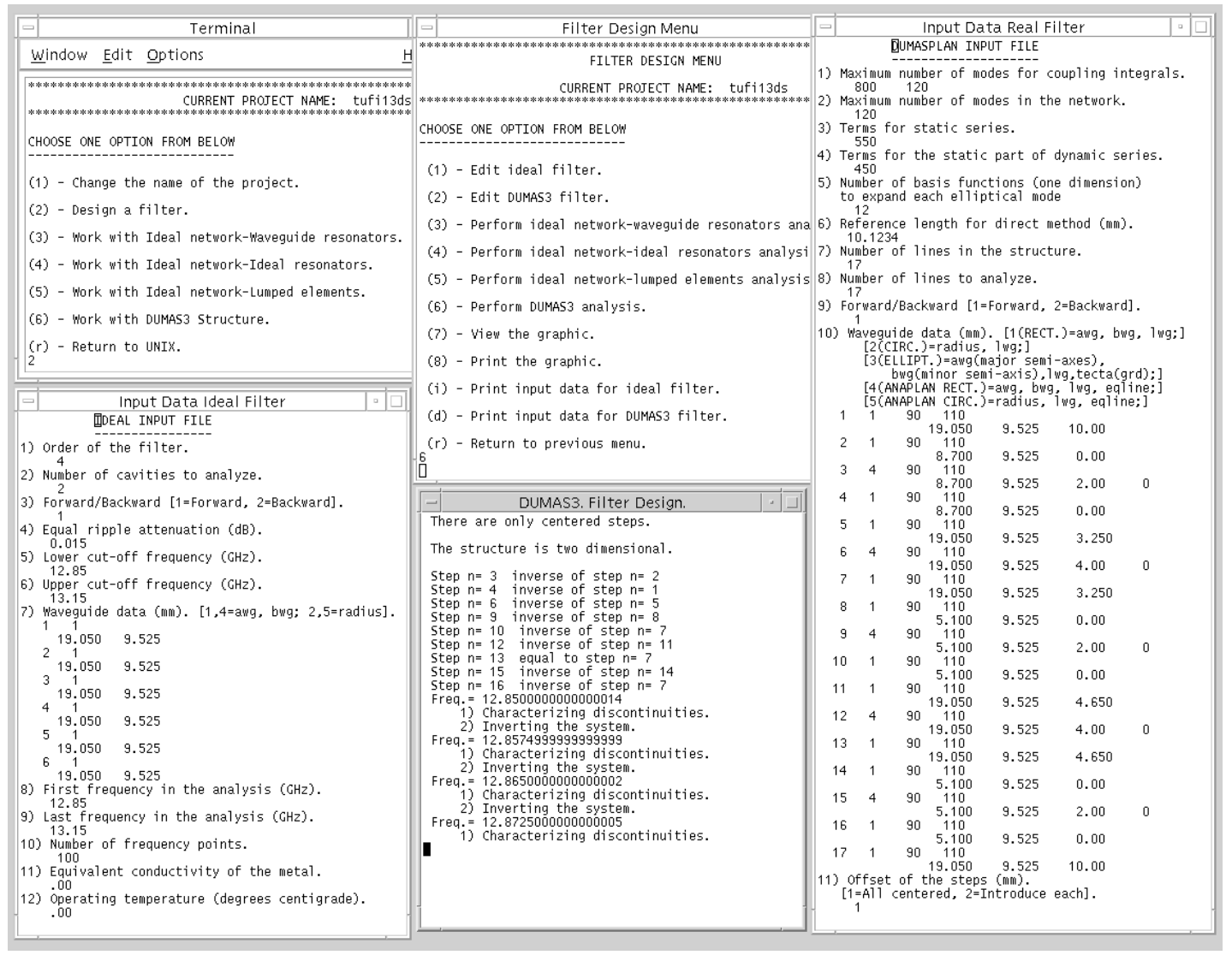

Figura B.2: Imagen del programa DUMAS 3.0 durante la etapa de diseño de un filtro paso banda empleando el primer tipo de red ideal; concretamente cuando se está analizando la respuesta de la estructura real para diseñar su segunda cavidad junto con sus respectivas ventanas de acoplo.

propuestos en el capítulo 3 (apartado 3.1.3), en concreto aquél que empleando tornillos de sintonía presenta una respuesta paso banda de $300 \mathrm{MHz}$ centrada a $13 \mathrm{GHz}$. El tipo de red ideal escogido para diseñar este filtro paso banda, debido a sus requerimientos de ancho de banda relativo (inferior al $3 \%$ ), corresponde pues al primer modelo planteado; que utiliza como se ha comentado guías reales para implementar los resonadores serie. En la figura B.2, se recoge una imagen de pantalla de una estación de trabajo; que corresponde al proceso de diseño del mencionado filtro empleando el programa DUMAS 3.0, reflejando en concreto dicha imagen el instante en el que se está simulando el comportamiento de las dos primeras cavidades de la estructura real para completar su diseño. La opción que permite diseñar filtros paso banda con DUMAS 3.0 se selecciona en dicho programa, tal y como puede observarse en la figura B.2, eligiendo la opción número 2 del menú principal; aquél mostrado en la ventana etiquetada como Terminal, al que se accede tras arrancar el programa ejecutando DUMAS3 en entorno UNIX e 
introducir el nombre asignado al proyecto en cuestión (en este caso denominado tufi13ds). En el mencionado menú principal del programa DUMAS 3.0, aparte de la citada opción número 2 correspondiente al diseño de filtros, se presentan asimismo el resto de posibles opciones que ofrece el programa; cuya descripción se recoge posteriormente. Así pues, tras seleccionar la opción número 2 en el menú principal del programa, se abre de forma automática una nueva ventana titulada Filter Design Menu (ver fig. B.2); con la que se podrá realizar el proceso de diseño expuesto en [40], que requiere diseñar cavidad tras cavidad comparando las respuestas que ofrecen la red ideal y la estructura real. Observando esta segunda ventana, se descubre la existencia de dos opciones, numeradas como la $1 \mathrm{y}$ la 2, que abren sendas ventanas tituladas Input Data Ideal Filter e Input Data Real Filter; en las que es posible editar respectivamente las características de la red ideal cuya respuesta sirve de modelo en el proceso de diseño a realizar, así como las dimensiones de la estructura real que se está diseñando. En el caso del fichero de entrada asociado a la red ideal, se descubre entre otros parámetros como se define el orden del filtro, la atenuación que debe presentar el rizado del parámetro $S_{21}$ del filtro en su banda de paso, o las frecuencias de corte inferior y superior de la banda de paso del filtro, y también lógicamente las guías a utilizar como resonadores serie en el prototipo paso banda. Un parámetro bastante importante en dicho fichero es aquél que define el número de resonadores serie a considerar en la red ideal para obtener su respuesta; número que comenzando en un valor igual a 1 debe incrementarse progresivamente para diseñar cavidad tras cavidad de la estructura real, comparando para ello las respuestas de las porciones de la estructura real y de la red ideal consideradas en cada caso. En el ejemplo recogido en la figura B.2, dicho parámetro presenta un valor igual a 2; puesto que, tal y como se ha mencionado anteriormente, dicho ejemplo corresponde al momento en el que se está diseñando la segunda cavidad (junto con sus ventanas inductivas) de la estructura real. En cuanto al fichero de datos asociado a la estructura real, en su respectiva ventana pueden observarse entre otros parámetros aquéllos que definen su geometría, permitiendo especificar para cada guía el tipo de la misma (ver posibles tipos en la sección B.3) y sus dimensiones junto a los mencionados números de modos accesibles y totales (accesibles más localizados) a considerar en dicha guía (recordar sección B.1), o aquellos parámetros más generales que definen por ejemplo el número de términos a emplear en la evaluación de las series estáticas de las diferentes uniones que integran la estructura (recordar apartado 2.2.1 del capítulo 2), y el número de sumandos a considerar durante el cálculo de las partes estáticas de las series dinámicas asociadas también a dichas uniones (recordar apartado 2.2.2 del capítulo 2). Una vez completados los ficheros de entrada de la red ideal y de la estructura real, debe analizarse en primer lugar el comportamiento de la red ideal cuya respuesta servirá de modelo con el que diseñar la estructura real; para lo cual en el menú de diseño de filtros debe seleccionarse la opción número 3, pues para diseñar el filtro propuesto se ha escogido la primera 


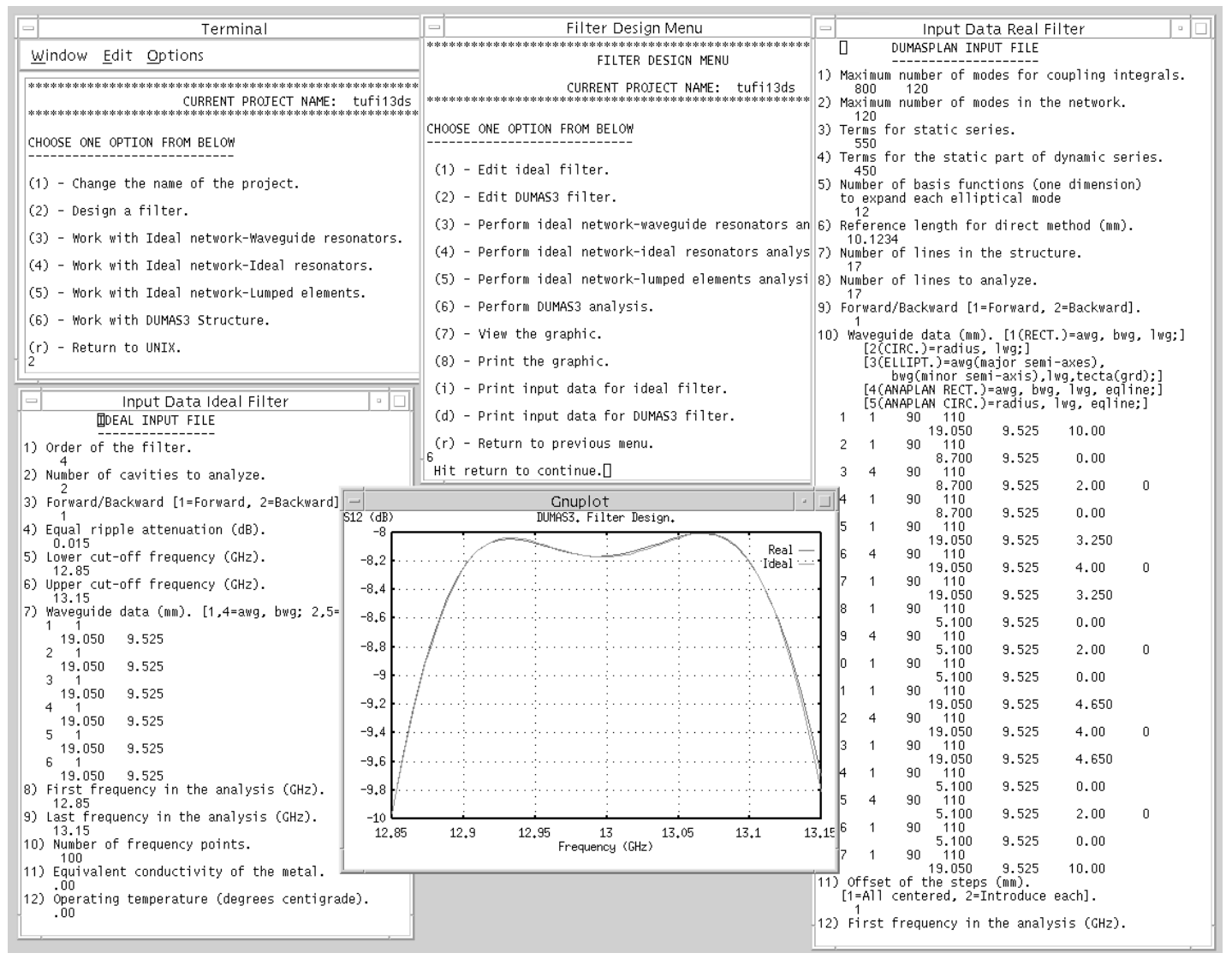

Figura B.3: Imagen del programa DUMAS 3.0 durante la etapa de diseño de un filtro paso banda empleando el primer tipo de red ideal; concretamente tras analizar la respuesta de la estructura real una vez finalizado el diseño de su segunda cavidad junto con sus respectivas ventanas de acoplo.

red ideal. A continuación, debe simularse la estructura real cuyas dimensiones físicas pretenden optimizarse; debiendo seleccionar para ello en el mismo menú mencionado previamente la opción número 6 , que analiza la estructura descrita en su fichero de datos empleando las técnicas de análisis eficiente descritas en el capítulo 2. La ejecución de este análisis produce varios mensajes mostrados en una nueva ventana que abre el programa bajo título DUMAS3. Filter Design (ver fig. B.2); entre los que se encuentran algunos relacionados con una comprobación previa que hace el programa de la geometría de la estructura, para evitar rehacer cálculos si la estructura presenta discontinuidades repetidas, así como mensajes que indican la frecuencia a la cual el programa está analizando la estructura.

Después de finalizar el análisis de la estructura considerada en el ejemplo anterior, el programa genera automáticamente una gráfica comparativa con las respuestas (parámetros $S_{21}$ ) de la red ideal y de la estructura real; tal y como se recoge en la figura B.3. Dicha gráfica, generada con un programa de libre 
distribución denominado Gnuplot, permite comparar las respuestas real e ideal; deduciendo pues de dicha comparación los cambios a realizar en el fichero de datos de la estructura real para que su nueva respuesta se aproxime más a la ideal, aunque en el caso mostrado en la figura B.3 ambas respuestas se encuentran superpuestas al haber culminado ya el diseño de la segunda cavidad. La mencionada gráfica no sólo aparece en pantalla tras realizar un análisis de la estructura real, como ocurre en el caso considerado en la figura B.3, sino que se genera también cuando se finaliza el análisis de la red ideal escogida para diseñar el filtro. Asimismo, sin necesidad de realizar ningún tipo de análisis, es posible recuperar la última de las mencionadas gráficas que apareció en pantalla eligiendo la opción número 7 del menú de diseño de filtros (ver fig. B.3); e incluso imprimir dicha gráfica, para lo cual debe seleccionarse ahora la opción número $8 \mathrm{del} \mathrm{mismo}$ menú recién mencionado.

Además de las dos redes ideales descritas con anterioridad, el programa DUMAS 3.0 incorpora un tercer tipo de red ideal bastante novedoso; que permite diseñar filtros paso banda implementados con cavidades evanescentes, aquéllas en las que todos los modos se encuentran al corte, y elementos de sintonía para implementar tanto los acoplos requeridos como las resonancias precisadas en las cavidades evanescentes. La nueva red ideal propuesta es bastante similar al segundo tipo de red ideal expuesto previamente; es decir, se basa en el mismo modelo paso bajo recogido en [32] que consta de inversores de impedancia e inductancias serie. Sin embargo, como en esta ocasión las cavidades del modelo ideal son evanescentes, no es necesario considerar ningún tipo de efecto dispersivo en los valores de las inductancias, ni tampoco en la transformación paso bajo a paso banda que debe implementarse para trasladar la respuesta ideal a la banda de frecuencias de interés; escogiendo pues en este caso una transformación clásica ofrecida en [32]. Este tipo de filtros con guías evanescentes y elementos de sintonía, cuya descripción se recoge por ejemplo en [86,87], pueden diseñarse con DUMAS 3.0 debido a que este programa permite simular en la estructura real el efecto de los elementos de sintonía requeridos por dichos filtros; empleando para ello, tal y como se explica en el apartado B.3, resultados generados por el programa comercial ANAPLAN-W. En cuanto a la nueva técnica que debe seguirse en el diseño de los mencionados filtros de guía evanescente, al igual que la técnica descrita en [40], conviene que divida el proceso de diseño de la estructura global en etapas más simples con objetivos concretos y claramente identificados; sin embargo, a diferencia del procedimiento empleado en [40] para diseñar filtros cuyas cavidades contienen al menos un modo propagándose, la nueva técnica de diseño de filtros con cavidades evanescentes no puede truncar la red ideal y la estructura real en cavidades intermedias al encontrarse éstas al corte. Por tanto, aunque la nueva técnica también diseñará cavidad tras cavidad, la red ideal y la estructura real manejadas en cada caso deben contener siempre una guía de entrada y salida que no estén al corte; obteniéndose pues ambas estructuras (ideal y real) 


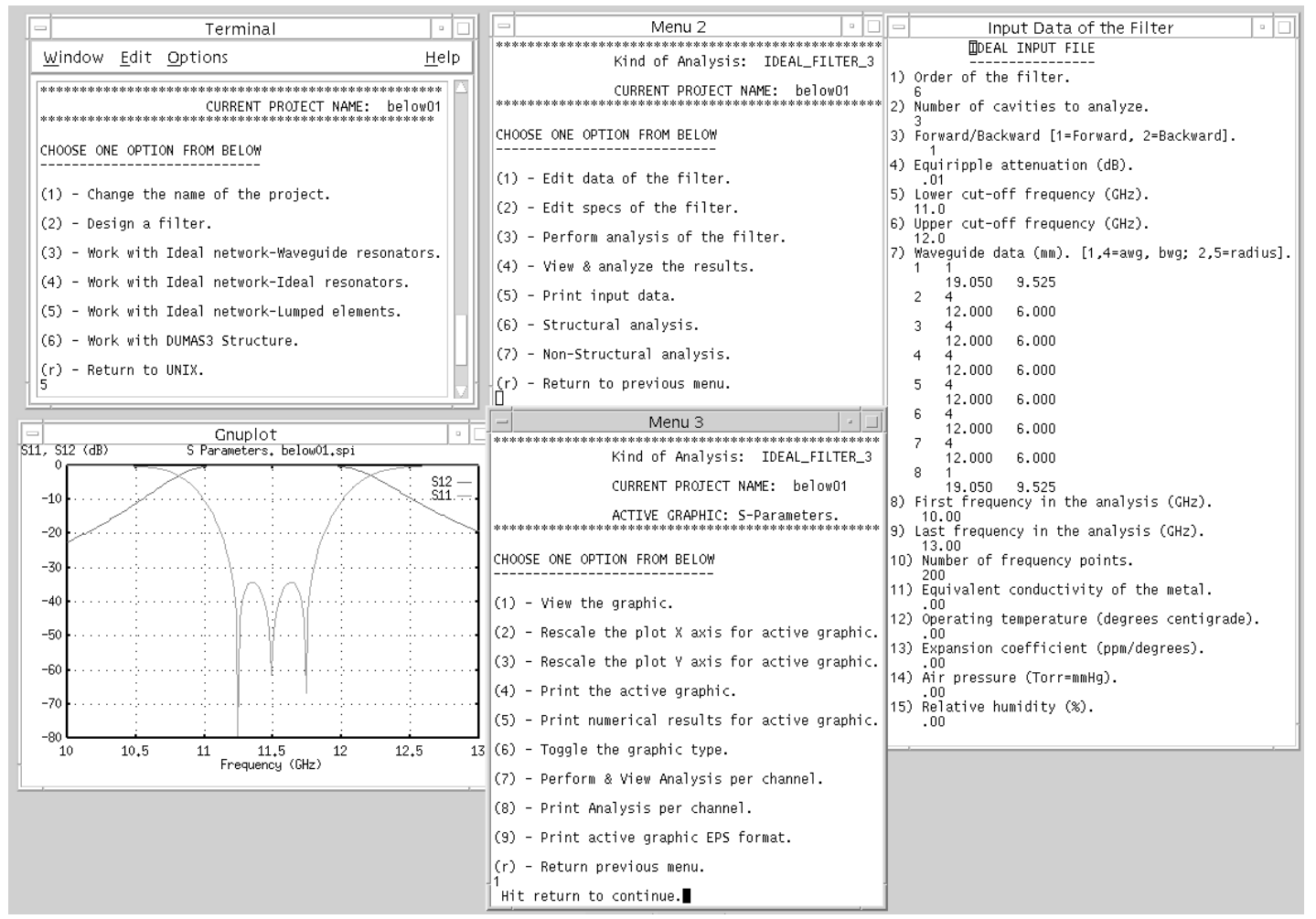

Figura B.4: Imagen del programa DUMAS 3.0 durante el análisis de una red ideal del tercer tipo; concretamente tras analizar la respuesta de dicha red ideal truncada de manera que contenga tres inductancias serie.

mediante expansiones sucesivas que siempre contendrán las respectivas guías de entrada y salida del filtro, en las cuales se propagarán lógicamentes sus respectivos modos fundamentales, los elementos manejados en las etapas previas, y aquellos elementos adicionales que se incorporan en cada nueva etapa siempre preservando simetría en las estructuras ideal y real consideradas. Procediendo de esta manera, será posible determinar respuestas electromagnéticas (parámetros $S_{21}$ ) tanto en la red ideal como en la estructura real, que estarán referidas lógicamente a los modos fundamentales de las guías de entrada y salida; y por tanto, tras realizar una comparación de dichas gráficas, optimizar las dimensiones físicas de la estructura real que se pretende diseñar.

Entre las diversas opciones que ofrece el programa DUMAS 3.0, éste permite analizar por separado el comportamiento de las tres redes ideales descritas en esta sección; básicamente con la intención de permitir al usuario determinar los parámetros que definen dicha red ideal de manera que su respuesta global satisfaga un conjunto de especificaciones. Para observar el aspecto de una de estas tres opciones de DUMAS 3.0, idéntico al ofrecido por las restantes dos opciones, en la figura B.4 se muestra una imagen capturada de la pantalla de una estación 
de trabajo que corresponde al programa DUMAS 3.0 analizando una red ideal del tercer tipo; habiendo escogido el tercer modelo ideal, descrito brevemente en el párrafo anterior, para poder comprobar asimismo como este nuevo tipo de red introducido recientemente en DUMAS 3.0 funciona de forma correcta. Con el objeto de acceder al módulo de análisis del tercer tipo de red ideal, debe arrancarse en primer lugar el programa DUMAS 3.0 de igual manera que se especificó en el ejemplo de diseño de filtros descrito previamente; es decir, debe ejecutarse el nombre del programa (DUMAS3) en entorno UNIX, y posteriormente a requerimiento del programa ha de introducirse el nombre asignado al proyecto con el que se va a trabajar (denominado en este caso below01). De esta manera, el programa genera un menú principal mostrado en una ventana de la figura B.4 titulada Terminal; menú principal donde se recogen, como ya se mencionó en el ejemplo considerado con anterioridad, todas las opciones básicas de funcionamiento del programa DUMAS 3.0. De entre todas estas opciones, aquéllas que se pretenden describir sucintamente con el presente ejemplo corresponden a las numeradas en el citado menú principal como opciones número 3 , número 4 y número 5 ; cuya elección permite implementar respectivamente el análisis de las redes ideales del primer tipo, del segundo tipo y del tercer tipo explicadas en la presente sección. Así pues, debido a que en el ejemplo considerado se pretende analizar una red ideal del tercer tipo, en el menú principal recién descrito debe seleccionarse la opción número 5 (ver fig. B.4), lo cual provoca inmediatamente que el programa produzca una nueva ventana bajo epígrafe Menu 2 (ver de nuevo fig. B.4); ventana que también habría sido generada en el caso de haber elegido en el menú principal bien la opción número 3 o la opción número 4, resultando posible identificar a que tipo de red ideal está asociada dicha ventana observando el mensaje presente en su cabecera (concretamente en el campo titulado Kind of Analysis). Con esta nueva ventana de título Menu 2, resulta posible implementar el análisis electromagnético del tipo de red ideal seleccionado, cuyas especificaciones particulares vendrán recogidas en el correspondiente fichero de datos asociado a la red ideal (recordar ejemplo anterior); que puede abrirse para ser modificado adecuadamente eligiendo en Menu 2 la opción número 1, presentando un aspecto que se recoge en la figura B.4 para el caso de la red ideal del tercer tipo considerada en el presente ejemplo. Seleccionando la opción 2 del menú mostrado en la ventana titulada Menu 2, se abre un nuevo fichero de datos asociado en este caso a las especificaciones del filtro que se pretende diseñar; en el que pueden introducirse diversas especificaciones a satisfacer tanto dentro de banda como fuera de banda por la respuesta del filtro a sintetizar, y en consecuencia que también debe satisfacer la respuesta de la red ideal utilizada como modelo al que ajustar la respuesta de la estructura real. Con las especificaciones recogidas en el mencionado fichero, el programa DUMAS 3.0 genera unas máscaras que muestra en la gráfica donde se presenta la respuesta de la red ideal definida en su correspondiente fichero de datos; para de esta forma poder comprobar gráficamente si la red ideal propuesta 
satisface los requerimientos exigidos o no, y en este último caso continuar modificando parámetros de la red ideal (orden del filtro, atenuación que presenta el rizado del parámetro $S_{21}$ del filtro en su banda de paso, o las frecuencias de corte inferior y superior de la banda de paso del filtro) hasta que la respuesta del mismo satisfaga todos los requisitos impuestos. En cuanto a la respuesta electromagnética que presenta la red ideal definida en cada instante, tras salvar su geometría en su respectivo fichero de datos, se obtiene una vez el programa ejecuta la opción número 3 del menú recogido en la citada ventana de título Menu 2 (ver fig. B.4); empleando códigos de programación diferentes para obtener dicha respuesta según el tipo de red ideal escogido. Con el objeto de mostrar gráficamente la respuesta obtenida al analizar el comportamiento de la red ideal propuesta, junto con las máscaras asociadas a las especificaciones si las hubiere, debe seleccionarse en el mismo menú recién mencionado la opción numerada como la 4; surgiendo, como consecuencia de dicha elección, una nueva ventana etiquetada como Menu 3 (ver fig. B.4), cuyo menú ofrece diversas opciones que permiten entre otras acciones visualizar la gráfica, imprimirla, reescalar los ejes de la misma, o incluso mostrar información de interés asociada a ciertos canales definidos previamente. En la figura B.4, se muestra una gráfica generada con el mencionado programa Gnuplot obtenida tras elegir la primera opción ofrecida en la nueva ventana denominada Menu 3; gráfica que corresponde al análisis de las tres primeras cavidades de una red ideal del tercer tipo cuyas características se ofrecen en su correspondiente fichero de datos (ver fig. B.4), y en la que no aparecen las mencionadas máscaras al haber finalizado ya el proceso de optimización de los parámetros definitorios de la red ideal.

\section{B.3 Análisis de Estructuras Pasivas de Micro- ondas}

La característica más destacada del programa DUMAS 3.0 es que permite analizar, de manera eficiente y precisa, el comportamiento electromagnético de estructuras pasivas de microondas constituidas por la conexión en cascada de diferentes tramos de guía uniforme; análisis que se lleva a cabo, tal y como se indica en el capítulo 2 (apartado 2.3.1), obteniendo las redes equivalentes multimodales de los tramos uniformes de guía y de las uniones planares que tienen lugar entre dichas guías. En cuanto a la obtención de las redes equivalentes multimodales de todas las uniones planares, basadas en sus correspondientes matrices de admitancias generalizadas, DUMAS 3.0 emplea la técnica acelerada de cálculo de ciertos elementos de dichas matrices descrita en el capítulo 2 (apartado 2.2.2); mientras en la resolución del sistema de ecuaciones lineales en banda, que surge al analizar el dispositivo global empleando las mencionadas matrices de admitancias generalizadas, DUMAS 3.0 utiliza la técnica recursiva eficiente detallada en 

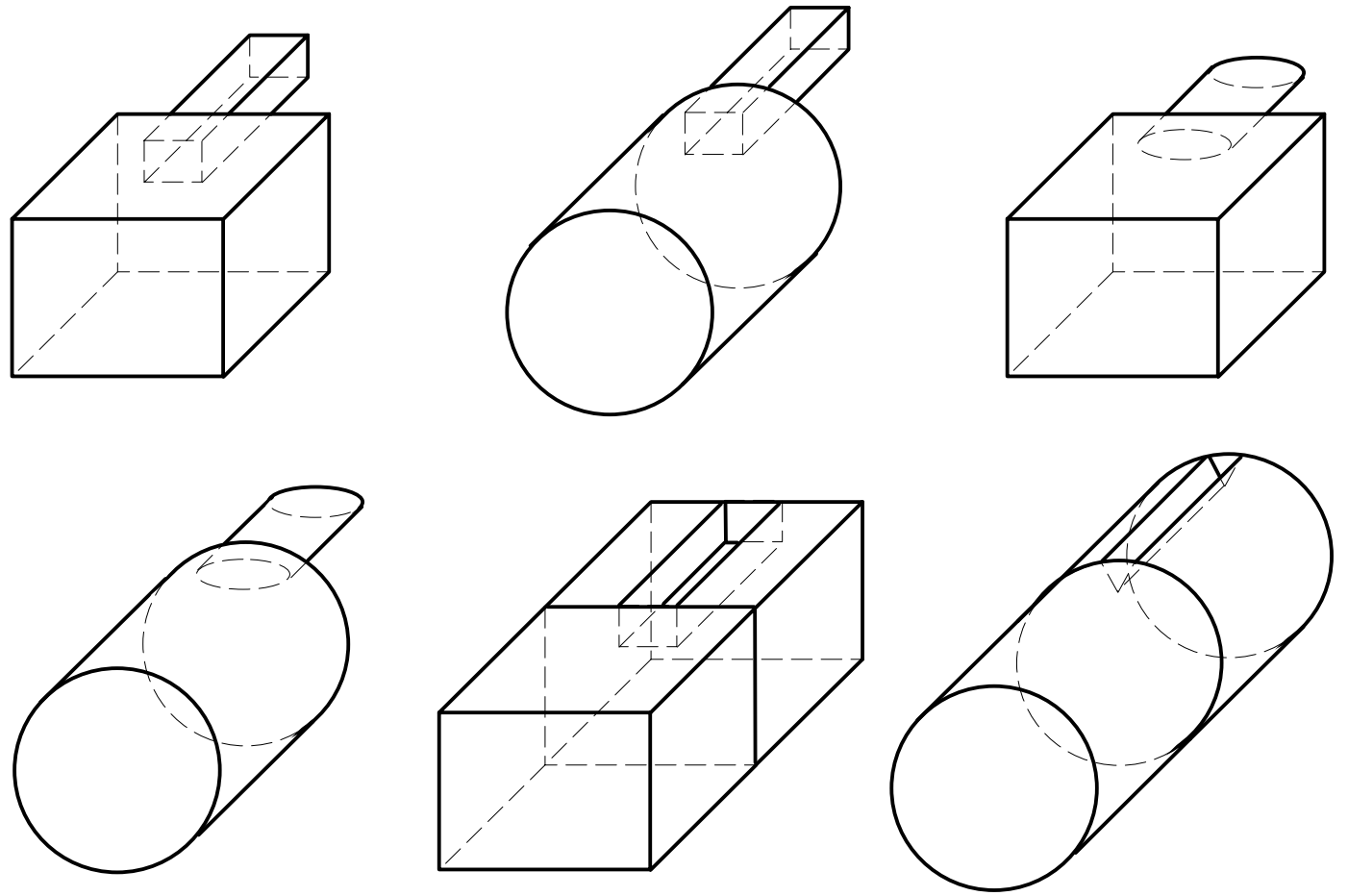

Figura B.5: Diferentes tipos de uniones planares que pueden caracterizarse con el programa DUMAS 3.0.

el capítulo 2 (apartado 2.3.3). El programa DUMAS 3.0 es capaz de analizar estructuras pasivas de microondas constituidas por guías rectangulares, circulares, elípticas, de contorno básico rectangular modificado, y de contorno básico circular modificado; ahora bien, dichas guías no pueden estar unidas entre sí de cualquier manera, pues de todas las posibles uniones que pueden producirse entre dichas guías DUMAS 3.0 sólo es capaz de analizar algunas de ellas. Los diferentes tipos de uniones planares que pueden caracterizarse mediante el programa DUMAS 3.0 se muestran gráficamente en la figura B.5; donde se encuentran representadas la unión planar entre guía rectangular grande y guía rectangular pequeña, la unión planar entre guía circular grande y guía rectangular pequeña, la unión planar entre guía rectangular grande y guía elíptica pequeña, la unión planar entre guía circular grande y guía elíptica pequeña, la unión planar entre guía rectangular y guía con el mismo contorno básico rectangular pero modificado, y finalmente la unión planar entre guía circular y guía con el mismo contorno básico circular pero modificado. En el análisis de las cuatro primeras discontinuidades recién mencionadas, tanto la obtención del espectro modal de las guías que integran cada una de dichas uniones, como el cálculo de las integrales de acoplamiento entre los modos de las correspondientes guías, constituyen operaciones implementadas directamente por el propio programa DUMAS 3.0. Por otra parte, al caracterizar 


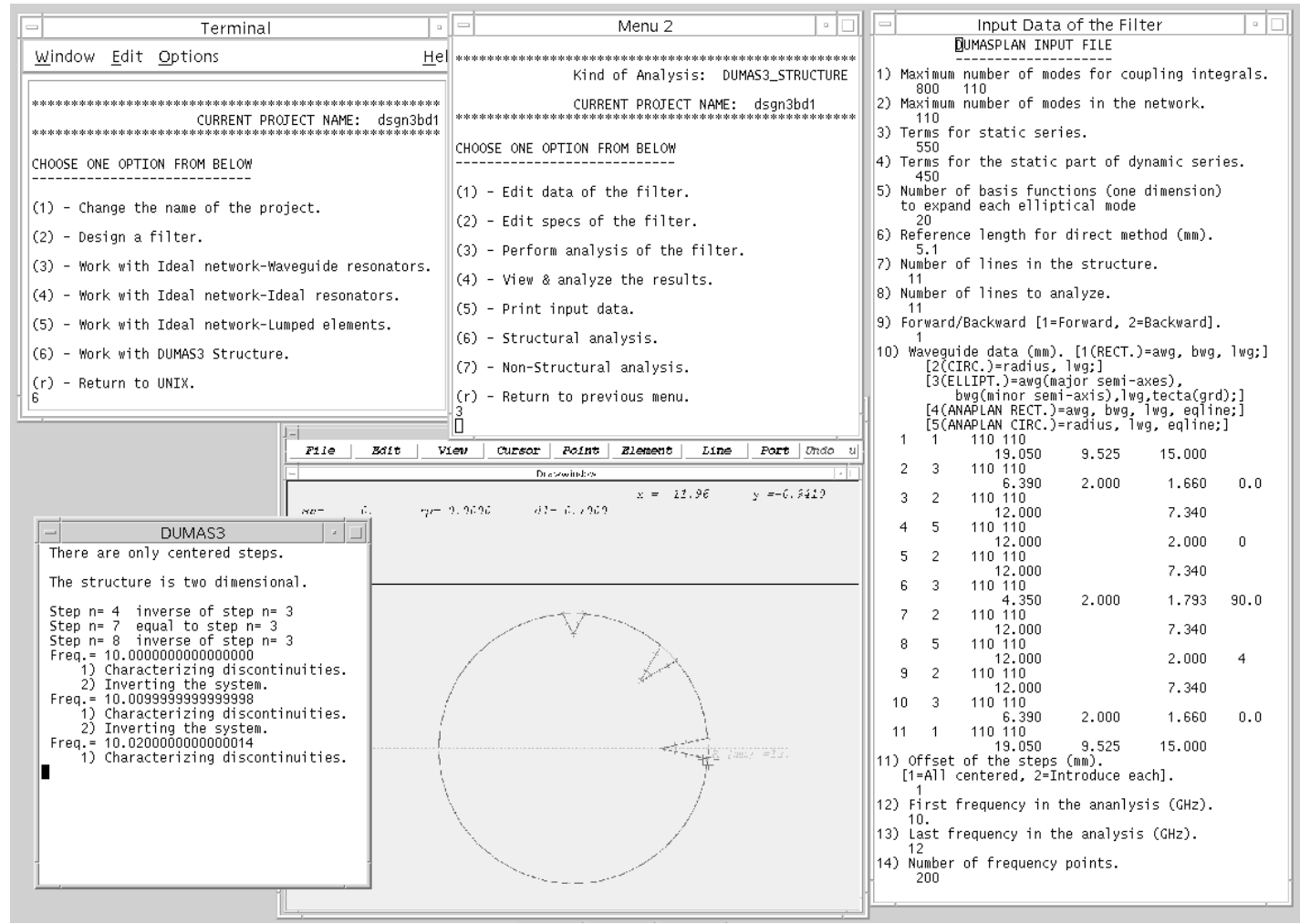

Figura B.6: Imagen del programa DUMAS 3.0 durante el análisis de un filtro de modo dual en guía circular con iris elípticos y guías circulares reentrantes, e imagen del editor gráfico del programa ANAPLAN-W con el contorno de las guías circulares reentrantes empleadas en el mencionado filtro.

las dos últimas uniones planares consideradas, aquéllas en las que intervienen guías de contorno básico rectangular o circular modificado, DUMAS 3.0 requiere que el programa comercial ANAPLAN-W le proporcione el espectro modal de dichas guías, así como las integrales de acoplamiento entre estos modos y aquéllos asociados a las respectivas guías de contorno básico rectangular o circular (según el caso); programa comercial que para obtener los citados modos e integrales emplea el método numérico muy eficiente descrito en el capítulo 3 (apartado 3.1.1). Con el objeto de mostrar el funcionamiento de la opción de análisis de estructuras que ofrece el programa DUMAS 3.0, a continuación se ofrece un ejemplo práctico correspondiente al análisis de un dispositivo que incorpora guías con contorno básico circular modificado; el cual permite asimismo comprobar como funciona DUMAS 3.0 en combinación con el programa comercial ANAPLAN-W.

Así pues, en la figura B.6 se ofrece una imagen capturada de la pantalla de una estación de trabajo; que corresponde al instante en el que el programa DUMAS 3.0 está analizando una estructura pasiva de microondas. En concreto, la estructura que se ha escogido en dicho análisis es el filtro de modo dual en guía 
circular, con iris elípticos y guías circulares reentrantes, descrito detalladamente en el capítulo 3 (apartado 3.2.3). La opción del programa DUMAS 3.0 que permite analizar estructuras pasivas de microondas viene definida como la opción número 6 del menú principal de dicho programa; menú mostrado en la ventana etiquetada como Terminal de la figura B.6, y al cual se accede desde el entorno UNIX de igual manera que en los ejemplos considerados en la sección anterior. Una vez elegida la opción 6 del citado menú principal, el programa genera automáticamente una nueva ventana etiquetada como Menu 2 (ver fig. B.6); que es idéntica a la del mismo nombre generada durante el análisis de cualquier tipo de red ideal (recordar último ejemplo considerado en la sección B.2), en cuyo campo Kind of Analysis se indica que dicha ventana está asociada en el presente ejemplo al análisis de una estructura real. Esta segunda ventana abierta (titulada Menu2) permite implementar el análisis electromagnético de estructuras pasivas de microondas; ofreciendo para ello un menú (ver fig. B.6) con diferentes opciones. La primera opción de dicho menú permite abrir el fichero de datos donde resulta posible definir la estructura real que se pretende analizar; mientras la segunda opción permite editar, de igual manera que en las opciones de análisis de redes ideales (recordar sección B.2), un fichero de datos con las especificaciones que debe observar en este caso la respuesta de la estructura real tanto dentro como fuera de banda. En el caso de existir dicho fichero, el programa DUMAS 3.0 traduce las mencionadas especificaciones en unas máscaras; que posteriormente se muestran en pantalla junto a los resultados procedentes del análisis de la estructura real, con el objeto de poder comprobar visualmente si la respuesta de la estructura satisface o no los requerimientos impuestos. En cuanto al fichero de datos asociado a la estructura real considerada, cuyo aspecto se muestra en la ventana con epígrafe Input Data of the Filter recogida también en la figura B.6, cabe destacar la presencia de numerosos parámetros; entre ellos por ejemplo, tal y como se mencionó brevemente en la sección B.2, los que permiten definir la geometría de cada guía junto con los modos accesibles y totales (accesibles más localizados) a considerar en dicha guía, o los que indican el número de sumandos a emplear en la evaluación de las series estáticas de las diferentes uniones que integran la estructura (recordar apartado 2.2.1 del capítulo 2), y el número de términos a utilizar en el cálculo de las partes estáticas de las series dinámicas asociadas también a dichas uniones (recordar apartado 2.2 .2 del capítulo 2). A modo indicativo, y para no extender en demasía el presente apéndice incluyendo una explicación detallada de todos los parámetros incluidos en el fichero de datos de la estructura real, a continuación se detalla tan sólo y de manera breve como definir las estructuras pasivas de microondas en DUMAS 3.0; para lo cual debe utilizarse el campo número 10 del citado fichero de datos (ver fig. B.6). Tal y como puede observarse en dicho campo, cada guía de la estructura real se define mediante dos líneas de parámetros; existiendo en el caso del fichero considerado un número total de 11 grupos de dos líneas, debido a que el filtro de modo dual 
detallado en dicho fichero está constituido por 11 guías. La primera de las dos citadas líneas consta de cuatro parámetros; el primero de ellos determina el orden que ocupa la correspondiente guía en la estructura global (empezando en este caso desde el número 1 hasta el número 11 lógicamente), el segundo parámetro define el tipo de guía mediante un código de números comprendidos entre el 1 y el 5 (ver significado de cada número en la explicación que incluye la cabecera del citado campo número 10 del fichero de datos), mientras el tercer y el cuarto parámetro se refieren respectivamente al número de modos accesibles y de modos totales (accesibles más localizados) asociados a la guía en cuestión (recordar definiciones de estos dos tipos de modos en la sección B.1). Por su parte, la segunda línea asociada a la definición de cada guía, según el tipo de la misma, contiene diferentes parámetros que determinan su geometría; número de parámetros y significado de los mismos que se encuentran resumidos también, para cada posible tipo de guía, en la cabecera del mencionado campo número 10 del fichero de datos de la estructura real. Observando las diferentes guías incluidas en el filtro de modo dual en guía circular considerado (ver el citado campo número $10 \mathrm{del}$ fichero de datos de la estructura real recogido en la figura B.6), se descubre que las guías número 4 y número 8 de la citada estructura corresponden a guías cuyo contorno básico circular está modificado (ambas llevan asociadas el código 5 en el segundo parámetro de sus respectivas primeras líneas); las cuales constituyen los tramos uniformes de guía circular reentrante requeridos por dicho filtro (recordar apartado 3.2.3 del capítulo 3). Ahora bien, como ya se ha explicado anteriormente, los modos de dichas guías junto a las integrales de acoplamiento entre estos modos y los de las guías circulares precedentes y siguientes deben determinarse con el programa comercial ANAPLAN-W; que requiere en primer lugar definir con su editor gráfico el contorno modificado de la guía bajo análisis, tal y como se recoge gráficamente en una ventana adicional (correspondiente al programa ANAPLAN-W) mostrada también en la figura B.6. Una vez definido el contorno de la nueva guía que se pretende caracterizar, debe lanzarse el módulo de análisis electromagnético del programa ANAPLAN-W; que generará unos ficheros, leídos posteriormente por DUMAS 3.0, con información relativa al espectro modal de la guía en cuestión, y con los valores que presentan las integrales de acoplamiento entre los modos de dicha guía y los modos de una guía con el mismo contorno básico (sin modificar) que el asociado a la nueva guía. Tras definir pues la estructura completa a caracterizar, lo cual debe incluir el análisis con ANAPLAN-W de las guías de dicha estructura que lo requieran, DUMAS 3.0 está en condiciones de analizar el comportamiento electromagnético de la estructura propuesta; para lo cual en el menú recogido en la mencionada ventana de título Menu 2 debe seleccionarse la opción número 3 (ver fig. B.6). Dicha elección produce, al igual que durante el análisis de la estructura real en el diseño de filtros (recordar sección B.2), una nueva ventana denominada en este caso DUMAS3; en la que junto a varios mensajes con información relativa a una comprobación previa que hace el 


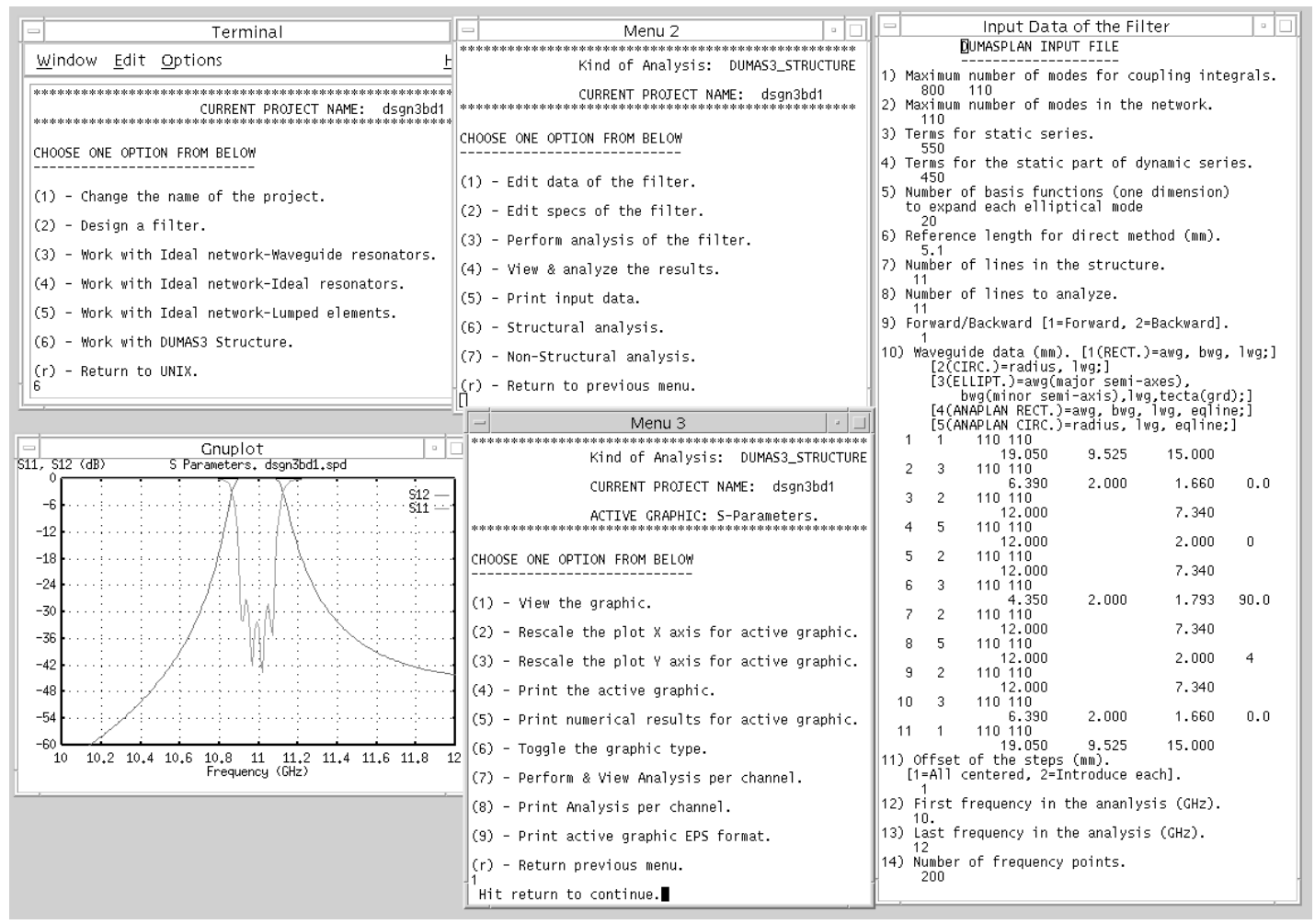

Figura B.7: Imagen del programa DUMAS 3.0 tras el análisis de un filtro de modo dual en guía circular con iris elípticos y guías circulares reentrantes; concretamente mostrando en una gráfica los resultados obtenidos con dicho análisis.

programa de la estructura a caracterizar, con el objeto de evitar repetir cálculos cuando la estructura consta de discontinuidades idénticas, se muestra también la frecuencia a la cual el programa está analizando la estructura considerada en cada instante.

Una vez que el programa DUMAS 3.0 finaliza el análisis de la estructura propuesta, la ventana denominada DUMAS3 donde se muestra el estado en el que se encuentra dicho análisis (recordar fig. B.6) desaparece también lógicamente; retornando el control del programa a la ventana con epígrafe Menu 2 (ver ahora fig. B.7) desde la cual se había lanzado el citado análisis. Con el objeto de observar gráficamente los resultados obtenidos con dicho análisis, junto a las máscaras que construye el programa para representar las especificaciones a cumplir por la estructura si las hubiere, debe seleccionarse la opción número 4 del menú ofrecido en la mencionada ventana con título Menu2; lo que provoca la inmediata aparición en pantalla de una nueva ventana denominada Menu 3 (ver fig. B.7), idéntica a la ventana que surge cuando se pretende visualizar los resultados obtenidos tras el análisis de cualquier red ideal (recordar fig. B.4 asociada al último ejemplo descrito en la sección B.2). Esta última ventana aparecida en pantalla 
contiene un menú (ver fig. B.7) con diferentes opciones; las cuales, entre otras posibles acciones, permiten visualizar una gráfica con los resultados de la estructura analizada (parámetros $S_{11}$ y $S_{21}$ o retardo de grupo), imprimir dicha gráfica, reescalar los ejes de la citada gráfica, e incluso obtener información de los resultados correspondientes a diferentes canales de interés definidos previamente en el fichero de datos con las especificaciones. Así por ejemplo, seleccionando la opción número 1 del menú recién descrito (ver fig. B.7), en la pantalla de la estación surge una gráfica generada con el programa Gnuplot; cuyo aspecto se recoge en la mencionada figura B.7, y en la que se muestran los resultados considerados en cada momento por el programa como activos (en el ejemplo de la figura B.7 son los parámetros $S_{11}$ y $S_{21}$, tal y como se indica en el campo ACTIVE GRAPHIC de la cabecera de la ventana Menu 3). 



\section{Apéndice C}

\section{Espectros Escalares de Ondas Planas y Cilíndricas. Transformaciones entre Espectros Plano y Cilíndrico}

La dispersión electromagnética producida por objetos dispersores en espacio libre se ha estudiado, en el presente trabajo, utilizando el concepto de la función de transferencia o matriz de caracterización asociada a cada objeto dispersor. Esta matriz relaciona, en términos espectrales, la incidencia existente sobre el objeto en cuestión con la dispersión que éste produce ante dicha incidencia. A una misma solución electromagnética en una región del espacio libre sin fuentes, le corresponden diferentes expresiones espectrales; que se obtienen tras resolver la misma ecuación de onda escalar, aunque expresada en diferentes sistemas de coordenadas. En la resolución de un mismo problema puede resultar necesario, por razones de conveniencia, utilizar diferentes expresiones espectrales asociadas a una misma solución electromagnética. En toda la parte del trabajo presentada para resolver problemas de dispersión en espacio libre, el concepto de la matriz de caracterización se ha particularizado al caso de objetos dispersores bidimensionales; habiendo escogido el espectro escalar de ondas cilíndricas para representar los campos incidente y dispersado correspondientes al problema bidimensional. Ahora bien, en determinadas situaciones, se ha necesitado utilizar el espectro escalar de ondas planas asociado al campo incidente; para lo cual se requiere tener conocimiento de la transformación, en ambos sentidos, entre dicho espectro y el espectro cilíndrico escogido en un principio al representar el campo incidente.

En consecuencia, este apéndice define inicialmente el concepto de espectro escalar asociado a la resolución de la correspondiente ecuación de onda; expresando la solución de dicha ecuación en términos de su espectro escalar de ondas planas, y también en función de su espectro escalar de ondas cilíndricas. Una vez 
presentadas estas expresiones espectrales, se particularizan al caso bidimensional que nos ocupa; introduciendo los conceptos de espectro escalar bidimensional plano y cilíndrico. Debido a la necesidad de utilizar ambos espectros en el análisis de ciertos problemas; se presentan posteriormente unas relaciones que permiten transformar, en ambos sentidos, los espectros bidimensionales de ondas planas y cilíndricas. Dichas relaciones, deducidas tras particularizar a un caso bidimensional las transformaciones genéricas entre espectros plano y cilíndrico recogidas en [14], se utilizan finalmente para encontrar el espectro cilíndrico de una onda plana incidente en situaciones bidimensionales; así como para obtener una matriz que permite transformar, de nuevo en problemas bidimensionales, un espectro cilíndrico asociado a un campo incidente en su espectro plano equivalente.

\section{C.1 Espectros Escalares. Concepto}

Un campo arbitrario en una región homogénea y libre de fuentes puede obtenerse resolviendo dos problemas complementarios; cuyas soluciones se las designa en [14] como campos $\mathrm{TM}^{\mathrm{z}}$ y $\mathrm{TE}^{\mathrm{z}}$, respectivamente. Siguiendo la notación empleada en [14], el campo $\mathrm{TM}^{\mathrm{z}}$ se obtiene tras resolver una ecuación de onda escalar definida para la componente según $\hat{z}$ del potencial vector magnético; pues en dicho caso se asume que el potencial vector eléctrico es nulo $(\vec{F}=0)$, y que el potencial vector magnético sólo tiene componente en $\hat{z}\left(\vec{A}=\psi^{\mathrm{TM}} \hat{z}\right)$. Por su parte, el campo $\mathrm{TE}^{\mathrm{z}}$ se determina tras solucionar una nueva ecuación de onda escalar, de aspecto idéntico al de la ecuación resuelta en el caso $T M^{z}$, aunque definida en esta ocasión para la componente según $\hat{z}$ del potencial vector eléctrico; ya que para obtener el campo $\mathrm{TE}^{\mathrm{z}}$ se asume la situación complementaria $\left(\vec{A}=0, \vec{F}=\psi^{\mathrm{TE}} \hat{z}\right)$ a la considerada anteriormente.

En consecuencia, cualquier campo arbitrario se obtiene superponiendo las soluciones a dos ecuaciones de onda escalares, también conocidas como ecuaciones escalares de Helmholtz. Dichas ecuaciones presentan infinitas soluciones, que se denominan funciones elementales o modos $\mathrm{TM}^{\mathrm{z}}$ y $\mathrm{TE}^{\mathrm{z}}$, cuya combinación lineal en cada caso constituye respectivamente la solución $\mathrm{TM}^{\mathrm{z}}$ y $\mathrm{TE}^{\mathrm{z}}$ de las mencionadas ecuaciones de onda escalares. Pues bien, los coeficientes que multiplican a los modos representan el espectro escalar asociado a la correspondiente solución de la ecuación de onda. Como ya se ha comentado previamente, la expresión de cualquier campo arbitrario requiere manejar dos espectros escalares; uno de ellos asociado a la componente en $\hat{z}$ del potencial vector magnético $\vec{A}$ para el campo $\mathrm{TM}^{\mathrm{z}}$, y el otro relacionado con la componente en $\hat{z}$ del potencial vector eléctrico $\vec{F}$ para el campo $\mathrm{TE}^{\mathrm{z}}$. La definición de estos espectros escalares se realiza en términos de las funciones elementales o modos; cuyo aspecto depende lógicamente del sistema de coordenadas en el que se expresan las dos ecuaciones escalares de Helmholtz. Como ambas ecuaciones, las planteadas para el caso $\mathrm{TM}^{\mathrm{z}}$ y para el 


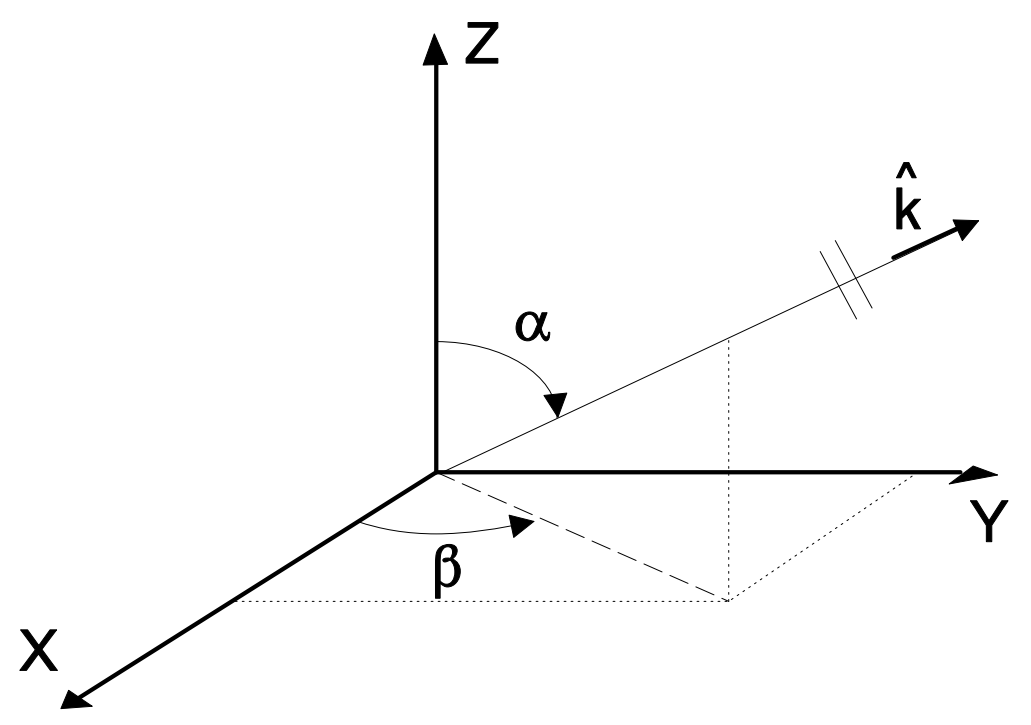

Figura C.1: Dirección de avance de una onda plana.

caso $\mathrm{TE}^{\mathrm{z}}$, son exactamente idénticas; a partir de este instante nos referiremos exclusivamente a una sola de estas ecuaciones, siendo válidas las expresiones deducidas para cualquiera de las dos.

En los dos próximos apartados, se muestra el aspecto concreto que presentan las funciones elementales o modos correspondientes a la ecuación de onda escalar, expresada respectivamente en un sistema de coordenadas cartesianas y cilíndricas. Asimismo, se introduce el concepto de espectro escalar asociado a la solución de la citada ecuación, obtenida al superponer las mencionadas funciones elementales deducidas para cada sistema de coordenadas; definiendo lo que se conoce como espectro escalar de ondas planas, y espectro escalar de ondas cilíndricas.

\section{C.1.1 Espectro Escalar de Ondas Planas}

Las funciones elementales o modos correspondientes a una ecuación de onda escalar, expresada en un sistema de coordenadas cartesianas, son ondas planas que se propagan en diferentes direcciones del espacio; tal y como se detalla en [14]. Cada uno de estos modos, designado como $\psi_{k_{x}, k_{y}, k_{z}}$, es una onda con amplitud constante y frente de fase plano; que avanza en una determinada dirección. La expresión analítica de estas funciones elementales presenta el siguiente aspecto

$$
\psi_{k_{x}, k_{y}, k_{z}}=e^{-j k_{x} x} e^{-j k_{y} y} e^{ \pm j k_{z} z}
$$

donde $k_{x}, k_{y}$ y $k_{z}$ son las tres componentes del vector $\vec{k}$, que define la dirección de avance del frente de fase plano. En la figura C.1 se representa la dirección de avance de una onda plana; observándose que dicha dirección puede definirse mediante el vector $\vec{k}$, o bien utilizando los ángulos directores $\alpha$ y $\beta$ de dicho 
vector. Las componentes $k_{x}, k_{y}$ y $k_{z}$ del vector $\vec{k}$ se definen, haciendo uso del módulo $k$ de este vector y de los ángulos $\alpha$ y $\beta$, del siguiente modo

$$
\begin{aligned}
& k_{x}=k \cos (\beta) \sin (\alpha) \\
& k_{y}=k \sin (\beta) \sin (\alpha) \\
& k_{z}=k \cos (\alpha) \\
& k^{2}=k_{x}^{2}+k_{y}^{2}+k_{z}^{2}=\left(\frac{2 \pi}{\lambda}\right)^{2}
\end{aligned}
$$

Una solución ${ }^{1}$ de la ecuación de onda escalar, designada como $\psi^{\tau}$, se puede obtener combinando linealmente los modos definidos en (C.1); que se propagan, con su correspondiente amplitud, en diferentes direcciones. Estas direcciones se consiguen variando los ángulos $\alpha$ y $\beta$, o de manera equivalente variando las componentes $k_{x}$ y $k_{y}$ del vector $\vec{k}$; pues según (C.2) tan sólo existen dos grados de libertad. Las amplitudes de las diferentes ondas planas se recogen en una función continua, representada como $p^{\tau}\left(k_{x}, k_{y}\right)$; que define el espectro de ondas planas asociado a la solución $\psi^{\tau}$ de la ecuación de onda escalar. De esta forma, dicha solución se define mediante la siguiente integral doble

$$
\psi^{\tau}(x, y, z)=\int_{k_{x}} \int_{k_{y}} p^{\tau}\left(k_{x}, k_{y}\right) e^{-j k_{x} x} e^{-j k_{y} y} e^{ \pm j k_{z} z} \mathrm{~d} k_{x} \mathrm{~d} k_{y}
$$

Puede resultar interesante referir la solución $\psi^{\tau}$, recogida en (C.3), a un sistema de coordenadas cilíndricas $(\rho, \phi, z)$. Para ello, en primer lugar, deben sustituirse en (C.3) las componentes cartesianas $k_{x}$ y $k_{y}$ del vector $\vec{k}$ por sus componentes cilíndricas equivalentes $k_{\rho}$ y $\beta$; haciendo uso de las siguientes relaciones

$$
\begin{aligned}
& k_{x}=k_{\rho} \cos (\beta) \\
& k_{y}=k_{\rho} \sin (\beta)
\end{aligned}
$$

Utilizando pues la conocida transformación entre las coordenadas rectangulares $(x, y)$ y las coordenadas cilíndricas $(\rho, \phi)$, así como las relaciones recién definidas en (C.4), es posible expresar las exponenciales que aparecen en (C.3) respecto de las nuevas coordenadas; tal y como se indica seguidamente

$$
e^{-j k_{x} x} e^{-j k_{y} y}=e^{-j k_{\rho} \rho \cos (\phi-\beta)}
$$

Finalmente, tan sólo resta por sustituir en (C.3) las variables de integración $k_{x}$ y $k_{y}$ por las componentes $k_{\rho}$ y $\beta$ del vector $\vec{k}$. Hallando el Jacobiano del cambio

\footnotetext{
${ }^{1}$ Esta solución $\left(\psi^{\tau}\right)$ de la ecuación de onda escalar representa la componente en $\hat{z}$ del potencial vector magnético $\left(\vec{A}=\psi^{\mathrm{TM}} \hat{z}\right)$ para el caso $\mathrm{TM}^{\mathrm{z}}$, y la componente en $\hat{z}$ del potencial vector eléctrico $\left(\vec{F}=\psi^{\mathrm{TE}} \hat{z}\right)$ para el caso $\mathrm{TE}^{\mathrm{z}}$.
} 
de variables realizado, se obtiene la siguiente relación

$$
\mathrm{d} k_{x} \mathrm{~d} k_{y}=k_{\rho} \mathrm{d} k_{\rho} \mathrm{d} \beta
$$

Siguiendo los pasos descritos hasta el momento, resumidos en (C.4), (C.5) y (C.6), la solución $\psi^{\tau}$ se refiere a un sistema de coordenadas cilíndricas mediante la siguiente expresión final

$$
\psi^{\tau}(\rho, \phi, z)=\int_{k_{\rho}} \int_{\beta} p^{\tau}\left(k_{\rho}, \beta\right) e^{-j k_{\rho} \rho \cos (\phi-\beta)} e^{ \pm j k_{z} z} k_{\rho} \mathrm{d} k_{\rho} \mathrm{d} \beta
$$

Por último, como en este trabajo se han considerado siempre problemas constituidos por objetos dispersores bidimensionales, se pretende particularizar la relación (C.7) al caso de un problema invariante según el eje $\hat{z}$. En dicha situación, las ondas planas están contenidas en el plano $X Y\left(\alpha=90^{\circ}\right)$. Empleando esta condición en (C.2), se deducen las siguientes nuevas expresiones para las componentes $k_{x}, k_{y}$ y $k_{z}$

$$
\begin{aligned}
& k_{x}=k \cos (\beta) \\
& k_{y}=k \sin (\beta) \\
& k_{z}=0 \\
& k^{2}=k_{x}^{2}+k_{y}^{2}=\left(\frac{2 \pi}{\lambda}\right)^{2}
\end{aligned}
$$

Al sustituir estas nuevas expresiones para $k_{x}$ y $k_{y}$ en (C.4), se comprueba que la variable de integración $k_{\rho}$ presente en (C.7) se convierte en una constante; tal y como se recoge a continuación

$$
k_{\rho}=\sqrt{k_{x}^{2}+k_{y}^{2}}=k
$$

Como consecuencia de (C.9), el espectro escalar de ondas planas $p^{\tau}\left(k_{\rho}, \beta\right)$ debe reemplazarse en (C.7) por su versión bidimensional $p^{\tau}(\beta)$; que depende tan sólo de la variable $\beta$. En este momento, conviene reseñar que en problemas bidimensionales la componente en $\hat{z}$ del correspondiente potencial vector, eléctrico o magnético según el tipo de polarización que se considere, está relacionada a través de constantes con la componente en $\hat{z}$ del campo eléctrico para polarización $\mathrm{TM}^{\mathrm{z}}$, y con la componente en $\hat{z}$ del campo magnético para polarización $\mathrm{TE}^{\mathrm{z}}$. Por tanto, en estos problemas pueden relacionarse directamente las componentes según $\hat{z}$ del campo ${ }^{2}$ eléctrico y magnético, designadas de forma genérica como $C_{z}$,

\footnotetext{
${ }^{2}$ Este campo $\left(C_{z}\right)$ representa la componente en $\hat{z}$ del campo eléctrico $\left(E_{z}\right)$, con espectro bidimensional de ondas planas $p^{\mathrm{TM}}(\beta)$, para polarización $\mathrm{TM}^{\mathrm{z}}$; y la componente en $\hat{z}$ del campo magnético $\left(H_{z}\right)$, con espectro bidimensional de ondas planas $p^{\mathrm{TE}}(\beta)$, para polarización $\mathrm{TE}^{\mathrm{z}}$.
} 
con sus propios espectros bidimensionales de ondas planas mediante la siguiente expresión; que se deduce sustituyendo en (C.7) las condiciones recogidas en las ecuaciones (C.8) y (C.9)

$$
C_{z}(\rho, \phi)=k \int_{\beta} p^{\tau}(\beta) e^{-j k \rho \cos (\phi-\beta)} \mathrm{d} \beta
$$

donde $p^{\tau}(\beta)$ representa el espectro bidimensional de ondas planas asociado directamente a la componente en $\hat{z}$ del campo eléctrico o magnético, según el tipo de polarización considerada; y por tanto es distinto del que se utiliza en las expresiones (C.3) y (C.7), asociado al potencial vector obtenido en problemas tridimensionales.

\section{C.1.2 Espectro Escalar de Ondas Cilíndricas}

Las funciones elementales o modos asociados a una ecuación de onda escalar, expresada en un sistema de coordenadas cilíndricas, son ondas cuyo frente de fase avanza en dirección radial; tal y como se recoge en [14]. Cada uno de estos modos, denominado $\psi_{k_{\rho}, n, k_{z}}^{(1,2)}$, se expresa analíticamente de la siguiente forma

$$
\psi_{k_{\rho}, n, k_{z}}^{(1,2)}=H_{n}^{(1,2)}\left(k_{\rho} \rho\right) e^{j n \phi} e^{ \pm j k_{z} z}
$$

donde $H_{n}^{(1)}\left(k_{\rho} \rho\right)$ y $H_{n}^{(2)}\left(k_{\rho} \rho\right)$ son respectivamente las funciones de Hankel de primera y segunda especie, también llamadas en [71] funciones de Bessel de tercera especie. Las funciones de Hankel de primera especie representan funciones cilíndricas cuyo frente de fase avanza, en dirección radial, desde el infinito hacia el origen de coordenadas. Por su parte, las funciones de Hankel de segunda especie representan funciones cilíndricas cuyo frente de fase avanza, nuevamente en dirección radial, pero desde el origen de coordenadas hacia el infinito. Las dos funciones de Hankel se definen, como puede observarse en la siguiente expresión, en términos de las funciones de Bessel de primera y segunda especie

$$
\begin{aligned}
& H_{n}^{(1)}\left(k_{\rho} \rho\right)=J_{n}\left(k_{\rho} \rho\right)+j Y_{n}\left(k_{\rho} \rho\right) \\
& H_{n}^{(2)}\left(k_{\rho} \rho\right)=J_{n}\left(k_{\rho} \rho\right)-j Y_{n}\left(k_{\rho} \rho\right)
\end{aligned}
$$

Las definiciones de las funciones de Hankel de primera y segunda especie, recogidas en (C.12), permiten constatar que la parte imaginaria de dichas funciones se obtiene a partir de la función de Bessel de segunda especie, también llamada en [71] función de Weber; que presenta un comportamiento singular en el origen de coordenadas.

En cuanto a las variables $k_{\rho}$ y $k_{z}$, que aparecen también en (C.11), representan dos componentes del vector $\vec{k}$ cuando éste se refiere a un sistema de coordenadas 
cilíndricas. Recordando las expresiones (C.2) y (C.4), las componentes $k_{\rho}$ y $k_{z}$ del vector $\vec{k}$ se definen del siguiente modo

$$
\begin{aligned}
& k_{\rho}=\sqrt{k_{x}^{2}+k_{y}^{2}}=k \sin (\alpha) \\
& k_{z}=k \cos (\alpha)
\end{aligned}
$$

Además, observando las expresiones recién deducidas en (C.13), se concluye que las componentes cilíndricas $k_{\rho}$ y $k_{z}$ del vector $\vec{k}$ deben satisfacer la siguiente condición

$$
k_{\rho}^{2}+k_{z}^{2}=k^{2}
$$

Utilizando pues las expresiones modales recogidas en (C.11), mediante una combinación lineal de las mismas, es posible obtener una solución ${ }^{3}$ de la ecuación de onda escalar; a la que designaremos nuevamente como $\psi^{\tau}$. Debido a la condición expresada en (C.14), tan sólo se dispone de dos grados de libertad para definir las distintas funciones elementales o modos; que se requieren, como se ha mencionado anteriormente, al construir la solución $\psi^{\tau}$. En este caso, se escogen como variables la componente $k_{\rho}$ del vector $\vec{k}$, y el índice $n$ que define la variación en azimut del correspondiente modo. Las amplitudes de las diferentes ondas cilíndricas se expresan a través de unos coeficientes que son función continua de la variable $k_{\rho}$, denominados $c_{i n}^{\tau}\left(k_{\rho}\right)$; los cuales constituyen el espectro de ondas cilíndricas asociado a la solución $\psi^{\tau}$ de la ecuación de onda escalar. Por tanto, haciendo uso de estos coeficientes, dicha solución se construye tras implementar la siguiente expresión

$$
\psi^{\tau}(\rho, \phi, z)=\sum_{i=1}^{2} \sum_{n=-\infty}^{\infty} \int_{k_{\rho}} c_{i n}^{\tau}\left(k_{\rho}\right) H_{n}^{(i)}\left(k_{\rho} \rho\right) e^{j n \phi} e^{ \pm j k_{z} z} \mathrm{~d} k_{\rho}
$$

Al igual que en el apartado C.1.1, resulta interesante particularizar la expresión recién presentada en (C.15) al caso de un problema bidimensional, considerado invariante según el eje $\hat{z}$. En este caso, las ondas cilíndricas se encuentran contenidas en el plano $X Y\left(\alpha=90^{\circ}\right)$; lo que se traduce, tras sustituir dicha condición en (C.13), en las siguientes nuevas expresiones para $k_{\rho} \mathrm{y} k_{z}$

$$
\begin{aligned}
& k_{\rho}=k \\
& k_{z}=0
\end{aligned}
$$

Como consecuencia del nuevo valor para $k_{\rho}$ recogido en (C.16), los coeficientes $c_{i n}^{\tau}\left(k_{\rho}\right)$ se convierten en unos coeficientes constantes denominados $c_{i n}^{\tau}$; que ya no

\footnotetext{
${ }^{3}$ Esta solución $\left(\psi^{\tau}\right)$ de la ecuación de onda escalar, obtenida cuando dicha ecuación se expresa en un sistema de coordenadas cilíndricas, representa la componente en $\hat{z}$ del potencial vector magnético $\left(\vec{A}=\psi^{\mathrm{TM}} \hat{z}\right)$ para el campo $\mathrm{TM}^{\mathrm{z}}$, y la componente en $\hat{z}$ del potencial vector eléctrico $\left(\vec{F}=\psi^{\mathrm{TE}} \hat{z}\right)$ para el campo $\mathrm{TE}^{\mathrm{z}}$.
} 
dependen de la variable $k_{\rho}$. Tal y como se ha mencionado en el apartado C.1.1 para problemas bidimensionales, es posible relacionar a través de constantes la componente en $\hat{z}$ del correspondiente potencial vector, eléctrico o magnético según el tipo de polarización considerada, con la componente en $\hat{z}$ del campo eléctrico para polarización $\mathrm{TM}^{\mathrm{z}}$, y con la componente en $\hat{z}$ del campo magnético para polarización $\mathrm{TE}^{\mathrm{z}}$. En conscuencia, dichas componentes según $\hat{z}$ del campo ${ }^{4}$ eléctrico y magnético, designadas genéricamente como $C_{z}$, pueden definirse en términos de sus correspondientes espectros bidimensionales de ondas cilíndricas a través de la siguiente expresión; la cual se deduce sustituyendo en (C.15) las condiciones reflejadas en la ecuación (C.16) para un problema bidimensional

$$
C_{z}(\rho, \phi)=\sum_{i=1}^{2} \sum_{n=-\infty}^{\infty} c_{i n}^{\tau} H_{n}^{(i)}(k \rho) e^{j n \phi}
$$

donde los coeficientes $c_{i n}^{\tau}$ constituyen el espectro bidimensional de ondas cilíndricas asociado directamente a la componente en $\hat{z}$ del campo eléctrico o magnético, dependiendo del tipo de polarización considerada; y por tanto dicho espectro es distinto de aquél utilizado en (C.15), que está asociado al correspondiente potencial vector deducido para problemas tridimensionales.

En el presente trabajo, se requiere expresar el campo que dispersa un objeto en un problema de dispersión bidimensional. Dicho campo, referido a un origen de coordenadas y válido fuera de la región con fuentes, puede desarrollarse en serie de funciones cilíndricas emergentes desde el citado origen hacia el infinito; por lo que el espectro cilíndrico asociado a este campo dispersado presenta unos coeficientes $c_{1 n}^{\tau}$ con valor nulo. Particularizando esta condición en el desarrollo mostrado anteriormente en (C.17), el campo dispersado se define mediante el siguiente sumatorio

$$
C_{z}^{d}(\rho, \phi)=\sum_{n=-\infty}^{\infty} c_{2 n}^{\tau} H_{n}^{(2)}(k \rho) e^{j n \phi}
$$

donde los coeficientes $c_{2 n}^{\tau}$ definen el espectro cilíndrico asociado a un campo dispersado por un objeto en problemas bidimensionales.

Por otra parte, resulta igualmente necesario expresar el campo incidente sobre un objeto dispersor en problemas bidimensionales. En este caso, como se supone que el campo incidente al objeto lo producen unas fuentes alejadas del origen de coordenadas, dicho campo no debe presentar un comportamiento singular en el citado origen. Debido a esta razón, los coeficientes $c_{1 n}^{\tau}$ y $c_{2 n}^{\tau}$ del espectro asociado a un campo incidente sobre un objeto deben escogerse iguales; para que de esta forma, recordando (C.12), se anulen las partes imaginarias de las funciones

\footnotetext{
${ }^{4}$ Este campo $\left(C_{z}\right)$ representa la componente en $\hat{z}$ del campo eléctrico $\left(E_{z}\right)$, con espectro bidimensional de ondas cilíndricas $c_{i n}^{\mathrm{TM}}$, para polarización $\mathrm{TM}^{\mathrm{z}}$; y la componente en $\hat{z}$ del campo magnético $\left(H_{z}\right)$, con espectro bidimensional de ondas cilíndricas $c_{i n}^{\mathrm{TE}}$, para polarización $\mathrm{TE}^{\mathrm{z}}$.
} 
de Hankel de primera y segunda especie, que son las responsables del comportamiento singular de dichas funciones en el origen de coordenadas. Sustituyendo esta condición en (C.17), el campo incidente sobre un objeto dispersor se expresa a través del siguiente desarrollo

$$
C_{z}^{i}(\rho, \phi)=\sum_{n=-\infty}^{\infty} i_{n}^{\tau} J_{n}(k \rho) e^{j n \phi}
$$

donde los coeficientes $i_{n}^{\tau}$, de valor $i_{n}^{\tau}=2 c_{1 n}^{\tau}=2 c_{2 n}^{\tau}$, constituyen el espectro cilíndrico asociado a un campo incidente sobre un objeto dispersor bidimensional; siempre y cuando las fuentes que producen dicho campo estén situadas lejos del origen de coordenadas.

\section{C.2 Transformaciones entre Espectros Plano y Cilíndrico}

En la sección C.1 (apartados C.1.1 y C.1.2), se han definido los espectros de ondas planas y cilíndricas; que corresponden a la misma solución de la ecuación de onda escalar, aunque expresada respectivamente en un sistema de coordenadas cartesianas y cilíndricas. La elección de una representación espectral u otra, para expresar la solución electromagnética en una región libre de fuentes, se realiza en base a cual de los dos espectros se adecua mejor a la geometría del problema bajo análisis. No obstante, existen problemas cuya resolución completa requiere utilizar las dos expresiones espectrales mencionadas anteriormente. En dichos problemas, será necesario emplear diversas transformaciones espectrales; que permitan transformar un espectro en otro para manejar en cada instante el más adecuado. Este tipo de transformaciones entre espectros de distinta naturaleza se catalogan en [14] como cruzadas. Concretamente, las transformaciones entre espectros plano y cilíndrico permiten relacionar, en ambos sentidos, el espectro escalar de ondas planas $p^{\tau}\left(k_{\rho}, \beta\right)$ y el espectro escalar de ondas cilíndricas $c_{i n}^{\tau}\left(k_{\rho}\right)$ mediante las siguientes expresiones; cuya deducción se detalla en [14]

$$
\begin{gathered}
c_{i n}^{\tau}\left(k_{\rho}\right)=j^{-n} k_{\rho} \frac{1}{2} \int_{\beta=0}^{2 \pi} p^{\tau}\left(k_{\rho}, \beta\right) e^{-j n \beta} \mathrm{d} \beta \\
p^{\tau}\left(k_{\rho}, \beta\right)=\frac{1}{\pi k_{\rho}} \sum_{n=-\infty}^{\infty} j^{n} c_{i n}^{\tau}\left(k_{\rho}\right) e^{j n \beta}
\end{gathered}
$$

En el presente trabajo, al analizar la dispersión electromagnética producida por objetos cuya geometría es invariante en una dimensión, la matriz de caracterización de cada objeto dispersor relaciona los espectros bidimensionales de ondas cilíndricas asociados a los campos incidente y dispersado. Al haberse elegido espectros cilíndricos en la definición de dicha matriz, no se elimina la necesidad de 
tener que recurrir puntualmente a las transformaciones entre espectros plano y cilíndrico; pues en la resolución de ciertos problemas de dispersión es necesario utilizar los espectros de ondas planas. Por ejemplo, al calcular la dispersión que produce un objeto ante la incidencia de una cierta onda plana, haciendo uso de la matriz de caracterización de dicho objeto, se requiere convertir previamente el espectro asociado a la onda plana en su espectro cilíndrico equivalente. Una segunda situación, en la que se requiere utilizar la misma transformación que en el ejemplo anterior pero en sentido inverso, se produce al intentar obtener mediante Optica Física la distribución de corrientes inducidas sobre la superficie del objeto dispersor. La técnica de Óptica Física precisa de un campo incidente sobre el objeto dispersor desarrollado a partir de su espectro de ondas planas; mientras el campo incidente en el problema de dispersión se describe en términos de su espectro de ondas cilíndricas. Por tanto, en este caso, el espectro cilíndrico asociado al campo incidente debe convertirse en un espectro plano equivalente.

Particularizando las relaciones entre espectros recogidas en (C.20) a un problema bidimensional, se obtienen las expresiones que permiten implementar las transformaciones entre espectros bidimensionales de ondas planas y cilíndricas. Dichas expresiones relacionan los espectros bidimensionales $p^{\tau}(\beta)$ y $c_{i n}^{\tau}$ del siguiente modo

$$
\begin{gathered}
c_{i n}^{\tau}=j^{-n} k \frac{1}{2} \int_{\beta=0}^{2 \pi} p^{\tau}(\beta) e^{-j n \beta} \mathrm{d} \beta \\
p^{\tau}(\beta)=\frac{1}{\pi k} \sum_{n=-\infty}^{\infty} j^{n} c_{i n}^{\tau} e^{j n \beta}
\end{gathered}
$$

Los dos apartados de esta sección, ofrecidos seguidamente, hacen uso de las expresiones recién definidas en (C.21) para calcular las transformaciones espectrales requeridas en ciertos problemas bidimensionales. Así pues, en el primer apartado se calcula el espectro cilíndrico bidimensional correspondiente a una onda plana incidente contenida en el plano $X Y$; mientras en el segundo apartado se obtiene una matriz de transformación de espectro cilíndrico bidimensional, asociado al campo incidente, en espectro bidimensional de ondas planas.

\section{C.2.1 Espectro Cilíndrico de una Onda Plana Incidente en Problemas Bidimensionales}

La componente ${ }^{5}$ según $\hat{z}$ del campo eléctrico o magnético incidente, designada como $C_{z}^{i}$, que corresponde a una onda plana bidimensional propagándose en el plano $X Y$, con dirección de avance $\phi=\beta_{0}$ (ver figura C.1), se expresa recordando

\footnotetext{
${ }^{5}$ Esta componente $\left(C_{z}^{i}\right)$ designa la componente según $\hat{z}$ del campo eléctrico incidente $\left(E_{z}^{i}\right)$ para polarización $\mathrm{TM}^{\mathrm{z}}$, y la componente según $\hat{z}$ del campo magnético incidente $\left(H_{z}^{i}\right)$ para polarización $\mathrm{TE}^{\mathrm{z}}$.
} 
los resultados recogidos en (C.5) y (C.9) como

$$
C_{z}^{i}(\rho, \phi)=e^{-j k \rho \cos \left(\phi-\beta_{0}\right)}
$$

El espectro bidimensional de ondas planas asociado a este campo $C_{z}^{i}$ se obtiene, despejando de forma adecuada en (C.10), mediante la siguiente expresión

$$
\begin{aligned}
p^{\tau}(\beta) & =\frac{1}{k} \frac{1}{2 \pi} \int_{\phi=0}^{2 \pi} C_{z}^{i}(\rho, \phi) e^{j k \rho \cos (\phi-\beta)} \mathrm{d} \phi \\
& =\frac{1}{k} \delta\left(\beta-\beta_{0}\right)
\end{aligned}
$$

Este resultado recién deducido en (C.23) indica que el espectro plano asociado a una onda plana bidimensional, que se propaga en la dirección $\phi=\beta_{0}$, es una función delta de Dirac; la cual está ubicada en la componente espectral $\beta=\beta_{0}$. El espectro cilíndrico correspondiente a dicha onda plana, aplicando la primera de las transformaciones recogidas en (C.21), se expresa de la siguiente manera

$$
\begin{aligned}
c_{i n}^{\tau} & =j^{-n} k \frac{1}{2} \int_{\beta=0}^{2 \pi} \frac{1}{k} \delta\left(\beta-\beta_{0}\right) e^{-j n \beta} \mathrm{d} \beta \\
& =j^{-n} \frac{1}{2} e^{-j n \beta_{0}}
\end{aligned}
$$

Del resultado obtenido en (C.24), se concluye que los coeficientes $c_{1 n}^{\tau}$ y $c_{2 n}^{\tau}$ presentan el mismo valor; lo cual es consecuente con el hecho de que el espectro cilíndrico $c_{i n}^{\tau}$ se encuentra asociado a un campo incidente (recordar apartado C.1.2), representado en este caso por la onda plana original. Consecuentemente, la onda plana puede desarrollarse como se indica en (C.19) utilizando unos coeficientes $i_{n}^{\tau}$ de valor $i_{n}^{\tau}=2 c_{1 n}^{\tau}=2 c_{2 n}^{\tau}=\left(j e^{j \beta_{0}}\right)^{-n}$; presentando dicho desarrollo el siguiente aspecto

$$
\begin{aligned}
C_{z}^{i}(\rho, \phi) & =e^{-j k \rho \cos \left(\phi-\beta_{0}\right)} \\
& =\sum_{n=-\infty}^{\infty}\left(j e^{j \beta_{0}}\right)^{-n} J_{n}(k \rho) e^{j n \phi}
\end{aligned}
$$

\section{C.2.2 Matriz de Transformación de Espectro Cilíndrico a Espectro Plano en Problemas Bidimensionales}

En el presente trabajo, el comportamiento dispersor de los objetos se ha representado utilizando matrices de caracterización; las cuales requieren que el campo incidente, así como el campo dispersado, se expresen a través de sus respectivos espectros de ondas cilíndricas. El cálculo de la matriz de caracterización de un 
objeto dispersor, tal y como se recoge en el capítulo 4 (sección 4.1), requiere de la obtención previa de una matriz de corrientes; que relaciona corrientes inducidas en la superficie del objeto dispersor con el espectro cilíndrico asociado al campo incidente. En el capítulo 4 (sección 4.2), se plantean dos posibles técnicas para obtener dicha matriz de corrientes; una de ellas basada en Óptica Física, y la otra en el Método de los Momentos. Pues bien, mientras la segunda técnica permite calcular directamente la matriz de corrientes a partir del espectro cilíndrico asociado al campo incidente; la primera de las técnicas, basada en Óptica Física, requiere en su correcta aplicación que el campo incidente se exprese a través de su correspondiente espectro de ondas planas. Por tanto, para poder aplicar dicha técnica es necesario expresar previamente un sumatorio de modos cilíndricos, como aquél recogido en (C.19), mediante una integral de ondas planas definida según (C.10); lo que sin duda representa una conversión de espectro cilíndrico a espectro plano. En este apartado, una vez discretizada la integral de ondas planas en un sumatorio equivalente, se definen los elementos de una matriz de transformación que permite implementar la citada conversión de espectros; garantizando de esta forma la posibilidad de obtener la matriz de corrientes mediante Óptica Física. Considerando el $p$-ésimo modo cilíndrico $\left(J_{p}(k \rho) e^{j p \phi}\right)$ del desarrollo recogido en (C.19) para expresar un campo incidente, se deduce que dicho modo presenta el siguiente espectro cilíndrico asociado

$$
J_{p}(k \rho) e^{j p \phi} \Longrightarrow c_{1 n}^{\tau}=c_{2 n}^{\tau}=\left\{\begin{array}{cc}
\frac{1}{2} & n=p \\
0 & n \neq p
\end{array}\right.
$$

El espectro de ondas planas correspondiente al espectro cilíndrico deducido en (C.26), tras aplicar la segunda de las transformaciones recogidas en (C.21), se expresa tal y como se indica a continuación

$$
p^{\tau}(\beta)=\frac{1}{2 \pi k}\left(j e^{j \beta}\right)^{p}
$$

A partir de este espectro de ondas planas asociado a la $p$-ésima componente modal $\left(J_{p}(k \rho) e^{j p \phi}\right)$ del campo incidente, y recordando la definición expresada en (C.10), es posible relacionar dicha componente modal con su espectro plano asociado del siguiente modo

$$
J_{p}(k \rho) e^{j p \phi}=k \int_{\beta=0}^{2 \pi}\left(\frac{1}{2 \pi k}\left(j e^{j \beta}\right)^{p}\right) e^{-j k \rho \cos (\phi-\beta)} \mathrm{d} \beta
$$

La integral que aparece en (C.28) representa una suma de infinitas ondas planas, con direcciones de avance $\beta$ comprendidas entre 0 y $2 \pi$, ponderadas cada una de ellas por el correspondiente valor del espectro en su dirección de avance; es decir, con una amplitud asociada de valor $p^{\tau}(\beta)$. Lógicamente, la implementación de dicha integral requiere su discretización previa en un sumatorio finito de ondas 
planas; transformando la expresión (C.28) de la siguiente manera

$$
\begin{aligned}
J_{p}(k \rho) e^{j p \phi} & =k \sum_{n=0}^{N_{\beta}-1}\left(\frac{1}{2 \pi k}\left(j e^{j \frac{2 \pi}{N_{\beta}} n}\right)^{p}\right) e^{-j k \rho \cos \left(\phi-\frac{2 \pi}{N_{\beta}} n\right)} \frac{2 \pi}{N_{\beta}} \\
& =\sum_{n=0}^{N_{\beta}-1}\left(\frac{1}{N_{\beta}}\left(j e^{j \frac{2 \pi}{N_{\beta}} n}\right)^{p}\right) e^{-j k \rho \cos \left(\phi-\frac{2 \pi}{N_{\beta}} n\right)}
\end{aligned}
$$

El desarrollo recogido en (C.29) demuestra que el modo cilíndrico $J_{p}(k \rho) e^{j p \phi}$ se ha expresado definitivamente mediante la suma de $N_{\beta}$ ondas planas, con direcciones de avance $\beta_{n}=2 \pi n / N_{\beta}$ variando $n$ desde 0 hasta $N_{\beta}-1$; y con una amplitud asociada a cada una de estas ondas igual a $\left(j e^{j \frac{2 \pi}{N_{\beta}} n}\right)^{p} / N_{\beta}$.

Recordando el desarrollo modal definido en (C.19), la componente ${ }^{6}$ según $\hat{z}$ del campo eléctrico o magnético incidente, designada como $C_{z}^{i}$, puede expresarse mediante un sumatorio de infinitos modos cilíndricos; los cuales se encuentran ponderados respectivamente por los coeficientes $i_{p}^{\tau}$ que constituyen el espectro asociado a dicho campo. A efectos prácticos, tal y como se indica en el capítulo 4 (sección 4.1), el sumatorio de infinitos modos se trunca en una serie con un número finito $\left(2 N_{i}+1\right)$ de modos; que permiten reconstruir correctamente el campo incidente sobre la superficie del objeto dispersor bajo análisis. Por tanto, utilizando la relación deducida en (C.29), el campo incidente $C_{z}^{i}$ expresado inicialmente como un sumatorio de modos cilíndricos puede escribirse, como se indica seguidamente, en términos de una suma finita de ondas planas

$$
\begin{aligned}
C_{z}^{i}(\rho, \phi) & =\sum_{p=-N_{i}}^{N_{i}} i_{p}^{\tau} J_{p}(k \rho) e^{j p \phi} \\
& =\sum_{p=-N_{i}}^{N_{i}} i_{p}^{\tau} \sum_{n=0}^{N_{\beta}-1}\left(\frac{1}{N_{\beta}}\left(j e^{j \frac{2 \pi}{N_{\beta}} n}\right)^{p}\right) e^{-j k \rho \cos \left(\phi-\frac{2 \pi}{N_{\beta}} n\right)} \\
& =\sum_{n=0}^{N_{\beta}-1}\left(\sum_{p=-N_{i}}^{N_{i}} i_{p}^{\tau} \frac{1}{N_{\beta}}\left(j e^{j \frac{2 \pi}{N_{\beta}} n}\right)^{p}\right) e^{-j k \rho \cos \left(\phi-\frac{2 \pi}{N_{\beta}} n\right)} \\
& =\sum_{n=0}^{N_{\beta}-1} p_{n}^{\tau} e^{-j k \rho \cos \left(\phi-\frac{2 \pi}{N_{\beta}} n\right)}
\end{aligned}
$$

donde cada uno de los coeficientes $p_{n}^{\tau}$, presentes en el último de los sumatorios recogidos en (C.30), representa la amplitud de la $n$-ésima componente del espectro

\footnotetext{
${ }^{6}$ Esta componente $\left(C_{z}^{i}\right)$ designa nuevamente la componente según $\hat{z}$ del campo eléctrico incidente $\left(E_{z}^{i}\right)$ para polarización $\mathrm{TM}^{z}$, y la componente según $\hat{z}$ del campo magnético incidente $\left(H_{z}^{i}\right)$ para polarización $\mathrm{TE}^{\mathrm{z}}$.
} 
plano asociado al campo incidente $C_{z}^{i}$; definiendo dicha componente espectral una onda plana con dirección de avance $\beta_{n}=2 \pi n / N_{\beta}$. En cuanto a los coeficientes $p_{n}^{\tau}$, cada uno de ellos se obtiene evaluando el espectro en un valor de la variable $\beta$ igual a $\beta_{n}$; es decir, $p_{n}^{\tau}=p^{\tau}\left(\beta=\beta_{n}=2 \pi n / N_{\beta}\right)$.

A partir de las relaciones recogidas en (C.30), es posible definir la transformación de espectro cilíndrico a plano en términos matriciales. Para ello, el espectro cilíndrico debe representarse mediante un vector columna con $2 N_{i}+1$ componentes de amplitud $i_{p}^{\tau}$, con $p$ variando desde $-N_{i}$ hasta $N_{i}$; mientras que el espectro plano se define a través de un vector columna constituido por $N_{\beta}$ componentes de valor $p_{n}^{\tau}$, con $n$ variando entre 0 y $N_{\beta}-1$. Finalmente, utilizando la transformación definida en (C.30), se consigue relacionar ambos vectores columna mediante el siguiente producto matricial

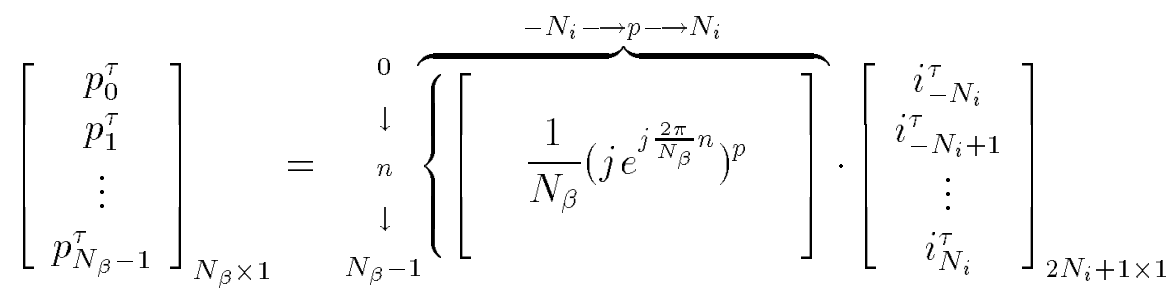

donde la matriz que multiplica al espectro de ondas cilindricas, designada de ahora en adelante como $D_{p c}$, constituye la buscada matriz de transformación de espectro cilíndrico a plano. 


\section{Apéndice D}

\section{Análisis del Giro de un Objeto Dispersor}

La matriz de caracterización individual de un objeto dispersor, calculada tanto para incidencia $\mathrm{TM}^{\mathrm{z}}$ como $\mathrm{TE}^{\mathrm{z}}$, depende por completo de la posición relativa que ocupa dicho objeto dispersor respecto de los ejes coordenados. Un simple giro del objeto, respecto de la posición en la que ha sido caracterizado inicialmente, provoca que el comportamiento dispersor del mismo objeto girado se represente mediante una nueva matriz de caracterización individual, diferente de aquélla obtenida para la posición inicial del objeto. En este apéndice, se muestra como obtener la nueva matriz a partir de la calculada originalmente; utilizando para ello las denominadas matrices de giro. Siguiendo el método propuesto en este apéndice, se evita la repetición del proceso de cálculo descrito en el capítulo 4 para obtener la nueva matriz de caracterización individual; que corresponde a un objeto dispersor ya caracterizado anteriormente, aunque girado respecto de su posición original.

En un principio, este apéndice describe un procedimiento genérico para calcular la nueva matriz de caracterización individual correspondiente a un objeto girado respecto de su posición original. Durante el desarrollo de este procedimiento, se introduce el concepto de las citadas matrices de giro; y asimismo se determina cómo calcular los elementos que integran dichas matrices. Posteriormente, las matrices de giro se aplican a la resolución de un problema práctico; consistente en calcular la dispersión producida por una tira metálica infinita, de grosor despreciable, y girada respecto de una posición original en la que previamente se había caracterizado su comportamiento dispersor. Los resultados obtenidos al resolver este problema utilizando la nueva matriz de caracterización de la tira girada, obtenida haciendo uso de las matrices de giro, validan completamente el procedimiento descrito en este apéndice para considerar el efecto que supone, en la matriz de caracterización individual del objeto dispersor, un giro de dicho objeto respecto de su posición original. 


\section{D.1 Matrices de Giro. Concepto}

En la presente sección, se introduce el concepto de las matrices de giro; las cuales surgen al evaluar el efecto que supone girar un objeto dispersor. Inicialmente, se considera un objeto dispersor bidimensional; cuya posición relativa respecto de los ejes coordenados se muestra en la figura D.1 (apartado a), y del que se conoce su función de transferencia o matriz de caracterización individual $D$ para dicha posición. Si este objeto se gira un ángulo $\phi_{g}$ respecto de su posición original, tal y como se ilustra en la figura D.1 (apartado b), el comportamiento dispersor de este mismo objeto girado se define en términos de una nueva matriz de caracterización individual, que designaremos como $D^{\prime}$. A continuación, se expone un método para obtener esta nueva matriz $D^{\prime}$; que utiliza las mencionadas matrices de giro, y la matriz $D$ asociada a la posición original del objeto. Es interesante reseñar que la aplicación de dicho método resulta independiente del tipo de incidencia, polarización $\mathrm{TM}^{\mathrm{z}}$ o $\mathrm{TE}^{\mathrm{z}}$, considerada en la definición de las matrices $D$ y $D^{\prime}$.

Recordando el apéndice C (apartado C.1.2), los campos ${ }^{1}$ incidente y dispersado correspondientes al problema original de la figura D.1 (apartado a), designados respectivamente como $C_{z}^{i}$ y $C_{z}^{d}$, se expresan mediante los siguientes desarrollos modales

$$
\begin{aligned}
C_{z}^{i}(\rho, \phi) & =\sum_{p=-N_{i}}^{N_{2}} i_{p}^{\tau} J_{p}(k \rho) e^{j p \phi} \\
C_{z}^{d}(\rho, \phi) & =\sum_{q=-N_{d}}^{N_{d}} c_{q}^{\tau} H_{q}^{(2)}(k \rho) e^{j q \phi}
\end{aligned}
$$

donde los coeficientes $i_{p}^{\tau}$ y $c_{q}^{\tau}$, que representan las amplitudes de los espectros asociados a los campos incidente y dispersado, se encuentran relacionados en términos matriciales mediante el siguiente producto

$$
\left[c_{q}^{\tau}\right]=D^{\tau} \cdot\left[i_{p}^{\tau}\right]
$$

En la expresión (D.2), $\left[i_{p}^{\tau}\right]$ y $\left[c_{q}^{\tau}\right]$ denotan sendos vectores columna constituidos respectivamente por los coeficientes espectrales $i_{p}^{\tau}$ y $c_{q}^{\tau}$; mientras la matriz $D$, como ya se ha mencionado anteriormente, caracteriza el comportamiento dispersor del objeto mostrado en la figura D.1 (apartado a).

Considerando ahora la nueva situación del objeto dispersor recogida en la figura D.1 (apartado b), donde el objeto está girado un ángulo $\phi_{g}$ respecto de su posición original, se define un nuevo sistema de ejes coordenados $\left(X^{\prime}, Y^{\prime}\right)$; cuya posición, relativa al objeto, es idéntica a la que ocupa el sistema de ejes $(X, Y)$ en

\footnotetext{
${ }^{1}$ Estos campos $\left(C_{z}^{i}\right.$ y $\left.C_{z}^{d}\right)$ representan campos eléctricos incidente y dispersado $\left(E_{z}^{i}\right.$ y $\left.E_{z}^{d}\right)$, con amplitudes espectrales $i_{p}^{\mathrm{TM}}$ y $c_{q}^{\mathrm{TM}}$, para polarización $\mathrm{TM}^{\mathrm{z}}$; y campos magnéticos incidente y dispersado $\left(H_{z}^{i}\right.$ y $\left.H_{z}^{d}\right)$, con amplitudes espectrales $i_{p}^{\mathrm{TE}}$ y $c_{q}^{\mathrm{TE}}$, para polarización $\mathrm{TE}^{\mathrm{z}}$.
} 


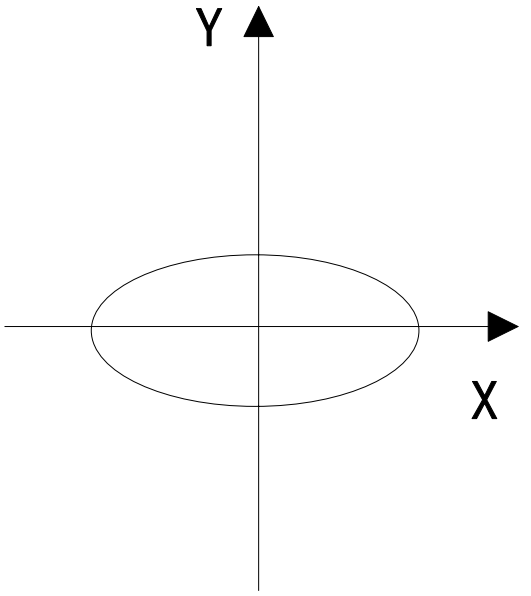

a)

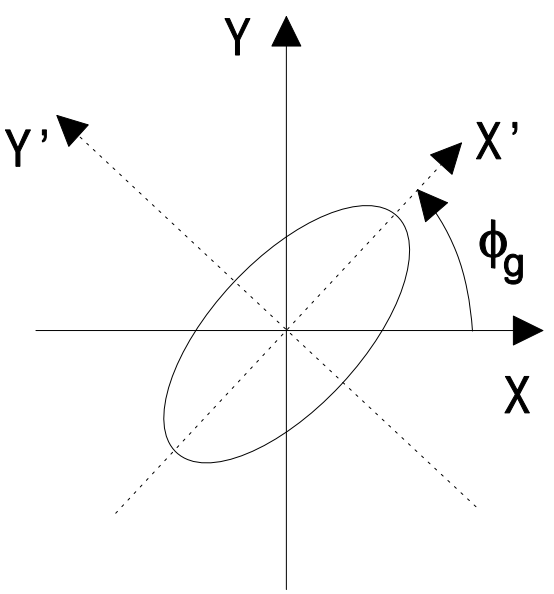

b)

Figura D.1: Posición original de un objeto dispersor en a), y posición de dicho objeto dispersor girado un ángulo $\phi_{g}$ respecto de la situación anterior en b).

la situación original (ver apartado a en la figura D.1). En este nuevo sistema de ejes $\left(X^{\prime}, Y^{\prime}\right)$, los campos incidente y dispersado asociados al problema constituido por el objeto dispersor girado, denominados en esta ocasión $C_{z}^{\prime i}$ y $C_{z}^{\prime d}$, se definen a través de las siguientes series de modos cilíndricos incidentes y dispersados

$$
\begin{aligned}
C_{z}^{\prime i}\left(\rho^{\prime}, \phi^{\prime}\right) & =\sum_{p=-N_{i}}^{N_{i}} i_{p}^{\prime \tau} J_{p}\left(k \rho^{\prime}\right) e^{j p \phi^{\prime}} \\
C_{z}^{\prime d}\left(\rho^{\prime}, \phi^{\prime}\right) & =\sum_{q=-N_{d}}^{N_{d}} c_{q}^{\prime \tau} H_{q}^{(2)}\left(k \rho^{\prime}\right) e^{j q \phi^{\prime}}
\end{aligned}
$$

donde los posibles significados atribuibles a los campos $C_{z}^{\prime i}$ y $C_{z}^{\prime d}$ son idénticos a los recogidos previamente para $C_{z}^{i}$ y $C_{z}^{d}$. Por su parte, los coeficientes $i_{p}^{\prime \tau}$ y $c_{q}^{\prime \tau}$ representan las amplitudes espectrales de los campos incidente y dispersado en la segunda situación (apartado b en la figura D.1); habiendo referido los desarrollos modales recogidos en (D.3) al nuevo sistema de ejes $\left(X^{\prime}, Y^{\prime}\right)$, posicionado en relación al objeto dispersor de igual manera que el sistema de ejes $(X, Y)$ en la situación original. Comparando pues los dos apartados de la figura D.1, se deduce que los vectores columna $\left[i_{p}^{\prime \tau}\right]$ y $\left[c_{q}^{\prime \tau}\right]$ se relacionan nuevamente, como se muestra a continuación, utilizando la misma matriz $D$ empleada al caracterizar el objeto dispersor en la situación original

$$
\left[c_{q}^{\prime \tau}\right]=D^{\tau} \cdot\left[i_{p}^{\prime \tau}\right]
$$

Ahora bien, para caracterizar el comportamiento dispersor del objeto en la segunda situación, los campos incidente y dispersado $\left(C_{z}^{\prime^{i}}\right.$ y $\left.C_{z}^{\prime d}\right)$ en dicha situación 
deben referise al mismo sistema de ejes $(X, Y)$ empleado en la situación original. Por tanto, dichos campos incidente y dispersado deben definirse mediante las siguientes nuevas expresiones

$$
\begin{aligned}
C_{z}^{\prime \prime}(\rho, \phi) & =\sum_{p=-N_{i}}^{N_{i}} i_{p}^{\tau} J_{p}(k \rho) e^{j p \phi} \\
C_{z}^{\prime d}(\rho, \phi) & =\sum_{q=-N_{d}}^{N_{d}} c_{q}^{\prime \prime \tau} H_{q}^{(2)}(k \rho) e^{j q \phi}
\end{aligned}
$$

donde al definir el campo incidente en la segunda situación, referido al sistema de ejes $(X, Y)$, se hace uso de los mismos coeficientes $i_{p}^{\tau}$ utilizados en (D.1) para describir el campo incidente en la situación original; pues dicho campo incidente se expresa de igual manera en ambas situaciones al referirse a un mismo sistema de ejes coordenados. Lógicamente, el campo dispersado por el objeto en la segunda de las dos situaciones consideradas, referido al sistema de ejes $(X, Y)$, es diferente de aquél generado por el mismo objeto en la situación original; de ahí que en el desarrollo modal del nuevo campo dispersado por el objeto girado se utilicen unos nuevos coeficientes, designados $\operatorname{como} c_{q}^{\prime \prime}$. De esta forma, la nueva matriz buscada para caracterizar el comportamiento del objeto dispersor girado, denominada $D^{\prime}$, debe relacionar del siguiente modo los vectores columna $\left[i_{p}^{\tau}\right]$ y $\left[c_{q}^{\prime \prime \tau}\right]$

$$
\left[c_{q}^{\prime \prime \tau}\right]=D^{\prime \tau} \cdot\left[i_{p}^{\tau}\right]
$$

Con la intención de expresar la matriz $D^{\prime}$, que define el comportamiento dispersor del objeto girado, en términos de la matriz $D$ obtenida en la situación original; resulta necesario establecer en primer lugar una relación entre los coeficientes $c_{q}^{\prime \prime \tau}$ y $c_{q}^{\prime \tau}$. Estos coeficientes pueden relacionarse igualando los desarrollos modales del campo dispersado recogidos en (D.3) y (D.5); aunque no es posible igualarlos directamente debido a que dichos desarrollos, correspondientes a un mismo campo, se encuentran referidos a distintos sistemas de ejes. Conviene pues elegir un mismo sistema de ejes al que referir ambos desarrollos, por ejemplo el sistema definido por los ejes $(X, Y)$; para lo cual debe emplearse el siguiente cambio de coordenadas

$$
\begin{aligned}
& \rho^{\prime}=\rho \\
& \phi^{\prime}=\phi-\phi_{g}
\end{aligned}
$$

Por tanto, utilizando el cambio de coordenadas recién expuesto en (D.7), es posible referir al sistema de ejes $(X, Y)$ el desarrollo recogido en (D.3) para el campo dispersado $C_{z}^{\prime d}$; e igualando posteriormente dicha expresión, referida al sistema de ejes $(X, Y)$, con el desarrollo definido según (D.5) para el mismo 
campo dispersado $C_{z}^{\prime d}$, se obtiene la siguiente ecuación

$$
\sum_{q=-N_{d}}^{N_{d}} e^{-j q \phi_{g}} c_{q}^{\prime \tau} H_{q}^{(2)}(k \rho) e^{j q \phi}=\sum_{q=-N_{d}}^{N_{d}} c_{q}^{\prime \prime \tau} H_{q}^{(2)}(k \rho) e^{j q \phi}
$$

de la que se despeja fácilmente el coeficiente $c_{q}^{\prime \prime^{\tau}}$, en función del coeficiente $c_{q}^{\prime \tau}$, a través de la expresión

$$
c_{q}^{\prime \prime \tau}=e^{-j q \phi_{g}} c_{q}^{\prime \tau}
$$

A partir de (D.9), es posible expresar en términos matriciales una relación entre los vectores columna $\left[c_{q}^{\prime{ }^{\tau}}\right]$ y $\left[c_{q}^{\prime \tau}\right]$; definida del siguiente modo

$$
\left[c_{q}^{\prime \prime \tau}\right]=G_{1} \cdot\left[c_{q}^{\prime \tau}\right]
$$

donde $G_{1}$ representa la primera matriz de giro, diagonal y de tamaño $2 N_{d}+1$; cuyo aspecto se ofrece a continuación

$$
G_{1}=\left[\begin{array}{cccc}
e^{j N_{d} \phi_{g}} & 0 & \cdots & 0 \\
0 & e^{j\left(N_{d}-1\right) \phi_{g}} & \cdots & 0 \\
\vdots & \vdots & \ddots & \vdots \\
0 & 0 & \cdots & e^{-j N_{d} \phi_{g}}
\end{array}\right]_{2 N_{d}+1 \times 2 N_{d}+1}
$$

La denominación de $G_{1}$ como matriz de giro obedece al hecho de que relaciona los coeficientes de un desarrollo modal, asociado a un cierto campo dispersado y referido a un sistema de ejes $(X, Y)$, con los coeficientes de otro desarrollo modal correspondiente al mismo campo dispersado; aunque este último desarrollo se refiere a un nuevo sistema de ejes $\left(X^{\prime}, Y^{\prime}\right)$, girado un cierto ángulo respecto del sistema de ejes $(X, Y)$ considerado inicialmente. Partiendo pues de la relación definida en (D.10) por la matriz de giro $G_{1}$, y utilizando aquella otra relación deducida en (D.4) a partir de la matriz $D^{\tau}$; resulta posible expresar el vector columna $\left[c_{q}^{\prime \prime \tau}\right]$, en función del vector columna $\left[i_{p}^{\prime \tau}\right]$, a través del siguiente producto de matrices

$$
\left[c_{q}^{\prime \prime \tau}\right]=G_{1} \cdot D^{\tau} \cdot\left[i_{p}^{\prime \tau}\right]
$$

Ahora bien, recordando (D.6), la matriz $D^{\prime}$ debe relacionar los vectores columna $\left[c_{q}^{\prime \prime}\right]$ e $\left[i_{p}^{\tau}\right]$; para lo cual en (D.12) tan sólo se requiere expresar el vector columna $\left[i_{p}^{\prime \tau}\right]$ en términos del vector columna $\left[i_{p}^{\tau}\right]$. Los coeficientes de dichos vectores permiten reconstruir el mismo campo incidente a partir de sendos desarrollos modales; que se encuentran referidos nuevamente a diferentes sistemas de ejes coordenados. El cambio de coordenadas, definido según (D.7), permite referir el desarrollo modal recogido en (D.3) para el campo incidente al sistema de ejes $(X, Y)$; que es el sistema utilizado para referir el desarrollo modal del mismo 
campo incidente recogido en (D.5). Igualando ambos desarrollos modales, una vez referidos al mismo sistema de ejes, se deduce la siguiente ecuación

$$
\sum_{p=-N_{i}}^{N_{i}} e^{-j p \phi_{g}} i_{p}^{\prime \tau} J_{p}(k \rho) e^{j p \phi}=\sum_{p=-N_{i}}^{N_{i}} i_{p}^{\tau} J_{p}(k \rho) e^{j p \phi}
$$

de la que se puede despejar en este caso el coeficiente $i_{p}^{\prime \tau}$, en función del coeficiente $i_{p}^{\tau}$, tal y como se recoge seguidamente

$$
i_{p}^{\prime \tau}=e^{j p \phi_{g}} i_{p}^{\tau}
$$

La relación entre los coeficientes $i_{p}^{\prime \tau}$ e $i_{p}^{\tau}$, recién deducida en (D.14), puede escribirse en términos matriciales; permitiendo relacionar los vectores columna $\left[i_{p}^{\prime \tau}\right]$ e $\left[i_{p}^{\tau}\right]$ de la siguiente manera

$$
\left[i_{p}^{\prime \tau}\right]=G_{2} \cdot\left[i_{p}^{\tau}\right]
$$

donde $G_{2}$ representa ahora la segunda matriz de giro, diagonal y de tamaño $2 N_{i}+1$, con el siguiente aspecto

$$
G_{2}=\left[\begin{array}{cccc}
e^{-j N_{i} \phi_{g}} & 0 & \cdots & 0 \\
0 & e^{-j\left(N_{i}-1\right) \phi_{g}} & \cdots & 0 \\
\vdots & \vdots & \ddots & \vdots \\
0 & 0 & \cdots & e^{j N_{i} \phi_{g}}
\end{array}\right]_{2 N_{i}+1 \times 2 N_{i}+1}
$$

Esta segunda matriz de giro $G_{2}$ recibe esta acepción debido a que, de igual manera que la matriz $G_{1}$, permite relacionar los coeficientes de sendos desarrollos modales referidos a dos sistemas de ejes girados el uno respecto del otro; aunque en este caso los desarrollos en serie de modos se encuentran asociados a un mismo campo incidente. Para finalizar, sustituyendo en (D.12) el vector columna $\left[i_{p}^{\prime \tau}\right]$ por la definición recogida en (D.15), se obtiene la relación buscada entre los vectores columna $\left[i_{p}^{\tau}\right]$ y $\left[c_{q}^{\prime \prime \tau}\right]$; que se expresa en función de las matrices $D, G_{1}$ y $G_{2}$ del siguiente modo

$$
\left[c_{q}^{\prime \prime \tau}\right]=G_{1} \cdot D^{\tau} \cdot G_{2} \cdot\left[i_{p}^{\tau}\right]
$$

Comparando (D.17) con (D.6), se deduce la siguiente expresión que relaciona la matriz de caracterización $D^{\prime}$ del objeto girado, a través de las correspondientes matrices de giro $G_{1}$ y $G_{2}$, con la matriz de caracterización $D$ asociada a la posición original del objeto

$$
D^{\prime \tau}=G_{1} \cdot D^{\tau} \cdot G_{2}
$$

Resumiendo, en esta sección se ha estudiado el efecto que supone el giro de un objeto dispersor en su matriz de caracterización individual asociada. El problema se ha resuelto definiendo unas matrices de giro; las cuales tienen en cuenta 
el cambio de orientación del objeto dispersor respecto de una posición original. A través de dichas matrices de giro se relaciona la nueva matriz de caracterización, que define el comportamiento dispersor del objeto girado, con la matriz de caracterización asociada a la posición original del objeto; evitando de esta forma tener que repetir el cálculo de la nueva matriz mediante el procedimiento descrito en el capítulo 4.

\section{D.2 Ejemplo de Aplicación de las Matrices de Giro}

Una vez estudiado teóricamente el giro de un objeto dispersor respecto de una posición original, se pretende aplicar dicho estudio a la resolución de un problema práctico de dispersión electromagnética; cuyos resultados permitan corroborar el método recién descrito en la sección D.1.

El problema a resolver consiste en evaluar la dispersión que produce una tira metálica infinita, de grosor despreciable y longitud $1 \lambda$, ante dos posibles orientaciones de la misma respecto del sistema de ejes $(X, Y)$ considerado fijo (recordar apartados a y b de la figura D.1). Inicialmente, se supone que la tira está orientada en la dirección del eje $\hat{y}$ de dicho sistema. El comportamiento dispersor de la tira en esta situación inicial se define a partir de su matriz de caracterización individual $D$, calculada para incidencia polarizada según $\mathrm{TM}^{\mathrm{z}}$ siguiendo el método descrito en el capítulo 4. Haciendo uso de dicha matriz $D$, se ha obtenido la respuesta de la citada tira ante un campo eléctrico incidente representado por una onda plana con dirección de avance $\beta=0$ (ver figura C.1); es decir, ante una onda plana que avanza en la dirección del eje $\hat{x}$ y en el sentido creciente del valor de la coordenada $x$. En la figura D.2 (apartado a), se muestra la amplitud del campo eléctrico total obtenido tras sumar los campos eléctricos incidente y dispersado; observando que detrás del objeto dispersor se produce la conocida zona de sombra al sumar dichos campos en contrafase, y que delante de la tira aparece una onda estacionaria como consecuencia del avance de ambos campos en sentidos opuestos. A continuación, se pretende caracterizar el comportamiento dispersor de la misma tira; pero girada un ángulo de valor $-\pi / 4$ con respecto a la situación anterior $\left(\phi_{g}=-\pi / 4\right.$ en el desarrollo teórico presentado en la sección D.1). La nueva matriz de caracterización individual de la misma tira en esta nueva orientación, obtenida para el mismo tipo de incidencia (polarización $\mathrm{TM}^{\mathrm{z}}$ ) y designada como $D^{\prime}$, se deduce fácilmente a partir de la matriz de caracterización individual $D$ utilizando la relación definida según (D.18); en la que las matrices de giro $G_{1}$ y $G_{2}$ deben particularizarse al problema concreto bajo análisis $\left(\phi_{g}=-\pi / 4\right)$. Empleando pues la nueva matriz $D^{\prime}$, se ha obtenido la respuesta de la misma tira en su nueva orientación ante el mismo campo eléctrico incidente del caso anterior; es decir, ante la misma onda plana con dirección de avance $\beta=0$. La amplitud 


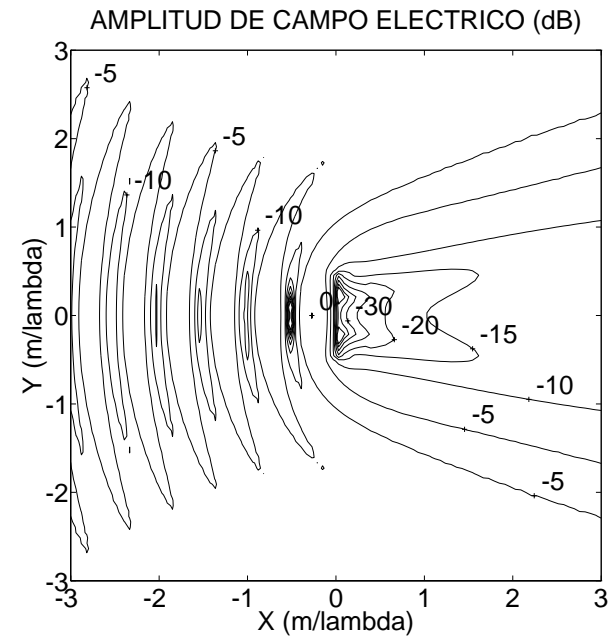

a)

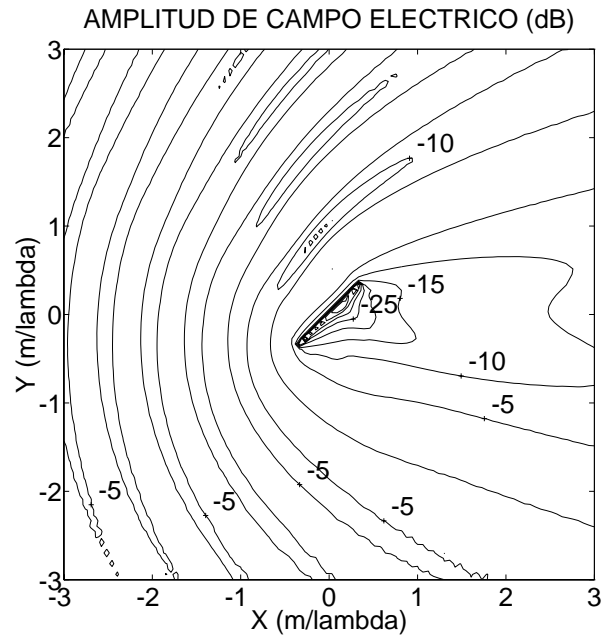

b)

Figura D.2: Amplitud del campo eléctrico total en un problema de dispersión electromagnética producida, ante la incidencia de una onda plana $(\beta=0)$, por una tira metálica infinita, de longitud $1 \lambda$; que se encuentra orientada según el eje $\hat{y}(\phi=\pi / 2)$ en a), y orientada según la dirección angular $\phi=\pi / 4$ en b).

del campo eléctrico total, calculado mediante la suma de los campos eléctricos incidente y dispersado, se representa en la figura D.2 (apartado b); donde se observa que la nueva zona de sombra y la nueva onda estacionaria se producen en las direcciones angulares previstas.

Los resultados ofrecidos en la figura D.2 (apartados a y b), deducidos respectivamente mediante el uso de las matrices de caracterización correspondientes a una misma tira con dos orientaciones diferentes, garantizan totalmente la validez del método presentado en este apéndice; que permite obtener la matriz de caracterización individual de un objeto girado respecto de una posición original, empleando las mencionadas matrices de giro, a partir de la matriz de caracterización individual asociada al mismo objeto en su orientación original. 


\section{Apéndice E}

\section{Traslación de Espectros Cilíndricos}

La caracterización de problemas bidimensionales de dispersión electromagnética, considerados en el presente trabajo, se ha realizado expresando los campos incidente y dispersado mediante sus correspondientes espectros de ondas cilíndricas. Dichos espectros correspondientes a los citados campos, tal y como se han definido en el apéndice C (apartado C.1.2), constituyen las amplitudes de sendos desarrollos modales; cuyos modos se refieren a un punto determinado. Al resolver ciertos problemas resulta necesario transformar algunos de estos desarrollos modales, referidos originalmente a ciertas posiciones espaciales, en nuevos desarrollos equivalentes de modos centrados en posiciones diferentes a las anteriores. Este tipo de transformaciones se conoce genéricamente como traslación de espectros cilíndricos, que en [14] constituye una de las llamadas transformaciones propias. Así, por ejemplo, la implementación del algoritmo recursivo descrito en el capítulo 6 requiere en determinados instantes expresar un desarrollo en serie de modos cilíndricos incidentes, referidos a un cierto punto, en un desarrollo equivalente del mismo tipo de funciones modales centradas en otro punto distinto; haciendo uso para ello de una matriz catalogada como matriz de traslación de espectro incidente a incidente. En otras situaciones, este mismo algoritmo recursivo precisa transformar una serie de modos cilíndricos dispersados por un objeto, referidos a un punto elegido normalmente en el centro de dicho objeto, en una serie equivalente de modos cilíndricos incidentes referidos al centro de otro objeto; definiendo en este caso una nueva matriz, denominada matriz de traslación de espectro emergente a incidente, que realiza esta última operación. Asimismo, la aplicación de dicho algoritmo recursivo a la resolución de problemas de dispersión múltiple con fuente interna al problema precisa, en ciertas ocasiones, trasladar a distintos puntos los desarrollos modales emergentes centrados inicialmente en otras posiciones; utilizando en dicha operación una tercera matriz conocida por matriz de traslación de espectro emergente a emergente. Por otra parte, una vez 
aplicado el algoritmo recursivo a la resolución de un problema de dispersión electromagnética producida por múltiples objetos, es necesario reconstruir el campo dispersado en diversas regiones del espacio; para lo cual, tal y como se recoge en el capítulo 6 (sección 6.5), deben utilizarse algunas de las anteriores matrices de traslación. Por último, conviene recordar que al construir la matriz de caracterización se utiliza una matriz de espectro (recordar capítulo 4); cuyo cálculo puede realizarse, como se describe en el capítulo 4 (apartado 4.3.2), mediante una técnica basada en el Teorema de Adición para las Funciones de Hankel. Esta técnica se basa en expresar a través de un único desarrollo modal, centrado en el origen de coordenadas, aquellos campos generados por fuentes que no están situadas en dicho origen; lo que supone trasladar a una nueva posición espacial, concretamente al origen de coordenadas, modos cilíndricos emergentes centrados en los puntos donde se sitúan las diferentes fuentes.

Las tres matrices de tralación de espectros cilíndricos mencionadas en el párrafo anterior, así como la matriz de espectro construida según la técnica comentada previamente, se deducen aplicando bien el Teorema de Adición para las Funciones de Bessel, o el Teorema de Adición para las Funciones de Hankel. En este apéndice, inicialmente se demuestran ambos Teoremas a partir del Teorema de Adición de Graf [71]; y usando dichos Teoremas se obtienen las expresiones de los elementos que constituyen las tres matrices de traslación de espectros cilíndricos, ampliamente utilizadas en la aplicación del algoritmo recursivo descrito en el capítulo 6. A continuación, empleando algunas de estas matrices de traslación, los espectros asociados a ciertos campos se transforman en nuevos espectros referidos a otras posiciones; reconstruyendo posteriormente los campos originales mediante estos espectros trasladados. De esta forma, se muestra la validez de las expresiones deducidas para construir las matrices de traslación de espectros cilíndricos; y asimismo se ponen de manifiesto las regiones donde es posible reconstruir correctamente los campos mediante los espectros trasladados. Finalmente, en relación con estas regiones, se observa una importante limitación asociada a la construcción de la matriz de traslación de espectro emergente a incidente; cuya no observación produce valores muy grandes en algunos elementos de dicha matriz, y consecuentemente que los sistemas de ecuaciones asociados al mencionado algoritmo recursivo no presenten solución al estar mal condicionados.

\section{E.1 Teorema de Adición para las Funciones de Bessel}

El campo incidente sobre un objeto dispersor bidimensional se expresa, tal y como se recoge en el apéndice C (apartado C.1.2), mediante un desarrollo en serie de modos cilíndricos incidentes referidos a un cierto punto. En determinadas situaciones, resulta necesario expresar cada uno de dichos modos cilíndricos incidentes 


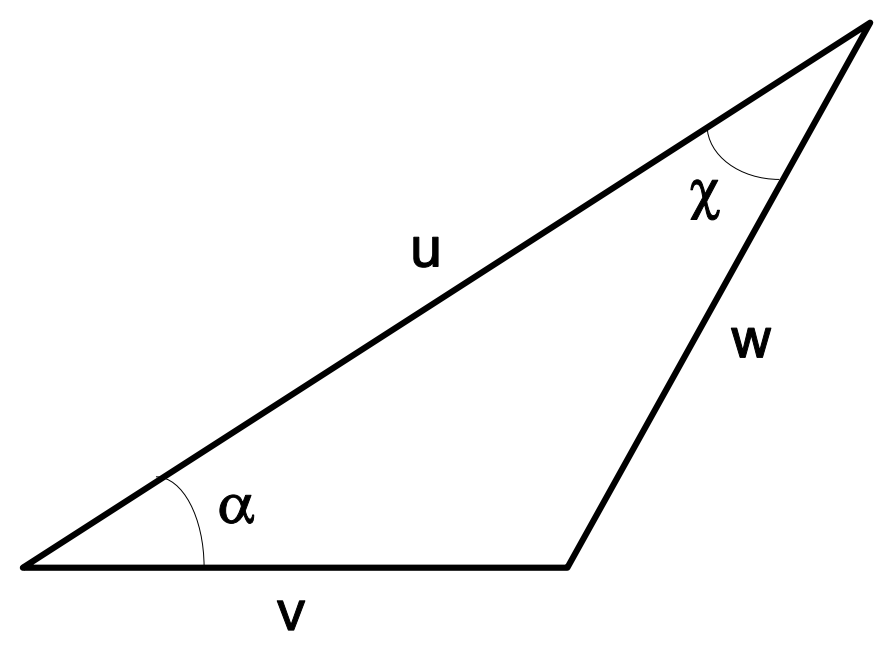

Figura E.1: Significado gráfico de las variables $u, v, w, \alpha$ y $\chi$; que aparecen en el Teorema de Adición de Graf.

mediante un sumatorio del mismo tipo de modos; aunque referidos a un nuevo punto. Esta operación se implementa haciendo uso del Teorema de Adición para las Funciones de Bessel; cuya demostración se ofrece seguidamente.

El Teorema de Adición para las Funciones de Bessel se demuestra a partir del Teorema de Adición de Graf [71]; que proporciona la siguiente relación general

$$
C_{\nu}(w) e^{j \nu \chi}=\sum_{r=-\infty}^{\infty} C_{\nu+r}(u) J_{r}(v) e^{j r \alpha} \quad\left|v e^{ \pm j \alpha}\right|<|u|
$$

La expresión del Teorema de Adición de Graf, recién ofrecida en (E.1), es válida cuando $C$ representa una función de Bessel de primera especie $J$, una función de Bessel de segunda especie $Y$, una función de Hankel de primera especie $H^{(1)}$, o una función de Hankel de segunda especie $H^{(2)}$; aunque la restricción impuesta $\left|v e^{ \pm j \alpha}\right|<|u|$ resulta innecesaria cuando $C$ denota una función de Bessel de primera especie $J$. Por lo que respecta a las variables $u, v, w, \alpha$ y $\chi$, presentes todas ellas en (E.1), se deben cumplir las siguientes relaciones

$$
\begin{aligned}
& w=\sqrt{u^{2}+v^{2}-2 u v \cos \alpha} \\
& u-v \cos \alpha=w \cos \chi \\
& v \sin \alpha=w \sin \chi
\end{aligned}
$$

a partir de las cuales es posible concluir que dichas variables están relacionadas como se indica gráficamente en la figura E.1.

Como el Teorema de Adición para las Funciones de Bessel pretende desarrollar un modo cilíndrico incidente genérico, referido a un cierto punto, en serie 
de modos cilíndricos incidentes centrados en un punto distinto; conviene particularizar el Teorema de Adición de Graf, recogido en (E.1), al caso en el que la función simbólica $C$ denota una función de Bessel de primera especie $J$. Bajo tal supuesto, a partir de la expresión planteada originalmente en (E.1), se deduce la siguiente nueva relación

$$
J_{\nu}(w) e^{j \nu \chi}=\sum_{r=-\infty}^{\infty} J_{\nu+r}(u) J_{r}(v) e^{j r \alpha}
$$

La deducción del Teorema de Adición para las Funciones de Bessel, utilizando la nueva relación ofrecida en (E.3), requiere una sustitución adecuada de las variables $u, v, w, \alpha$ y $\chi$ que aparecen en dicha expresión. Para ello, se considera una situación como la mostrada en la figura E.2; constituida por dos objetos dispersores $O_{1}$ y $O_{2}$ sobre los que incide un cierto campo incidente. Este campo se expresa en principio mediante un desarrollo en serie de modos cilíndricos incidentes referidos al punto $C_{1}$, elegido como origen local de un sistema de ejes $\left(X_{1}, Y_{1}\right)$ asociado al objeto $O_{1}$ (ver figura E.2). El Teorema de Adición para las Funciones de Bessel pretende expresar la $\nu$-ésima componente modal del anterior desarrollo mediante una suma de infinitos modos; aunque dichos modos deben referirse en esta ocasión al punto $C_{2}$, considerado como el origen local del sistema de ejes $\left(X_{2}, Y_{2}\right)$ asociado al segundo objeto $O_{2}$ (ver figura E.2). Para elegir correctamente los valores de las variables presentes en (E.3), es preciso identificar en el caso que nos ocupa (recordar figura E.2) un triángulo similar al mostrado en la figura E.1. Como tercer vértice de este nuevo triángulo, que debe acompañar a los otros dos definidos por los puntos $C_{1}$ y $C_{2}$, se elige un punto genérico $P$ (ver figura E.2); que se define por sus coordenadas cilíndricas $\left(\rho_{1}, \phi_{1}\right)$ respecto del sistema de ejes $\left(X_{1}, Y_{1}\right)$, y por sus coordenadas cilíndricas $\left(\rho_{2}, \phi_{2}\right)$ respecto del segundo sistema de ejes $\left(X_{2}, Y_{2}\right)$. Comparando pues ambos triángulos (recogidos en las figuras E.1 y E.2) de forma apropiada, se deducen las siguientes expresiones para las variables $u, v, w, \alpha$ y $\chi$

$$
\begin{aligned}
u & =d_{21} \\
v & =\rho_{2} \\
w & =\rho_{1} \\
\alpha & =\phi_{21}-\phi_{2} \\
\chi & =\pi+\phi_{1}-\phi_{21}
\end{aligned}
$$

donde los parámetros $d_{21}$ y $\phi_{21}$ constituyen las coordenadas cilíndricas del punto $C_{1}$ respecto del sistema de ejes $\left(X_{2}, Y_{2}\right)$, tal y como se indica de manera gráfica en la figura E.2. Utilizando las coordenadas cartesianas $\left(x_{c_{1}}, y_{c_{1}}\right)$ y $\left(x_{c_{2}}, y_{c_{2}}\right)$ de los 


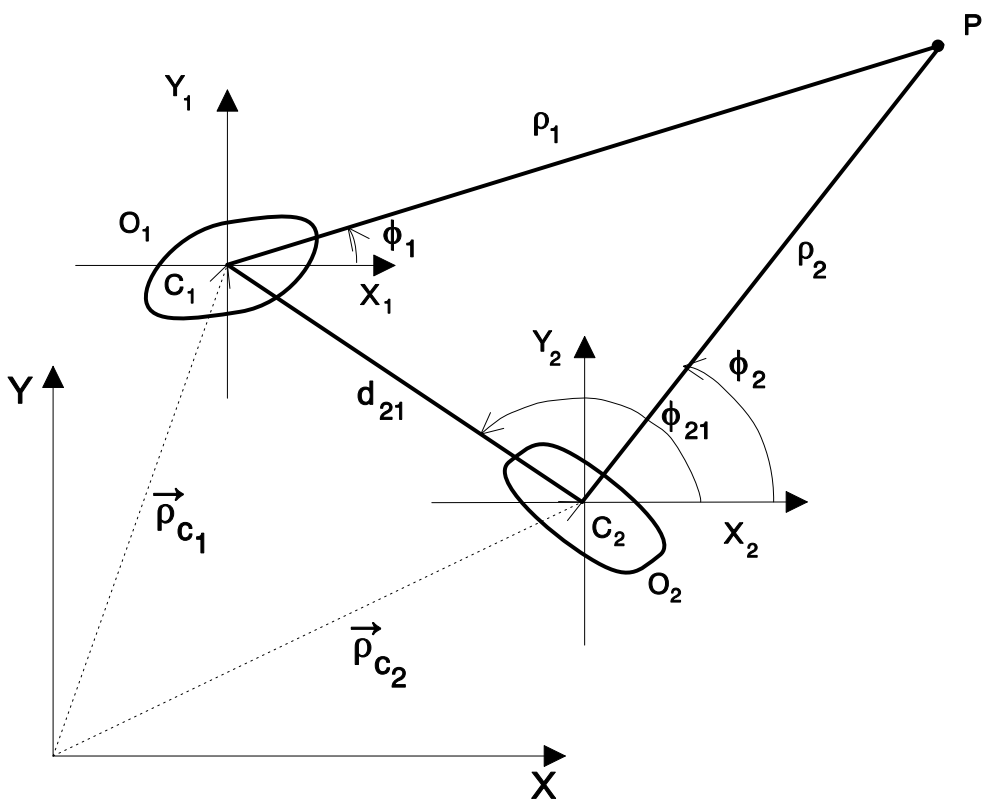

Figura E.2: Geometría de un sistema constituido por dos objetos dispersores $O_{1}$ y $O_{2}$; que permite determinar los valores concretos de las variables $u, v, w, \alpha$ y $\chi$ presentes en el Teorema de Adición de Graf.

puntos $C_{1}$ y $C_{2}$, referidas ambas al sistema global de ejes $(X, Y)$, es posible obtener los parámetros $d_{21}$ y $\phi_{21}$ mediante las expresiones que se ofrecen a continuación

$$
\begin{aligned}
& d_{21}=\sqrt{\left(x_{c_{1}}-x_{c_{2}}\right)^{2}+\left(y_{c_{1}}-y_{c_{2}}\right)^{2}} \\
& \phi_{21}=\arctan \left(\frac{y_{c_{1}}-y_{c_{2}}}{x_{c_{1}}-x_{c_{2}}}\right)
\end{aligned}
$$

Seguidamente, se impone sustituir en (E.3) las variables $u, v, w, \alpha$ y $\chi$ por sus respectivos valores recogidos en (E.4); transformando del siguiente modo la relación inicial definida según (E.3)

$$
J_{\nu}\left(\rho_{1}\right) e^{j \nu\left(\pi+\phi_{1}-\phi_{21}\right)}=\sum_{r=-\infty}^{\infty} J_{\nu+r}\left(d_{21}\right) J_{r}\left(\rho_{2}\right) e^{j r\left(\phi_{21}-\phi_{2}\right)}
$$

El argumento de las funciones de Bessel, cuando éstas forman parte de los modos cilíndricos incidentes, debe estar constituido por el producto de dos factores; uno de ellos el número de onda $k$, y el otro la distancia entre el punto donde se evalúa el modo y el origen local al que se refiere dicho modo. Como puede observarse en (E.6), el mencionado número de onda $k$ está ausente de todos los argumentos de las funciones de Bessel que aparecen en dicha expresión. La inclusión del factor $k$, en todos los argumentos anteriores, se consigue multiplicando 
simplemente los tres lados del triángulo mostrado en la figura E.2 por el citado número de onda; lo que no altera en absoluto los valores utilizados en (E.6) para las variables angulares $\alpha$ y $\chi$. Considerando pues el nuevo triángulo, cuyos lados han sido escalados por el factor $k$, y desarrollando asimismo las funciones exponenciales presentes en (E.6); se consigue que la relación entre las funciones de Bessel, definida inicialmente en (E.6), presente el siguiente aspecto

$$
(-1)^{\nu} J_{\nu}\left(k \rho_{1}\right) e^{j \nu \phi_{1}}=\sum_{r=-\infty}^{\infty} J_{\nu+r}\left(k d_{21}\right) e^{j(r+\nu) \phi_{21}} J_{r}\left(k \rho_{2}\right) e^{-j r \phi_{2}}
$$

Cuando las funciones exponenciales forman parte de los modos cilíndricos incidentes, el signo que acompaña al exponente de dichas funciones debe ser positivo. Por tanto, se impone realizar un cambio de signo en el índice del sumatorio presente en (E.7); para de esta forma poder expresar el $\nu$-ésimo modo cilíndrico incidente, referido al punto $C_{1}$, en una serie infinita de modos cilíndricos incidentes referidos al punto $C_{2}$. Así pues, recordando además la conocida relación genérica $J_{-n}(z)=(-1)^{n} J_{n}(z)$ expuesta en [71], es posible expresar el desarrollo modal recogido en (E.7) del siguiente modo

$$
(-1)^{\nu} J_{\nu}\left(k \rho_{1}\right) e^{j \nu \phi_{1}}=\sum_{r=-\infty}^{\infty} J_{\nu-r}\left(k d_{21}\right) e^{-j(r-\nu) \phi_{21}}(-1)^{r} J_{r}\left(k \rho_{2}\right) e^{j r \phi_{2}}
$$

Finalmente, con el objeto de simplificar la expresión presentada en (E.8), resulta conveniente utilizar una relación del tipo $J_{\nu-r}(z)=(-1)^{r-\nu} J_{r-\nu}(z)$; que se deduce a partir de una relación genérica, recogida en [71], entre funciones de Bessel con órdenes cambiados de signo. Por consiguiente, implementando la nueva relación en (E.8), se obtiene la siguiente expresión definitiva del Teorema de Adición para las Funciones de Bessel

$$
J_{\nu}\left(k \rho_{1}\right) e^{j \nu \phi_{1}}=\sum_{r=-\infty}^{\infty} J_{r-\nu}\left(k d_{21}\right) e^{-j(r-\nu) \phi_{21}} J_{r}\left(k \rho_{2}\right) e^{j r \phi_{2}}
$$

La validez de la expresión analítica del Teorema de Adición para las Funciones de Bessel, recién deducida en (E.9), puede corroborarse comparando dicha expresión con aquella presentada en [81] para este mismo Teorema; concluyendo que ambas expresiones son exactamente idénticas. Este Teorema, mediante la relación recogida en (E.9), garantiza el desarrollo de cualquier modo cilíndrico incidente, referido a un cierto punto, en términos de una serie de infinitos modos cilíndricos incidentes; que, tal y como se pretendía, están referidos a otro punto distinto del mencionado anteriormente. En determinadas situaciones, puede resultar interesante sin embargo transformar un desarrollo modal asociado a un cierto campo incidente, con los modos centrados en un punto determinado, en otro desarrollo modal asociado al mismo campo incidente; aunque en esta ocasión 
los modos de este último desarrollo deben estar centrados en otro punto distinto del anterior. En el siguiente apartado, haciendo uso del Teorema de Adición para las Funciones de Bessel demostrado previamente, se construye una matriz de traslación de espectro incidente a incidente; que permite implementar la mencionada operación de transformación de espectros cilíndricos incidentes.

\section{E.1.1 Matriz de Traslación de Espectro Incidente a Inci- dente}

En el análisis de la dispersión electromagnética producida por múltiples objetos bidimensionales, utilizando el algoritmo recursivo descrito en el capítulo 6 , se requiere expresar cada campo incidente considerado mediante su correspondiente espectro cilíndrico bidimensional; el cual debe referirse en cada caso a un punto determinado. En numerosas circunstancias, el espectro asociado a un campo incidente se encuentra referido a un punto distinto del requerido por el mencionado algoritmo recursivo. La solución a este problema consiste en trasladar al punto requerido el espectro asociado al anterior campo incidente; para lo cual se presenta en este apartado la matriz de traslación de espectro incidente a incidente. Asimismo, una vez construida dicha matriz, se detalla la región donde tiene validez la reconstrucción de un campo incidente a partir de su espectro trasladado. Finalmente, haciendo uso de la citada matriz de traslación, se ofrecen dos ejemplos prácticos en los que se trasladan espectros cilíndricos incidentes. Estos dos ejemplos confirman la correcta definición de la matriz de traslación de espectro incidente a incidente; y además muestran gráficamente la región donde es posible reconstruir un campo incidente, de manera correcta, utilizando su espectro cilíndrico trasladado.

Con la intención de obtener los elementos de la matriz de traslación de espectro incidente a incidente; se considerará la existencia de un cierto campo incidente ${ }^{1}$, denominado $C_{z}^{i}$. Dicho campo incidente, tal y como se recoge en el apéndice C (apartado C.1.2), se expresa mediante un desarrollo en serie de modos cilíndricos incidentes; los cuales se refieren inicialmente a un punto definido por sus coordenadas cartesianas globales $\left(x_{c_{1}}, y_{c_{1}}\right)$. En la figura E.3, se hace referencia a dicho punto; que representa el origen local del sistema de ejes $\left(X_{1}, Y_{1}\right)$. Evaluando este desarrollo modal en un punto genérico del espacio, representado por sus coordenadas cilíndricas $\left(\rho_{1}, \phi_{1}\right)$ respecto del sistema de ejes $\left(X_{1}, Y_{1}\right)$, se obtiene la siguiente expresión para el valor del campo $C_{z}^{i}$ en dicho punto

$$
C_{z}^{i}\left(\rho_{1}, \phi_{1}\right)=\sum_{p=-N_{i_{1}}}^{N_{i_{1}}} i_{p}^{\tau} J_{p}\left(k \rho_{1}\right) e^{j p \phi_{1}}
$$

\footnotetext{
${ }^{1}$ Este campo incidente $\left(C_{z}^{i}\right)$ representa campo eléctrico incidente $\left(E_{z}^{i}\right)$ para incidencia polarizada según $\mathrm{TM}^{\mathrm{z}}$, y campo magnético incidente $\left(H_{z}^{i}\right)$ para incidencia polarizada según $\mathrm{TE}^{\mathrm{z}}$.
} 


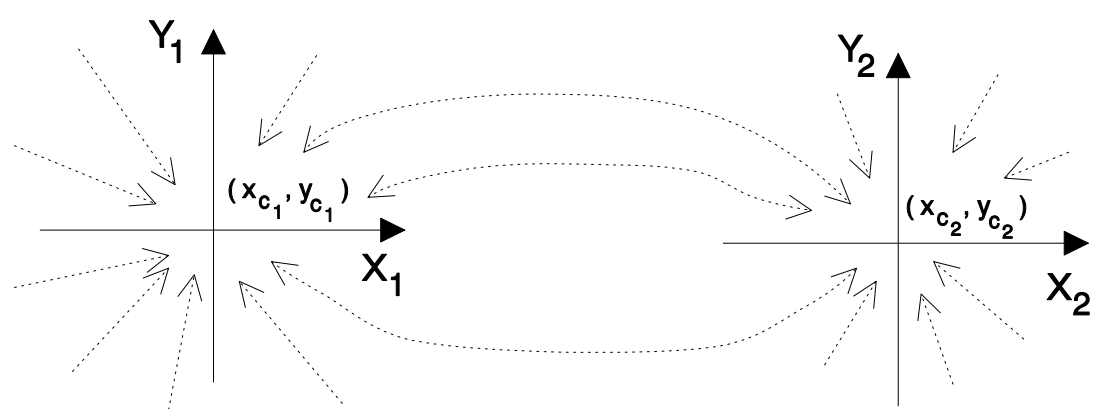

Figura E.3: Expresión de un campo incidente mediante dos espectros cilíndricos referidos a distintos puntos; que se definen a través de sus coordenadas cartesianas globales.

En el desarollo modal presentado en (E.10), los coeficientes $i_{p}^{\tau}(\tau=$ TM para polarización $\mathrm{TM}^{\mathrm{z}}$ y $\tau=\mathrm{TE}$ para polarización $\mathrm{TE}^{\mathrm{z}}$ ) constituyen el espectro cilíndrico bidimensional, referido al punto $\left(x_{c_{1}}, y_{c_{1}}\right)$, del campo incidente $C_{z}^{i}$. En cuanto a los límites del sumatorio presente en (E.10); éstos se escogen, recordando el capítulo 4 (sección 4.1), para reconstruir de manera correcta el campo incidente en el interior de una circunferencia, con centro en el punto $\left(x_{c_{1}}, y_{c_{1}}\right)$, y de radio el valor del cociente $N_{i_{1}} / k$.

Ahora bien, puede resultar necesario expresar el mismo campo incidente $C_{z}^{i}$ mediante un desarrollo similar al recogido en (E.10); pero constituido por unos modos que se refieran a otro punto, definido concretamente por sus coordenadas cartesianas globales $\left(x_{c_{2}}, y_{c_{2}}\right)$. Dicho punto representa, como puede observarse en la figura E.3, el origen local de un nuevo sistema de ejes $\left(X_{2}, Y_{2}\right)$. Al evaluar pues este nuevo desarrollo modal en el mismo punto donde se evaluó el desarrollo recogido en (E.10), utilizando lógicamente las coordenadas cilíndricas $\left(\rho_{2}, \phi_{2}\right)$ de dicho punto respecto del nuevo sistema de ejes $\left(X_{2}, Y_{2}\right)$, se deduce el siguiente valor para el campo $C_{z}^{i}$

$$
C_{z}^{i}\left(\rho_{2}, \phi_{2}\right)=\sum_{m=-N_{i_{2}}}^{N_{i_{2}}} i_{m}^{\prime \tau} J_{m}\left(k \rho_{2}\right) e^{j m \phi_{2}}
$$

donde, en este caso, los coeficientes $i_{m}^{\prime \tau}\left(\tau=\right.$ TM para polarización $\mathrm{TM}^{\mathrm{z}}$ y $\tau=\mathrm{TE}$ para polarización $\mathrm{TE}^{\mathrm{Z}}$ ) constituyen el espectro cilíndrico bidimensional, referido ahora al punto $\left(x_{c_{2}}, y_{c_{2}}\right)$, del mismo campo incidente $C_{z}^{i}$. Por lo que respecta a los límites del sumatorio que aparece en (E.10), éstos se escogen con la intención de reconstruir correctamente el campo incidente en el interior de una circunferencia; que en esta ocasión tiene su centro en el punto $\left(x_{c_{2}}, y_{c_{2}}\right)$, y presenta un valor para el radio igual al cociente $N_{i_{2}} / k$.

La obtención de los coeficientes $i_{m}^{\prime \tau}$ del desarrollo modal recogido en (E.11), a partir de los coeficientes $i_{p}^{\tau}$, requiere expresar en (E.10) cada uno de los modos cilíndricos incidentes, centrados en el punto $\left(x_{c_{1}}, y_{c_{1}}\right)$, mediante una serie de 
modos cilíndricos incidentes centrados en el punto $\left(x_{c_{2}}, y_{c_{2}}\right)$. Dicha relación, proporcionada por el Teorema de Adición para las Funciones de Bessel recogido en (E.9), presenta en el caso que nos ocupa el siguiente aspecto

$$
J_{p}\left(k \rho_{1}\right) e^{j p \phi_{1}}=\sum_{m=-\infty}^{\infty} J_{m-p}\left(k d_{21}\right) e^{-j(m-p) \phi_{21}} J_{m}\left(k \rho_{2}\right) e^{j m \phi_{2}}
$$

Sustituyendo en (E.10) la relación recién deducida en (E.12), el campo incidente $C_{z}^{i}$ se evalúa en el punto de coordenadas cilíndricas $\left(\rho_{2}, \phi_{2}\right)$, definidas respecto del sistema de ejes $\left(X_{2}, Y_{2}\right)$, mediante el siguiente desarrollo modal

$$
\begin{gathered}
C_{z}^{i}\left(\rho_{2}, \phi_{2}\right)=\sum_{p=-N_{i_{1}}}^{N_{i_{1}}} i_{p}^{\tau} \sum_{m=-\infty}^{\infty} J_{m-p}\left(k d_{21}\right) e^{-j(m-p) \phi_{21}} J_{m}\left(k \rho_{2}\right) e^{j m \phi_{2}} \\
=\sum_{m=-\infty}^{\infty}\left(\sum_{p=-N_{i_{1}}}^{N_{i_{1}}} i_{p}^{\tau} J_{m-p}\left(k d_{21}\right) e^{-j(m-p) \phi_{21}}\right) J_{m}\left(k \rho_{2}\right) e^{j m \phi_{2}}
\end{gathered}
$$

Truncando el sumatorio presente en (E.13) para el índice $m$ en un número finito de modos, como en el desarrollo modal del campo incidente recogido en (E.11), es posible identificar la siguiente relación entre los coeficientes $i_{m}^{\prime \tau}$ e $i_{p}^{\tau}$

$$
i_{m}^{\prime \tau}=\sum_{p=-N_{i_{1}}}^{N_{i_{1}}} i_{p}^{\tau} J_{m-p}\left(k d_{21}\right) e^{-j(m-p) \phi_{21}} \quad \forall m, m \in\left[-N_{i_{2}}, N_{i_{2}}\right]
$$

Si se consideran dos vectores columna, constituidos respectivamente por los coeficientes $i_{m}^{\prime \tau}$ e $i_{p}^{\tau}$, la relación expresada en (E.14) entre dichos coeficientes puede escribirse en términos matriciales del siguiente modo

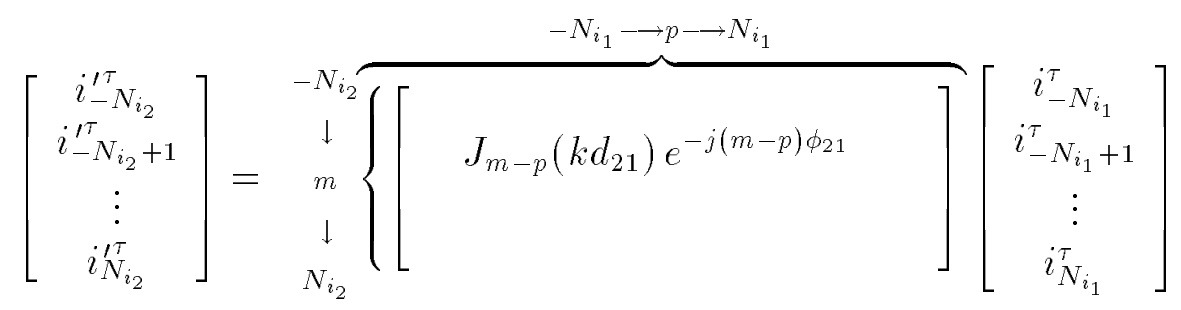

donde la matriz implicada representa la matriz de traslación de espectro incidente a incidente, designada como $T J_{21}$. Esta designación obedece al hecho de que dicha matriz permite trasladar un espectro cilíndrico incidente, referido inicialmente al punto $\left(x_{c_{1}}, y_{c_{1}}\right)$, en un nuevo espectro cilíndrico incidente asociado al mismo campo; aunque el nuevo espectro se refiere en esta ocasión al punto $\left(x_{c_{2}}, y_{c_{2}}\right)$.

La región donde es posible reconstruir de forma correcta un campo incidente, a partir de una versión trasladada del espectro asociado originalmente a dicho 


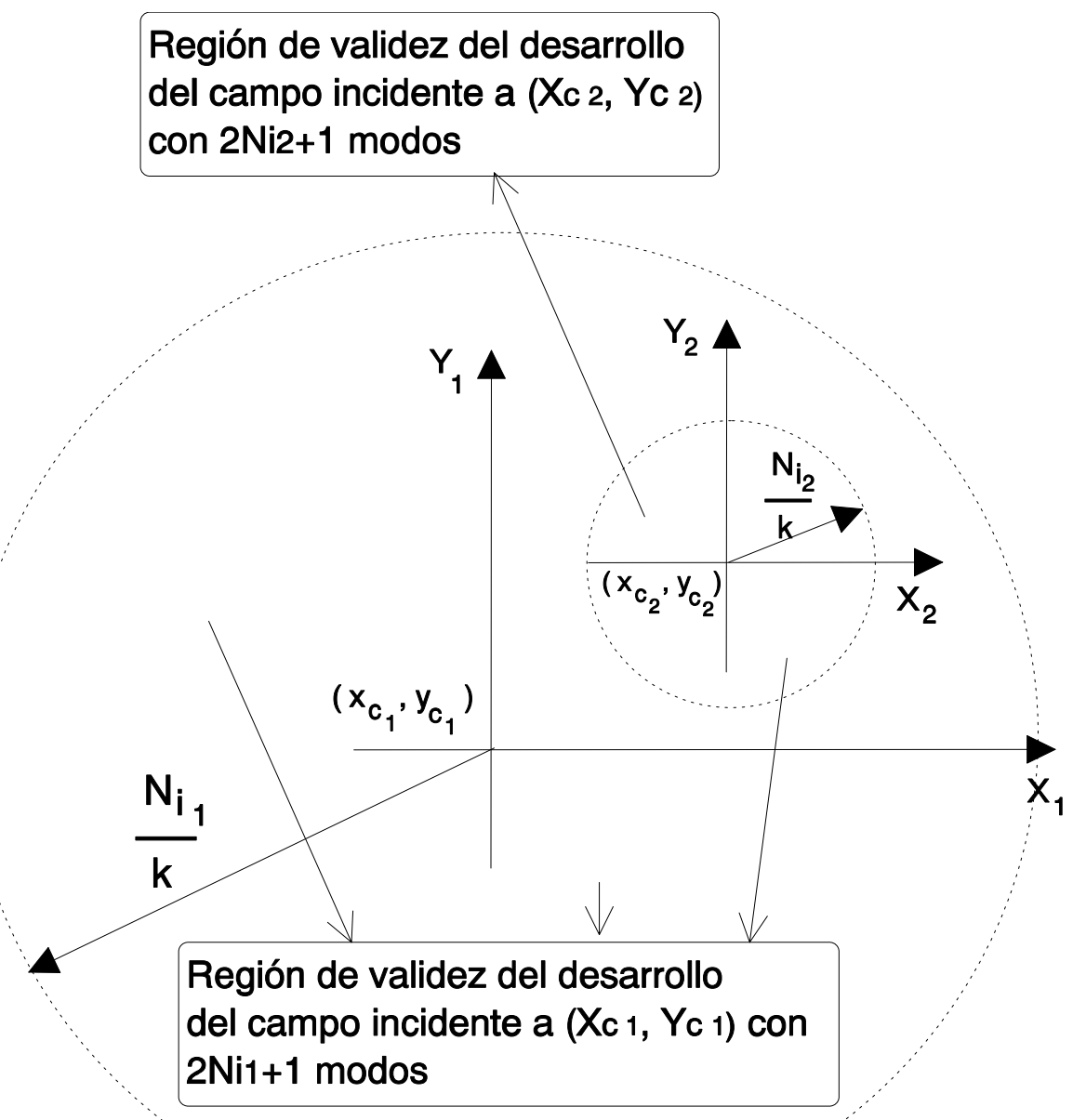

Figura E.4: Región de validez para la reconstrucción de un cierto campo incidente a partir de una versión trasladada del espectro asociado originalmente a dicho campo.

campo, depende no sólo de la zona de reconstrucción correcta definida por el nuevo espectro trasladado; sino también de la zona en la que utilizando el espectro original se reconstruye correctamente el campo incidente. Así por ejemplo, el campo incidente considerado en la situación anterior (recordar figura E.3) se reconstruye en principio de manera correcta, tal y como se indica en la figura E.4, dentro de una circunferencia centrada en $\left(x_{c_{1}}, y_{c_{1}}\right)$ y de radio el cociente $N_{i_{1}} / k$; pues el espectro original asociado al campo incidente, que se utiliza en (E.10) para desarrollar dicho campo, se refiere al punto $\left(x_{c_{1}}, y_{c_{1}}\right)$ y consta de $2 N_{i_{1}}+1$ componentes. Si este espectro original se traslada a un nuevo punto, por ejemplo al representado por las coordenadas $\left(x_{c_{2}}, y_{c_{2}}\right)$, el nuevo espectro trasladado permite a lo sumo una reconstrucción correcta del campo incidente dentro de la anterior 
circunferencia; para lo cual sería necesario considerar, como se indica en (E.13), infinitas componentes espectrales en el nuevo espectro trasladado. Por contra, si el espectro original se traslada implementando el producto matricial expuesto en (E.15); el espectro trasladado queda referido de nuevo al punto $\left(x_{c_{2}}, y_{c_{2}}\right)$, pero en este caso sólo consta de $2 \mathrm{~N}_{i_{2}}+1$ componentes. Consecuentemente, empleando dicho espectro trasladado es posible reconstruir de forma correcta el campo incidente, como se muestra nuevamente en la figura E.4, dentro de una circunferencia con centro en el punto $\left(x_{c_{2}}, y_{c_{2}}\right)$ y radio el valor del cociente $N_{i_{2}} / k$. En el supuesto caso de que una porción de esta última circunferencia quedase fuera de aquélla asociada al espectro original, el campo incidente podría obtenerse igualmente a partir del anterior espectro trasladado; aunque el valor de este campo carece de interés, pues la zona mencionada no pertenece a la región donde el espectro original permite reconstruir de manera correcta el campo incidente.

A continuación, se ofrecen los resultados correspondientes a dos ejemplos de traslación de espectros cilíndricos incidentes; con los que se pretende corroborar, de forma práctica, el concepto de la matriz de traslación de espectro incidente a incidente. Asimismo, ambos ejemplos sirven para poner de manifiesto dónde es posible reconstruir un campo incidente, de manera correcta, a partir de una versión trasladada del espectro asociado originalmente a dicho campo. En los dos ejemplos, se considera como campo eléctrico incidente (polarización $\mathrm{TM}^{\mathrm{z}}$ ) una onda plana con dirección de avance $\beta=0$ (recordar figura C.1); es decir, una onda de amplitud constante cuyo frente de fase avanza en la dirección del eje $\hat{x}$ y en el sentido creciente de la coordenada $x$. El espectro cilíndrico asociado originalmente a dicha onda plana, cuyo cálculo se deduce en el apéndice C (apartado C.2.1), queda referido en ambos casos al origen de coordenadas $(0,0)$; habiendo escogido $2 N_{i_{1}}+1$ componentes espectrales con $N_{i_{1}}=k 5 \lambda$. La reconstrucción de la amplitud y la fase de la mencionada onda plana, utilizando su espectro cilíndrico original en el desarrollo modal recogido en (E.10), se representa en la figura E.5; donde se observa, como era de esperar, que el campo eléctrico incidente se reconstruye como una onda plana $(\beta=0)$ dentro de una circunferencia con centro en el punto $(0,0)$ y de radio $5 \lambda$. En dicha región, cuyo contorno se representa en la figura E.5 mediante un círculo punteado, se tiene un valor constante para la amplitud del campo eléctrico incidente, mientras que el frente de fase avanza en la dirección del eje $\hat{x}$ y en el sentido creciente de la coordenada $x$; tal y como corresponde a la amplitud y a la fase de la onda plana considerada $(\beta=0)$.

En el primer ejemplo, utilizando la matriz de traslación de espectro incidente a incidente definida según (E.15), se transforma el espectro cilíndrico original asociado a la onda plana incidente $(\beta=0)$, referido al punto $(0,0)$ con $N_{i_{1}}=k 5 \lambda$, en un nuevo espectro cilíndrico asociado a la misma onda plana; aunque referido en este caso al punto de coordenadas cartesianas normalizadas $(2,0)$, y constituido por $2 N_{i_{2}}+1$ componentes espectrales con $N_{i_{2}}=k 2 \lambda$. Esta traslación de espectros cilíndricos se realiza con la intención de poder reconstruir la misma onda plana 

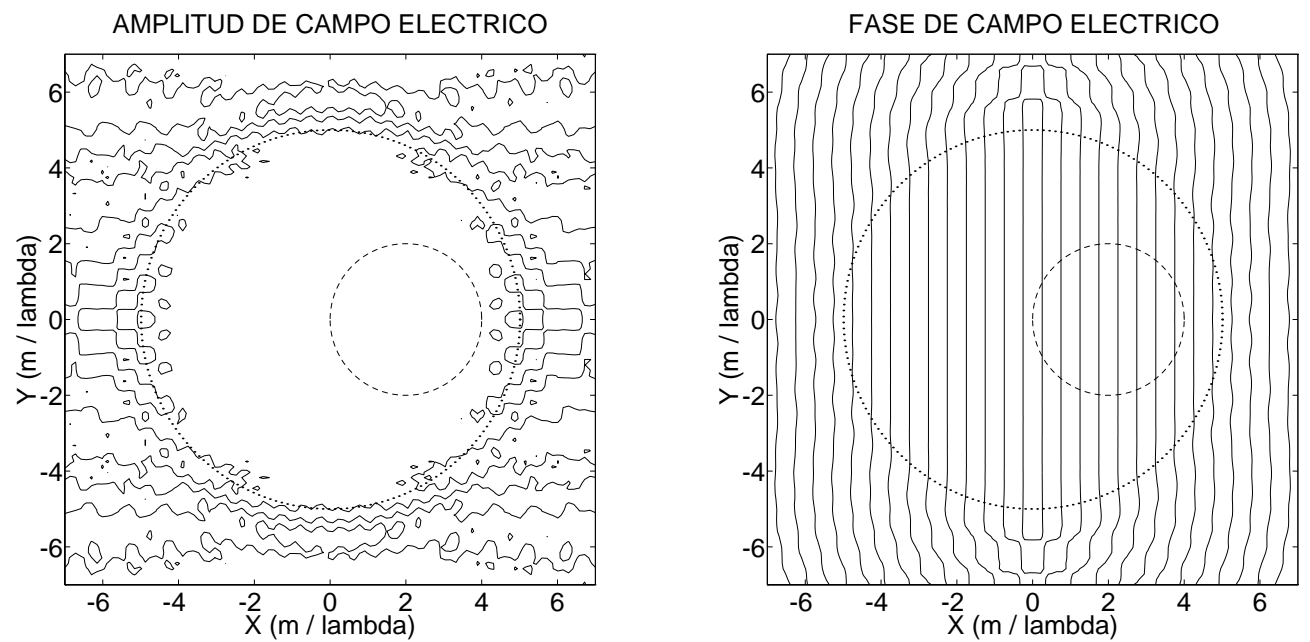

Figura E.5: Amplitud y fase de una onda plana incidente $(\beta=0)$. Reconstrucción a partir de su espectro cilíndrico centrado en $(0,0)$ con $N_{i_{1}}=k 5 \lambda$.
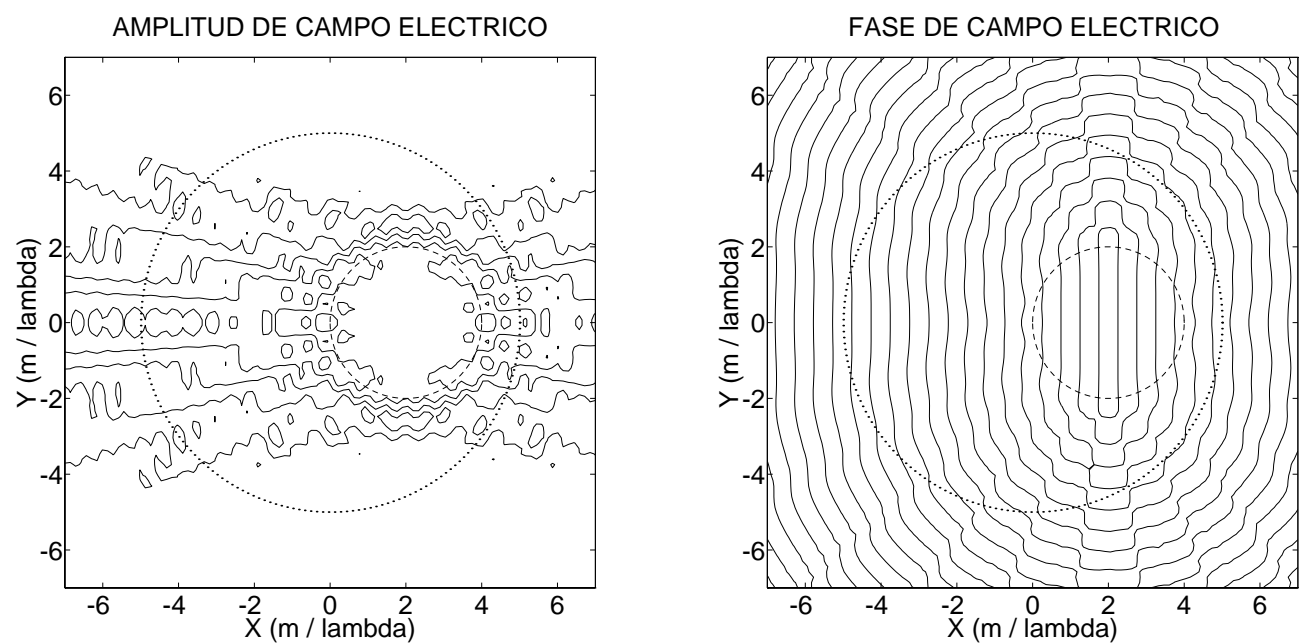

Figura E.6: Amplitud y fase de una onda plana incidente $(\beta=0)$. Reconstrucción a partir de su espectro cilíndrico centrado en $(2,0)$ con $N_{i_{2}}=k 2 \lambda$; obtenido mediante una traslación del espectro cilíndrico original centrado en $(0,0)$ con $N_{i_{1}}=k 5 \lambda$.

incidente $(\beta=0)$, a partir del espectro trasladado, en la región contenida dentro de la circunferencia con centro en el punto $(2,0)$ y de radio $2 \lambda$. Empleando pues el espectro cilíndrico trasladado, se reconstruye el campo eléctrico incidente mediante el desarrollo modal recogido en (E.11). La amplitud y la fase correspondientes a este campo eléctrico incidente se muestran en la figura E.6; donde se observa, como era de prever, que la onda plana incidente se reconstruye perfectamente 

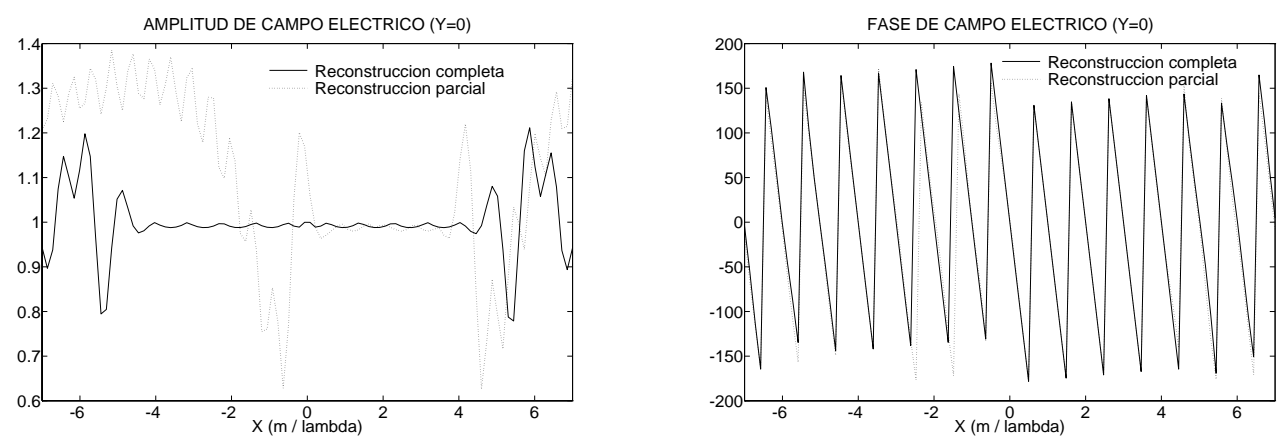

Figura E.7: Amplitud y fase de una onda plana incidente, con dirección de avance $\beta=0$, a lo largo del eje $\hat{x}(y=0)$. Reconstrucción completa a partir del espectro cilíndrico original asociado a dicha incidencia, y reconstrucción parcial a partir de la versión de dicho espectro trasladada al punto $(2,0)$ con $N_{i_{2}}=k 2 \lambda$.

dentro de la circunferencia centrada en el punto $(2,0)$ y de radio $2 \lambda$. El contorno que define esta nueva región se representa en las figuras E.5 y E.6 mediante sendos círculos de trazo discontinuo. La situación de este círculo en relación al de trazo punteado, que determina la región donde es posible reconstruir correctamente la onda plana incidente mediante su espectro cilíndrico original, revela que este primer ejemplo coincide con la situación mostrada en la figura E.4. Con el objeto de observar con más detalle el grado de reconstrucción conseguido en la onda plana incidente utilizando el espectro cilíndrico trasladado, en la figura E.7 se comparan cortes realizados a lo largo del eje $\hat{x}$ en las diversas gráficas mostradas en las figuras E.5 y E.6. En dicha figura comparativa, se representa con línea continua la reconstrucción (denominada completa en fig. E.7) de la onda plana incidente a lo largo del eje $\hat{x}(y=0)$, obtenida a partir del espectro cilíndrico original de la onda plana centrado en el punto $(0,0)$ con $N_{i_{1}}=k 5 \lambda$; mientras que con línea punteada se representa la reconstrucción (denominada parcial en fig. E.7) de la misma onda plana incidente a lo largo del eje $\hat{x}(y=0)$, utilizando sin embargo en esta segunda reconstrucción la versión del anterior espectro cilíndrico original trasladada al punto $(2,0)$ con $N_{i_{2}}=k 2 \lambda$. Lógicamente, ambas reconstrucciones son muy similares en aquellos puntos que satisfacen la condición $x \in[0,4]$; pues pertenecen a la región en la que se garantiza la reconstrucción correcta de la onda plana a partir del espectro cilíndrico trasladado. Si se pretendiera reconstruir de forma correcta la onda plana incidente, haciendo uso de una versión del espectro cilíndrico original trasladada al punto $(2,0)$, en una región mayor a la definida por la circunferencia centrada en el punto $(2,0)$ y de radio $2 \lambda$; debería aumentarse el número de componentes asociadas al espectro cilíndrico trasladado que se ha utilizado para obtener los resultados mostrados en la figura E.6.

En el segundo ejemplo, usando nuevamente una matriz de traslación de espectro incidente a incidente como la definida en (E.15), se transforma el espectro 

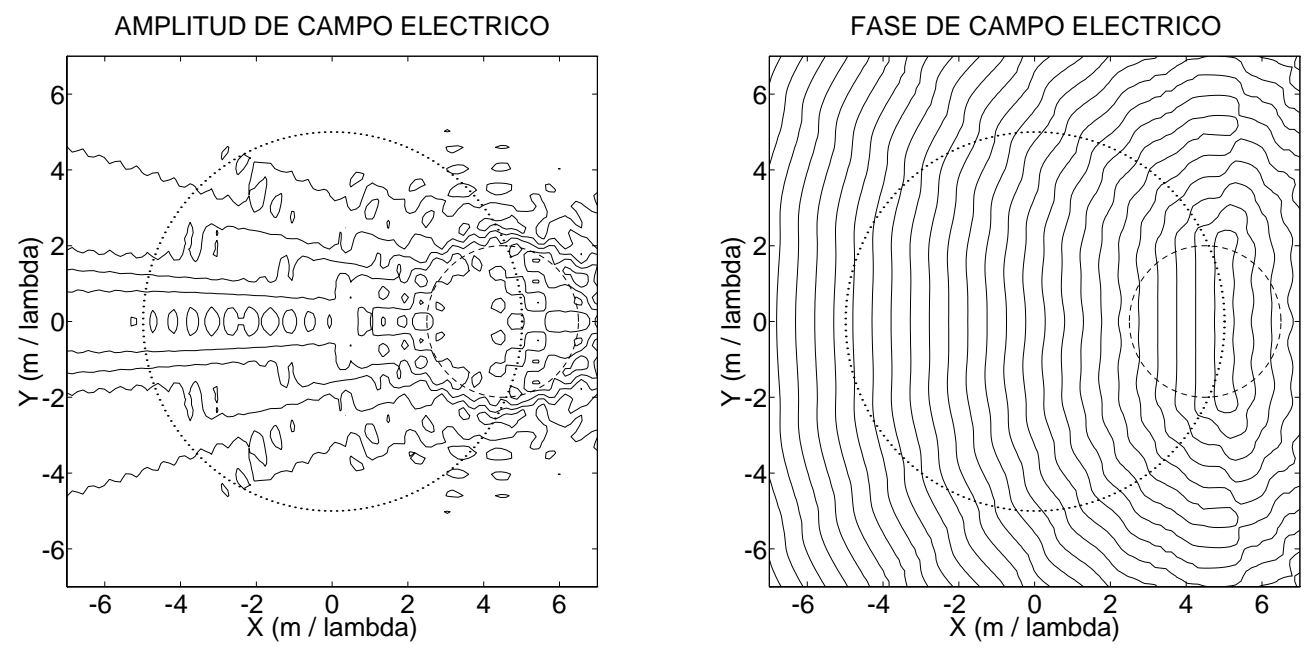

Figura E.8: Amplitud y fase de una onda plana incidente $(\beta=0)$. Reconstrucción a partir de su espectro cilíndrico centrado en $(4.5,0)$ con $N_{i_{2}}=k 2 \lambda$; obtenido mediante una traslación del espectro cilíndrico original centrado en $(0,0)$ con $N_{i_{1}}=k 5 \lambda$.

cilíndrico asociado originalmente a la onda plana incidente $(\beta=0)$, referido al punto $(0,0)$ con $N_{i_{1}}=k 5 \lambda$, en un nuevo espectro cilíndrico centrado ahora en el punto de coordenadas cartesianas normalizadas $(4.5,0)$; pero considerando de nuevo en dicho espectro $2 N_{i_{2}}+1$ componentes con $N_{i_{2}}=k 2 \lambda$. La amplitud y la fase del campo eléctrico incidente, calculadas empleando los coeficientes del nuevo espectro cilíndrico trasladado en el desarrollo modal recogido en (E.11), se muestran en la figura E.8; de la que puede concluirse, tras comparar con la figura E.5 donde se representa la reconstrucción del campo incidente a partir del espectro original, que el campo incidente se reconstruye totalmente a partir del nuevo espectro trasladado dentro de la circunferencia centrada en el punto $(4.5,0)$ y de radio $2 \lambda$. No obstante, dentro de esta región delimitada en la figura E. 8 por un círculo de trazo discontinuo, la onda plana incidente $(\beta=0)$ tan sólo se reconstruye en aquella parte de la citada región contenida además dentro de otro círculo, dibujado en este caso en la figura E.8 con trazo punteado, que acota la zona donde es posible reconstruir la onda plana incidente mediante el espectro considerado originalmente. Con el objeto de confirmar esta interpretación de los resultados ofrecidos en la figura E.8, se han realizado diversos cortes a lo largo del eje $\hat{x}$ de la amplitud y la fase recogidas en las figuras E.5 y E.8; que se exponen conjuntamente en la figura E.9. En dicha figura, con línea continua se representa la reconstrucción (denominada completa en fig. E.9) del campo eléctrico incidente a lo largo del eje $\hat{x}(y=0)$, deducida a partir del espectro cilíndrico asociado originalmente a dicho campo; mientras con línea punteada se ofrece la reconstrucción (denominada parcial en fig. E.9) del mismo campo eléctrico incidente a lo 

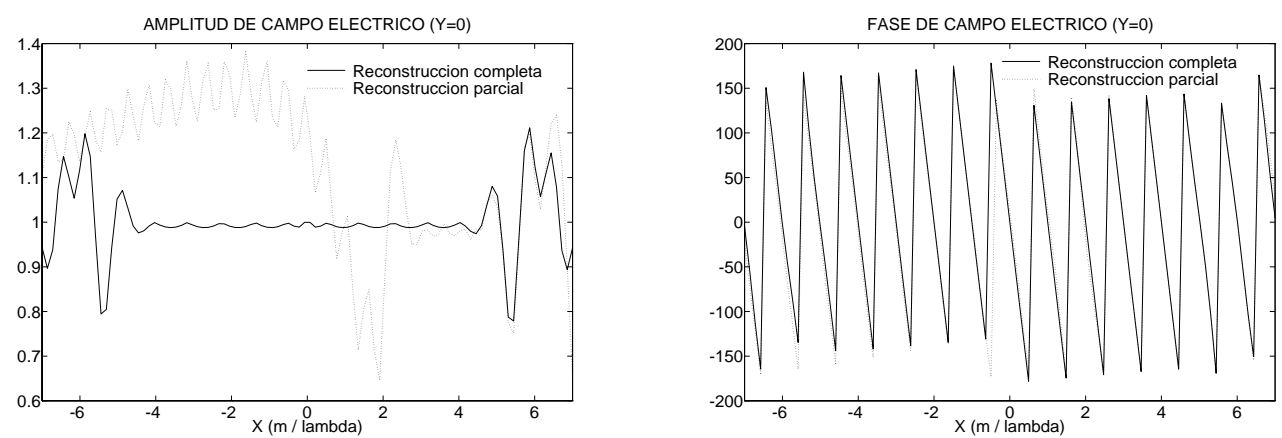

Figura E.9: Amplitud y fase de una onda plana incidente, con dirección de avance $\beta=0$, a lo largo del eje $\hat{x}(y=0)$. Reconstrucción completa a partir del espectro cilíndrico original asociado a dicha incidencia, y reconstrucción parcial a partir de la versión de dicho espectro trasladada al punto $(4.5,0)$ con $N_{i_{2}}=k 2 \lambda$.

largo del eje $\hat{x}(y=0)$, pero empleando en dicha reconstrucción la versión del espectro cilíndrico original trasladada al punto $(4.5,0)$ con $N_{i_{2}}=k 2 \lambda$. Este estudio comparativo, recogido en la figura E.9, revela que el espectro cilíndrico trasladado permite reconstruir el campo incidente en todo el intervalo $x \in[2.5,6.5]$; que pertenece a la región contenida en el círculo dibujado con trazo discontinuo en la figura E.8, definido por el número de componentes del espectro cilíndrico trasladado. Ahora bien, como se desprende de la figura E.9, utilizando el espectro trasladado sólo resulta posible reconstruir correctamente la onda plana incidente en el intervalo $x \in[2.5,5]$; ya que dicho intervalo pertenece además al círculo de trazo punteado (ver figura E.8) en cuyo interior, a partir del espectro cilíndrico original, se garantiza la reconstrucción correcta de la citada onda plana.

Los resultados correspondientes a los dos ejemplos anteriores permiten extraer diversas conclusiones sobre la reconstrucción de un cierto campo incidente, definido inicialmente mediante un espectro cilíndrico asociado, empleando versiones de dicho espectro trasladadas a distintas posiciones. En principio, el campo incidente se reconstruye correctamente mediante su espectro original en el interior de una determinada zona; que está delimitada por el número de componentes espectrales asociadas a dicho espectro. No obstante, el espectro cilíndrico original también determina fuera de dicha zona ciertos valores para el campo incidente, obtenidos mediante el correspondiente desarrollo modal, que sin embargo carecen de significado e interés en el problema donde se considera el mencionado campo incidente. Pues bien, cuando se traslada el espectro original a una nueva posición; este nuevo espectro también define una nueva zona de reconstrucción del campo incidente, que está asociada en este caso al número de componentes del nuevo espectro trasladado. En el interior de toda esta nueva zona se obtendrá, a partir del nuevo espectro trasladado, unos valores de campo incidente idénticos o muy parecidos a los conseguidos mediante el espectro cilíndrico original; debido 
a que la aplicación del Teorema de Adición para las Funciones de Bessel, definido anteriormente en esta sección, no presenta ninguna restricción espacial. Sin embargo, el campo incidente sólo podrá reconstruirse correctamente, empleando el espectro cilíndrico trasladado, en aquella parte de la zona asociada a dicho espectro trasladado que pertenezca además a la zona definida inicialmente por el espectro cilíndrico original. Esta limitación está causada lógicamente por el hecho de que el espectro trasladado se obtiene, utilizando la matriz de traslación de espectro incidente a incidente descrita en este apartado, a partir del espectro asociado originalmente al campo incidente.

\section{E.2 Teorema de Adición para las Funciones de Hankel}

El campo dispersado por un objeto bidimensional ante una cierta incidencia, así como el campo generado por una fuente puntual, se expresan según se recoge en el apéndice C (apartado C.1.2) mediante un desarrollo en serie de modos cilíndricos emergentes referidos a un cierto punto. En algunas ocasiones, se requiere transformar cada uno de estos modos emergentes en un sumatorio de modos cilíndricos incidentes referidos a otro punto; mientras en otras situaciones, conviene expresar cada uno de los modos emergentes mediante un sumatorio del mismo tipo de modos, pero referidos a otra posición. Cada una de estas operaciones se implementa utilizando la correspondiente versión del Teorema de Adición para las Funciones de Hankel; cuya deducción completa se ofrece a continuación.

La primera versión del Teorema de Adición para las Funciones de Hankel permite desarrollar un modo cilíndrico emergente, referido a un cierto punto, en serie de modos cilíndricos incidentes centrados en otro punto distinto del anterior. Dicha versión se deduce de nuevo a partir del Teorema de Adición de Graf [71], que se encuentra perfectamente descrito en la sección E.1 del presente apéndice. Al pretender desarrollar en este caso un modo cilíndrico emergente, en la expresión general del citado Teorema de Adición de Graf, recogida en (E.1), debe sustituirse la función simbólica $C$ por la correspondiente función de Hankel de segunda especie $H^{(2)}$; obteniendo pues la siguiente nueva relación

$$
H_{\nu}^{(2)}(w) e^{j \nu \chi}=\sum_{r=-\infty}^{\infty} H_{\nu+r}^{(2)}(u) J_{r}(v) e^{j r \alpha} \quad\left|v e^{ \pm j \alpha}\right|<|u|
$$

donde el significado de las variables $u, v, w, \alpha$ y $\chi$ es idéntico al deducido en la sección E.1 para las mismas variables; el cual se expresa de forma analítica mediante las relaciones definidas en (E.2), y se recoge de forma gráfica en la figura E.1. No obstante, resulta conveniente reseñar que el desarrollo recogido en (E.16) para la función de Hankel de segunda especie es válido tan sólo cuando se 
satisface la condición $\left|v e^{ \pm j \alpha}\right|<|u|$; a diferencia del desarrollo presentado en (E.3) para las funciones de Bessel, que no presenta restricción alguna.

La deducción de la primera versión del Teorema de Adición para las Funciones de Hankel requiere, al igual que en la demostración del Teorema de Adición para las Funciones de Bessel, identificar de forma adecuada las variables $u, v, w, \alpha \mathrm{y}$ $\chi$ presentes en (E.16). Para ello, puede considerase de nuevo la situación mostrada en la figura E.2; aunque la interpretación de dicha figura es diferente a la ofrecida en la sección E.1. En el presente caso, se supone que el objeto $O_{1}$ dispersa un campo; el cual, tras propagarse, se convierte en un campo incidente sobre el objeto $O_{2}$. El campo dispersado por $O_{1}$ se expresa en un principio, como bien es sabido, mediante un desarrollo de modos cilíndricos emergentes centrados en el punto $C_{1}$. Por lo que respecta a este campo expresado en serie de modos cilíndricos emergentes, referidos al punto $C_{1}$, puede considerarse también que está generado por una o varias fuentes puntuales; en lugar de suponer, como se acaba de mencionar, que representa un campo dispersado por un objeto ante una cierta incidencia. La primera versión del Teorema de Adición para las Funciones de Hankel pretende expresar el $\nu$-ésimo modo cilíndrico emergente del anterior desarrollo, centrado en el punto $C_{1}$, en términos de una serie constituida por infinitos modos cilíndricos incidentes; que lógicamente deben referirse a algún punto del objeto $O_{2}$, representado en la figura E.2 por el punto $C_{2}$. Por su parte, el punto $C_{2}$ también puede representar tan sólo una posición espacial a la que se desean referir los modos cilíndricos incidentes; no siendo necesario que dicho punto pertenezca, tal y como se termina de comentar, a algún objeto dispersor. El significado de los puntos $C_{1}$ y $C_{2}$, que en la figura E.2 constituyen respectivamente los orígenes locales de los sistemas de ejes $\left(X_{1}, Y_{1}\right)$ y $\left(X_{2}, Y_{2}\right)$, es idéntico al asignado en la sección E.1 para estos mismos puntos. En cuanto a la elección de las variables implicadas en la expresión recogida en (E.16), recordando nuevamente la sección E.1, se requiere identificar en la figura E.2 un triángulo con el que poder comparar aquél mostrado en la figura E.1. Como tercer vértice del triángulo a construir en la figura E.2, se escoge otra vez el punto $P$; cuyas coordenadas cilíndricas respecto de los sistemas de ejes $\left(X_{1}, Y_{1}\right)$ y $\left(X_{2}, Y_{2}\right)$ se designan de igual manera que en la sección E.1. De esta forma, las variables en (E.16) deben sustituirse por las mismas expresiones, recogidas en (E.4), que se deducen en la sección E.1 para $u, v, w, \alpha$ y $\chi$; transformando pues del siguiente modo la relación definida inicialmente según (E.16)

$$
H_{\nu}^{(2)}\left(\rho_{1}\right) e^{j \nu\left(\pi+\phi_{1}-\phi_{21}\right)}=\sum_{r=-\infty}^{\infty} H_{\nu+r}^{(2)}\left(d_{21}\right) J_{r}\left(\rho_{2}\right) e^{j r\left(\phi_{21}-\phi_{2}\right)} \quad \rho_{2}<d_{21}
$$

donde los parámetros $d_{21}$ y $\phi_{21}$, representados gráficamente en la figura E.2, se calculan utilizando las expresiones deducidas en (E.5); mientras $\left(\rho_{1}, \phi_{1}\right)$ y $\left(\rho_{2}, \phi_{2}\right)$ definen, como se ha indicado anteriormente, las coordenadas cilíndricas del punto genérico $P$ respecto de los sistemas de ejes $\left(X_{1}, Y_{1}\right)$ y $\left(X_{2}, Y_{2}\right)$. En cuanto a la 
restricción asociada al cumplimiento de la igualdad recogida en (E.17), se observa que ha desaparecido el término exponencial presente en (E.16); pues el valor de la variable $\alpha$, tal y como se desprende de la figura E.2, es real.

Considerando en principio la relación definida según (E.17), y tras aplicar sucesivamente en dicha relación las mismas operaciones, definidas en la sección E.1, que han permitido obtener las ecuaciones (E.7), (E.8) y (E.9); se deduce la siguiente expresión definitiva de la primera versión del Teorema de Adición para las Funciones de Hankel

$$
H_{\nu}^{(2)}\left(k \rho_{1}\right) e^{j \nu \phi_{1}}=\sum_{r=-\infty}^{\infty} H_{r-\nu}^{(2)}\left(k d_{21}\right) e^{-j(r-\nu) \phi_{21}} J_{r}\left(k \rho_{2}\right) e^{j r \phi_{2}} \quad \rho_{2}<d_{21}
$$

Esta primera versión del Teorema de Adición para las Funciones de Hankel expresada en (E.18), corroborada tras compararla con la versión presentada en [81] y concluir que ambas son idénticas, permite desarrollar cualquier modo cilíndrico emergente, referido a un cierto punto, en una serie de infinitos modos cilíndricos incidentes centrados en un nuevo punto; aunque la región de validez de dicho desarrollo, tal y como indica la restricción presente en (E.18), se circunscribe al interior de una circunferencia centrada en el nuevo punto al que se refiere el desarrollo modal incidente, y de radio la distancia entre dicho punto y aquél al que se refiere el modo cilíndrico emergente.

Por otra parte, la segunda versión del Teorema de Adición para las Funciones de Hankel pretende expresar un modo cilíndrico emergente, referido a un punto determinado, mediante una serie de modos cilíndricos emergentes centrados en otro punto distinto del anterior. Esta segunda versión del Teorema de Adición para las Funciones de Hankel no puede deducirse directamente, tal y como se ha realizado en las anteriores demostraciones recogidas en el presente apéndice, a partir del Teorema de Adición de Graf; sino que se obtiene tras combinar de forma adecuada dos expresiones, cada una de las cuales se deduce por separado del Teorema de Adición de Graf. Concretamente, la primera de estas dos expresiones es idéntica a la ofrecida por el Teorema de Adición para las Funciones de Bessel, que permite relacionar funciones de Bessel de primera especie; mientras la segunda expresión debe ocuparse de relacionar, de igual manera que la anterior expresión, funciones de Bessel de segunda especie. La deducción de estas dos expresiones, cuya combinación permite obtener la segunda versión del Teorema de Adición para las Funciones de Hankel, se ofrece a continuación de forma paralela.

La primera de las mencionadas expresiones, que debe relacionar funciones de Bessel de primera especie, se deduce directamente del Teorema de Adición de Graf recogido en (E.1); mientras la segunda de las expresiones, que debe mostrar una relación entre funciones de Bessel de segunda especie, se demuestra a partir de una versión del Teorema de Adición de Graf ligeramente modificada respecto de aquélla ofrecida en (E.1). Esta nueva versión del Teorema de Adición de Graf se obtiene sustituyendo en (E.1) el índice $r$ del sumatorio por un nuevo índice, 
definido mediante la suma $\nu+r$; de manera que las dos versiones del Teorema de Adición de Graf, aquélla considerada inicialmente y la nueva versión modificada, presentan el siguiente aspecto

$$
\begin{aligned}
& C_{\nu}(w) e^{j \nu \chi}=\sum_{r=-\infty}^{\infty} C_{\nu+r}(u) J_{r}(v) e^{j r \alpha} \quad\left|v e^{ \pm j \alpha}\right|<|u| \\
& C_{\nu}(w) e^{j \nu \chi}=\sum_{r=-\infty}^{\infty} C_{r}(u) J_{r-\nu}(v) e^{j(r-\nu) \alpha} \quad\left|v e^{ \pm j \alpha}\right|<|u|
\end{aligned}
$$

Estas dos versiones del Teorema de Adición de Graf, recogidas en (E.19), siguen siendo válidas cuando $C$ designa una función de Bessel de primera especie $J$, una función de Bessel de segunda especie $Y$, una función de Hankel de primera especie $H^{(1)}$, o una función de Hankel de segunda especie $H^{(2)}$. En (E.19), las restricciones que afectan a las dos versiones del Teorema de Adición de Graf son también innecesarias cuando $C$ designa una función de Bessel de primera especie $J$; mientras las variables $u, v, w, \alpha$ y $\chi$ se encuentran relacionadas en ambas expresiones de igual manera, según se recoge de forma analítica en (E.2) y gráficamente en la figura E.1.

La primera de las expresiones buscadas debe relacionar, tal y como se ha indicado previamente, funciones de Bessel de primera especie; mientras la segunda expresión debe establecer una relación, en los mismos términos que la primera expresión, entre funciones de Bessel de segunda especie. Consecuentemente, para deducir la primera expresión conviene particularizar la versión original del Teorema de Adición de Graf, ofrecida en primer lugar en (E.19), al caso en el que la función simbólica $C$ designa una función de Bessel de primera especie $J$; y con el objeto de encontrar la segunda de las expresiones buscadas debe particularizarse la versión modificada del Teorema de Adición de Graf, presentada en segundo lugar en (E.19), al caso en el que la función simbólica $C$ designa una función de Bessel de segunda especie $Y$. Bajo dichas circunstancias, las relaciones definidas inicialmente en (E.19) se reescriben del siguiente modo

$$
\begin{aligned}
& J_{\nu}(w) e^{j \nu \chi}=\sum_{r=-\infty}^{\infty} J_{\nu+r}(u) J_{r}(v) e^{j r \alpha} \\
& Y_{\nu}(w) e^{j \nu \chi}=\sum_{r=-\infty}^{\infty} Y_{r}(u) J_{r-\nu}(v) e^{j(r-\nu) \alpha} \quad\left|v e^{ \pm j \alpha}\right|<|u|
\end{aligned}
$$

donde, como era de esperar, la primera de las dos relaciones carece de restricción alguna; pues relaciona funciones de Bessel de primera especie.

Los valores a utilizar en cada una de las relaciones recogidas en (E.20) para las variables $u, v, w, \alpha$ y $\chi$, con la intención de obtener las dos expresiones buscadas, pueden deducirse considerando nuevamente la situación mostrada en la 
figura E.2; aunque deben tenerse en cuenta ciertas apreciaciones en la interpretación de dicha figura. Inicialmente, se supone que existe un campo expresado en serie de modos cilíndricos emergentes, referidos al punto $C_{1}$, que ha sido generado por una o varias fuentes puntuales. Por diversas razones mencionadas anteriormente, puede resultar interesante centrar el desarrollo modal emergente asociado a dicho campo en un nuevo punto; por ejemplo, en aquél denominado $C_{2}$ en la figura E.2. La segunda versión del Teorema de Adición para las Funciones de Hankel pretende desarrollar la $\nu$-ésima componente modal del anterior desarrollo, referida al punto $C_{1}$, en serie de infinitos modos cilíndricos emergentes centrados en el punto $C_{2}$. La deducción de esta segunda versión del Teorema de Adición para las Funciones de Hankel requiere obtener, como se ha mencionado anteriormente, dos expresiones adecuadas a partir de las relaciones definidas en (E.20); para lo cual deben identificarse, en cada una de las citadas relaciones, los valores de las variables $u, v, w, \alpha$ y $\chi$ mediante la construcción en la figura E.2 de un triángulo similar al mostrado en la figura E.1. El tercer vértice del triángulo en la figura E.2, que debe acompañar a los otros dos situados respectivamente en los puntos $C_{1}$ y $C_{2}$, se elige en un punto genérico denominado $P$ (ver fig. E.2); cuyas coordenadas cilíndricas respecto de los sistemas de ejes $\left(X_{1}, Y_{1}\right)$ y $\left(X_{2}, Y_{2}\right)$, con orígenes locales en los puntos $C_{1}$ y $C_{2}$, se designan de igual manera que en la sección E.1. Así pues, los valores de las variables $u, v, w, \alpha$ y $\chi$ a emplear en cada una de las relaciones recogidas en (E.20) se deducen tras comparar, de manera apropiada en cada caso, los triángulos mostrados respectivamente en las figuras E.1 y E.2.

En cuanto a la primera de las relaciones descritas en (E.20), tras comparar de forma adecuada los dos triángulos mencionados anteriormente, se deducen para las variables $u, v, w, \alpha$ y $\chi$ los mismos valores empleados en la sección E.1 y recogidos en (E.4). Sustituyendo pues dichos valores en la primera de las relaciones definidas en (E.20), e implementando en dicha relación las mismas operaciones descritas en la sección E.1; se obtiene directamente la primera de las dos expresiones requeridas para demostrar la segunda versión del Teorema de Adición para las Funciones de Hankel. Esta primera expresión, que relaciona funciones de Bessel de primera especie, es idéntica a la utilizada en la definición del Teorema de Adición para las Funciones de Bessel; cuyo aspecto, recordando (E.9), se reproduce a continuación

$$
J_{\nu}\left(k \rho_{1}\right) e^{j \nu \phi_{1}}=\sum_{r=-\infty}^{\infty} J_{r-\nu}\left(k d_{21}\right) e^{-j(r-\nu) \phi_{21}} J_{r}\left(k \rho_{2}\right) e^{j r \phi_{2}}
$$

donde los parámetros $d_{21}$ y $\phi_{21}$, con el mismo significado que en la sección E.1 (recordar fig. E.2), se calculan mediante las expresiones presentadas en (E.5); mientras $\left(\rho_{1}, \phi_{1}\right)$ y $\left(\rho_{2}, \phi_{2}\right)$ designan respectivamente, como en la sección E.1, las coordenadas cilíndricas del punto genérico $P$ respecto de los sistemas de ejes $\left(X_{1}, Y_{1}\right)$ y $\left(X_{2}, Y_{2}\right)$. Por último, al igual que en el Teorema de Adición para 
las Funciones de Bessel, la expresión recogida en (E.21) no presenta restricción espacial alguna en cuanto a su validez.

Por lo que respecta a los valores de las variables $u, v, w, \alpha$ y $\chi$ a emplear en la segunda de las relaciones descritas en (E.20); éstos deben elegirse para obtener una segunda expresión, análoga a la recogida en (E.21), que sin embargo relacione funciones de Bessel de segunda especie. Con este objetivo, una vez comparados de forma adecuada los dos triángulos mostrados en las figuras E.1 y E.2, se deducen unos valores para dichas variables diferentes a los recogidos en (E.4); definiéndose estos nuevos valores de la siguiente manera

$$
\begin{aligned}
u & =\rho_{2} \\
v & =d_{21} \\
w & =\rho_{1} \\
\alpha & =\phi_{21}-\phi_{2} \\
\chi & =\phi_{2}-\phi_{1}
\end{aligned}
$$

Todos estos parámetros, a partir de los cuales se definen en (E.22) los nuevos valores de las variables $u, v, w, \alpha$ y $\chi$, presentan respectivamente los mismos significados (recordar fig. E.2) que los parámetros utilizados al deducir la primera de las expresiónes buscadas, recogida en (E.21). Igualmente, las expresiones que permiten calcular los parámetros $d_{21}$ y $\phi_{21}$, presentes ambos en (E.22), se encuentran recogidos nuevamente en (E.5). A continuación, sustituyendo en la segunda de las relaciones recogidas en (E.20) las variables $u, v, w, \alpha$ y $\chi$ por sus respectivos valores definidos en (E.22), se obtiene la siguiente nueva relación entre las funciones de Bessel de segunda especie

$$
Y_{\nu}\left(\rho_{1}\right) e^{j \nu\left(\phi_{2}-\phi_{1}\right)}=\sum_{r=-\infty}^{\infty} Y_{r}\left(\rho_{2}\right) J_{r-\nu}\left(d_{21}\right) e^{j(r-\nu)\left(\phi_{21}-\phi_{2}\right)} \quad d_{21}<\rho_{2}
$$

Siguiendo el mismo proceso deductivo empleado en la sección E.1 al demostrar el Teorema de Adición para las Funciones de Bessel, resulta conveniente introducir el número de onda $k$ en todos los argumentos de las funciones de Bessel de segunda especie presentes en (E.23). Para ello, simplemente deben multiplicarse por el citado número de onda los tres lados del triángulo mostrado en la figura E.2; lo cual no afecta en absoluto a los valores empleados en (E.23) para las variables angulares $\alpha$ y $\chi$. En lo referente a la restricción espacial que determina la zona de validez de la igualdad recogida en (E.23), tampoco se ve afectada por el hecho de multiplicar los lados del triángulo por el número de onda $k$; pues los dos términos que constituyen la desigualdad asociada a la restricción presente en (E.23) quedan multiplicados por el mismo factor $k$. De esta manera, y tras 
desarrollar adecuadamente las funciones exponenciales que aparecen en (E.23), se obtiene la siguiente relación entre funciones de Bessel de segunda especie

$$
Y_{\nu}\left(k \rho_{1}\right) e^{-j \nu \phi_{1}}=\sum_{r=-\infty}^{\infty} J_{r-\nu}\left(k d_{21}\right) e^{j(r-\nu) \phi_{21}} Y_{r}\left(k \rho_{2}\right) e^{-j r \phi_{2}} \quad \rho_{2}>d_{21}
$$

Con el objeto de encontrar la relación buscada entre las funciones de Bessel de segunda especie, análoga a la deducida en (E.21) entre las funciones de Bessel de primera especie, se requiere tener un signo positivo en los exponentes de las funciones exponenciales que en (E.24) multiplican a las funciones $Y$. Realizando pues un simple cambio de signo en los índices $\nu$ y $r$ presentes en (E.24), y recordando asimismo la conocida propiedad $Y_{-n}(z)=(-1)^{n} Y_{n}(z)$ expuesta en [71]; es posible transformar la relación recogida en (E.24) del siguiente modo

$$
(-1)^{\nu} Y_{\nu}\left(k \rho_{1}\right) e^{j \nu \phi_{1}}=\sum_{r=-\infty}^{\infty} J_{\nu-r}\left(k d_{21}\right) e^{-j(r-\nu) \phi_{21}}(-1)^{r} Y_{r}\left(k \rho_{2}\right) e^{j r \phi_{2}} \quad \rho_{2}>d_{21}
$$

Por último, con la intención de simplificar el desarrollo recién deducido en (E.25), puede utilizarse una relación del tipo $J_{\nu-r}(z)=(-1)^{r-\nu} J_{r-\nu}(z)$; que también se ha empleado en la sección E.1 al deducir el Teorema de Adición para las Funciones de Bessel recogido en (E.9). Haciendo uso de la anterior relación en (E.25), se obtiene la segunda de las expresiones requeridas en la demostración del Teorema de Adición para las Funciones de Hankel. Esta segunda expresión relaciona, como se indica seguidamente, funciones de Bessel de segunda especie

$$
Y_{\nu}\left(k \rho_{1}\right) e^{j \nu \phi_{1}}=\sum_{r=-\infty}^{\infty} J_{r-\nu}\left(k d_{21}\right) e^{-j(r-\nu) \phi_{21}} Y_{r}\left(k \rho_{2}\right) e^{j r \phi_{2}} \quad \rho_{2}>d_{21}
$$

Es interesante observar que la relación entre las funciones de Bessel de segunda especie, recién deducida en (E.26), es completamente análoga a la relación presentada en (E.21) entre funciones de Bessel de primera especie; salvo que mientras esta última relación es válida en cualquier región del espacio, la anterior relación sólo tiene validez fuera de una circunferencia centrada en $C_{2}$, y de radio la distancia entre los puntos $C_{1}$ y $C_{2}$ (recordar fig. E.2). Utilizando estas dos relaciones, definidas respectivamente en (E.21) y (E.26), es posible demostrar fácilmente la segunda versión del Teorema de Adición para las Funciones de Hankel. En dicha demostración, en primer lugar debe multiplicarse la expresión recogida en (E.26) por el número imaginario $j$, y posteriormente debe sustraerse dicha expresión multiplicada por $j$ de aquella primera expresión mostrada en (E.21); para de esta forma, recordando la definición de las funciones de Hankel de segunda especie presentada en (C.12), obtener la siguiente expresión definitiva de la segunda versión del Teorema de Adición para las Funciones de Hankel

$$
H_{\nu}^{(2)}\left(k \rho_{1}\right) e^{j \nu \phi_{1}}=\sum_{r=-\infty}^{\infty} J_{r-\nu}\left(k d_{21}\right) e^{-j(r-\nu) \phi_{21}} H_{r}^{(2)}\left(k \rho_{2}\right) e^{j r \phi_{2}} \quad \rho_{2}>d_{21}
$$


La restricción espacial presente en (E.27) se debe al hecho de haber obtenido dicha expresión mediante la suma de otras dos; una de las cuales, concretamente la definida según (E.26), tiene asociada dicha restricción. Así pues, la segunda versión del Teorema de Adición para las Funciones de Hankel, recogida en (E.27), permite desarrollar un modo cilíndrico emergente genérico, referido a un punto determinado, en una serie de infinitos modos cilíndricos emergentes referidos a un nuevo punto; aunque la región donde tiene validez este desarrollo modal, de acuerdo con la restricción espacial mostrada en (E.27), se corresponde con la zona situada fuera de una circunferencia centrada en el punto al que se refiere el nuevo desarrollo modal emergente, y de radio la distancia entre dicho punto y aquél al que se refiere el modo cilíndrico emergente original. Como era de esperar, esta restricción espacial es complementaria con aquélla recogida en (E.18) para la primera versión del Teorema de Adición para las Funciones de Hankel.

Resumiendo, las dos versiones del Teorema de Adición para las Funciones de Hankel demostradas en esta sección permiten desarrollar cualquier modo cilíndrico emergente, referido a una determinada posición espacial, en serie de modos cilíndricos incidentes centrados en otra posición con validez en una cierta región del espacio; y en serie de modos cilíndricos emergentes centrados en la misma posición con validez en la región complementaria a la anterior. En determinadas situaciones, como se verá a continuación, resulta necesario desarrollar una serie de modos cilíndricos emergentes, centrados en un cierto punto, bien en serie de modos cilíndricos incidentes centrados en otro punto; o bien en serie de modos cilíndricos emergentes centrados en otra posición. Estas dos operaciones de transformación de espectros cilíndricos se definen, tal y como puede observarse en los dos próximos apartados, mediante sendas matrices conocidas como matriz de traslación de espectro emergente a incidente, y matriz de traslación de espectro emergente a emergente; que se deducen respectivamente a partir de las dos versiones del Teorema de Adición para las Funciones de Hankel demostradas anteriormente en la presente sección.

\section{E.2.1 Matriz de Traslación de Espectro Emergente a In- cidente}

Al resolver problemas de dispersión electromagnética producida por múltiples objetos dispersores bidimensionales, siguiendo el algoritmo recursivo descrito en el capítulo 6, es necesario considerar ciertos campos generados por una o varias fuentes puntuales como campos incidentes. En términos espectrales, dicha consideración requiere transformar espectros cilíndricos emergentes, referidos a determinadas posiciones, en espectros cilíndricos incidentes centrados en otras posiciones distintas a las anteriores. En el presente apartado, haciendo uso de la primera versión del Teorema de Adición para las Funciones de Hankel, se deduce la matriz de traslación de espectro emergente a incidente; que permite imple- 
mentar de forma matricial la transformación de espectros cilíndricos mencionada anteriormente. A su vez, debido a la restricción espacial asociada a la primera versión del Teorema de Adición para las Funciones de Hankel, se describe la región donde es posible reconstruir un campo generado por una o varias fuentes puntuales, cuyo espectro emergente original se refiere a un cierto punto, utilizando un espectro cilíndrico incidente trasladado; que se obtiene a partir del espectro emergente original mediante la correspondiente matriz de traslación de espectro emergente a incidente. A continuación, usando la citada matriz de traslación, se ofrecen dos ejemplos prácticos de transformación de espectro cilíndrico emergente en espectro cilíndrico incidente; que corroboran por completo la definición ofrecida en este apartado para la citada matriz de traslación de espectros, y asimismo confirman dónde puede aplicarse el espectro cilíndrico incidente trasladado que se obtiene con dicha matriz. Finalmente, a partir de los resultados correspondientes a uno de los dos ejemplos considerados, se descubre que la restricción espacial asociada a la primera versión del Teorema de Adición para las Funciones de Hankel debe traducirse, de manera consecuente, en una limitación de las dimensiones de la matriz de traslación de espectro emergente a incidente; cuya no observación provoca que ciertos elementos de la citada matriz presenten valores muy elevados. En tales circunstancias, el principal inconveniente surge cuando dichas matrices se utilizan en el algoritmo recursivo descrito en el capítulo 6; pues provocan que los sistemas de ecuaciones asociados a este algoritmo estén mal condicionados, y por tanto que no tengan solución.

Para obtener los elementos de la matriz de traslación de espectro emergente a incidente, se considerará una situación como la mostrada en la figura E.10; que está constituida por dos objetos dispersores, denominados respectivamente $O_{1} \mathrm{y}$ $O_{2}$. En un principio, se supone que el objeto $O_{1}$ genera un campo dispersado ${ }^{2}$, denominado genéricamente $C_{z}^{d}$. Recordando el apéndice C (apartado C.1.2), este campo dispersado se expresa mediante un desarrollo en serie de modos cilíndricos emergentes; los cuales se refieren a un punto del objeto $O_{1}$, normalmente al centro de dicho objeto, que representa el origen local del sistema de ejes $\left(X_{1}, Y_{1}\right)$ mostrado en la figura E.10. Así pues, el valor del campo dispersado por el objeto $O_{1}$ en un punto genérico, definido por sus coordenadas cilíndricas $\left(\rho_{1}, \phi_{1}\right)$ respecto del sistema de ejes $\left(X_{1}, Y_{1}\right)$, se recoge en la siguiente expresión

$$
C_{z}^{d}\left(\rho_{1}, \phi_{1}\right)=\sum_{q=-N_{d_{1}}}^{N_{d_{1}}} c_{q}^{\tau} H_{q}^{(2)}\left(k \rho_{1}\right) e^{j q \phi_{1}}
$$

donde los coeficientes $c_{q}^{\tau}\left(\tau=\right.$ TM para polarización $\mathrm{TM}^{\mathrm{z}}$ y $\tau=$ TE para polarización $\mathrm{TE}^{\mathrm{z}}$ ) determinan el espectro cilíndrico bidimensional, referido al origen local del sistema de ejes $\left(X_{1}, Y_{1}\right)$, que corresponde al campo dispersado $C_{z}^{d}$. Por lo que

\footnotetext{
${ }^{2}$ Este campo dispersado $\left(C_{z}^{d}\right)$ representa campo eléctrico dispersado $\left(E_{z}^{d}\right)$ para polarización $\mathrm{TM}^{\mathrm{z}}$, y campo magnético dispersado $\left(H_{z}^{d}\right)$ para polarización $\mathrm{TE}^{\mathrm{z}}$.
} 


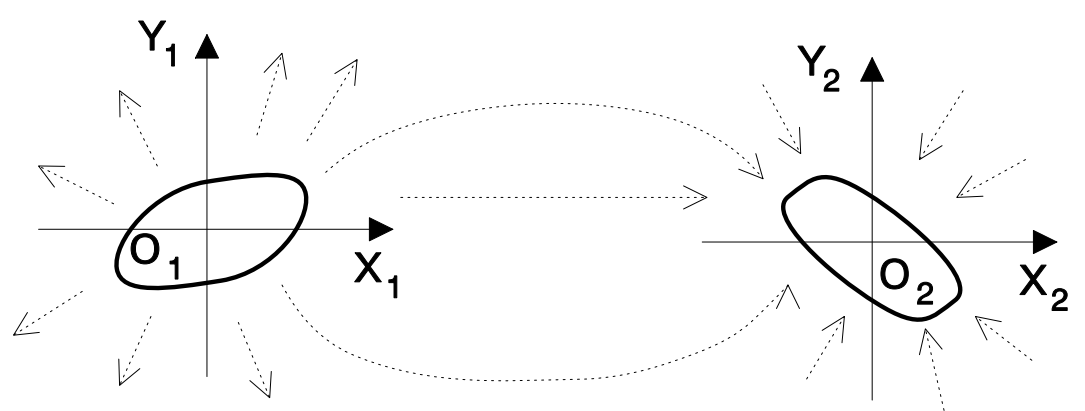

Figura E.10: Expresión del campo dispersado por $O_{1}$, definido por su espectro cilíndrico emergente, como incidencia sobre $O_{2}$ utilizando el correspondiente espectro cilíndrico incidente.

respecta al número de modos cilíndricos emergentes considerados en (E.28); éste se elige con la intención de calcular correctamente el campo dispersado por $O_{1}$ en una región situada fuera de la mínima circunferencia, con centro en el origen local del sistema de ejes $\left(X_{1}, Y_{1}\right)$, que contiene a todas las fuentes de corriente inducidas en el contorno del objeto $O_{1}$

No obstante, el campo dispersado $C_{z}^{d}$ por el objeto $O_{1}$ se propaga hasta convertirse en campo incidente ${ }^{3}$, denominado $C_{z}^{i}$, sobre el objeto $O_{2}$ (ver figura E.10). Este campo incidente se expresa, según el apéndice C (apartado C.1.2), mediante un desarrollo de modos cilíndricos incidentes; aunque referidos en esta ocasión a un punto del objeto $O_{2}$, normalmente al centro de dicho objeto, que constituye el origen local del sistema de ejes $\left(X_{2}, Y_{2}\right)$ representado en la figura E.10. Tras evaluar pues este desarrollo modal incidente en el mismo punto donde se evaluó el campo dispersado $C_{z}^{d}$ recogido en (E.28), definiendo dicho punto por sus coordenadas cilíndricas $\left(\rho_{2}, \phi_{2}\right)$ respecto del sistema de ejes $\left(X_{2}, Y_{2}\right)$, se obtiene una expresión modal para $C_{z}^{i}$ que presenta el siguiente aspecto

$$
C_{z}^{i}\left(\rho_{2}, \phi_{2}\right)=\sum_{p=-N_{i_{2}}}^{N_{i_{2}}} i_{p}^{\tau} J_{p}\left(k \rho_{2}\right) e^{j p \phi_{2}}
$$

donde los coeficientes $i_{p}^{\tau}\left(\tau=\right.$ TM para polarización $\mathrm{TM}^{\mathrm{z}}$ y $\tau=$ TE para polarización $\mathrm{TE}^{\mathrm{z}}$ ) constituyen el espectro cilíndrico bidimensional incidente, referido al origen local del sistema de ejes $\left(X_{2}, Y_{2}\right)$, que está asociado al campo incidente $C_{z}^{i}$ sobre el objeto $\mathrm{O}_{2}$. En lo referente al número de términos escogidos para implementar el sumatorio presente en (E.29), éste se determina (recordar sección 4.1 del capítulo 4) con el objeto de reconstruir de manera correcta el campo incidente $C_{z}^{i}$, procedente del campo dispersado $C_{z}^{d}$, en el interior de una circunferencia con centro en el origen local del sistema de ejes $\left(X_{2}, Y_{2}\right)$, y de radio el cociente $N_{i_{2}} / k$.

\footnotetext{
${ }^{3}$ Este campo incidente $\left(C_{z}^{i}\right)$ representa campo eléctrico incidente $\left(E_{z}^{i}\right)$ para polarización $\mathrm{TM}^{\mathrm{z}}$, y campo magnético incidente $\left(H_{z}^{i}\right)$ para polarización $\mathrm{TE}^{\mathrm{z}}$.
} 
Como los campos dispersado $C_{z}^{d}$ e incidente $C_{z}^{i}$ hacen referencia a un mismo campo, debe ser posible relacionar las amplitudes espectrales $c_{q}^{\tau}$ e $i_{p}^{\tau}$ presentes en los desarrollos modales recogidos en (E.28) y (E.29); para lo cual, se necesita expresar cada uno de los modos cilíndricos emergentes empleados en (E.28), referidos al origen local del sistema de ejes $\left(X_{1}, Y_{1}\right)$, en serie de modos cilíndricos incidentes centrados en el origen local del sistema de ejes $\left(X_{2}, Y_{2}\right)$. Esta relación se corresponde con la primera versión del Teorema de Adición para las Funciones de Hankel, definida en (E.18); que se particulariza al caso que nos ocupa del siguiente modo

$$
H_{q}^{(2)}\left(k \rho_{1}\right) e^{j q \phi_{1}}=\sum_{p=-\infty}^{\infty} H_{p-q}^{(2)}\left(k d_{21}\right) e^{-j(p-q) \phi_{21}} J_{p}\left(k \rho_{2}\right) e^{j p \phi_{2}} \quad \rho_{2}<d_{21}
$$

donde los parámetros $d_{21}$ y $\phi_{21}$, cuyo significado se muestra explícitamente en la figura E.2, están asociados en (E.30) a los orígenes locales de los sistemas de ejes $\left(X_{1}, Y_{1}\right)$ y $\left(X_{2}, Y_{2}\right)$ recogidos en la figura E.10. Así pues, haciendo uso en (E.28) de la relación recién presentada en (E.30), el valor del campo dispersado $C_{z}^{d}$ en un punto de coordenadas cilíndricas $\left(\rho_{2}, \phi_{2}\right)$, referidas al sistema de ejes $\left(X_{2}, Y_{2}\right)$, se expresa mediante el siguiente desarrollo modal incidente

$$
\begin{aligned}
& C_{z}^{d}\left(\rho_{2}, \phi_{2}\right)=\sum_{q=-N_{d_{1}}}^{N_{d_{1}}} c_{q}^{\tau} \sum_{p=-\infty}^{\infty} H_{p-q}^{(2)}\left(k d_{21}\right) e^{-j(p-q) \phi_{21}} J_{p}\left(k \rho_{2}\right) e^{j p \phi_{2}} \\
= & \sum_{p=-\infty}^{\infty}\left(\sum_{q=-N_{d_{1}}}^{N_{d_{1}}} c_{q}^{\tau} H_{p-q}^{(2)}\left(k d_{21}\right) e^{-j(p-q) \phi_{21}}\right) J_{p}\left(k \rho_{2}\right) e^{j p \phi_{2}} \quad \rho_{2}<d_{21}
\end{aligned}
$$

El campo dispersado $C_{z}^{d}$, de acuerdo con la expresión recién ofrecida en (E.31), puede desarrollarse en términos de una serie constituida por infinitos modos cilíndricos incidentes. Si dicho número de modos se trunca en un valor finito, igual al número de modos empleados en el desarrollo modal recogido en (E.29), es posible comparar ambas expresiones para deducir la siguiente relación entre los coeficientes $i_{p}^{\tau}$ y $c_{q}^{\tau}$

$$
i_{p}^{\tau}=\sum_{q=-N_{d_{1}}}^{N_{d_{1}}} c_{q}^{\tau} H_{p-q}^{(2)}\left(k d_{21}\right) e^{-j(p-q) \phi_{21}} \quad \forall p, \quad p \in\left[-N_{i_{2}}, N_{i_{2}}\right]
$$

La expresión analítica que permite relacionar los coeficientes $i_{p}^{\tau}$ y $c_{q}^{\tau}$, detallada en (E.32), puede escribirse en términos matriciales; para lo cual, es necesario que los citados coeficientes constituyan los elementos de sendos vectores columna. Bajo dicha suposición, las amplitudes espectrales $i_{p}^{\tau}$ se obtienen a partir de las 
amplitudes espectrales $c_{q}^{\tau}$ implementando la siguiente operación

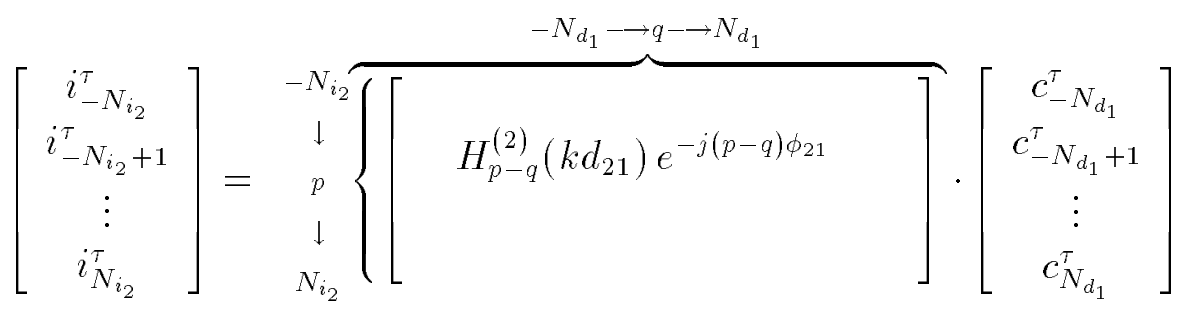

donde la matriz que interviene representa la matriz de traslación de espectro emergente a incidente, designada en este caso como $T_{21}$. Esta designación se debe a que dicha matriz permite transformar un espectro cilíndrico emergente, asociado a un cierto campo dispersado y referido al origen local del sistema de ejes $\left(X_{1}, Y_{1}\right)$, en un espectro cilíndrico incidente asociado al mismo campo; aunque este nuevo espectro incidente se encuentra centrado en el origen local del sistema de ejes $\left(X_{2}, Y_{2}\right)$.

La región donde es posible reconstruir de manera correcta un campo generado por una o varias fuentes puntuales, utilizando un espectro cilíndrico incidente trasladado a un punto distinto de aquél al que se refiere el espectro emergente del citado campo, depende no sólo de la región de correcta reconstrucción determinada por el espectro cilíndrico incidente; sino también de la restricción espacial asociada a la primera versión del Teorema de Adición para las Funciones de Hankel, que se utiliza para transformar el espectro cilíndrico emergente original en el espectro cilíndrico incidente trasladado. Por ejemplo, el campo dispersado $C_{z}^{d}$ considerado en el desarrollo anterior puede reconstruirse correctamente, como se indica en la figura E.11, en la zona situada fuera de la mínima circunferencia centrada en el origen local del sistema de ejes $\left(X_{1}, Y_{1}\right)$, que además encierra completamente todo el contorno del objeto $O_{1}$; ya que el espectro emergente asociado originalmente al campo dispersado por dicho objeto se refiere al mencionado origen local, y el número de componentes espectrales consideradas garantiza la reconstrucción correcta de este campo en aquella región espacial ausente de fuentes de corriente inducidas. El anterior campo dispersado, tras propagarse, se convierte en un campo incidente sobre el objeto $\mathrm{O}_{2}$; y por tanto puede resultar interesante expresar dicho campo mediante un espectro cilíndrico incidente, centrado en el origen local del sistema de ejes $\left(X_{2}, Y_{2}\right)$. La región donde es posible reconstruir correctamente el campo dispersado por $O_{1}$, a partir del citado espectro cilíndrico incidente obtenido según la transformación espectral definida en (E.31), se encuentra acotada por la restricción espacial presente en dicha expresión. Concretamente, dicha restricción limita la correcta reconstrucción del campo dispersado por $O_{1}$, considerando como se indica en (E.31) infinitas componentes en el espectro cilíndrico incidente, a la región contenida en el interior de una circunferencia con centro en el origen local del sistema de ejes $\left(X_{2}, Y_{2}\right)$, y de radio la distancia entre dicho origen y el del sistema de ejes $\left(X_{1}, Y_{1}\right)$; tal y como 


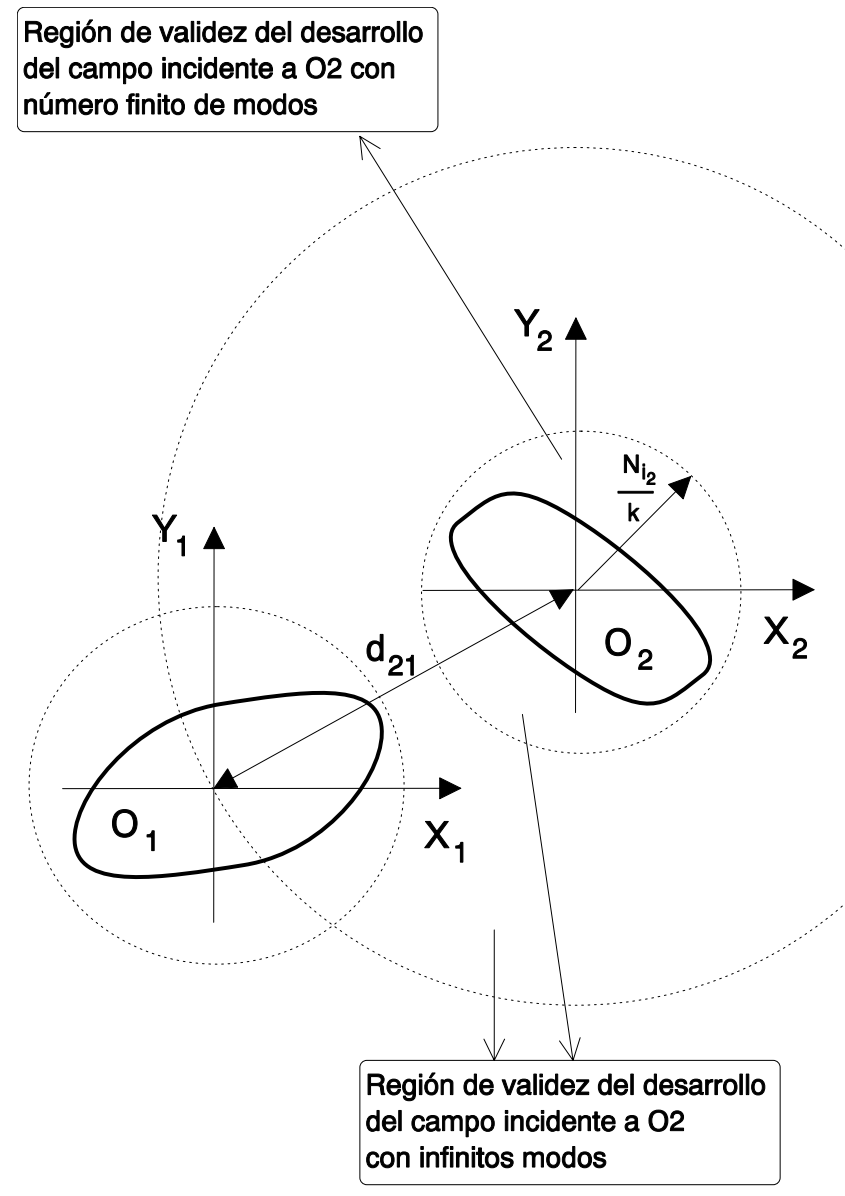

Figura E.11: Región de validez para la reconstrucción de un campo dispersado por $O_{1}$, definido inicialmente en términos de su correspondiente espectro cilíndrico emergente, a partir de un espectro cilíndrico incidente centrado en el objeto $\mathrm{O}_{2}$.

se resume gráficamente en la figura E.11. Ahora bien, en la mayoría de las situaciones sólo es necesario reconstruir correctamente el campo dispersado, a partir del correspondiente espectro cilíndrico incidente trasladado, en las proximidades del contorno del objeto $\mathrm{O}_{2}$; por lo que dicho espectro incidente puede obtenerse, a partir del espectro emergente original, implementando la operación descrita en (E.33). De esta manera, el nuevo espectro cilíndrico incidente está constituido tan sólo por $2 \mathrm{~N}_{i_{2}}+1$ componentes; y por tanto, como se indica en la figura E.11, el campo dispersado por $O_{1}$ se reconstruye correctamente a partir del espectro incidente en el interior de una circunferencia, centrada nuevamente en el origen local del sistema de ejes $\left(X_{2}, Y_{2}\right)$, pero en esta ocasión con un radio igual al cociente $\mathrm{N}_{i_{2}} / k$. Por otra parte, como consecuencia de la restricción espacial asociada a la primera versión del Teorema de Adición para las Funciones de Hankel, no resulta posible incrementar el número de modos cilíndricos incidentes, centrados 
en el origen local del sistema de ejes $\left(X_{2}, Y_{2}\right)$, con la intención de reconstruir el campo dispersado original fuera de la máxima zona permitida por la mencionada restricción; resultando por tanto ineficiente, en términos computacionales, el empleo de un número de modos incidentes superior al correspondiente a dicha zona máxima de correcta reconstrucción.

Seguidamente, se ofrecen los resultados obtenidos en dos ejemplos de traslación de espectro emergente a incidente; que permiten validar el concepto de la matriz de traslación de espectro emergente a incidente, utilizada al resolver ambos ejemplos, y confirmar asimismo la región donde es posible reconstruir, de forma correcta, un campo generado por una o varias fuentes puntuales a partir del correspondiente espectro cilíndrico incidente trasladado. De igual manera, estos resultados permiten identificar una limitación asociada a la construcción de la mencionada matriz de traslación de espectro emergente a incidente; cuya no observación provoca un efecto indeseado, descrito a continuación, al emplear dicha matriz en el algoritmo recursivo descrito en el capítulo 6. En ambos ejemplos, se pretende reconstruir correctamente el campo eléctrico dispersado por un cilindro metálico infinito, de radio $2 \lambda$ y centrado en el punto de coordenadas cartesianas normalizadas $(-4,0)$, ante un campo eléctrico incidente (polarización $\mathrm{TM}^{\mathrm{z}}$ ) representado por una onda plana $(\beta=0)$. El espectro asociado al campo eléctrico dispersado por el cilindro se ha obtenido, recordando el capítulo 4, a partir de la matriz de caracterización individual que define el comportamiento dispersor del cilindro considerado. Dicho espectro, referido lógicamente al centro del cilindro situado en el punto $(-4,0)$, está constituido por el número de componentes necesarias (recordar sección 4.1 del capítulo 4) para reconstruir, de manera correcta, el campo dispersado fuera del contorno definido por el propio cilindro. La amplitud y la fase correspondientes al campo eléctrico dispersado por el cilindro, cuyo cálculo se ha realizado utilizando el anterior espectro cilíndrico emergente en el desarrollo modal recogido en (E.28), se encuentran recogidas en la figura E.12; donde se observa que el campo no se ha representado en el interior del cilindro metálico dispersor, pues el desarrollo modal utilizado proporciona valores correctos de campo dispersado fuera de la circunferencia que encierra a las fuentes de corriente inducidas.

En el primer ejemplo, se pretende reconstruir correctamente el campo eléctrico dispersado por el cilindro mediante un espectro cilíndrico incidente, centrado en el punto de coordenadas cartesianas normalizadas $(3,0)$; que se obtiene, utilizando la matriz de traslación de espectro emergente a incidente definida en (E.33), a partir del espectro cilíndrico emergente asociado originalmente al campo dispersado por el cilindro. La región donde se pretende reconstruir este campo dispersado, empleando en (E.29) el espectro cilíndrico incidente trasladado, se corresponde con el interior de una circunferencia centrada en el punto $(3,0)$ y de radio $4 \lambda$; por lo que dicho espectro incidente está constituido por $2 N_{i_{2}}+1$ componentes con $N_{i_{2}}=k 4 \lambda$. La amplitud y la fase correspondientes a la reconstrucción del citado 

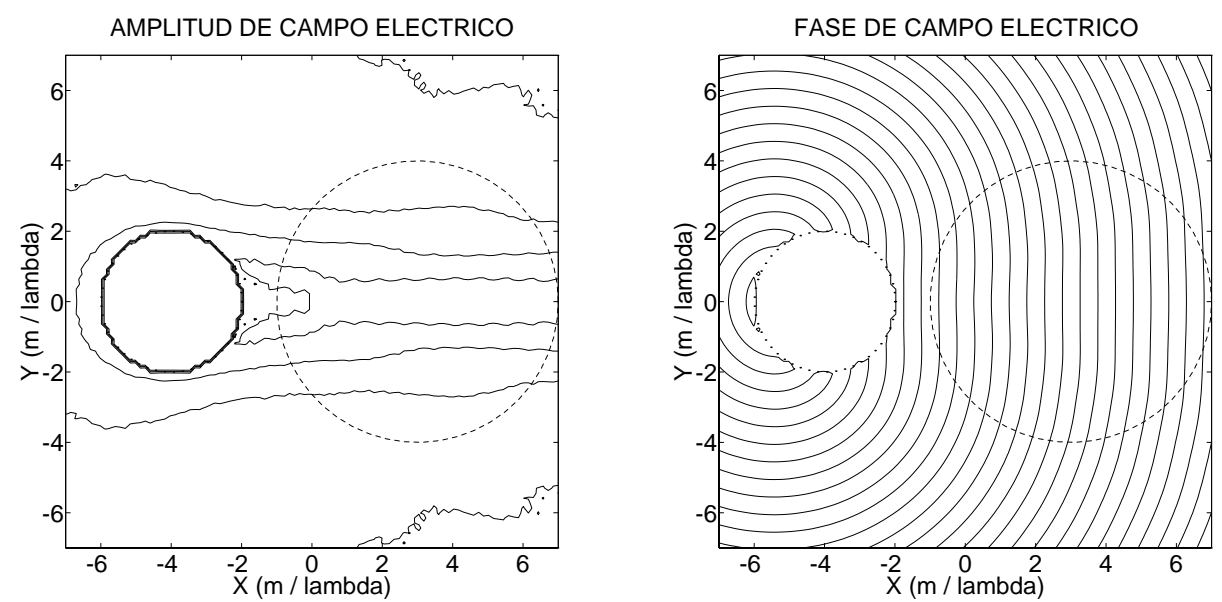

Figura E.12: Amplitud y fase del campo eléctrico dispersado por un cilindro metálico infinito, de radio $2 \lambda$ y centrado en $(-4,0)$, ante la incidencia de una onda plana $(\beta=0)$. Reconstrucción a partir de su espectro cilíndrico emergente centrado en $(-4,0)$.
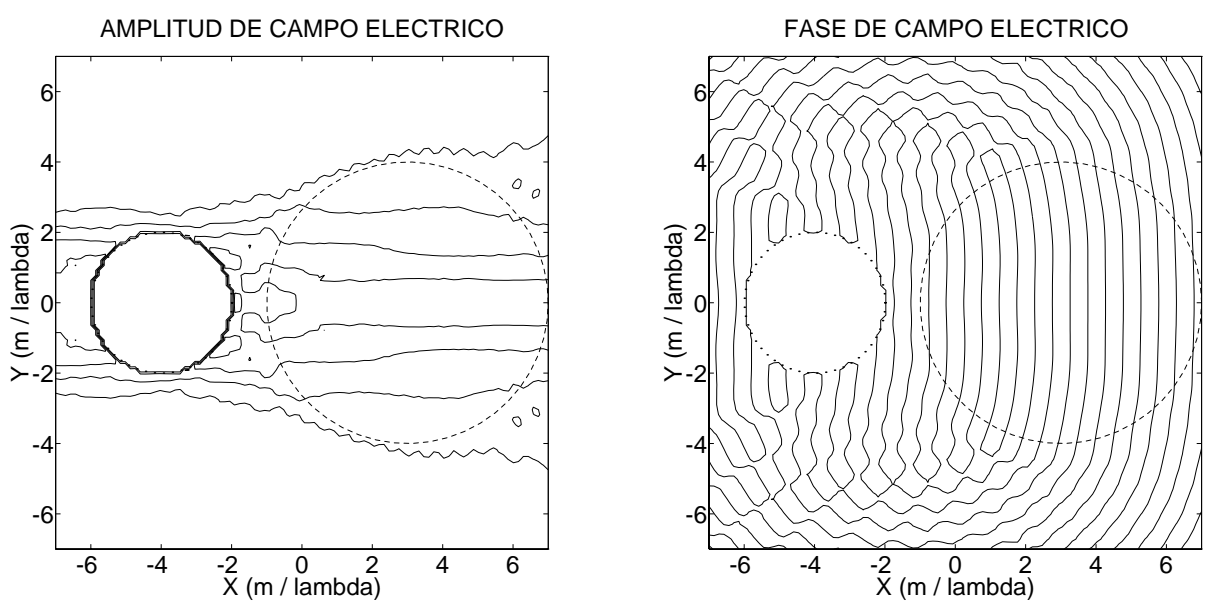

Figura E.13: Amplitud y fase del campo eléctrico dispersado por un cilindro metálico infinito, de radio $2 \lambda$ y centrado en $(-4,0)$, ante la incidencia de una onda plana $(\beta=0)$. Reconstrucción a partir del espectro cilíndrico incidente centrado en $(3,0)$, y constituido por $2 N_{i_{2}}+1$ componentes espectrales con $N_{i_{2}}=k 4 \lambda$.

campo eléctrico, dispersado originalmente por el cilindro, se representan ambas en la figura E.13; donde con trazo discontinuo, al igual que en la figura E.12, se ha dibujado el contorno de la región en la que se pretende reconstruir correctamente, mediante el mencionado espectro cilíndrico incidente, el campo eléctrico dispersado por el cilindro. Es interesante observar que en la figura E.13 la posición del círculo de trazo discontinuo, relativa al círculo que encierra a las fuentes 

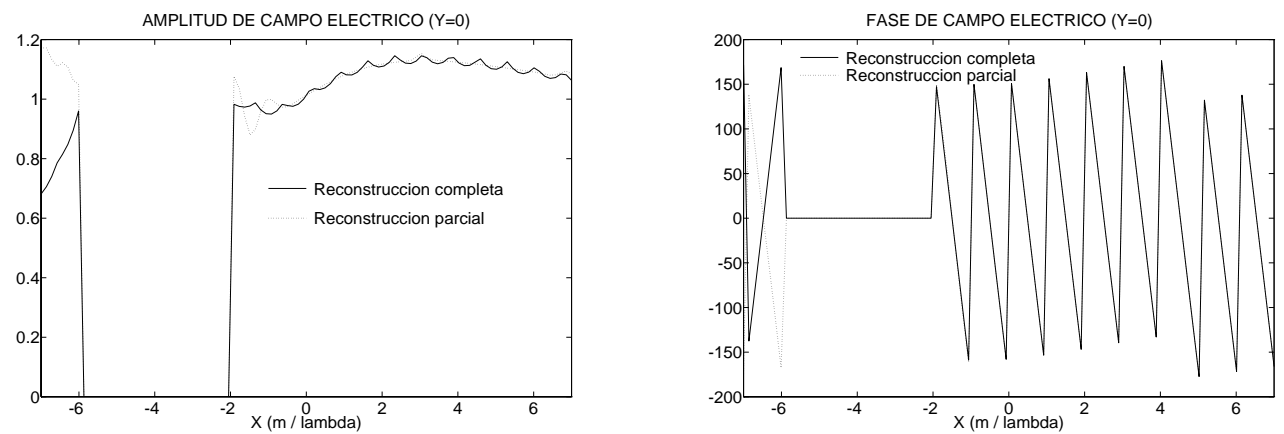

Figura E.14: Amplitud y fase del campo eléctrico dispersado por el cilindro, ante una onda plana $(\beta=0)$, a lo largo del eje $\hat{x}(y=0)$. Reconstrucción completa a partir del espectro emergente asociado originalmente a dicho campo, y reconstrucción parcial a partir del espectro incidente centrado en el punto $(3,0)$ con $N_{i_{2}}=k 4 \lambda$.

puntuales de corriente inducidas, es completamente análoga a la posición relativa entre estos mismos círculos mostrada en la figura E.11. Comparando pues las figuras E.12 y E.13, se concluye que el campo dispersado por el cilindro se ha reconstruido correctamente, a partir del espectro incidente trasladado, en la región contenida dentro del círculo dibujado en dichas figuras con trazo discontinuo; lo cual confirma los comentarios realizados anteriormente acerca de la región donde es posible reconstruir, de manera correcta, un campo generado por una o varias fuentes puntuales mediante un espectro cilíndrico incidente trasladado. Nuevamente, como puede observarse en la figura E.13, el campo eléctrico dispersado no se ha reconstruido en el interior del cilindro metálico dispersor; pues en dicha zona no es posible reconstruir el mencionado campo, ni tan siquiera a partir de su espectro emergente original. Con la intención de observar más detalladamente el grado de reconstrucción del campo eléctrico dispersado, empleando el espectro cilíndrico incidente trasladado, se han realizado diversos cortes a lo largo del eje $\hat{x}$ en la amplitud y la fase mostradas en las figuras E.12 y E.13; representando estos cortes de forma conjunta en la figura E.14. En dicha figura, se representa con línea continua la reconstrucción (denominada completa en fig. E.14) del campo eléctrico dispersado por el cilindro a lo largo del eje $\hat{x}(y=0)$, deducida a partir del espectro emergente asociado originalmente a dicho campo; mientras con línea punteada se ofrece la reconstrucción (denominada parcial en fig. E.14) del mismo campo dispersado, nuevamente a lo largo del eje $\hat{x}(y=0)$, pero calculada mediante el espectro cilíndrico incidente centrado en el punto $(3,0)$. Como era de esperar, las dos reconstrucciones son prácticamente coincidentes en el intervalo $x \in[-1,7]$; que pertenece a la región de correcta reconstrucción del campo dispersado, delimitada en las figuras E.12 y E.13 por un círculo de trazo discontinuo. Si el campo dispersado por el cilindro se pretende reconstruir correctamente, utilizando el espectro cilíndrico incidente centrado en el punto $(3,0)$, en una re- 

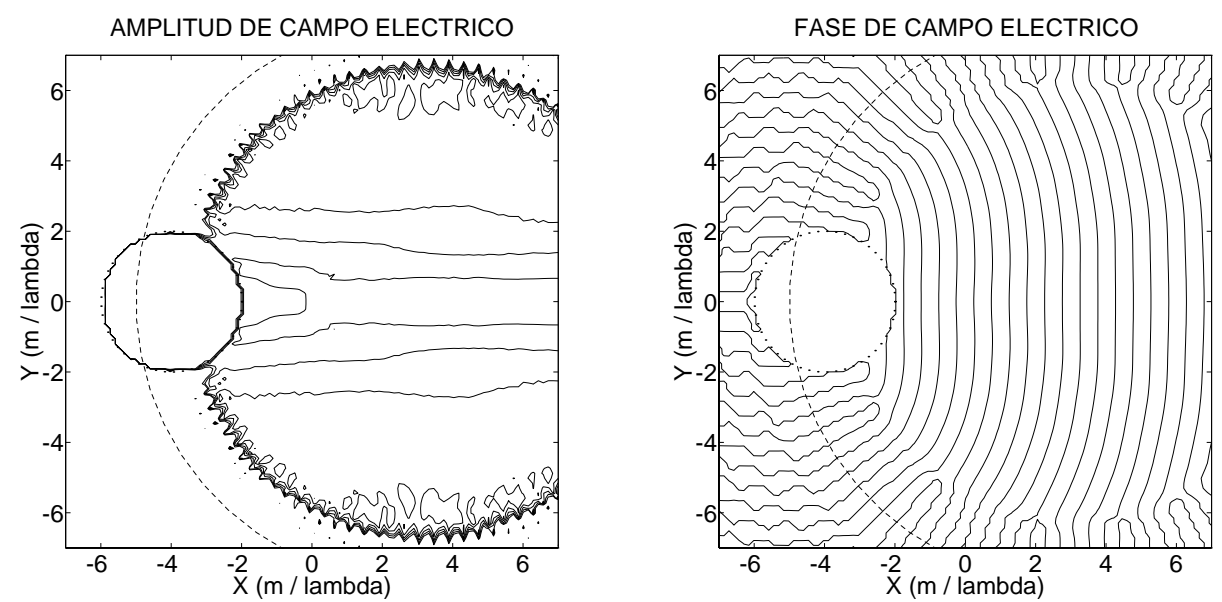

Figura E.15: Amplitud y fase del campo eléctrico dispersado por un cilindro metálico infinito, de radio $2 \lambda$ y centrado en $(-4,0)$, ante la incidencia de una onda plana $(\beta=0)$. Reconstrucción a partir del espectro cilíndrico incidente centrado en $(3,0)$, y constituido por $2 N_{i_{2}}+1$ componentes espectrales con $N_{i_{2}}=k 8 \lambda$.

gión mayor a la definida por una circunferencia con centro en $(3,0)$ y radio $4 \lambda$; resulta necesario incrementar el número de componentes espectrales asociadas al espectro cilíndrico incidente.

En el segundo ejemplo, ofrecido a continuación, se pretende reconstruir el campo dispersado por el cilindro en una región mayor a la considerada en el ejemplo anterior; para lo cual, haciendo uso de una matriz de traslación de espectro emergente a incidente como la definida según (E.33), se transforma el espectro cilíndrico emergente asociado originalmente al campo dispersado en un espectro cilíndrico incidente, centrado de nuevo en el punto $(3,0)$, pero constituido en este caso por $2 N_{i_{2}}+1$ componentes con $N_{i_{2}}=k 8 \lambda$. La reconstrucción de la amplitud y la fase del campo eléctrico dispersado por el cilindro, utilizando en (E.29) los coeficientes del nuevo espectro cilíndrico incidente trasladado, se presenta en la figura E.15; en la que con trazo discontinuo se dibuja el contorno de la región donde, debido al número de componentes espectrales que constituyen el nuevo espectro cilíndrico incidente, debiera ser posible reconstruir el campo dispersado original a partir del nuevo espectro. Esta región, como es lógico, se encuentra acotada en la figura E.15 por una circunferencia centrada en $(3,0)$ y de radio $8 \lambda$. No obstante, tal y como puede apreciarse en la figura E.15, el campo dispersado por el cilindro tan sólo se reconstruye correctamente, mediante el mencionado espectro cilíndrico incidente trasladado, en el interior de una circunferencia con centro en $(3,0)$ y radio $7 \lambda$; que corresponde en este ejemplo al valor de la distancia, denominada $d_{21}$ en la figura E.11, presente en la restricción espacial introducida por la primera versión del Teorema de Adición para las Funciones de Hankel. 

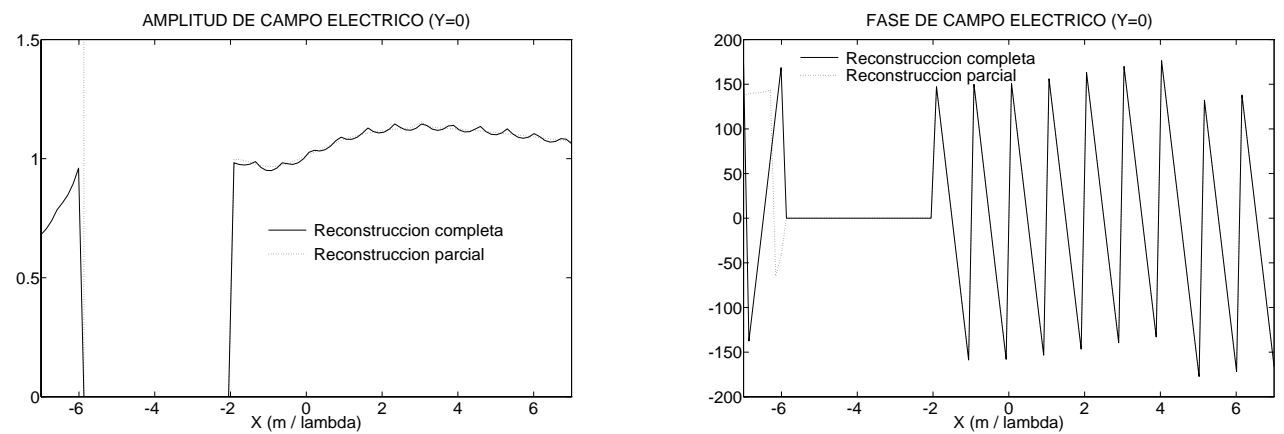

Figura E.16: Amplitud y fase del campo eléctrico dispersado por el cilindro, ante una onda plana $(\beta=0)$, a lo largo del eje $\hat{x}(y=0)$. Reconstrucción completa a partir del espectro emergente asociado originalmente a dicho campo, y reconstrucción parcial a partir del espectro incidente centrado en el punto $(3,0)$ con $N_{i_{2}}=k 8 \lambda$.

Adicionalmente, al igual que en el ejemplo anterior, el campo dispersado por el cilindro sigue sin poder reconstruirse correctamente en su interior; pues dicha zona contiene a las fuentes que han generado originalmente el campo dispersado utilizado en ambos ejemplos. Con el objeto de corroborar la zona de reconstrucción correcta del campo dispersado determinada anteriormente, se han realizado diversos cortes a lo largo del eje $\hat{x}$ en las gráficas ofrecidas en las figuras E.12 y E.15; que se representan conjuntamente, por razones comparativas, en la figura E.16. Concretamente, dicha figura muestra con línea continua la reconstrucción (denominada completa en fig. E.16) del campo eléctrico dispersado originalmente por el cilindro, a lo largo del eje $\hat{x}(y=0)$, empleando el espectro emergente original de dicho campo; mientras con línea punteada se recoge en la misma figura la reconstrucción (denominada parcial en fig. E.16) del mismo campo eléctrico dispersado, nuevamente a lo largo del eje $\hat{x}(y=0)$, pero utilizando el nuevo espectro cilíndrico incidente trasladado al punto $(3,0)$ con $N_{i_{2}}=k 8 \lambda$. Esta figura comparativa revela un alto grado de reconstrucción del mencionado campo dispersado, a partir del nuevo espectro incidente trasladado, en el intervalo $x \in[-2,7]$; que está contenido en el interior de la nueva zona prevista anteriormente para reconstruir correctamente el campo dispersado, definida por la circunferencia centrada en $(3,0)$ y de radio la distancia $d_{21}$ de valor igual en este caso a $7 \lambda$. Sin embargo, fuera de esta región, se observa que la reconstrucción parcial del campo eléctrico dispersado presenta valores de campo cuya amplitud es muy elevada, como puede observarse en la figura E.16 para las posiciones $x<-6$; donde la amplitud del campo eléctrico es mucho mayor que aquélla reconstruida en el ejemplo anterior (recordar figura E.14) utilizando menos modos cilíndricos incidentes.

A partir de estos resultados descubiertos en el ejemplo anterior, observando la matriz de traslación de espectro emergente a incidente utilizada en dicho ejemplo, se descubre que los valores tan elevados en la amplitud del campo dispersado 


\begin{tabular}{|c|c|c|}
\hline \hline Radio $\left(N_{i_{2}} / k\right)$ & Margen con $d_{21}$ & Elemento de mayor amplitud \\
\hline 2 & 5 & 0.1451 \\
\hline 2.5 & 4.5 & 0.1543 \\
\hline 3 & 4 & 0.1685 \\
\hline 3.5 & 3.5 & 0.1941 \\
\hline 4 & 3 & 0.2941 \\
\hline 4.5 & 2.5 & 0.6190 \\
\hline 5 & 2 & 2.2402 \\
\hline 5.5 & 1.5 & 12.5295 \\
\hline 6 & 1 & 96.8612 \\
\hline 6.5 & 0.5 & 977.2143 \\
\hline 7 & 0 & $1.242810^{4}$ \\
\hline 7.5 & -0.5 & $5.073810^{5}$ \\
\hline 8 & -1 & $1.016810^{7}$ \\
\hline 8.5 & -1.5 & $2.413310^{8}$ \\
\hline \hline
\end{tabular}

Tabla E.1: Evolución de la amplitud asociada al elemento más grande de la matriz de traslación de espectro emergente a incidente en función del número de modos cilíndricos incidentes considerados.

reconstruido, obtenidos fuera de la región de correcta reconstrucción del campo dispersado, están causados por el hecho de que algunos elementos de la matriz de traslación empleada presentan también amplitudes muy grandes. Estas amplitudes tan elevadas en algunos elementos de la matriz de traslación, descubiertas en el ejemplo anterior, surgen por haber utilizado un número de modos cilíndricos incidentes superior al determinado por la restricción espacial de la primera versión del Teorema de Adición para las Funciones de Hankel, utilizada al construir la citada matriz de traslación. Aunque los valores elevados en la amplitud del campo se producen fuera de la zona donde interesa reconstruir correctamente el campo dispersado original, y realmente no afectan a la correcta reconstrucción del campo en dicha zona (recordar fig. E.16), pueden surgir problemas cuando este tipo de matrices de traslación se utilizan para resolver, aplicando el algoritmo recursivo descrito en el capítulo 6 , problemas de dispersión electromagnética producida por múltiples objetos. Al aplicar el citado algoritmo recursivo, es necesario resolver diversos sistemas de ecuaciones matriciales; que pueden estar mal condicionados, e incluso no tener solución, debido a la utilización de matrices de traslación de espectro emergente a incidente cuyos elementos presentan amplitudes muy elevadas. Con el objeto de tener una idea acerca del orden de magnitud de estas amplitudes elevadas en algunos elementos de la matriz de traslación, se ha construido la 
tabla E.1; en la que se muestra, para la misma situación considerada en los ejemplos anteriores, la evolución de la amplitud del elemento más grande de la matriz de traslación en función del número de modos cilíndricos incidentes considerados. La primera columna de la citada tabla muestra el radio $\left(N_{i_{2}} / k\right)$ de la zona donde supuestamente se debe reconstruir de manera correcta el campo dispersado original; utilizando en dicha reconstrucción un espectro cilíndrico incidente centrado en el punto $(3,0)$ y constituido por $2 N_{i_{2}}+1$ componentes espectrales. La segunda columna indica el margen entre dicho radio y el máximo permitido, de valor en el presente ejemplo igual a $7 \lambda$, que determina la restricción espacial asociada a la primera versión del Teorema de Adición para las Funciones de Hankel. Finalmente, la tercera columna de la tabla E.1 muestra, para cada valor del número de modos cilíndricos incidentes considerados, la amplitud del elemento más grande de la mencionada matriz de traslación de espectro emergente a incidente. $\mathrm{Ob}-$ servando los resultados recogidos en la tabla E.1, se descubre que la matriz de traslación no presenta elementos con amplitudes muy elevadas cuando el margen del radio $N_{i_{2}} / k$ con la distancia $d_{21}$ es positivo; mientras que cuando dicho margen se hace negativo, al pretender reconstruir el campo dispersado en el punto donde se centra el espectro emergente asociado originalmente a dicho campo, los elementos de la citada matriz de traslación presentan amplitudes muy elevadas. La razón de estas amplitudes tan elevadas se debe a que el campo a reconstruir, definido originalmente en términos de un espectro emergente centrado en el punto $(-4,0)$, presenta precisamente en dicho punto un comportamiento singular; que pretende reconstruirse mediante un espectro cilíndrico incidente trasladado a otra posición, haciendo uso para ello de la mencionada matriz de traslación. De esta discusión, se desprende una limitación importante a observar en la construcción de la matriz de traslación de espectro emergente a incidente, relacionada con la elección del número de modos cilíndricos incidentes; número que debe ser inferior al determinado por la restricción espacial asociada a la primera versión del Teorema de Adición para las Funciones de Hankel. Este número debe ser tal que no introduzca en la zona de posible reconstrucción correcta del campo dispersado original, deducida a partir del espectro cilíndrico incidente trasladado, un margen negativo entre el radio $N_{i_{2}} / k$ de dicha zona y la distancia límite $d_{21}$ (recordar tabla E.1). En caso contrario, tal y como se recoge en la tabla E.1, la citada matriz de traslación presenta elementos con amplitudes muy elevadas; lo cual se traduce en que los sistemas de ecuaciones matriciales asociados a la resolución, mediante el algoritmo recursivo descrito en el capítulo 6 , de problemas de dispersión producida por múltiples objetos estén mal condicionados e incluso no presenten solución. Además, como ya se ha comentado anteriormente, el empleo de un número de modos superior al máximo recomendado por la distancia $d_{21}$ no permite reconstruir correctamente el campo dispersado original, utilizando el espectro cilíndrico incidente trasladado, en una región mayor a la máxima permitida por la restricción espacial determinada por la distancia $d_{21}$; razón que se une 
a la anterior para desestimar el uso de un número de modos cilíndricos incidentes superior al impuesto por la distancia $d_{21}$.

\section{E.2.2 Matriz de Traslación de Espectro Emergente a Emer- gente}

Al resolver problemas de dispersión electromagnética, como los analizados en el presente trabajo, resulta necesario expresar cada campo generado por una o varias fuentes puntuales de corriente mediante un espectro cilindrico emergente; que debe referirse en cada situación a un punto determinado. En ciertas ocasiones, dichos espectros están centrados en posiciones diferentes a las deseadas; siendo por tanto imprescindible una traslación de estos espectros a los puntos requeridos en cada caso. Así, por ejemplo, el comportamiento dispersor de múltiples objetos en problemas con fuente interna se caracteriza, siguiendo el algoritmo descrito en la sección 6.4, para una determinada posición espacial de la fuente interna al problema. Cuando la fuente que genera el campo incidente del problema se cambia a otra posición, con el objeto de no tener que caracterizar nuevamente el comportamiento dispersor de los objetos, conviene trasladar el espectro emergente asociado a la nueva fuente al punto donde estaba situada la fuente considerada en el primer análisis del problema; y de esta forma resolver el nuevo problema utilizando las mismas matrices de caracterización conjunta obtenidas inicialmente. Una segunda situación en la que se trasladan espectros emergentes a nuevas posiciones se produce, una vez resuelto un problema de dispersión múltiple mediante el algoritmo recursivo descrito en el capítulo 6 , cuando se pretende reconstruir el campo dispersado por todos los objetos fuera de la mínima circunferencia que los contiene a todos ellos (recordar sección 6.5 del capítulo 6). Como bien es sabido, la matriz de caracterización conjunta de cada objeto dispersor proporciona, tras multiplicar al espectro de campo incidente, un espectro emergente que se refiere usualmente al centro del citado objeto. En este caso, si los distintos espectros emergentes centrados en cada objeto se trasladan a un punto común, normalmente al punto medio de los centros de todos los objetos, el campo dispersado total puede calcularse a partir de un único desarrollo modal emergente centrado en el mencionado punto común; lo cual sin duda resulta más eficiente, desde el punto de vista computacional, que el cálculo por separado del campo dispersado por cada uno de los objetos y su posterior suma. En el presente apartado, se describe como implementar en términos matriciales las anteriores operaciones de traslación de espectros emergentes; para lo cual se define una matriz de traslación de espectro emergente a emergente, deducida a partir de la segunda versión del Teorema de Adición para las Funciones de Hankel. Esta nueva matriz de traslación permite transformar un espectro emergente asociado a un campo generado por una o varias fuentes, referido a un punto determinado, en un nuevo espectro emergente asociado al mismo campo; pero centrando este nuevo espectro en 

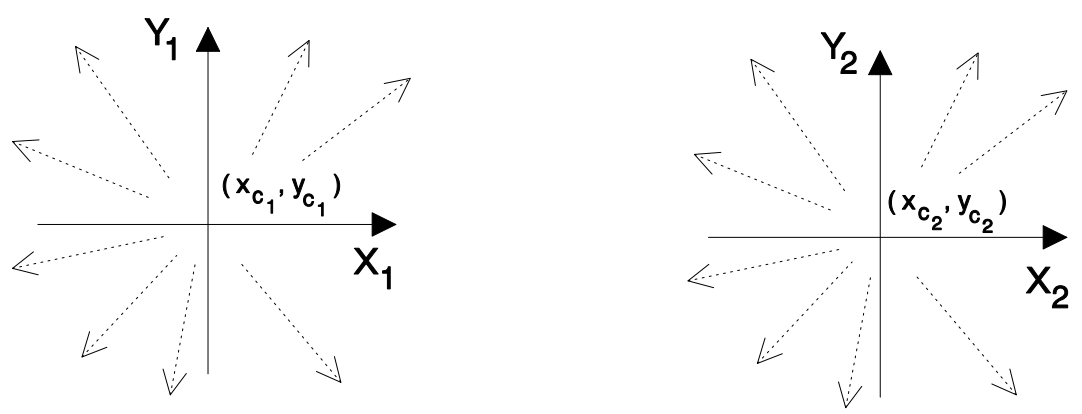

Figura E.17: Expresión de un campo generado por una o varias fuentes mediante dos espectros cilíndricos emergentes referidos a distintos puntos; que se definen a través de sus coordenadas cartesianas globales.

otro punto distinto de aquél al que se refería el espectro original. Finalmente, observando la restricción espacial asociada a la segunda versión del Teorema de Adición para las Funciones de Hankel, se describe la región donde es posible reconstruir, de manera correcta, un campo generado por una o varias fuentes a partir de una versión trasladada del espectro emergente original, obtenida utilizando la mencionada matriz de traslación de espectro emergente a emergente; y asimismo, se discute el número de modos cilíndricos emergentes trasladados que requiere el nuevo espectro.

Para obtener los elementos de la matriz de traslación de espectro emergente a emergente, se considera la existencia de un cierto campo ${ }^{4}$ generado por una o varias fuentes puntuales de corriente, designado de forma genérica como $C_{z}^{d}$. Este campo se expresa, al igual que los campos dispersados definidos en el apartado C.1.2 del apéndice C, mediante un desarrollo en serie de modos cilíndricos emergentes; que se refieren originalmente al punto de coordenadas cartesianas globales $\left(x_{c_{1}}, y_{c_{1}}\right)$, representado en la figura E.17 como el origen local del sistema de ejes $\left(X_{1}, Y_{1}\right)$. Al evaluar este desarrollo modal en un punto genérico del espacio, definido por sus coordenadas cilíndricas $\left(\rho_{1}, \phi_{1}\right)$ respecto del sistema de ejes $\left(X_{1}, Y_{1}\right)$, se obtiene la siguiente expresión

$$
C_{z}^{d}\left(\rho_{1}, \phi_{1}\right)=\sum_{q=-N_{d_{1}}}^{N_{d_{1}}} c_{q}^{\tau} H_{q}^{(2)}\left(k \rho_{1}\right) e^{j q \phi_{1}}
$$

donde los coeficientes $c_{q}^{\tau}\left(\tau=\right.$ TM para polarización $\mathrm{TM}^{\mathrm{z}}$ y $\tau=$ TE para polarización $\mathrm{TE}^{\mathrm{z}}$ ) determinan el espectro cilíndrico emergente bidimensional, referido al punto $\left(x_{c_{1}}, y_{c_{1}}\right)$, que está asociado al campo $C_{z}^{d}$ generado por una o varias fuentes puntuales de corriente. En cuanto a los límites del sumatorio presente en (E.34), se escogen de manera que el número de modos considerados $\left(2 N_{d_{1}}+1\right)$ permite

\footnotetext{
${ }^{4}$ Este campo $\left(C_{z}^{d}\right)$ representa campo eléctrico $\left(E_{z}^{d}\right)$ para polarización $\mathrm{TM}^{\mathrm{z}}$, y campo magnético $\left(H_{z}^{d}\right)$ para polarización $\mathrm{TE}^{\mathrm{z}}$.
} 
reconstruir correctamente el campo $C_{z}^{d}$ fuera de la mínima circunferencia, centrada en el punto $\left(x_{c_{1}}, y_{c_{1}}\right)$, que contiene a todas las fuentes puntuales de corriente generadoras del campo $C_{z}^{d}$ a reconstruir.

Por las razones ya comentadas anteriormente, puede resultar necesario expresar el mismo campo $C_{z}^{d}$ mediante un nuevo desarrollo modal emergente; cuyos modos se refieren en este caso al punto de coordenadas cartesianas globales $\left(x_{c_{2}}, y_{c_{2}}\right)$, que en la figura E.17 representa el origen local del nuevo sistema de ejes $\left(X_{2}, Y_{2}\right)$. Si este nuevo desarrollo modal emergente se evalúa en el mismo punto donde se evaluó el anterior desarrollo recogido en (E.34), pero utilizando lógicamente las coordenadas cilíndricas $\left(\rho_{2}, \phi_{2}\right)$ de dicho punto respecto del sistema de ejes $\left(X_{2}, Y_{2}\right)$, el valor del campo $C_{z}^{d}$ puede expresarse del siguiente modo

$$
C_{z}^{d}\left(\rho_{2}, \phi_{2}\right)=\sum_{n=-N_{d_{2}}}^{N_{d_{2}}} c_{n}^{\prime \tau} H_{n}^{(2)}\left(k \rho_{2}\right) e^{j n \phi_{2}}
$$

donde los nuevos coeficientes $c_{n}^{\prime \tau}\left(\tau=\right.$ TM para polarización $\mathrm{TM}^{\mathrm{z}}$ y $\tau=$ TE para polarización $\mathrm{TE}^{\mathrm{z}}$ ) constituyen el espectro cilíndrico emergente bidimensional, centrado en el punto $\left(x_{c_{2}}, y_{c_{2}}\right)$, que corresponde al mismo campo $C_{z}^{d}$ generado por una o varias fuentes puntuales de corriente. Por lo que respecta a los nuevos límites del sumatorio utilizado en (E.35), éstos se determinan para que el nuevo número de modos considerados $\left(2 N_{d_{2}}+1\right)$ garantice la correcta reconstrucción del campo $C_{z}^{d}$ fuera de una circunferencia, centrada en el punto $\left(x_{c_{2}}, y_{c_{2}}\right)$; y cuyo radio se determina, tal y como se detalla a continuación, a partir de la restricción espacial asociada a la segunda versión del Teorema de Adición para las Funciones de Hankel.

La relación buscada entre los coeficientes $c_{n}^{\prime \tau}$ y $c_{q}^{\tau}$ no puede encontrarse directamente comparando los desarrollos modales recogidos en (E.34) y (E.35); pues dichos desarrollos utilizan coordenadas cilíndricas de un mismo punto referidas a dos sistemas de ejes diferentes. Con el objeto de poder comparar los citados desarrollos, y poder expresar los coeficientes $c_{n}^{\prime \tau}$ en función de los coeficientes $c_{q}^{\tau}$, es necesario sustituir en (E.34) cada modo cilíndrico emergente, referido al punto $\left(x_{c_{1}}, y_{c_{1}}\right)$, por una serie de modos cilíndricos emergentes centrados en el punto $\left(x_{c_{2}}, y_{c_{2}}\right)$. Esta sustitución se implementa mediante la segunda versión del Teorema de Adición para las Funciones de Hankel recogida en (E.27); que, tras particularizarse al problema bajo análisis, presenta el siguiente aspecto

$$
H_{q}^{(2)}\left(k \rho_{1}\right) e^{j q \phi_{1}}=\sum_{n=-\infty}^{\infty} J_{n-q}\left(k d_{21}\right) e^{-j(n-q) \phi_{21}} H_{n}^{(2)}\left(k \rho_{2}\right) e^{j n \phi_{2}} \quad \rho_{2}>d_{21}
$$

Esta relación, recién presentada en (E.36), se particulariza al caso en el que el orden $q$ es cero para su utilización, ante incidencia $\mathrm{TM}^{\mathrm{z}}$, en la técnica basada en el Teorema de Adición para las Funciones de Hankel; que permite construir, recordando el apartado 4.3.2 del capítulo 4, la matriz de espectro necesaria en la 
obtención de la matriz de caracterización individual de un objeto dispersor. En dicha técnica, los campos generados por distintas fuentes puntuales de corriente situadas fuera del origen de coordenadas, expresados mediante funciones de Hankel de segunda especie y orden cero, deben desarrollarse en series de modos cilíndricos emergentes centrados en el origen de coordenadas; las cuales pueden deducirse, como se indica en el apartado 4.3.2, a partir de la expresión recogida en (E.36).

Por otra parte, en (E.36), los parámetros $d_{21}$ y $\phi_{21}$ tienen el mismo significado recogido gráficamente en la figura E.2; aunque en este ejemplo hacen referencia a los puntos $\left(x_{c_{1}}, y_{c_{1}}\right)$ y $\left(x_{c_{2}}, y_{c_{2}}\right)$ considerados en la figura E.17, donde representan respectivamente los orígenes locales de los sistemas de ejes $\left(X_{1}, Y_{1}\right)$ y $\left(X_{2}, Y_{2}\right)$. A continuación, utilizando en (E.34) la relación mostrada anteriormente en (E.36), es posible transformar el campo $C_{z}^{d}$ considerado originalmente en (E.34) de la siguiente manera

$$
\begin{gathered}
C_{z}^{d}\left(\rho_{2}, \phi_{2}\right)=\sum_{q=-N_{d_{1}}}^{N_{d_{1}}} c_{q}^{\tau} \sum_{n=-\infty}^{\infty} J_{n-q}\left(k d_{21}\right) e^{-j(n-q) \phi_{21}} H_{n}^{(2)}\left(k \rho_{2}\right) e^{j n \phi_{2}} \\
=\sum_{n=-\infty}^{\infty}\left(\sum_{q=-N_{d_{1}}}^{N_{d_{1}}} c_{q}^{\tau} J_{n-q}\left(k d_{21}\right) e^{-j(n-q) \phi_{21}}\right) H_{n}^{(2)}\left(k \rho_{2}\right) e^{j n \phi_{2}} \quad \rho_{2}>d_{21}
\end{gathered}
$$

Con el objeto de poder comparar la expresión recogida en (E.37) para el campo $C_{z}^{d}$ con aquélla presentada en (E.35), e identificar de esta forma la relación entre los coeficientes $c_{n}^{\prime \tau}$ y $c_{q}^{\tau}$, resulta necesario truncar en (E.37) el sumatorio para el índice $n$ de igual manera que en (E.35); deduciendo de esta forma la relación que se ofrece seguidamente

$$
c_{n}^{\prime \tau}=\sum_{q=-N_{d_{1}}}^{N_{d_{1}}} c_{q}^{\tau} J_{n-q}\left(k d_{21}\right) e^{-j(n-q) \phi_{21}} \quad \forall n, \quad n \in\left[-N_{d_{2}}, N_{d_{2}}\right]
$$

Considerando dos vectores columna constituidos respectivamente por los coeficientes $c_{n}^{\prime \tau}$ y $c_{q}^{\tau}$; es posible expresar en términos matriciales, tal y como se indica a continuación, la relación entre dichos coeficientes recién presentada en (E.38)

$$
\left.\left[\begin{array}{c}
c_{-N_{d_{2}}}^{\prime \tau} \\
c_{-N_{d_{2}}+1}^{\prime \tau} \\
\vdots \\
c_{N_{d_{2}}}^{\prime \tau}
\end{array}\right]=\begin{array}{c}
{ }^{\prime} \\
\downarrow \\
\downarrow \\
\downarrow \\
N_{d_{2}}
\end{array}\right] \overbrace{\left[\begin{array}{l}
J_{n-q}\left(k d_{21}\right) e^{-j(n-q) \phi_{21}} \\
\end{array}\right]}^{-N_{d_{1}} \rightarrow q-\rightarrow N_{d_{1}}}\left[\begin{array}{c}
c_{-N_{d_{1}}}^{\tau} \\
c_{-N_{d_{1}}+1}^{\tau} \\
\vdots \\
c_{N_{d_{1}}}^{\tau}
\end{array}\right]
$$

donde la matriz que interviene constituye la matriz de traslación de espectro emergente a emergente, denominada en este caso $T H_{21}$. Esta designación se 


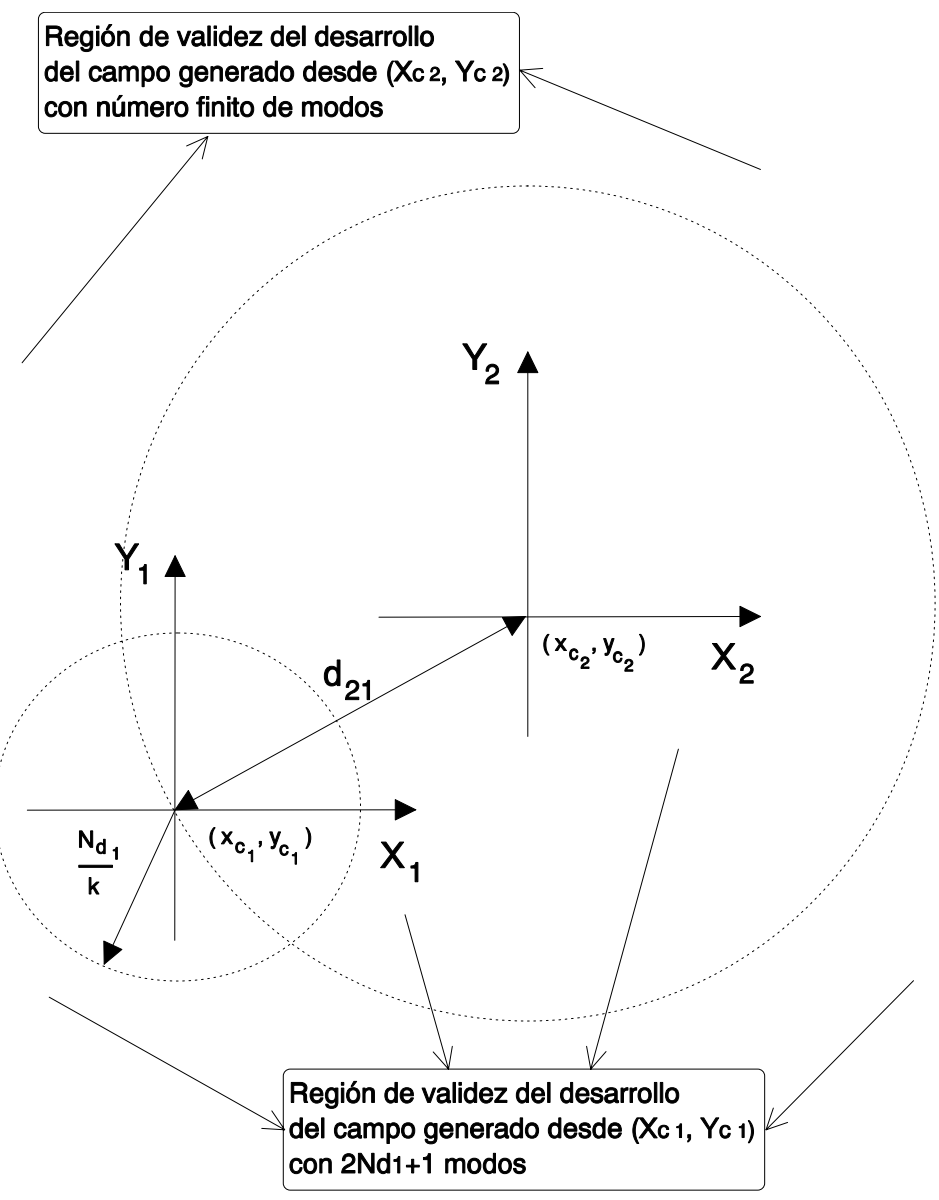

Figura E.18: Región de validez para la reconstrucción de un campo generado por una o varias fuentes de corriente, definido inicialmente en términos de su espectro cilíndrico emergente centrado en el punto $\left(x_{c_{1}}, y_{c_{1}}\right)$, a partir de una versión de dicho espectro trasladada al punto $\left(x_{c_{2}}, y_{c_{2}}\right)$.

debe a que dicha matriz permite transformar un espectro cilíndrico emergente, asociado a un campo generado por una o varias fuentes puntuales y referido al punto $\left(x_{c_{1}}, y_{c_{1}}\right)$, en un nuevo espectro cilíndrico emergente correspondiente al mismo campo; pero referido en esta ocasión al punto $\left(x_{c_{2}}, y_{c_{2}}\right)$. Es interesante reseñar, tras comparar la matriz definida según (E.39) con aquélla construida según (E.15), que los elementos de la nueva matriz de traslación de espectro emergente a emergente se construyen de igual manera que los de la matriz de traslación de espectro incidente a incidente.

La región donde es posible reconstruir un campo generado por una o varias fuentes puntuales de corriente, empleando una versión trasladada del espectro cilíndrico emergente asociado originalmente a dicho campo, depende única y exclusivamente de la restricción espacial asociada a la segunda versión del Teorema 
de Adición para las Funciones de Hankel; que se utiliza en la construcción de la matriz de traslación de espectro emergente a emergente presentada en este apartado. Considerando, por ejemplo, el anterior campo $C_{z}^{d}$; éste se reconstruye correctamente a partir de su espectro emergente original centrado en el punto $\left(x_{c_{1}}, y_{c_{1}}\right)$, como puede observarse en la figura E.18, fuera de la mínima circunferencia centrada en dicho punto que además encierra a las fuentes generadoras del campo $C_{z}^{d}$. Si el espectro original se traslada al punto $\left(x_{c_{2}}, y_{c_{2}}\right)$, empleando la transformación espectral definida según (E.37), la correcta reconstrucción del mismo campo $C_{z}^{d}$ mediante el nuevo espectro trasladado, debido a la restricción espacial presente en (E.37), sólo es posible fuera de la circunferencia centrada en $\left(x_{c_{2}}, y_{c_{2}}\right)$ y de radio la distancia entre los puntos $\left(x_{c_{1}}, y_{c_{1}}\right)$ y $\left(x_{c_{2}}, y_{c_{2}}\right)$; utilizando para ello, tal y como se recoge en (E.37), infinitas componentes en el nuevo espectro trasladado. Ahora bien, esta misma reconstrucción fuera de la citada circunferencia puede obtenerse igualmente, a partir del nuevo espectro trasladado, empleando un número finito de modos cilíndricos emergentes trasladados; que vendrá determinado por el radio de la anterior circunferencia, definido como la distancia $d_{21}$ que existe entre los puntos $\left(x_{c_{1}}, y_{c_{1}}\right)$ y $\left(x_{c_{2}}, y_{c_{2}}\right)$. Concretamente, empleando un número de modos cilíndricos trasladados $\left(2 N_{d_{2}}+1\right)$ tal que $N_{d_{2}} \geq k d_{21}$; el campo $C_{z}^{d}$ generado por una o varias fuentes puntuales de corriente podrá reconstruirse correctamente, a partir de una versión de su espectro emergente original trasladada a un nuevo punto, fuera de la mínima circunferencia centrada en dicho punto y que además contiene el punto al que se refiere el espectro original. 



\section{Apéndice $\mathbf{F}$}

\section{Publicaciones en Revistas Internacionales}

En el presente apéndice, se recogen las publicaciones de mayor importancia relacionadas con el trabajo realizado en la presente tesis; ofreciendo en concreto aquéllas que en este momento ya se encuentran publicadas (o aceptadas para su inmediata publicación) en revistas internacionales.

Así pues, en primer lugar se muestra un trabajo bajo título" Accelerated Computation of Admittance Parameters for Planar Waveguide Junctions"; aceptado para su publicación en fechas próximas (durante 1997) en el "Special Issue on Passive and Active Filters and Multiplexers" de la revista International Journal of Microwave and Millimeter-Wave Computer-Aided Engineering.

A continuación, se presenta un artículo titulado "A Generalized Method for Characterizing Two-Dimensional Scattering Problems with Spectral Techniques"; publicado recientemente en la revista Microwave and Optical Technology Letters, volumen 14, número 1 (Enero 1997), páginas 6-9.

Seguidamente, el apéndice recoge una nueva publicación con título "A Generalized Method for Two Dimension Scattering Problems under TE Incidence with the Use of Spectral Techniques"; aparecida también recientemente en la revista Microwave and Optical Technology Letters, volumen 13, número 6 (Diciembre 1996), páginas $354-358$.

Para finalizar el presente apéndice, se ofrece el artículo denominado "Generalised Iterative Method for Solving 2D Multiscattering Problems using Spectral Techniques"; publicado en fechas muy recientes en la revista IEE Proceedings on Microwaves, Antennas and Propagation, volumen 144, número 2 (Abril 1997), páginas $73-80$. 



\section{Bibliografía}

[1] R.F. Harrington, Time-Harmonic Electromagnetic Fields. McGraw-Hill Book Company, Inc., New York, 1961.

[2] A. Wexler, "Solution of Waveguide Discontinuities by Modal Analysis", IEEE Transactions on Microwave Theory and Techniques, vol. MTT-15, no. 9, pp. 508-517, Sept. 1967.

[3] P.H. Mastermann y P.J.B. Clarricoats, "Computer Field-Matching Solution of Waveguide Transverse Discontinuities", Proceedings of the IEE, vol. 118pp. 51-63, 1971.

[4] R. Mittra y S.W. Lee, Analytic Techniques in the Theory of Guided Waves. The Macmillan Company, New York, 1971.

[5] H. Patzelt y F. Arndt, "Double-Plane Steps in Rectangular Waveguides and their Applications for Transformers, Irises and Filters", IEEE Transactions on Microwave Theory and Techniques, vol. MTT-30, no. 5, pp. 771-776, Mayo 1982.

[6] S.W. Lee, W.R. Jones, y J.J. Campbell, "Convergence of Numerical Solutions of Iris-Type Discontinuity Problems", IEEE Transactions on Microwave Theory and Techniques, vol. MTT-19, no. 6, pp. 528-536, Junio 1971.

[7] R. Mittra, T. Itoh, y T.S. Li, "Analytical and Numerical Studies of the Relative Convergence Phenomenon Arising in the Solution of an Integral Equation by the Moment Method", IEEE Transactions on Microwave Theory and Techniques, vol. MTT-20, no. 2, pp. 96-104, Feb. 1972.

[8] A. Alvarez, G. Connor, y M. Guglielmi, "New Simple Procedure for the Computation of the Multimode Admittance or Impedance Matrix of Planar Waveguide Junctions", IEEE Transactions on Microwave Theory and Techniques, vol. MTT-44, no. 3, pp. 413-418, Marzo 1996.

[9] E.D. Sharp, "An Exact Calculation for a T-Junction of Rectangular Waveguides Having Arbitrary Cross Sections", IEEE Transactions on Microwave Theory and Techniques, vol. MTT-15, no. 2, pp. 109-116, Feb. 1967. 
[10] A.D. Yaghjian, "Near-Field Antenna Measurements on a Cylindrical Surface; a Source Scattering Matrix Formulation", NBS Technical Note, , no. 696, , Sept. 1977.

[11] R. Lewis, "Spherical-Wave Source-Scattering Matrix Analysis of Coupled Antennas; a General System Two-Port Solution", IEEE Transactions on Antennas and Propagation, vol. 35, no. 12, pp. 1375-1380, Dic. 1987.

[12] J.E. Hansen, Spherical Near-Field Antenna Measurements. Peter Peregrinus Ltd., London, 1988.

[13] J.C. Cruellas, Análisis de la Difracción de Objetos Dieléctricos mediante Elementos Finitos y Realimentación Modal, Tesis Doctoral, Universidad Politécnica de Cataluña, Barcelona, 1989.

[14] M. Baquero, Transformaciones Espectrales y Aplicaciones a Sintesis de Ondas, Medida de Antenas y Difracción, Tesis Doctoral, Universidad Politécnica de Valencia, Valencia, 1994.

[15] N. Marcuvitz, Waveguide Handbook. Peter Peregrinus Ltd., London, 1986.

[16] A. Alvarez y M. Guglielmi, "Multimode Network Representation of Two Dimensional Steps in Rectangular Waveguides", En Proceedings of the $24^{\text {th }}$ European Microwave Conference, vol. 2, pp. 1943-1948, Cannes, France, Sept. 1994.

[17] J.M. Rebollar, J. Esteban, y J.E. Page, "Fullwave Analysis of Three and Four-Port Rectangular Waveguide Junctions", IEEE Transactions on Microwave Theory and Techniques, vol. MTT-42, no. 2, pp. 256-263, Feb. 1994.

[18] V.E. Boria y M. Guglielmi, "Accelerated Computation of Admittance Parameters for Planar Waveguide Junctions", International Journal of Microwave and Millimeter-Wave Computer-Aided Engineering, 1997, Aceptado para publicación en fechas próximas en el "Special Issue on Passive and Active Filters and Multiplexers".

[19] Y. Leviatan, P.G. Li, A.T. Adams, y J. Perini, "Single-Post Inductive Obstacle in Rectangular Waveguide", IEEE Transactions on Microwave Theory and Techniques, vol. MTT-31, no. 10, pp. 806-811, Oct. 1983.

[20] M. Guglielmi y A.A. Oliner, "Multimode Network Description of a Planar Periodic Metal-Strip Grating at a Dielectric Interface-Part I: Rigorous Network Formulations", IEEE Transactions on Microwave Theory and Techniques, vol. MTT-37, no. 3, pp. 534-541, Marzo 1989. 
[21] G. Conciauro, M. Bressan, y C. Zuffada, "Waveguide Modes via an Integral Equation Leading to a Linear Matrix Eigenvalue Problem", IEEE Transactions on Microwave Theory and Techniques, vol. MTT-32, no. 11, pp. 1495-1504, Nov. 1984.

[22] P. Arcioni, "Fast Evaluation of Modal Coupling Coefficients of Waveguide Step Discontinuities", IEEE Microwave and Guided Wave Letters, vol. 6, no. 6, pp. 232-234, Junio 1996.

[23] W.H. Press, S.A. Teukolsky, W.T. Vetterling, y B.P. Flannery, Numerical Recipes in Fortran: The Art of Scientific Computing, Second Edition. Cambridge University Press, Cambridge, 1992.

[24] J.M. Rebollar y J.A. Encinar, "Field Theory Analysis of MultiportMultidiscontinuity Structures: Application to Short-Circuited E-Plane Septum", IEE Proceedings on Microwaves, Antennas and Propagation, vol. 135, no. 1, pp. 1-7, Feb. 1988.

[25] R.E. Collin, Foundations for Microwave Engineering. McGraw-Hill Book Company, Inc., Singapore, 1992.

[26] L. Tarricone, M. Dionigi, y R. Sorrentino, "A Strategy for the Efficient Mode Matching Analysis of Complex Waveguide Networks", En Proceedings of the $25^{\text {th }}$ European Microwave Conference, vol. 1, pp. 425-429, Bologna, Italy, Sept. 1995.

[27] M. Dionigi, A. Esposito, L. Tarricone, y R. Sorrentino, "Optimum Port Numbering in the Electromagnetic Simulation of Complex Networks", En Proceedings of the 1996 IEEE MTT-S International Microwave Symposium, vol. 1, pp. 189-192, San Francisco, U.S.A., Junio 1996.

[28] V.E. Boria, G. Gerini, y M. Guglielmi, "An Efficient Inversion Technique for Banded Linear Systems", En Proceedings of the 1997 IEEE MTT-S International Microwave Symposium, Denver, U.S.A., Junio 1997, Comunicación aceptada.

[29] F. Arndt, J. Bornemann, C. Piontek, y H. Schüller, "Shunt-InductanceCoupled Waveguide Filters with Expanded Second Stopband", IEE Electronic Letters, vol. 21pp. 238-239, 14 de Marzo 1985.

[30] J-F. Liang, H-C. Chang, y K.A. Zaki, "Design and Tolerance Analysis of Thick Iris Waveguide Bandpass Filters", IEEE Transactions on Magnetics, vol. 29, no. 2, pp. 1605-1608, Marzo 1993. 
[31] M. Guglielmi, F. Montauti, L. Pellegrini, y P. Arcioni, "Implementing Transmission Zeros in Inductive-Window Bandpass Filters", IEEE Transactions on Microwave Theory and Techniques, vol. MTT-43, no. 8, pp. 1911-1915, Agosto 1995.

[32] G. Matthaei, L. Young, y E.M.T. Jones, Microwave Filters, ImpedanceMatching Networks, and Coupling Structures. Artech House, Inc., U.S.A., 1964.

[33] M.H. Chen, "Short-Circuit Tuning Methods for Singly Terminated Filters", IEEE Transactions on Microwave Theory and Techniques, vol. MTT-25, no. 12, pp. 1032-1036, Dic. 1977.

[34] H-W. Yao, K.A. Zaki, A.E. Atia, y R. Hershtig, "Full Wave Modeling of Conducting Posts in Rectangular Waveguides and Its Applications to Slot Coupled Combline Filters", IEEE Transactions on Microwave Theory and Techniques, vol. MTT-43, no. 12, pp. 2824-2830, Dic. 1995.

[35] A.D. Yaghjian, "Electric Dyadic Green's Functions in the Source Region", Proceedings of the IEEE, vol. 28, no. 2, pp. 248-263, Feb. 1980.

[36] M. Bressan y G. Conciauro, "Rapidly Converging Expressions for Dyadic Green's Functions in Two-Dimensional Resonators of Circular and Rectangular Cross Section", Alta Frequenza (Special Issue on Applied Electromagnetics), vol. 52, no. 3, pp. 188-190, 1983.

[37] P. Arcioni, M. Bressan, y G. Conciauro, "A New Algorithm for the WideBand Analysis of Arbitrarily Shaped Planar Circuits", IEEE Transactions on Microwave Theory and Techniques, vol. MTT-36, no. 10, pp. 1426-1437, Oct. 1988.

[38] P. Arcioni, M. Bressan, y G. Conciauro, "Wide-Band Analysis of Planar Waveguide Circuits", Alta Frequenza (Special Issue on Focus on Computer Oriented Design Techniques for Microwave Circuits), vol. 57, no. 5, pp. 217226, Junio 1988.

[39] P.M. Morse y H. Feshbach, Methods of Theoretical Physics. McGraw-Hill Book Company, Inc., New York, 1953.

[40] M. Guglielmi, "Simple CAD Procedure for Microwave Filters and Multiplexers", IEEE Transactions on Microwave Theory and Techniques, vol. MTT42, no. 7, pp. 1347-1352, Julio 1994.

[41] A.E. Williams, "A Four-Cavity Elliptic Waveguide Filter", IEEE Transactions on Microwave Theory and Techniques, vol. MTT-18, no. 12, pp. 11091114, Dic. 1970. 
[42] A.E. Atia y A.E. Williams, "Narrow Bandpass Waveguide Filters", IEEE Transactions on Microwave Theory and Techniques, vol. MTT-20, no. 4, pp. 258-265, Abril 1972.

[43] H-C. Chang y K.A. Zaki, "Evanescent-Mode Coupling of Dual-Mode Rectangular Waveguide Filters", IEEE Transactions on Microwave Theory and Techniques, vol. MTT-39, no. 8, pp. 1307-1312, Agosto 1991.

[44] C. Kudsia, R. Cameron, y W-C. Tang, "Innovations in Microwave Filters and Multiplexing Networks for Communications Satellite Systems", IEEE Transactions on Microwave Theory and Techniques, vol. MTT-40, no. 6, pp. 1133-1149, Junio 1992.

[45] H.A. Bethe, "Theory of Diffraction of Small Holes", Physical Review, vol. 66pp. 163-182, Oct. 1944.

[46] S.B. Cohn, "Determination of Aperture Parameters by Electronic Tank Measurements", Proceedings IRE, vol. 39, no. 11, pp. 1416-1421, Nov. 1951.

[47] S.B. Cohn, "Microwave Coupling by Large Apertures", Proceedings IRE, vol. 40, no. 6, pp. 696-699, Junio 1952.

[48] S.B. Cohn, "The Electric Polarizability of Apertures of Arbitrary Shape", Proceedings IRE, vol. 40, no. 9, pp. 1069-1071, Sept. 1952.

[49] N.A. McDonald, "Electric and Magnetic Coupling Through Small Apertures in Shield Walls of Any Thickness", IEEE Transactions on Microwave Theory and Techniques, vol. MTT-20, no. 10, pp. 689-695, Oct. 1972.

[50] N.A. McDonald, "Polynomial Approximations for the Transverse Magnetic Polarizabilities of Some Small Apertures", IEEE Transactions on Microwave Theory and Techniques, vol. MTT-35, no. 1, pp. 20-23, Enero 1987.

[51] K.A. Zaki, C. Chen, y A.E. Atia, "Canonical and Longitudinal Dual-Mode Dielectric Resonator Filters Without Iris", IEEE Transactions on Microwave Theory and Techniques, vol. MTT-35, no. 12, pp. 1130-1135, Dic. 1987.

[52] R. Ihmels y F. Arndt, "Rigorous Modal S-Matrix Analysis of the Cross-Iris in Rectangular Waveguides", IEEE Microwave and Guided Wave Letters, vol. 2, no. 10, pp. 400-402, Oct. 1992.

[53] P. Couffignal, H. Baudrand, y B. Théron, "A New Rigorous Method for the Determination of Iris Dimensions in Dual-Mode Cavity Filters", IEEE Transactions on Microwave Theory and Techniques, vol. MTT-42, no. 7, pp. 1314-1320, Julio 1994. 
[54] M. Guglielmi, R.C. Molina, y A. Alvarez, "Dual-Mode Circular Waveguide Filters Without Tuning Screws", IEEE Microwave and Guided Wave Letters, vol. 2, no. 11, pp. 457-458, Nov. 1992.

[55] R. Beyer y F. Arndt, "Field-Theory Design of Circular Waveguide DualMode Filters by a Combined Mode-Matching Finite Element Method", En Proceedings of the $24^{\text {th }}$ European Microwave Conference, vol. 1, pp. 294-303, Cannes, France, Sept. 1994.

[56] R. Beyer y F. Arndt, "Efficient Modal Analysis of Waveguide Filters Including the Orthogonal Mode Coupling Elements by an MM/FE Method", IEEE Microwave and Guided Wave Letters, vol. 5, no. 1, pp. 9-11, Enero 1995.

[57] X-P. Liang, K.A. Zaki, y A.E. Atia, "Dual Mode Coupling by Square Corner Cut in Resonators and Filters", IEEE Transactions on Microwave Theory and Techniques, vol. MTT-40, no. 12, pp. 2294-2302, Dic. 1992.

[58] R. Ihmels y F. Arndt, "Field Theory CAD of L-Shaped Iris Coupled Mode Launchers and Dual-Mode Filters", En Proceedings of the 1993 IEEE MTT-S International Microwave Symposium, pp. 765-768, San Diego, U.S.A., Junio 1993.

[59] J-F. Liang, X-P. Liang, K.A. Zaki, y A.E. Atia, "Dual-Mode Dielectric or Air-Filled Rectangular Waveguide Filters", IEEE Transactions on Microwave Theory and Techniques, vol. MTT-42, no. 7, pp. 1330-1336, Julio 1994.

[60] S. Moretti, F. Alessandri, y R. Sorrentino, "Field Theory Design of a Novel Circular Waveguide Dual-Mode Filter", En Proceedings of the $25^{\text {th }}$ European Microwave Conference, vol. 2, pp. 779-783, Bologna, Italy, Sept. 1995.

[61] R. Orta, P. Savi, R. Tascone, y D. Trinchero, "Rectangular Waveguide Dual-Mode Filters Without Discontinuities Inside the Resonators", IEEE Microwave and Guided Wave Letters, vol. 5, no. 9, pp. 302-304, Sept. 1995.

[62] R. Orta, P. Savi, R. Tascone, y D. Trinchero, "A New Configuration of DualMode Rectangular Waveguide Filters", En Proceedings of the $25^{\text {th }}$ European Microwave Conference, vol. 1, pp. 538-542, Bologna, Italy, Sept. 1995.

[63] B. Gimeno y M. Guglielmi, "Full Wave Network Representation for Rectangular, Circular, and Elliptical to Elliptical Waveguide Junctions", IEEE Transactions on Microwave Theory and Techniques, vol. MTT-45, no. 3, pp. 376-384, Marzo 1997. 
[64] V.E. Boria, M. Guglielmi, y P. Arcioni, "Accurate CAD for Dual Mode Filters in Circular Waveguide Including Tuning Elements", En Proceedings of the 1997 IEEE MTT-S International Microwave Symposium, Denver, U.S.A., Junio 1997, Comunicación aceptada.

[65] A.B. Carlson, Communication Systems. An Introduction to Signals and Noise in Electrical Communication. McGraw-Hill Book Company, Inc., New York, 1986.

[66] H. Esteban, J.M. López, V.E. Boria, M. Baquero, y M. Ferrando, ” A Generalized Method for Characterizing Two-Dimensional Scattering Problems with Spectral Techniques", Microwave and Optical Technology Letters, vol. 14, no. 1, pp. 6-9, Enero 1997.

[67] J.M. López, V.E. Boria, M. Baquero, y M. Ferrando, " A Generalized Method for Two Dimension Scattering Problems under TE Incidence with the Use of Spectral Techniques", Microwave and Optical Technology Letters, vol. 13, no. 6, pp. 354-358, Dic. 1996.

[68] H. Esteban, V.E. Boria, M. Baquero, y M. Ferrando, "Generalised Iterative Method for Solving 2D Multiscattering Problems using Spectral Techniques", IEE Proceedings on Microwaves, Antennas and Propagation, vol. 144, no. 2, pp. 73-80, Abril 1997.

[69] C.A. Balanis, Advanced Engineering Electromagnetics. John Wiley \& Sons, Inc., New York, 1989.

[70] R.F. Harrington, Field Computation by Moment Methods. The Macmillan Company, New York, 1968.

[71] M. Abramowitz y I.A. Stegun, Handbook of Mathematical Formulas with Formulas, Graphs and Mathematical Tables. Dover Publications, Inc., New York, 1970.

[72] A.V. Oppenheim y R.W. Schafer, Discrete-Time Signal Processing. PrenticeHall International, Inc., Englewood Cliffs, N.J., 1989.

[73] V. Twersky, "Multiple Scattering of Radiation by Arbitrary Planar Configuration of Parallel Cylinders and Parallel Cylinders", Journal of Applied Physics, vol. 23, no. 407, , 1952.

[74] J. Bruning, Multiple Scattering by Spheres, Tesis Doctoral, University of Illinois, 1969. 
[75] V.E. Boria, M. Baquero, y M. Ferrando, "Analysis of Multireflector Antenna Clusters by Spectral Methods", En Proceedings of the $24^{\text {th }}$ European Microwave Conference, vol. 1, pp. 870-875, Cannes, France, Sept. 1994.

[76] B. Peterson y S. Ström, "T-Matrix for Electromagnetic Scattering from an Arbitrary Number of Scatterers and Representations of E(3)", Physical Review D, vol. 8, no. 10, pp. 3661-3678, Nov. 1973.

[77] Y.M. Wang y W.C. Chew, "An Efficient Algorithm for Solution of a Scattering Problem", Microwave and Optical Technology Letters, vol. 3, no. 3, pp. 102-106, Marzo 1990.

[78] W.C. Chew y Y.M. Wang, "A Fast Algorithm for Solution of a Scattering Problem Using a Recursive Aggregate $\bar{\tau}$ Matrix Method", Microwave and Optical Technology Letters, vol. 3, no. 5, pp. 164-169, Mayo 1990.

[79] Y.M. Wang y W.C. Chew, "Application of the Fast Recursive Algorithm to a Large Inhomogeneous Scatterer for TM Polarization", Microwave and Optical Technology Letters, vol. 4, no. 4, pp. 155-157, Marzo 1991.

[80] W.C Chew, L. Gürel, Y.M. Wang, G. Otto, R.L. Wagner, y Q.H. Liu, " A Generalized Recursive Algorithm for Wave-Scattering Solutions in Two Dimensions", IEEE Transactions on Microwave Theory and Techniques, vol. 40, no. 4, pp. 716-723, Abril 1992.

[81] A.Z. Elsherbeni, M. Hamid, y G. Tian, "Iterative Scattering of a Gaussian Beam by an Array of Circular Conducting and Dielectric Cylinders", Journal of Electromagnetic Waves and Applications, vol. 7, no. 10, pp. 1323-1342, 1993.

[82] C.A. Balanis, Antenna Theory. Analysis and Design. John Wiley \& Sons, Inc., New York, 1982.

[83] T.E. Rozzi y W.F.G. Mecklenbraüker, "Wide-Band Network Modelling of Interacting Inductive Irises and Steps", IEEE Transactions on Microwave Theory and Techniques, vol. MTT-23, no. 2, pp. 235-245, Feb. 1975.

[84] M.S. Navarro, T.E. Rozzi, y Y.T. Lo, "Propagation in a Rectangular Waveguide Periodically Loaded With Resonant Irises", IEEE Transactions on Microwave Theory and Techniques, vol. MTT-28, no. 8, pp. 857-865, Agosto 1980.

[85] F. Alessandri, G. Bartolucci, y R. Sorrentino, "Admittance Matrix Formulation of Waveguide Discontinuity Problems: Computer-Aided Design of Branch Guide Directional Couplers", IEEE Transactions on Microwave Theory and Techniques, vol. MTT-36, no. 2, pp. 394-403, Feb. 1988. 
[86] G.F. Craven y C.K. Mok, "The Design of Evanescent Mode Waveguide Bandpass Filters for a Prescribed Insertion Loss Characteristic", IEEE Transactions on Microwave Theory and Techniques, vol. MTT-19, no. 3, pp. 295-308, Marzo 1971.

[87] D. Willems, "Evanescent-Mode Waveguide Filters Built in a Day", Microwaves \& RF, vol. MTT-28, no. 8, pp. 117-124, Julio 1987. 